DOI suffix: 10.36962/NEC61022021

INTERNATIONAL SCIENTIFIC-

PRACTICAL QUARTERLY JOURNAL, PRECISE AND REVIEWED

Economy is Everything!

ISSN 2667-9752 [DNLLNE] ISSN 1512-4649 [PRINT]

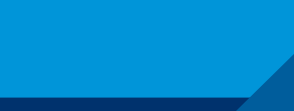

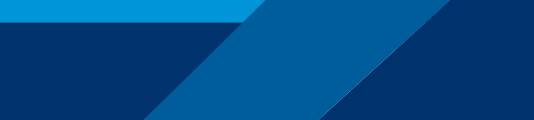
N2, 2021, VOL 16, ISSUE 2

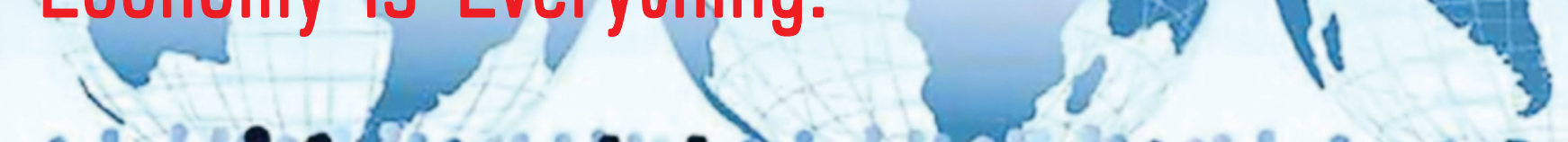
sili,
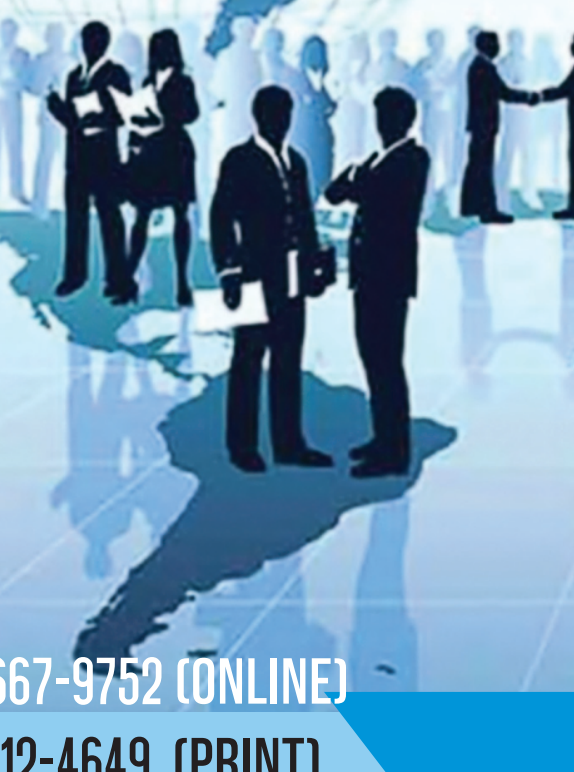

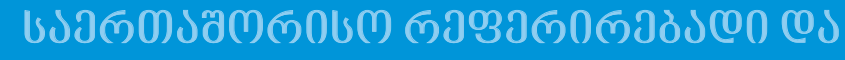

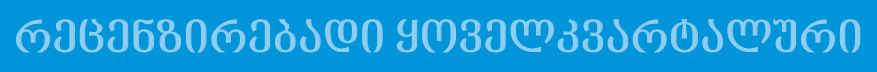

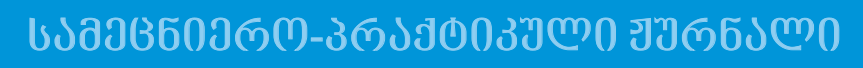


The Magazine "The New Economist" HAS BEEN REVIEWED:

In "Georgian Reviewed Magazine" P.L.L.E. Institute of Techinform. Identifiable in the Google Scholar and Research Gate bases.

\section{ACKNOWLEDGED TO BE REVIEWED BY:}

- Georgian Academy of Business Sciences;

- Georgian Academy of Economical Sciences;

- I. Javakhishvili Tbilisi State University;

- Ilia State University;

- Georgian Technical University;

- Grigol Robakidze University;

- Tbilisi Free University, (Business School ESM);

- Caucasus University;

- Sukhumi State University;

- Shota Rustaveli State University;

- Samtskhe-Javakheti State University;

- Shota Meskhia State University of Zugdidi;

- Akaki Tsereteli State University;

- Saint Andrew the First Called Georgian University of the Patriarchate of Georgia;

- East European University;

- Odessa Mechnikov National University (Odessa, Ukraine);

- Institute of Strategic Studies of the Caucassus

(Baku, Azerbaijan);

- State University of Belarus (Minsk);

- University of Kazakhstan of Economy,

Finances and International Trade (Nursultan).

ADDRESS: 5 A. Jorjadze str., 0105, Tbilisi, Georgia.

Tel.: +995 (32) 2990 576;

Mob.: +995 55277 554; +995 (58) 944800.

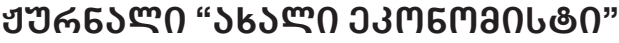

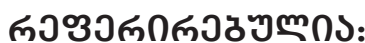

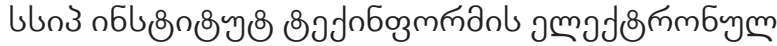

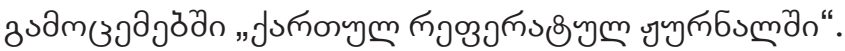

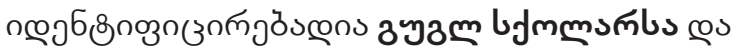

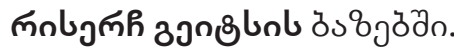

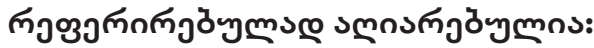

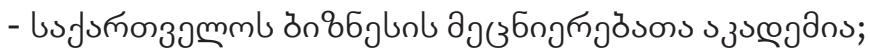

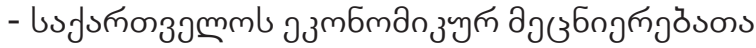

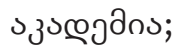

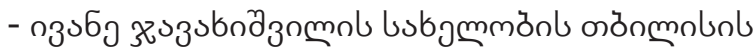

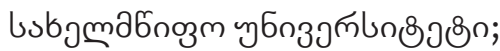

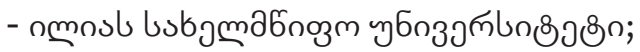

- Lujuños

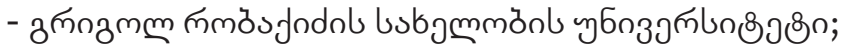

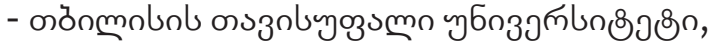

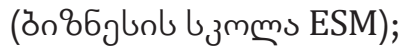

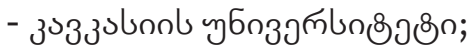

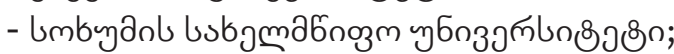

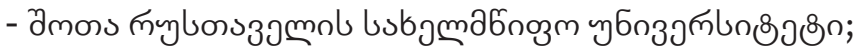

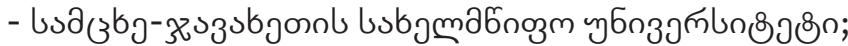

- amons ajubnsl byaœn@ol lusbjma6nozm

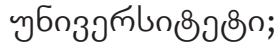

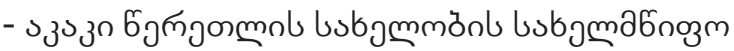

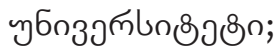

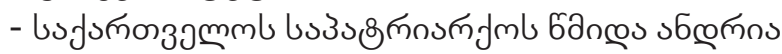

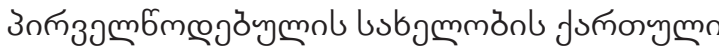
yбกзวศ̆

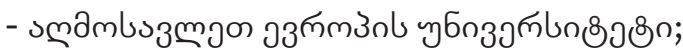

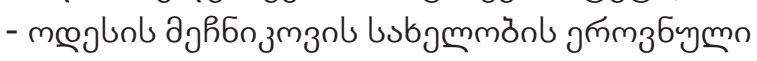

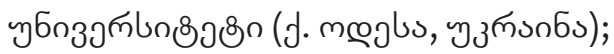

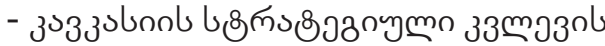

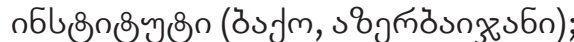

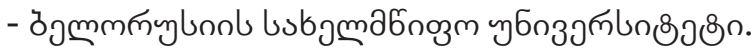
(anбlз3n);

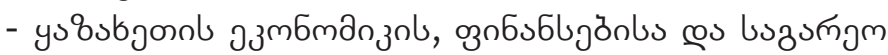

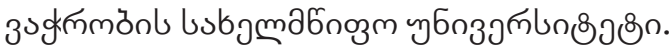

(6y nெuymsu6n).

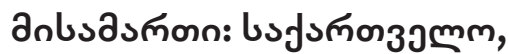

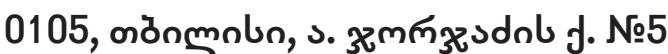

$$
\begin{aligned}
& \text { 8ิэm.: +995 (32) } 2990 \text { 576; } \\
& \text { amỏ.: +995 (55) } 277 \text { 554; +995 (58) } 944800 .
\end{aligned}
$$

\section{E-mail: editor@neweconomist.com.ge; loidk@yahoo.com}

web-site: www.neweconomist.com.ge; www.loi.com. ge 


\section{The new \\ ECONOMIST \\ INTERNATIONAL SCIENTIFIC-PRACTICAL QUARTERLY JOURNAL,}

PRECISE AND REVIEWED N2(61), 2021, Vol. 16, Issue 2.

LOID KARCHAVA - Editor-in-Chief and Head of Editorial Board;

Doctor of Business Administration,

Professor of Georgian Technical University;

Head of the Council of Scientists of Georgia;

Academician of the Georgian Academy of Business Sciences;

Academician of the Georgian Academy of Fazisi Sciences.

\section{EDITORIAL BOARD}

Doctors of science, Professors:

GIORGI ABASHISHVILI; RAMAZ ABESADZE; NINO ABESADZE; ANZOR ABRALAVA; IURI ANANIASHVILI; TEIMURAZ BABUNASHVILI (Prezident of the Georgian Academy of Business Sciences; EVGENI BARATASHVILI; ELBERD BATIASHVILI; GIVI BEDIANASHVILI; ROSTOM BERIDZE; SIMON GELASHVILI; RAMAZ GERLIANI; REVAZ GVELESIANI; VLADIMIR GRIGOLAIA (Moscow, Russian Federation); ALEKSEY DANILCHENKO (Belarusia State University); WOLFGANG WENG (Berlin, Garmany); SHOTA VESHAPIDZE; NUGZAR TODUA; GOCHA TUTBERIDZE (Rector of Kutaisi University); NATIA TURNAVA (Minister of Economy and Sustainable Development of Georgia); SERGEY YAKUBOVSKIY (Odesa I.I.Mechnikov Odessa National University, Ukraine); NAMIG YSAZADE; MERAB KAKULIA; REVAZ KVATASHIDZE; ALEKSANDRE KVASHILAVA; GIA KVASHILAVA; MURTAZ KVIRKVAIA (Editor of the Scientific part, Dean of Business and Management Scool of G. Robakidze University); VASIL KIKUTADZE; IRAKLI KOVZANADZE (Head of Budget and Finance Committee of Parliament of Georgia); KAKHA KORZAIA (Rector of Caucasus International University); PAATA KOGUASHVILI (member of Georgian Academy of Agriculture); HARALD KUNZ (Brandenburg, Garmany); ENVER LAGVILAVA; The Rt Hon STEVEN LORD LLOYDBAGRATIONI (University of Oxford, England); REVAZ LORDKIPANIDZE; SHALVA MACHAVARIANI (Vice-Prasidenrt of Caucasus University in sciens field); LASHA MGELADZE; ELGUJA MEQVABISHVILI; DAVID NARMANIA (Georgian
National Energy and Water Supply Regulatory Commission (GNERC), Chairman); SOLOMON PAVLIASHVILI (Deputy Minister of Environmental Protection and Agriculture of Georgia); VAKHTANG SARTANIA; ETER SARJVELADZE; AVTANDIL SILAGADZE (Member of Georgian National Academy of Science); ALEKSANDRE SICHINAVA; GELA SVIRAVA; LASHA TABATADZE (Risponsible Secretary); DAMETKEN TUREKULOVA (Academician of a Esthetical Science Academy of Kazakhstan); SLAVA FETELAVA; LEVAN KISTAURI (Dean of the School of Business, Computing and Social Sciences of Saint Andrew the First Called Georgian University of the Patriarchate of Georgia); RUSUDAN KUTATELADZE (Dean of Business-engineering Faculty of Georgian Tecnical University); TEMUR SHENGELIA; GIORGI SHIKHASHVILI; FAIA SHULENBAYEVA (University of Kazakhstan of Economy, Finances and International Trade); LALI CHAGELISHVILI; DAVID CHERKEZISHVILI (Rector of East European University); MICHAEL CHIKVILADZE; NIKO CHIKHLADZE; AVTANDIL CHUTLASHVILI; ELENE KHARABADZE; ETER KHARAISHVILI; REVAZ KHAREBAVA; VASIL KHIZANISHVILI; DAVID JALAGONIA (Dean of Economics and Buziness Faculty of Sukhumi State University); IVANE JAGODNISHVILI (Professro of Georgian Technical University); JAMLET JANJGAVA (Deputy of Editor-in-Chief); DIMITRI JAPARIDZE (Ilia State University, Dean of the Faculty Business, Technology and Education); MICHAEL JIBUTI (Prezident of the Georgian Academy of Economics Sciences); NANA JGHARKAVA. 


\section{$\operatorname{sov} \sqrt{2}$ \\ วзПбПวกเชก}

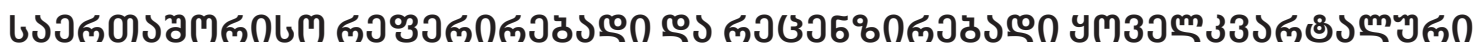

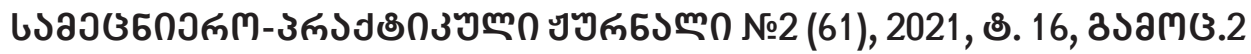

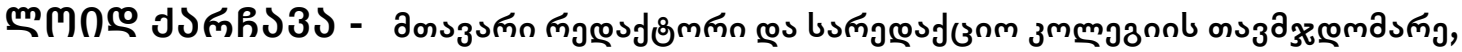

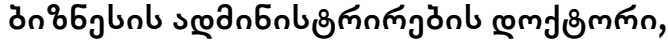

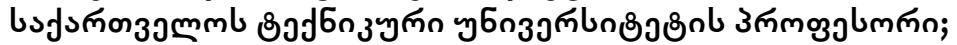

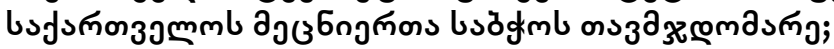

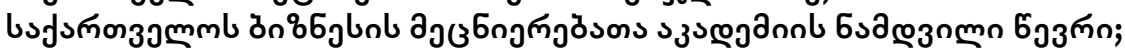

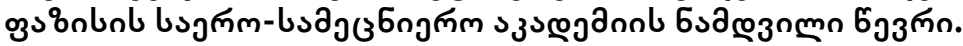

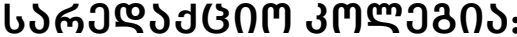

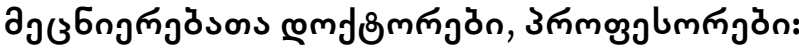

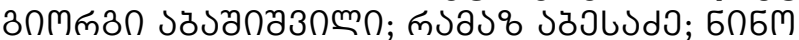

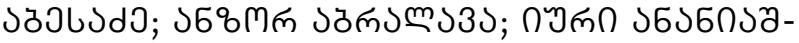

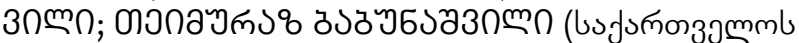

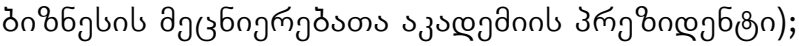

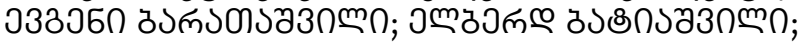

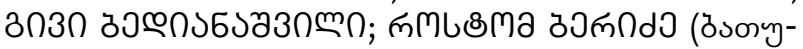

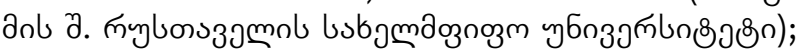

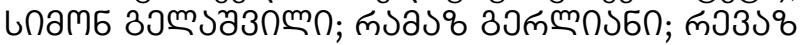

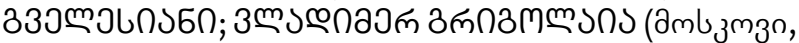

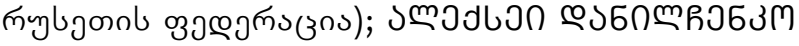

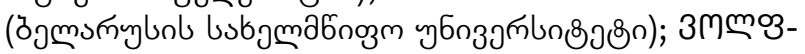

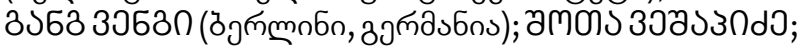

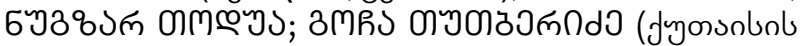

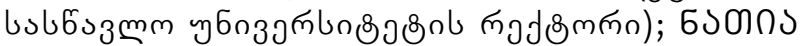

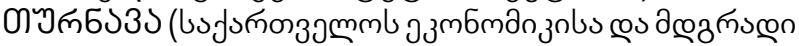

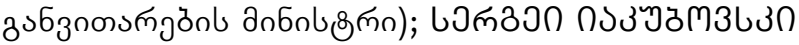

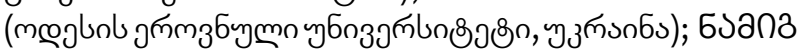

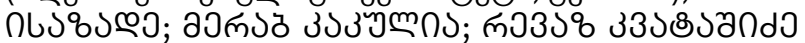

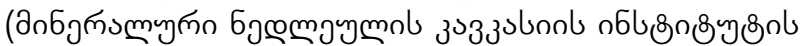

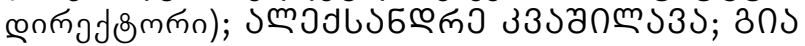

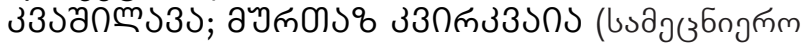

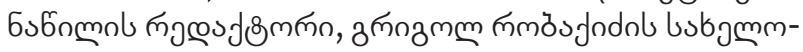

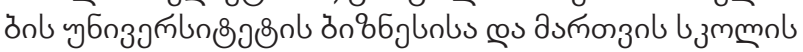

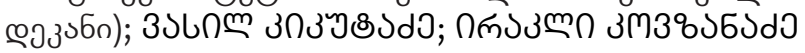

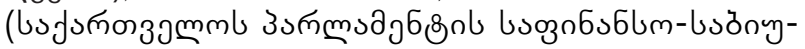

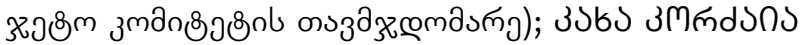

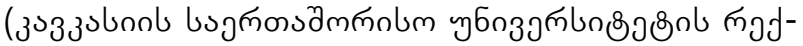

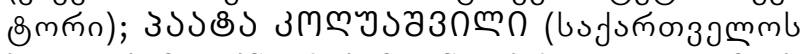
bmozmol a jønб

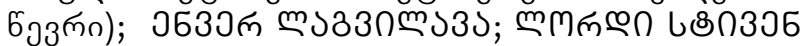

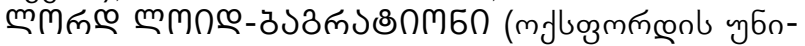

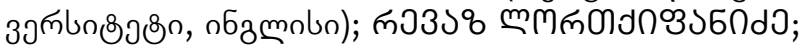

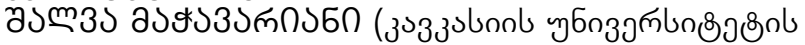

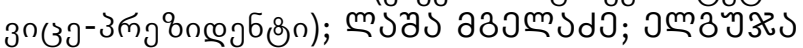

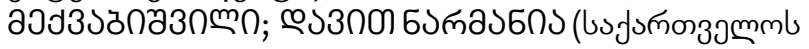

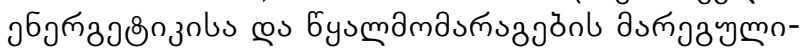

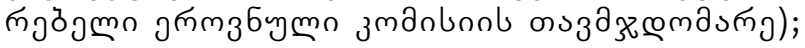

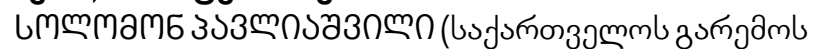

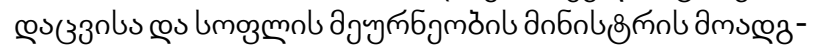
nmง); Зง68

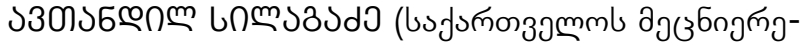

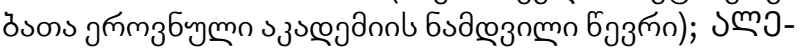

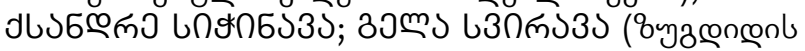

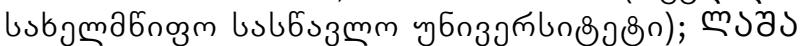

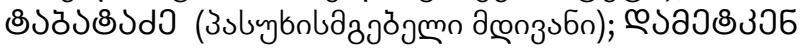

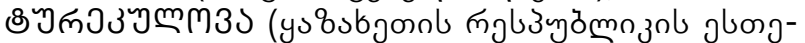

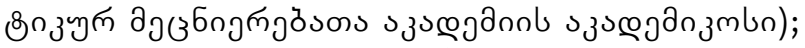
し

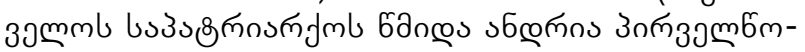

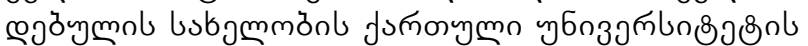

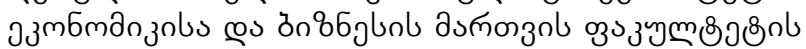

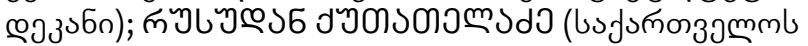

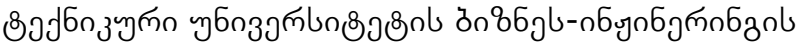

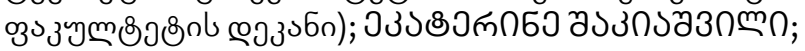

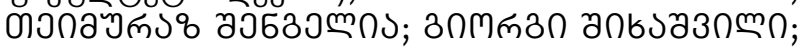

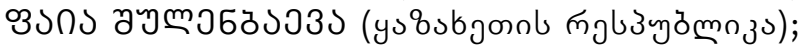

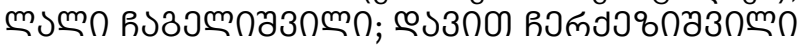

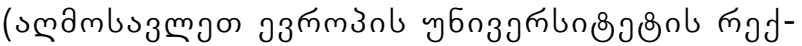

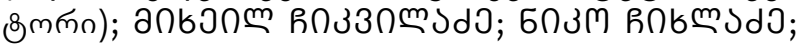

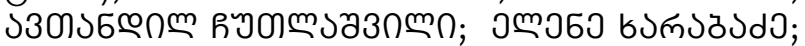

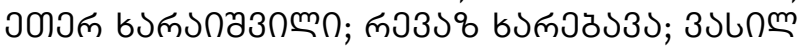

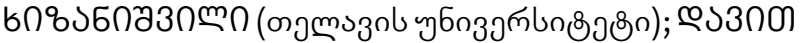

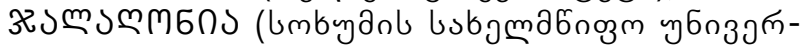
un\&)

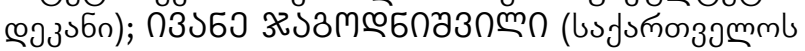

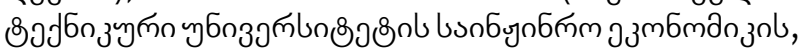

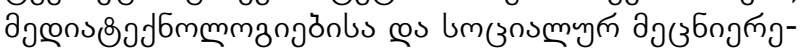

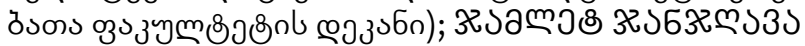

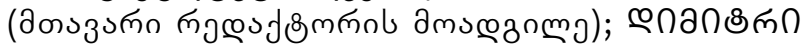

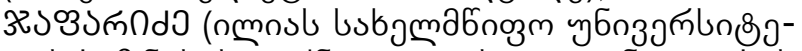

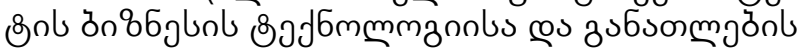

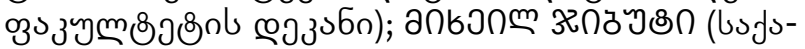

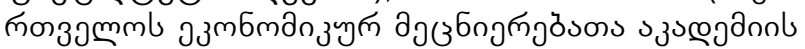

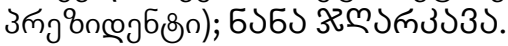

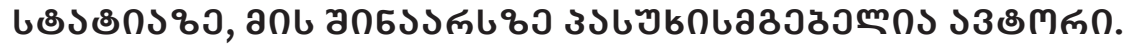

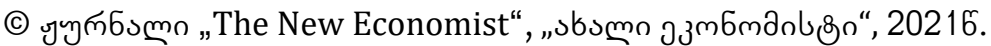

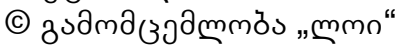

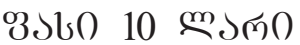




\section{INTERWIEV}

Interview with the Director of the Rural Development Agency under the Ministry of Environment Protection and Agricultute of Georgia Mr. Ilia Tamarashvili

\section{SCIENCE}

\section{GIORGI ABASHISHVILI}

Benefits and challenges of applying e-learning in the Georgian higher education system

\section{SHOTA VESHAPIDZE, GIA ZOIDZE}

Institutional Evolution of Higher Education in Georgia

\section{KRISTINA JGANJGAVA}

The world pandemic- experiment developing deglobalisation

GIORGI ABASHISHVILI, SALOME LIPARTELIANI

Georgia's Transit Potential - Country's Competitive Advantage-

\section{NATIA TERTERASHVILI}

Early effects of the COVID-19 pandemic on the real estate market in Georgia

\section{MARIAM GALDAVA}

Influence of Basic Actors in Globalization in Developing

and Post-Soviet Countries on the Example of Georgia

\section{GVANTSA CHKHEIDZE, IRAKLI MEKVEVRISHVILI}

The Geopolitical Dimension of the Conflicts in Abkhazia and the Tskhinvali Region

\section{TSIALA MELADZE}

Polling stations - the main cornerstone of elections

NEW BOOCKS

Basic paradigms of institutional economics

Review of Irakli Jangavadze's textbook: "Fundamentals of Innovative Economy"

\section{CONGLATURATION}




\section{ก68ి0ต30ๆ}

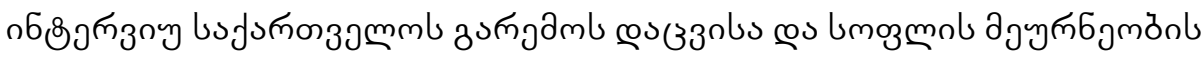

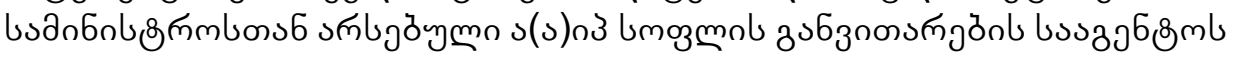

œоп̆

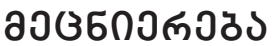

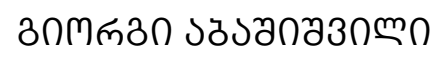

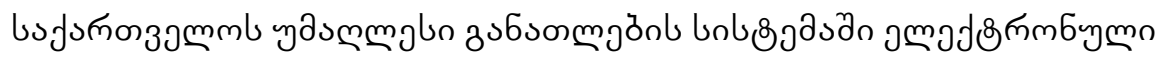

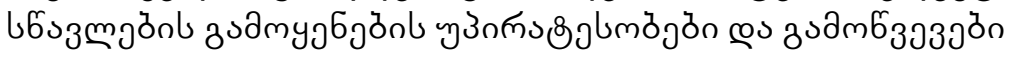

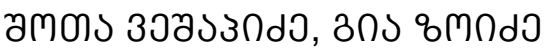

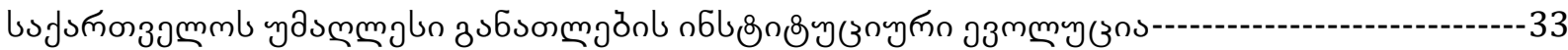

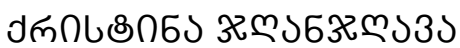

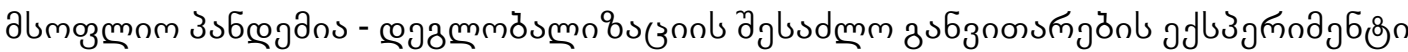

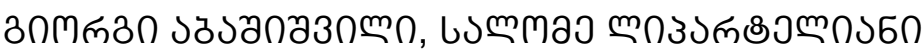

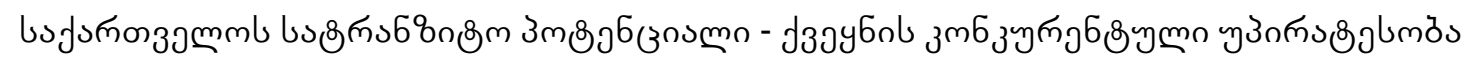

б১Mกง ৪ว

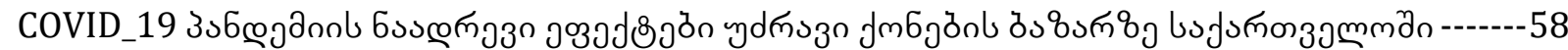

a১mก১a З১mূs

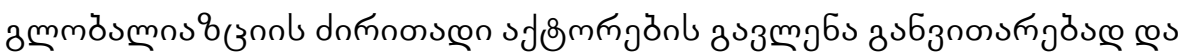

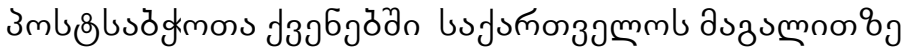

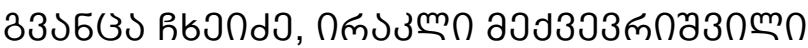

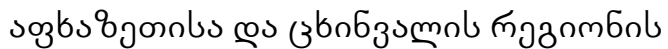

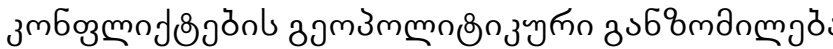

(3ก)m วอm

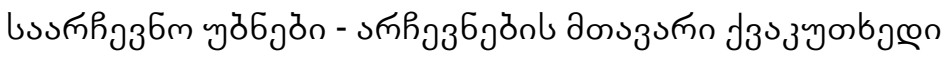
$-76$

\section{s৮smn 6036030}

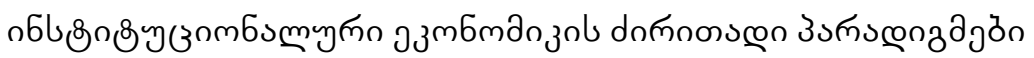

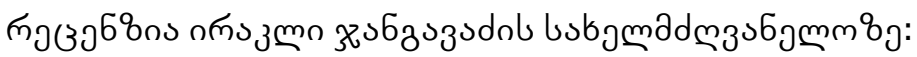

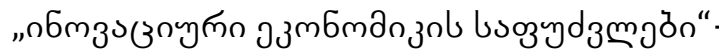

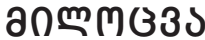

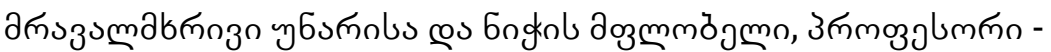

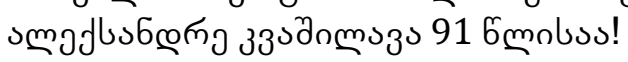




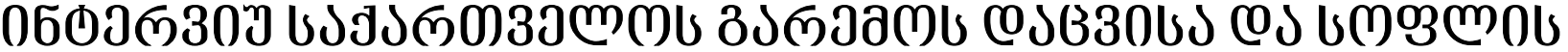

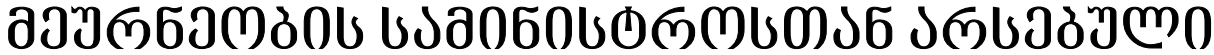

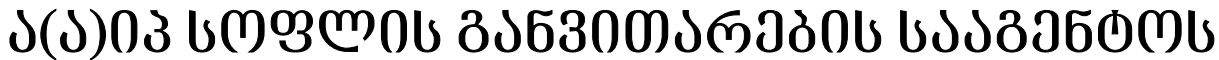

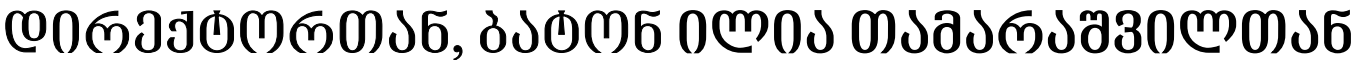

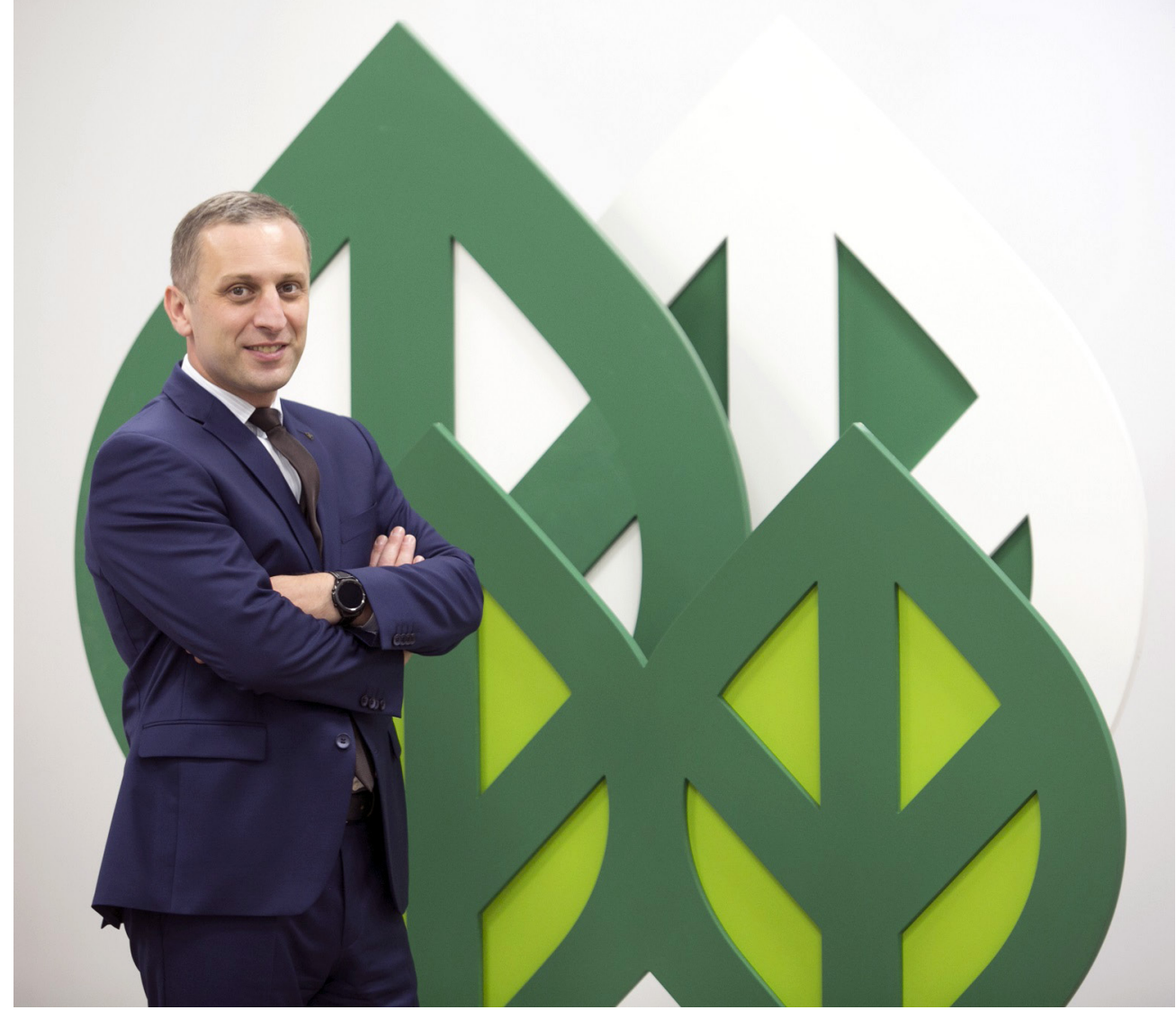

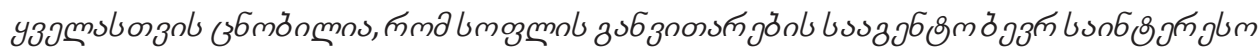

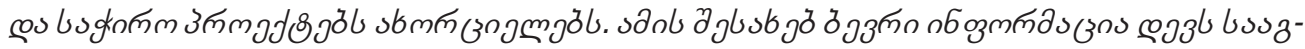

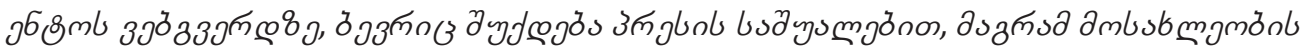

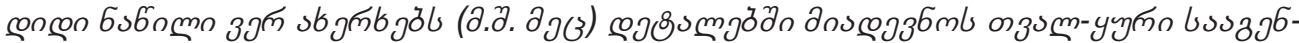

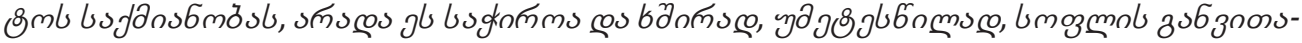

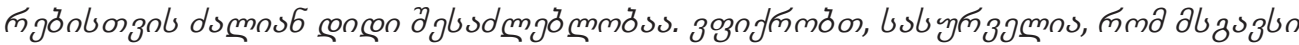

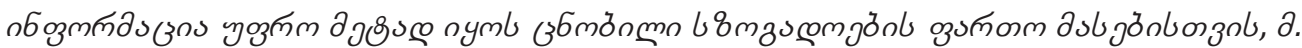

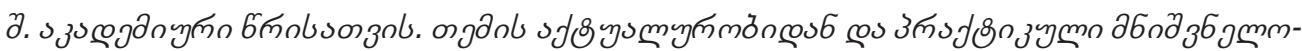

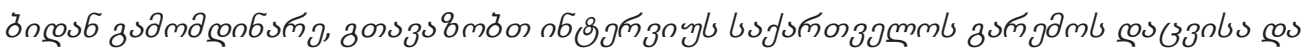

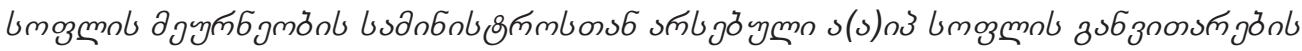

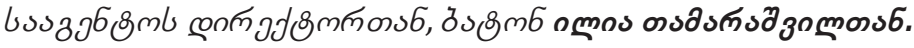

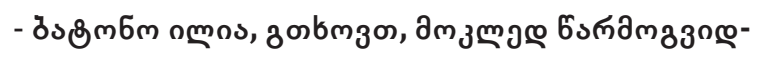

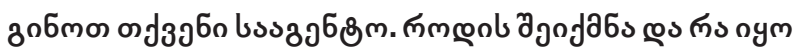

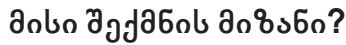

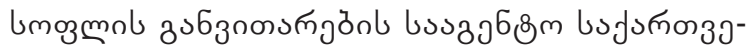

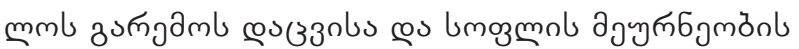

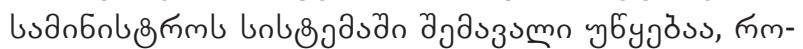

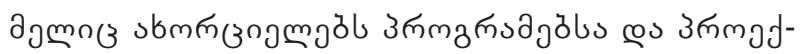

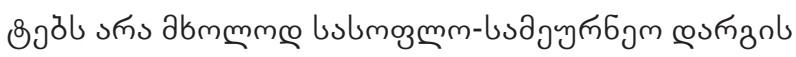

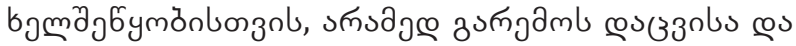

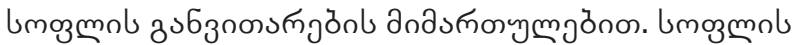

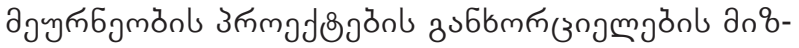

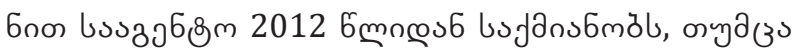

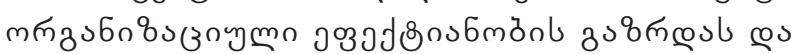

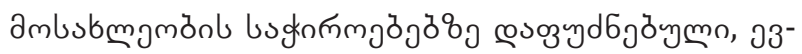




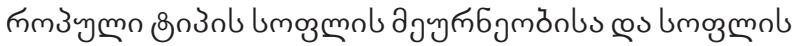

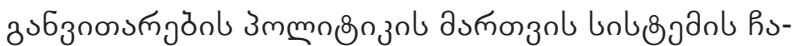

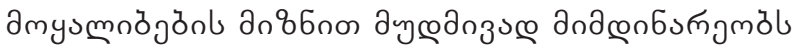

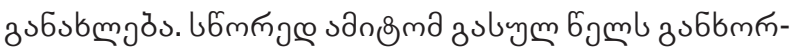

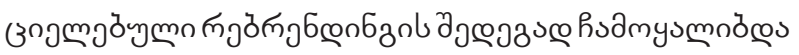

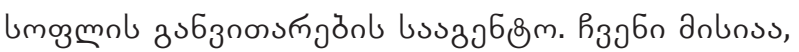

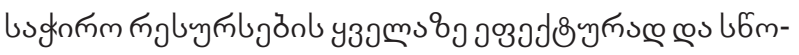

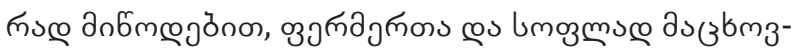

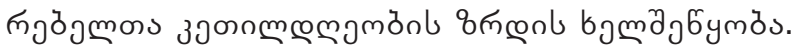

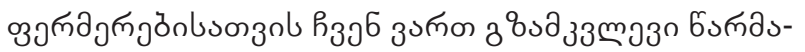

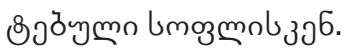

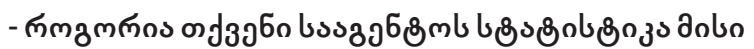
১ங

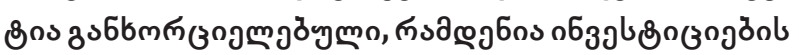

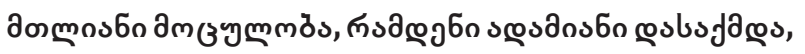

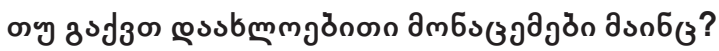

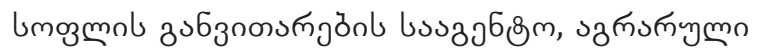

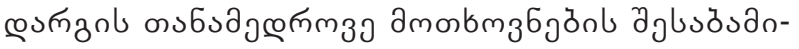

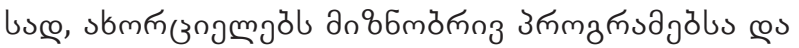

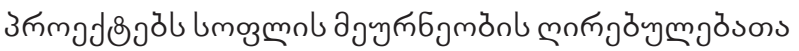

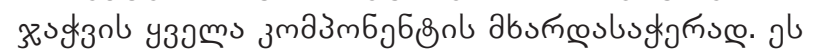

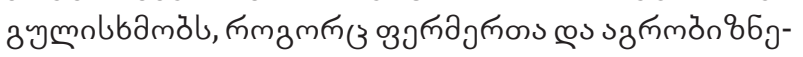

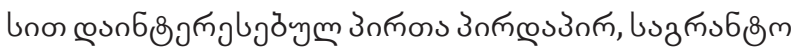

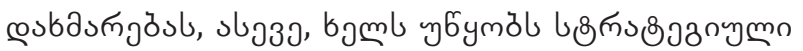

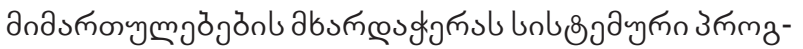

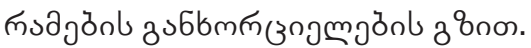

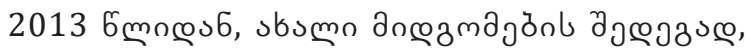

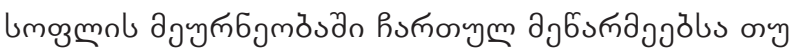

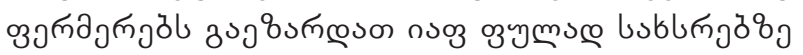

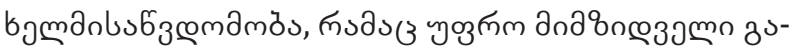

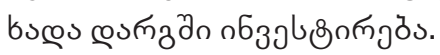

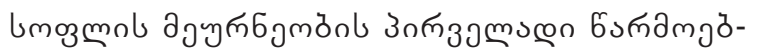

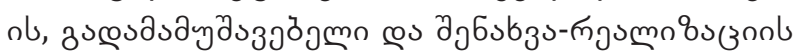

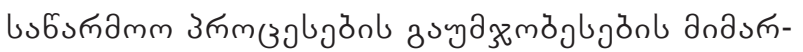

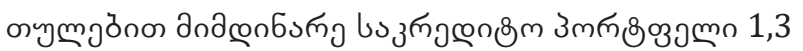

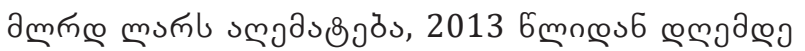

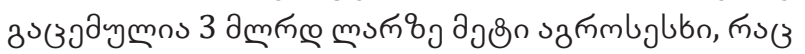

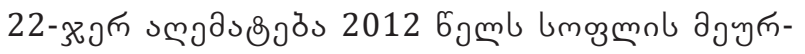

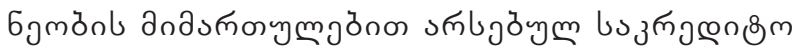

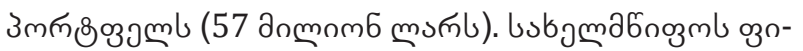

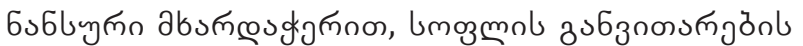

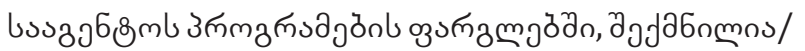

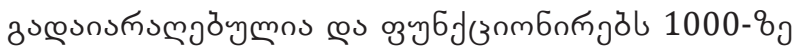

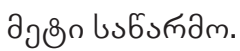

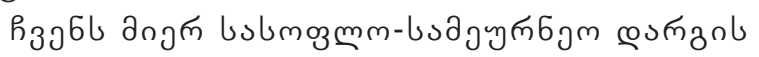

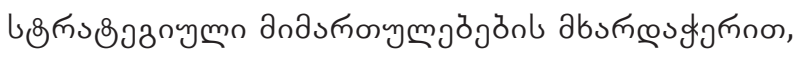

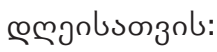

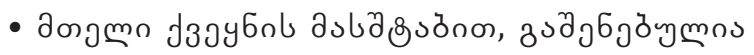

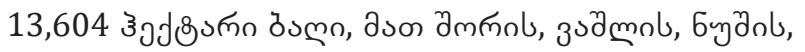

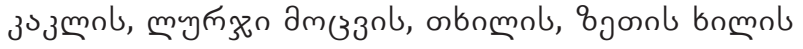
œ๐ ১.ð.

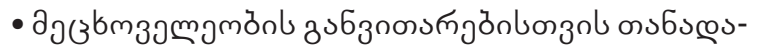

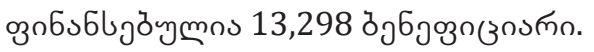

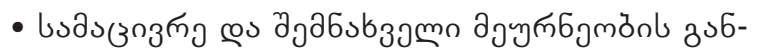

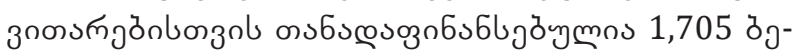

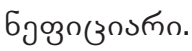

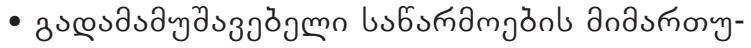

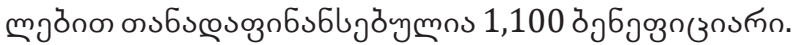

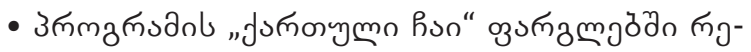

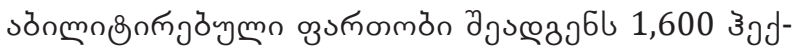

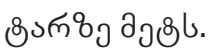

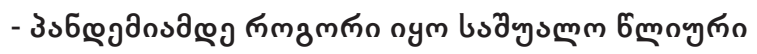

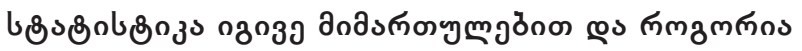

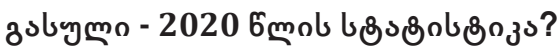

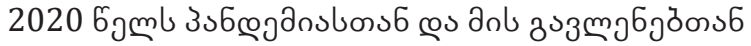

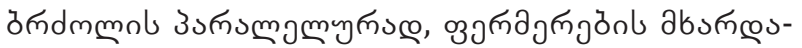

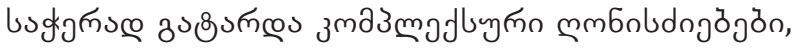

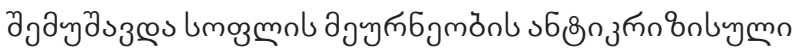

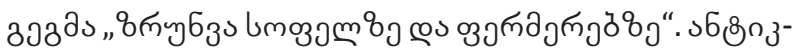

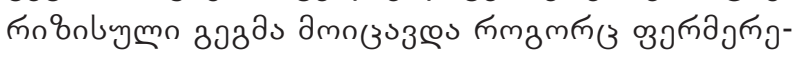

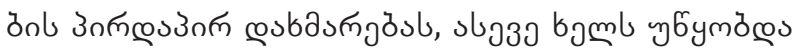

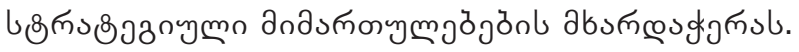

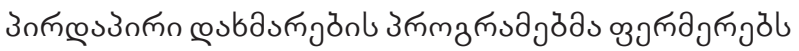

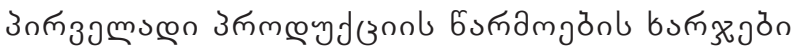
วбпдзб

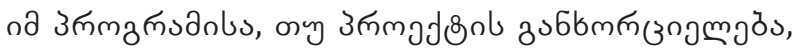

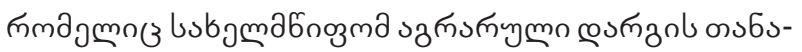

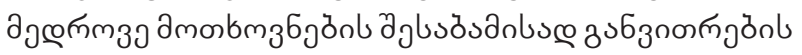

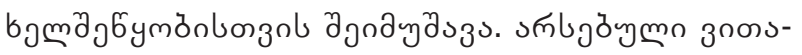

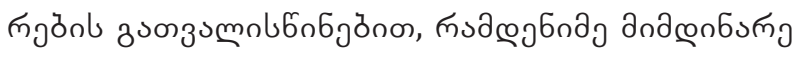

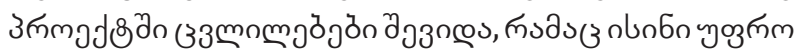
amj6nmo zงbsœs.

umoुmol ajynб

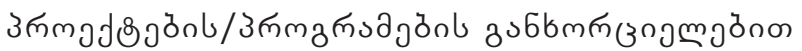
ววœววงณ:

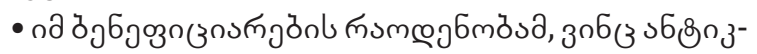

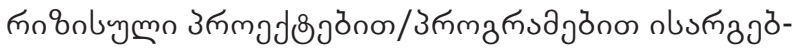

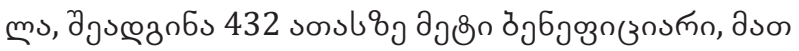

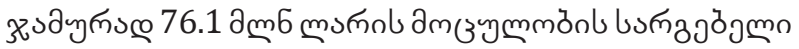
annglo;

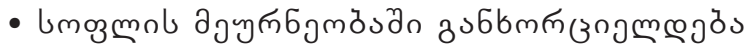

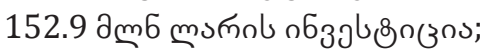

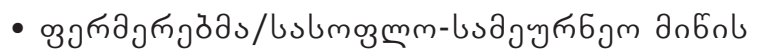

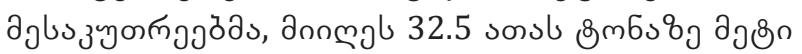

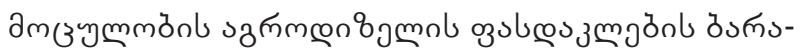
ตรठัก;

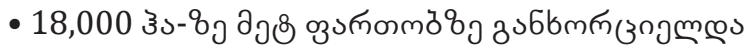

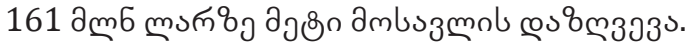




\section{ISSN 2667-9752(Online)}

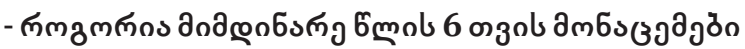
œ১ Зпмяб

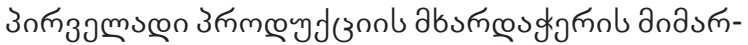

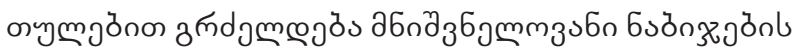

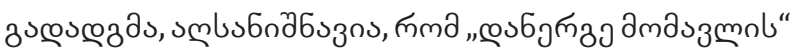

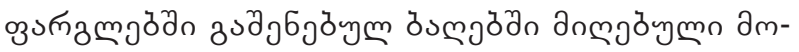

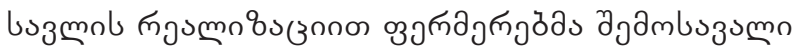

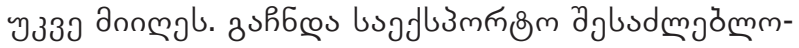

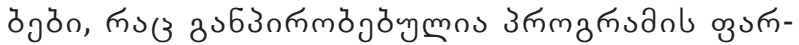

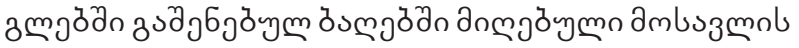

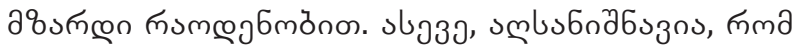

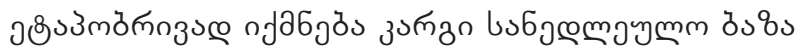

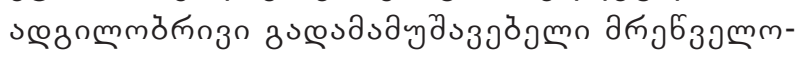

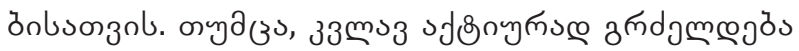

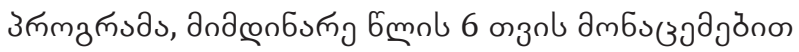

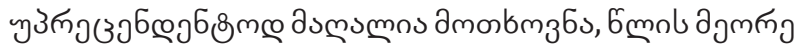

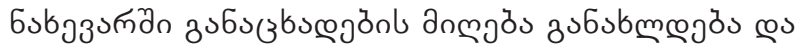

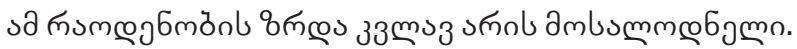

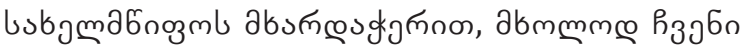

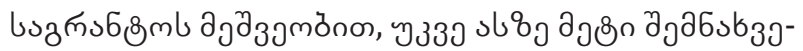

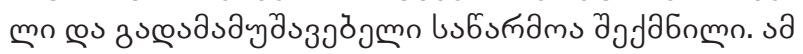

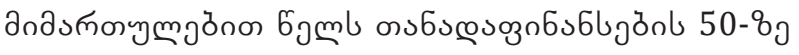

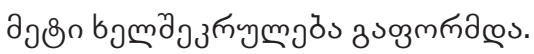

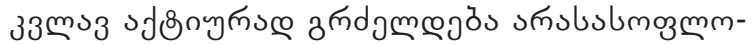

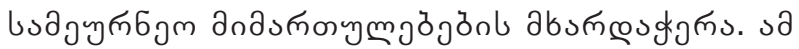

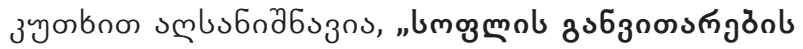

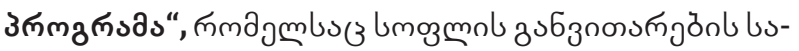

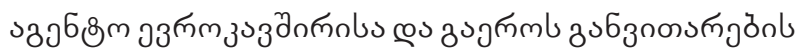

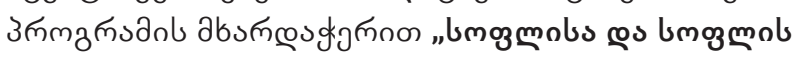

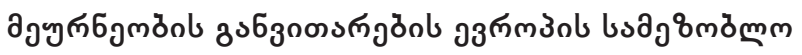

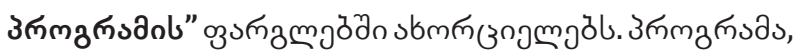

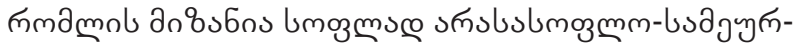

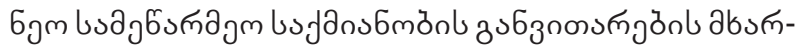

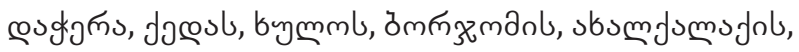

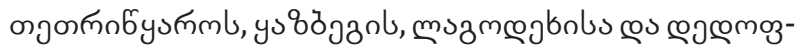

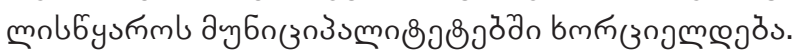

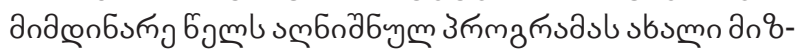

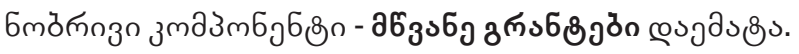

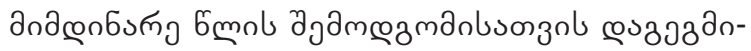

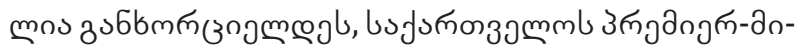

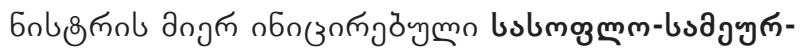

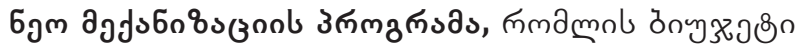

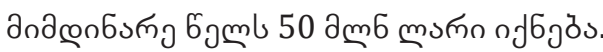

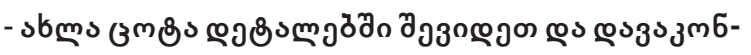
उ б

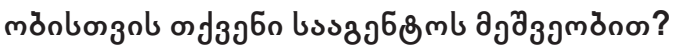

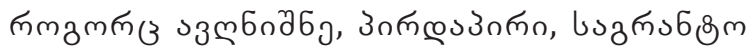

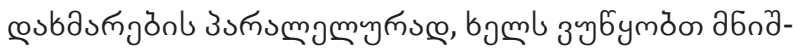

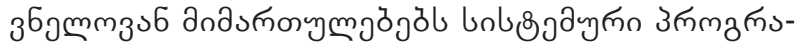

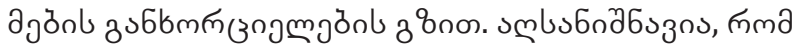

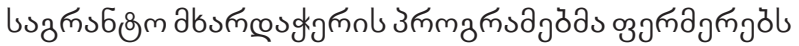

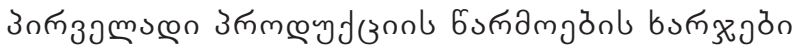

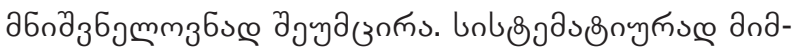

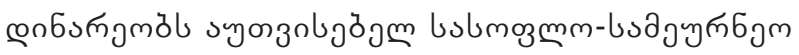

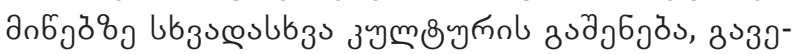

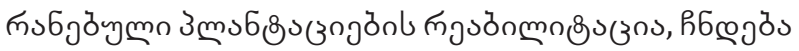

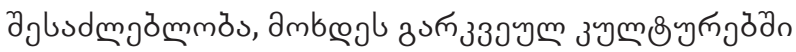
nазмп в

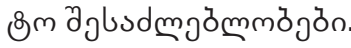

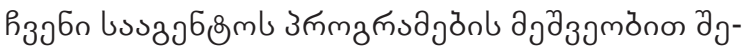
しsdmృ

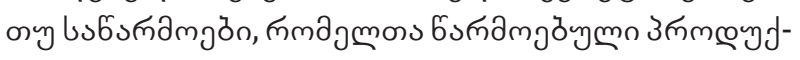

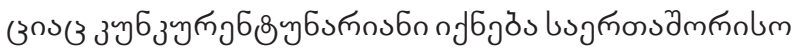

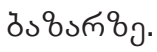

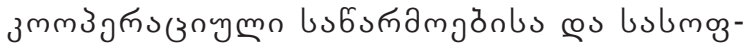

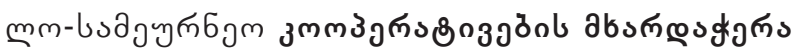

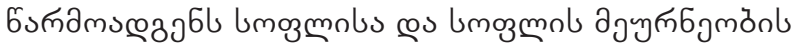

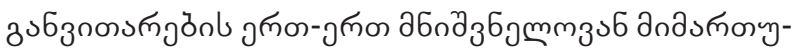

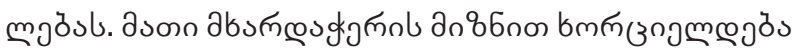

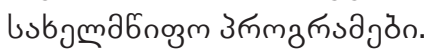

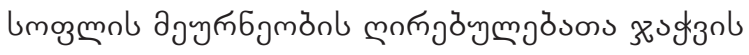

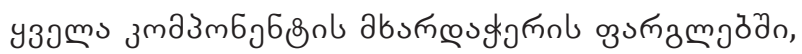

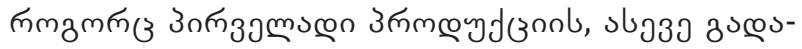

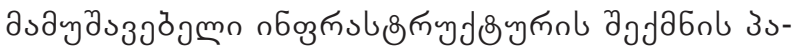

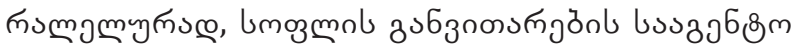

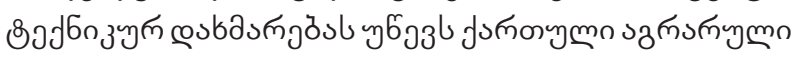

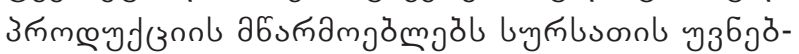

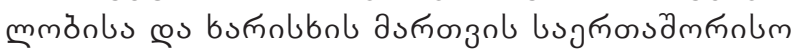

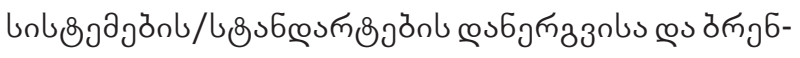

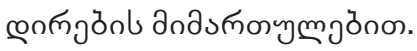

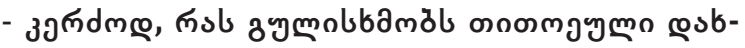

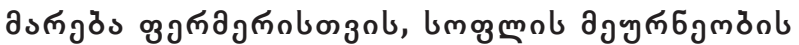

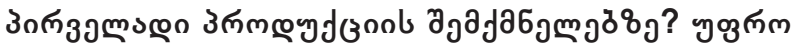

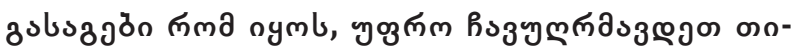

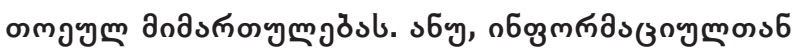

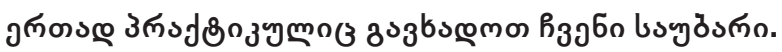

umozmol zง

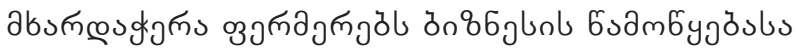

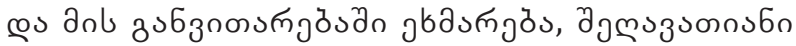

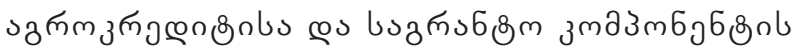

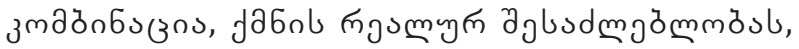

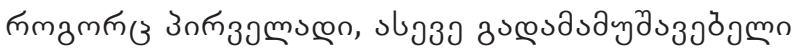

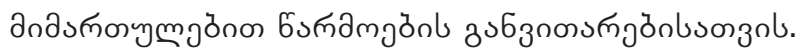

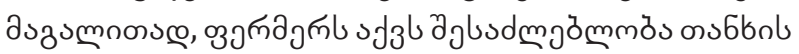

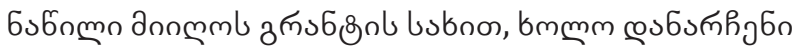

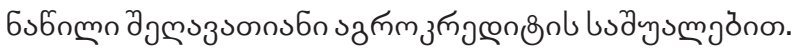

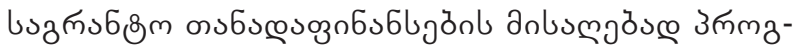

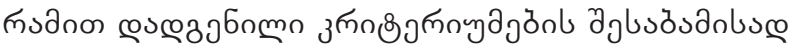




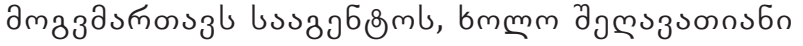

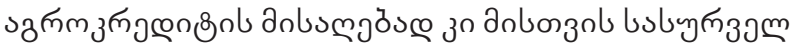

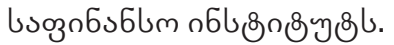

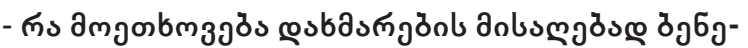

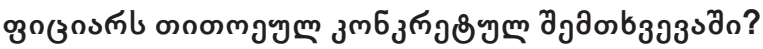

umozmol z

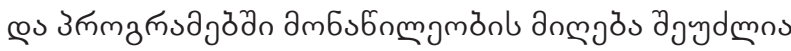

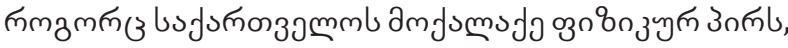

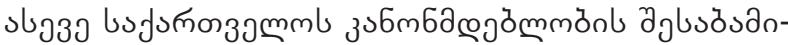

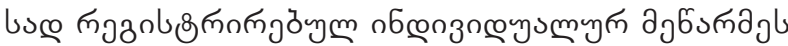

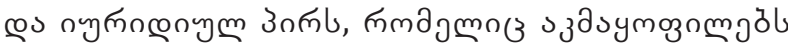

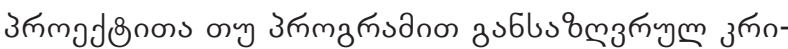

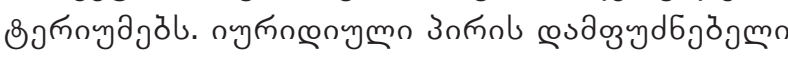

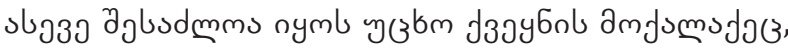

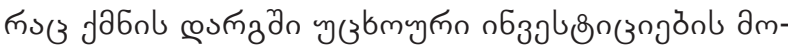

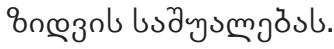

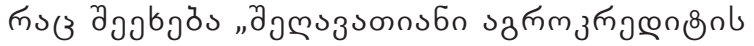

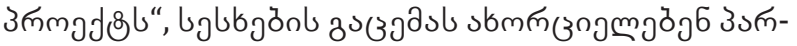

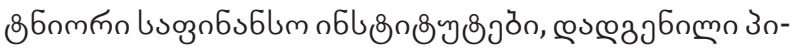

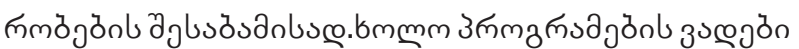

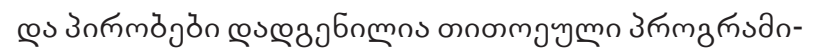

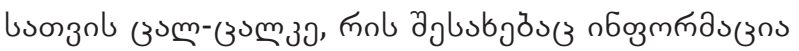

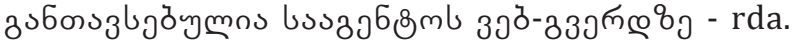
gov.ge.

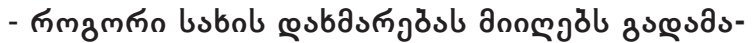

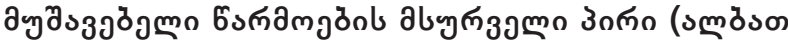

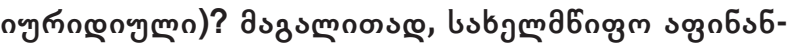

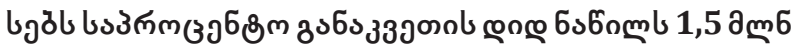

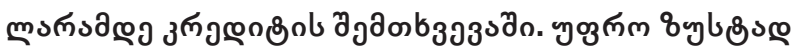

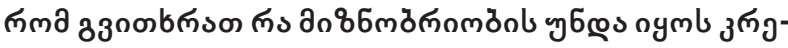

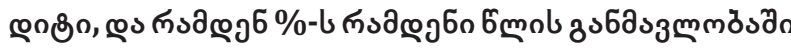

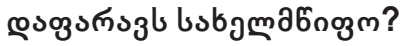

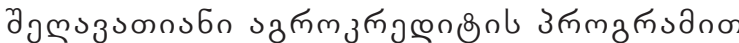

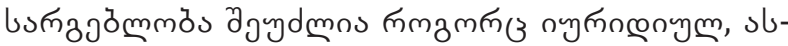

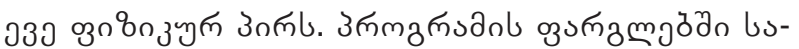

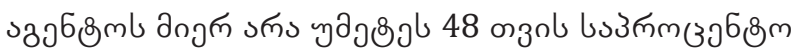

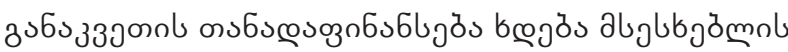

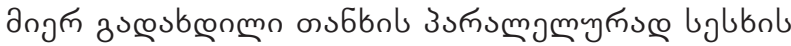

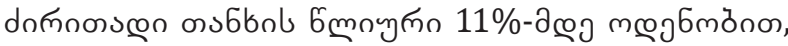

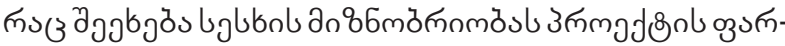

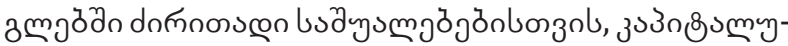

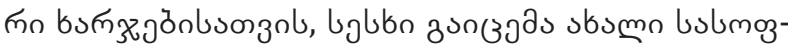

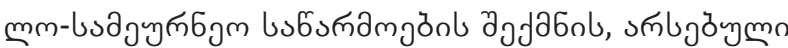

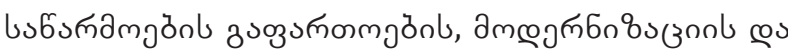
sங்

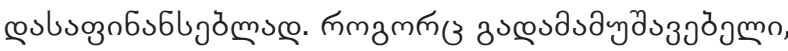

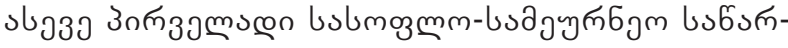

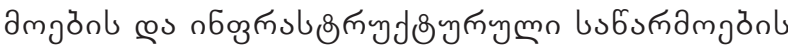

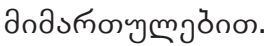

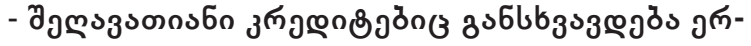

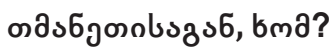

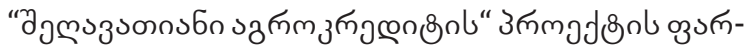

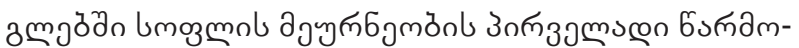

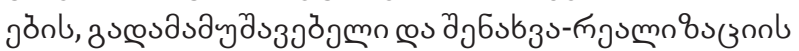

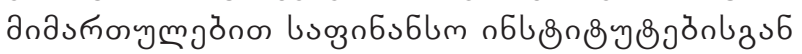

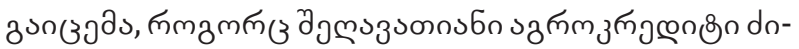

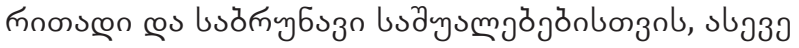
১љпмmовобаn.

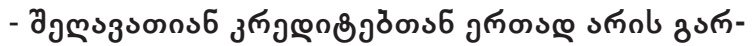

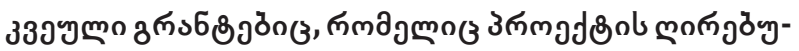

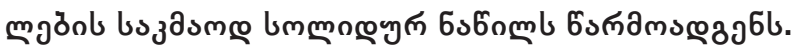

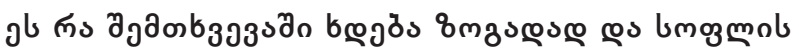

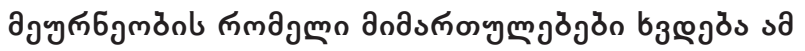

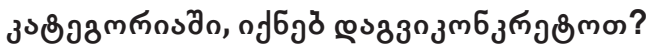

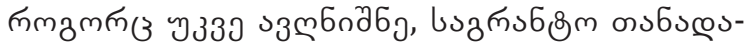

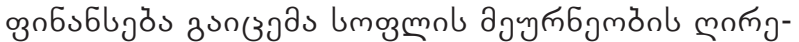

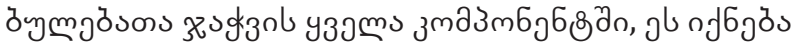

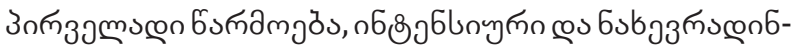

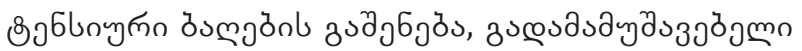

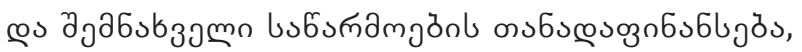

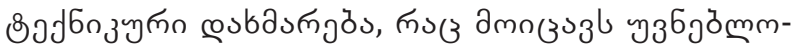

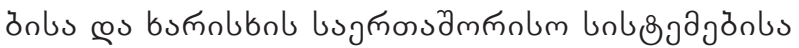

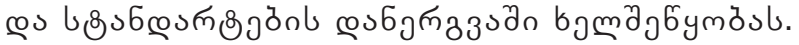

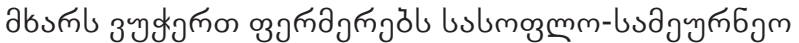

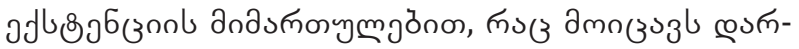

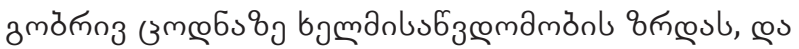

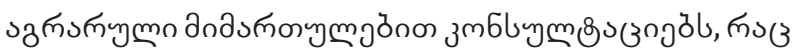

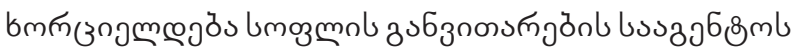

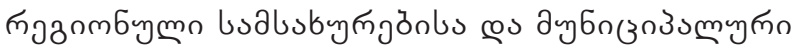

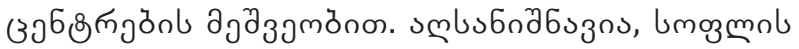

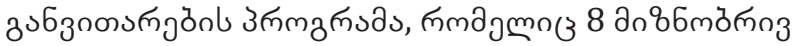

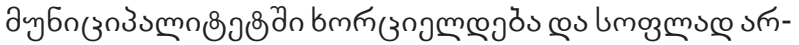

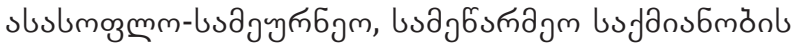

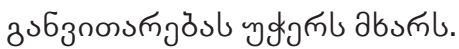

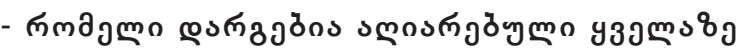
उмомповి

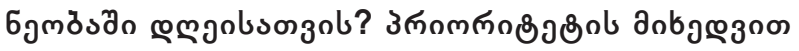

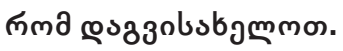

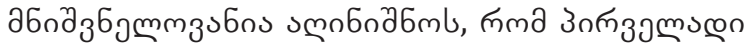

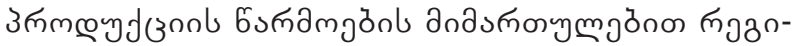

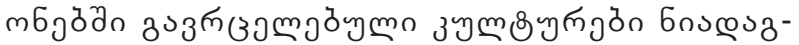

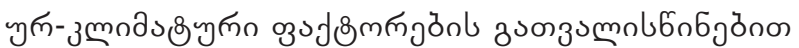

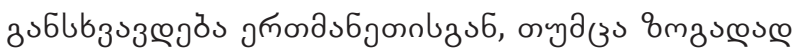

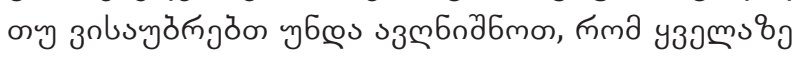

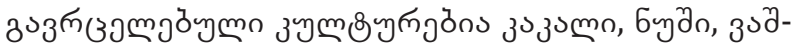

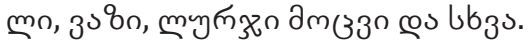

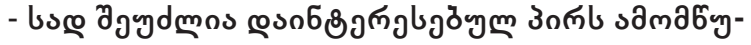

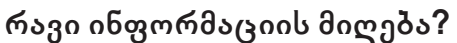




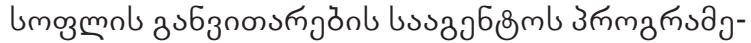

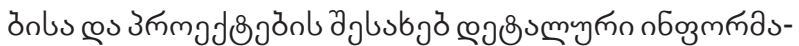

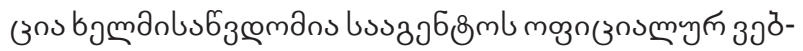

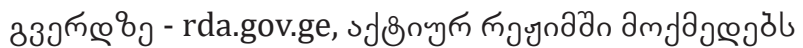

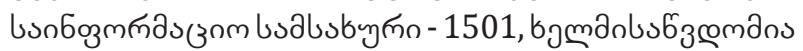

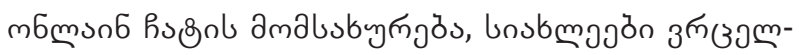

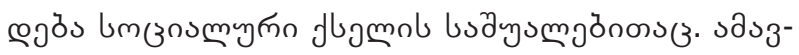

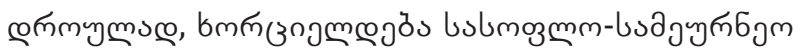

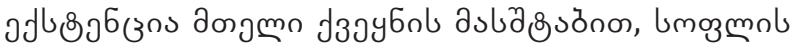

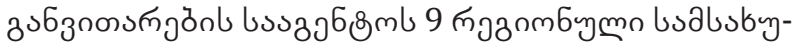

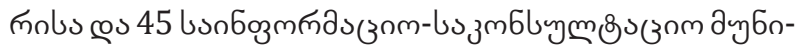

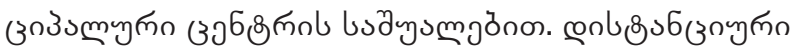

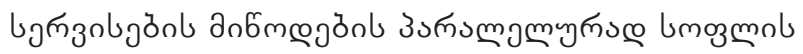
3ง6з

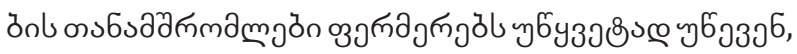

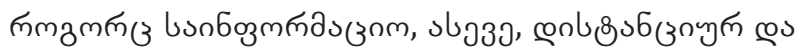

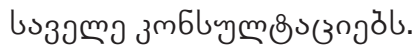

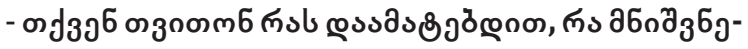

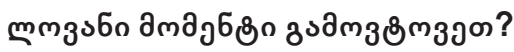

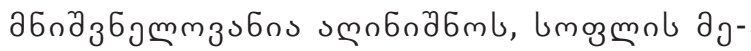

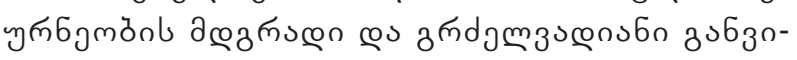

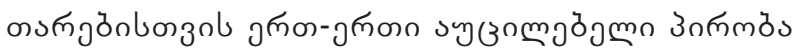

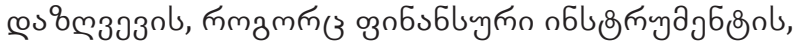

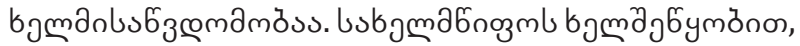

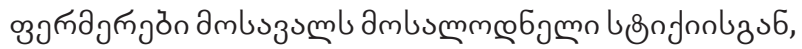

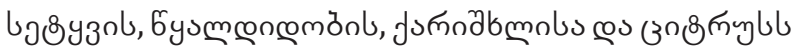

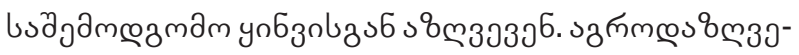

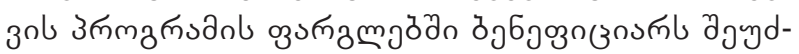

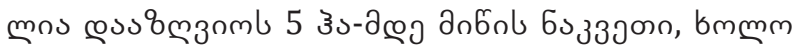

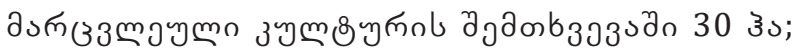

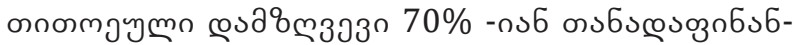

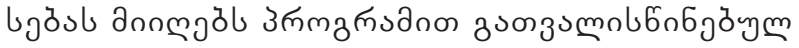

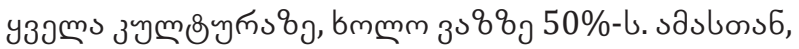

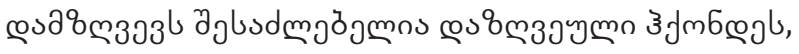

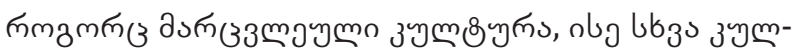

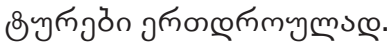

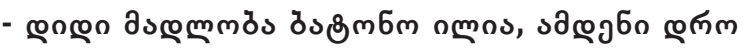

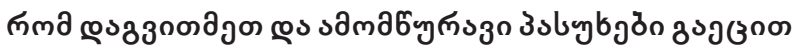

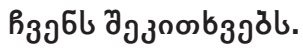

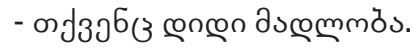

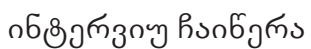

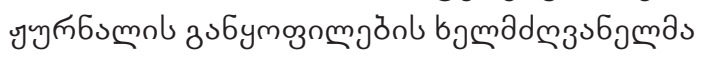

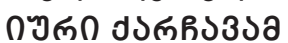




\section{BENEFITS AND CHALLENGES OF APPLYING E-LEARNING IN THE GEORGIAN HIGHER EDUCATION SYSTEM}

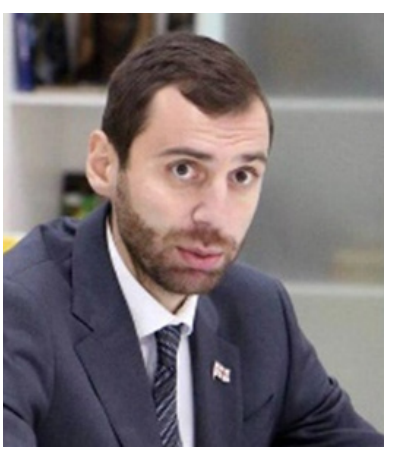

Giorgi Abashishvili,

$\mathrm{PhD}$ in Business Administration, Chancellor, Georgian Institute of Public Affairs (GIPA)

E.mail: giorgi.abashishvili@gmail.com

Cell:(+995 599) 089988

\section{DOI suffix: 10.36962/NEC6102202112}

E-learning has an increasingly important role within the ever-growing tertiary education system in many developed countries. While the research on e-learning is still relatively a novel discipline, with even a universally accepted definition being absent, there are numerous indications pointing to its increasing importance. For example, in the US alone, some $35 \%$ of university students take at least one online degree, while the ratio has been steadily increasing in the recent years.

There are numerous underlying factors which support the intensification of e-learning. Most countries cannot keep up with the increasing demand for tertiary education by merely expanding their traditional universities - be it because of high needed fixed investments, or because or elevated costs of engaging the relatively scarce teaching staff. In the same time, the ICT revolution - as well as the ongoing COVID outbreak - both facilitate and require shifts to a delocalized contact between students and the teaching staff. In sum, this provides many developing countries with a mechanism of provision of tertiary education to large masses of prospective students without having to invest in physical infrastructure.

However, this is not a process without challenges. Regulation in many countries is only yet to cope with these technology and demography-induced shifts in education. Some academic fields are not yet appropriate for distance learning. Cheating and plagiarism could be widespread if not tackled with appropriate strategies and technological solutions. This document examines these elements by providing an overview of the experiences in some of the countries where the e-learning system already took deep roots.

Georgia has much to gain if it includes e-learning in its tertiary education system. Georgia at this moment is, seemingly, one of the few relatively developed countries which still do not have a fully-fledged and accredited elearning platform within its tertiary education system. However, as World Bank data show, some 64\% of Georgia's high school graduates successfully enroll to a university, which is approx. 10 percentage points lower than OECD average, or as much as 25-30 percentage points lower than some of the world's top education performers, such as Finland, the Netherlands or South Korea. While this gap needs to be bridged if Georgia is to tap the potential of the ongoing technological revolution, introduction of e-learning to its system may be of significant help, while it would not incur large additional costs. Indeed, numerous international examples show that in many countries, the number of students enrolled to universities soared following the introduction of e-learning, while the quality of education has not declined. In terms of increasing the base of potential enrollments, in Georgia's case it is important to underline that e-learning may also be a mean of reaching out and connecting with members of the numerous Georgian diaspora. Also, setting up an e-learning platform also helps the universities to engage top lecturers in many educational domains at relatively low cost, meaning that more students may be given a higher quality education.

COVID-19 outbreak is a case in point. The ongoing pandemics outbreak has shown, among other, that true business continuity for many education institutions, at all education levels, could have only been reached by employing adequate e-learning procedures. This means that those who have already instituted some forms of e-learning had fewer difficulties in overcoming the operative issues, while continuing to deliver education.

Keywords: Higher education, E-lerning. 


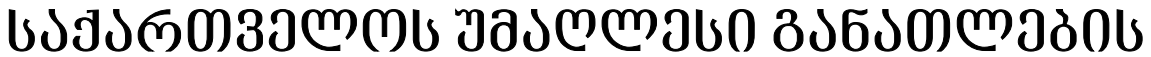

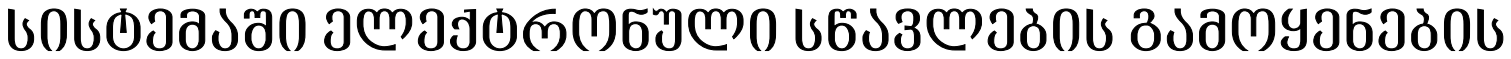

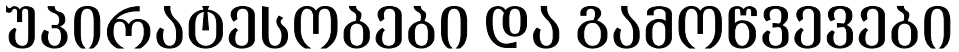

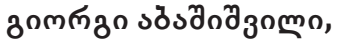

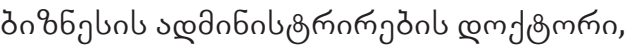

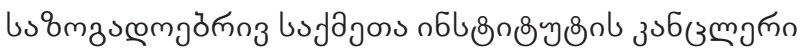 \\ эm.oुmb\&s: giorgi.abashishvili@gmail.com \\ amő:(+995 599) 089988
}

\section{LM\&SЗก১}

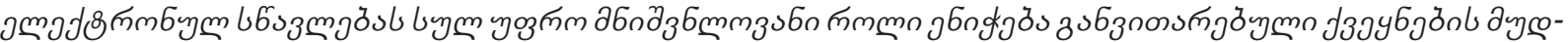

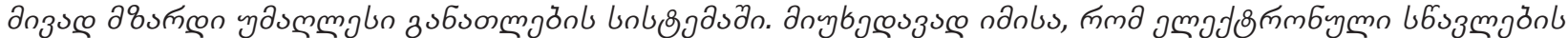

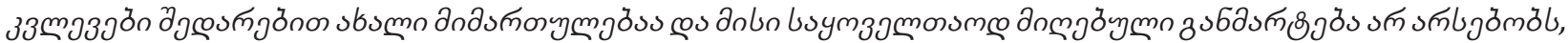
дn

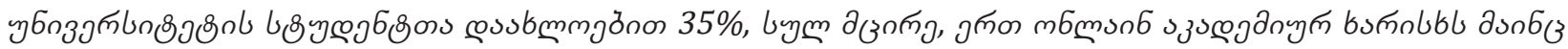

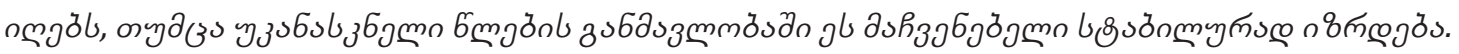

долммп ппзо òn

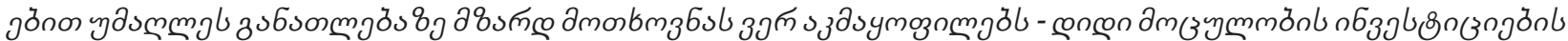

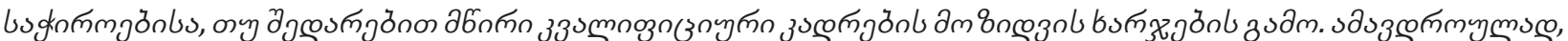

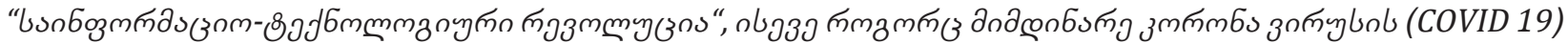

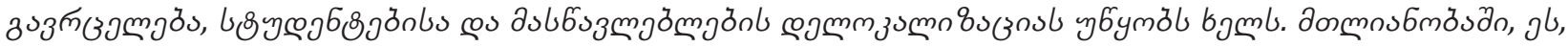

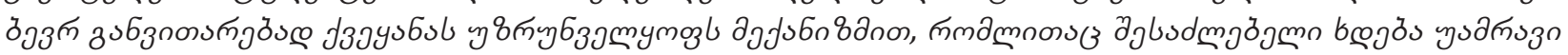

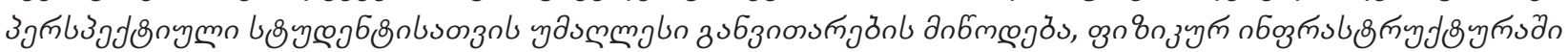

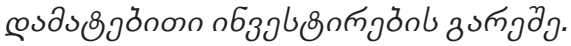

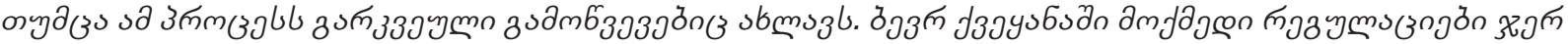

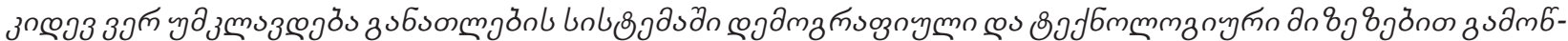

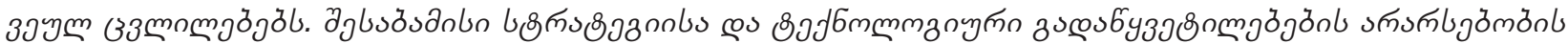

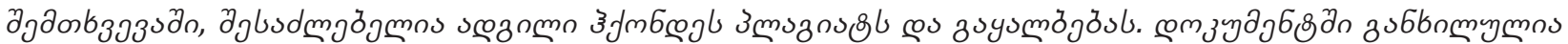

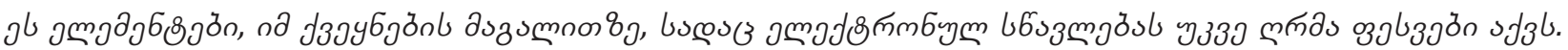

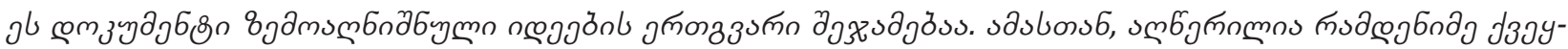

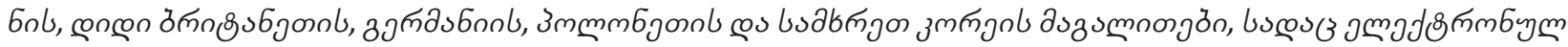

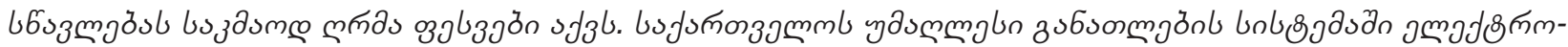

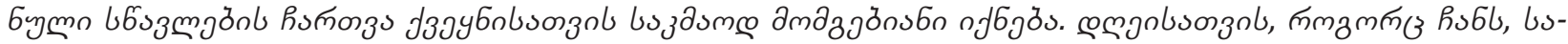

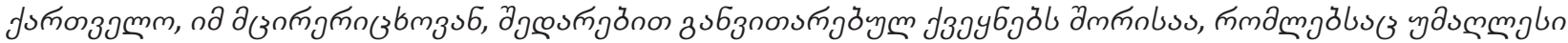
з उ6sonm

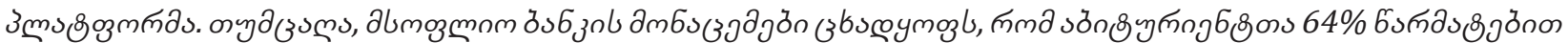

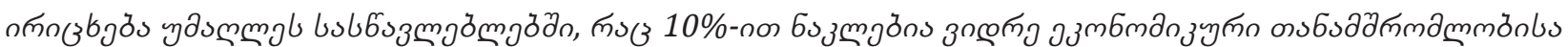

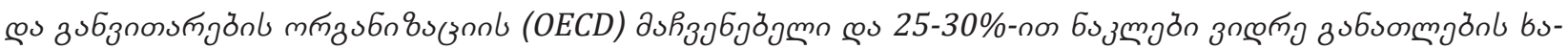

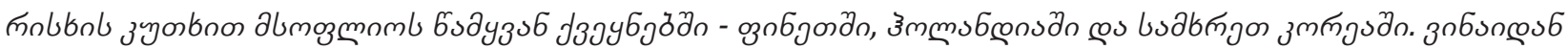

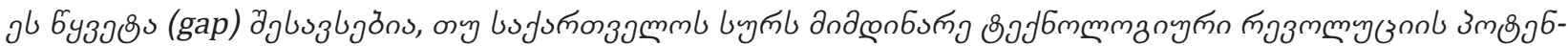

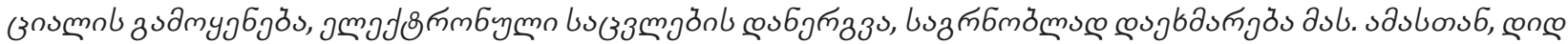

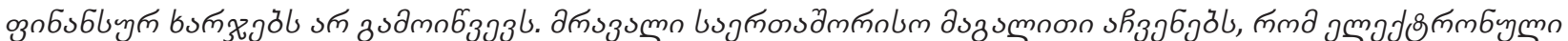

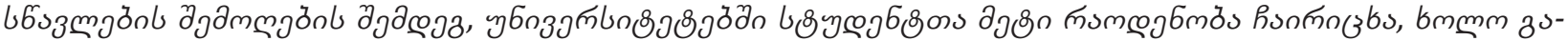

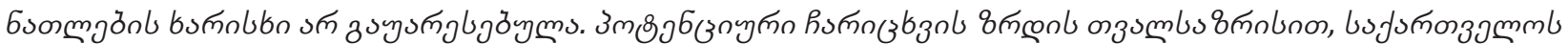

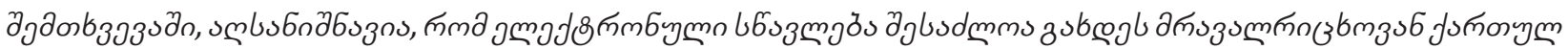

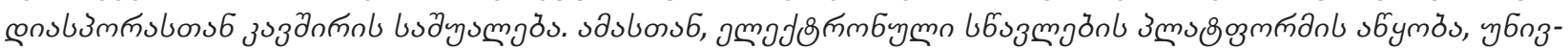

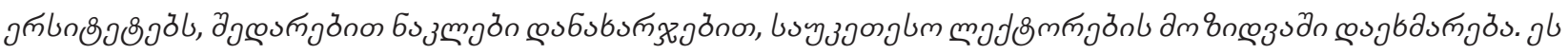




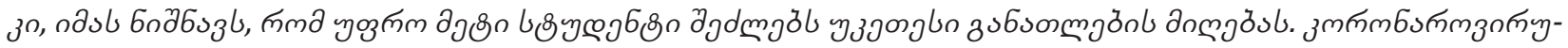

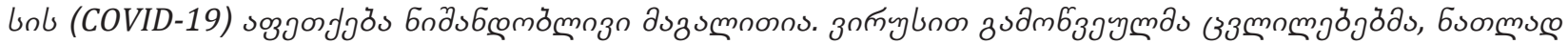

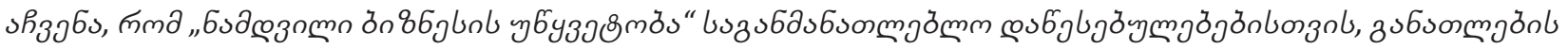

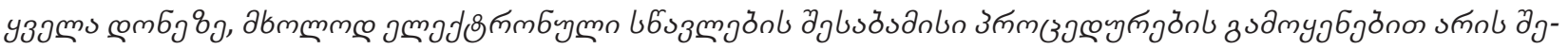

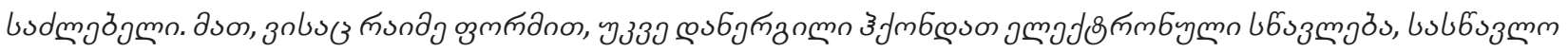

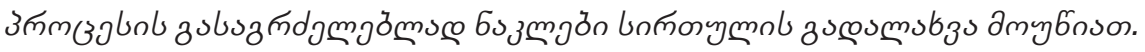

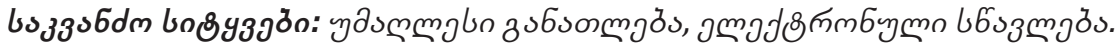

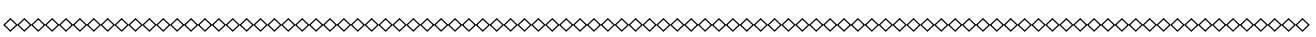

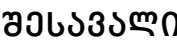

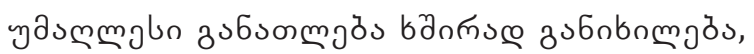

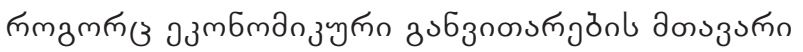

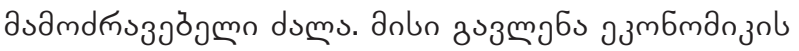

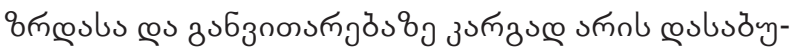

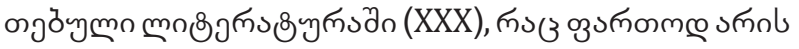

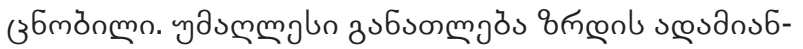

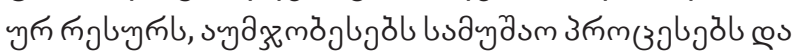

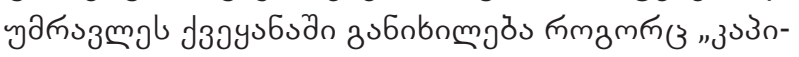

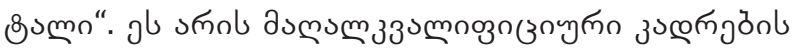

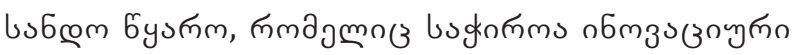

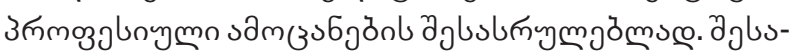

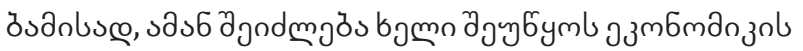

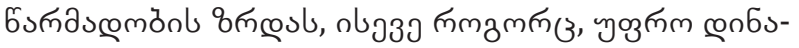

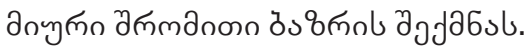

ๆзsб

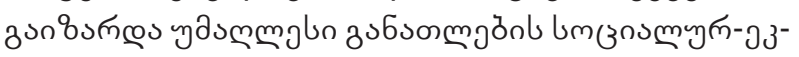

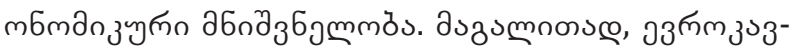

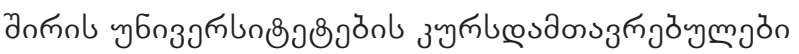

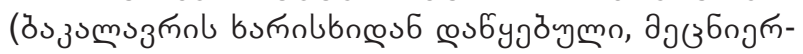

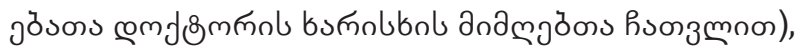

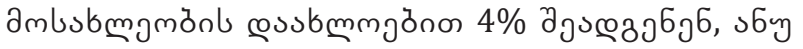

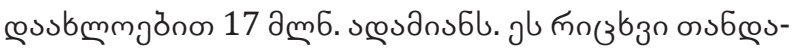

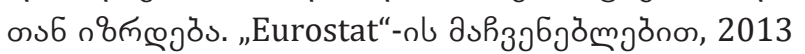

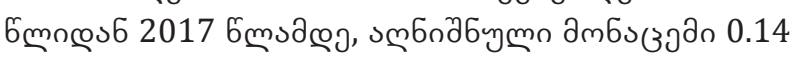

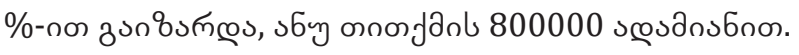

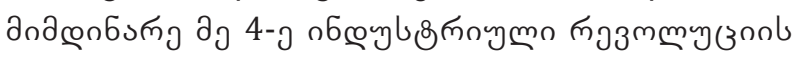

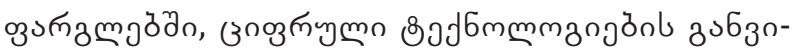

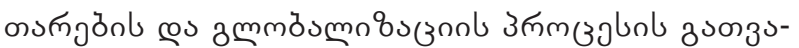

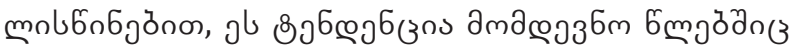

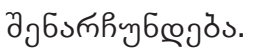

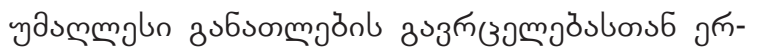

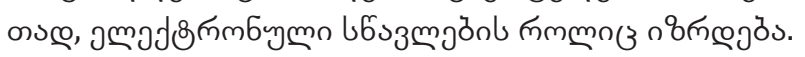

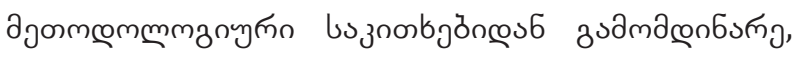

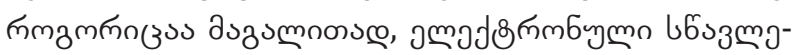

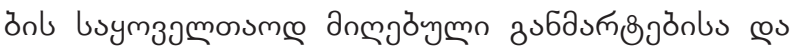

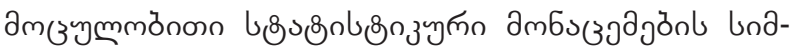

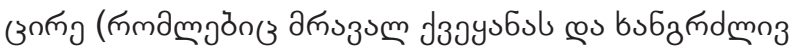

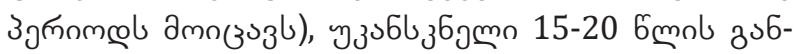

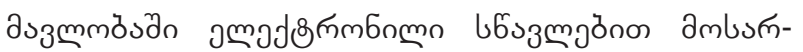

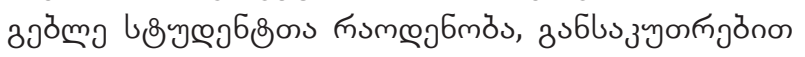

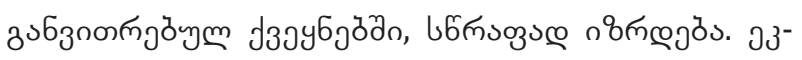

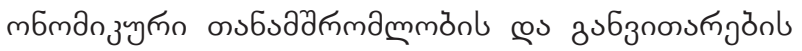

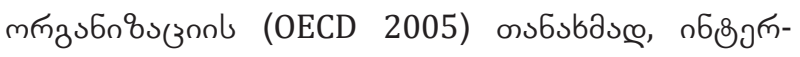

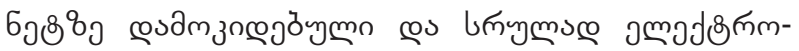

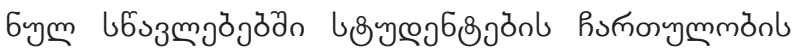

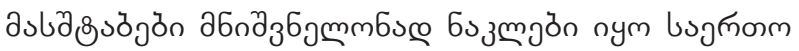

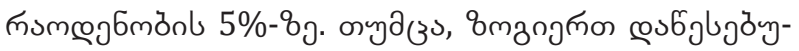

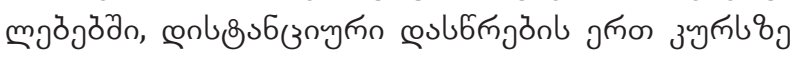

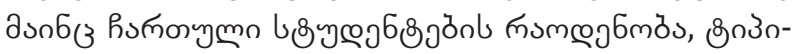

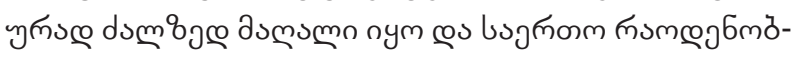

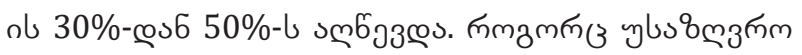

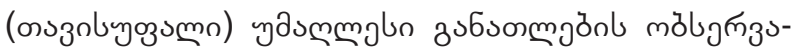

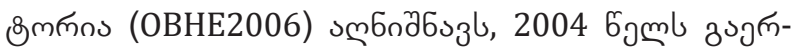

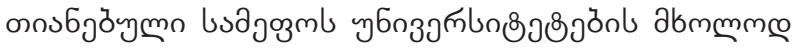

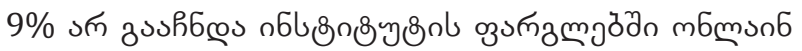

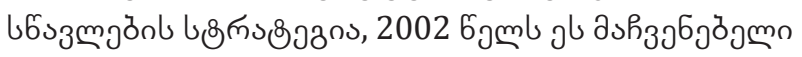

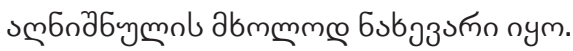

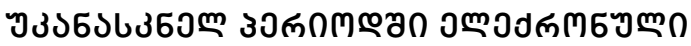 し6১з}

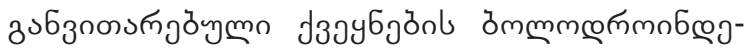

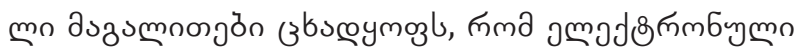

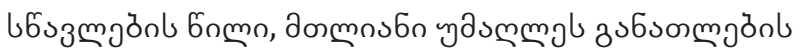

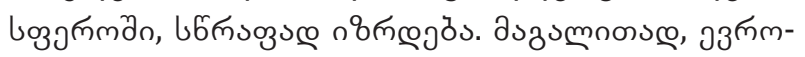

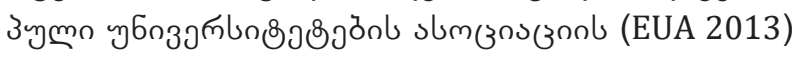

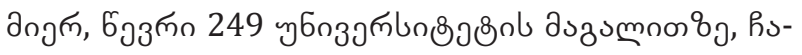

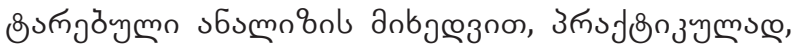

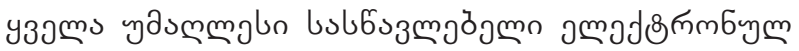

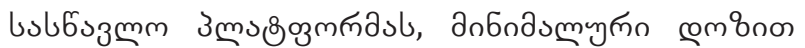

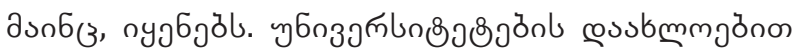

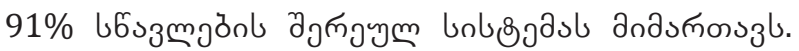

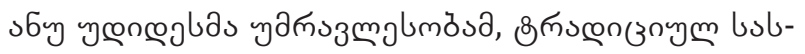

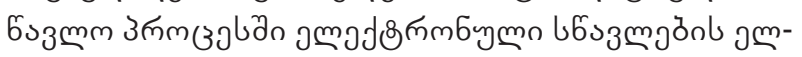

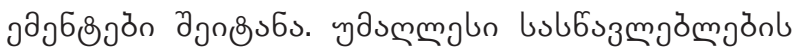

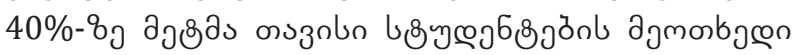

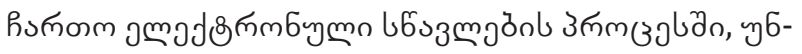

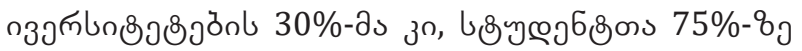

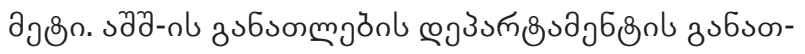

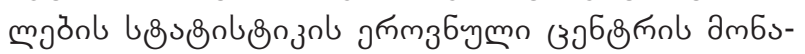
३эว

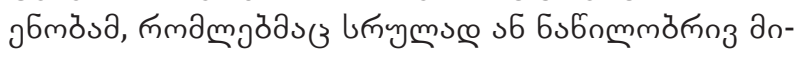




\section{ISSN 2667-9752(Online)}

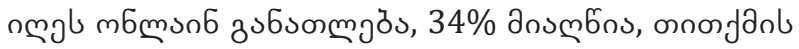

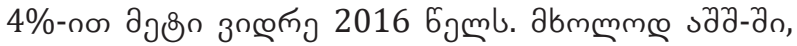

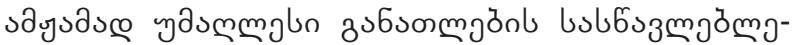

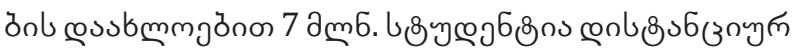

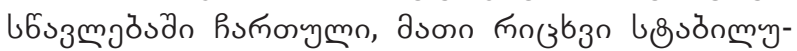

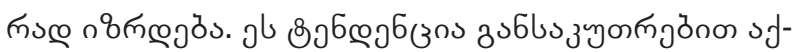

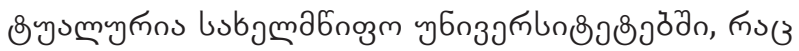

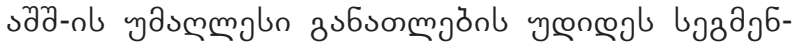

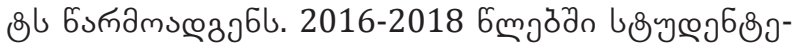

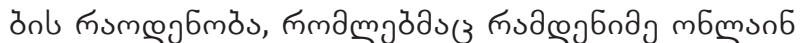

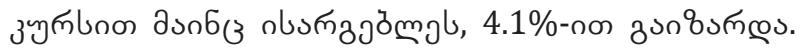

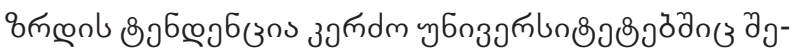

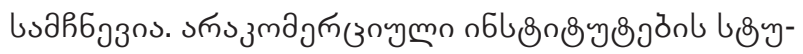

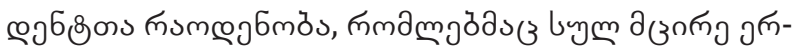

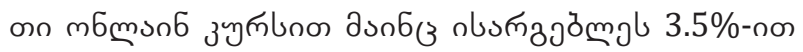

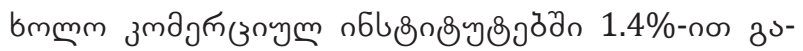

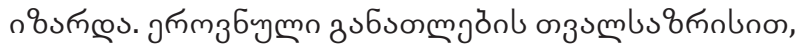

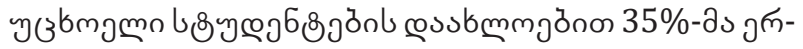

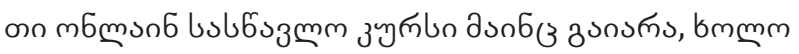

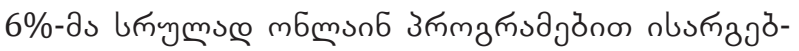

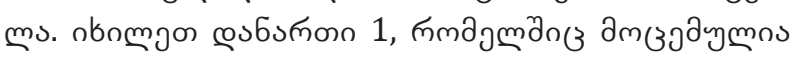

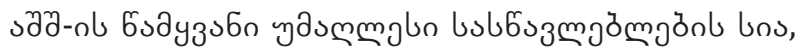

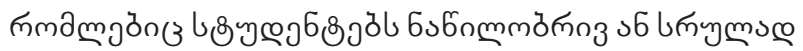

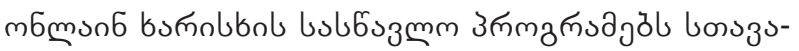

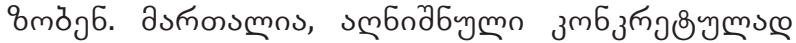

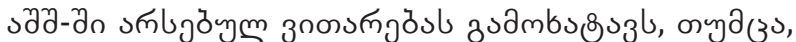
إ

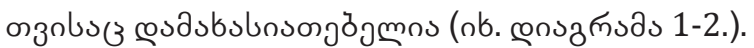

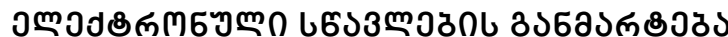

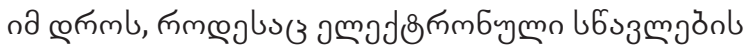

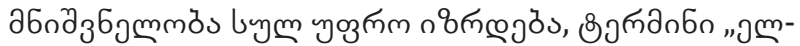

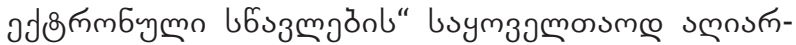

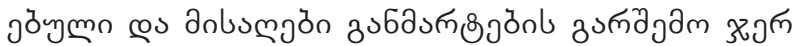

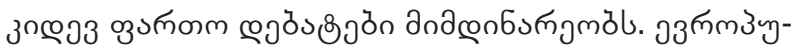
mo zmanbns (European Commission 2001) mb mun6

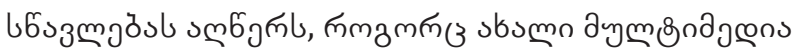

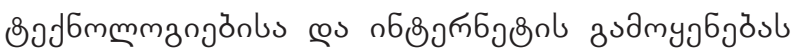

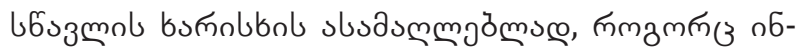

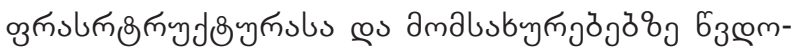

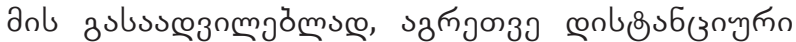

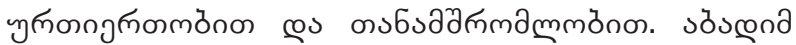

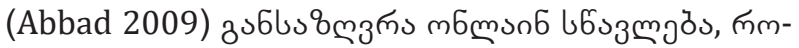

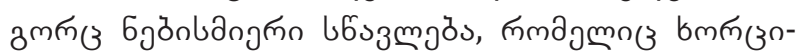

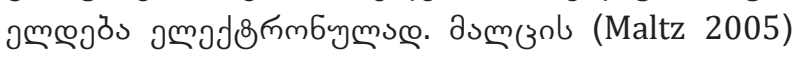

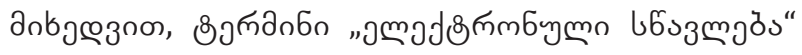

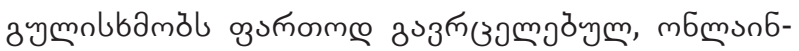

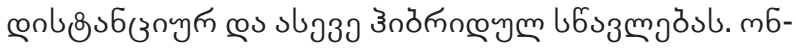

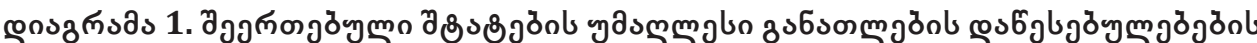

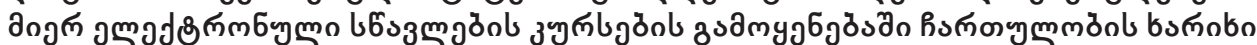

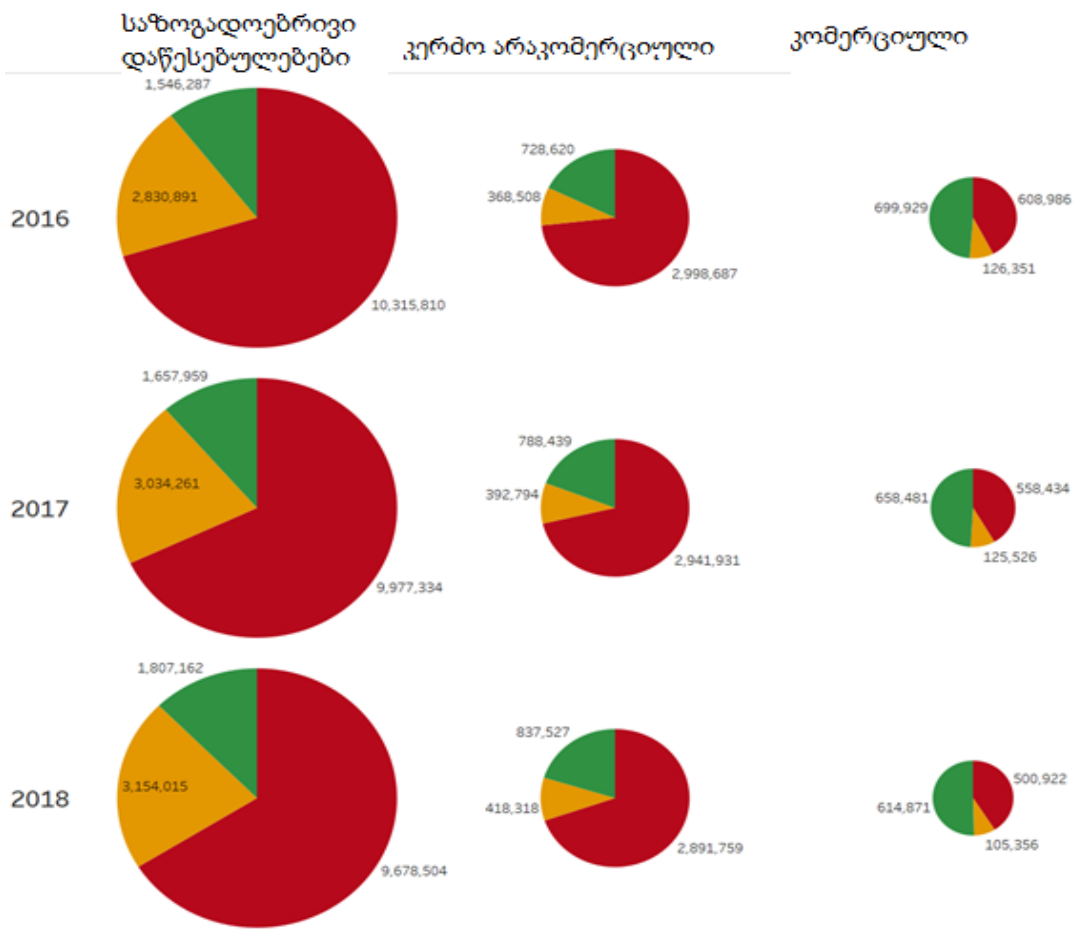

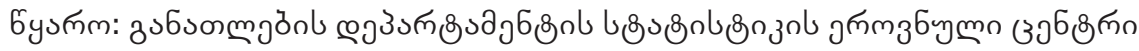




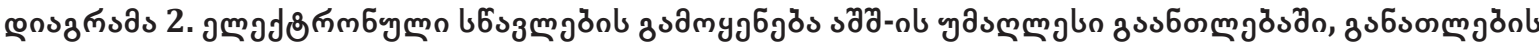

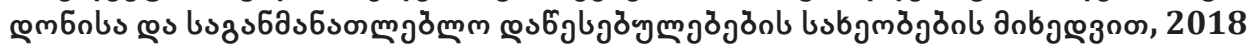
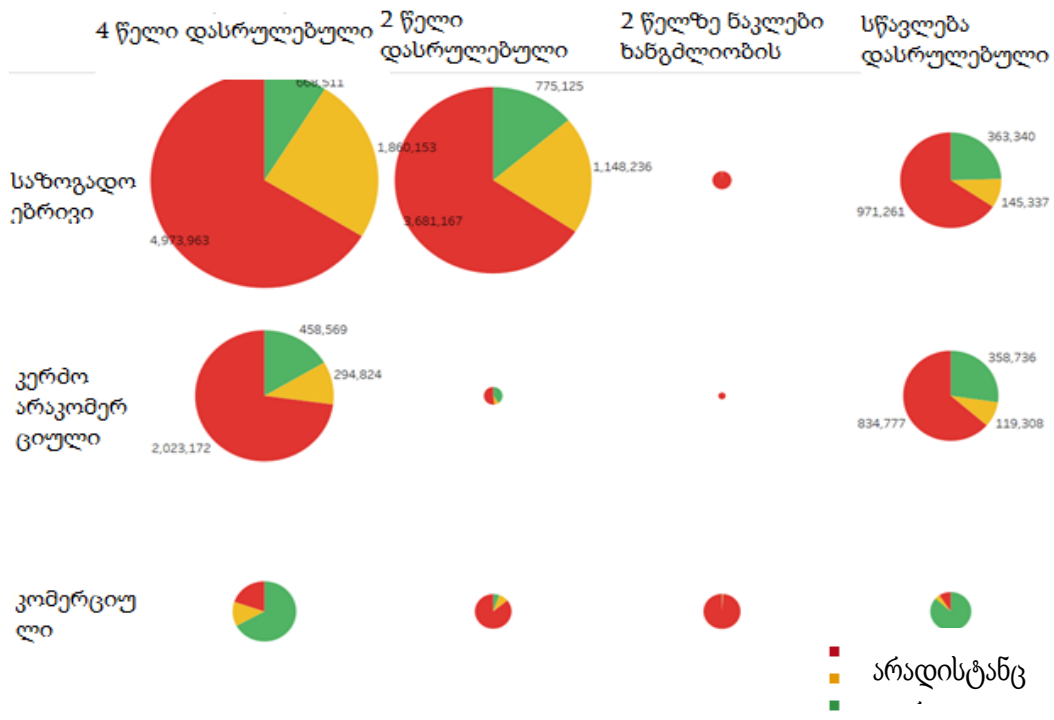

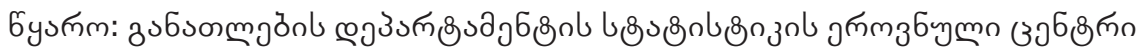

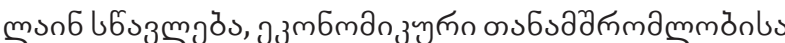

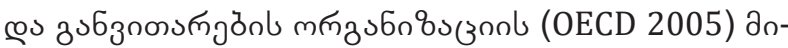

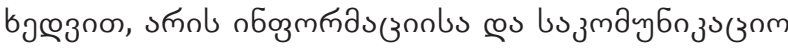

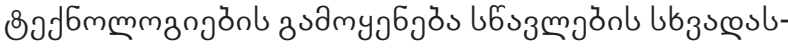

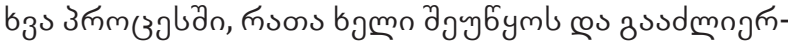

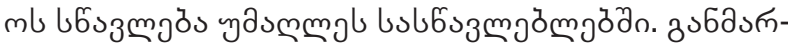

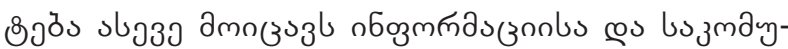

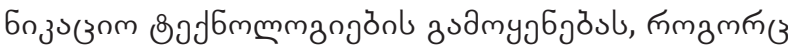

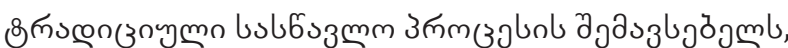

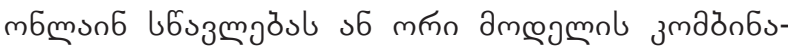

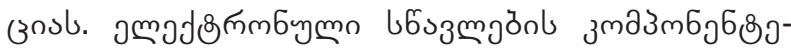

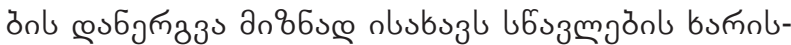

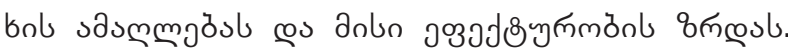

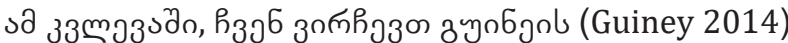

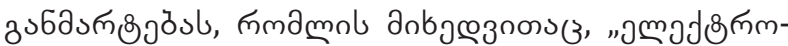

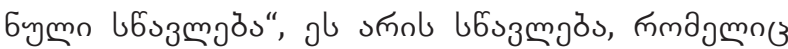

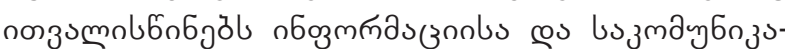

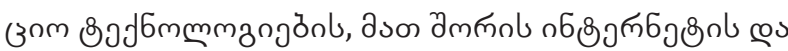

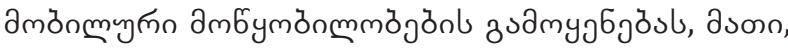

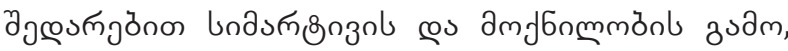

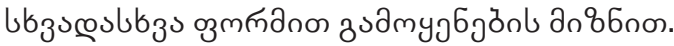

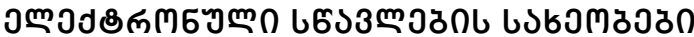

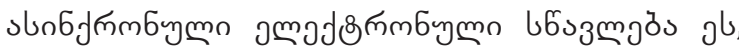

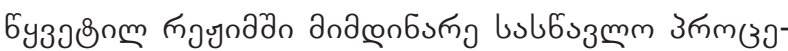

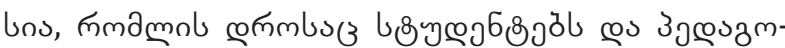

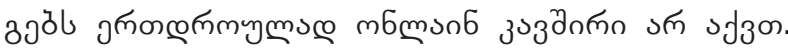

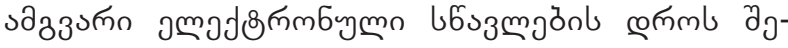

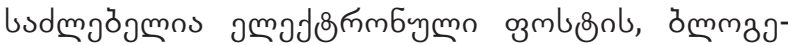

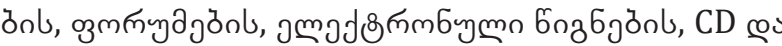

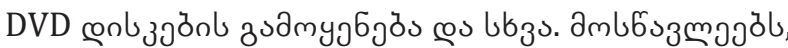
asonomzo buluyn

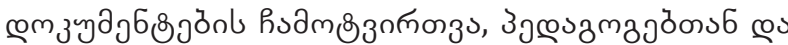

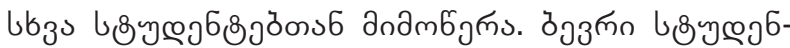

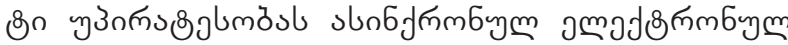

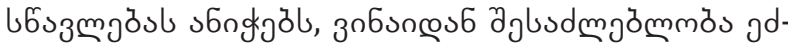

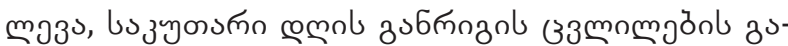

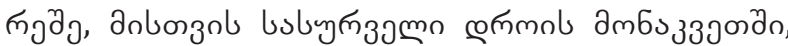

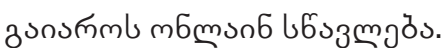

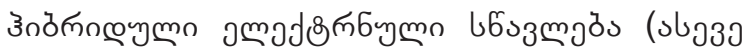

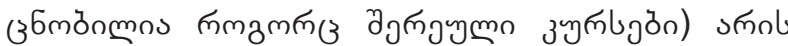

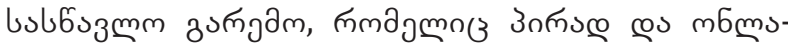

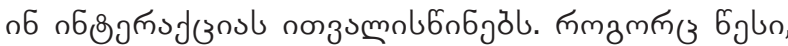

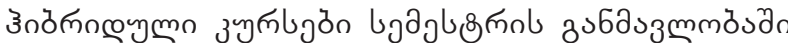

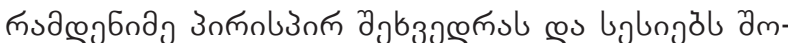

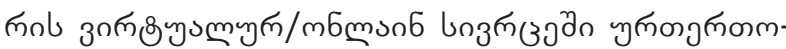
ò aymobtamől.

\section{ว

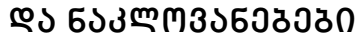

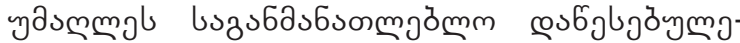

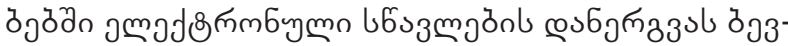

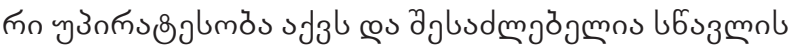

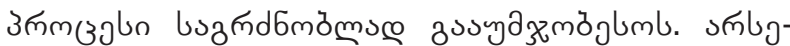

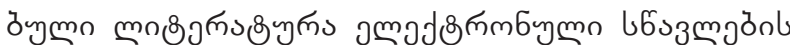

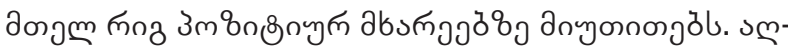

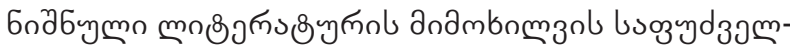

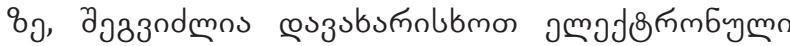

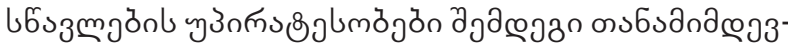
๓mmठ̀no: 


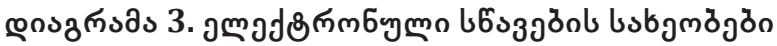

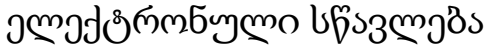

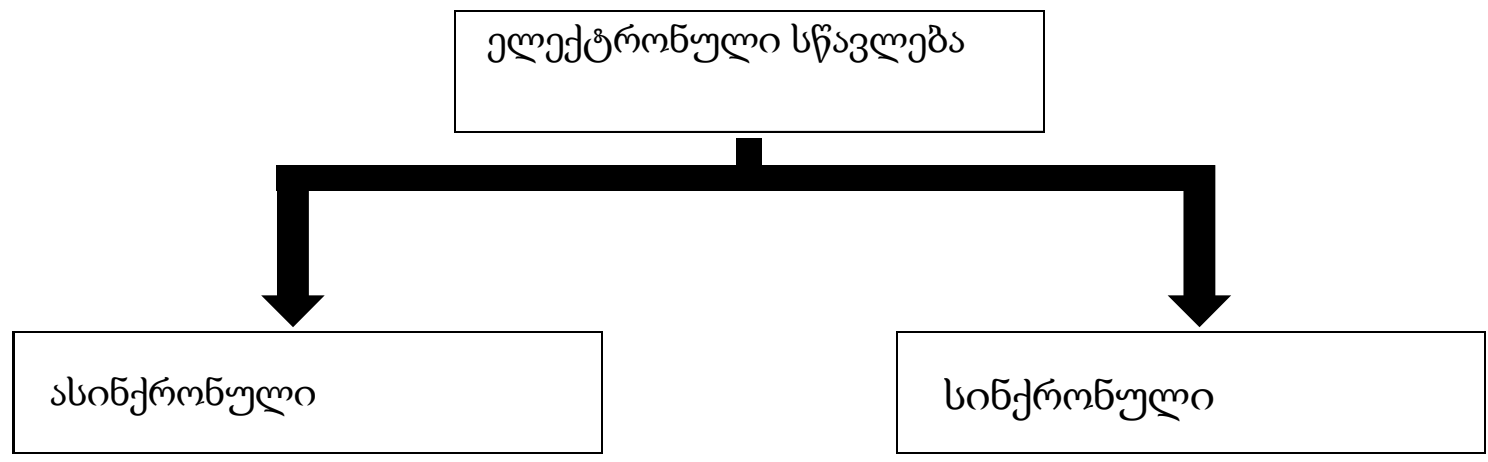

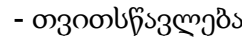

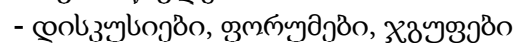

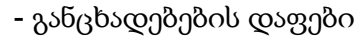

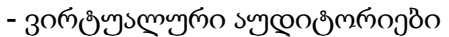

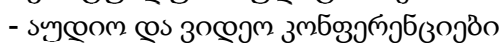

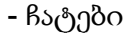

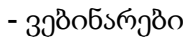

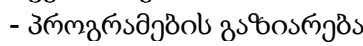

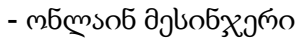

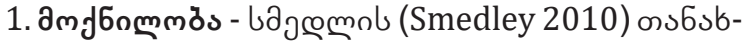

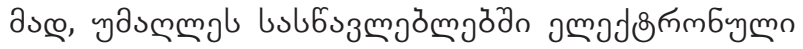

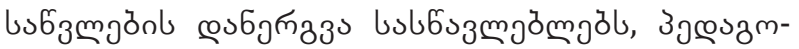

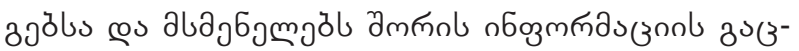

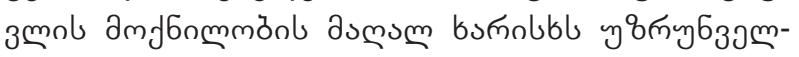
ymozl.

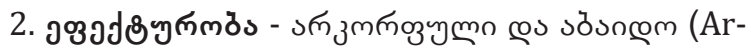

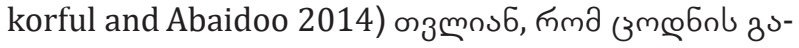

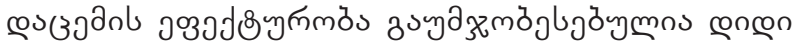

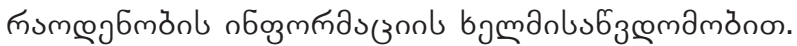

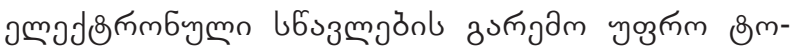

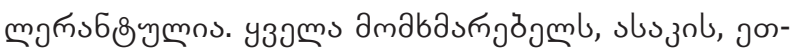

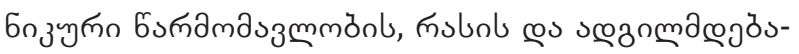

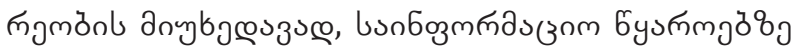
6зœмаn

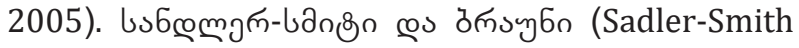

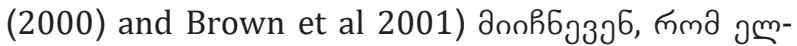

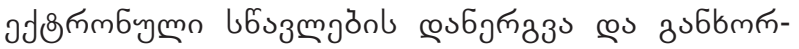

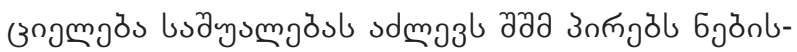

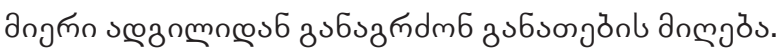

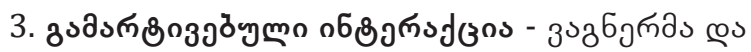

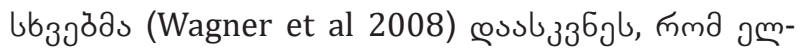

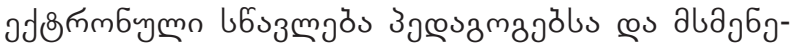

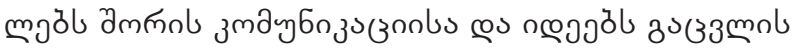

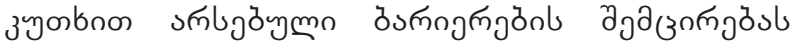

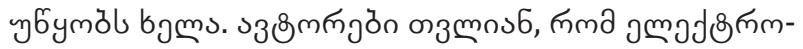

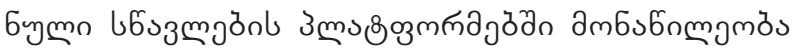

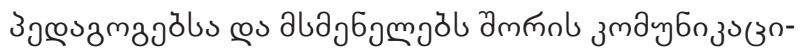

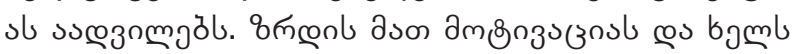

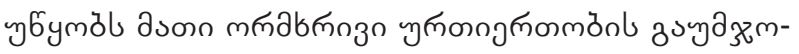

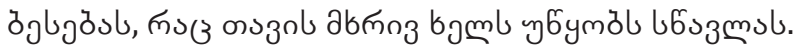

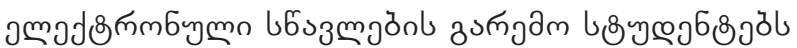

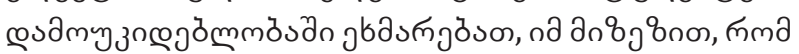

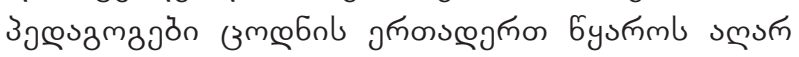

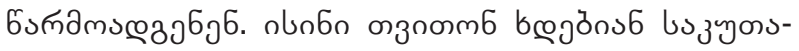

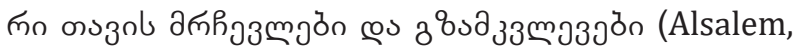
2004). hजु

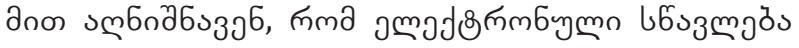

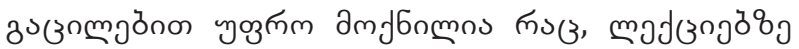

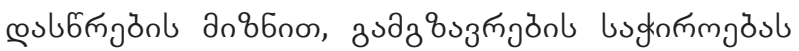

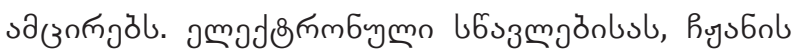

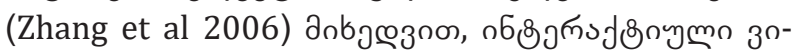

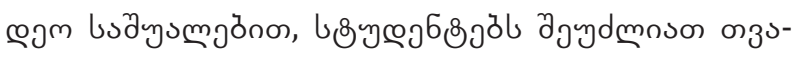

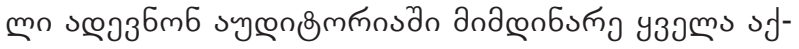

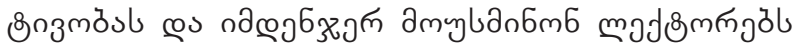

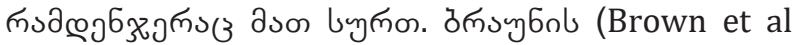
2008) œ১ nyœunmob (Judahil et al 2007) dnbgœ-

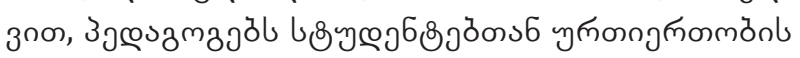

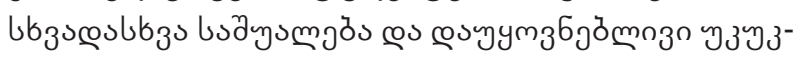
उ

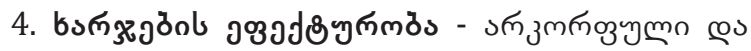

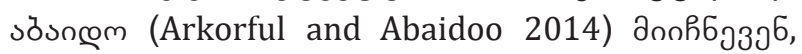

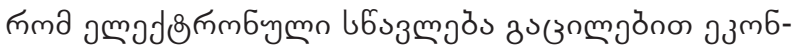

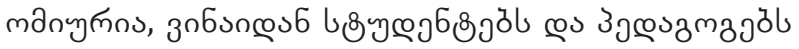

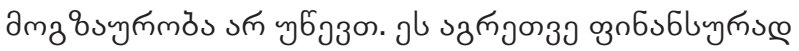

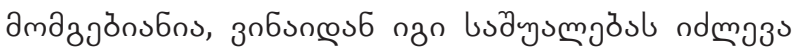

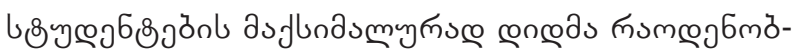

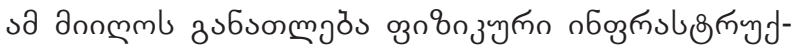

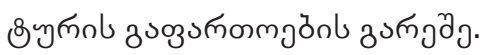

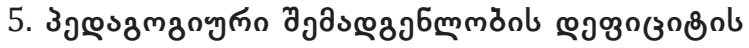

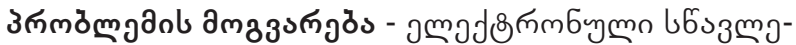

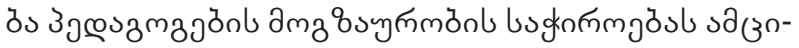




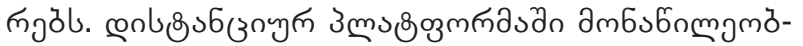

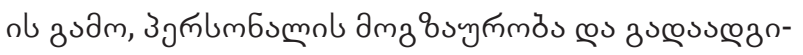

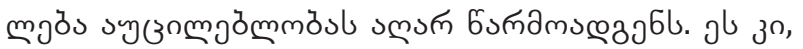

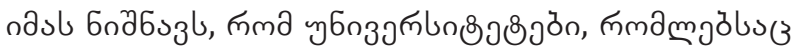

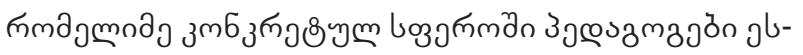

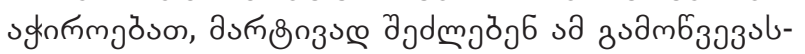

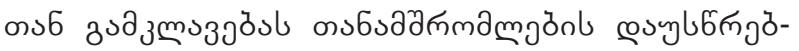

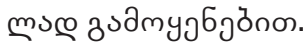

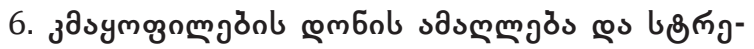

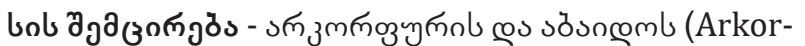

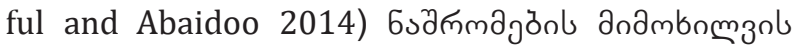

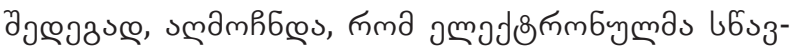

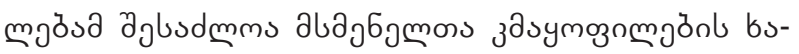

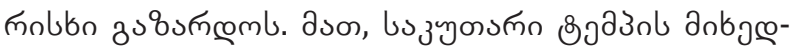

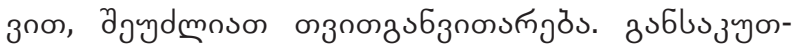

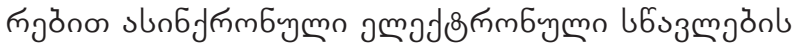

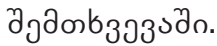

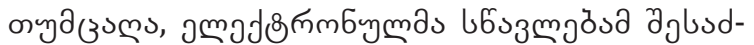

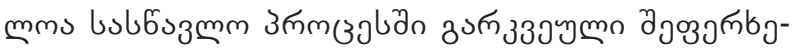

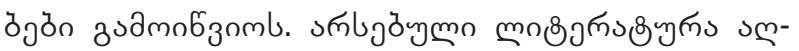

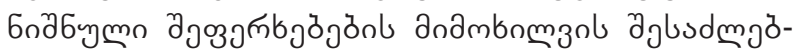

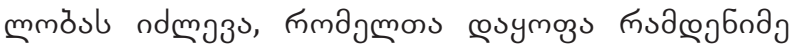

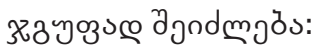

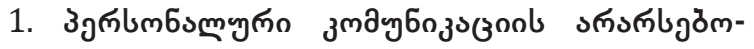

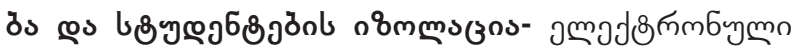

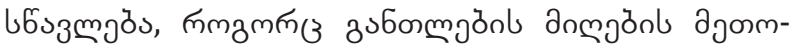

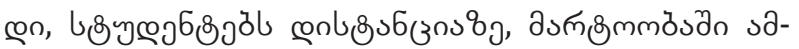

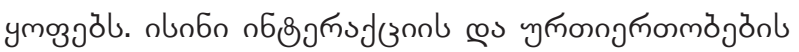

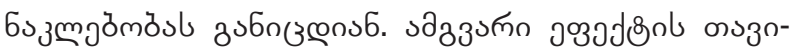

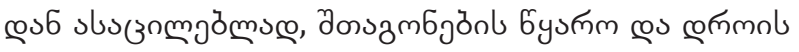

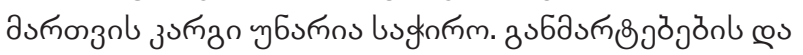

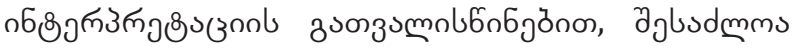

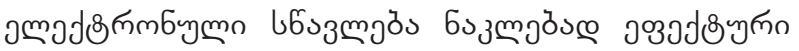

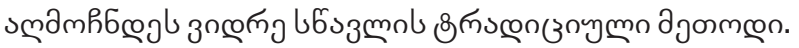

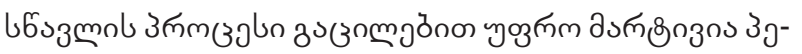

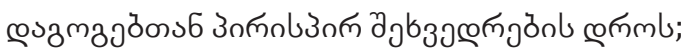

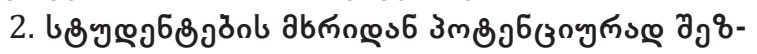

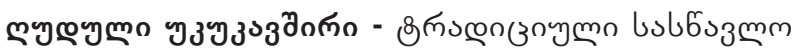

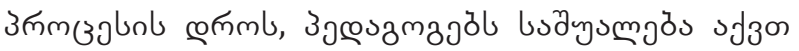

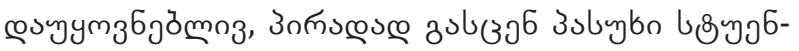

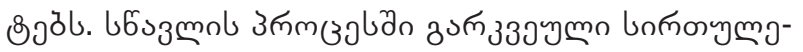

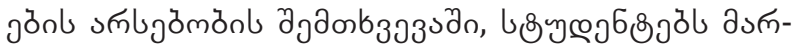

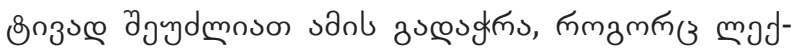

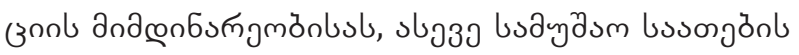

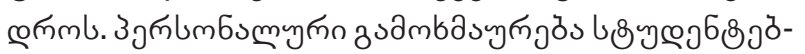

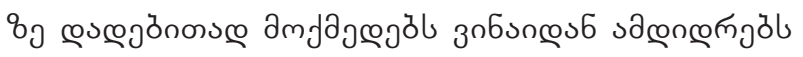

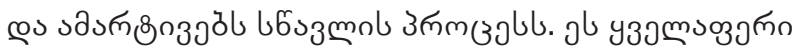

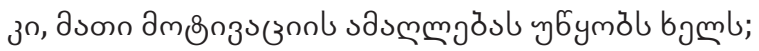

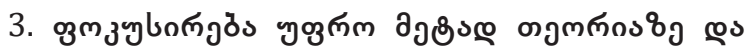

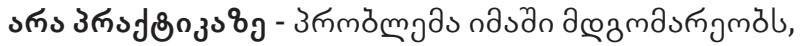

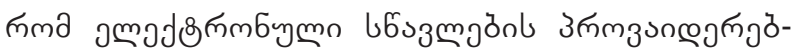

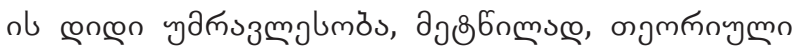

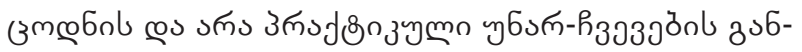

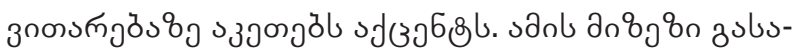

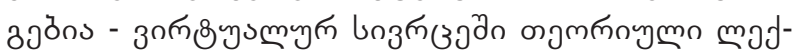

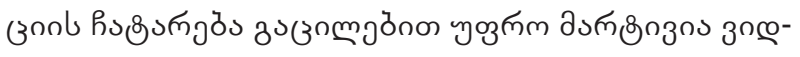

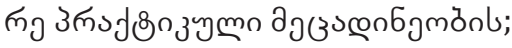

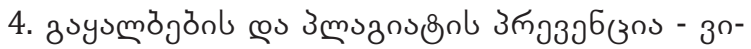

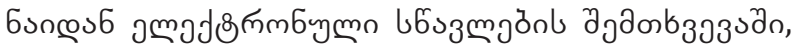

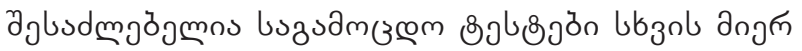

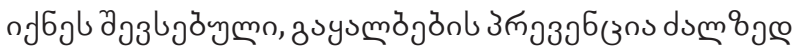

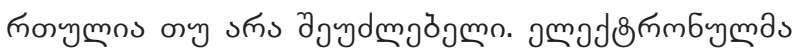

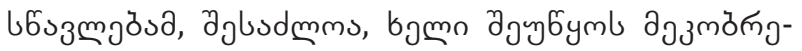

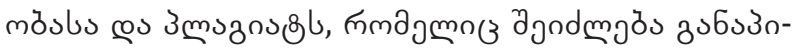

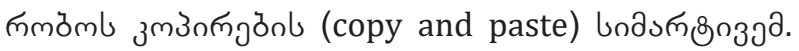

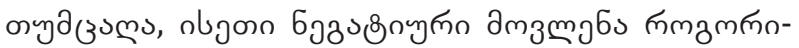

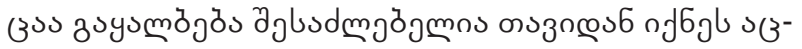

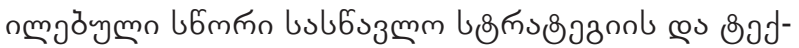

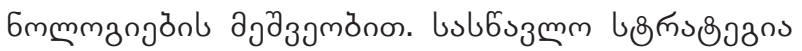

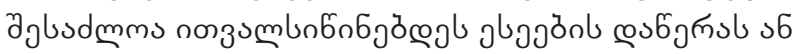

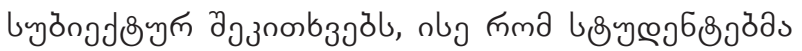

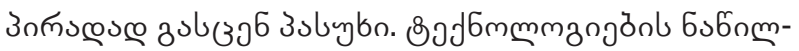

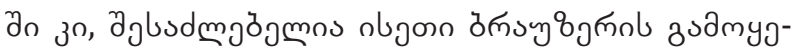

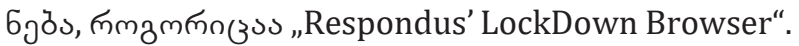

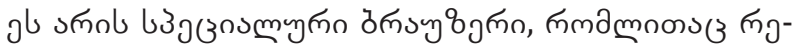

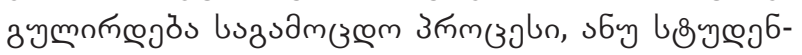

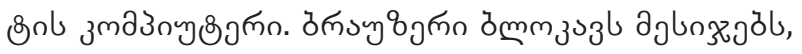

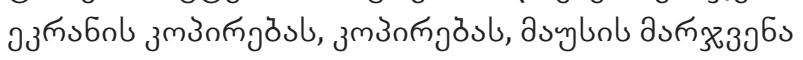

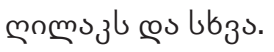

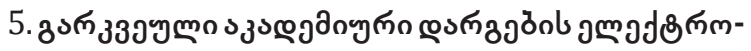

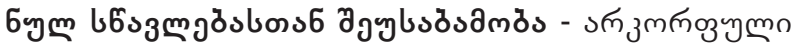

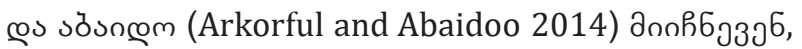

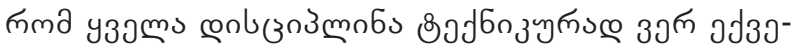

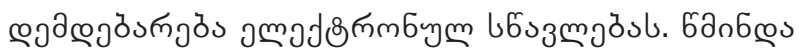

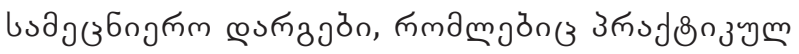

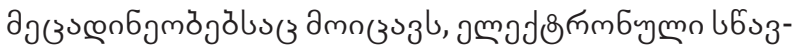

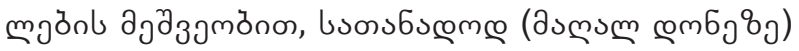

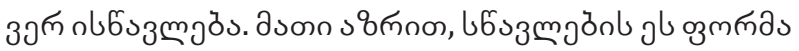

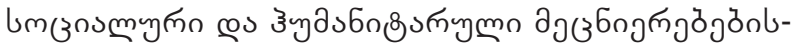

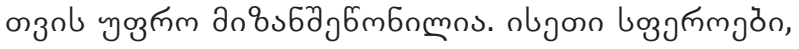

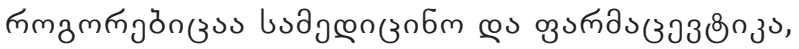

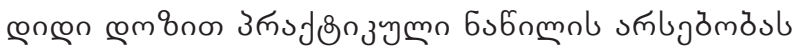

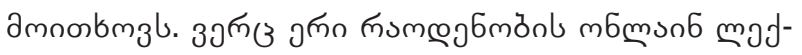

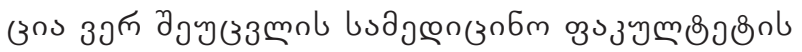

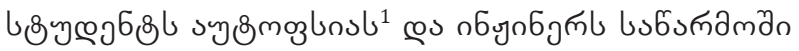

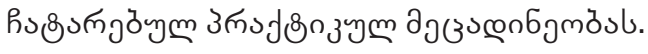

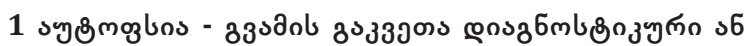

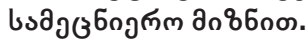




\section{ISSN 2667-9752(Online)}

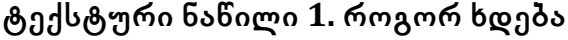

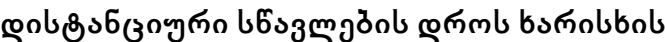

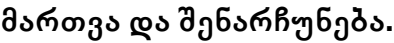

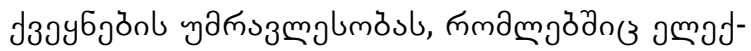

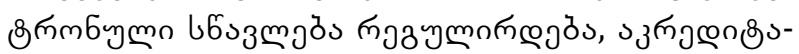

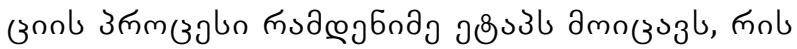
змб\&ळmm

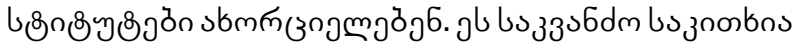

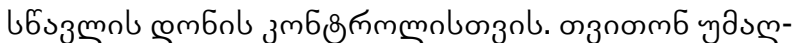

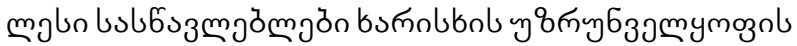

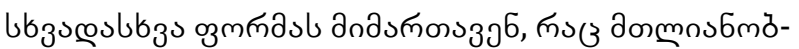

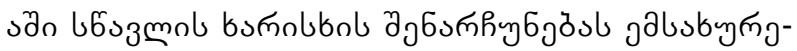

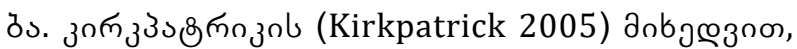

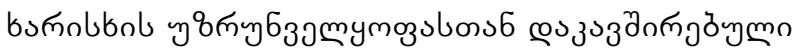

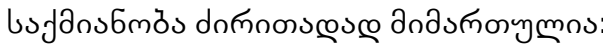

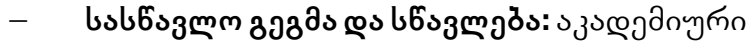

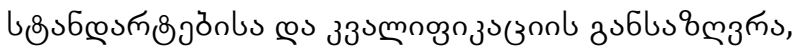

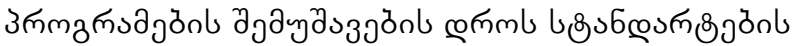

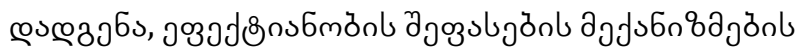

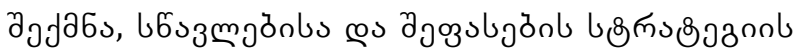

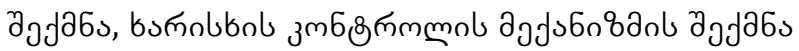

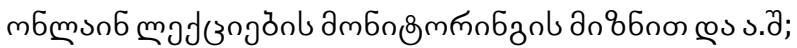

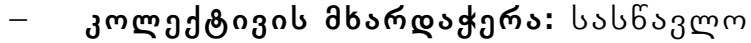

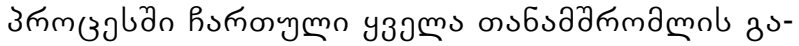

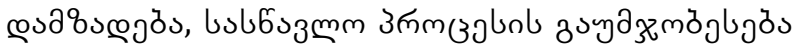

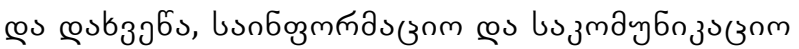

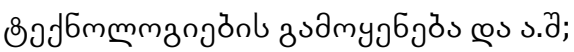

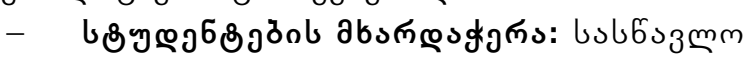

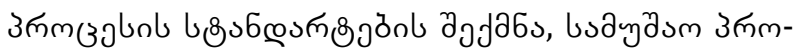

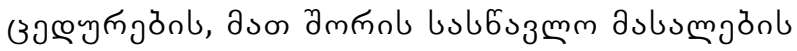

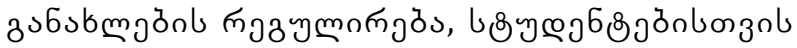

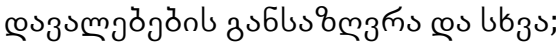

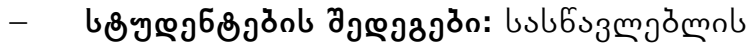

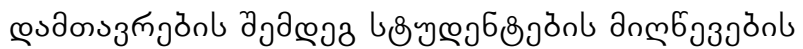

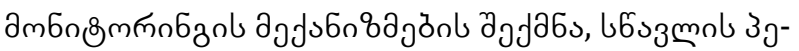

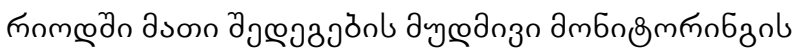
д

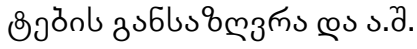

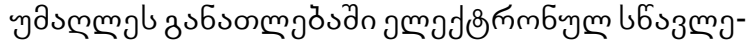

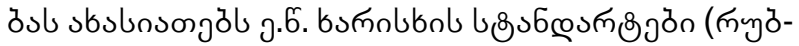

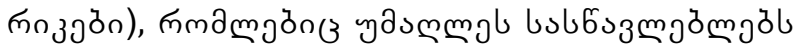

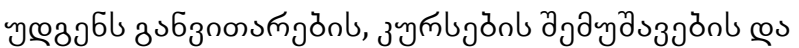

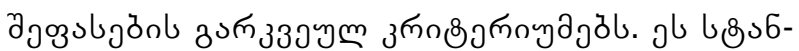

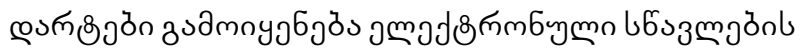

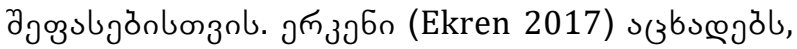

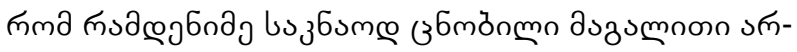

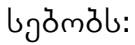

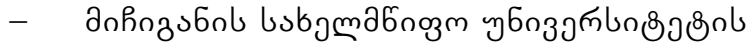

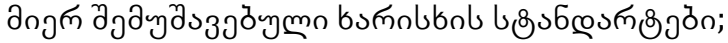

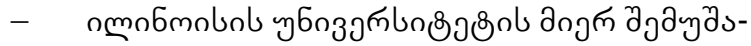

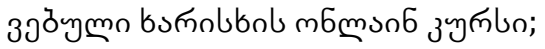

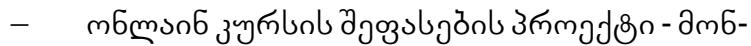

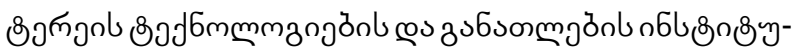
$8 \cap$;

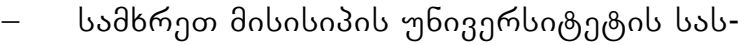

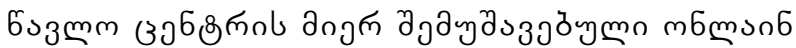

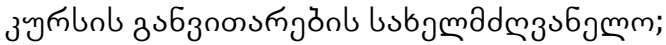

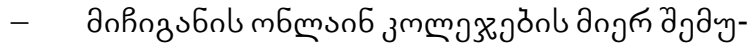

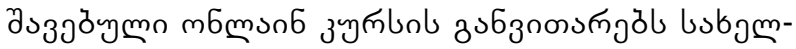
дамзงбјмmm;

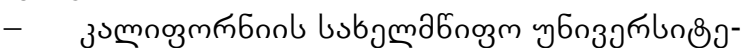

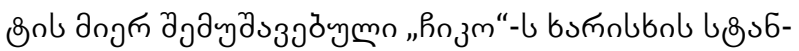

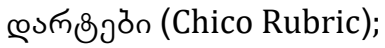

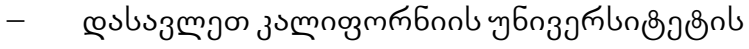

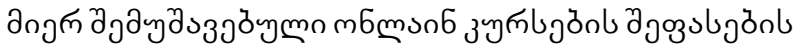

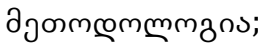

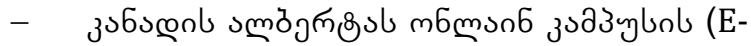
campus Alberta) jmod8 mozว.

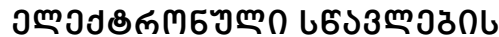 \&03ง3}

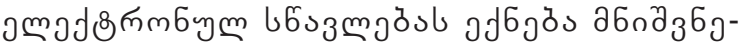

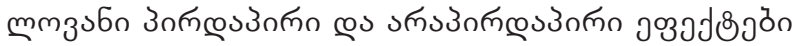

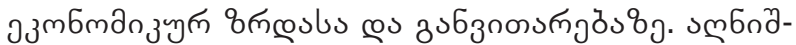

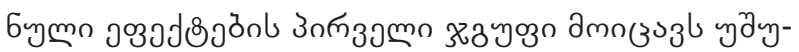

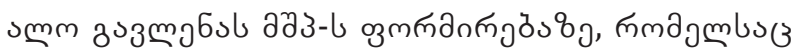

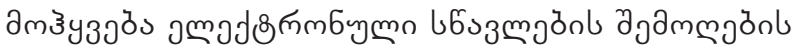

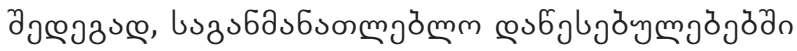

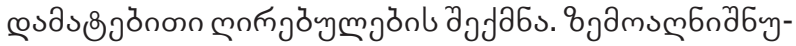

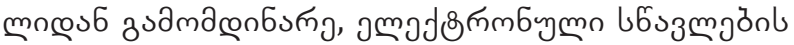

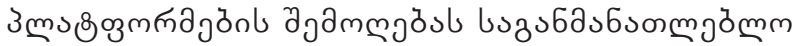
œs6б

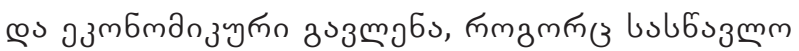

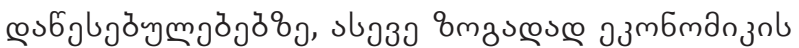

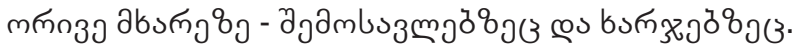

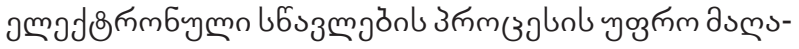

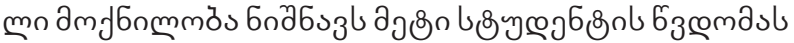

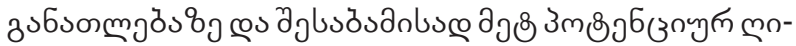

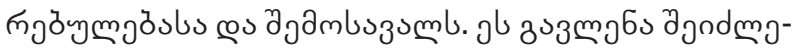

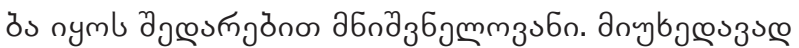

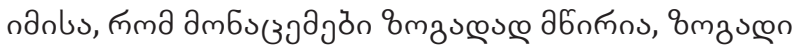

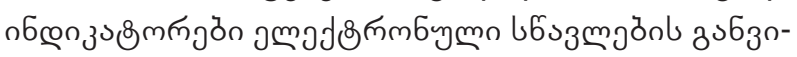

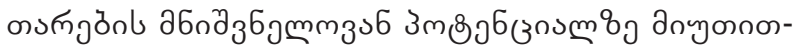

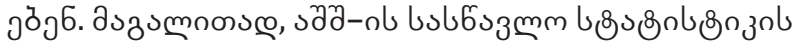

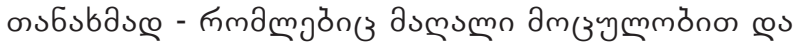

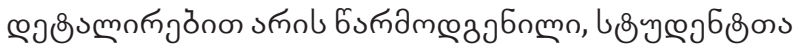




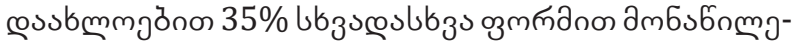

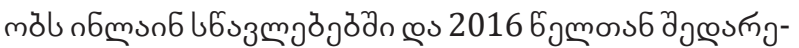

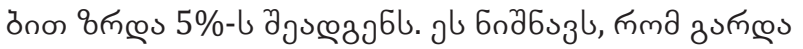

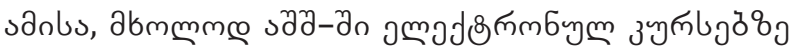

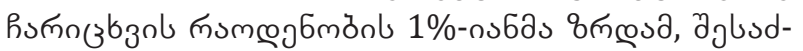

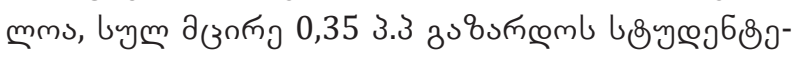

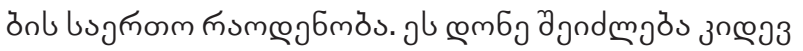

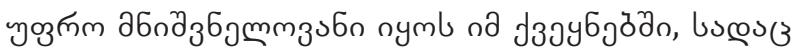

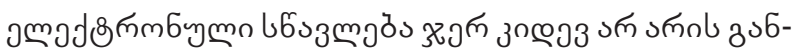

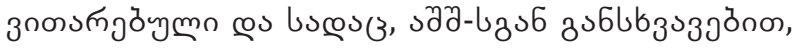

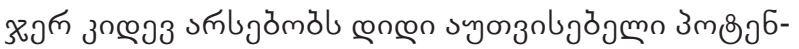

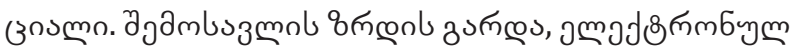

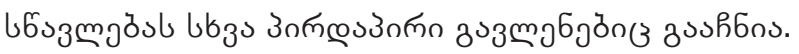

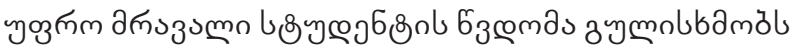

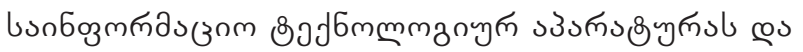

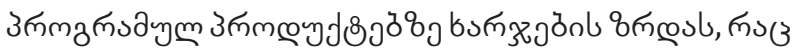

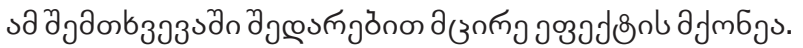

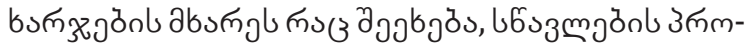

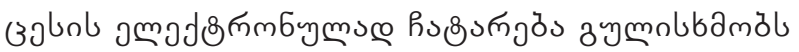

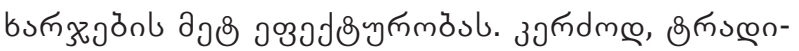

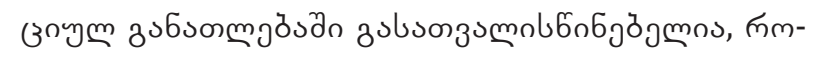

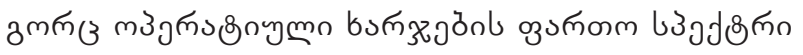

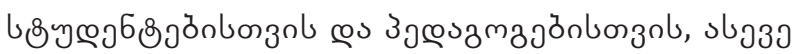

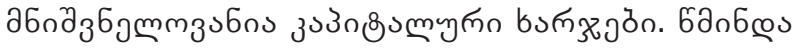

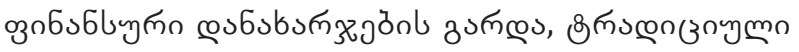

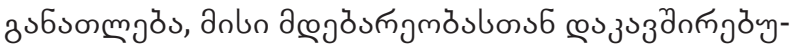

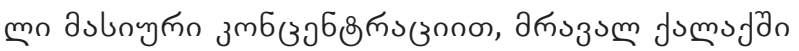

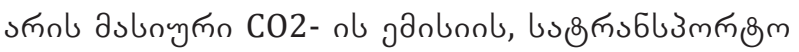

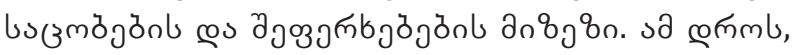

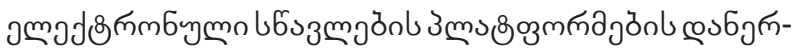

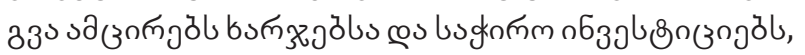

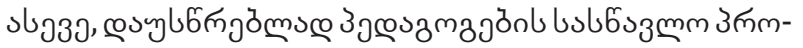
३з

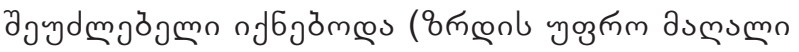

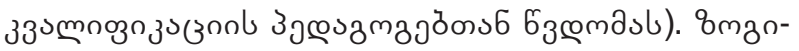

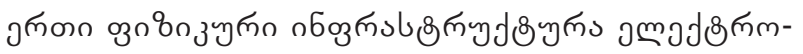

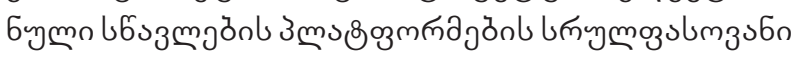
לا

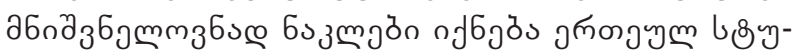

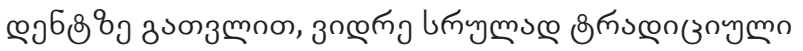

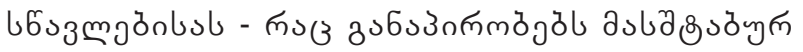

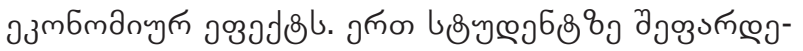

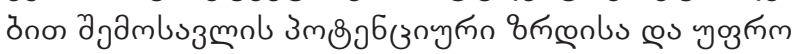

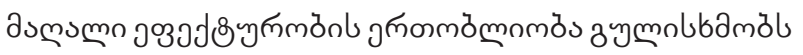

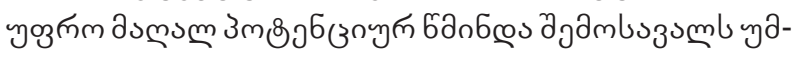

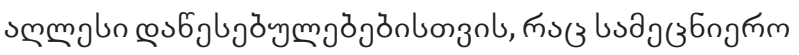

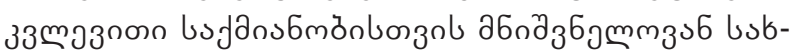

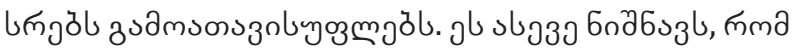

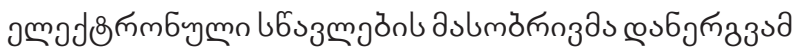

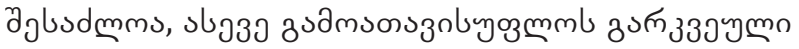

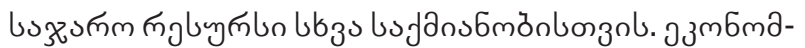

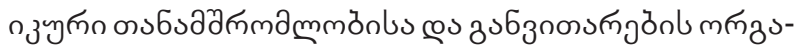

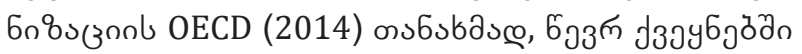

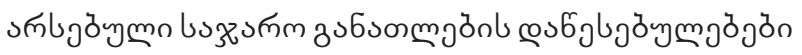

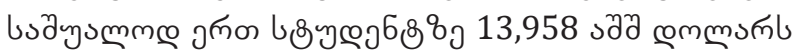

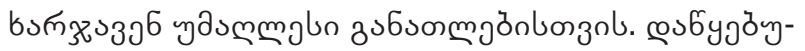

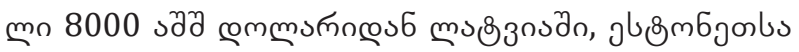

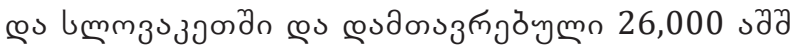

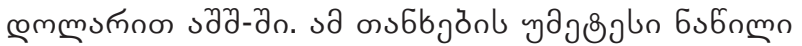

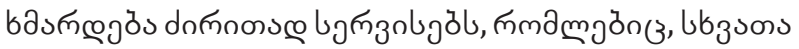

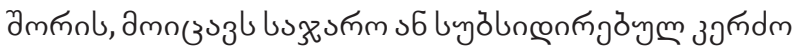

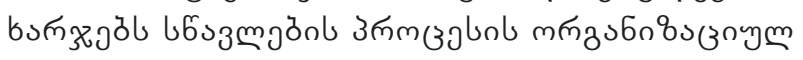

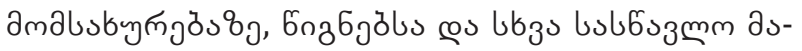

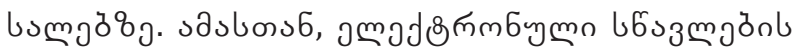

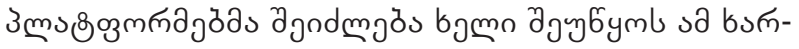

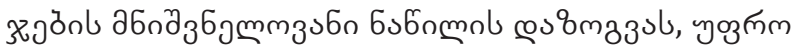

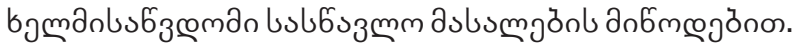

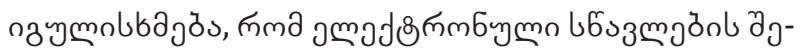

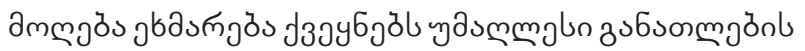

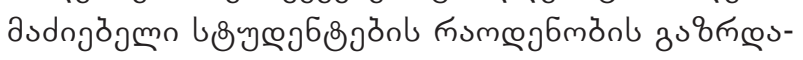

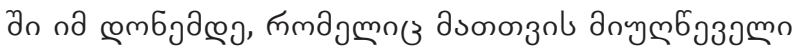

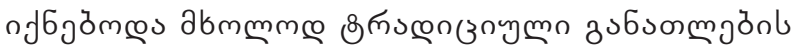

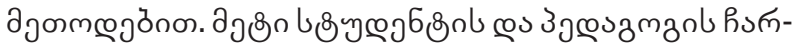

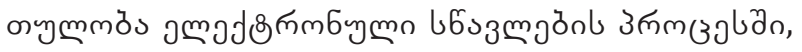

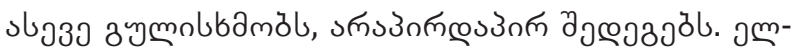

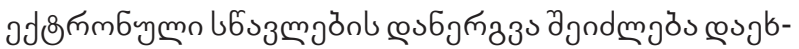

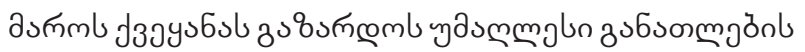

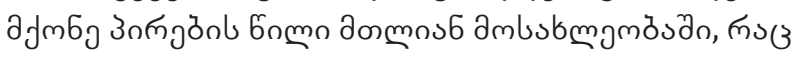

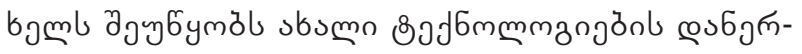
33د. ة

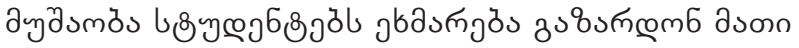

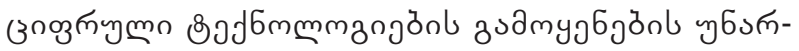

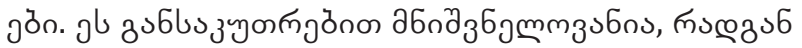

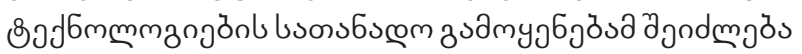

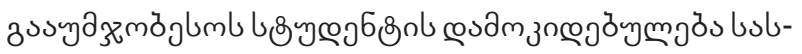

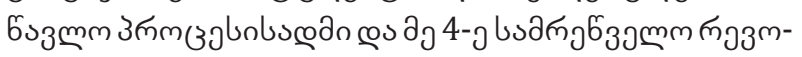

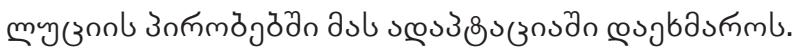

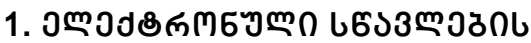

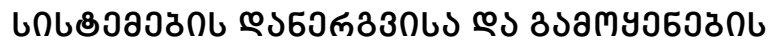

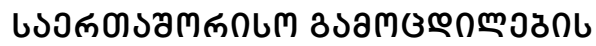

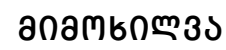

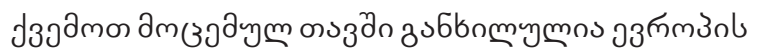

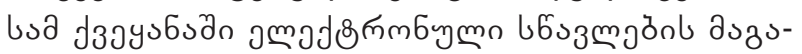

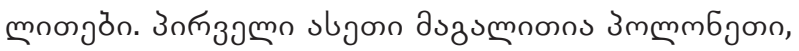

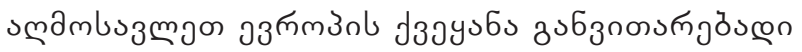

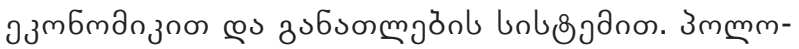

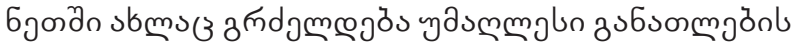




\section{ISSN 2667-9752(Online)}

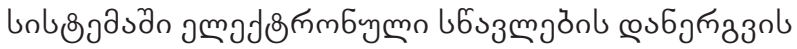

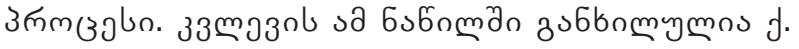

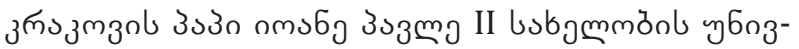

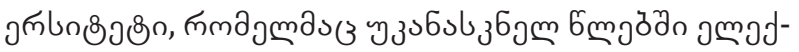

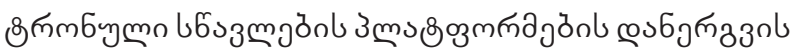

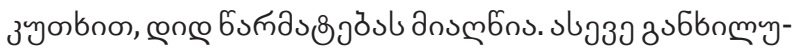
mos эmo

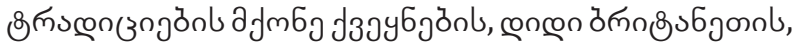

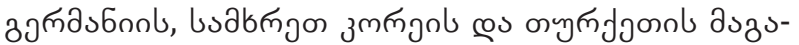

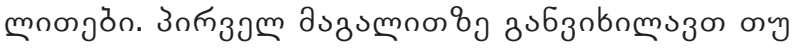

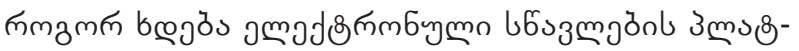

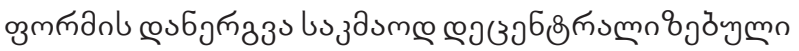

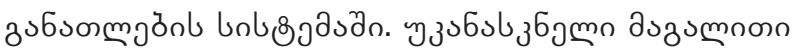

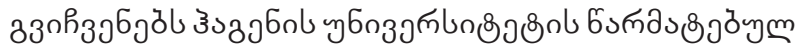

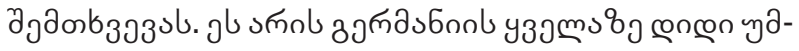
sмmmglo bub6sз

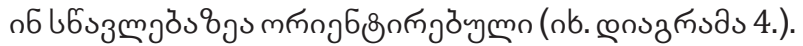

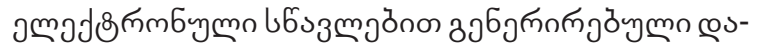

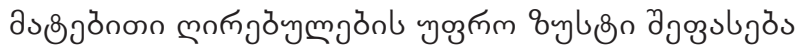

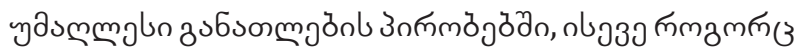

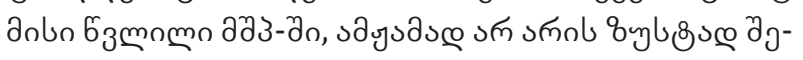

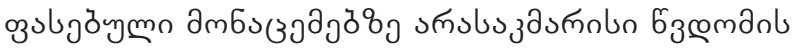

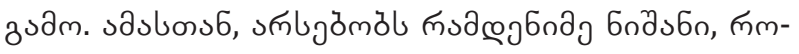

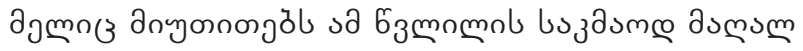
œs วquпূ

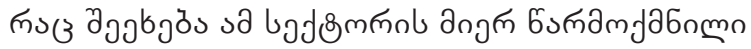

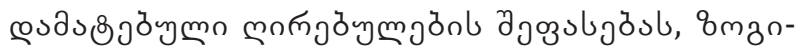

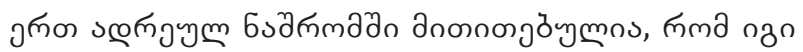

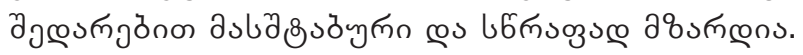

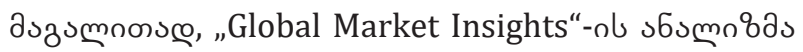

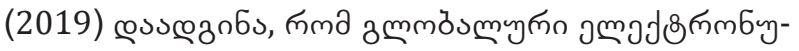

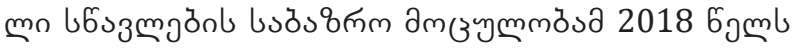

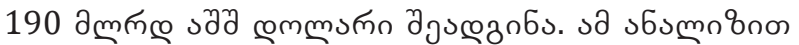

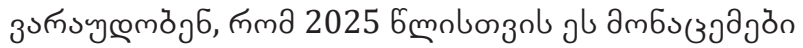

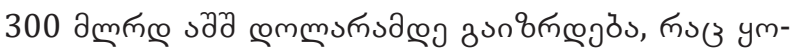

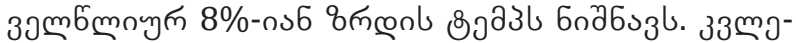

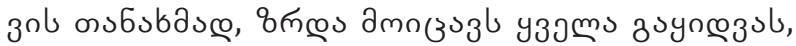

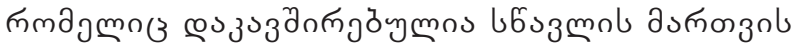

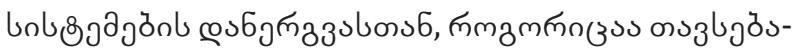

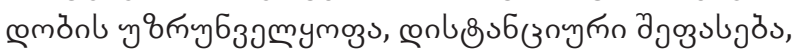

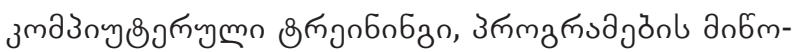

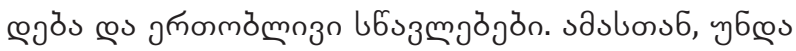

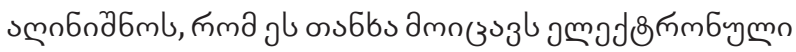

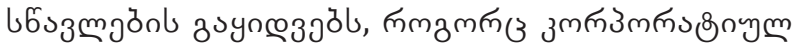

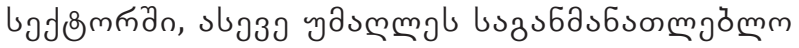

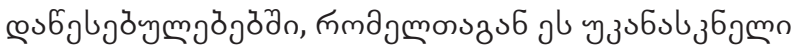

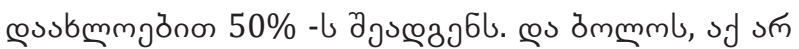

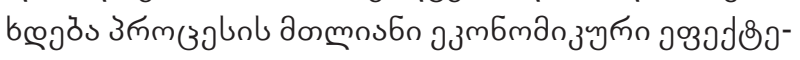

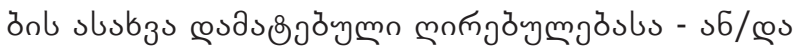

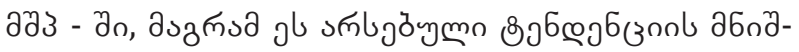

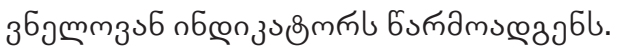

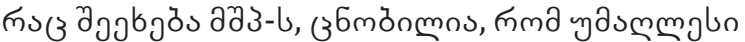

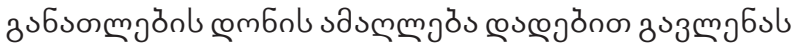

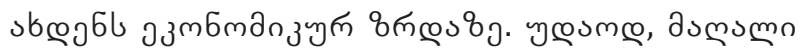

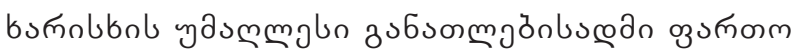

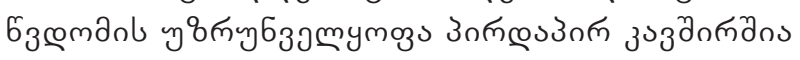

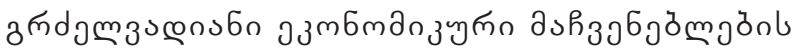

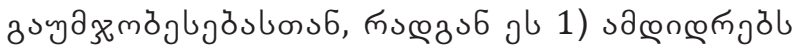

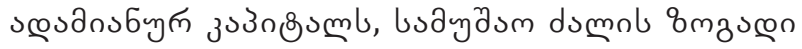

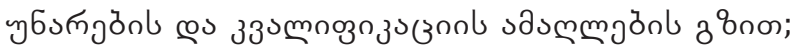

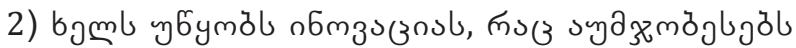

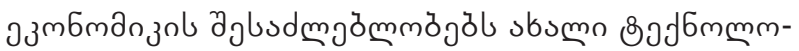

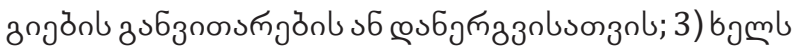

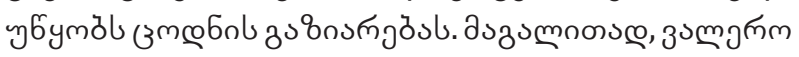
œs 3ง6 mo6nбol (Valero and Van Reenen 2019) ๓s-

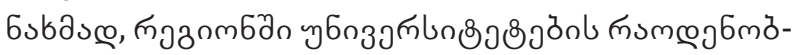

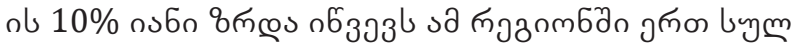

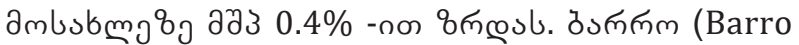

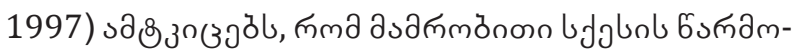
дっœа

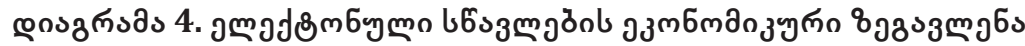

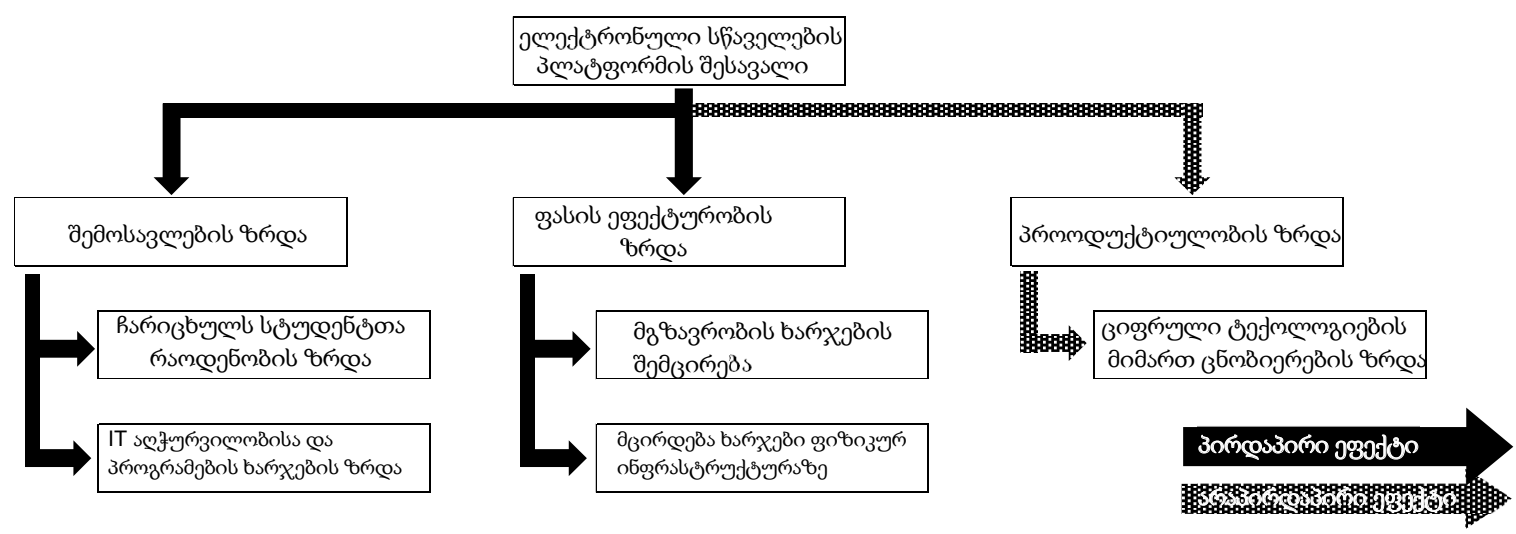

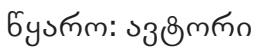




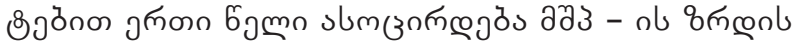

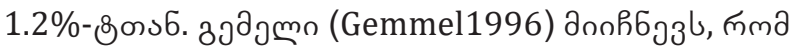

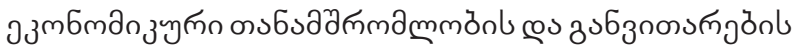

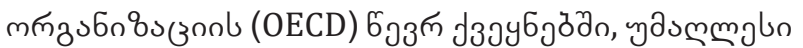

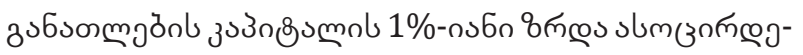

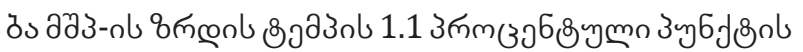

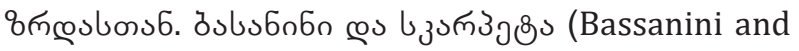

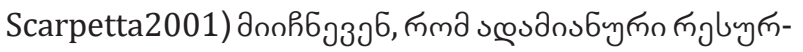

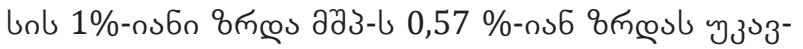

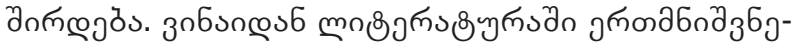

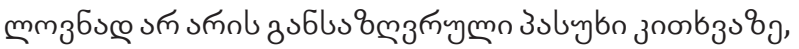
s

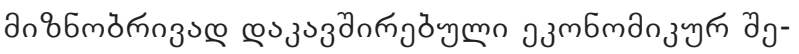

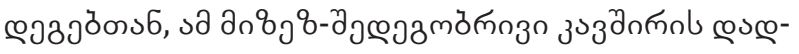

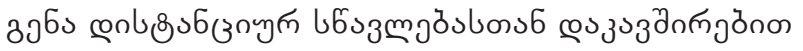

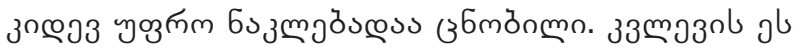

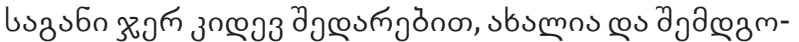

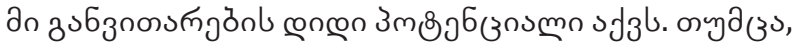

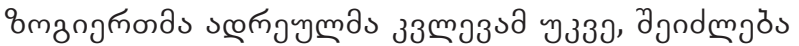

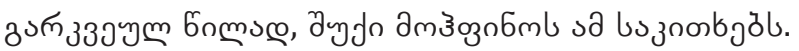

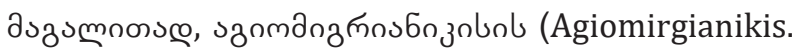

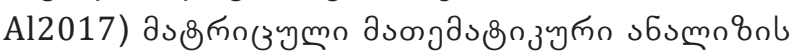

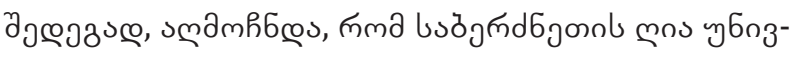
j

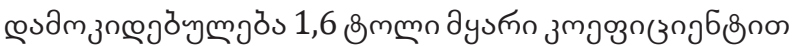
bubnsong

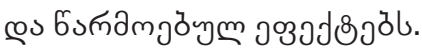

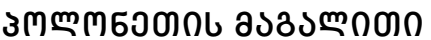

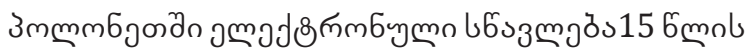

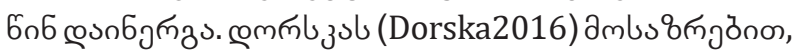

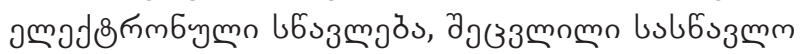

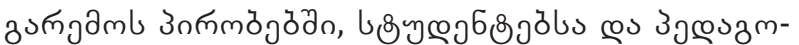

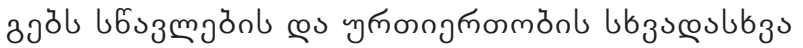

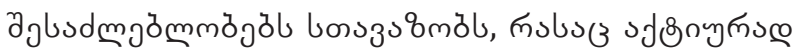

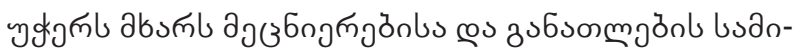

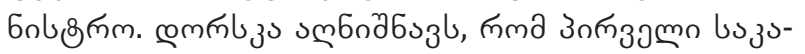

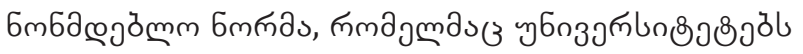

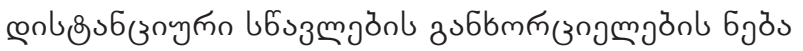

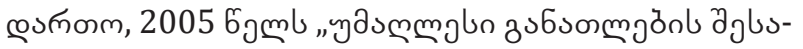

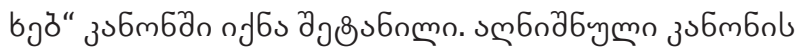

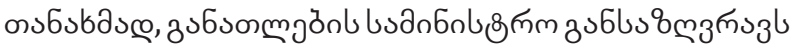
amonkmзб

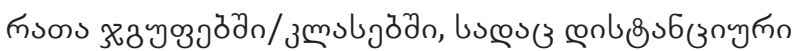

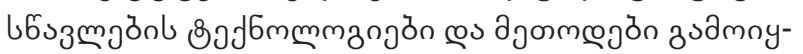
jб

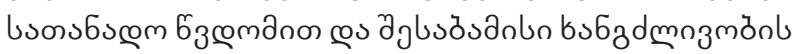

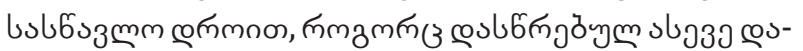

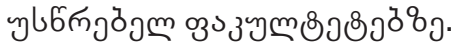

3mmmbjonnb yaummgln zubsonmgönb bsan6nb-

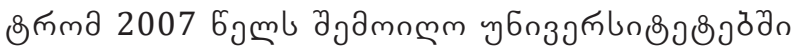

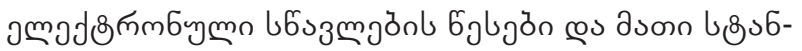

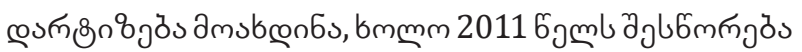

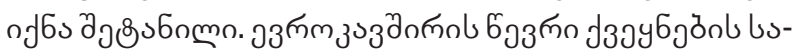

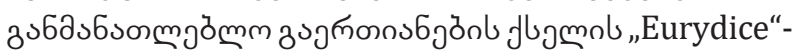

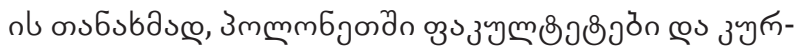

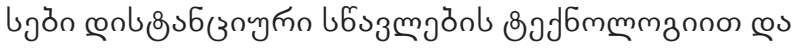

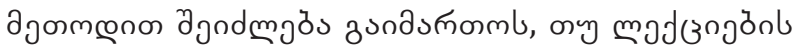

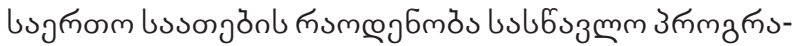

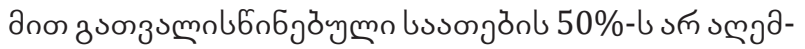

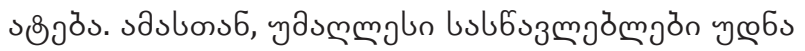

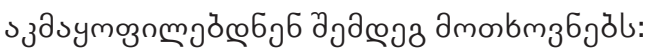

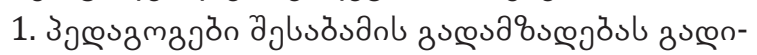

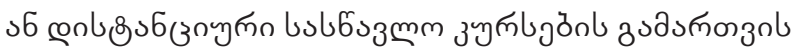

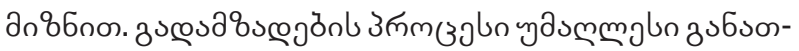

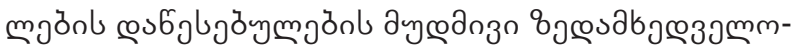

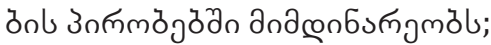

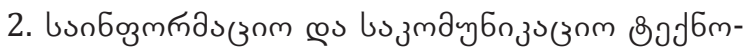

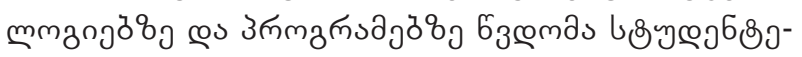

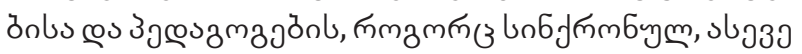

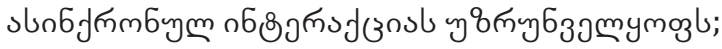

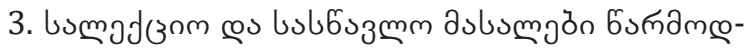

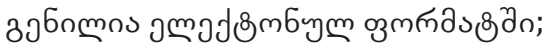

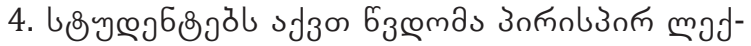

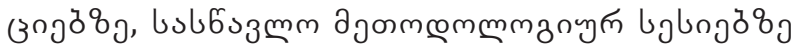

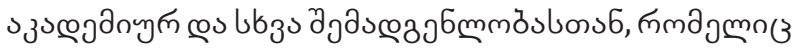

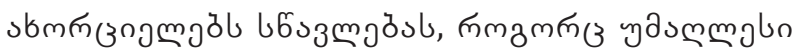

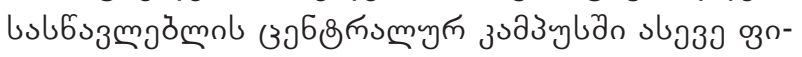
moumgàdn;

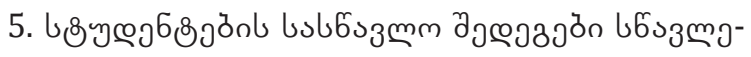
on u उmmang

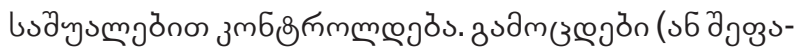

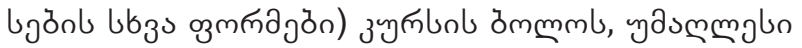

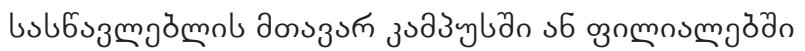
৪ัฒ๓๓

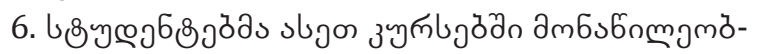

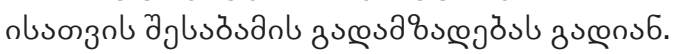

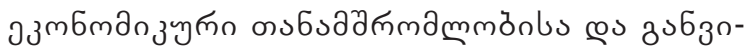

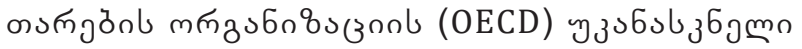

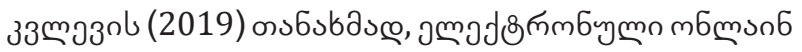

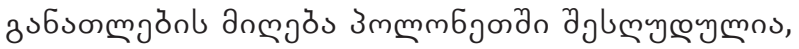

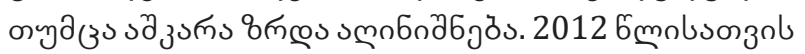

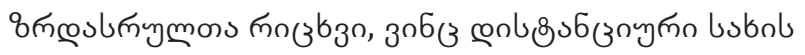

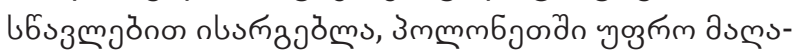

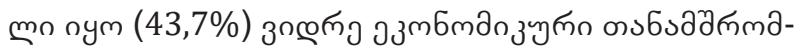

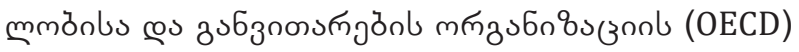

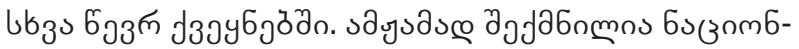

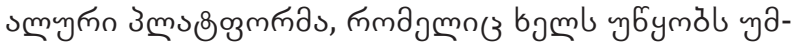




\section{ISSN 2667-9752(Online)}

sмm

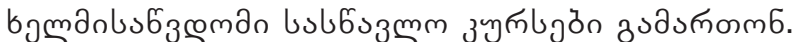

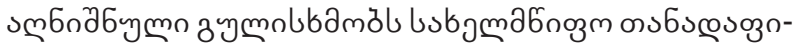

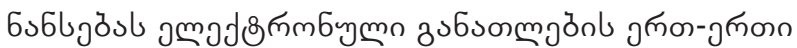

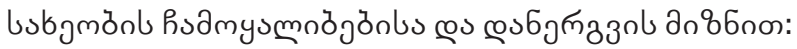

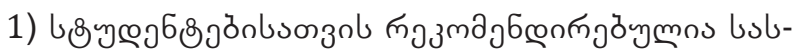

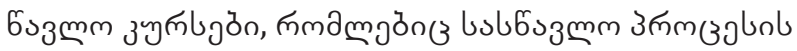
œ১а১৪ృ

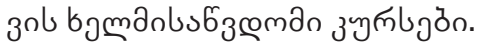

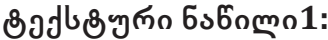

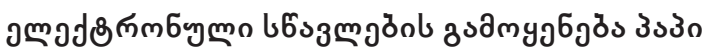

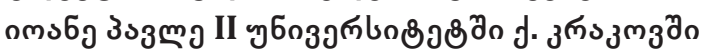

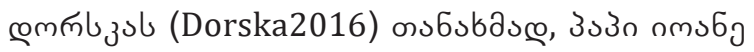

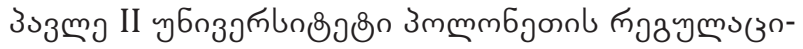

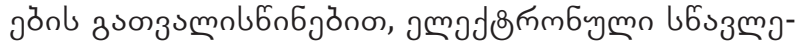

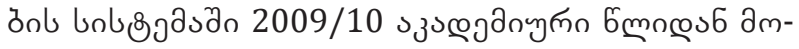

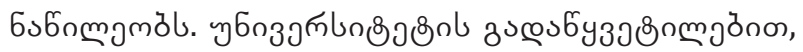

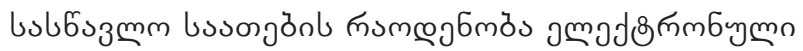

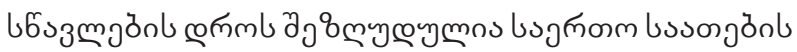

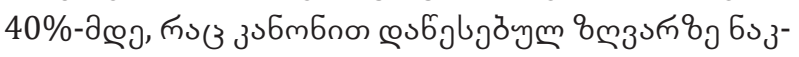

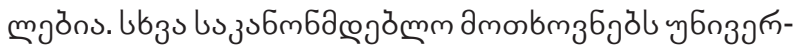

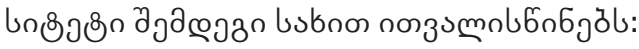

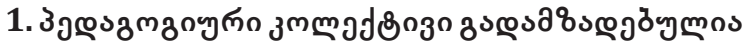
œob8

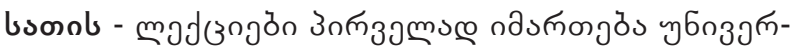
un\&

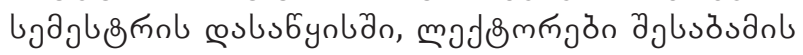

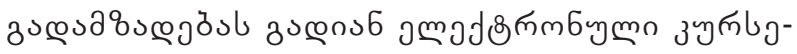

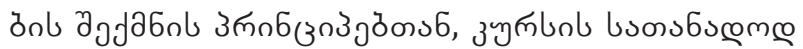
ho

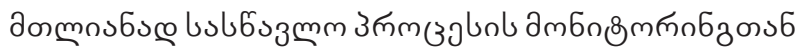

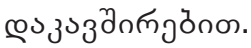

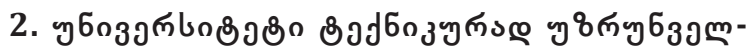

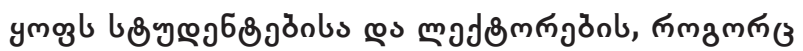

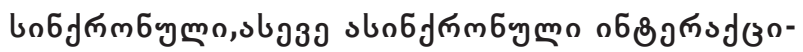

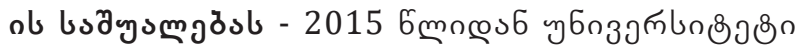

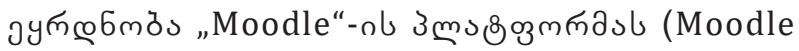
platform - Modular Object-Oriented Dynamic Learning

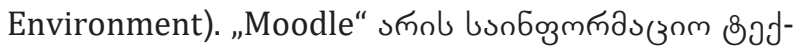

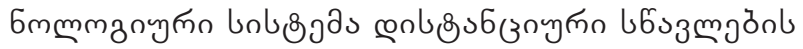

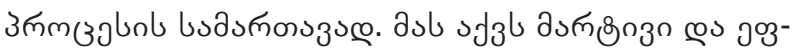

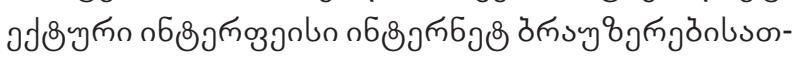

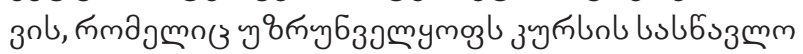

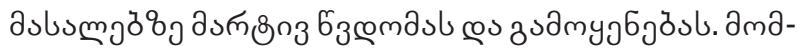

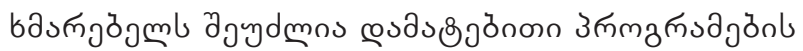

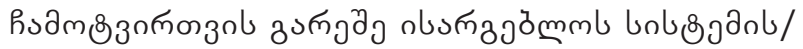

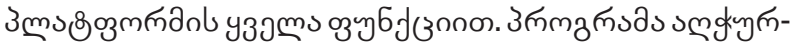

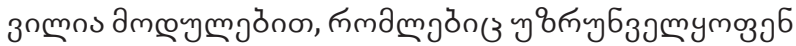

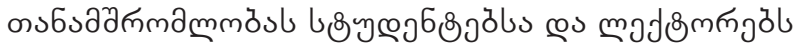

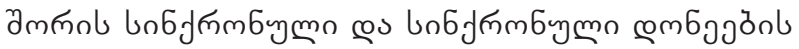

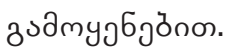

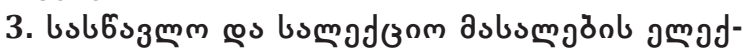

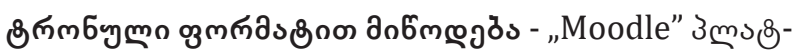
озмпда \&

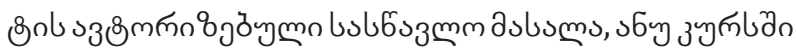

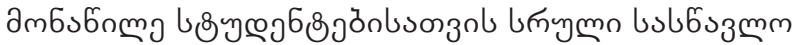

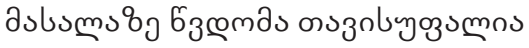

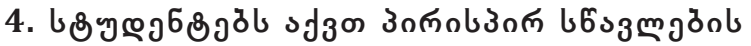

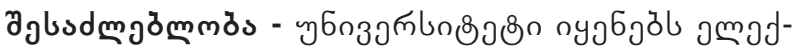

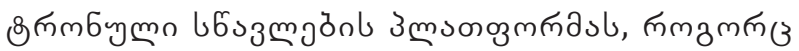

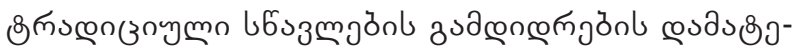

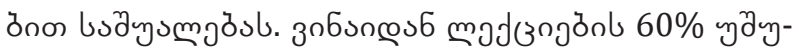

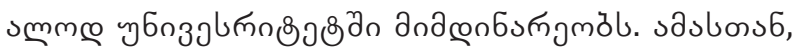

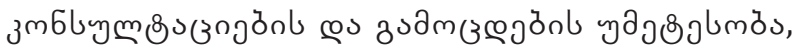

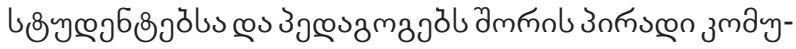

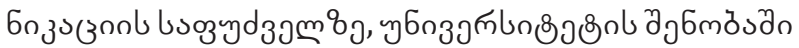
nдงmogòs.

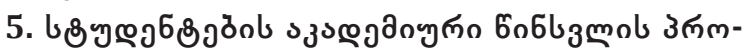

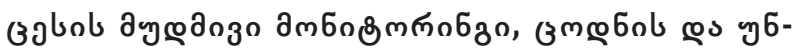

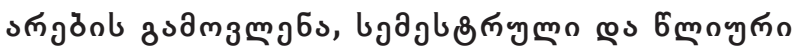

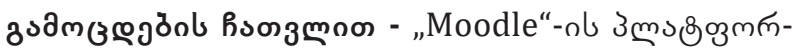

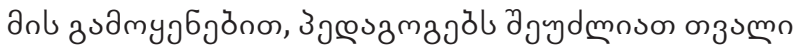
งœว

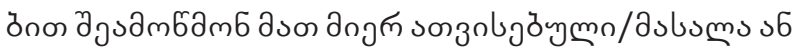

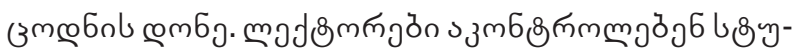

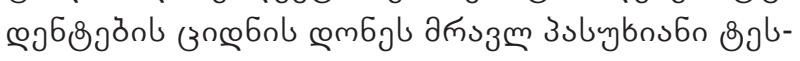

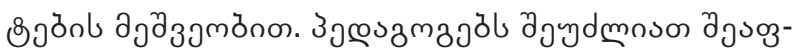

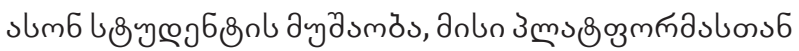

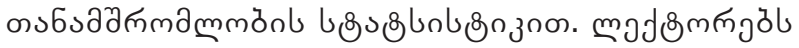

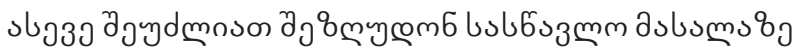

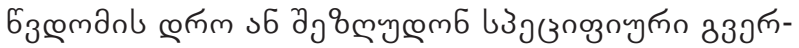

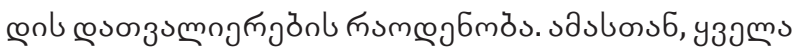

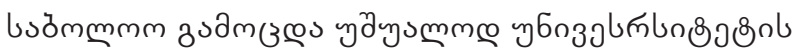

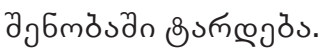

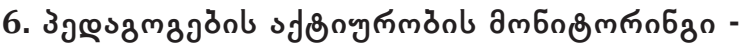

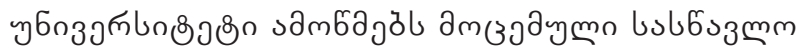

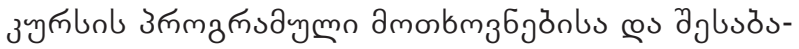

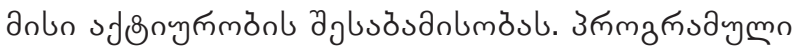

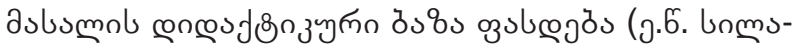

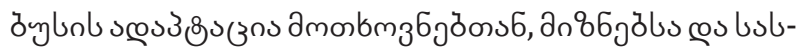

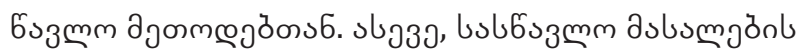

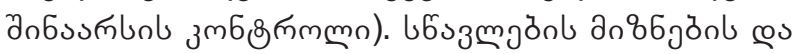

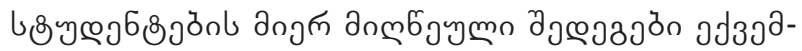

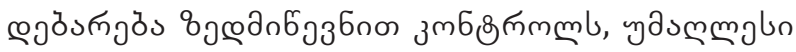

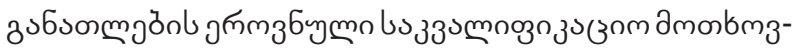

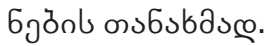




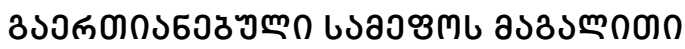

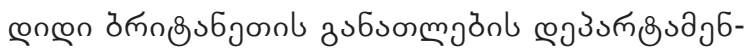

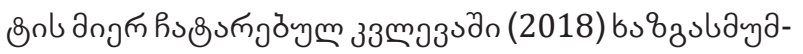

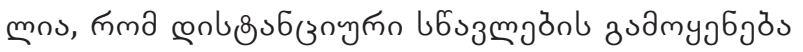

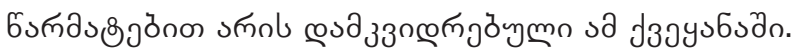

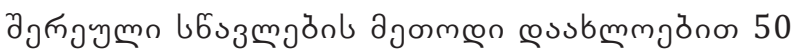

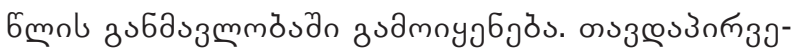

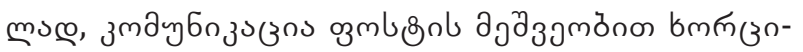

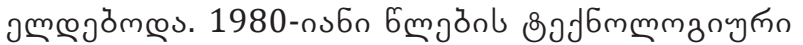

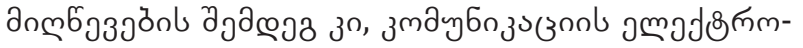

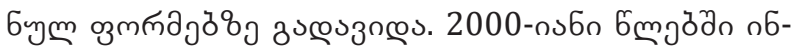

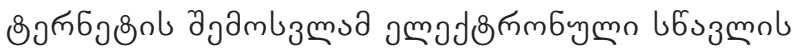

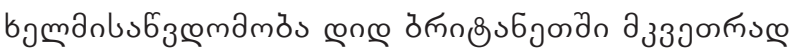

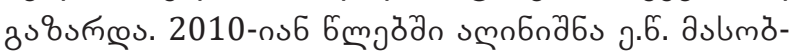

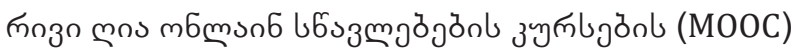

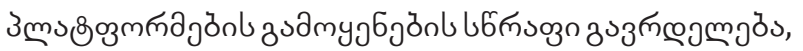

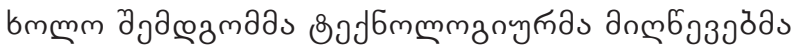

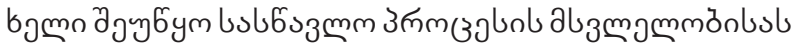

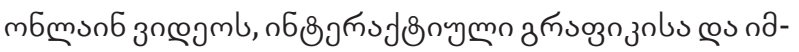

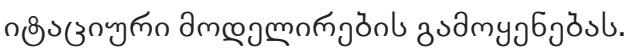

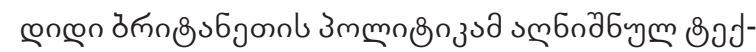

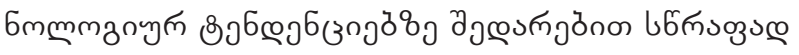

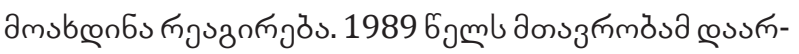

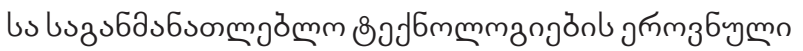

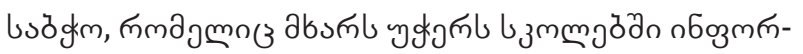

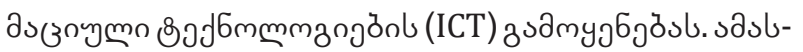

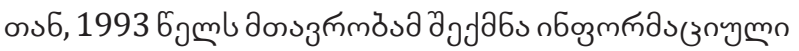

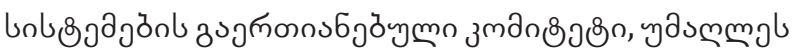

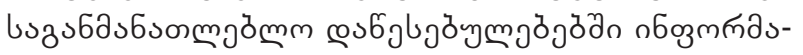

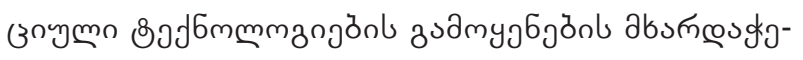

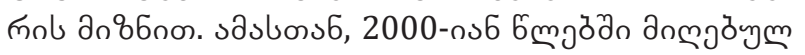

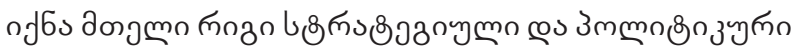
œмзудаб

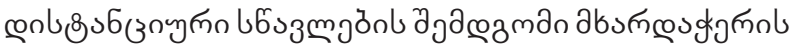
วก86กn.

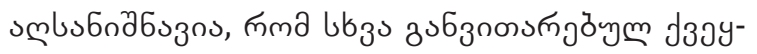

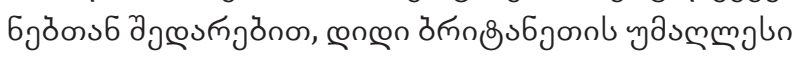

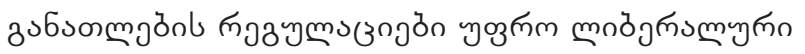

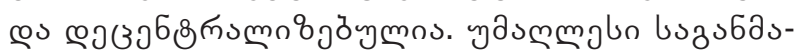

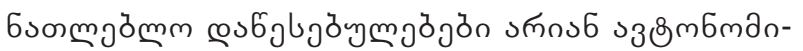

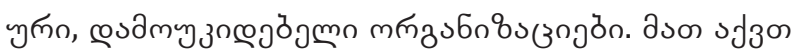

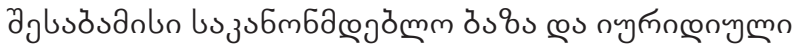

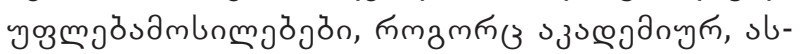

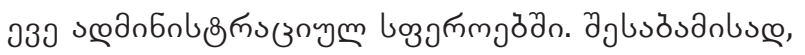

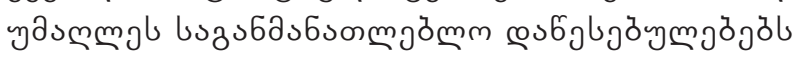

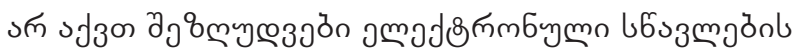

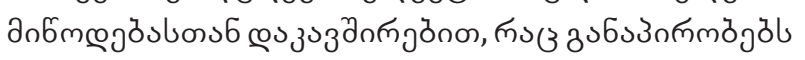

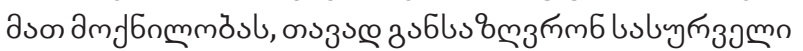
дпœамал пma

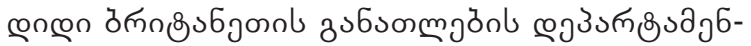

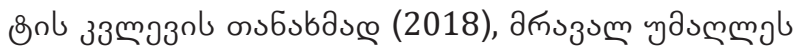

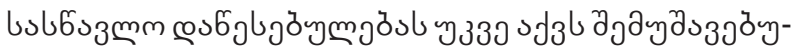

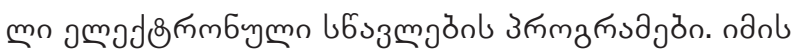

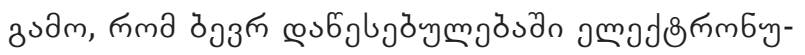

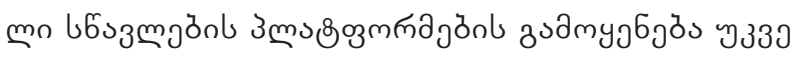

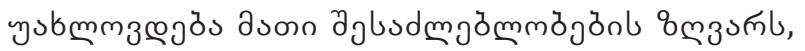

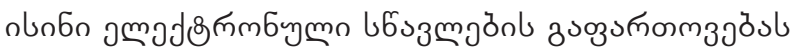

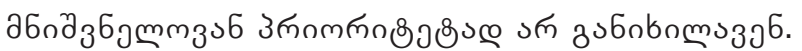

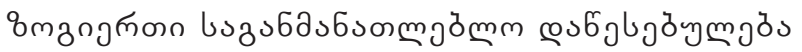

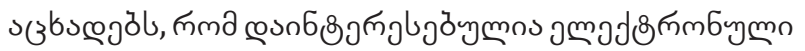

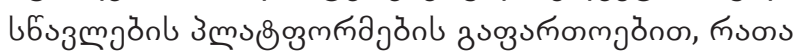

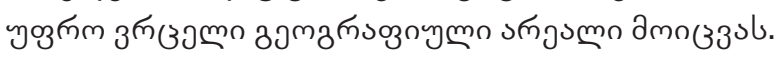

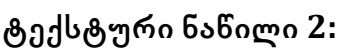

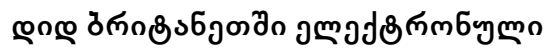

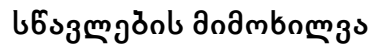

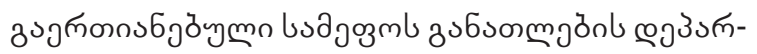

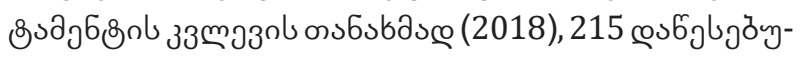

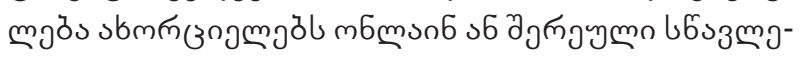
òn amalsubyng

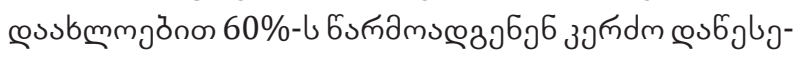

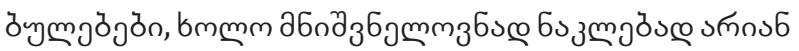

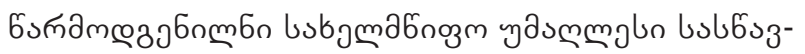

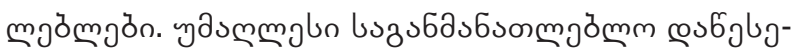

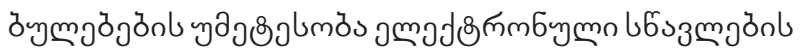

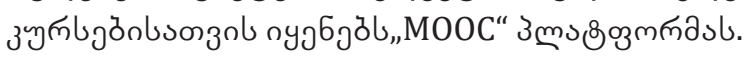

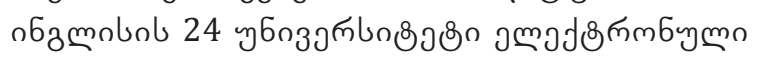

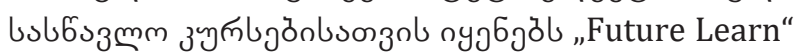

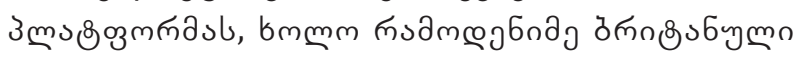

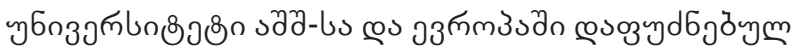

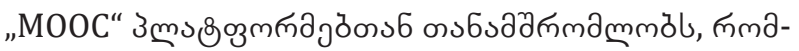

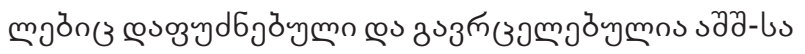

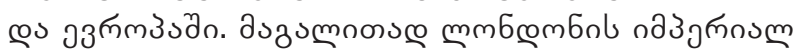

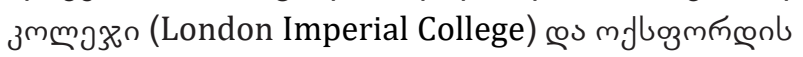

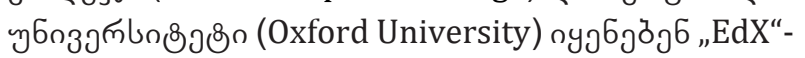

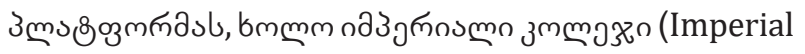

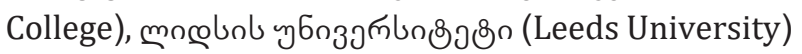

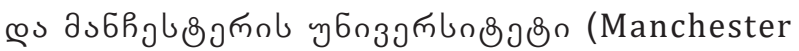

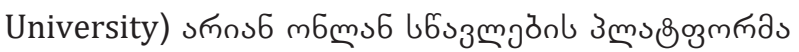

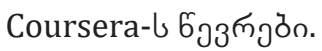

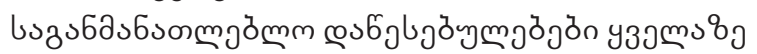

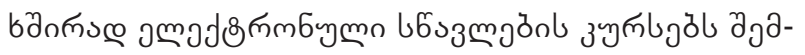

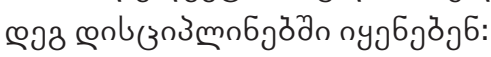

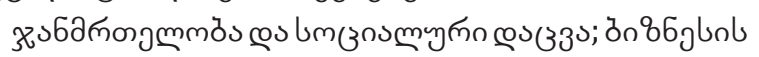

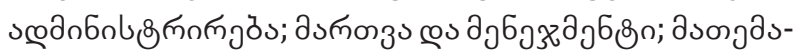

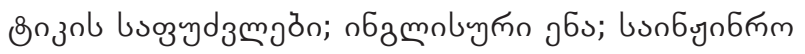

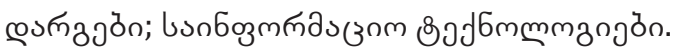




\section{ISSN 2667-9752(Online)}

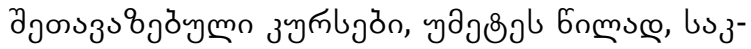

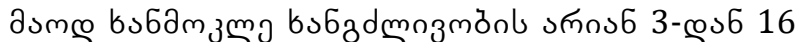

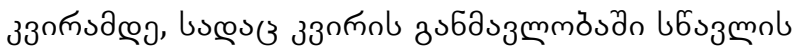

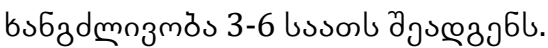

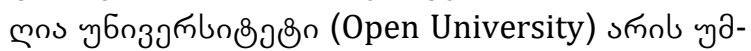
৮6зпмm

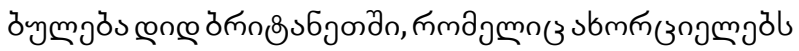

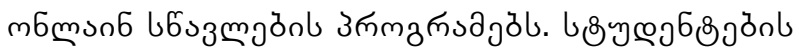

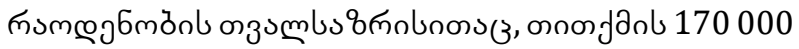

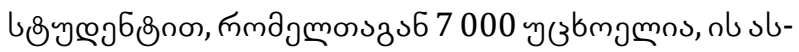

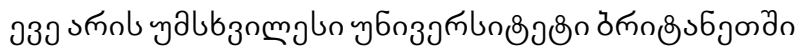

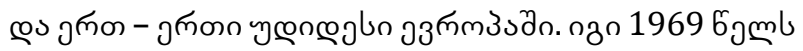

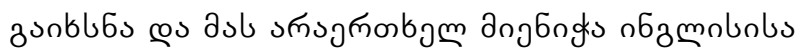

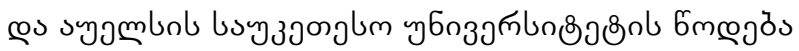

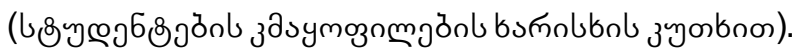

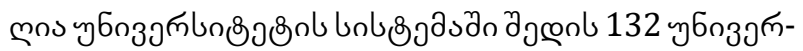

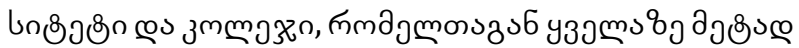

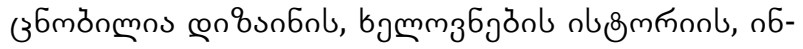

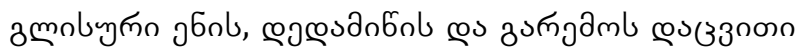
а

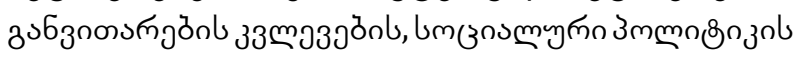

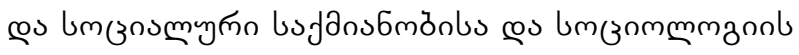

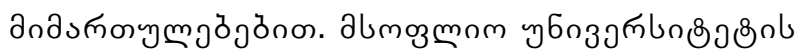

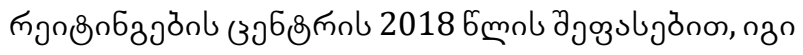

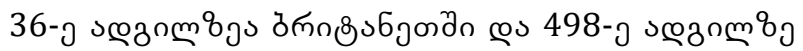
almoुmomàn.

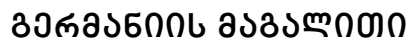

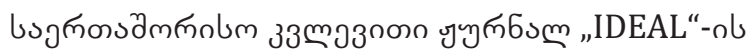

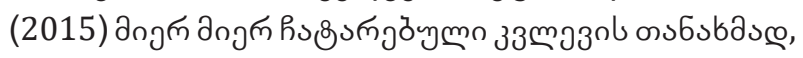

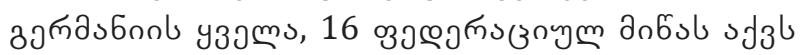

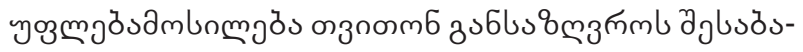
an

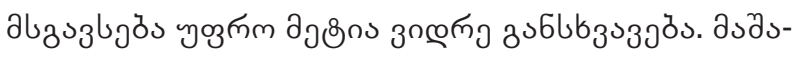

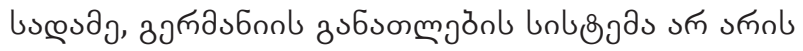

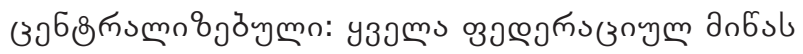

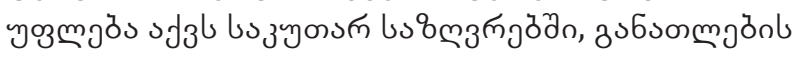

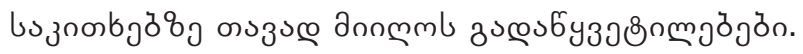

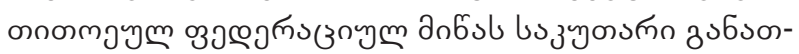

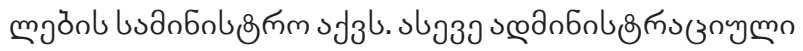

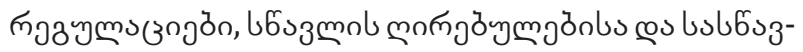

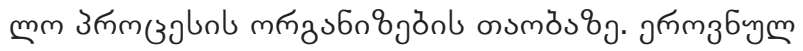

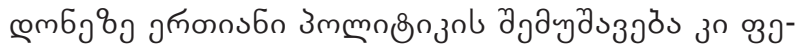

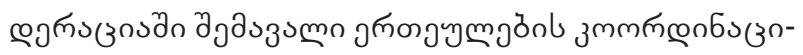

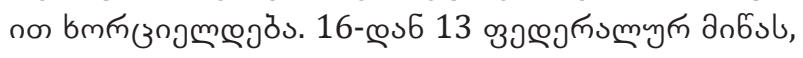

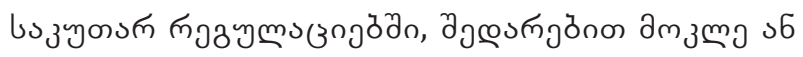

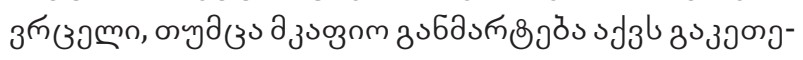

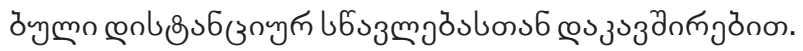

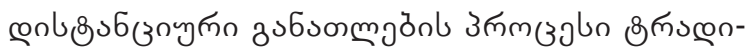

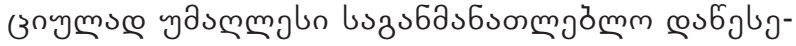

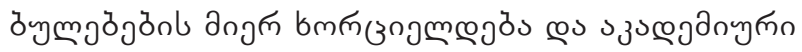

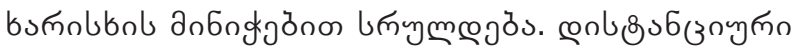

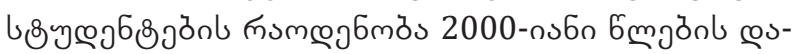

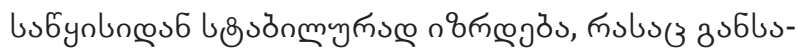

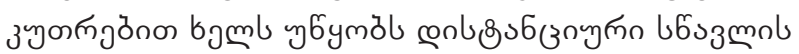

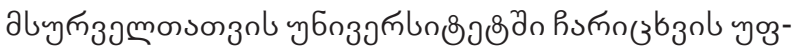

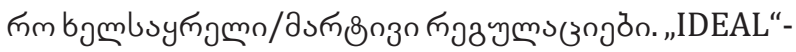

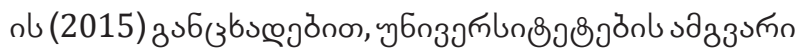

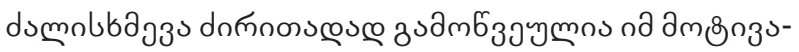

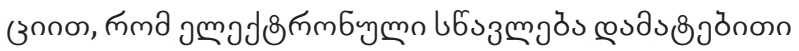

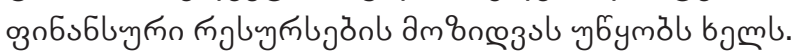

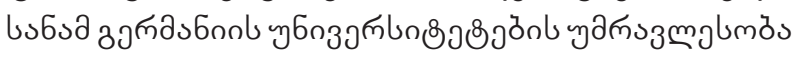

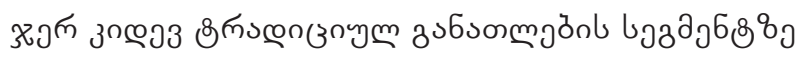

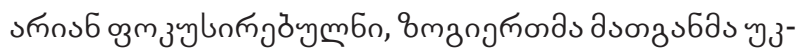

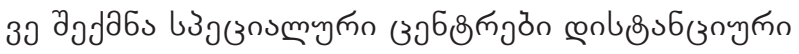

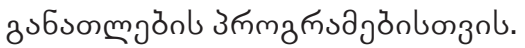

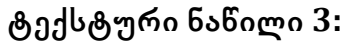

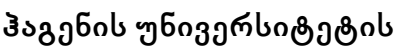

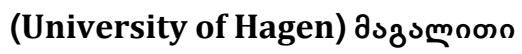

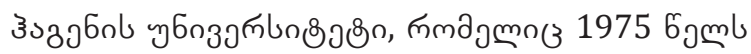

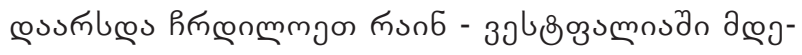

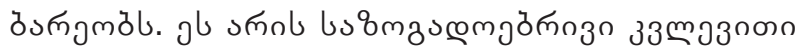

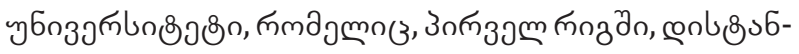

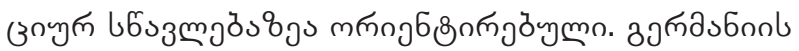

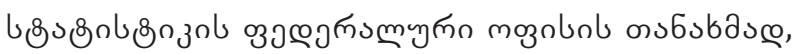

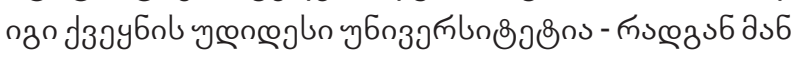

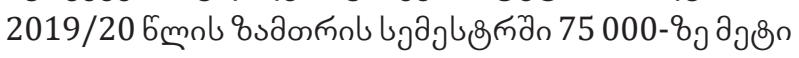

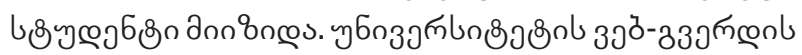

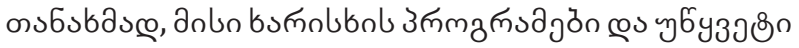

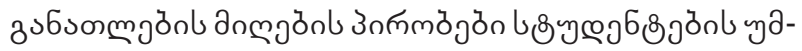

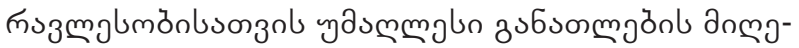
bu bymanbu6 з

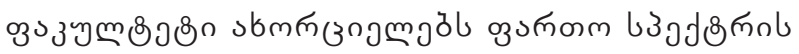

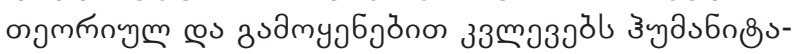

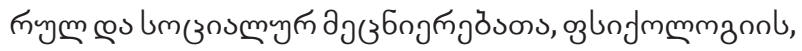

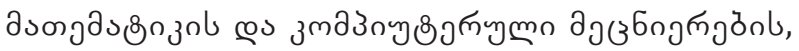

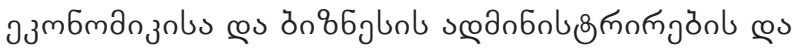

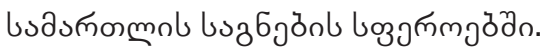

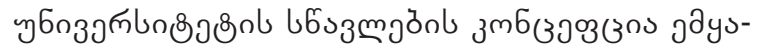

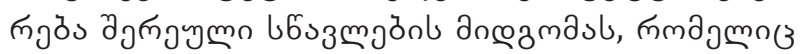

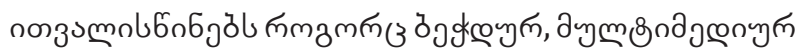

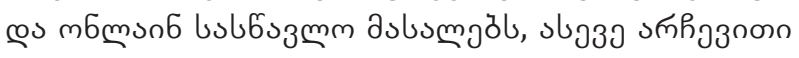

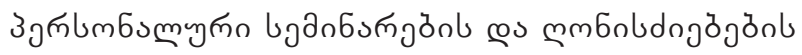

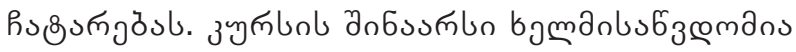

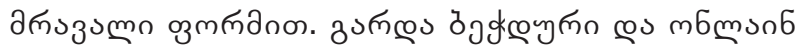

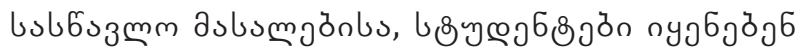




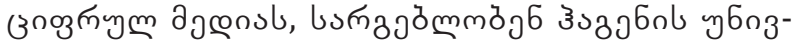
gศ̆

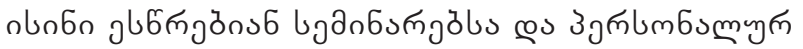

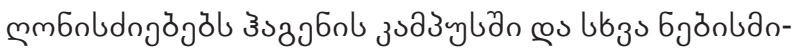

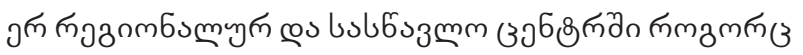

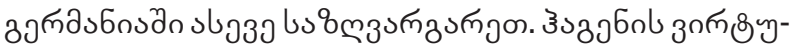

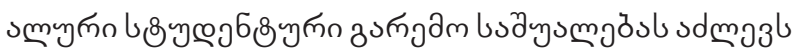

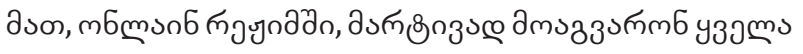

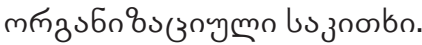

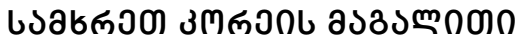

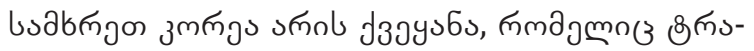

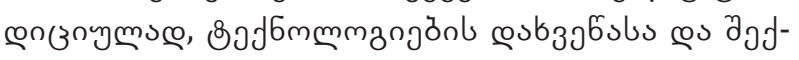

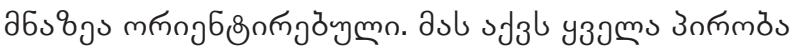

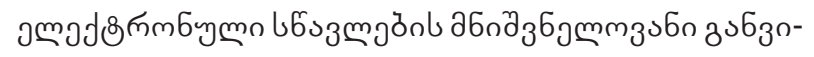

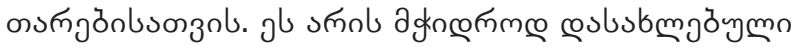

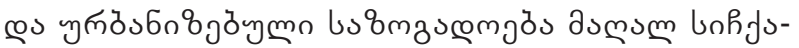

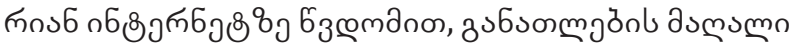

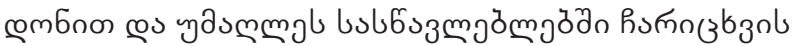

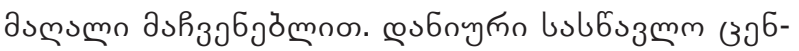

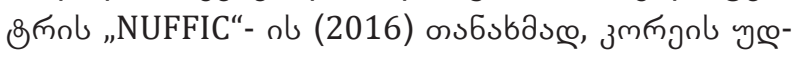
пœj

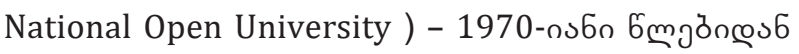

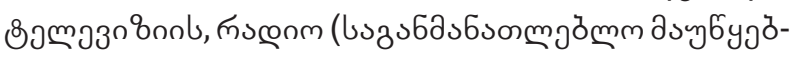

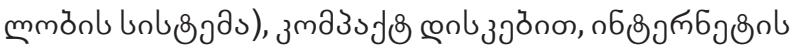

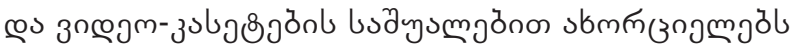

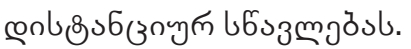

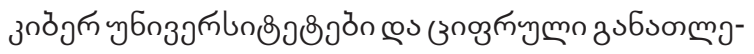

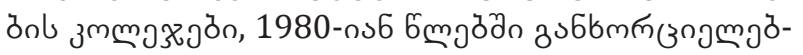

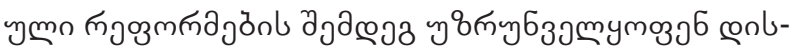

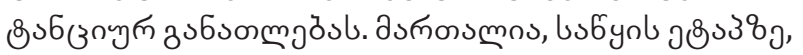

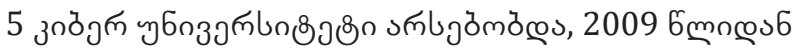
asonn mn

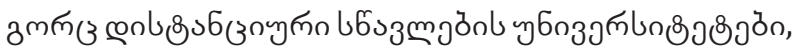

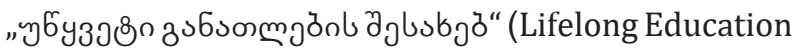

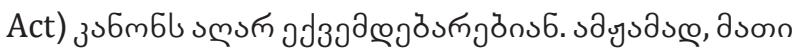

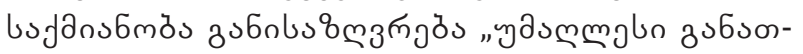

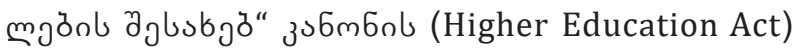

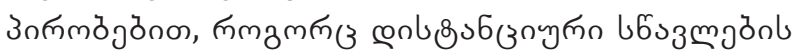

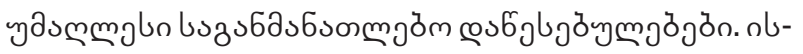

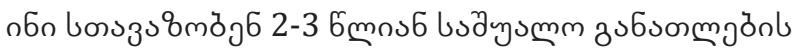

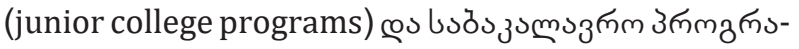

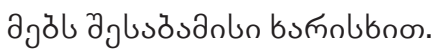

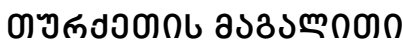

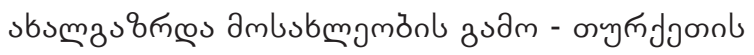

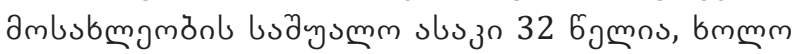

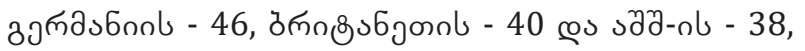

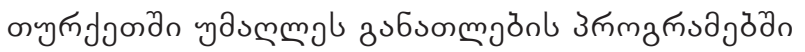

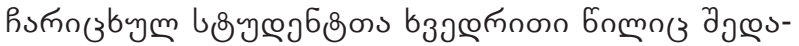

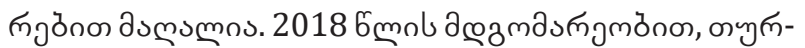

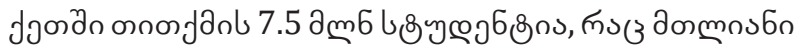

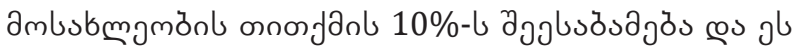

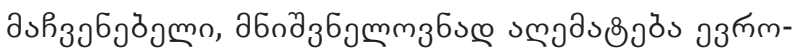

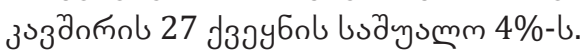

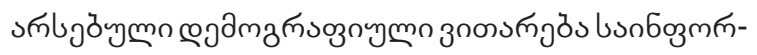

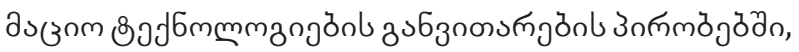

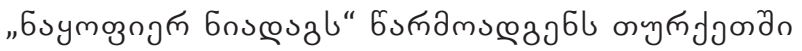

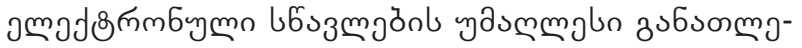

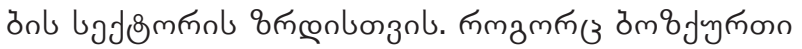

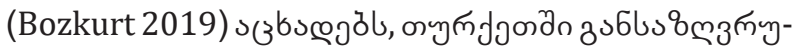

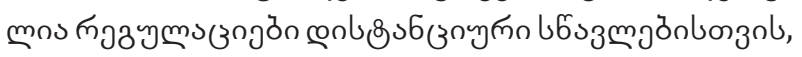

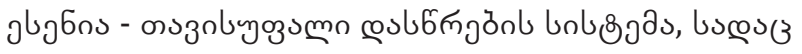

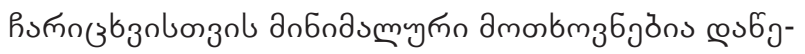

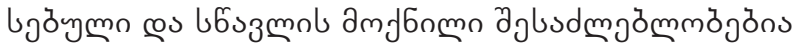

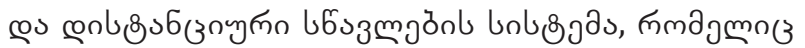

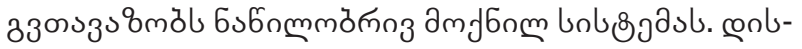

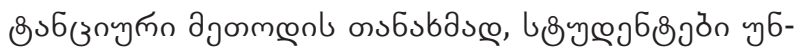

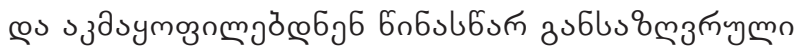

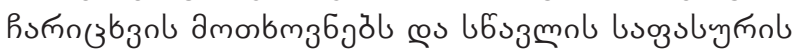

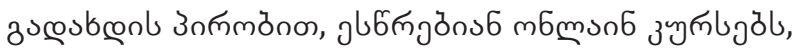

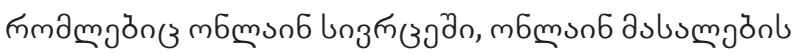

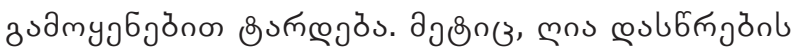

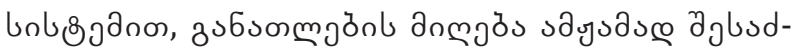

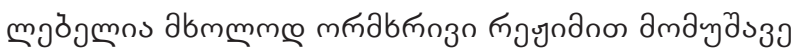

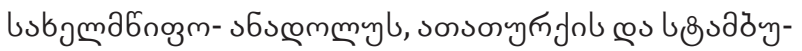
mol (Anadolu, Atatürk. Istanbul Universities) yбก弓jங̆ ᄂ6ззм

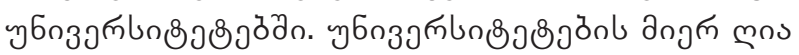

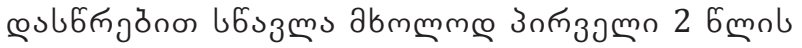

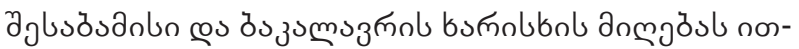

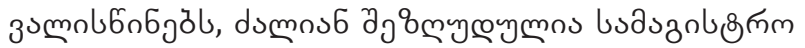

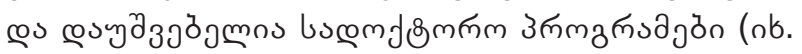

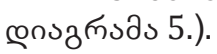

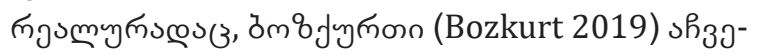
бృ

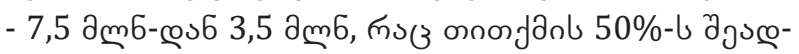

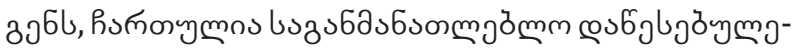

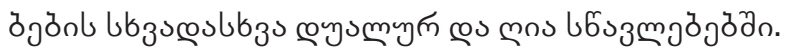

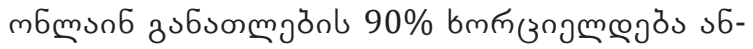

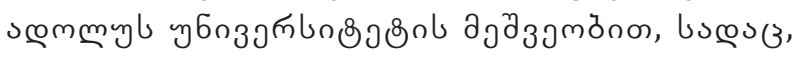

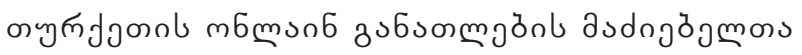

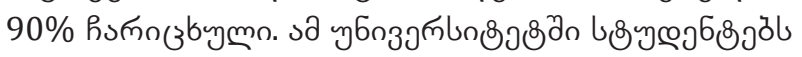

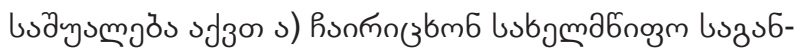

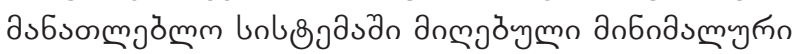

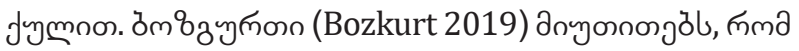

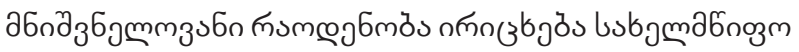




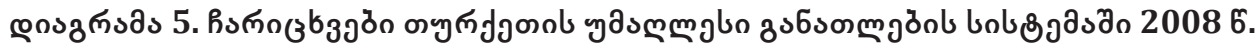

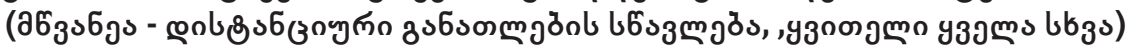

sumzongன்⿻上丨
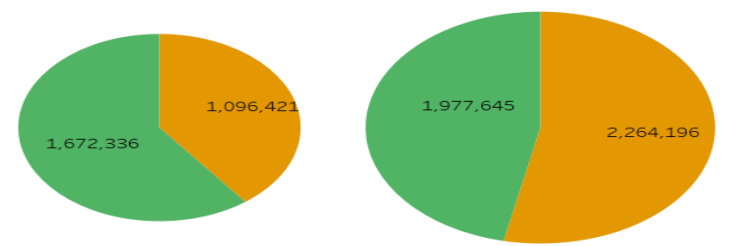

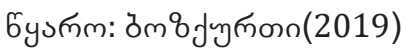

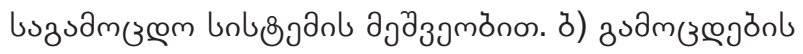

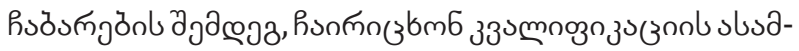

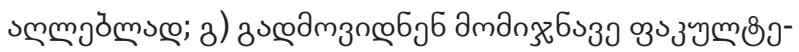

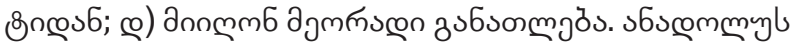

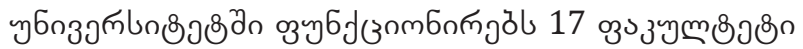

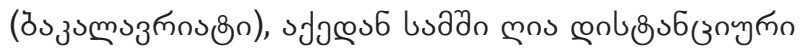

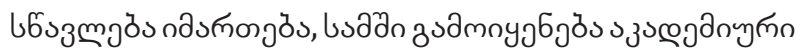

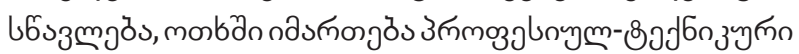

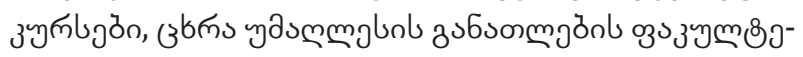

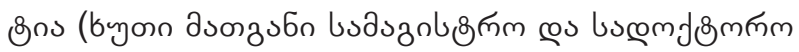

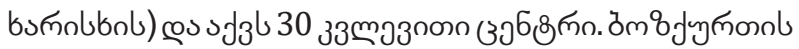

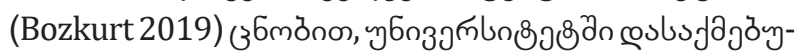

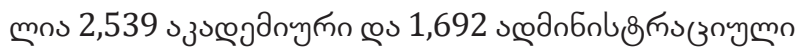

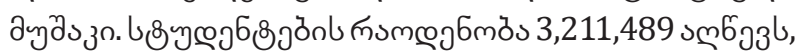

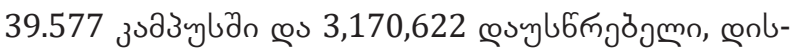

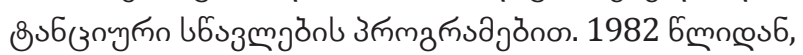

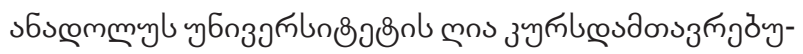

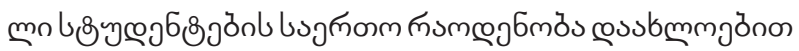
2.8 dnmombns.

\section{உ১৬336১}

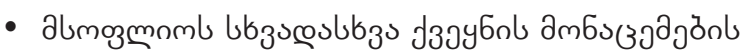

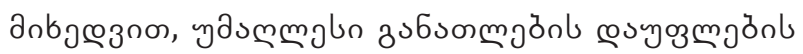

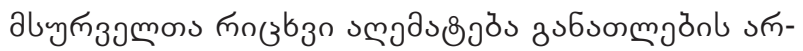

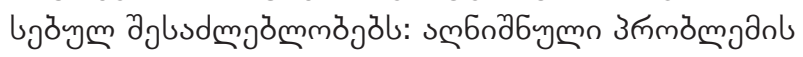

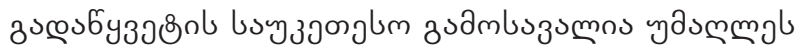

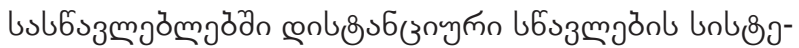
anl cos6gпззs.

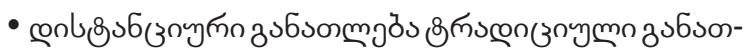

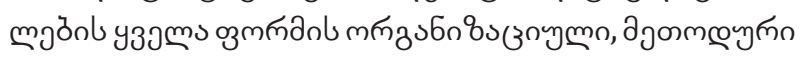

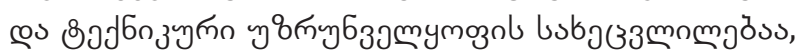

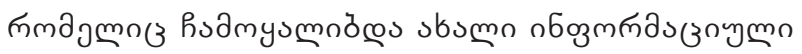

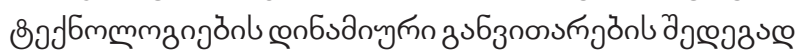

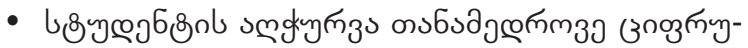

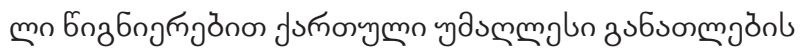

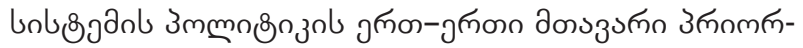

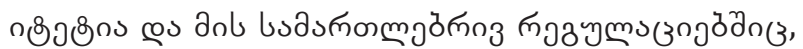

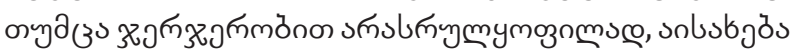

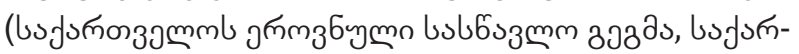

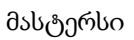

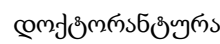

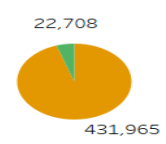

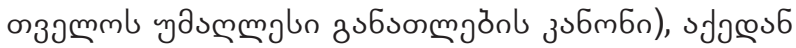

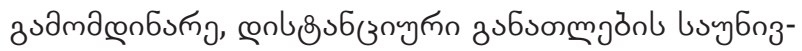

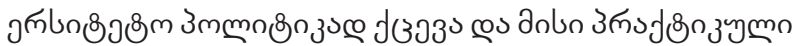

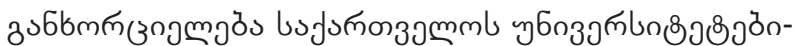

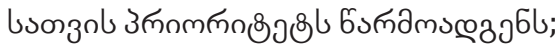

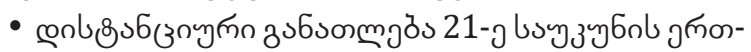

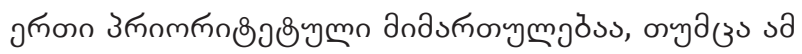

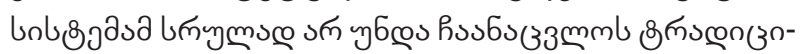

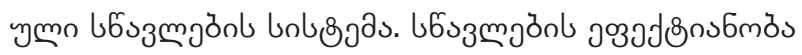

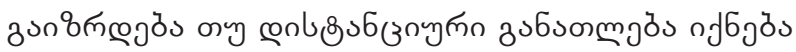

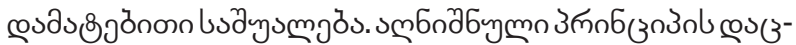
3

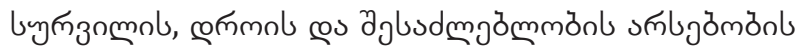

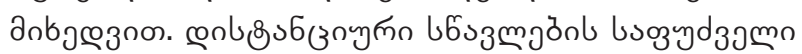

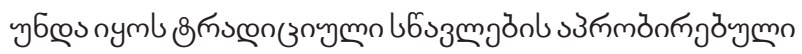

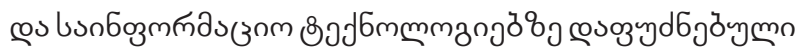

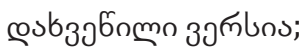

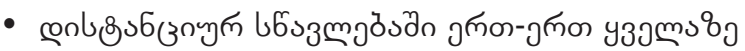
26пдз

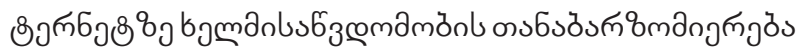

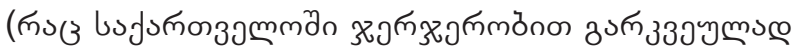

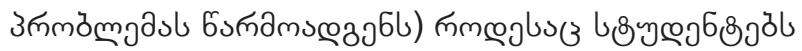

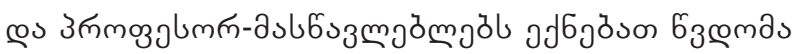

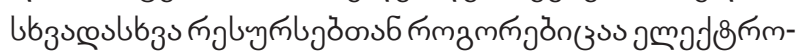

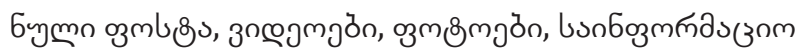

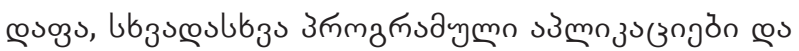

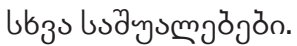

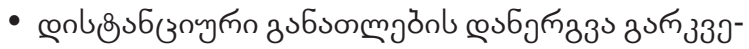

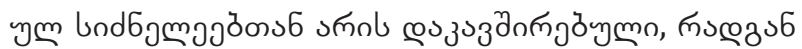

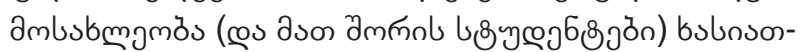

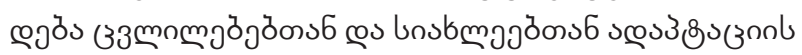

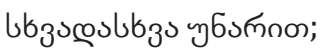

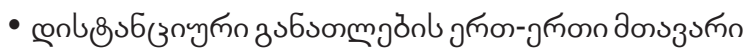

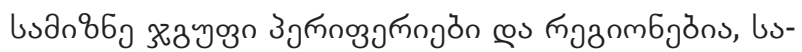

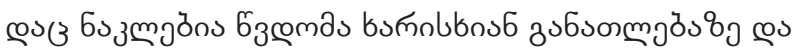

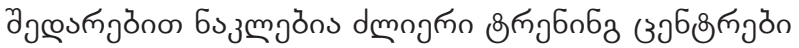

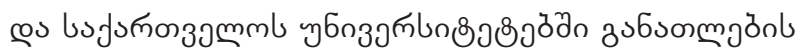

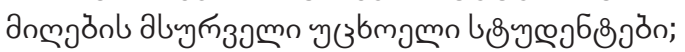

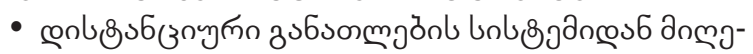




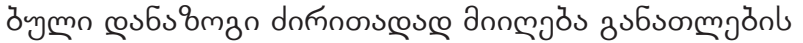

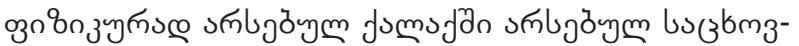

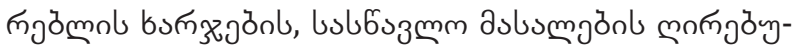

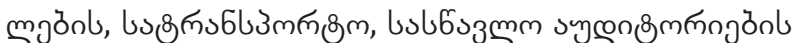

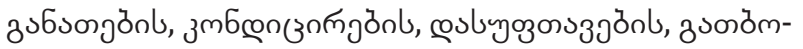

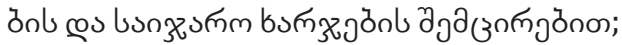

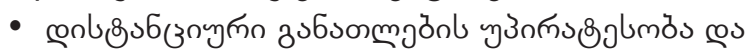

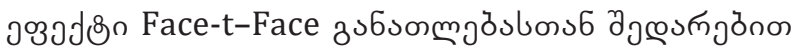

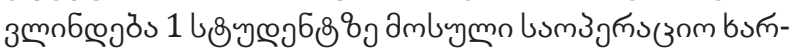
з

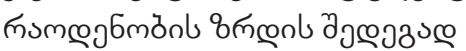

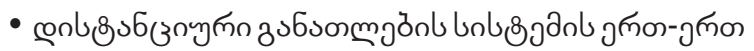

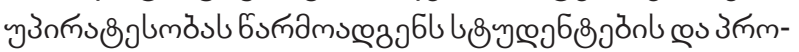

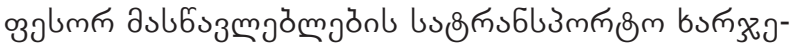

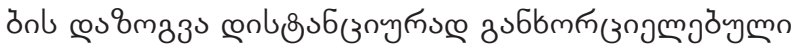

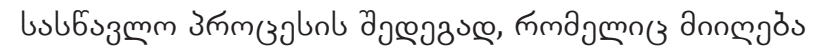

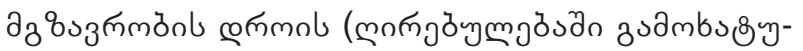

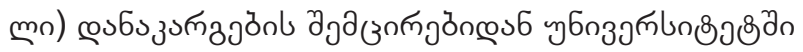

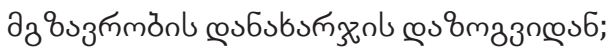

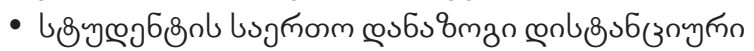

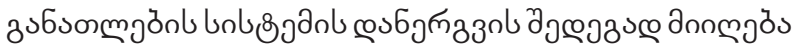

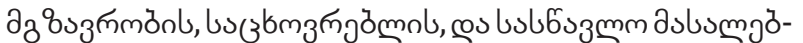

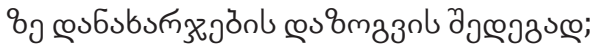

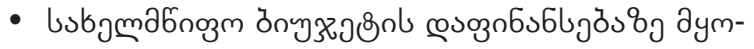

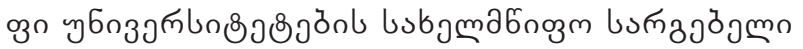

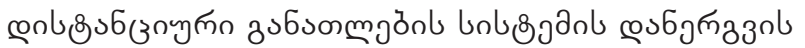

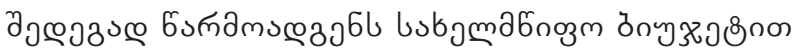

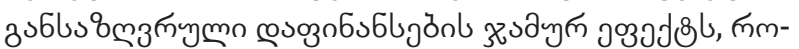
ajmna zuamnbs \&

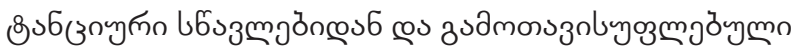

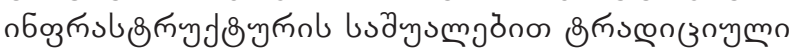
3งбsonm

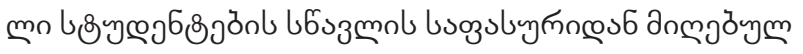

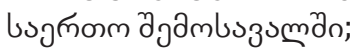

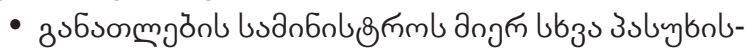

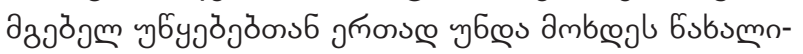

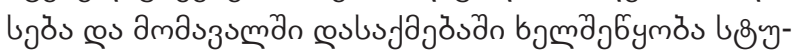

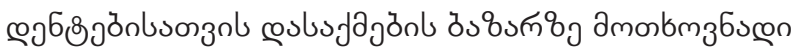

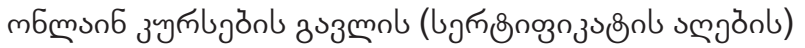

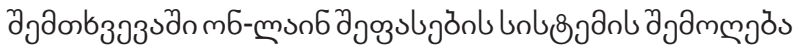

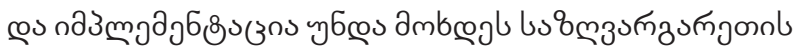

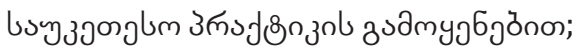

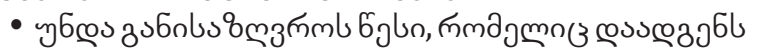

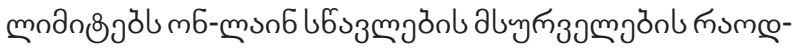

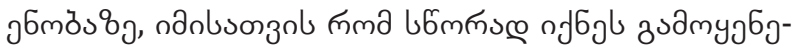

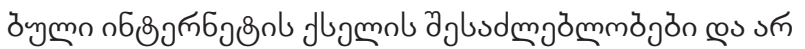

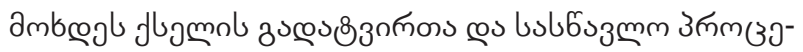

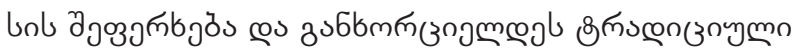
(Face2face) ৬6َsว

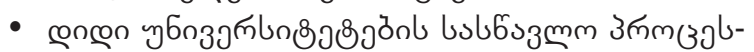

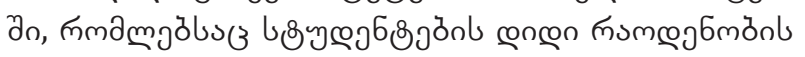

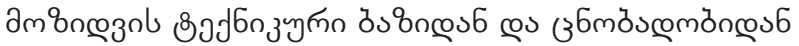

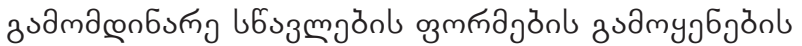

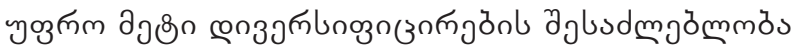

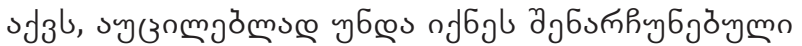

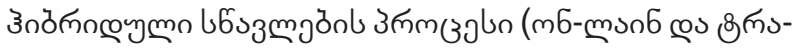

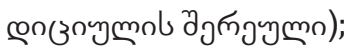

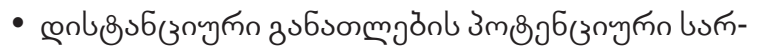
3

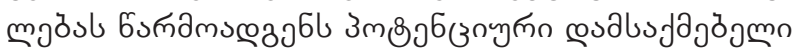

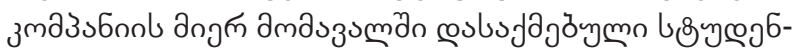

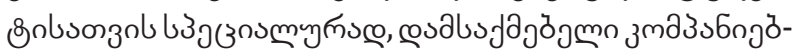

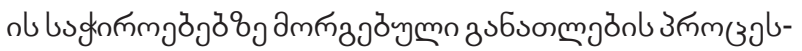

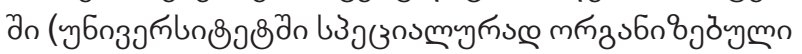

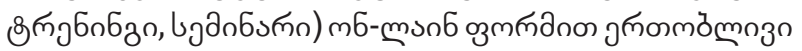

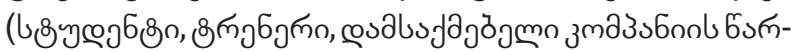

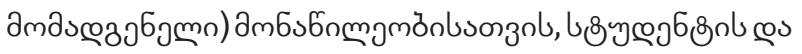

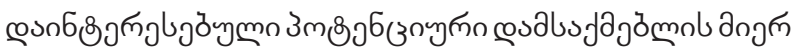

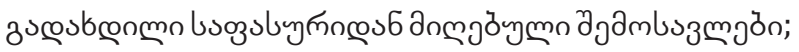

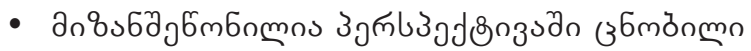

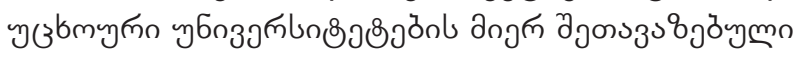

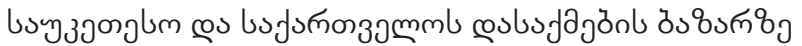

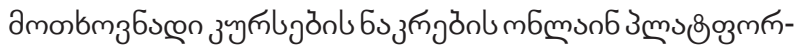

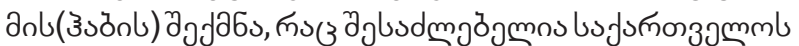

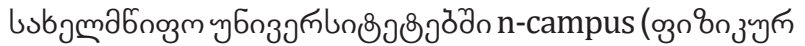

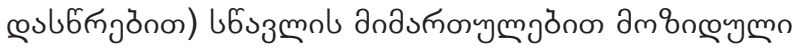

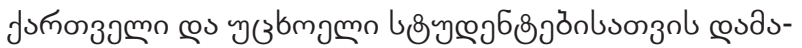

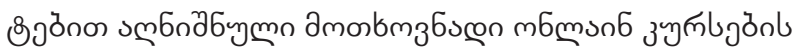
дjons 3u

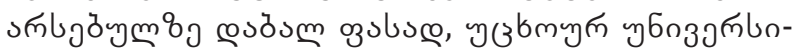

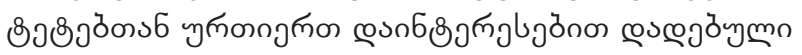

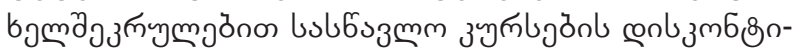

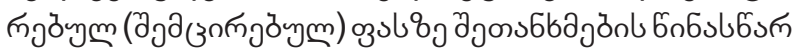

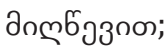

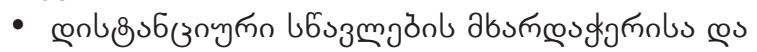

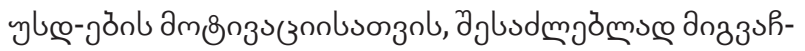

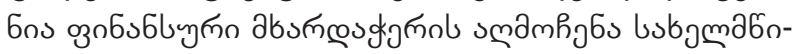

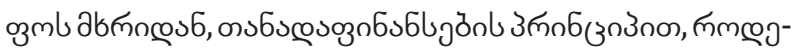
lsзз yб

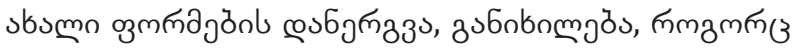

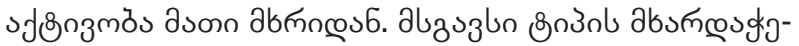

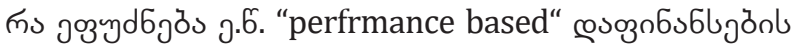

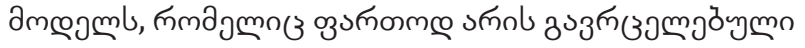

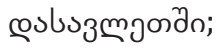

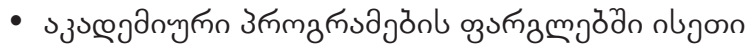
змазиббб

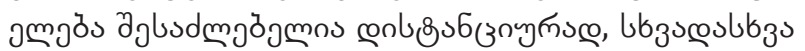

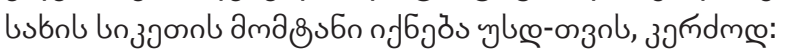

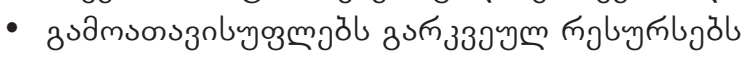

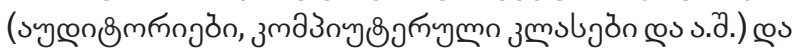

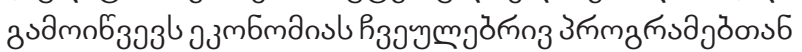

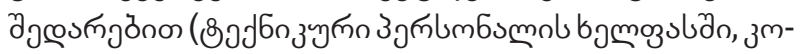




\section{ISSN 2667-9752(Online)}

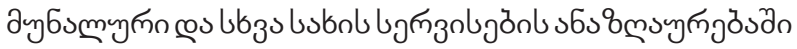

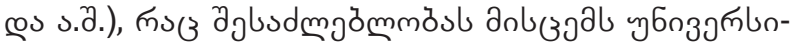

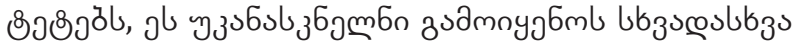

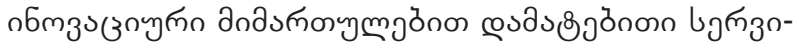

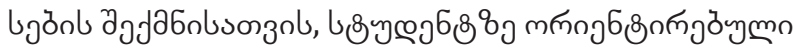

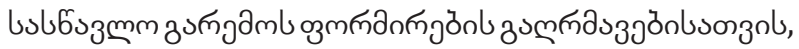

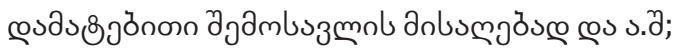

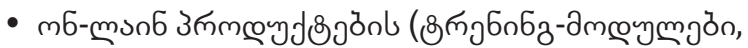

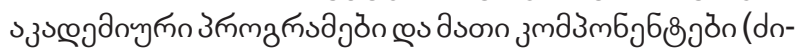

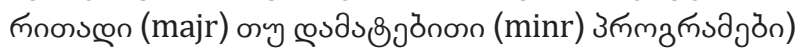

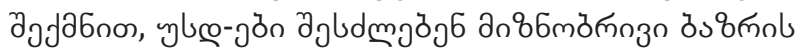

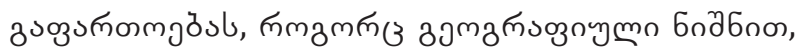

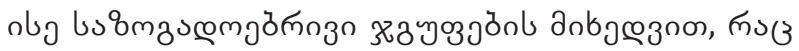
دsonoz

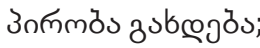

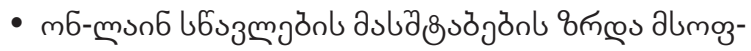

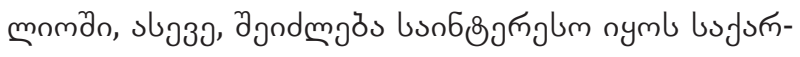

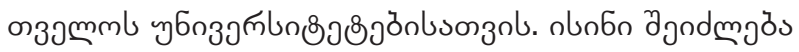

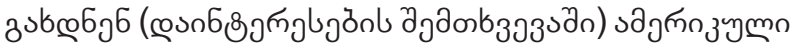

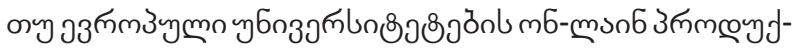

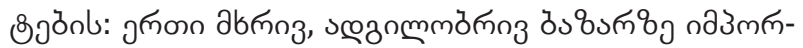

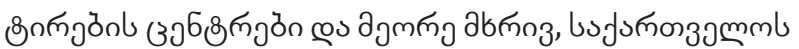

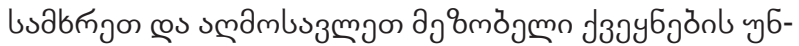

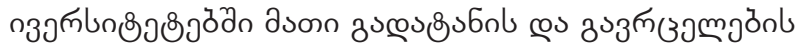

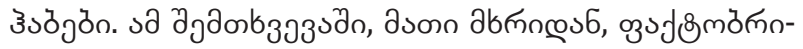

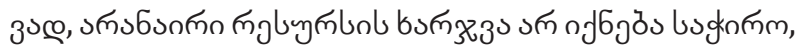

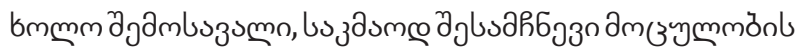

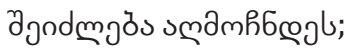

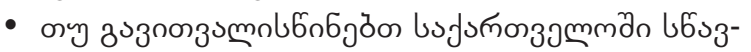

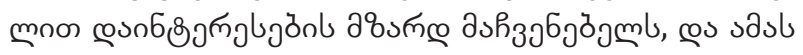

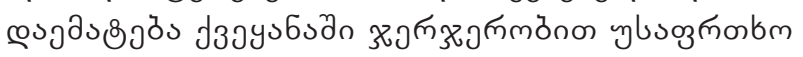

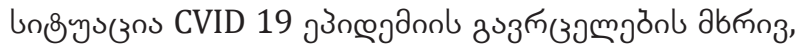

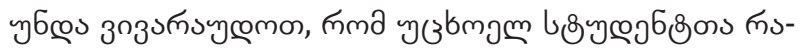

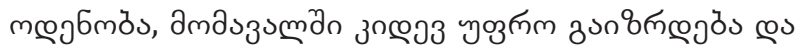

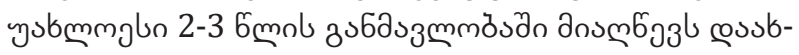
mmgठ̆no1 15,0-17,0 sonubl.

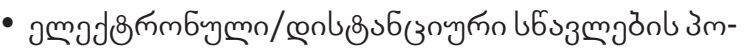

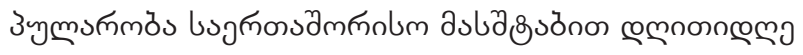

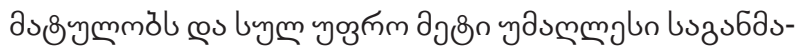

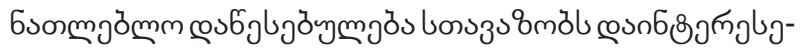

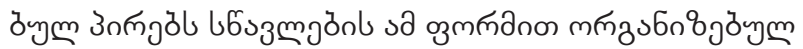

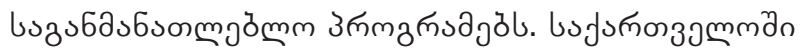

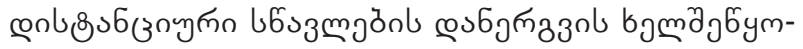

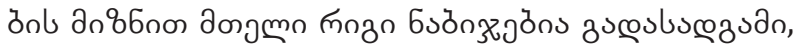

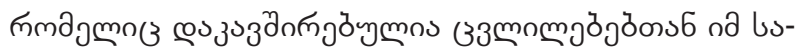

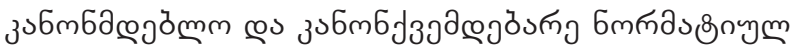

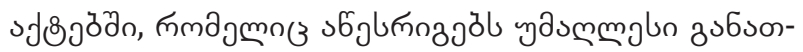
mjönb lnbهjaub:

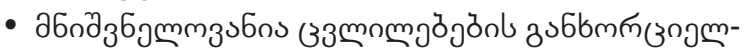

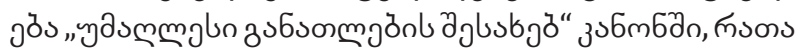

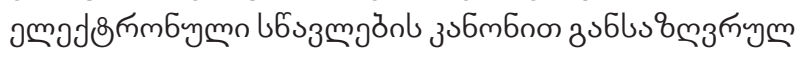

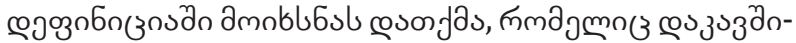

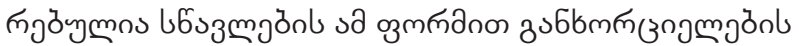

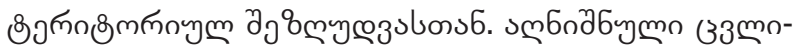

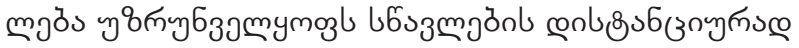

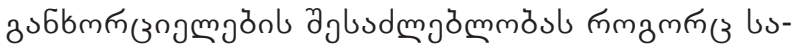

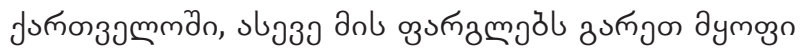

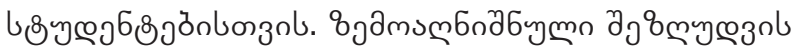

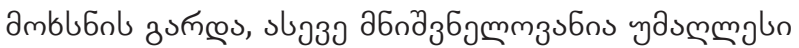

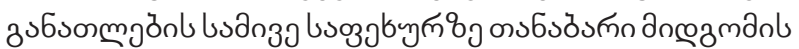

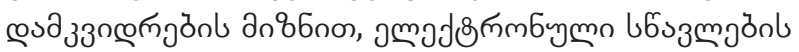

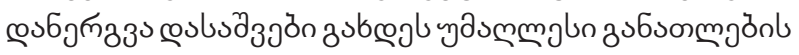

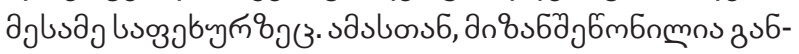

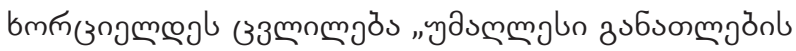

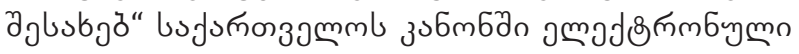

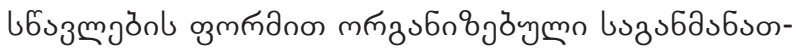

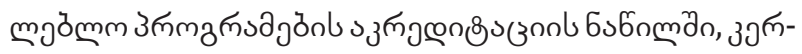

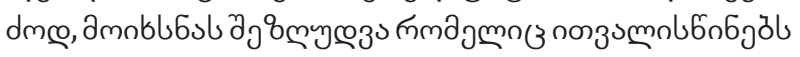

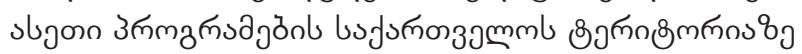

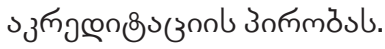

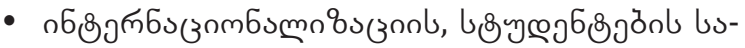

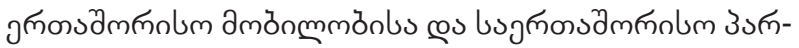

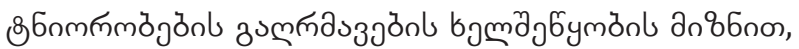

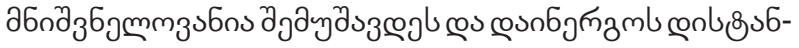

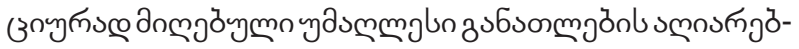

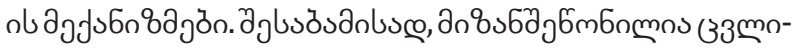

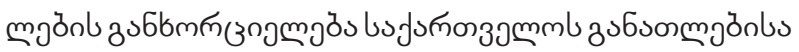
œs a

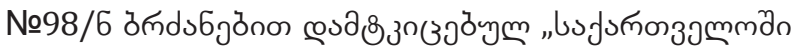

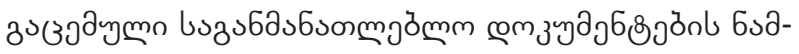

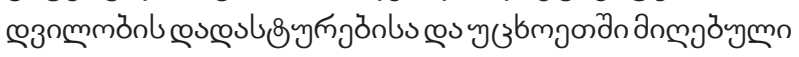

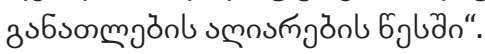

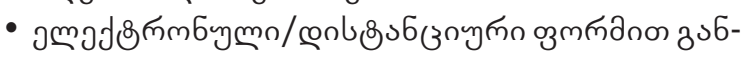

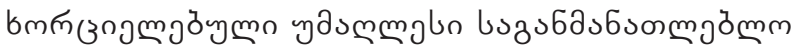

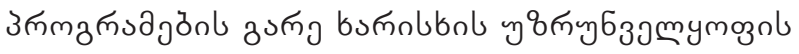

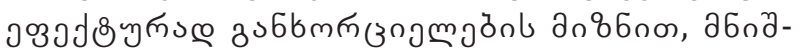

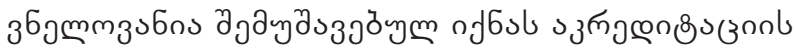

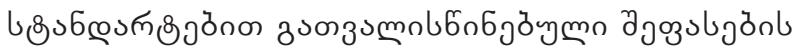

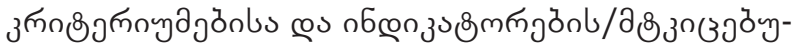

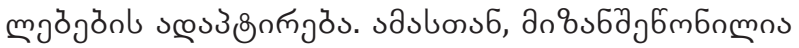

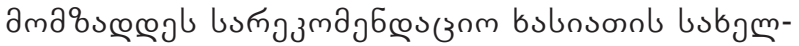

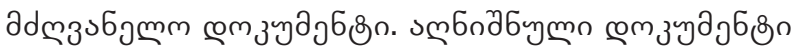

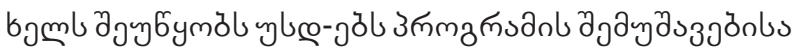

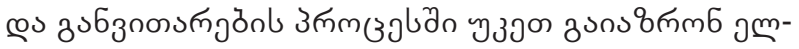

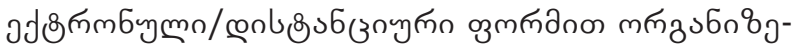

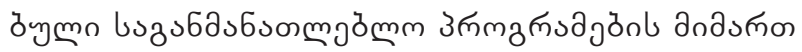

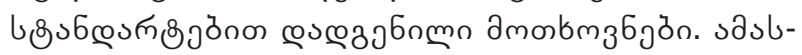
๓s6, ప6пдз

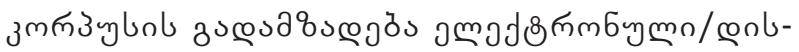

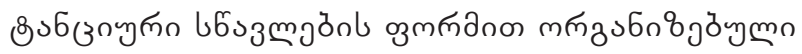

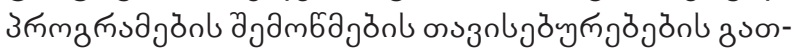

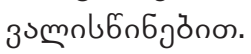




\section{REFERENCES:}

1. Abaidoo N, Arkorful V, (2014), "The role of e-learning, the advantages and disadvantages of its adoption in Higher Education", International journal of education and research, vol. 2, No. 12, pp. 397-410;

2. Abbad, M. M., Morris, D., \& de Nahlik, C, 2009, "Looking under the Bonnet: Factors Affecting Student Adoption of E-Learning Systems in Jordan", The International Review of Research in Open and Distance Learning;

3. Alsalem A, 2004, Educational Technology and E-learning, Riyadh

4. Barro R, 1997, Determinants of economic growth: a cross country empirical study, MIT press, Cambridge (USA);

5. Bassanini A, Scarpetta S, Hemmings P, 2001, "Economic growth: the role of policies and institutions. Panel data evidence from OECD countries", OECD Economics Department Working Papers, No. 283;

6. Bozkurt A, 2019, "From Distance Education to Open and Distance Learning: A Holistic Evaluation of History, Definitions, and Theories", Handbook of Research on Learning in the Age of Transhumanism, pp. 252-273;

7. Brown D, Cromby J, Standen P, 2001, "The effective use of virtual environments in the education and rehabilitation of students with intellectual disabilities", British Journal of Educational Technology, 32 (3), pp. 289-299;

8. Dublin L, 2003, "If you only look under the street lamps.......Or nine e-Learning Myths", The e-Learning developers journal;

9. European Commission, 2001, The eLearning Action Plan: Designing tomorrow's education, http://www. elearningeuropa.info;

10. European university association, 2013, Annual report 2013, Brussels;

11. Gemmel N, 1996, "Evaluating the impacts of human capital stocks and accumulation on economic growth: some new evidence", Oxford bulleting of economics and statistics, vol. 58, pp. 9-28;;

12. Gorska D, 2016, "E-learning in higher education", The person and the challenges, vol.6, p. 35-43

13. Guiney P, 2015, E-learning in the workplace - an annotated bibliography, Auckland (New Zealand);

14. IDEAL, 2015, Distance education in European higher education: Germany case study, Oslo (Norway);;

15. JuhadiI N, Samah A, Sarah H, 2007, "Use of Technology, Job Characteristics and work outcomes: A case of Unitary Instructors", International Review of business Research papers, 3(2), vol. 184-203

16. Khan B, 2005, Managing E-learning: Design, Delivery, Implementation and Evaluation, Hershey (USA);

17. Kirkpatrick D, 2005, Accreditation and assuring quality in open and distance learning, Vancouver;

18. Maltz, L., Deblois, P. \& The EDUCAUSE Current Issues Committee, 2005, “Top Ten IT Issues", EDUCAUSE Review, 40 (1), pp. 15-28;

19. NUFFIC, 2015, Education system in South Korea, The Hague;

20. OBHE, 2007, Online learning in Universities: Selected data from the 2006 Observatory survey, London;

21. OECD, 2005, E-learning in tertiary education, Paris;

22. Sadler-Smith E, 2000, "Modern learning methods: rhetoric and reality", Personnel Review, 29(4), pp. 474-490;

23. UK Department for education, 2018, Review of the online learning and artificial intelligence education market, London;

24. Valero A, Van Reenen J, 2019, "The economic impact of universities: Evidence from across the globe", Economics of Education Review, vol. 68(C), pp. 53-67;

25. Wagner N, Hassanein K, Head M, 2008, "Who is responsible for E-learning in Higher Education? A Stakeholders' Analysis", Educational Technology \& Society, 11 (3), 26-36;

26. Zhang D, Zhou L, Briggs R. \& Nunamaker J, 2006, "Instructional video in e-learning: Assessing the impact of interactive video on learning effectiveness", Information \& Management, 43 (1), pp. 15-27;

27. Law of Georgia on General Education, 2005, Legislative Herald of Georgia.

28. Veshapidze, S., (2020). Religion and national values in Georgia. International Scientific Collection, Volume 3, pp. 33-36.

29. Veshapidze, S., Mchedlishvili, Z., (2020). From Ilia Chavchavadze's Economic Views: Competition, Private Property and International Trade. Ecoforum Journal 9 (2).

30. Veshapidze, S., (2017). THE TRADITIONS ORIENTATED ON EUROPEAN VALUES IN GEORGIA. Sciences of Europe. VOL 4, No 13 (13)

31. Sichinava, A., Chikava, M., Veshapidze, S., Sekhniashvili, D., Pailodze, N., (2013). Realities of internationalization of higher education in Georgia. PRADEC Conference. Internationalisation in Higher Education: Evaluating concepts, challenges and strategies, 25-26 April, 2013 Proceedings Volume 2 | Issue 1 | August 2013 |pp.95-98] 


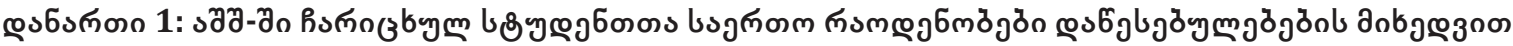

\begin{tabular}{|c|c|c|c|c|c|c|c|c|}
\hline & & & & 2018 & 2017 & & 2015 & \\
\hline 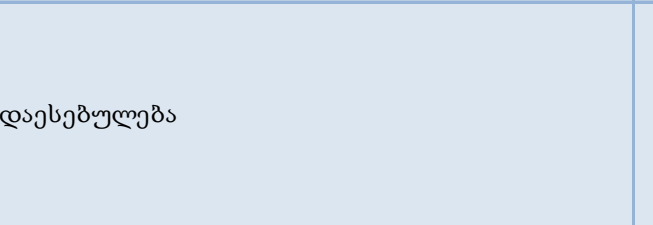 & 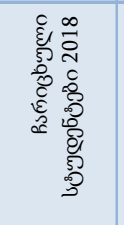 & 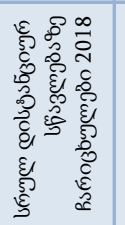 & 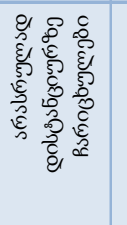 & 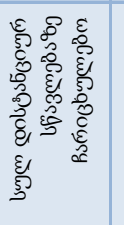 & 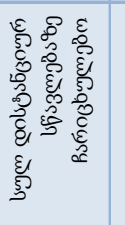 & 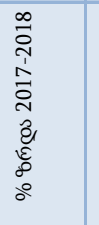 & 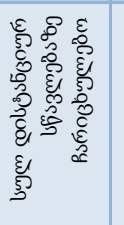 & 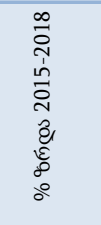 \\
\hline 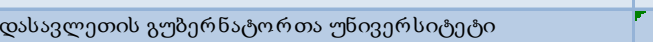 & $121,437^{\prime}$ & 121,437 & & $121,437^{\prime}$ & $98,627^{\circ}$ & $23.1 \%$ & $70,504^{r}$ & $72.2 \%$ \\
\hline 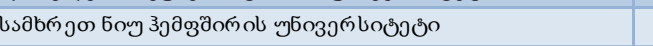 & 104,068 & 96,912 & 500 & 97,412 & 84,550 & $15.2 \%$ & 56,371 & $72.8 \%$ \\
\hline 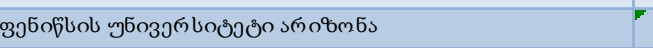 & $95,777^{\prime}$ & 94,472 & 342 & $94,814^{7}$ & $102,495^{\circ}$ & $-7.5 \%$ & 162,003 & $-41.5 \%$ \\
\hline 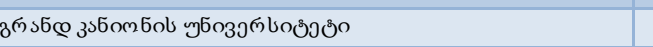 & 90,253 & 70,295 & 10,704 & 80,999 & 75,154 & $7.8 \%$ & $54,543^{\top}$ & $48.5^{\circ}$ \\
\hline 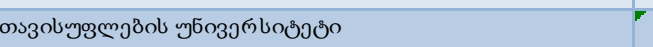 & $79,152^{\prime}$ & $64,006^{\prime}$ & $7,106^{\top}$ & 71,112 & 67,312 & $5.6 \%$ & $72,519^{\top}$ & $-1.9 \%$ \\
\hline 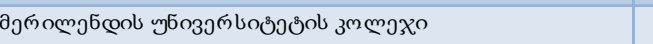 & 60,603 & $48,432^{\prime}$ & $5,996^{\prime}$ & 54,428 & 53,080 & 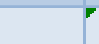 & 48,677 & $11.8 \%$ \\
\hline 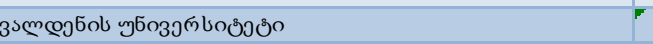 & $50,360^{\prime}$ & 50,360 & $r$ & $50,360^{\circ}$ & $49,680^{\circ}$ & $1.4 \%$ & $52,799^{\circ}$ & $-4.6 \%$ \\
\hline 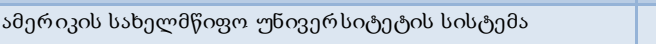 & 46,088 & 46,088 & $F$ & 46,088 & $46,420^{\circ}$ & $-0.7 \%$ & 52,361 & $-12.0 \%$ \\
\hline 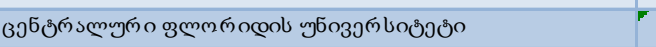 & $68,475^{\prime}$ & $10,269^{\prime}$ & $29,001^{\prime}$ & 39,270 & 38,073 & $3.1 \%$ & $33,034^{r}$ & $18.9 \%$ \\
\hline 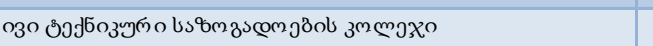 & $72,006^{\prime}$ & $15,010^{\prime}$ & 23,627 & 38,637 & 36,640 & $5.5 \%$ & 34,103 & $13.3 \%$ \\
\hline 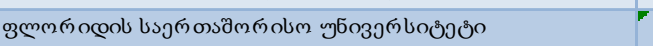 & $57,942^{\prime}$ & $11,785^{\circ}$ & $25,978^{-12}$ & 37,763 & 34,281 & $10.2 \%$ & 26,341 & $43.4 \%$ \\
\hline 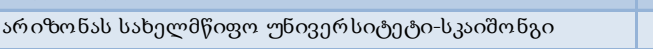 & $38,540^{\prime}$ & 37,000 & 3051 & 37,305 & 30,422 & $22.6 \%$ & 19,094 & $95.4^{\circ}$ \\
\hline 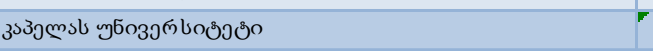 & $37,171^{\prime}$ & 37,171 & $r$ & 37,171 & 36,284 & $2.4 \%$ & $34,365^{-}$ & $8.2 \%$ \\
\hline 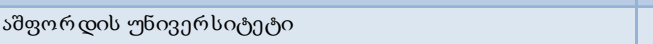 & $34,710 \mathrm{i}^{\prime}$ & 34,705 & 5 & $34,710^{\circ}$ & 36,452 & $-4.8 \%$ & $42,046^{\circ}$ & $-17.4 \%$ \\
\hline 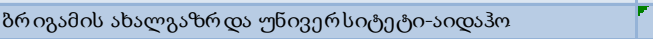 & $42,341^{\prime}$ & $21,053^{\prime}$ & $11,395^{\prime}$ & 32,448 & $42,057^{7}$ & $-22.8 \%$ & $33,551^{\circ}$ & $-3.3 \%$ \\
\hline 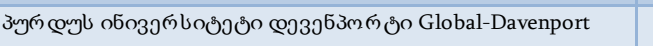 & 30,512 & 30,512 & 0 & 30,512 & 33,287 & $-8.3 \%$ & 45,268 & $-32.6 \%$ \\
\hline 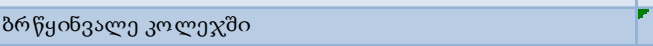 & $30,008^{\prime}$ & 30,008 & $\boldsymbol{r}$ & 30,008 & 34,022 & $-11.8 \%$ & $43,123^{\prime}$ & $-30.4 \%$ \\
\hline 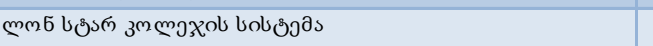 & $73,499^{\prime}$ & 11,524 & 17,317 & 28,841 & 29,340 & $-1.7 \%$ & 21,811 & $32.2 \%$ \\
\hline 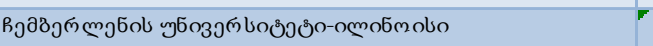 & $27,629^{\prime}$ & $25,033^{\prime}$ & $1,317^{\top}$ & $26,350^{r}$ & $25,757^{7}$ & $2.3 \%$ & 22,114 & $19.2 \%$ \\
\hline 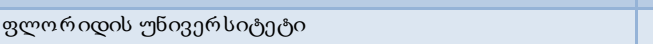 & 52,218 & $4,766^{\prime}$ & 21,513 & $26,279^{\prime}$ & $30,795^{\circ}$ & $-14.7 \%$ & $28,838^{\circ}$ & $-8.9^{\circ}$ \\
\hline 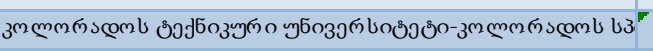 & $26,184^{\prime}$ & 25,038 & $886^{\prime}$ & 25,924 & 25,093 & $3.3 \%$ & $900^{\circ}$ & $2780.4 \%$ \\
\hline 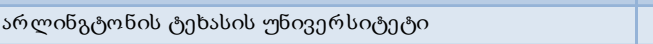 & 47,899 & 18,425 & 6,439 & 24,864 & 23,362 & $6.4 \%$ & 17,541 & $41.7 \%$ \\
\hline 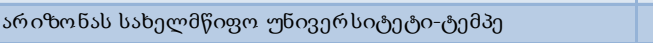 & 51,585 & 268 & 23,569 & 23,837 & 23,723 & $0.5 \%$ & 22,809 & $4.5 \%$ \\
\hline 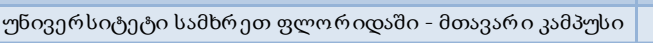 & 43,846 & 5,468 & 18,317 & 23,785 & 22,876 & $4.0 \%$ & 20,993 & $13.3 \%$ \\
\hline 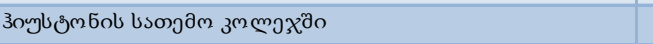 & 57,200 & 8,384 & 13,186 & 21,570 & 20,344 & $6.0 \%$ & 19,111 & $12.9 \%$ \\
\hline 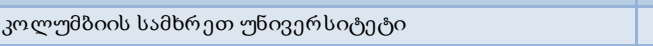 & 21,104 & 21,104 & & 21,104 & 20,818 & $1.4 \%$ & 20,823 & $1.3 \%$ \\
\hline 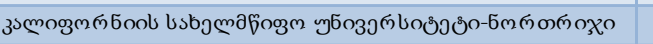 & 40,212 & 1,304 & 19,451 & 20,755 & 20,127 & $3.1 \%$ & 16,130 & 28.7 \\
\hline 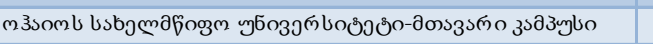 & 61,170 & 2,284 & 18,037 & 20,321 & 16,439 & $23.6 \%$ & 11,747 & $73.0^{\circ}$ \\
\hline 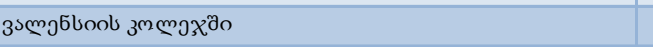 & 46,521 & 7,987 & 12,318 & 20,305 & 18,746 & $8.3 \%$ & 17,216 & $17.9 \%$ \\
\hline 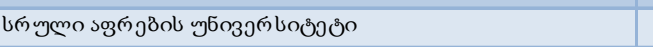 & 20,170 & 14,554 & 5,168 & 19,722 & 18,212 & $8.3 \%$ & 19,939 & $-1.1 \%$ \\
\hline 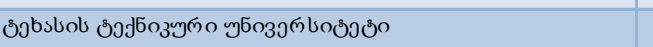 & 38,209 & 3,513 & 15,508 & 19,021 & 17,369 & $9.5 \%$ & 14,826 & $28.3^{\circ}$ \\
\hline 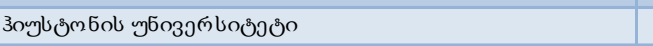 & 46,324 & 1,646 & 16,613 & 18,259 & 16,558 & $10.3 \%$ & 12,961 & $40.9^{\circ}$ \\
\hline ட๓ ஹомल & 38,241 & 3,902 & 13,820 & 17,722 & 15,821 & $12.0 \%$ & 12,517 & $41.6 \%$ \\
\hline 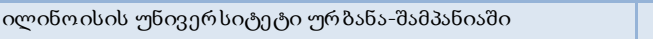 & 49,702 & 4,767 & 12,503 & 17,270 & 14,551 & $18.7 \%$ & 10,720 & $61.1 \%$ \\
\hline 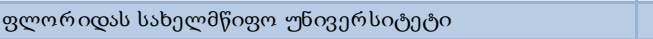 & 41,005 & 2,738 & 14,515 & 17,253 & 16,355 & $5.5 \%$ & 12,858 & $34.2 \%$ \\
\hline 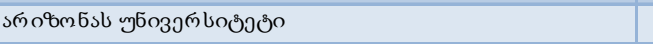 & 44,097 & 4,242 & 12,701 & & 15,443 & $9.7 \%$ & 9,660 & $75.4 \%$ \\
\hline 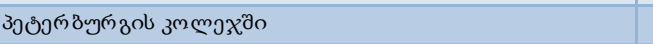 & 29,183 & 10,068 & 6,814 & 16,882 & 16,409 & $2.9 \%$ & 16,501 & 2.3 \\
\hline 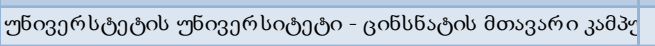 & 37,886 & 6,280 & 10,520 & 16,800 & 15,358 & $9.4 \%$ & 13,992 & $20.1^{\circ}$ \\
\hline 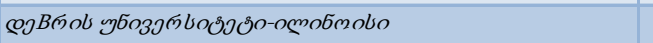 & 17,364 & 15,837 & 941 & 16,778 & 16,119 & $4.1 \%$ & 20,458 & $-18.0 \%$ \\
\hline 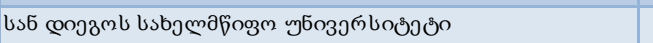 & 35,303 & 646 & 15,788 & 16,434 & 14,170 & $16.0 \%$ & 9,634 & $70.6 \%$ \\
\hline 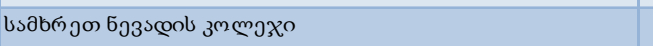 & 34,169 & 6,621 & 9,291 & 15,912 & 15,368 & $3.5 \%$ & 14,906 & $6.7^{\circ}$ \\
\hline 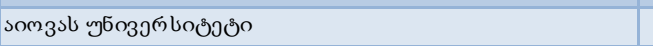 & 1,656 & 3,022 & 12,073 & 15,095 & 9,363 & $61.2 \%$ & 12,784 & 18. \\
\hline 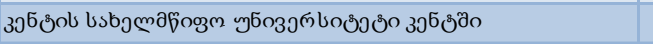 & 28,122 & 3,953 & 11,117 & 15,070 & 14,235 & $5.9 \%$ & 13,754 & 0 \\
\hline 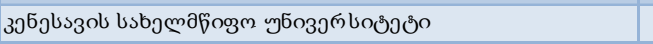 & $35,420^{\prime}$ & 3,899 & 11,083 & 14,982 & 14,204 & $5.5 \%$ & 10,056 & 14 \\
\hline 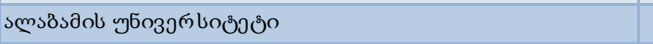 & 38,390 & 3,786 & 11,148 & 14,934 & 11,697 & $27.7 \%$ & 9,658 & $54.6 \%$ \\
\hline 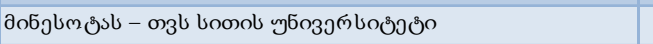 & 50,734 & 1,095 & 13,571 & 14,666 & 13,689 & $7.1 \%^{\prime}$ & 10,037 & $46.1 \%$ \\
\hline 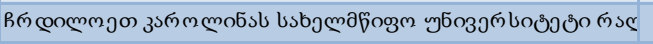 & 35,479 & 2,986 & 11,555 & 14,541 & 14,199 & $2.4 \%$ & 12,321 & $18.0 \%$ \\
\hline 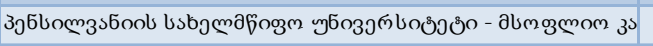 & 14,458 & 14,458 & & 14,458 & 14,301 & $1.1 \%$ & 12,242 & $18.1 \%$ \\
\hline 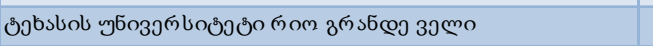 & 28,644 & 2,434 & 11,935 & 14,369 & 12,741 & $12.8 \%$ & 9,914 & $44.9 \%$ \\
\hline 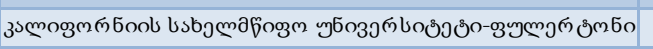 & 40,280 & 2,161 & 12,133 & 14,294 & 13,957 & $2.4 \%$ & 11,148 & 28. \\
\hline 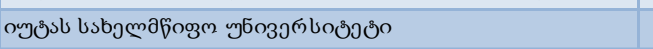 & 27,932 & 4,905 & 8,947 & 13,852 & 13,148 & $5.4 \%$ & 13,360 & $3.7 \%$ \\
\hline 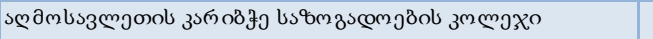 & 13,940 & 12,576 & 1,1251 & 13,701 & 6,781 & $102.0 \%$ & 1,091 & $155.8^{\circ}$ \\
\hline
\end{tabular}




\begin{tabular}{|c|c|c|c|c|c|c|c|c|}
\hline 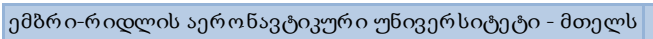 & 14,168 & 12,201 & 1,483 & 13,684 & 13,820 & $-1.0 \%$ & 12,857 & $6.4 \%$ \\
\hline 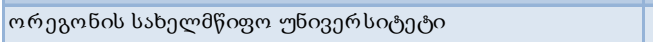 & 30,986 & 6,565 & 6,917 & 13,482 & 12,725 & $5.9 \%$ & 10,148 & $32.9 \%$ \\
\hline 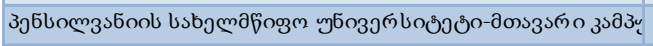 & 46,810 & 89 & 13,255 & 13,344 & 16,064 & $-16.9 \%$ & 14,355 & $-7.0 \%$ \\
\hline 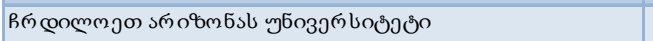 & 31,066 & 6,304 & 7,024 & 13,328 & 13,094 & $1.8 \%$ & 11,769 & $13.2 \%$ \\
\hline 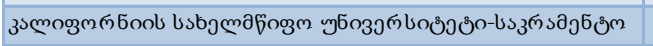 & 31,902 & 578 & 12,655 & 13,233 & 11,659 & $13.5 \%$ & 7,511 & $76.2 \%$ \\
\hline мЗెsంmb эбం & 28,480 & 7,975 & 5,0301 & 13,005 & 12,909 & $0.7 \%$ & 10,828 & $20.1 \%$ \\
\hline 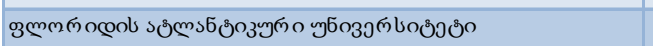 & 29,772 & 3,170 & 9,807 & 12,977 & 13,332 & $-2.7 \%$ & 10,433 & $24.4 \%$ \\
\hline 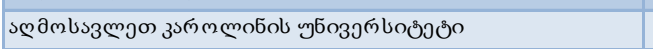 & 28,718 & 6,812 & 6,148 & 12,960 & 12,348 & $5.0 \%$ & 12,011 & $7.9 \%$ \\
\hline 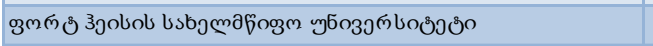 & 15,523 & 11,012 & 1,864 & 12,876 & 12,264 & $5.0 \%$ & 10,950 & $17.6 \%$ \\
\hline 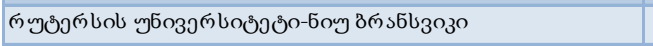 & 50,254 & 2,501 & 10,368 & 12,869 & 14,072 & $-8.5 \%$ & 10,652 & $20.8 \%$ \\
\hline Хм๓ & 37,316 & 2,164 & 10,589 & 12,753 & 10,199 & $25.0 \%$ & 7,901 & $61.4 \%$ \\
\hline 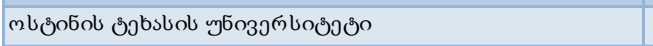 & 51,832 & 154 & 12,546 & 12,700 & 11,329 & $12.1 \%$ & 7,021 & $80.9 \%$ \\
\hline 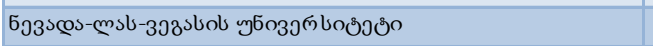 & 30,457 & 1,492 & 11,188 & 12,680 & 12,520 & $1.3 \%$ & 10,319 & $22.9 \%$ \\
\hline ంyరికt 3ృ & 39,931 & 3,097 & 9,476 & 12,573 & 11,219 & $12.1 \%$ & 9,557 & $31.6 \%$ \\
\hline 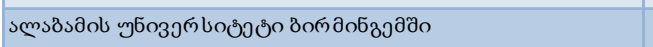 & 21,923 & 5,603 & 6,935 & 12,538 & 11,409 & $9.9 \%$ & 8,659 & $44.8 \%$ \\
\hline 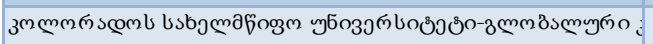 & 12,476 & 12,476 & & 12,476 & 12,381 & $0.8 \%$ & 9,838 & $26.8 \%$ \\
\hline 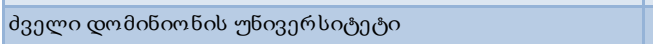 & 24,176 & 6,011 & 6,385 & 12,396 & 11,705 & $5.9 \%$ & 9,343 & $32.7 \%$ \\
\hline 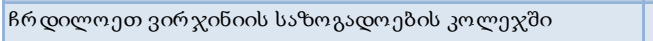 & 50,929 & 5,373 & 6,948 & 12,321 & 13,110 & $-6.0 \%$ & 13,421 & $-8.2 \%$ \\
\hline 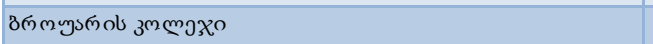 & 40,784 & 5,162 & 7,000 & 12,162 & 11,328 & $7.4 \%$ & 10,923 & $11.3 \%$ \\
\hline 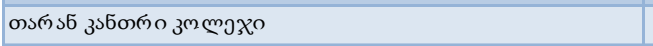 & 51,100 & 5,824 & 6,036 & 11,860 & 11,921 & $-0.5 \%$ & 10,377 & $14.3 \%$ \\
\hline 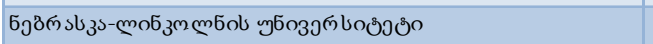 & 25,820 & 1,649 & 10,178 & 11,827 & 10,896 & $8.5 \%$ & 7,911 & $49.5 \%$ \\
\hline 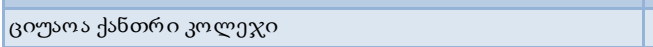 & 23,440 & 5,150 & 6,6291 & 11,779 & 11,617 & $1.4 \%$ & 12,266 & $-4.0 \%$ \\
\hline 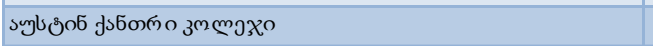 & 40,799 & 4,760 & 6,988 & 11,748 & 11,222 & $4.7 \%$ & 9,896 & $18.7 \%$ \\
\hline 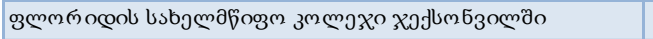 & 23,413 & 1,734 & 9,902 & 11,636 & 14,595 & $-20.3 \%$ & 11,611 & $0.2 \%$ \\
\hline 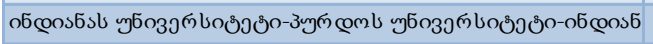 & 29,579 & 2,233 & 9,387 & 11,620 & 11,185 & $3.9 \%$ & 9,807 & $18.5 \%$ \\
\hline 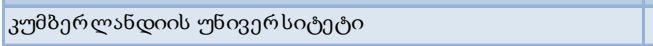 & 13,472 & 6,473 & 5,046 & 11,519 & 8,214 & $40.2 \%$ & 4,752 & $142.4 \%$ \\
\hline 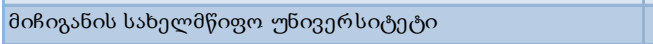 & 50,351 & 2,196 & 9,273 & 11,469 & 12,002 & $-4.4 \%$ & 9,901 & $15.8 \%$ \\
\hline 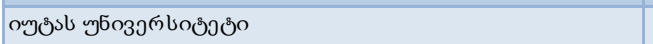 & 33,023 & 1,476 & 9,847 & 11,323 & 10,720 & $5.6 \%$ & 8,598 & $31.7 \%$ \\
\hline 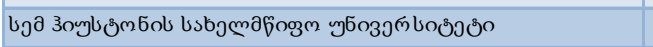 & 21,213 & 3,489 & 7,776 & 11,265 & 10,547 & $6.8 \%$ & 9,278 & $21.4 \%$ \\
\hline 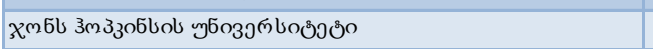 & 26,152 & 8,722 & 2,521 & 11,243 & 9,822 & $14.5 \%$ & 8,119 & $38.5 \%$ \\
\hline Змп өీ & 27,046 & 5,064 & 6,070 & 11,134 & 11,019 & $1.0 \%$ & 10,849 & $2.6 \%$ \\
\hline 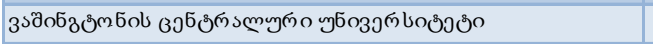 & 12,342 & 3,180 & 7,872 & 11,052 & 4,770 & $131.7 \%$ & 3,816 & $189.6 \%$ \\
\hline 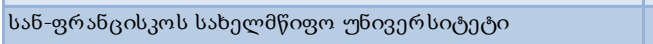 & 29,778 & 418 & 10,593 & 11,011 & 10,264 & $7.3 \%$ & 6,557 & $67.9 \%$ \\
\hline 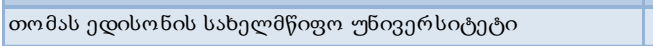 & 10,934 & 10,836 & 98 & 10,934 & 11,945 & $-8.5 \%{ }^{\prime}$ & 13,093 & $-16.5 \%$ \\
\hline 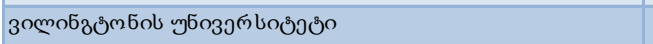 & 14,786 & 7,750 & 3,123 & 10,873 & 8,839 & $23.0 \%$ & 12,745 & $-14.7 \%$ \\
\hline 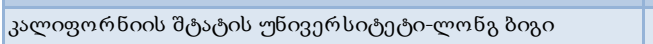 & 37,466 & 443 & 10,391 & 10,834 & 9,591 & $13.0 \%$ & 6,084 & $78.1 \%$ \\
\hline 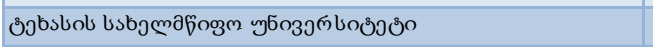 & 38,644 & 1,376 & 9,406 & 10,782 & 8,513 & $26.7 \%$ & 5,798 & $86.0 \%$ \\
\hline 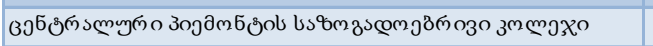 & 18,824 & 4,1391 & 6,617 & 10,756 & 10,649 & $1.0 \%$ & 10,177 & $5.7 \%$ \\
\hline 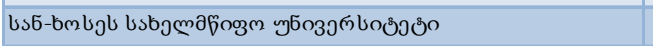 & 35,400 & 2,633 & 8,114 & 10,747 & 10,125 & $6.1 \%$ & 6,426 & $67.2 \%$ \\
\hline 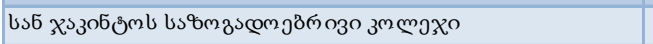 & 32,137 & 3,529 & 7,207 & 1,736 & 9,493 & $13.1 \%$ & 7,961 & $34.9 \%$ \\
\hline 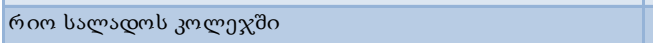 & 18,304 & 10,146 & 537 & 10,683 & 10,737 & $-0.5 \%$ & 12,092 & $-11.7 \%$ \\
\hline 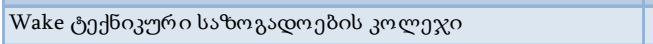 & 21,872 & 5,474 & 5,077 & 10,551 & 10,158 & $3.9 \%$ & & $22.1 \%$ \\
\hline 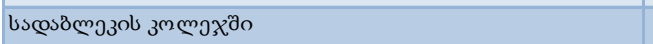 & 19,421 & 5,475 & 5,064 & 10,539 & 9,675 & $8.9 \%$ & 8,514 & $23.8 \%$ \\
\hline 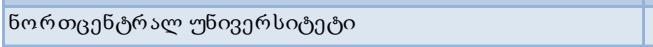 & 10,535 & 10,535 & & 10,535 & 10,788 & $-2.3 \%$ & 11,029 & $-4.5 \%$ \\
\hline 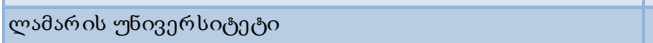 & 14,783 & 7,109 & 3,409 & 10,518 & 9,501 & $10.7 \%$ & 9,120 & $15.3 \%$ \\
\hline 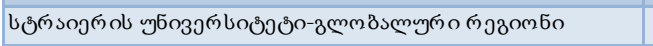 & 10,498 & 10,389 & 36 & 10,425 & 8,789 & $18.6 \%$ & 6,885 & $51.4 \%$ \\
\hline 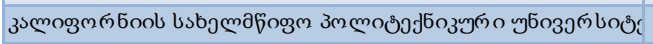 & 26,598 & 100 & 10,261 & 10,361 & 9,216 & $12.4 \%$ & 6,298 & $64.5 \%$ \\
\hline
\end{tabular}

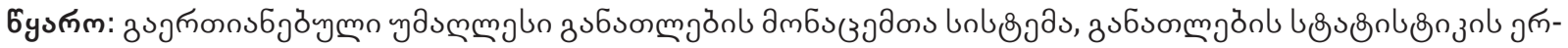

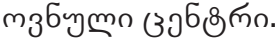




\section{INSTITUTIONAL EVOLUTION OF HIGHER EDUCATION IN GEORGIA}

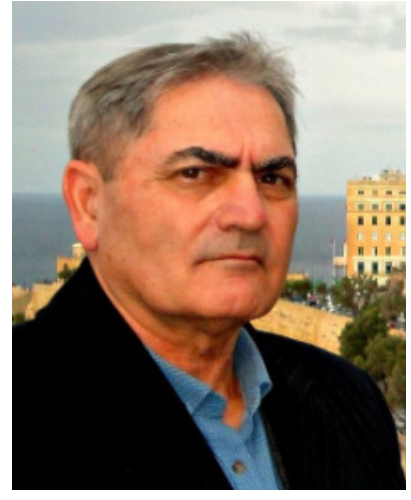

Shota Veshapidze

Ivane Javakhishvili Tbilisi State University, Associate professor, Georgia E-mail: Shota46@yahoo.com

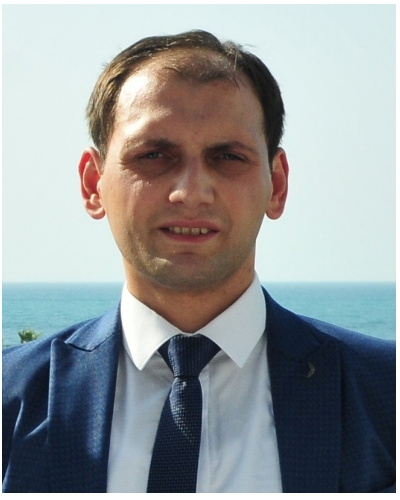

Gia Zoidze

Batumi Shota Rustaveli State University, Batumi State Maritime Academy, Invitee Lector, Georgia. E-mail: giazoidze@yahoo.com

DOI suffix: 10.36962/NEC6102202133

\section{ABSTRACT}

Education policy is an important direction of Georgia's security policy, of which higher education is an integral part. Institutions occupy an important place in its development. Regulatory norms, institutions, values, cultural patterns, forms of ownership are recognized directions for establishing progress in the education system, its arrangement and institutionalization.

In conditions when there is no orderly, social protection-oriented system, as well as any clearly defined priorities, all forms of discrimination in the labor system in the education system are excluded by the Georgian legislation. In this field, sufficient experience has been accumulated in public and private higher education institutions of Georgia. This approach was developed by the adoption of the Organic Law of Georgia on the Labor Code of Georgia.

Georgia needs the development of institutions that will enable us to effectively realize the potential of the higher education system. It is also important to implement long-term education reform, which is openly supported by the World Bank.

Keywords: Institute, Institutionalization, Education Policy, Higher Education, European Standard Education System.

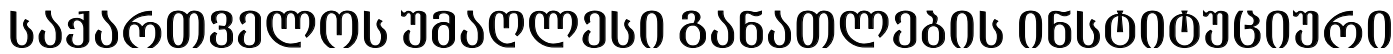

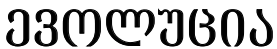

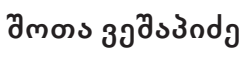

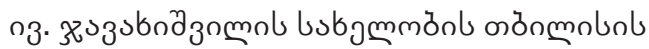

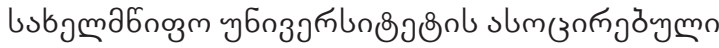

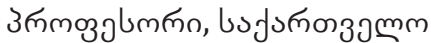

\author{
zos 8modo

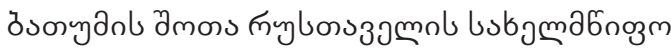

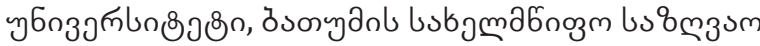

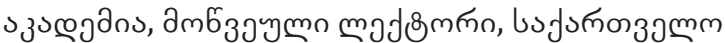

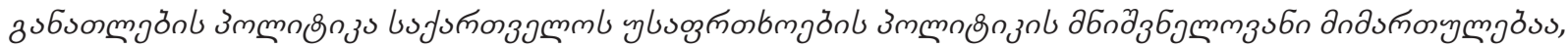

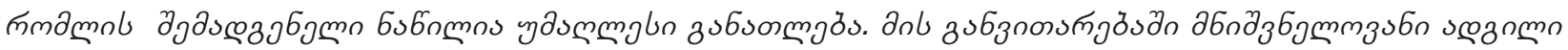




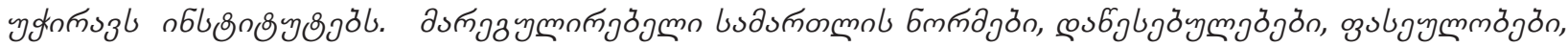

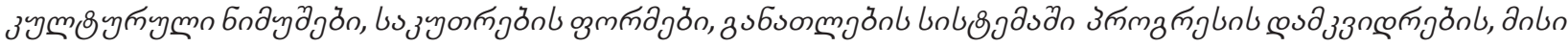

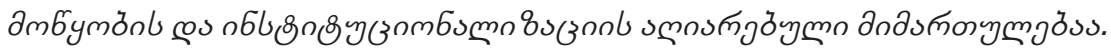

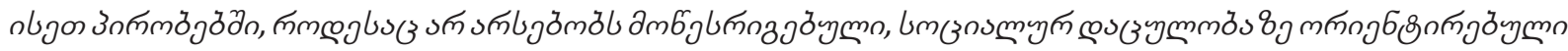

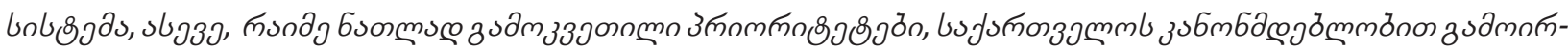

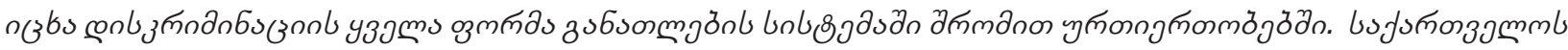

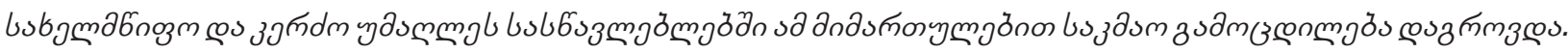

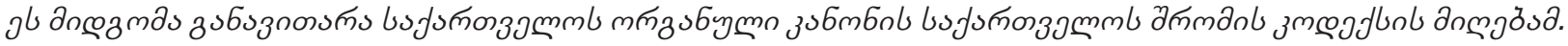

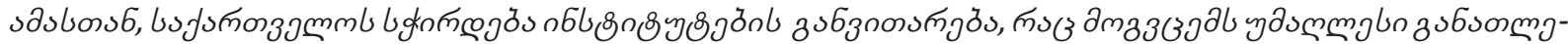

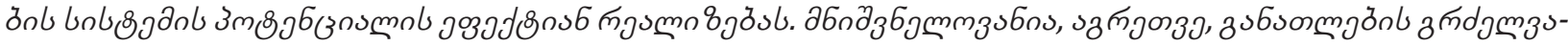

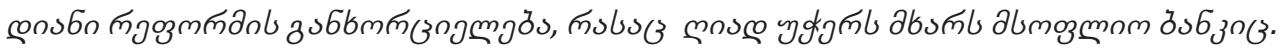

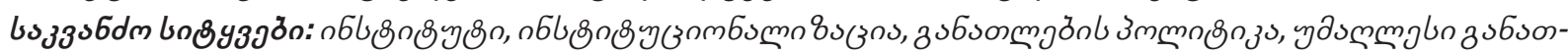

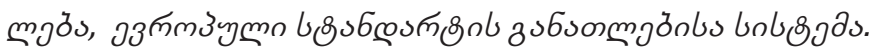

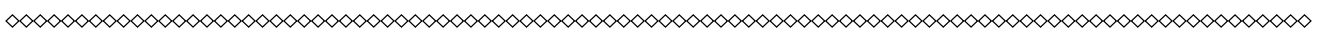

\section{วอ৬১3১m0}

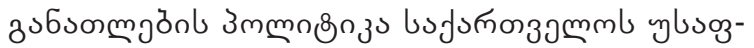

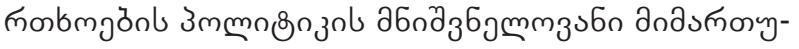

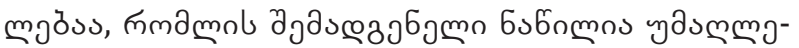

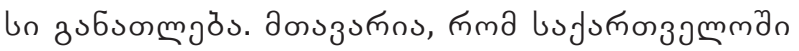

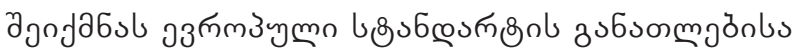

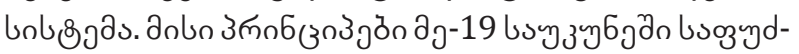

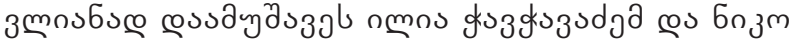

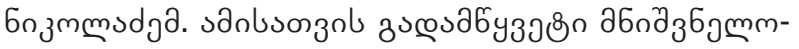

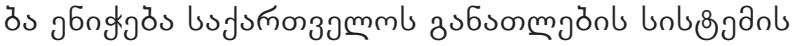

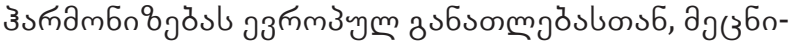

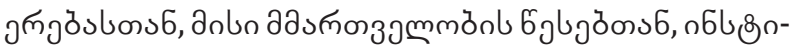

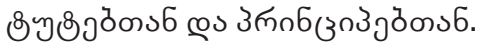

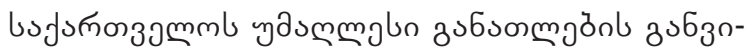

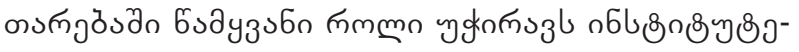

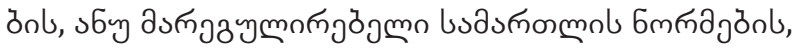

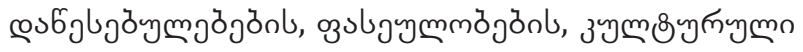

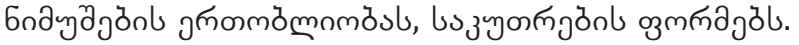

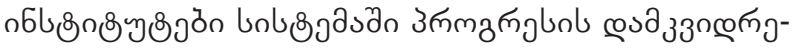

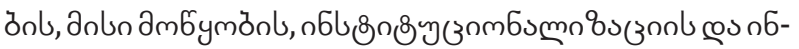

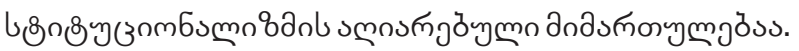

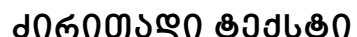

bufunnas

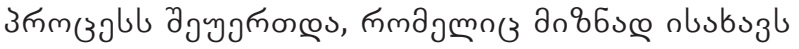

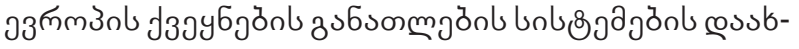

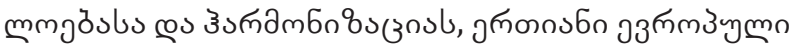

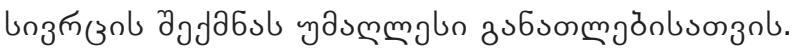

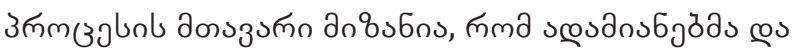

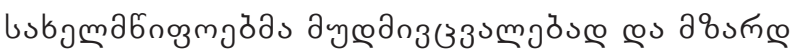

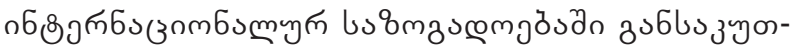

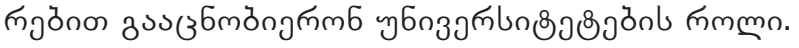

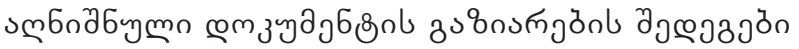

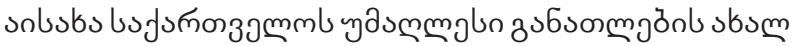

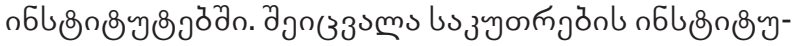

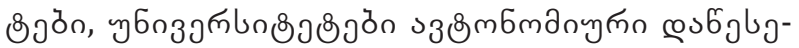

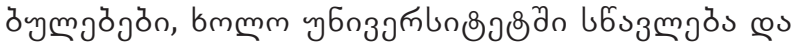

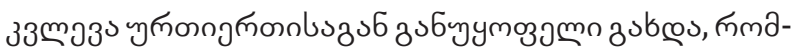

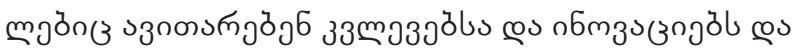

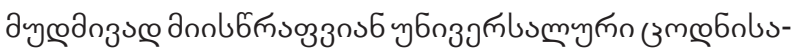

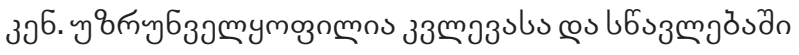

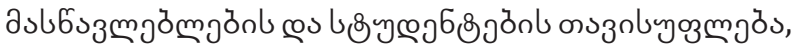

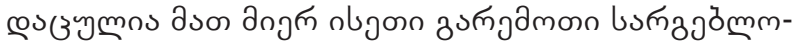

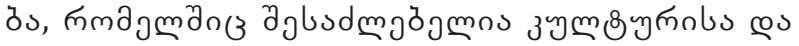

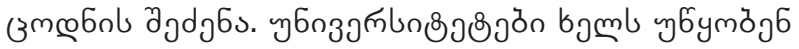

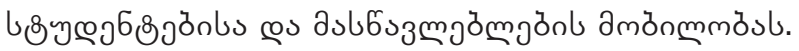

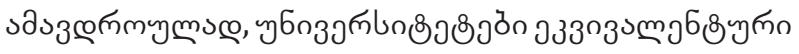

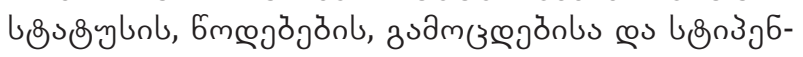

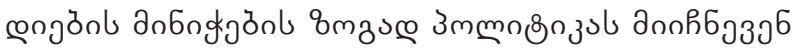

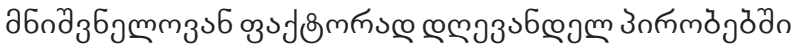

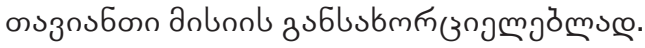

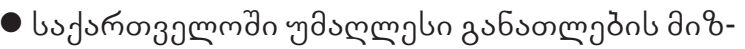

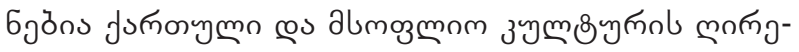

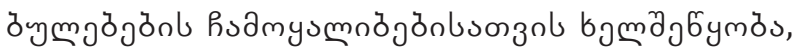

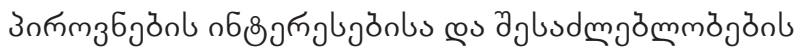

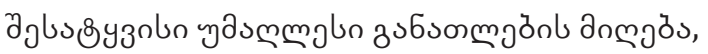

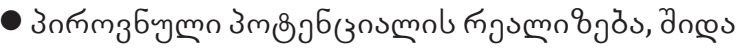

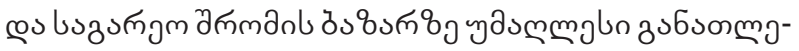

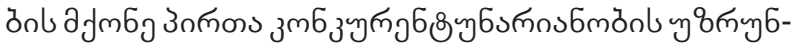

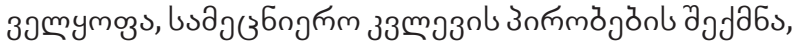

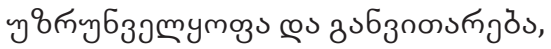

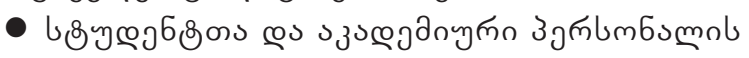

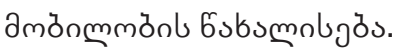

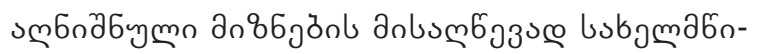

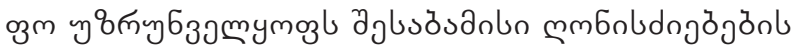

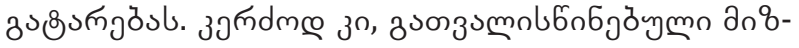

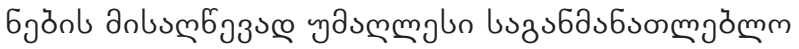
œu6 


\section{ISSN 2667-9752(Online)}

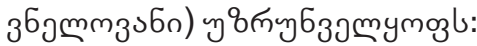

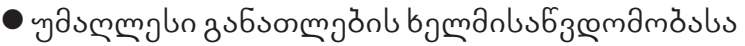
@o nnomósul,

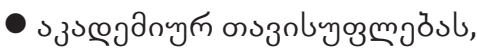

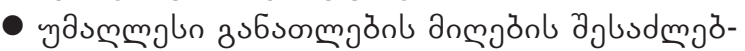

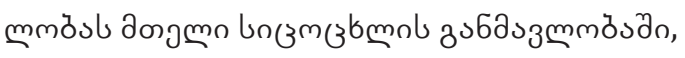

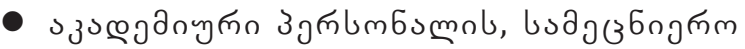

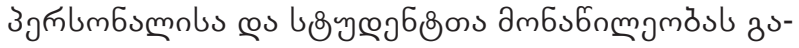

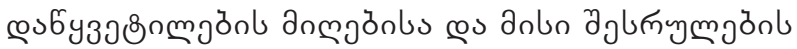

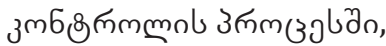

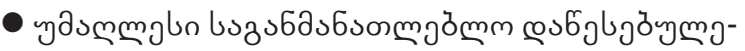

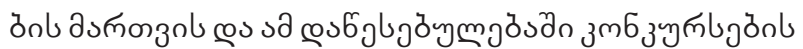

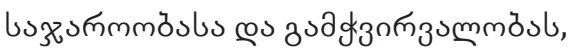

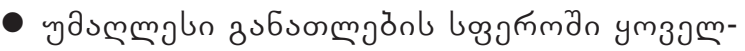

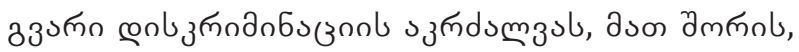

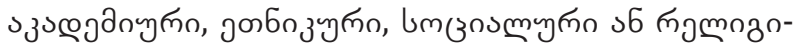

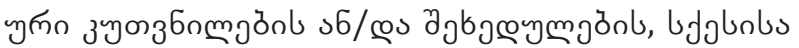
œu เ6з3 6กว6กо.

аง

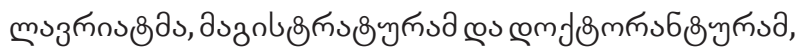

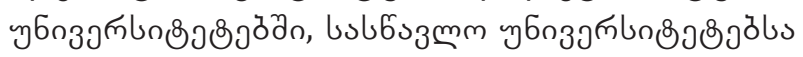

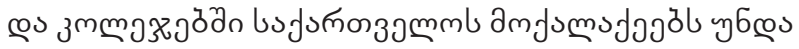

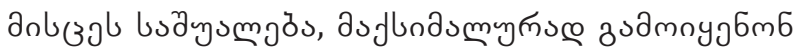

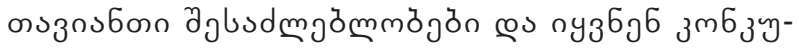

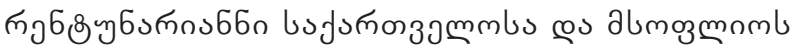

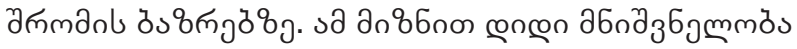
sلj

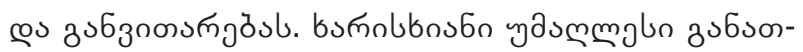

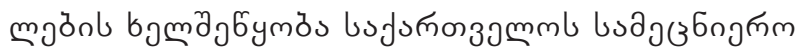
Зм৪ј

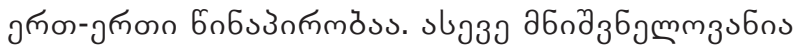

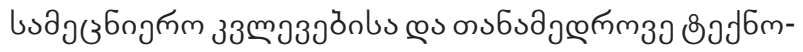

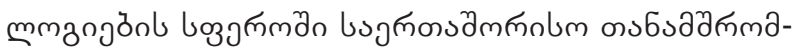

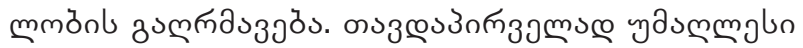

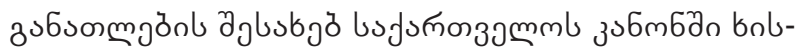

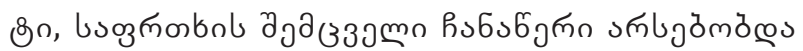

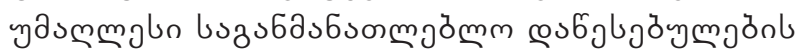

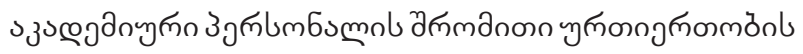

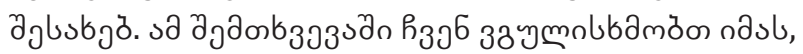

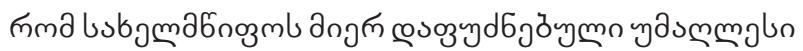

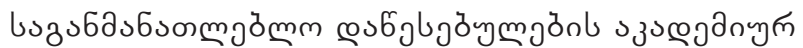

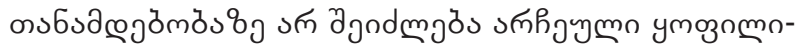

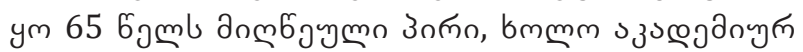

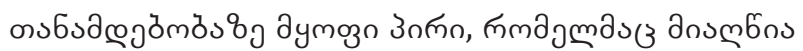

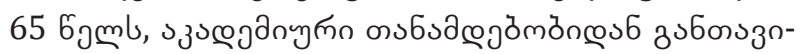

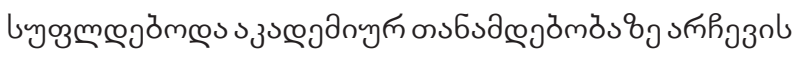

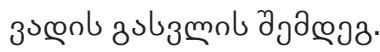

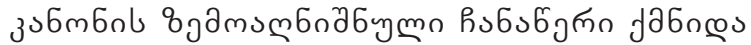

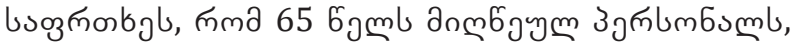

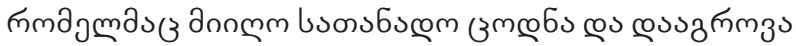

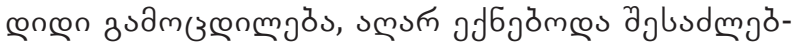

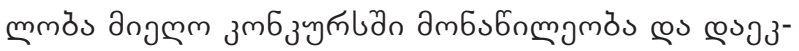

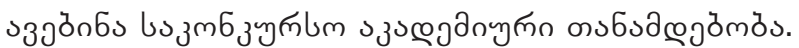

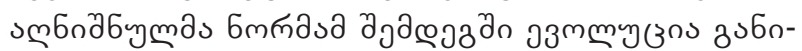

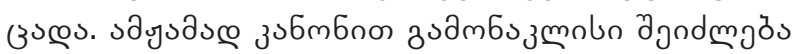

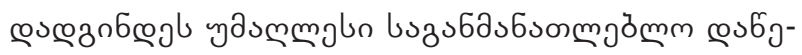

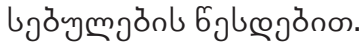

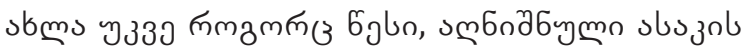

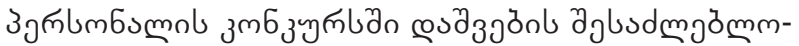

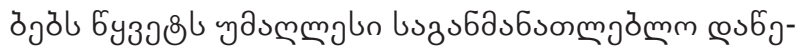

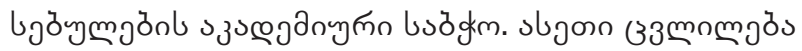

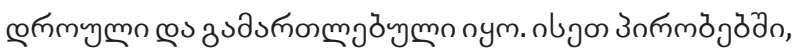

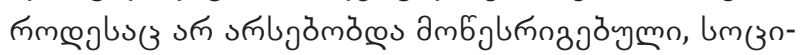

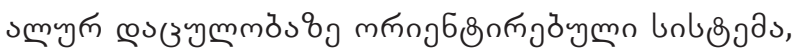

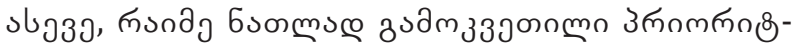

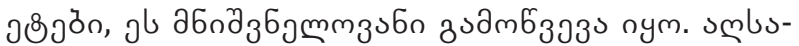

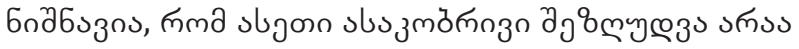

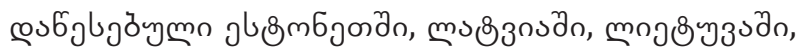

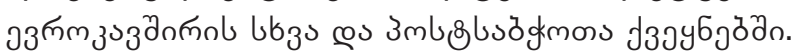

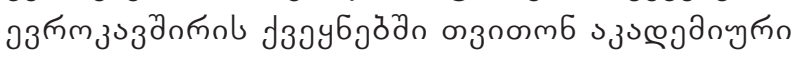

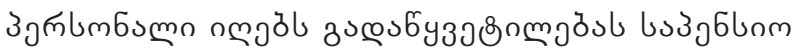

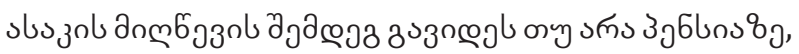

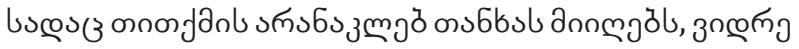

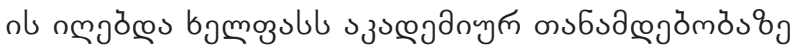

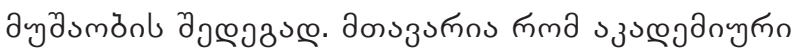

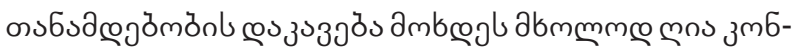

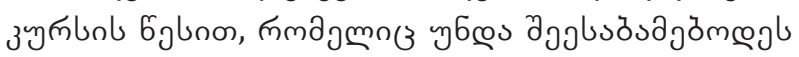

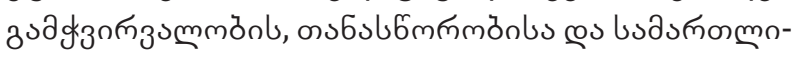
sб з з

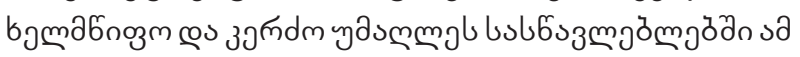

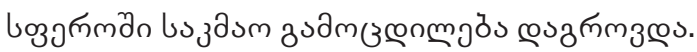

j ا

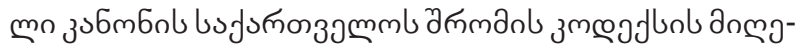

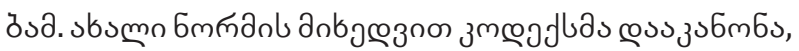

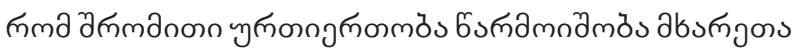

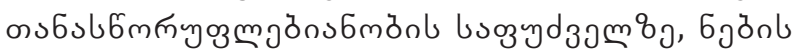

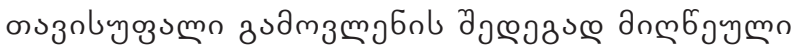

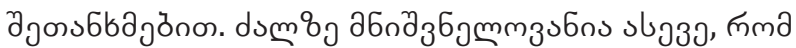

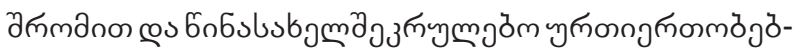

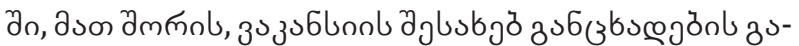
amj3əyб

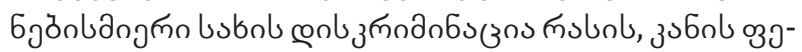

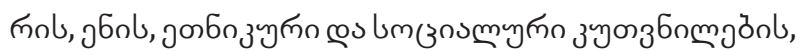

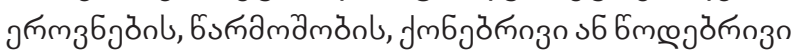

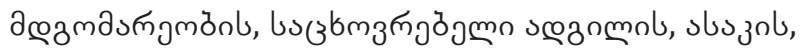

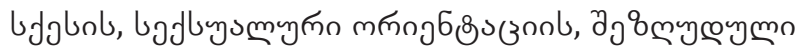

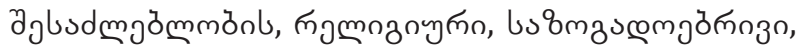

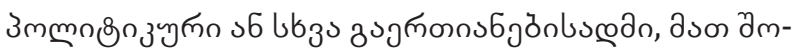




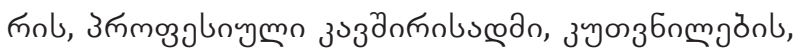

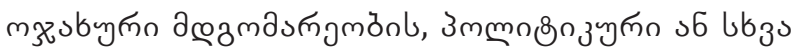

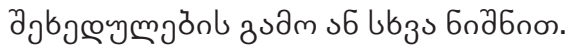

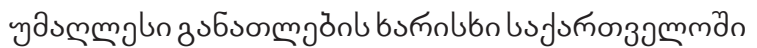

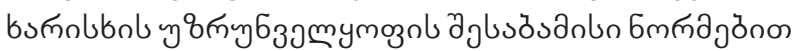

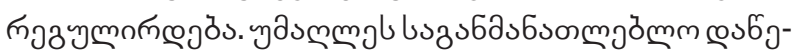
৮ว

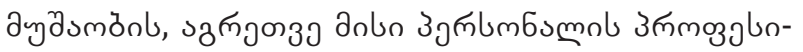

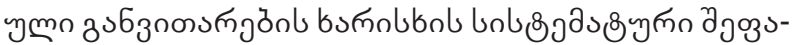

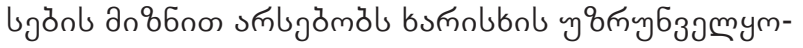

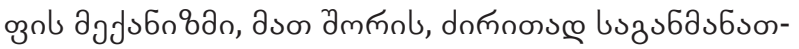

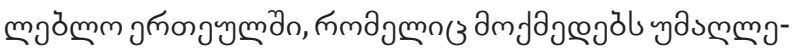

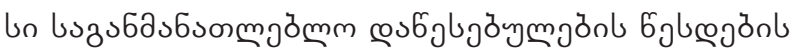

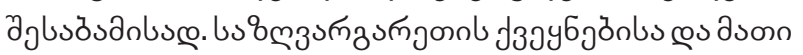

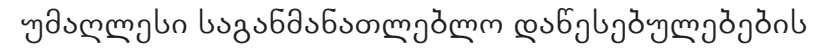

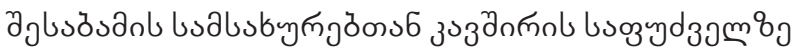

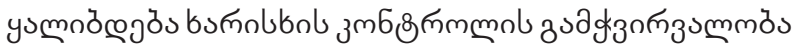
œs ason ฑ

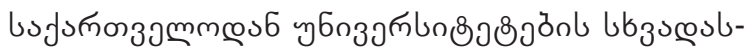

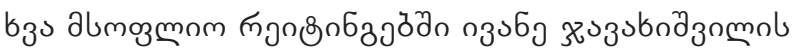

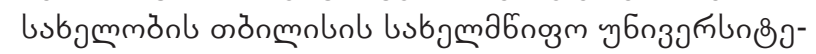

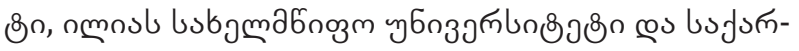

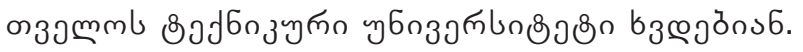

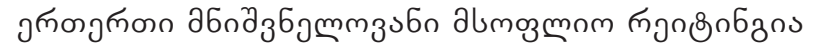

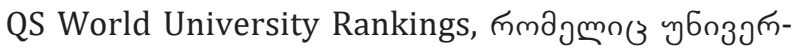

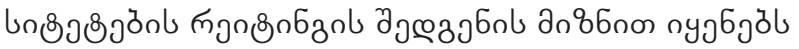

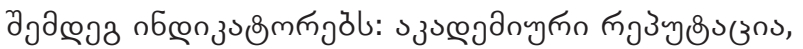
œsаاs

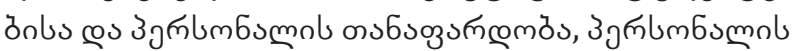

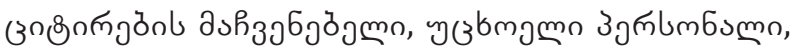

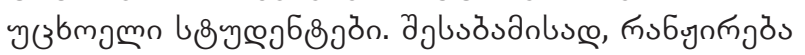

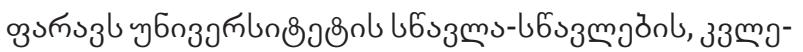

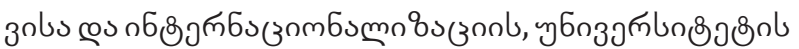

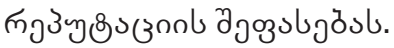

QS World University Rankings - b 20216 mol

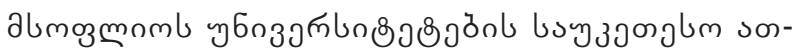

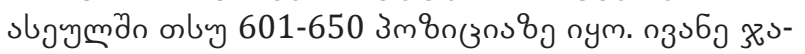

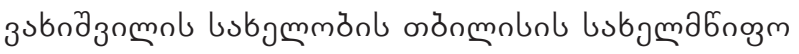
y6กзวங்

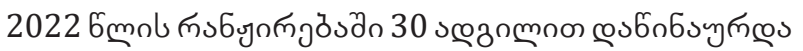

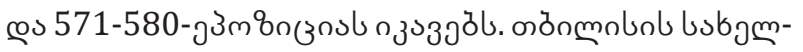

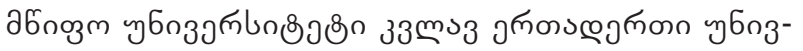
jங̆

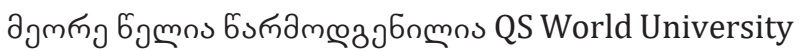

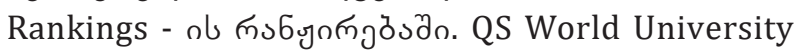

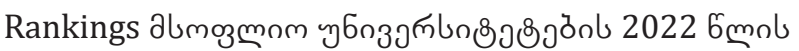

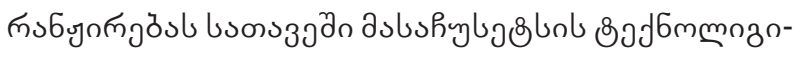

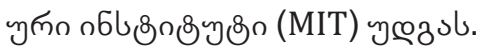

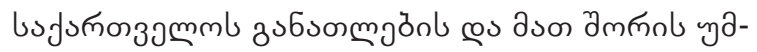

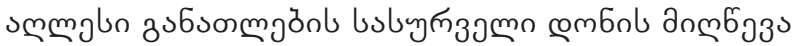
абпдз

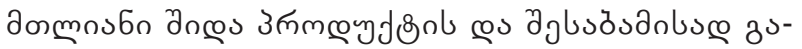

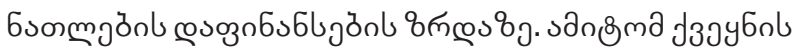

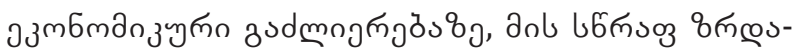

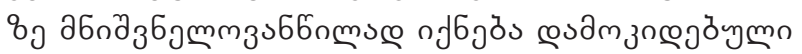

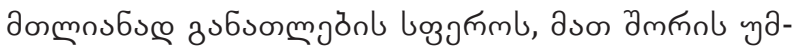

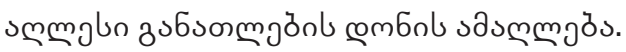

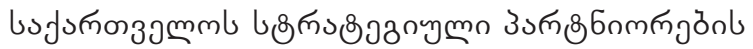

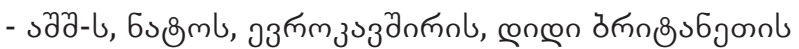

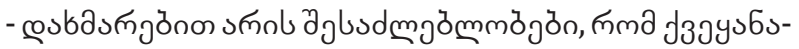
Әn sasмmœ

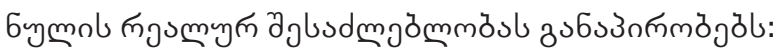

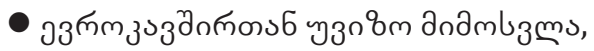

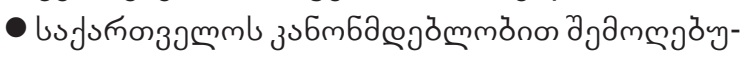

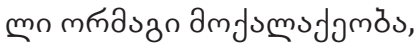

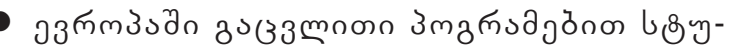

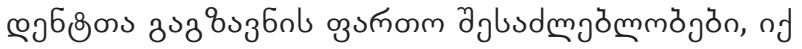

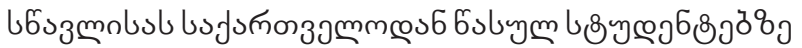

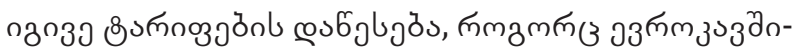
mol amjumsjojơ⿱宀o.

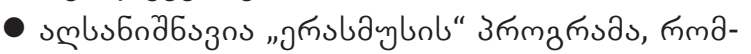
mnoss h hృб amfums

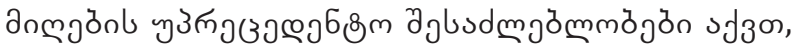

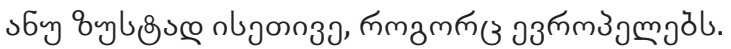

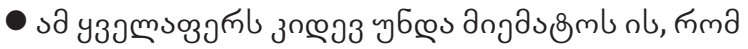

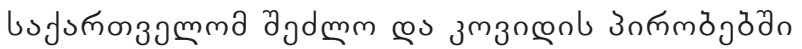

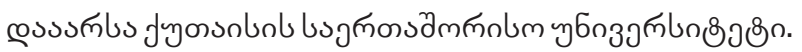

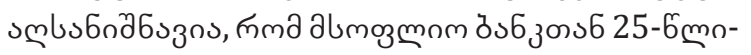

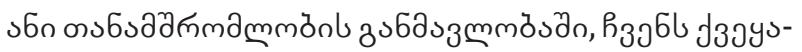

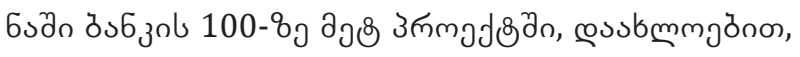

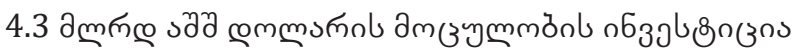

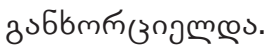

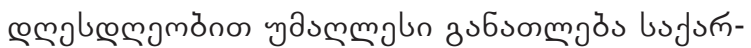

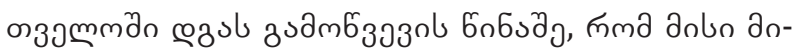

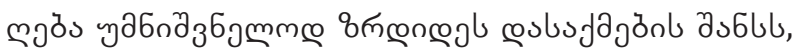

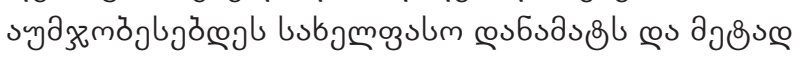

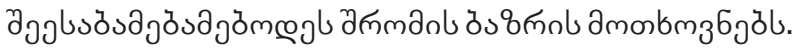

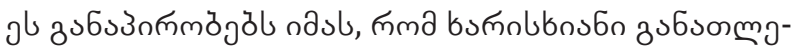

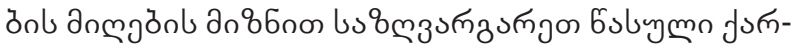

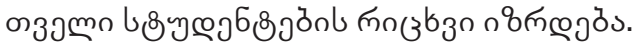

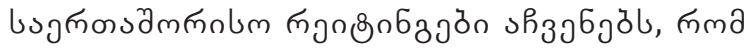

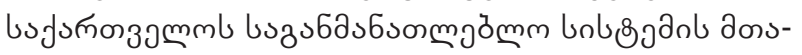

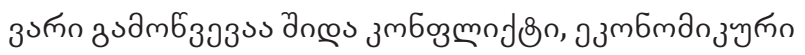

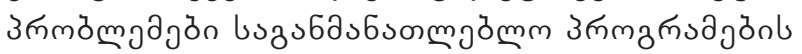

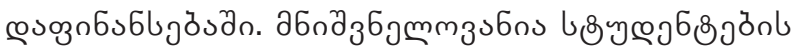

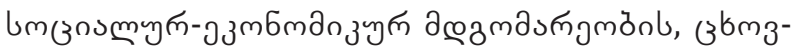

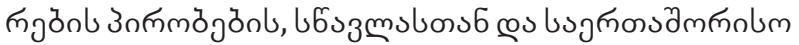

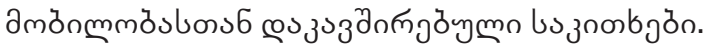




\section{6১}

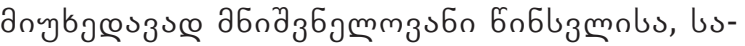

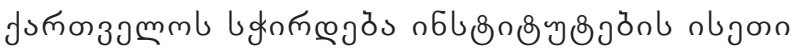

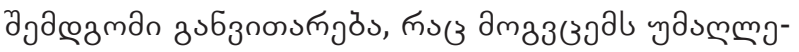

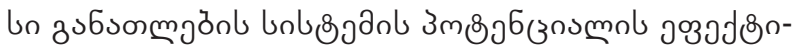

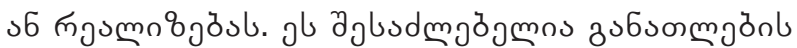

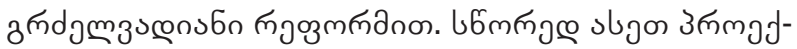

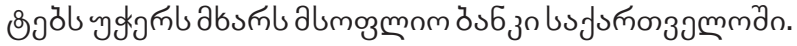

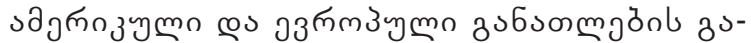

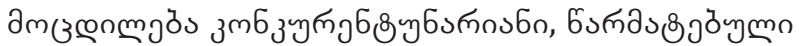

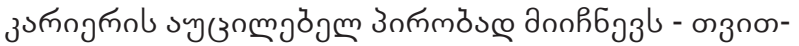

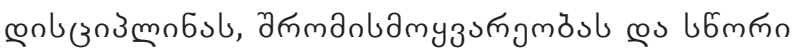

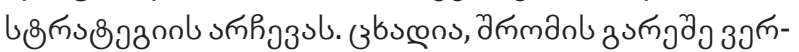

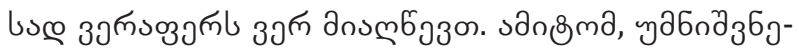

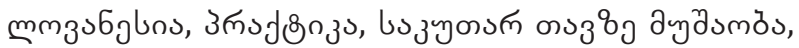

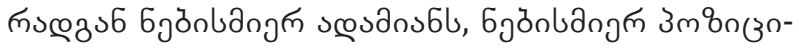

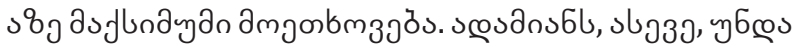

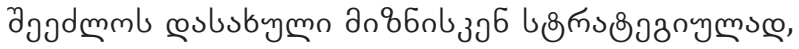

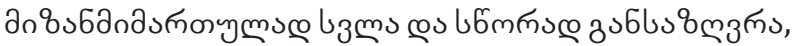

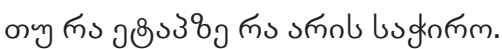

26пว

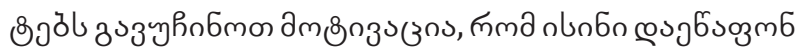

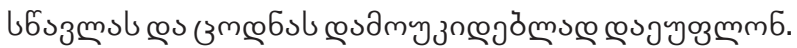

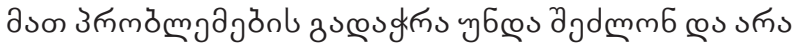

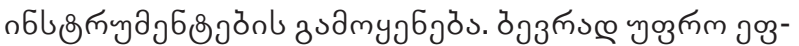

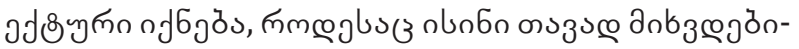

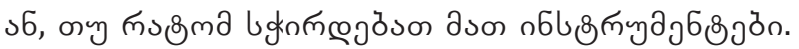

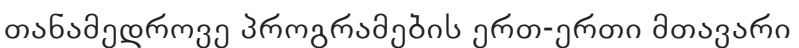

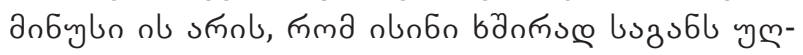

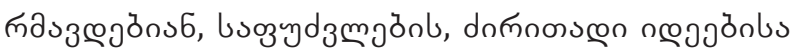

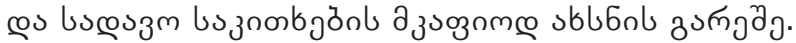

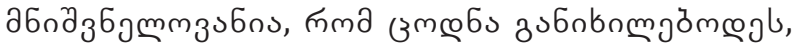

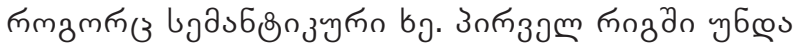

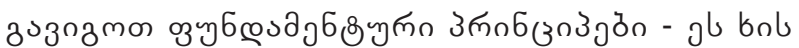

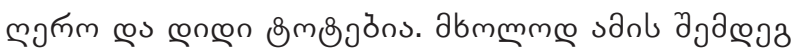

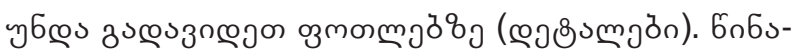

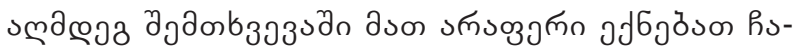

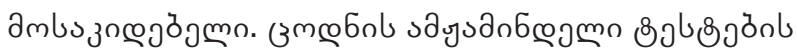

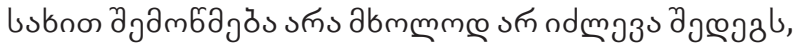

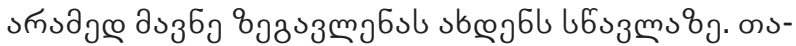

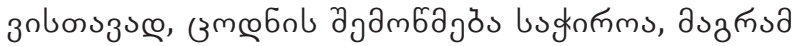

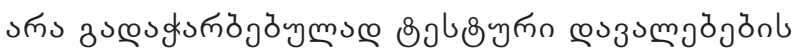

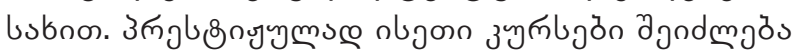

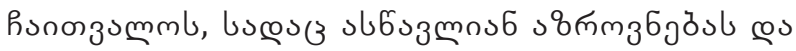

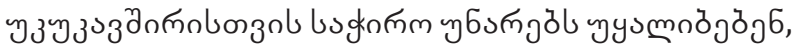

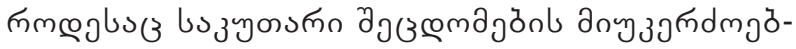

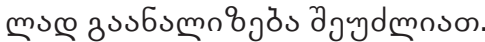

almozmom ơ

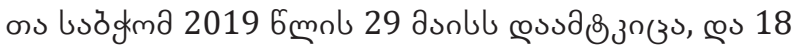

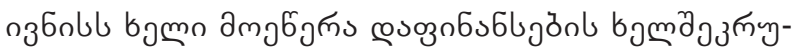

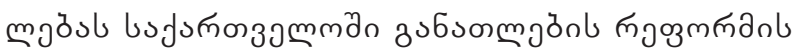

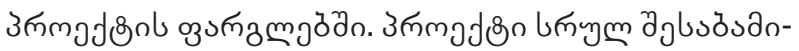

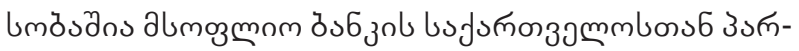

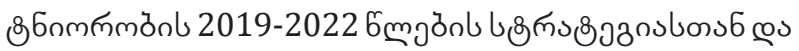

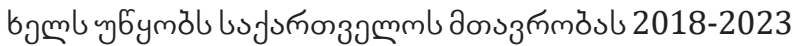

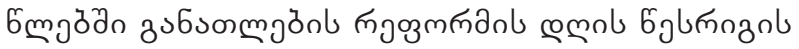

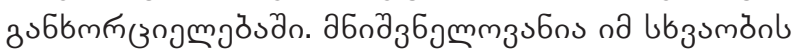

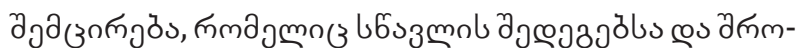

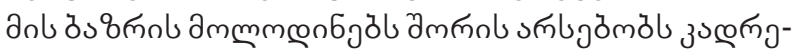

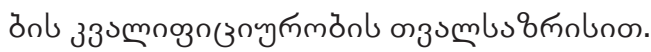

\section{REFERENCES:}

1. Sichinava, A., Chikava, M., Veshapidze, S., Sekhniashvili, D., Pailodze, N., (2013). Realities of internationalization of higher education in Georgia. PRADEC Conference. Internationalisation in Higher Education: Evaluating concepts, challenges and strategies, 25-26 April, 2013 Proceedings Volume 2 | Issue 1 | August 2013 |pp.95-98

2. Veshapidze, S., Zubiashvili, T., (2020). About the Origins of Modern Geoeconomic Foundations of Georgia. Ecoforum Journal 9 (2).

3. Veshapidze, S., (2017). THE TRADITIONS ORIENTATED ON EUROPEAN VALUES IN GEORGIA. Sciences of Europe. VOL 4, No 13 (13)

4. Veshapidze, Sh., Karalashvili, Sh., (2018). Pension Reform in Georgia. Refereed International Journal Ecoforum. Vol. 7. №2.

5. Veshapidze, S., Darbaidze, M., Beridze, T., (2016). Euro-Atlantic values: what tie up us. Universal. pp. 230-235.

6. Veshapidze, S., (2020). Religion and national values in Georgia. International Scientific Collection, Volume 3, pp. 33-36.

7. Veshapidze, S., Mchedlishvili, Z., (2020). From Ilia Chavchavadze's Economic Views: Competition, Private Property and International Trade. Ecoforum Journal 9 (2). 
8. Veshapidze S., Babuadze N., Beridze T., EUROPEAN VALUES AND CHOICE OF GEORGIA (HUMAN DIGNITY, LAWFUL SOVEREIGNTY, DEMOCRACY AND WELLBEING). ACTUAL PROBLEMS OF SUSTAINABLE DEVELOPMENT OF NATIONAL ECONOMIES. (10-11 July, 2015). PUBLISHING HOUSE OF PAATA GUGUSHVILI INSTITUTE OF ECONOMICS OF IVANE JAVAKHISHVILI TBILISI STATE UNIVERSITY. Tbilisi, 2015, pp. 79-83.

9. Zoidze, G., (2021). Basic Paradigms of Institutional Economics. Universali, Tbilisi. p. 202

10. Zoidze, G., (2020). GEORGIAN AGRO-SPHERE DEVELOPMENT PRIORITIES AND WAYS OF CARRYING OUT SOME IMPROVEMENTS. Myśl Ekonomiczna i Polityczna, (2 (69)), 55-71. DOI: 10.26399/ meip.2(69).2020.10/g.zoidze

11. Zoidze, G., (2020). Risks Caused by Pandemics and Anti-crisis Management. Ivane Javakhishvili Tbilisi State University, Paata Gugushvili Institute of Economics, Proceedings of Materials of International Scientific Conference Dedicated to the 115th Birth Anniversary of Academician Paata Gugushvili, 190-195

12. Galt \& Taggart, Education Sector in Georgia. Georgia Education, Sector Review July 28, 2020. Bochorishvili E., Peranidze N. (In Georgian) Available at: https://galtandtaggart.com/upload/reports/14673. pdf (Accessed: 1st July 2021).

13. International Bank for Reconstruction and Development / World Bank, 2020. Survival, Learning, Prosperity: A Strategic Investment in Human Capital for Georgia's Potential Development. (In Georgian) Available at: https://documents1.worldbank.org/curated/en/217471597681506927/Survive-LearnThrive-Strategic-Human-Capital-Investments-to-Unlock-Georgias-Potential.pdf (Accessed: 1st July 2021).

14. Legislative Herald of Georgia, Convention for the Protection of Human Rights and Fundamental Freedoms. (In Georgian) Available at: https://matsne.gov.ge/ka/document/view/1208370?publication=0 (Accessed: 1st July 2021).

15. Legislative Herald of Georgia, International Covenant on Economic, Social and Cultural Rights. (In Georgian) Available at: https://matsne.gov.ge/ka/document/view/1483577?publication=0 (Accessed: 1st July 2021).

16. Legislative Herald of Georgia, European Social Charter (Revised) Strasbourg, 3.V. 1996 (in Georgian) Available at: https://matsne.gov.ge/ka/document/view/1392164?publication=0 (Accessed: 1 st July 2021).

17. Legislative Herald of Georgia, Law of Georgia on Higher Education. (In Georgian) Available at: https://matsne.gov.ge/ka/document/view/32830?publication=85. (Accessed: 1st July 2021).

18. Legislative Herald of Georgia, Organic Law of Georgia Labor Code of Georgia. (In Georgian) Available at: https://matsne.gov.ge/document/view/1155567?publication=10 (Accessed: 1st July 2021).

19. Legislative Herald of Georgia, Constitution of Georgia. (In Georgian) Available at: https://matsne. gov.ge/ka/document/view/30346?publication=36 (Accessed: 1st July 2021).

20. Ministry of Education and Science of Georgia, project "Innovation, Inclusive Education and Quality". (In Georgian)Available at: https://mes.gov.ge/content.php?id=10452\&lang=geo (Accessed: 1st July 2021).

21. Society and banks, education for economic growth. (In Georgian) Available at: http://sab.ge/ge/ news/880-ganatleba-ekonomikuri-zrdistvis (Accessed: 1st July 2021).

22. UN, Universal Declaration of Human Rights, 60th Anniversary, Special Edition 1948-2008. (In Georgian) Available at: http://www.supremecourt.ge/files/upload-file/pdf/aqtebi3.pdf (Accessed: 1st July 2021).

23. United Nations General Assembly Resolution A / RES / 70/1 of 25 September 2015 - "Transforming Our World: A 2030 Agenda for Sustainable Development". (In Georgian) Available at: https://undocs. org/A/RES/70/1 (Accessed: 1st July 2021). 


\title{
THE WORLD PANDEMIC- EXPERIMENT DEVELOPING DEGLOBALISATION
}

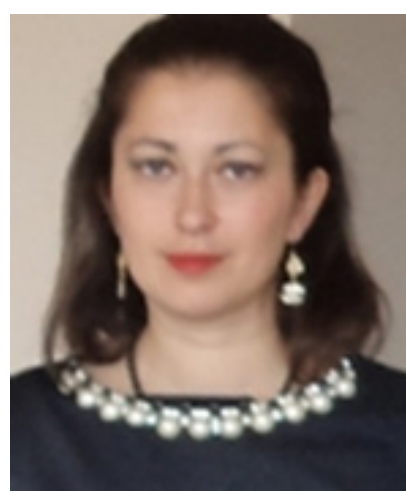

\author{
Kristina Jganjgava \\ Doctor of Business Administration Science \\ E-mail: kjganjgava@yahoo.com \\ Cell: (+995) 579088612
}

DOI suffix: 10.36962/NEC6102202139

\begin{abstract}
Globalization is the result of the development of civilization. This process has helped reduce transportation costs around the world, resulting in an increase in travelers around the world. Travel is even recognized as an important determinant of the spread of the disease. Therefore, globalization has become the mechanism of transmission of the infectious disease coronavirus (Covid-19) worldwide. Thus, coronavirus-related events pose additional practical and symbolic challenges to the globalization agenda. The purpose of this article is to determine the potential impact of the pandemic on the future direction of globalization.

Keywords: Covid-19, Deglobalisation, Economic Impact.
\end{abstract}

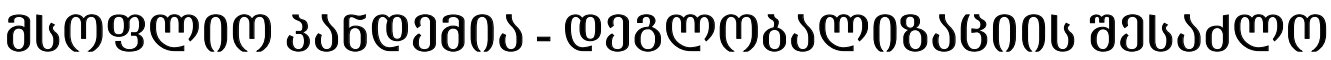

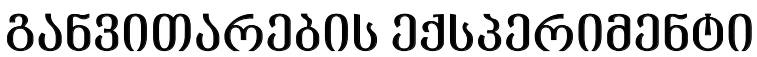

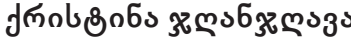

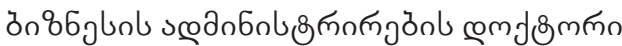 \\ E-mail:kjganjgava@yahoo.com
}

\begin{abstract}
১бП৪১৫০১

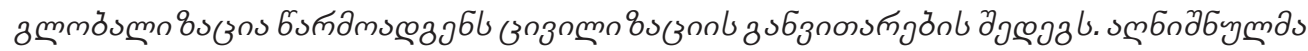

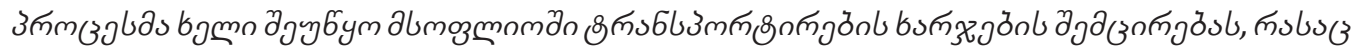

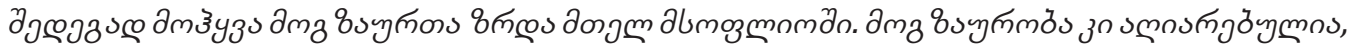

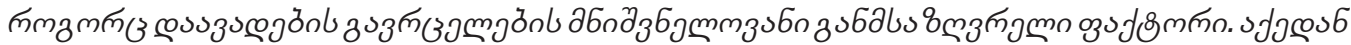

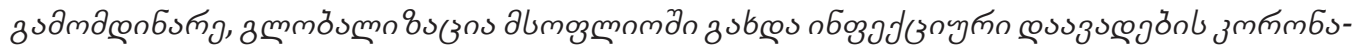

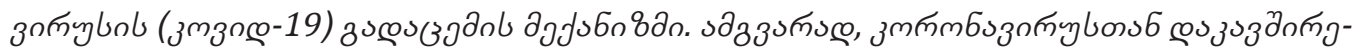

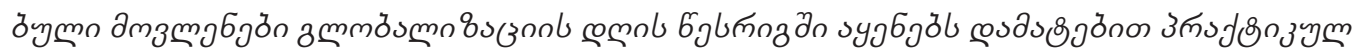

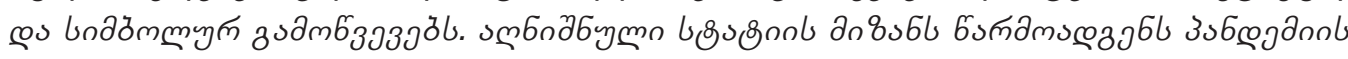

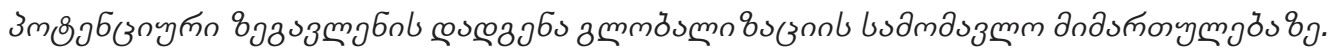

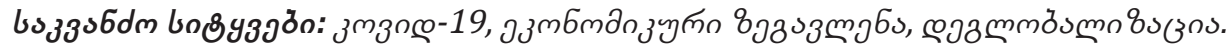




\section{วอ৬১૩১ল}

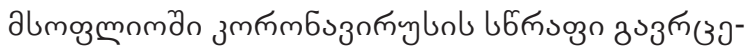
mృ

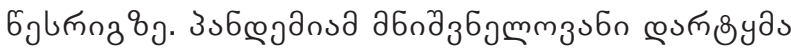

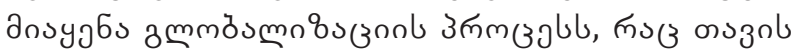

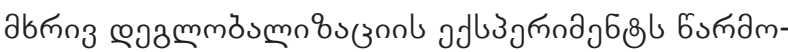

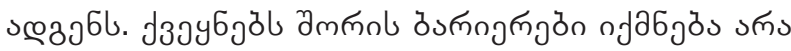

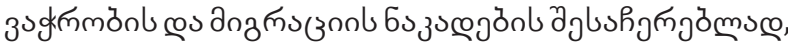

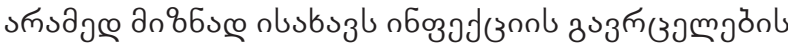

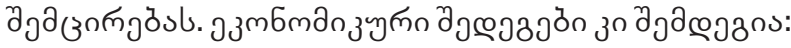

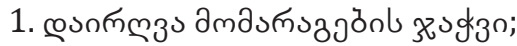

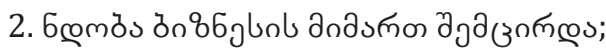

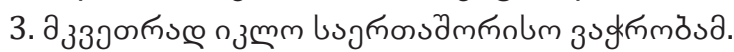

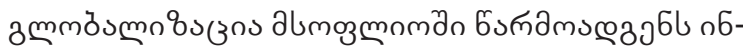

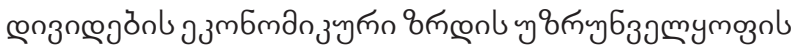

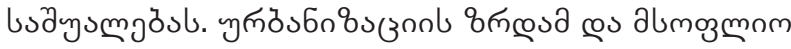

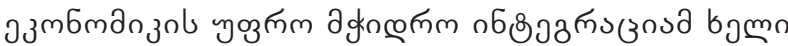

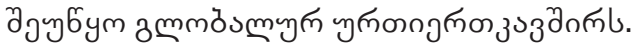

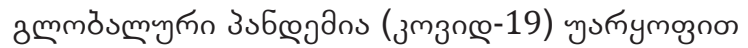

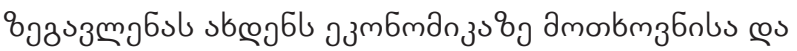

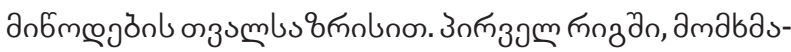

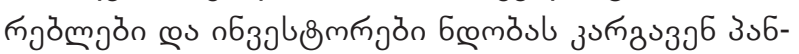

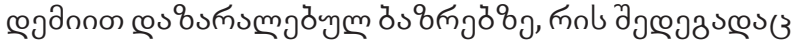
a उnпূ@

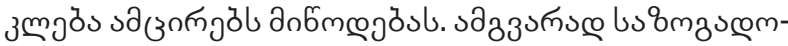

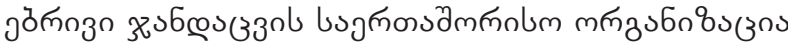

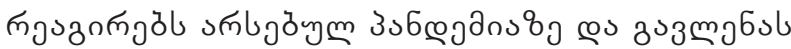

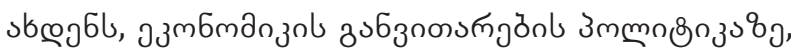

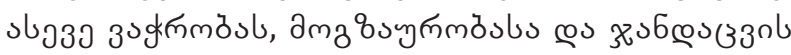
เoзgตm

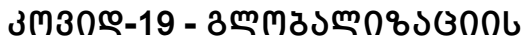

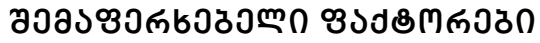

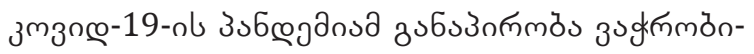

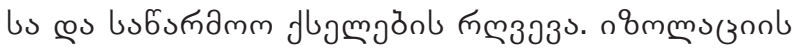

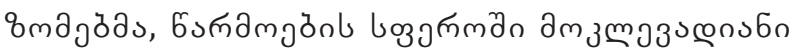

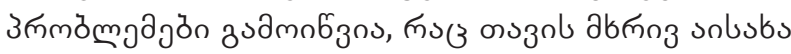

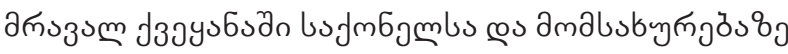

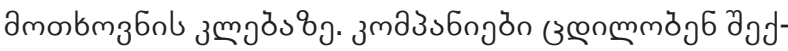

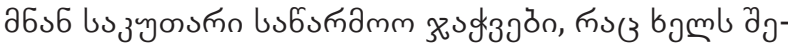

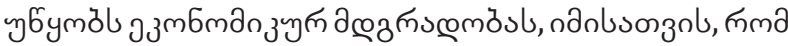

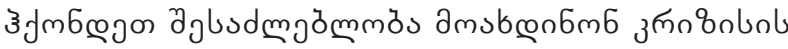

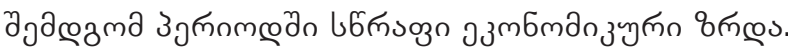

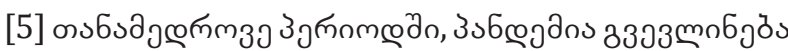

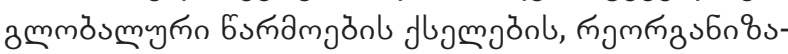

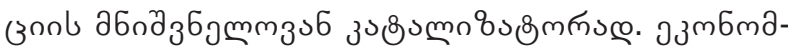

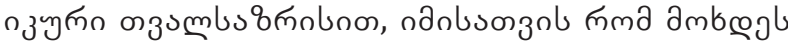

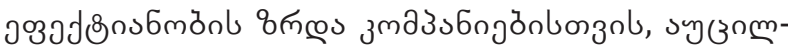

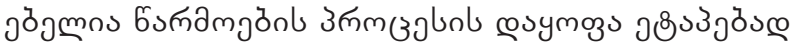

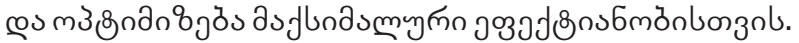

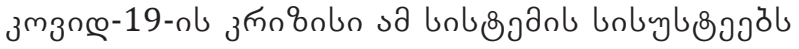

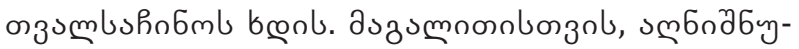

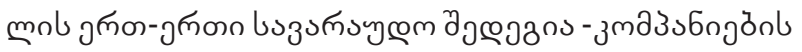

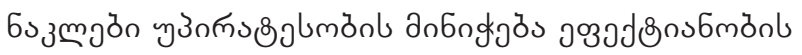

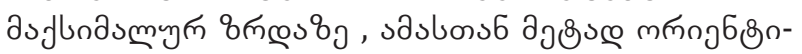

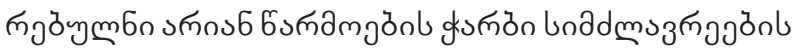

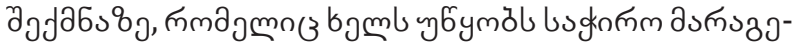

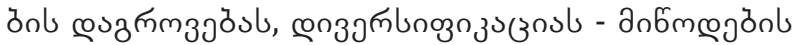

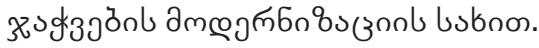

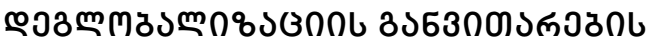

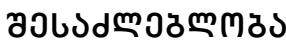

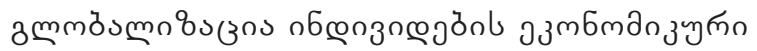
œs зуyм

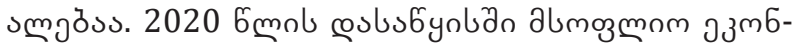

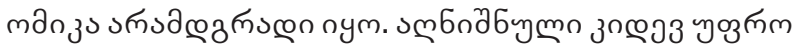

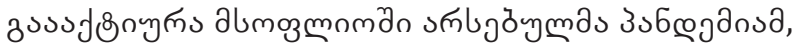

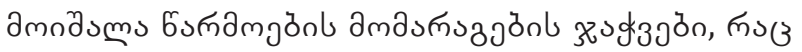

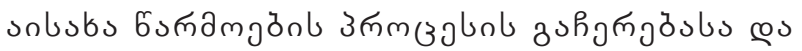

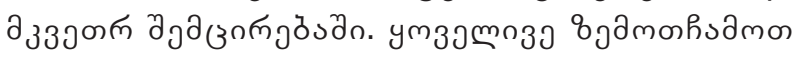

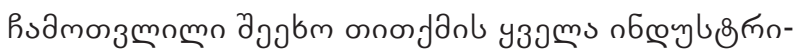

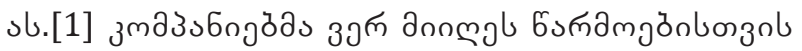

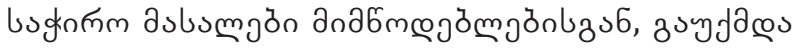
วбпдз

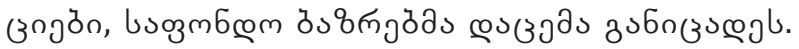

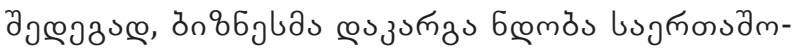

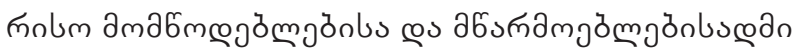

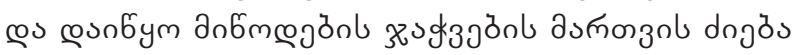

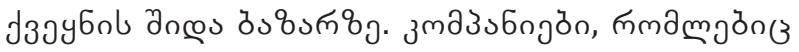

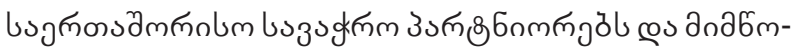

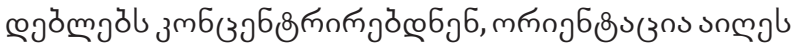

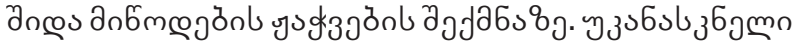

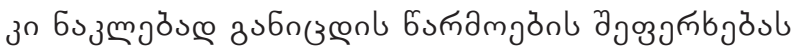

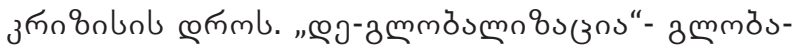

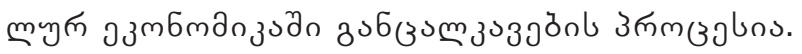

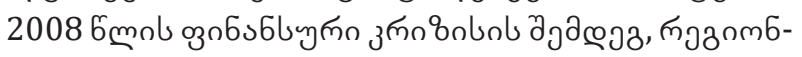

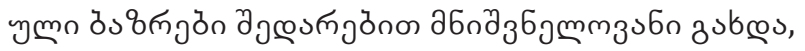

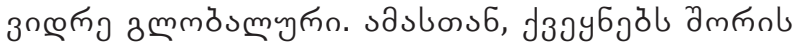

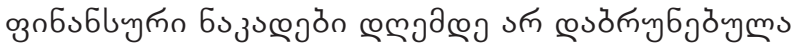

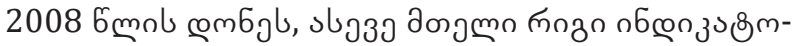

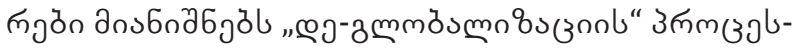

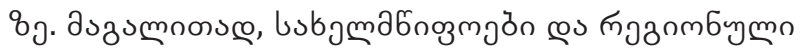

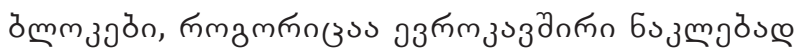

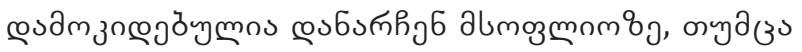

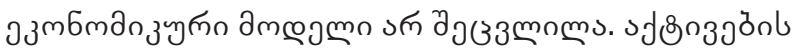

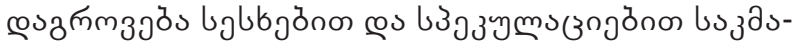

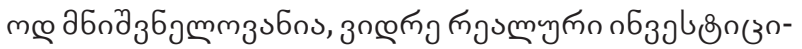

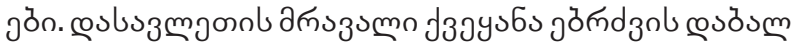




\section{ISSN 2667-9752(Online)}

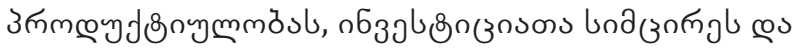

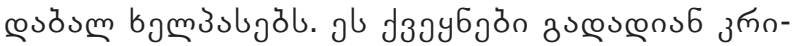

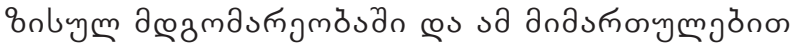

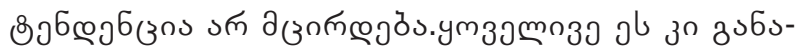

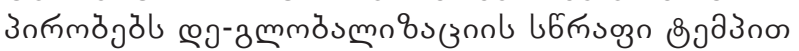

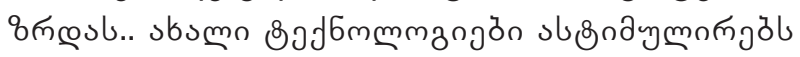

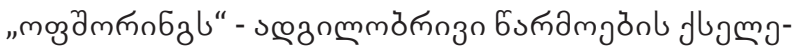

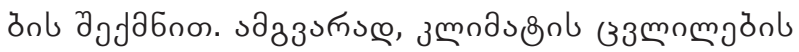

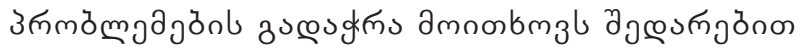

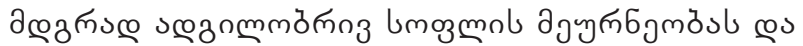

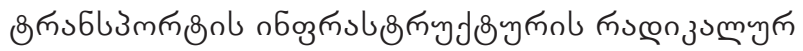

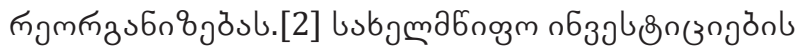

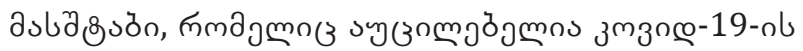

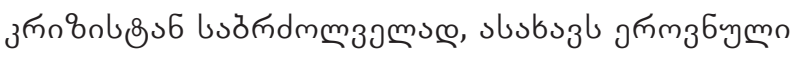

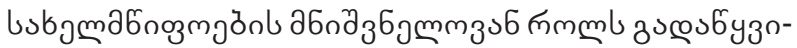

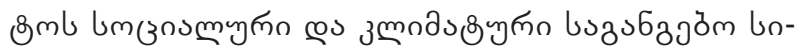

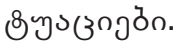

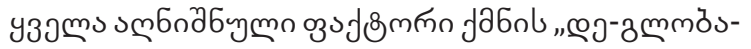

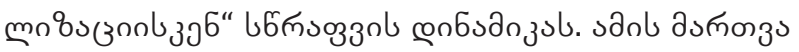

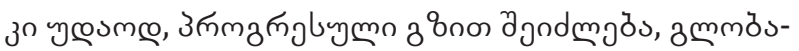

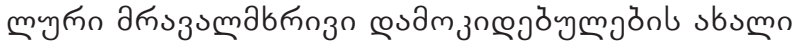

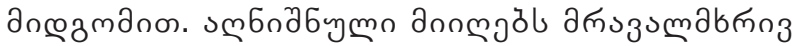

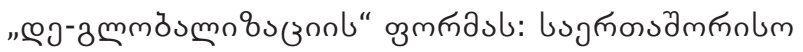

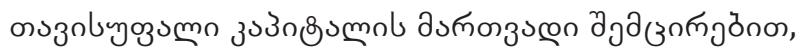

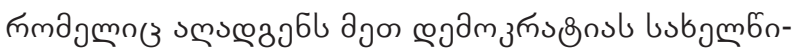

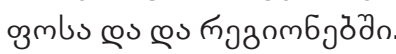

\section{6ง}

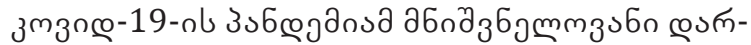

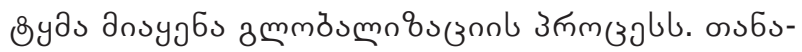

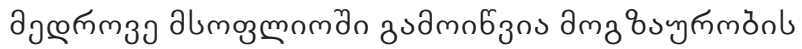

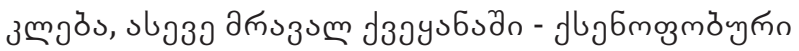

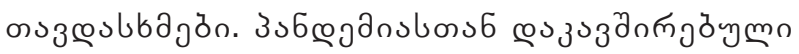

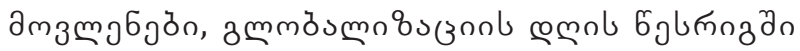

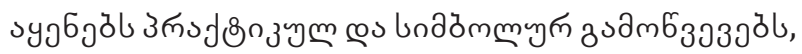

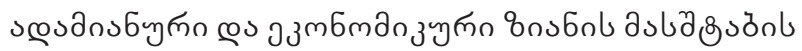

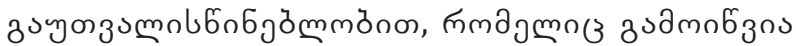

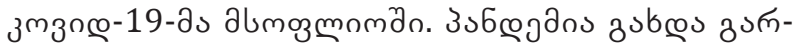

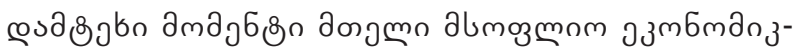
obozol.

\section{REFERENCES:}

1. «Potential Economic impact of an Avian Flu Pandemic on Asia”, E.Bloom, V.De Wit, M.J. Carangal, 2005;

2. „Towards Digital Globalisation and the corona-19 challenge”,D. Schiliro,may, 2020

3. Globalisation and Global health, G.Berlinguer, 2020

4. ThE COVID Impact on Business Globalisation: what does the future Hold? The European Business Review, octomber 14, 2020

5. Global Trade Alert ${ }^{\mathrm{h},}$ Global Trade Alert Report - Going It Alone? Trade Policy After Three Years of Populism, 2019

6. Obstfeld M. Globalization Cycles, CEPR Discussion Paper, 2020

7. Coronavirus and the crisis of Globalisation," L.Cooper, 2020 


\title{
GEORGIA'S TRANSIT POTENTIAL - COUNTRY'S COMPETITIVE ADVANTAGE
}

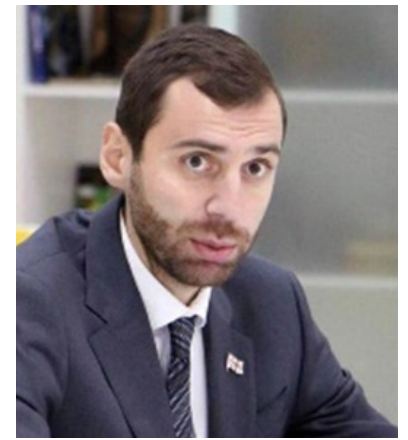

Giorgi Abashishvili,

$\mathrm{PhD}$ in Business Administration, Chancellor, Georgian Institute of Public Affairs (GIPA)

E.mail: giorgi.abashishvili@gmail.com

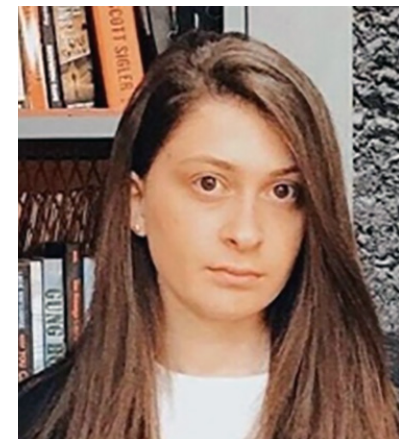

Salome Liparteliani, MBA student, Georgian Institute of Public Affairs

DOI suffix: 10.36962/NEC6102202142

\section{ABSTRACT}

The recent years witnessed the repeated urgency of Georgia's transport potential. In parallel, Georgia's role in the BRI initiative has become clear. Consequently, Georgia's capabilities have become clearer, however, there are still issues in this regard, the study of which will greatly help the country to achieve SDGs and increase efficiency.

To achieve the above objective, complex recommendations have been developed within the scope of the survey that will significantly increase the country's transport potential and attractiveness; however, it should also be noted that the impact of the transit corridor on the achievement of the Sustainable Development Goals (SDGs) to promote inclusive economic development/growth in the long-term perspective should be considered.

The survey is dedicated to the analysis of Georgia's transit potential, opportunities, existing problems, challenges and prospects, as well as recommendations for the use of this potential to achieve the country's Sustainable Development Goals (SDGs), which analyzes the country's top priorities, current risks and economic development opportunities.

Keywords: Transit potential

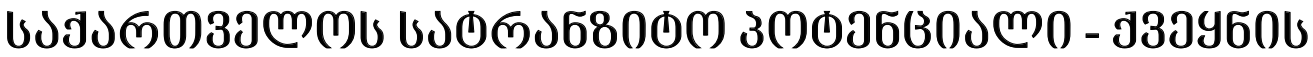

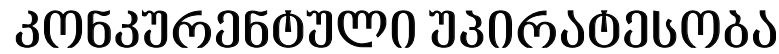

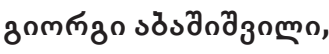

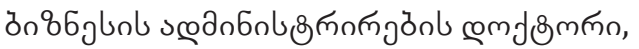

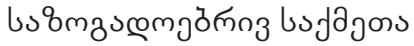

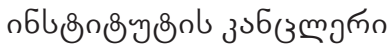

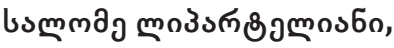

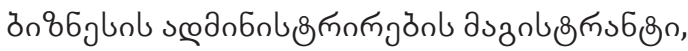

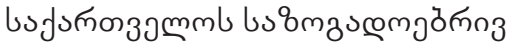

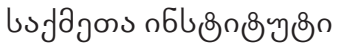

\begin{abstract}
ग6M\&S30ડ

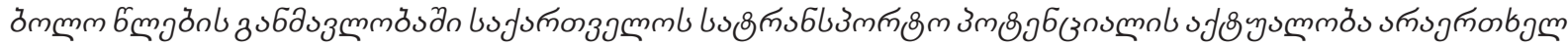

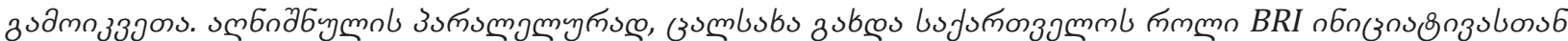

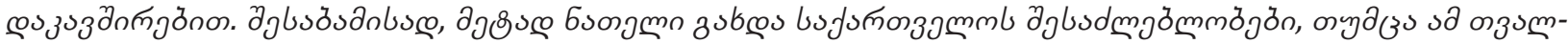

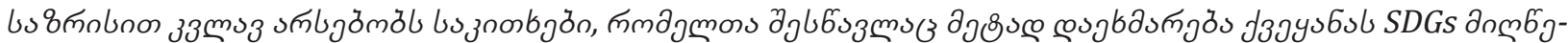

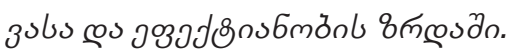

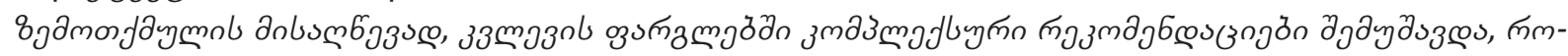




\section{ISSN 2667-9752(Online)}

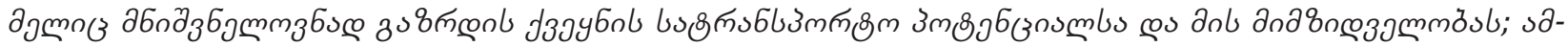

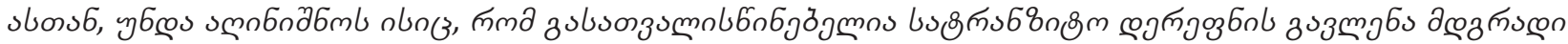

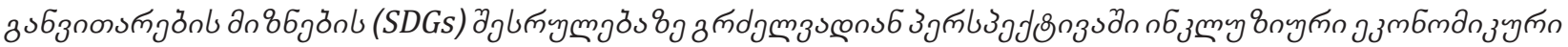

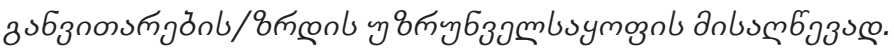

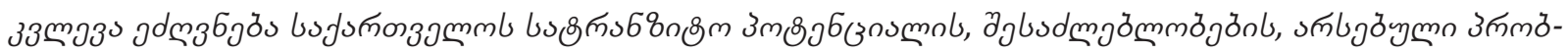

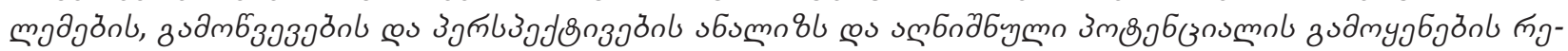

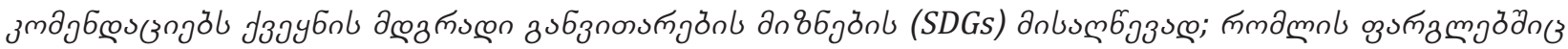

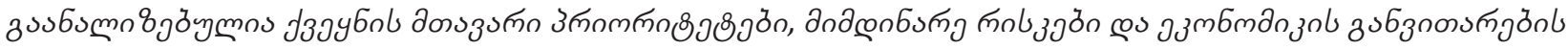

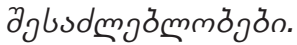

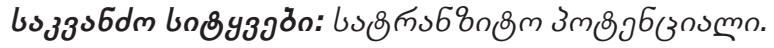

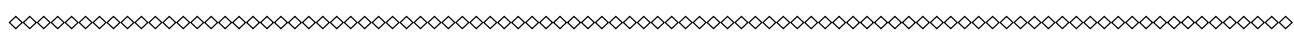

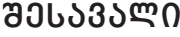

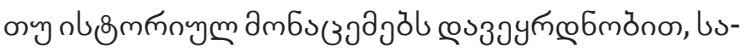
jงпоззлмm

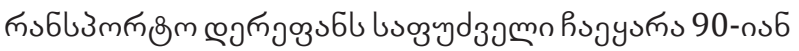

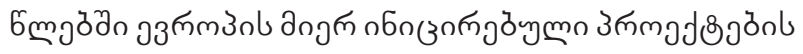

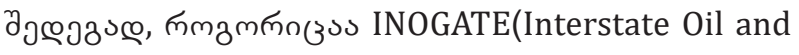
GAs Transportation to Europe) @๖ TRACECA(Transport Corridor Europe-Caucasus-Asia).

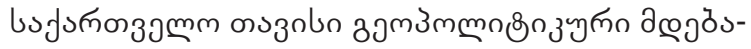

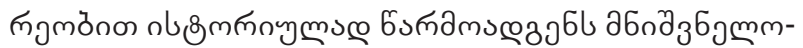

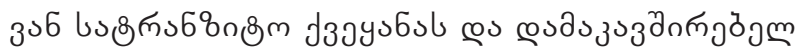

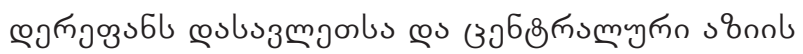
ذ3әyб

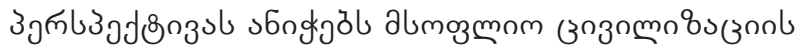
зпмзз

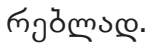

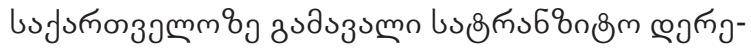

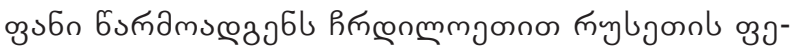

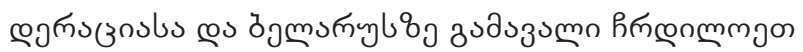

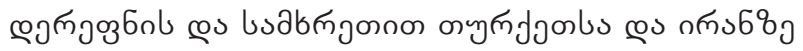

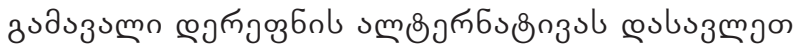

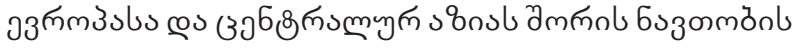

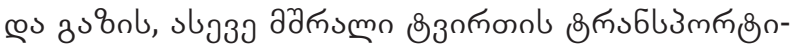
mjönbonzol.

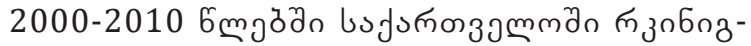
8non zuœu

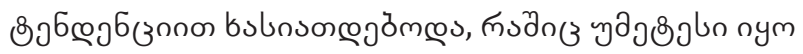

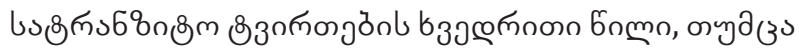

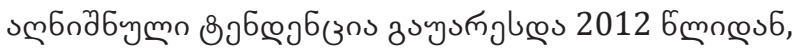

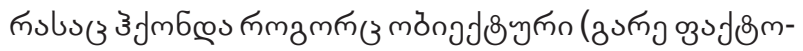

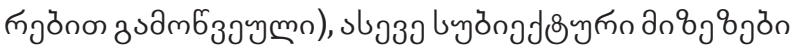

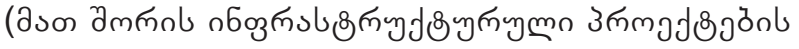

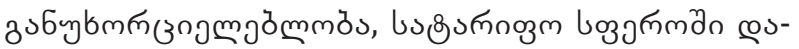

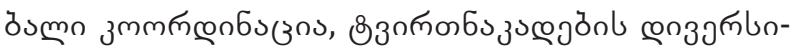

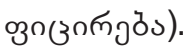

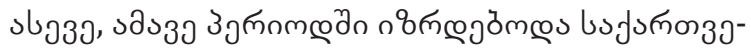

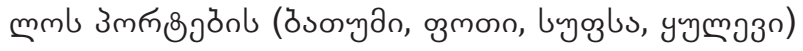

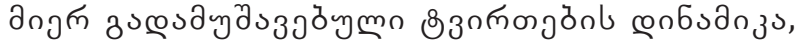

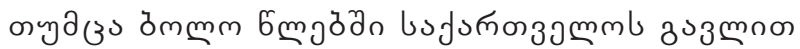

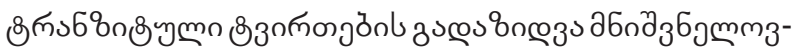

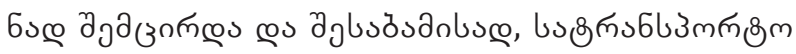

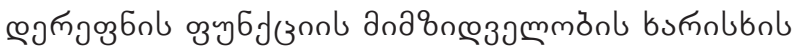

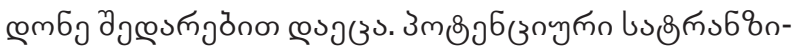

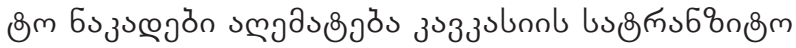

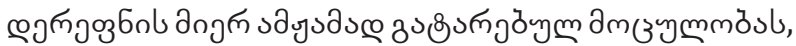

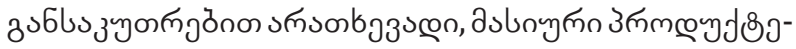

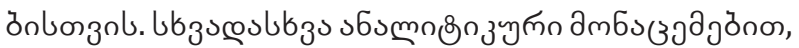

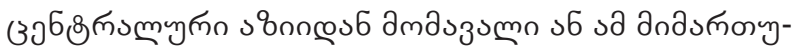

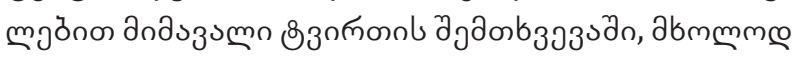
उмఠ९

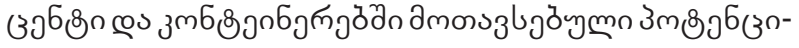
ymn ls 8 m

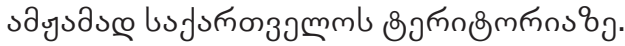

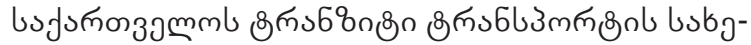

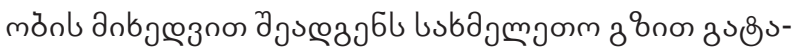

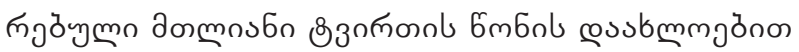

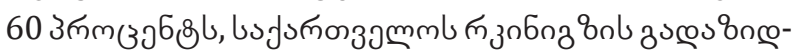

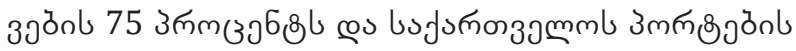

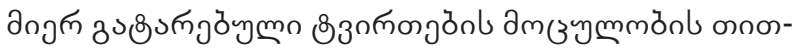

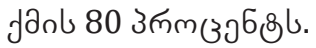

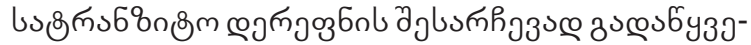

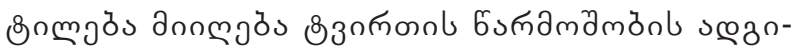

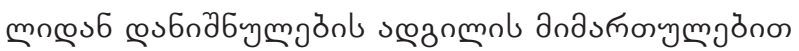

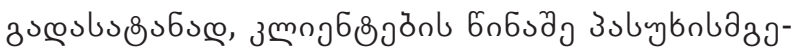

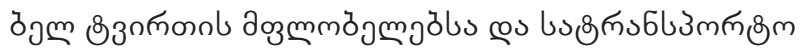

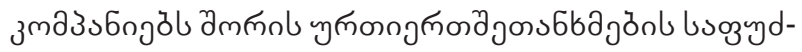

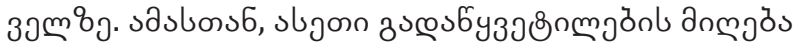

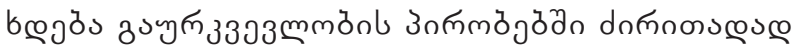

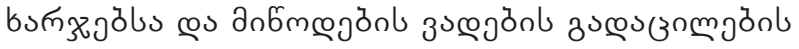
molul

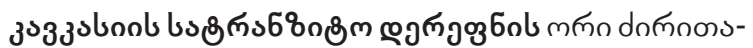

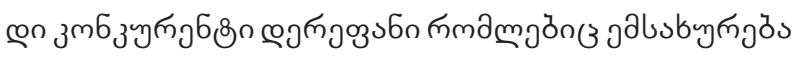

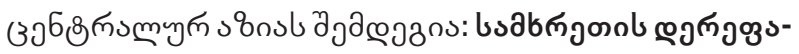

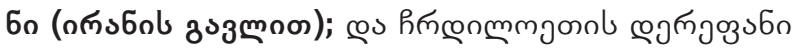




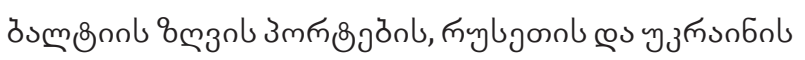
зง 3 mnon.

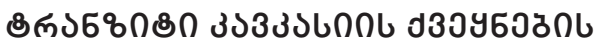

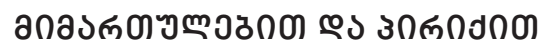

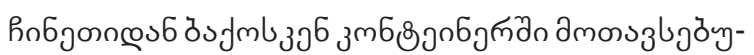

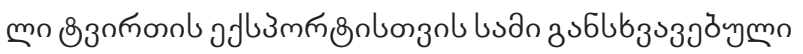

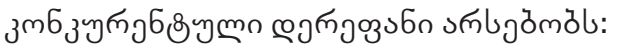

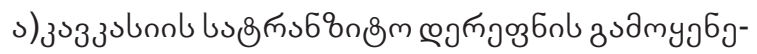

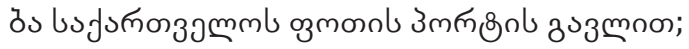

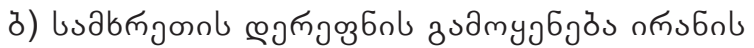

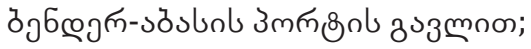

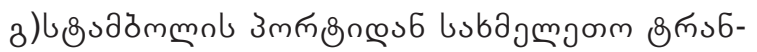

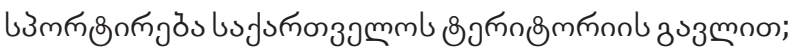

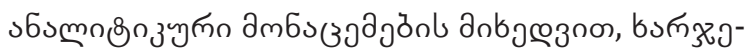

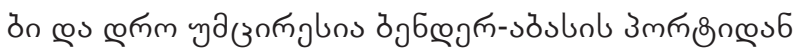

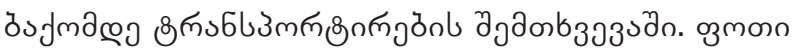

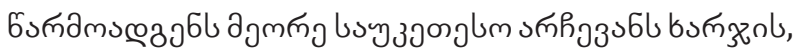

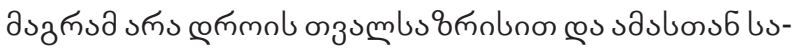

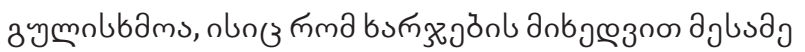

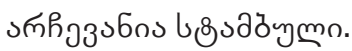

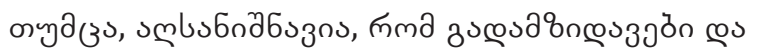

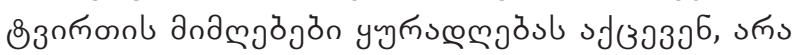

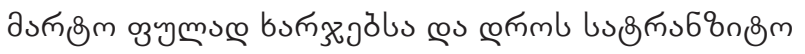

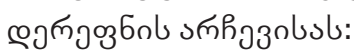

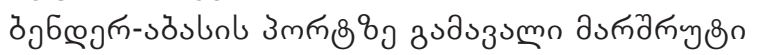

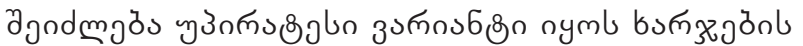

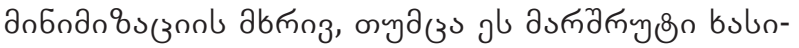

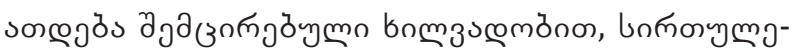

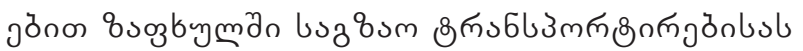

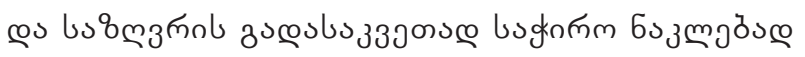

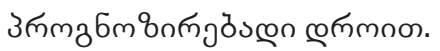

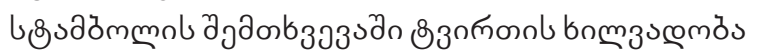

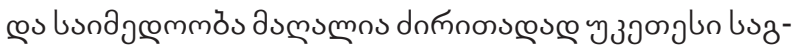

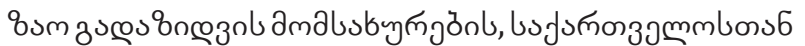

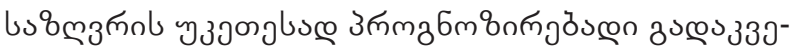

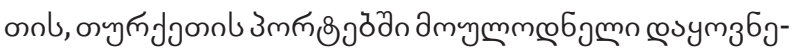

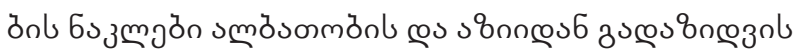

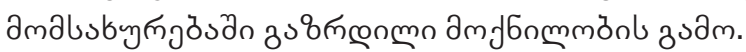

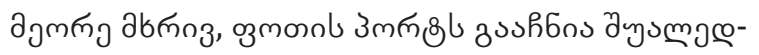

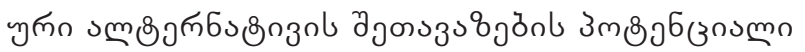

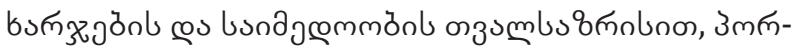

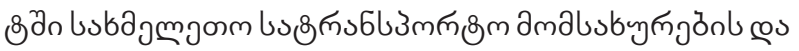
৬ง

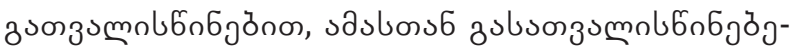

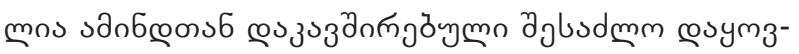
6ృ

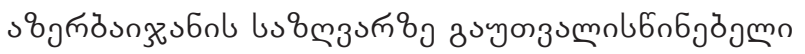

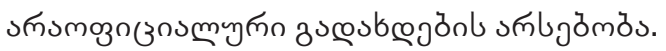

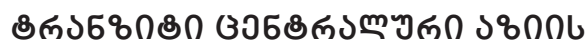

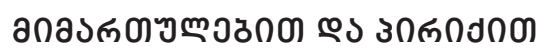

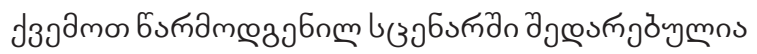

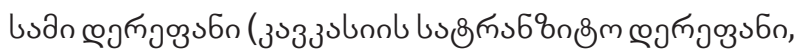

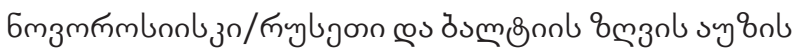

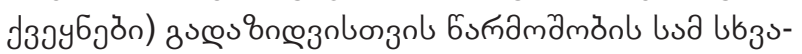

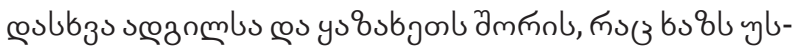

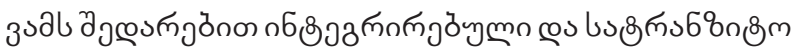
dsmozol a6nz̧б

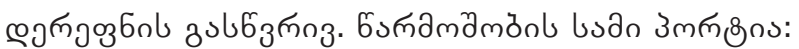

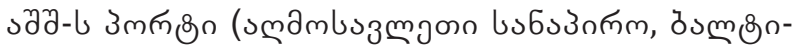

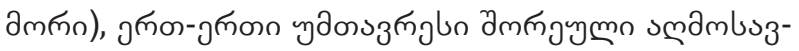

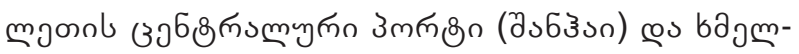
๓лду

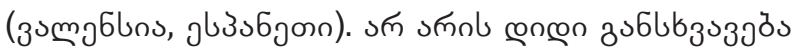

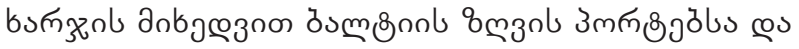

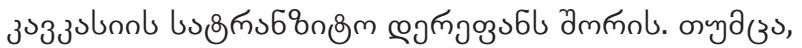
зsূs

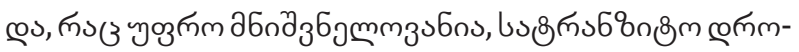

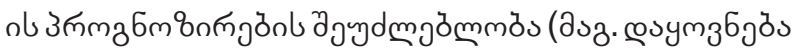

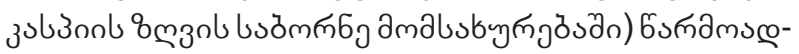

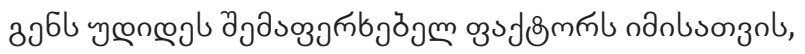

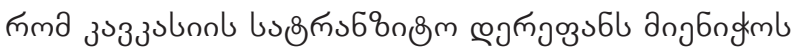

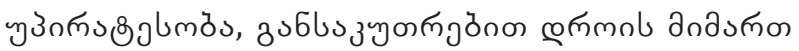

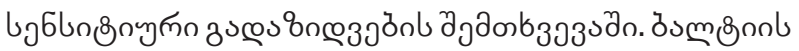

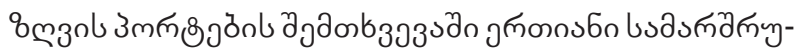

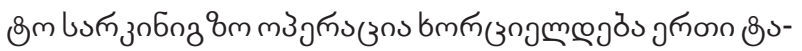

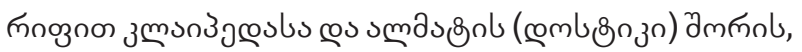
œ১৪ి

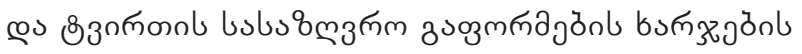

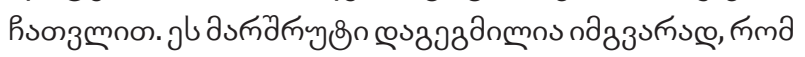

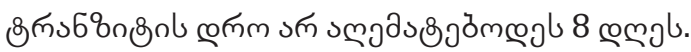

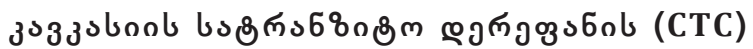

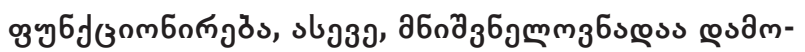

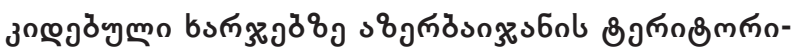

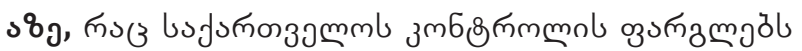

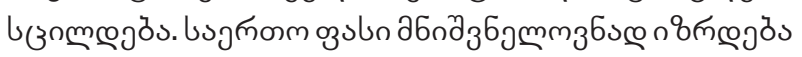

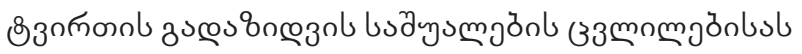

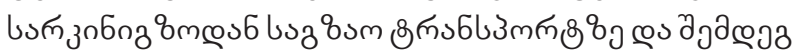

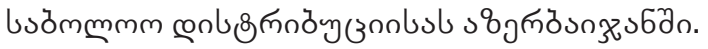

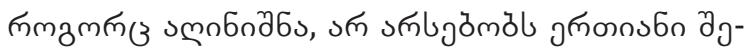

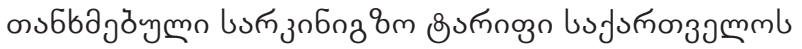

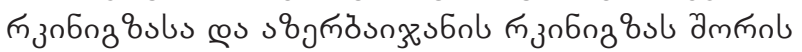

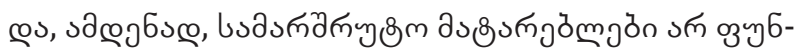

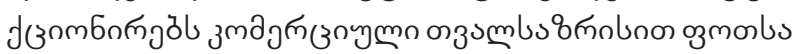
œu ठ̀ Jmb ปेmmol.

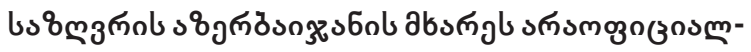

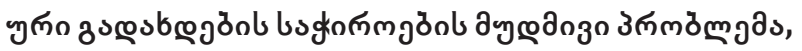

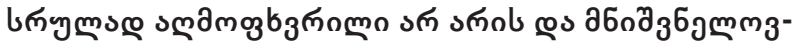




\section{ISSN 2667-9752(Online)}

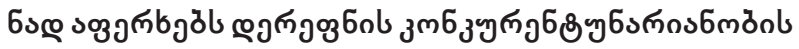
sasmmgósl.

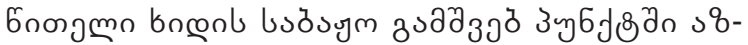

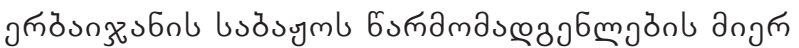

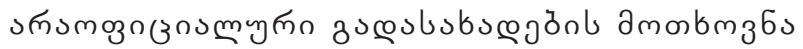
aбпдзб

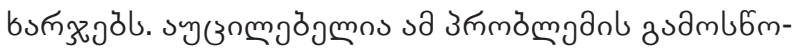

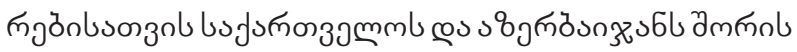

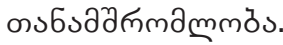

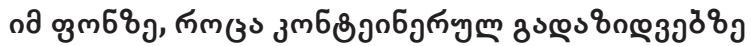
2mobmзб

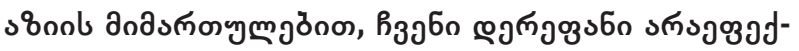

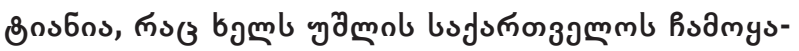

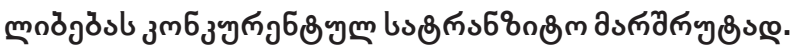

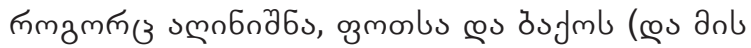

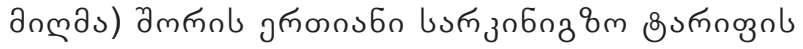

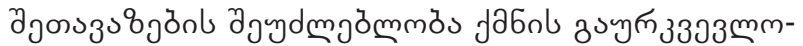

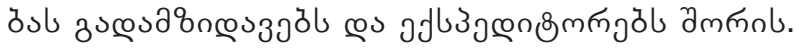

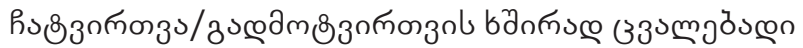

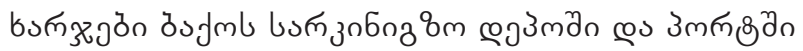

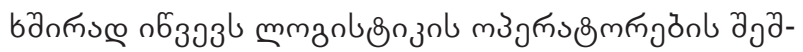

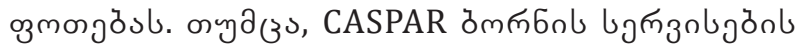

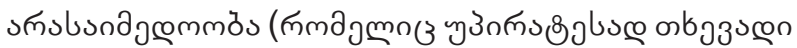

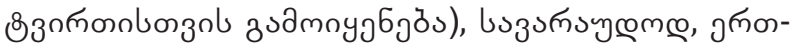

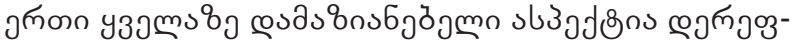

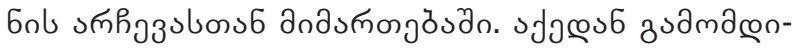

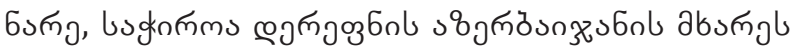
১ங்

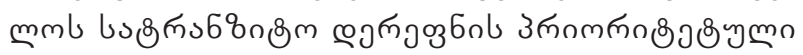

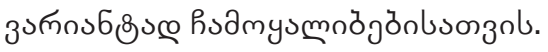

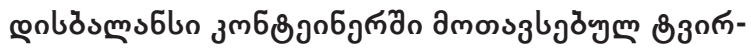

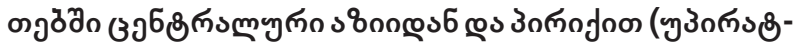

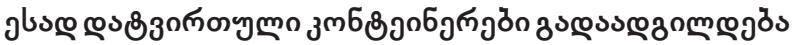

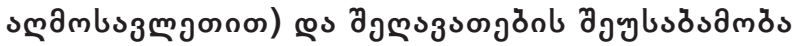

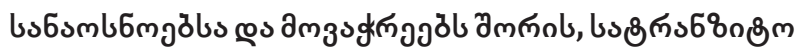

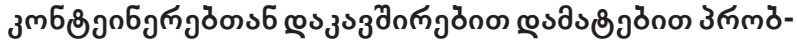

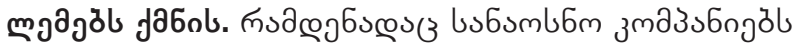

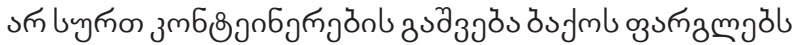

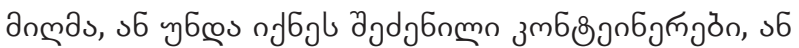

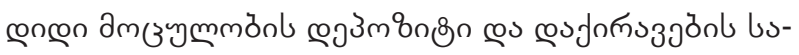

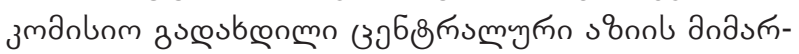

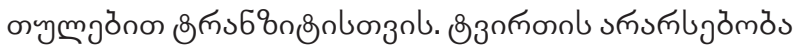

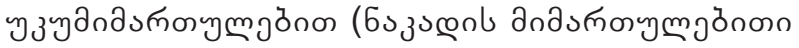

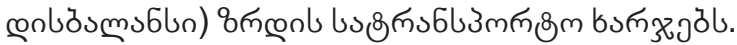

bujunnз

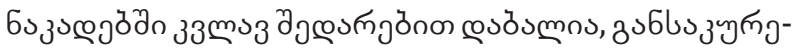

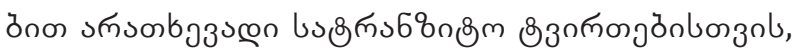
๓mamjön

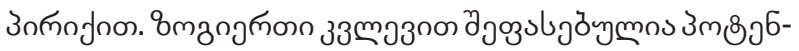

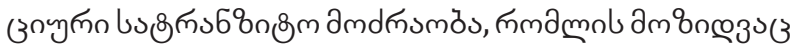

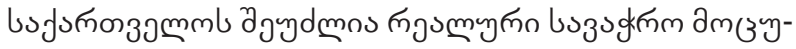

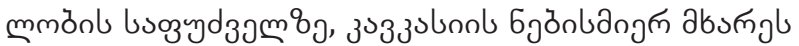

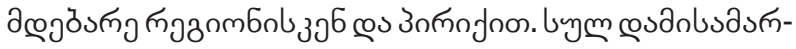

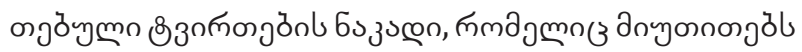

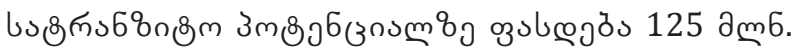

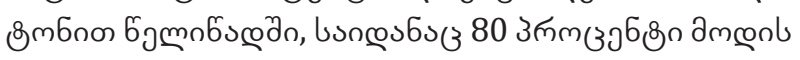

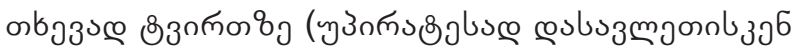

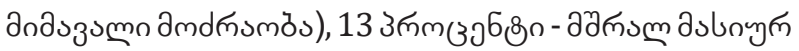

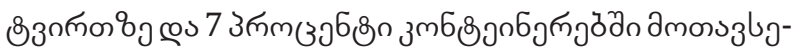

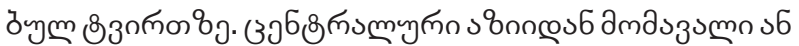

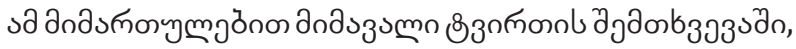

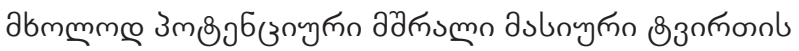

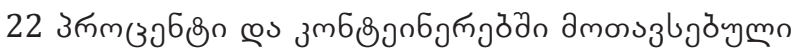
Зм \&

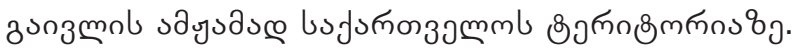

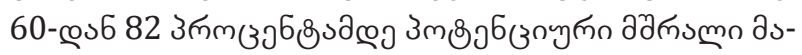

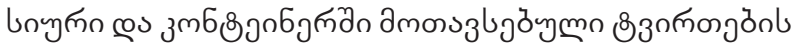

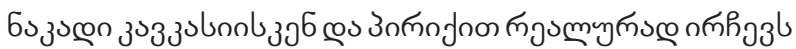

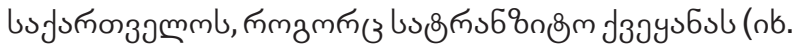

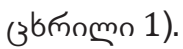

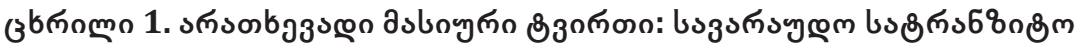
Зм 8े

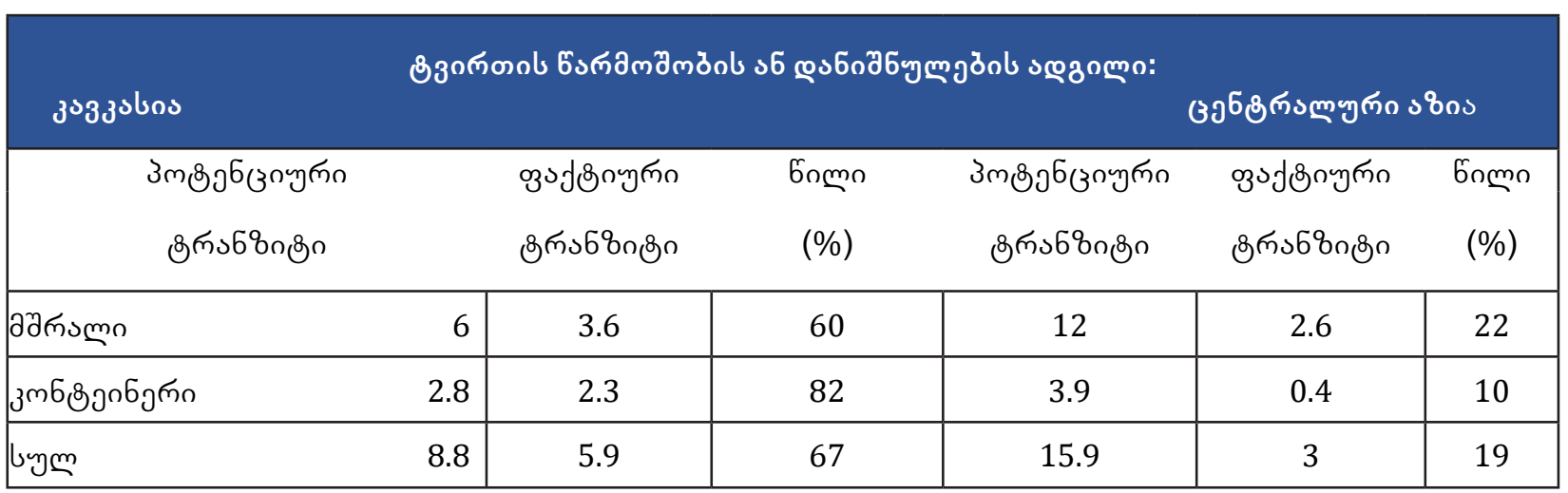

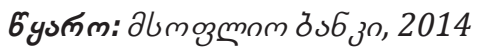

№2(61), 2021, Vol. 16, Issue 2. 


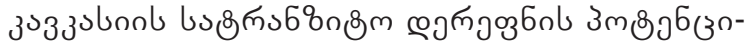

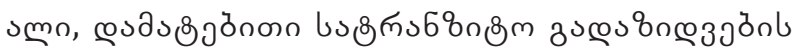

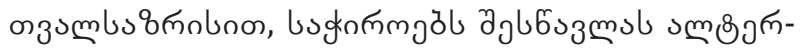

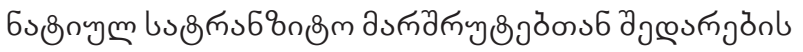

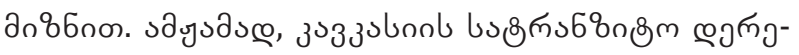

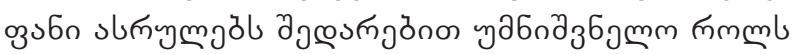

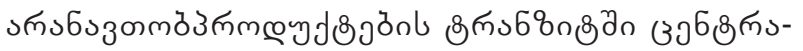

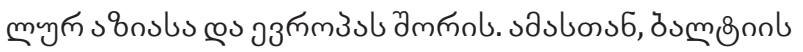

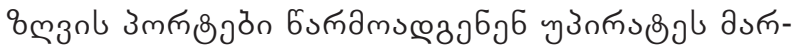

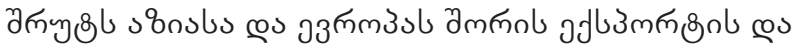

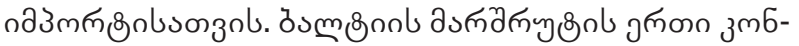

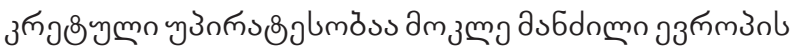

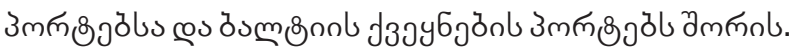

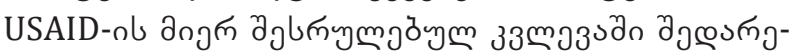

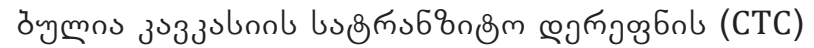

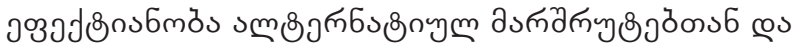

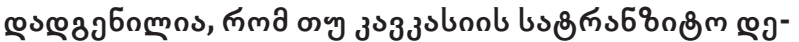

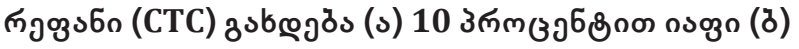

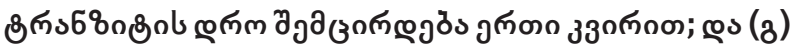

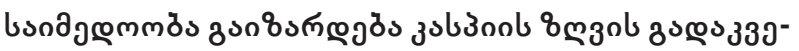

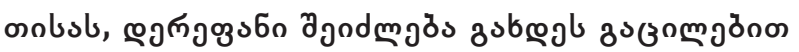

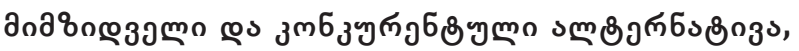

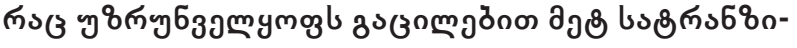

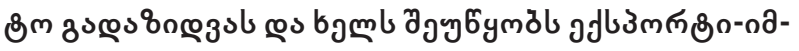

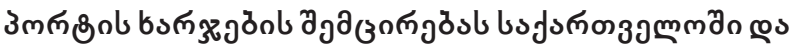
วə3-ob 8nূ̣ul.

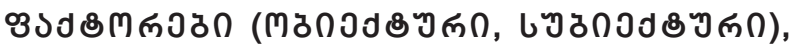

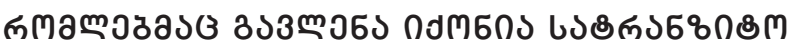

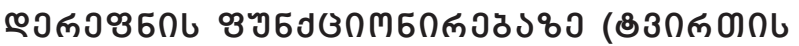

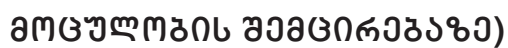

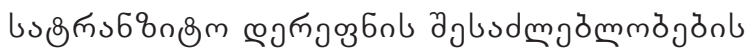

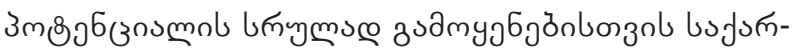

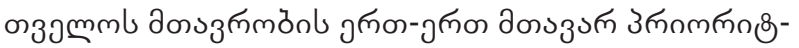

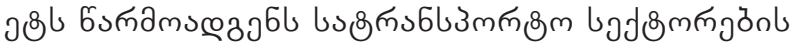

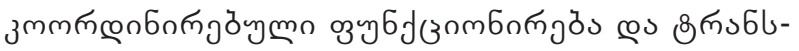

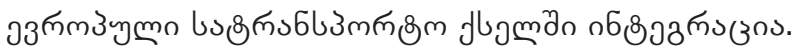

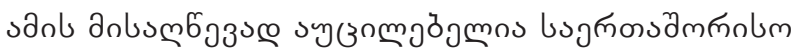

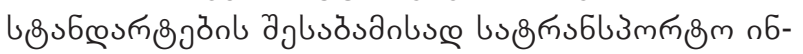

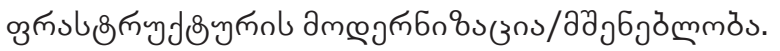

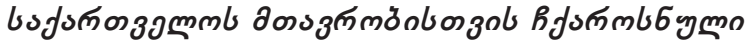

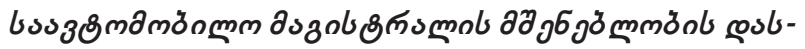

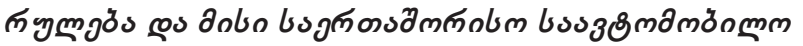

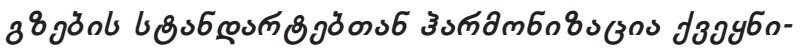

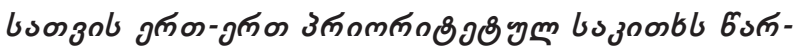
amuূ̧aj6l.

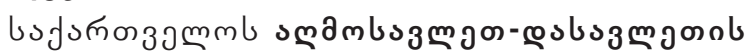

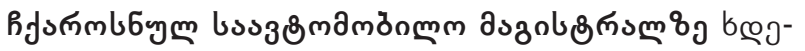

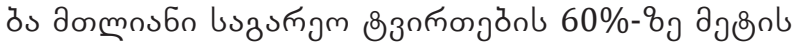

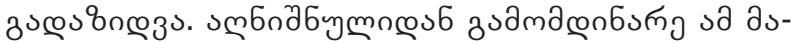

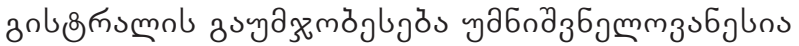

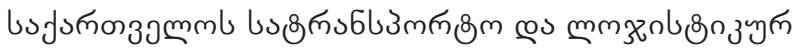

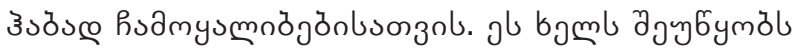

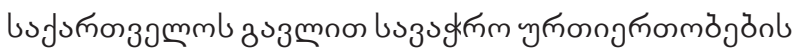

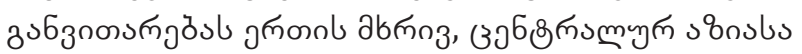

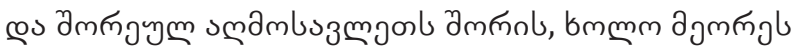

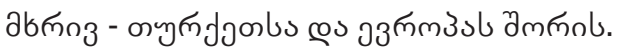

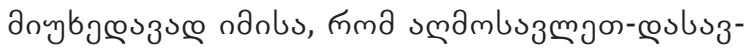

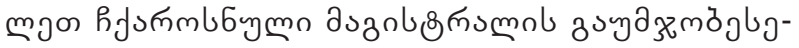

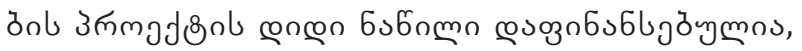

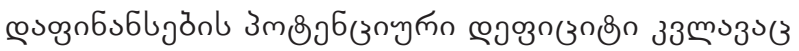

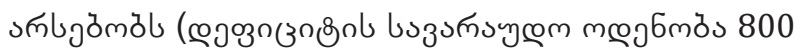

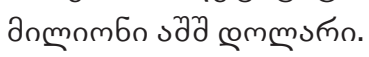

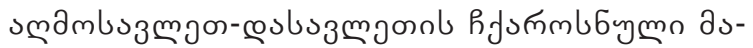

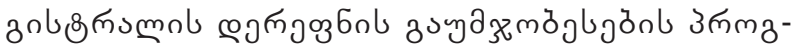

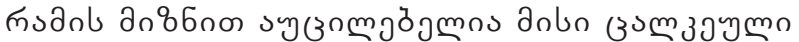

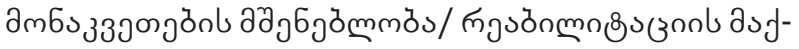

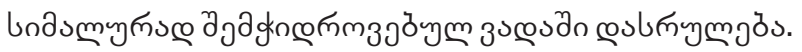

ठmmm lsa 6 б mo6

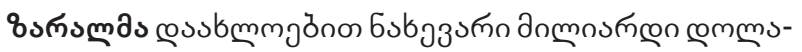

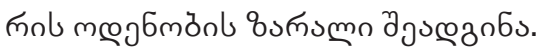

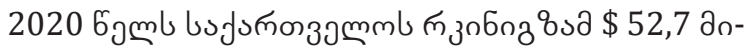

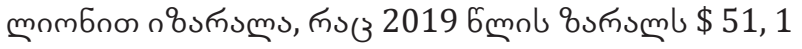

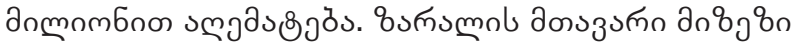

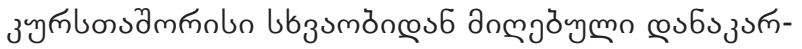

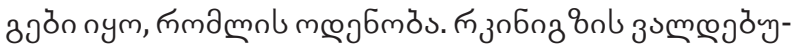

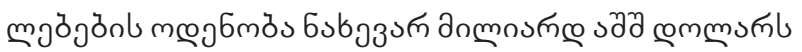

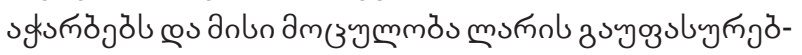

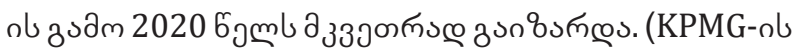

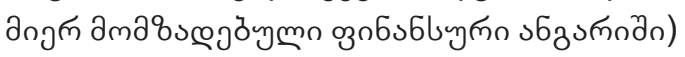

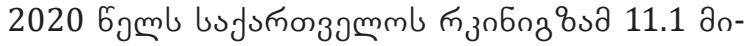

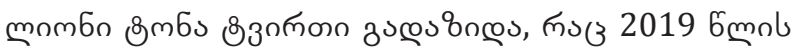

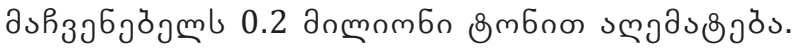

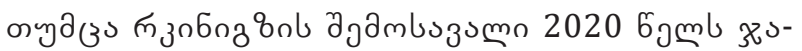

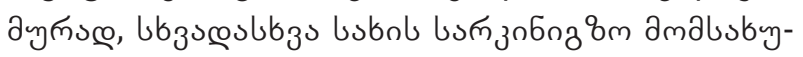

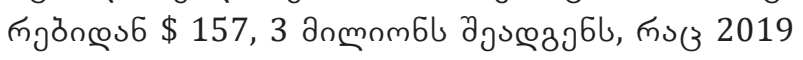

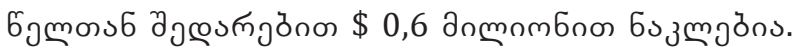

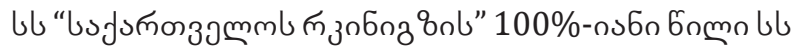

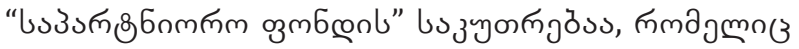

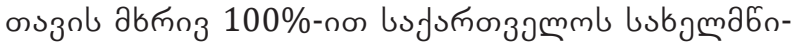
ogmb jзyonzकol.

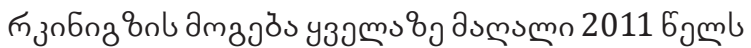

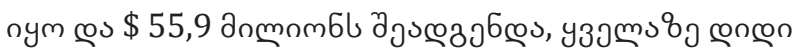

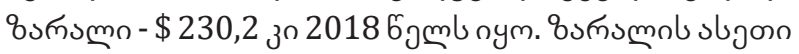

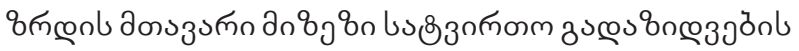
zృ

2012 6 jmb 的

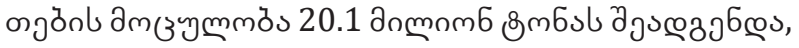




\section{ISSN 2667-9752(Online)}

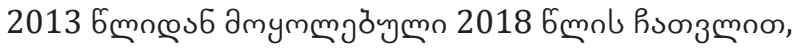

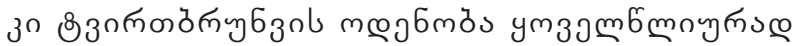

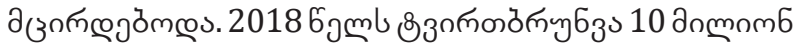

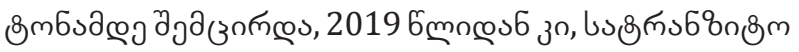

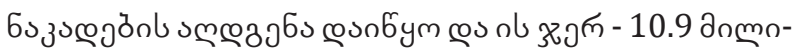

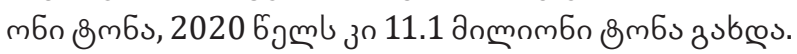

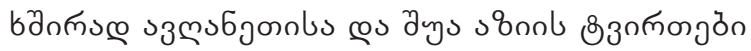

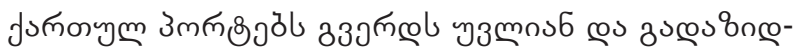

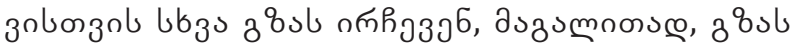

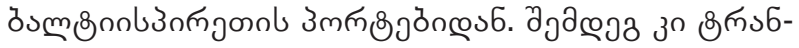
lзмm

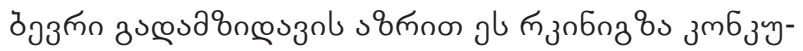

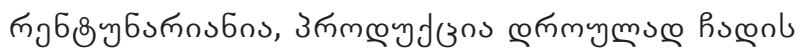

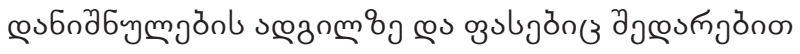
œu’̉smos.

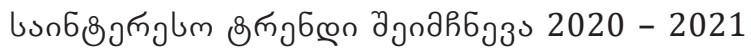

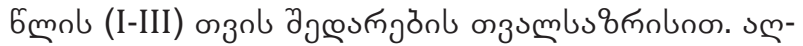

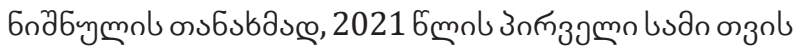

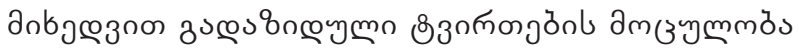

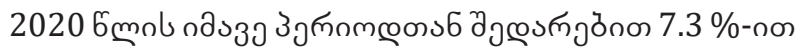

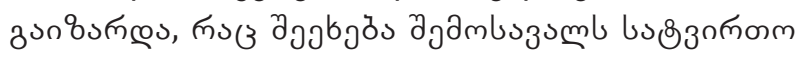

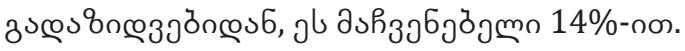

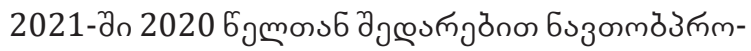

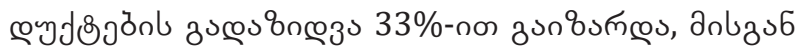

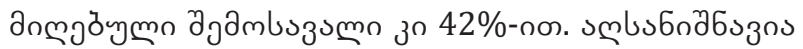

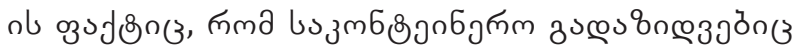

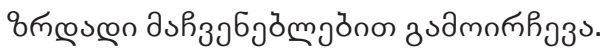

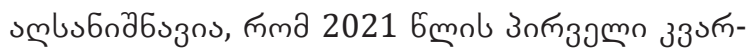

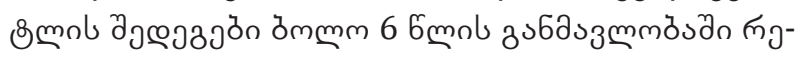

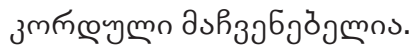

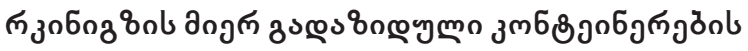

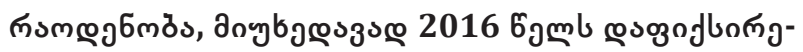

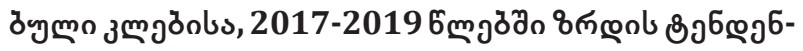

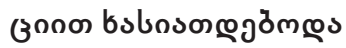

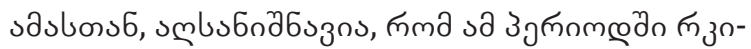

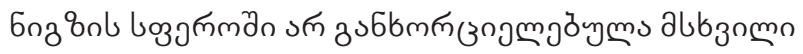

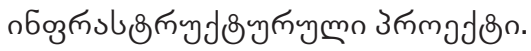

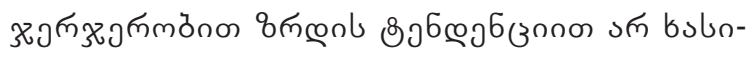

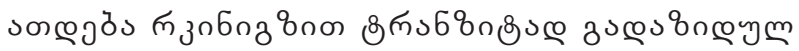

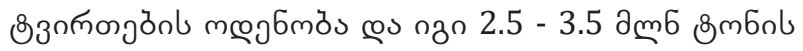

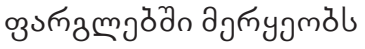

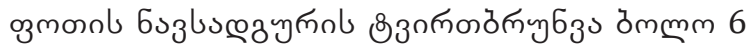

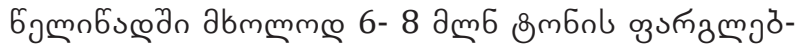

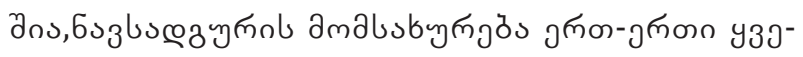

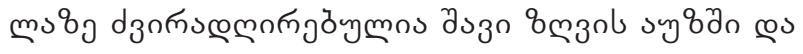

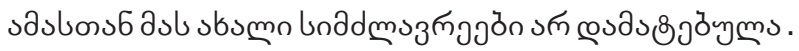

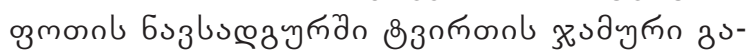

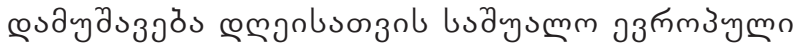

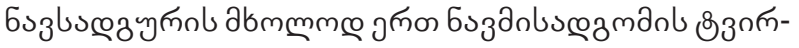

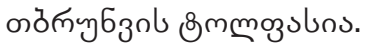

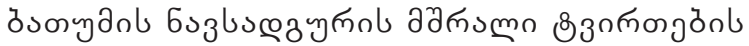

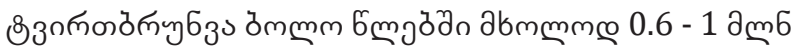

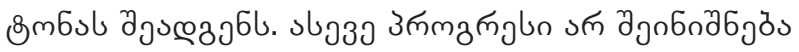

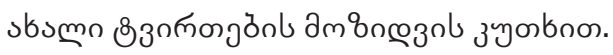

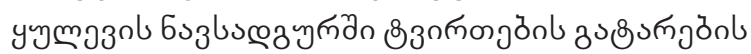

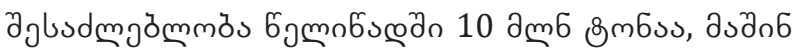
пмщо

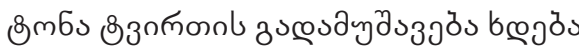

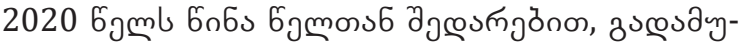

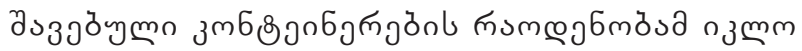

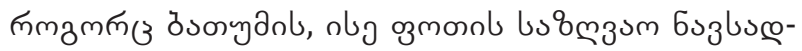

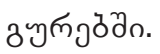

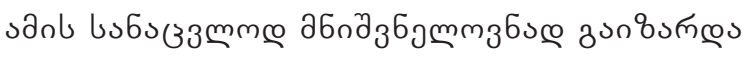

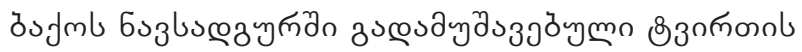

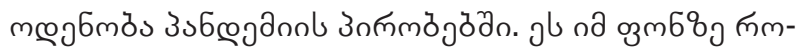

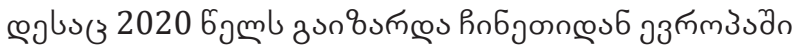

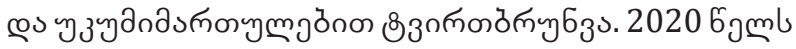

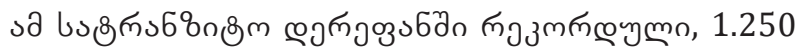

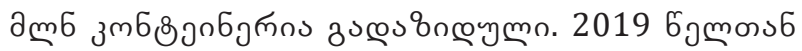

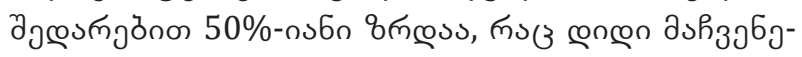

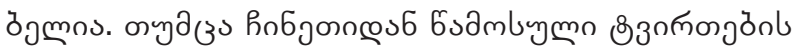

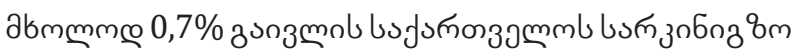

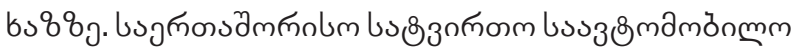
зงœu

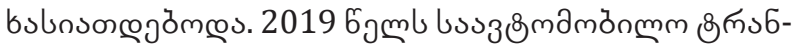

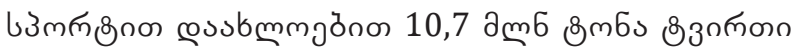

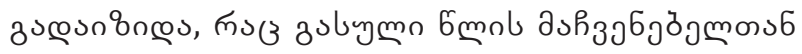

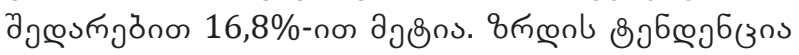

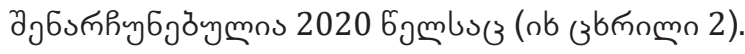

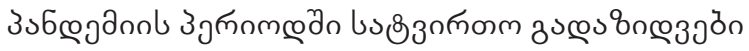

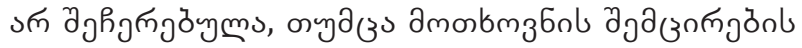

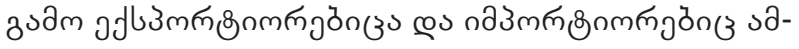

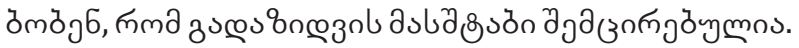

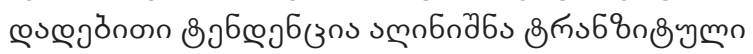

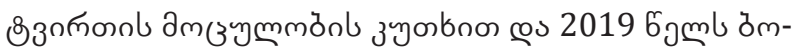

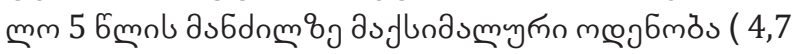

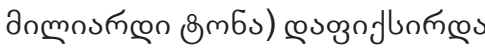

उs6œృ

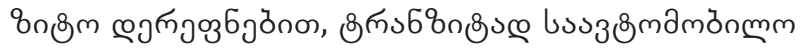

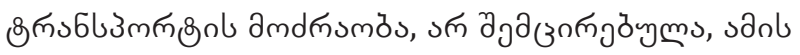

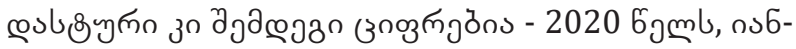

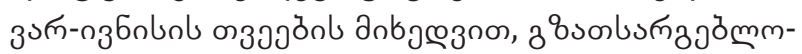

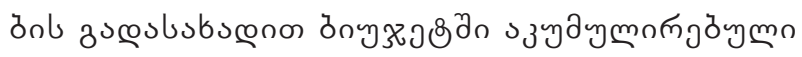

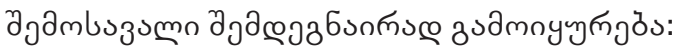

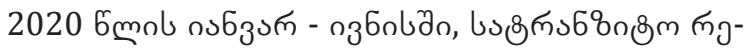

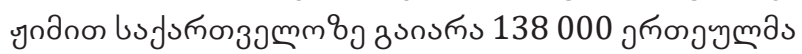

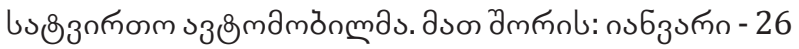

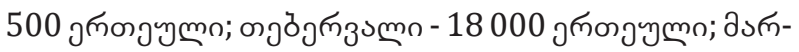




\begin{tabular}{|c|c|c|c|c|c|c|c|c|}
\hline \multicolumn{9}{|c|}{ 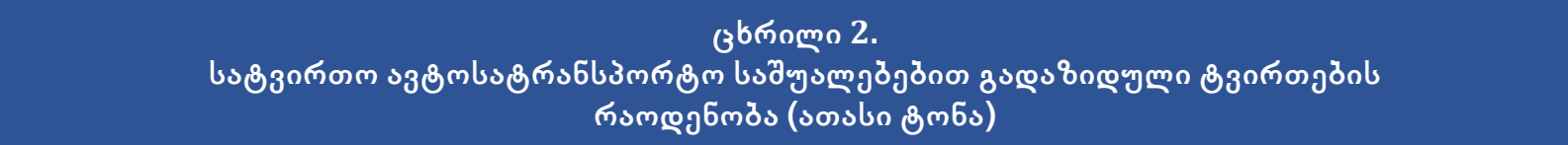 } \\
\hline 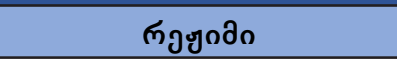 & 2014 & 2015 & 2016 & 2017 & 2018 & 2019 & $2019 / 6$ & $2020 / 6$ \\
\hline 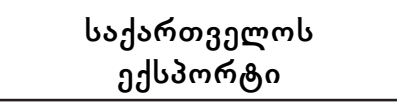 & 692.0 & 910.6 & 968.9 & $1,430.8$ & $1,253.7$ & $1,513.0$ & 723.9 & 619.5 \\
\hline 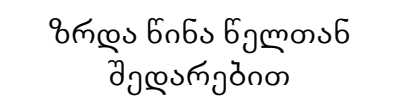 & - & $31.6 \%$ & $6.4 \%$ & $47.7 \%$ & $-12.4 \%$ & $20.7 \%$ & - & $-14.4 \%$ \\
\hline 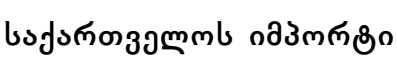 & $2,975.8$ & $2,952.1$ & $3,299.0$ & $3,705.0$ & $3,894.7$ & $4,376.6$ & $1,871.7$ & $1,972.9$ \\
\hline 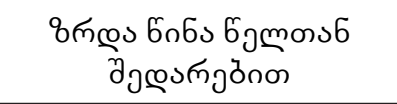 & - & $-0.8 \%$ & $11.8 \%$ & $12.3 \%$ & $5.1 \%$ & $12.4 \%$ & - & $5.4 \%$ \\
\hline 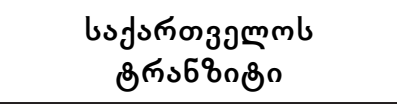 & $2,638.3$ & $2,708.4$ & $2,886.1$ & $3,628.5$ & $3,992.5$ & $4,783.2$ & $2,209.5$ & $2,509.2$ \\
\hline 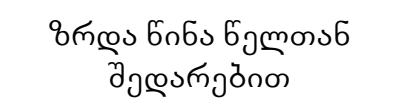 & - & $2.7 \%$ & $6.6 \%$ & $25.7 \%$ & $10.0 \%$ & $19.8 \%$ & - & $13.6 \%$ \\
\hline by๗ & $6,306.1$ & $6,571.1$ & $7,154.0$ & $8,764.3$ & $9,141.0$ & $10,672.7$ & $4,805.1$ & $5,101.6$ \\
\hline 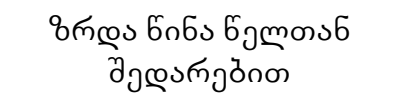 & - & $4.2 \%$ & $8.9 \%$ & $22.5 \%$ & $4.3 \%$ & $16.8 \%$ & - & $6.2 \%$ \\
\hline
\end{tabular}

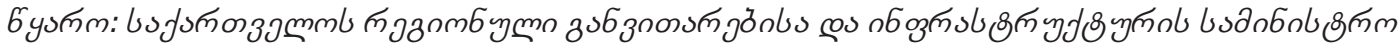

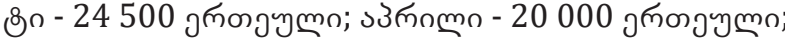

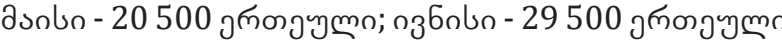

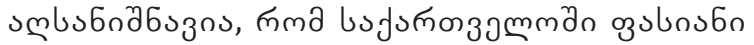

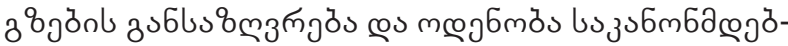

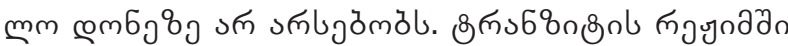

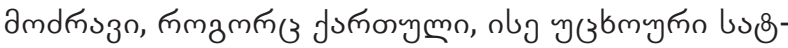

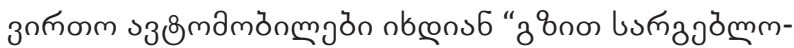

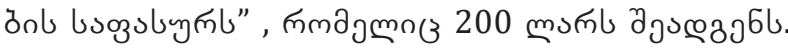

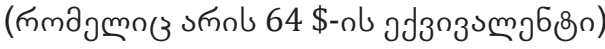

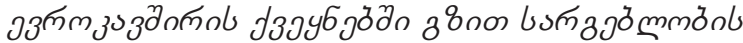

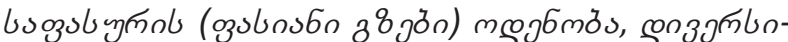

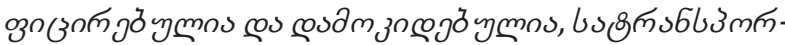

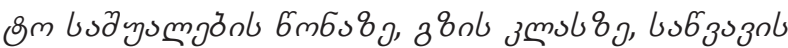

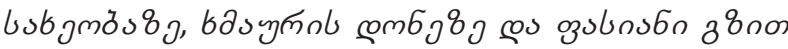
ப й

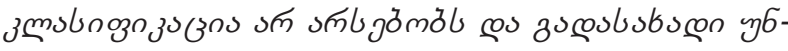

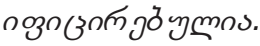

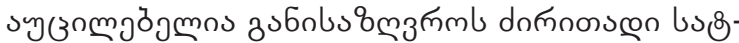

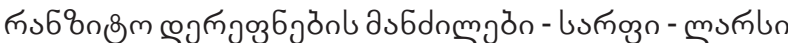

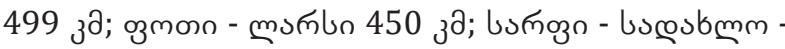

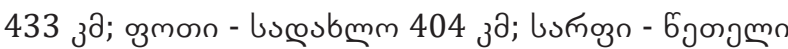

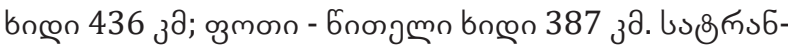

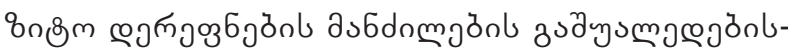

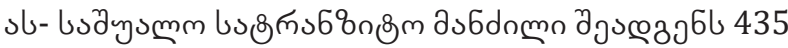
за-८; øу a

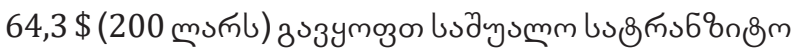
дง6dnm

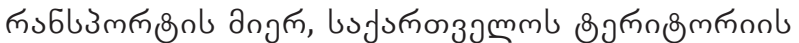

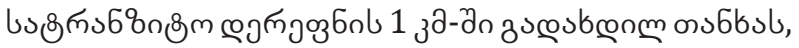

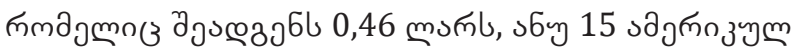

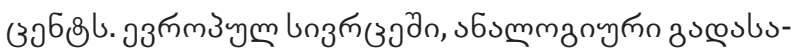

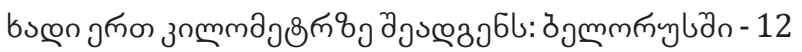

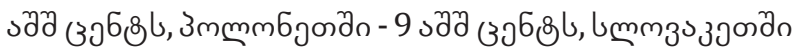
21 s

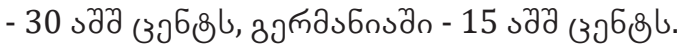

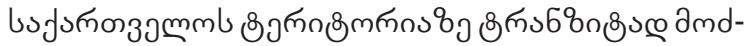

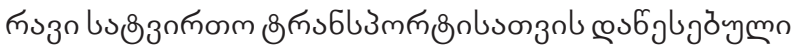

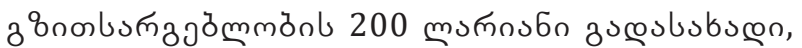

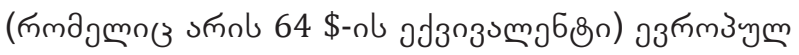

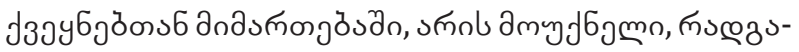

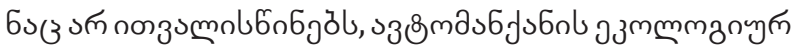

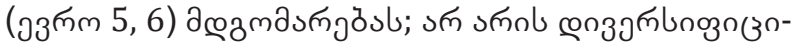

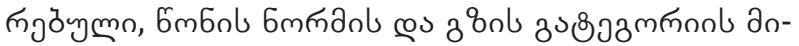

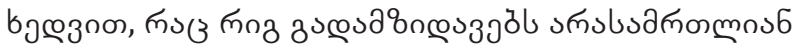

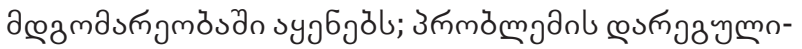

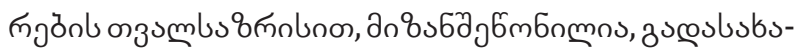

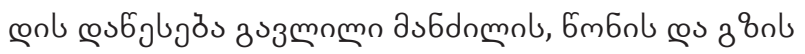

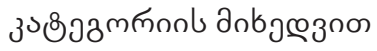

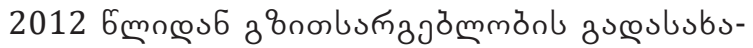

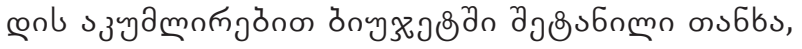




\section{ISSN 2667-9752(Online)}

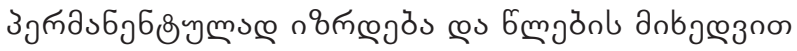

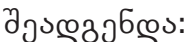

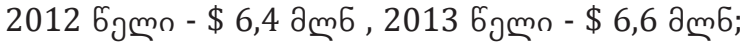

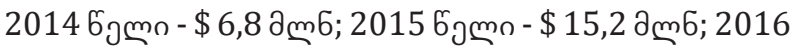

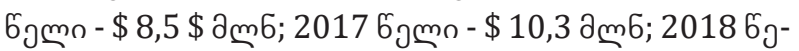

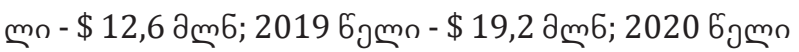
(8 пзэ) - \$ 12.3 дm6.

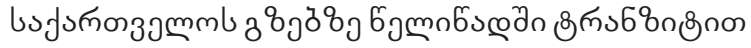

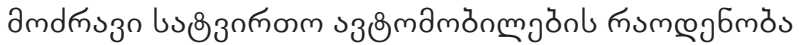

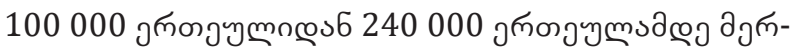
yอmỏb.

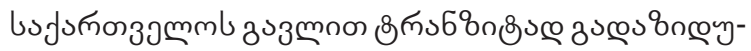

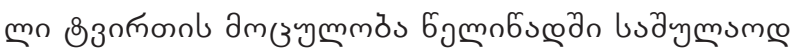

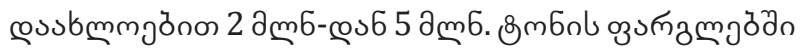
ал๓улмыे.

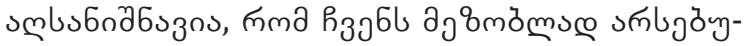

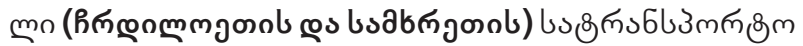

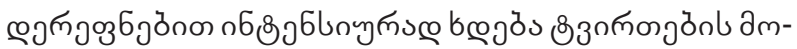

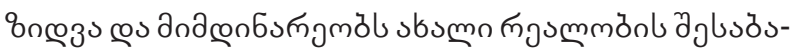

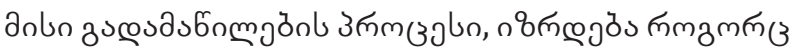

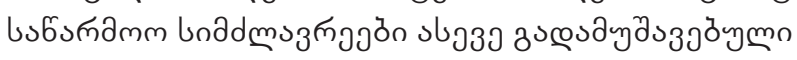

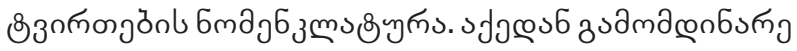

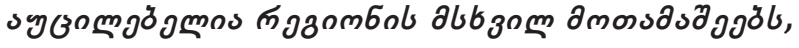

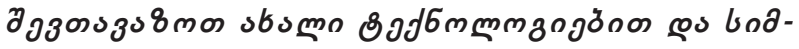

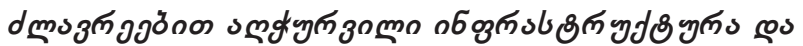

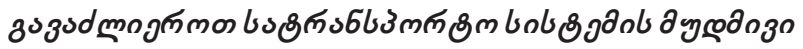

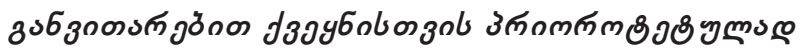

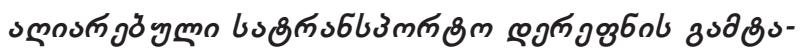

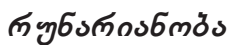

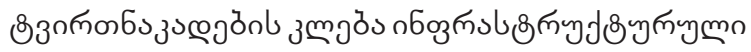

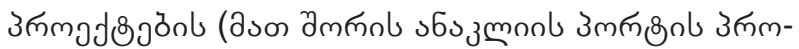

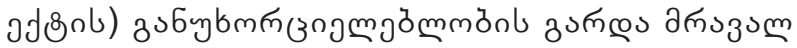

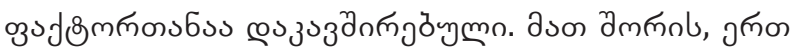

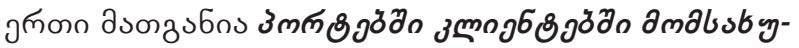

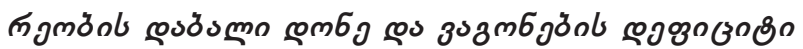

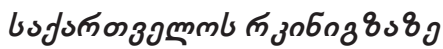

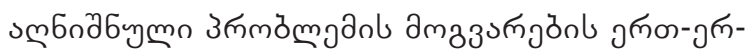
๓ก z

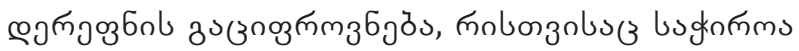

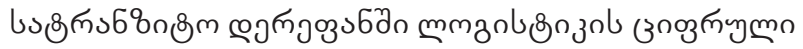

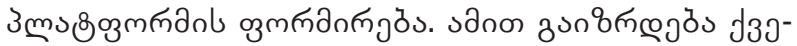

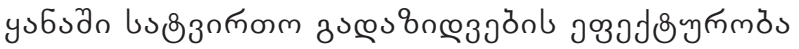

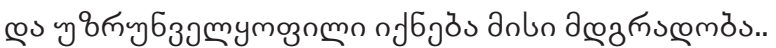

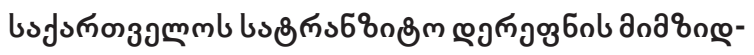

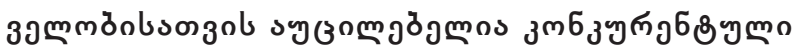

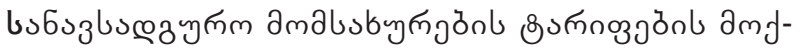

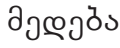

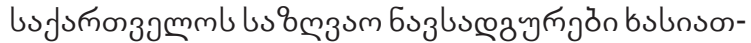

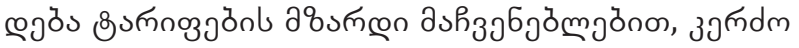

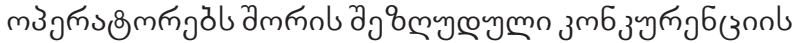

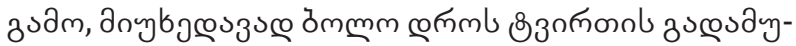

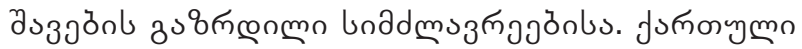

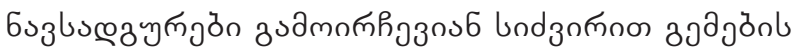

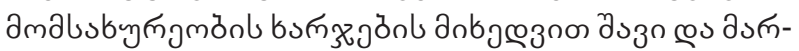

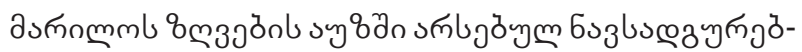

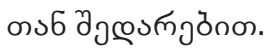

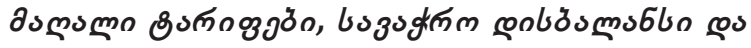

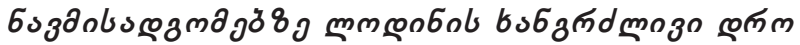

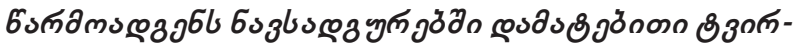

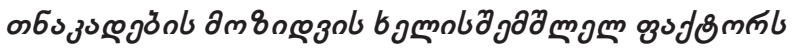

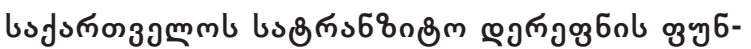

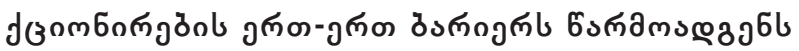

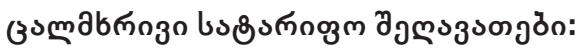

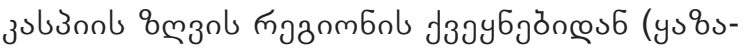

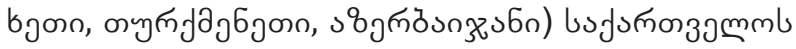

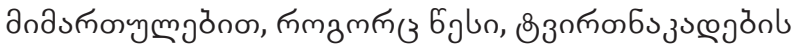

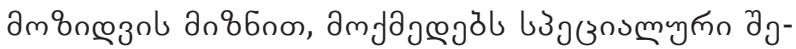

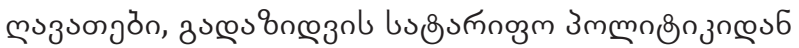

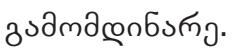

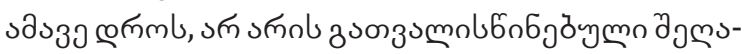

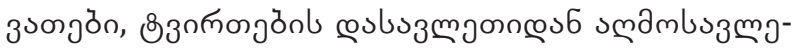

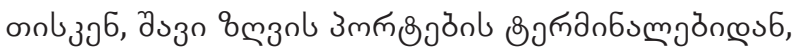

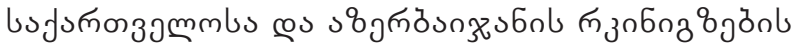

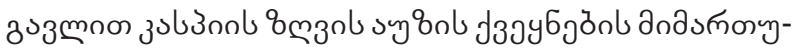

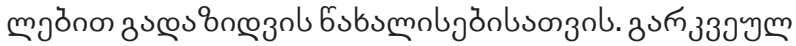

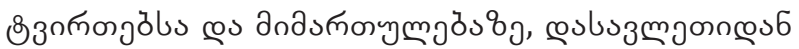

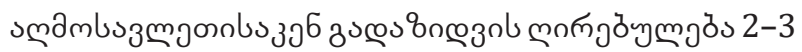

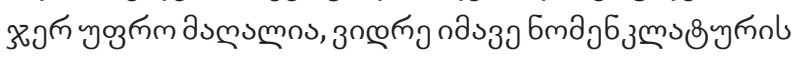

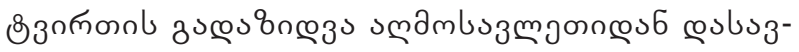

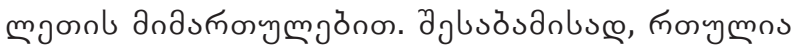

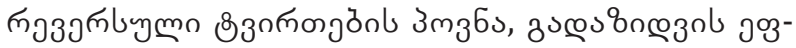

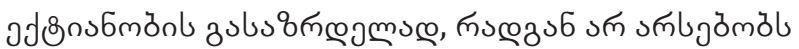
วృ

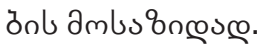

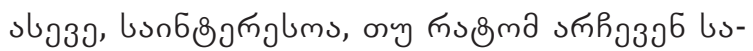

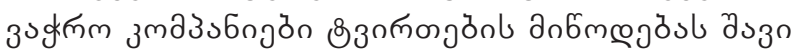

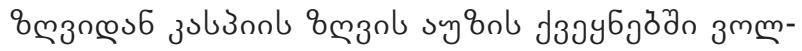

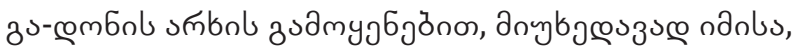

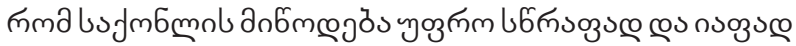

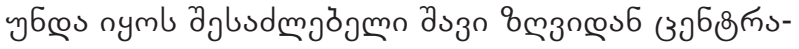

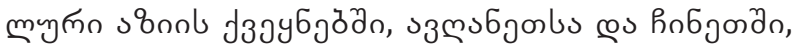

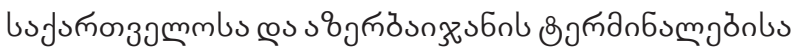

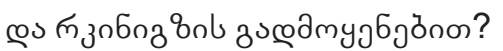

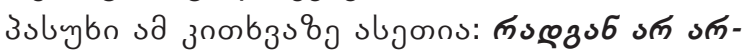

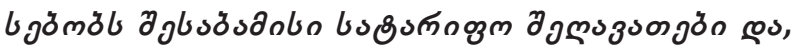

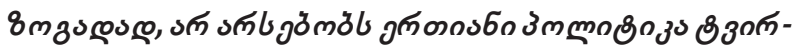

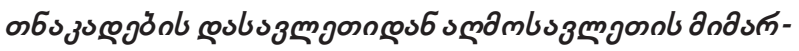

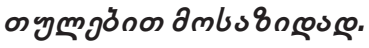




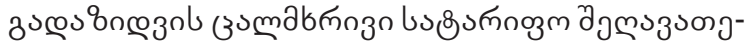

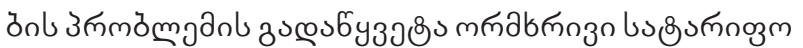

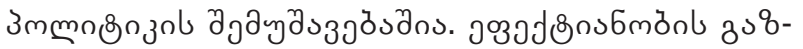

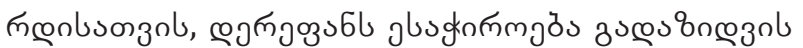

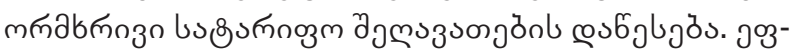

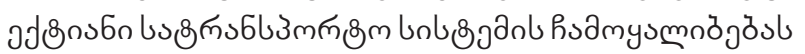

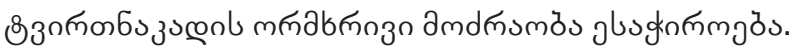

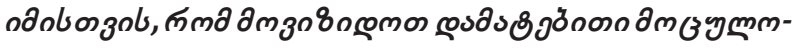
ర்

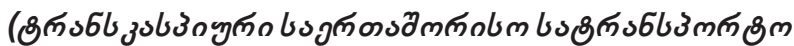

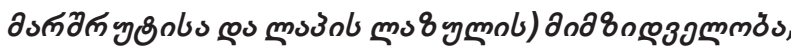

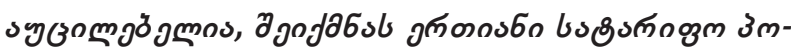

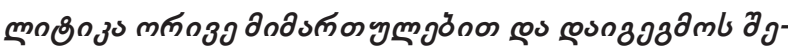

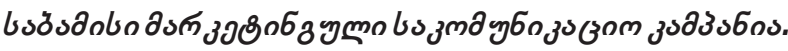

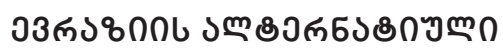

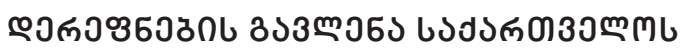

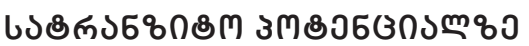

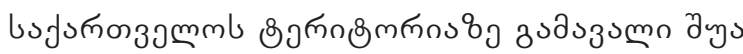

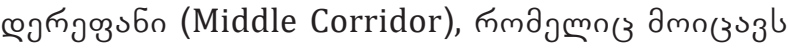

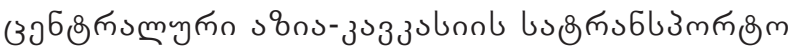

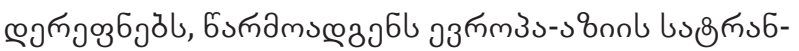

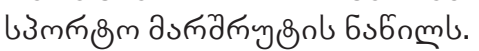

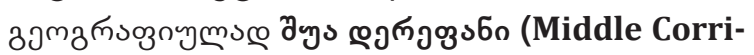

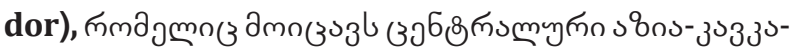

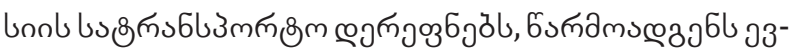

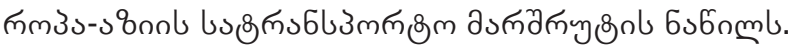

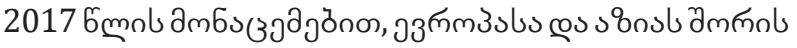
зงূs

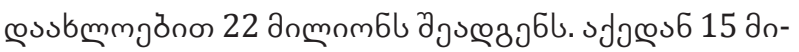

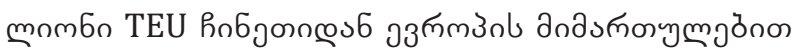

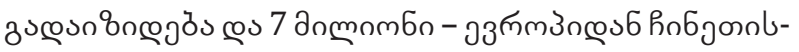

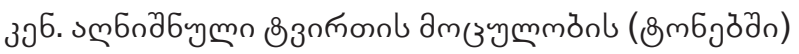
97 з

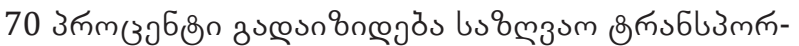

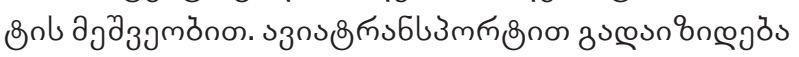

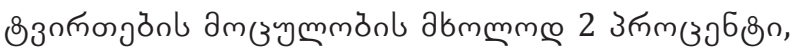

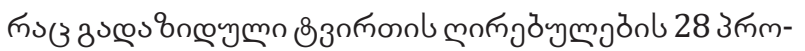

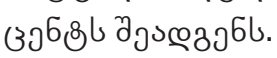

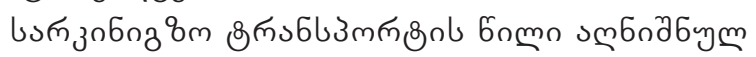

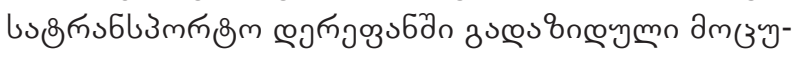

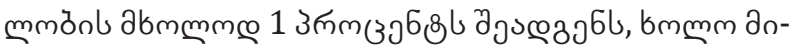

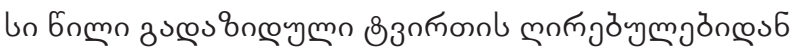

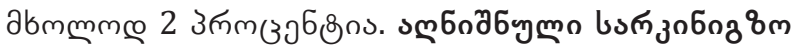

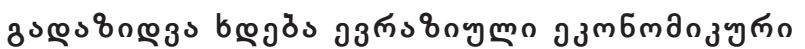

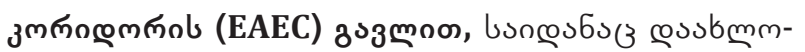

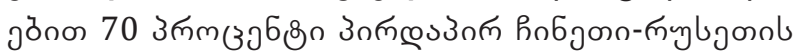

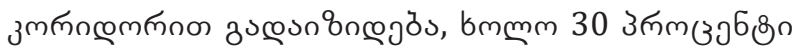

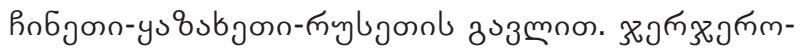

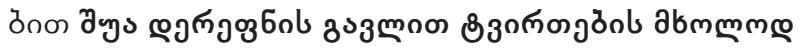
ฑวбодз

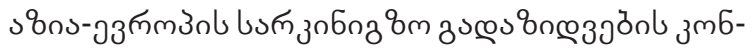

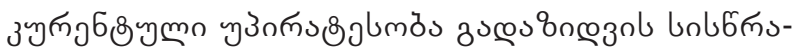

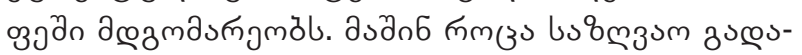

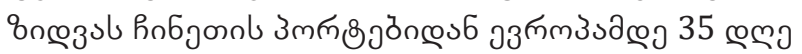

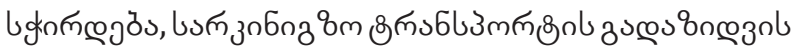

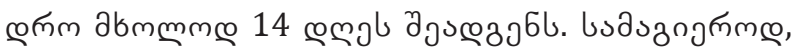

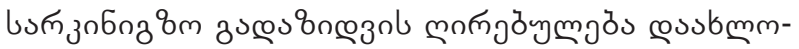

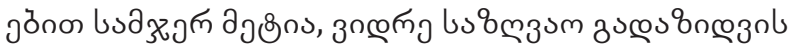

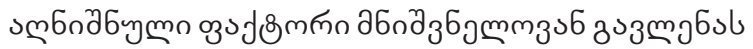

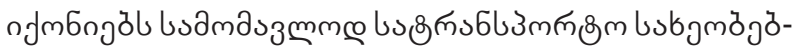

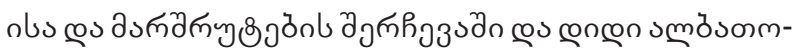

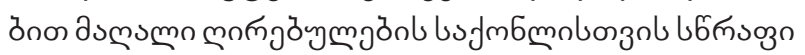

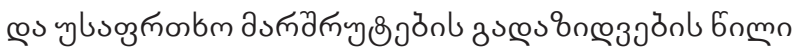

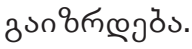

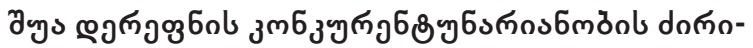

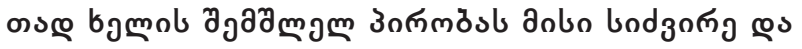

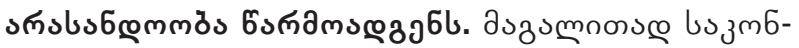

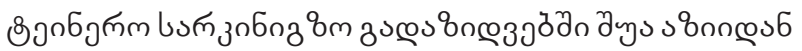

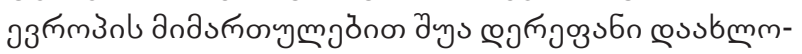

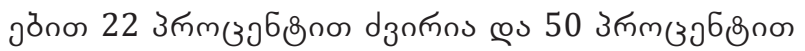

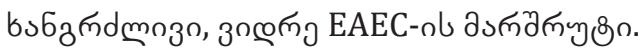

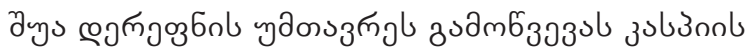

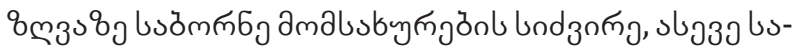

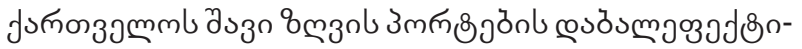

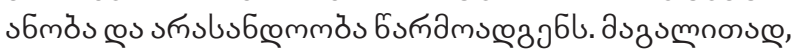

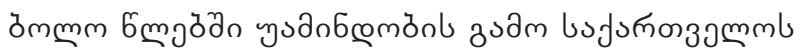

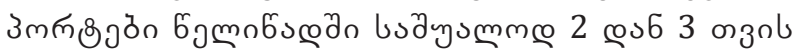

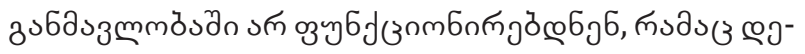

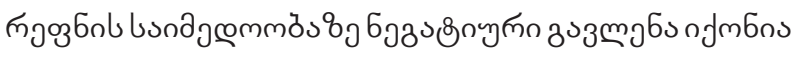
œs zง

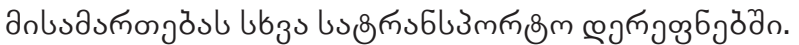

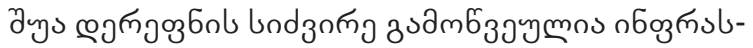

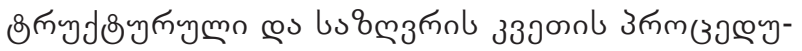

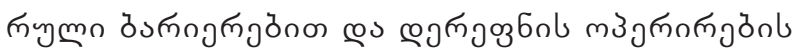

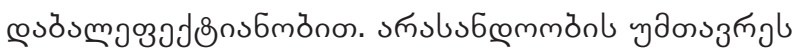

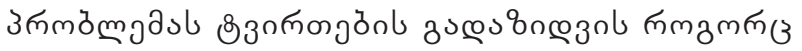

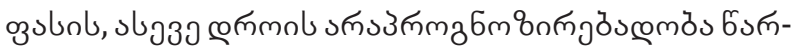
amsূa

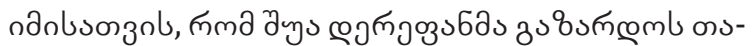

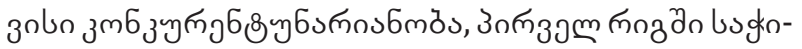

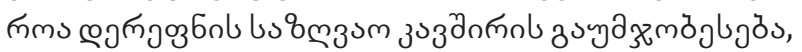

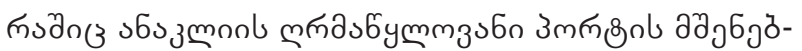

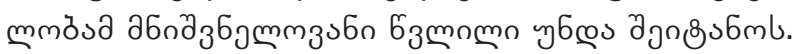

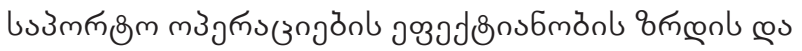

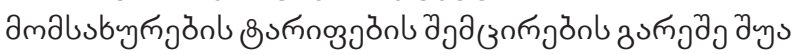

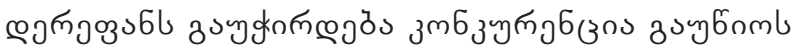

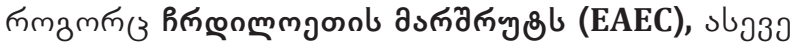




\section{ISSN 2667-9752(Online)}

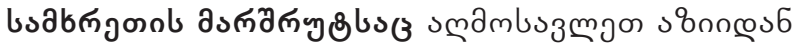

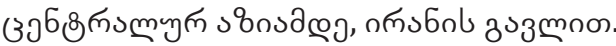

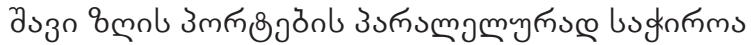

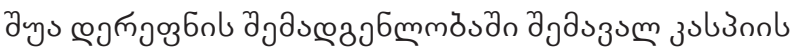

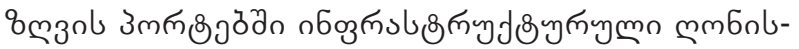

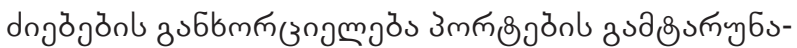

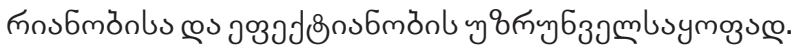

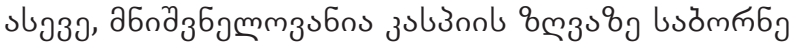

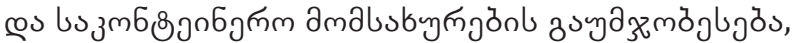

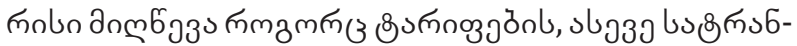

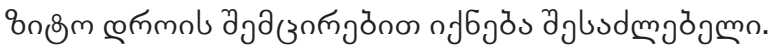

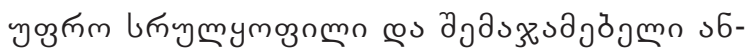

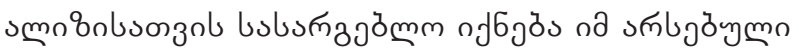

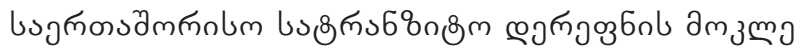

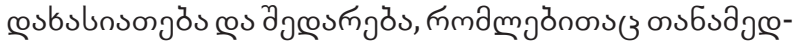
mm3ว

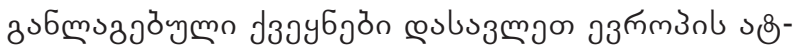

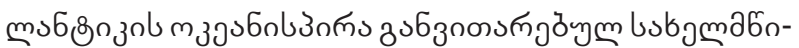

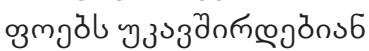

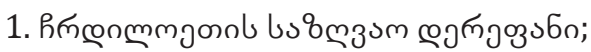

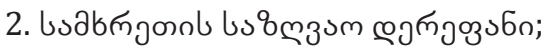

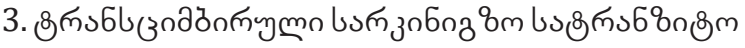

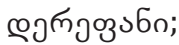

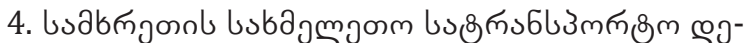

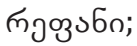

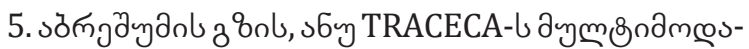

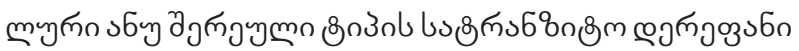

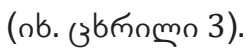

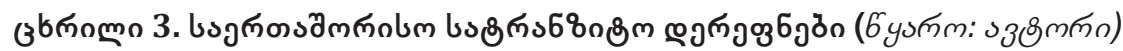

\begin{tabular}{|c|c|c|c|}
\hline & 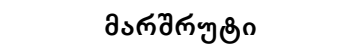 & అకెంగుఠి & 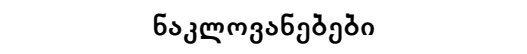 \\
\hline 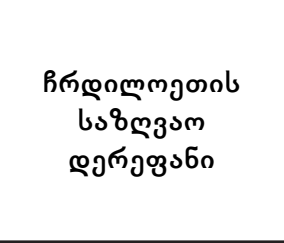 & 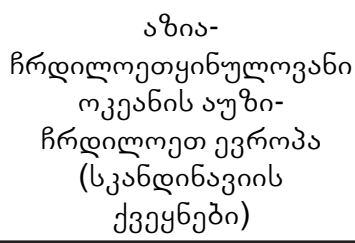 & 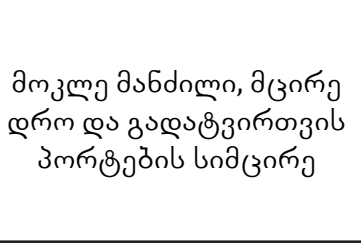 & 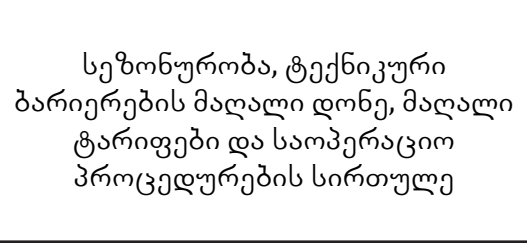 \\
\hline 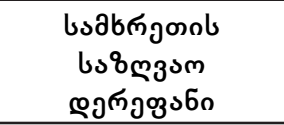 & 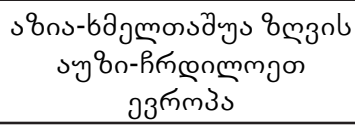 & 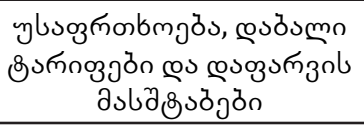 & 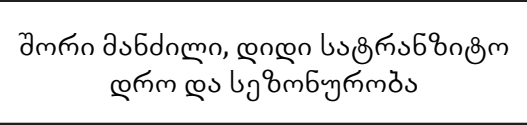 \\
\hline 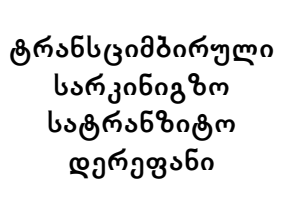 & 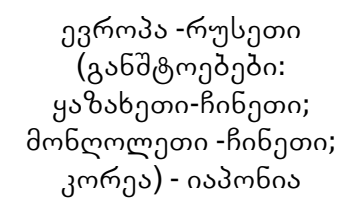 & 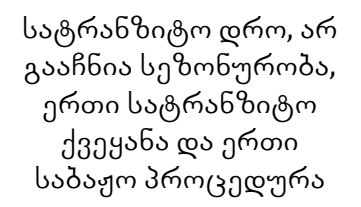 & 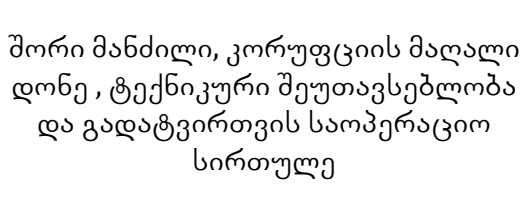 \\
\hline 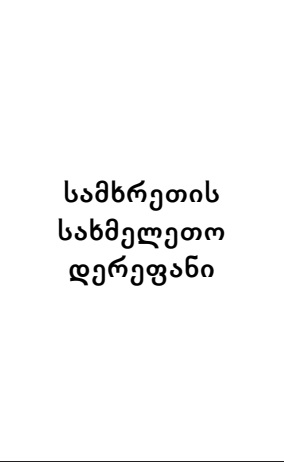 & 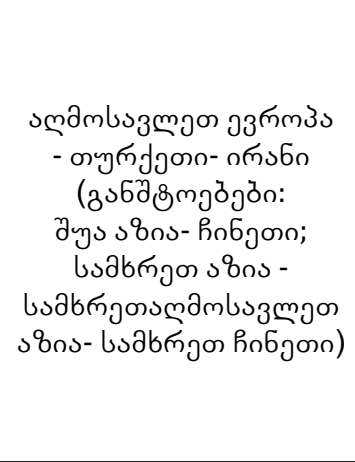 & 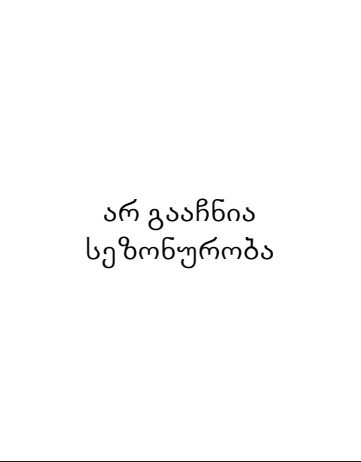 & 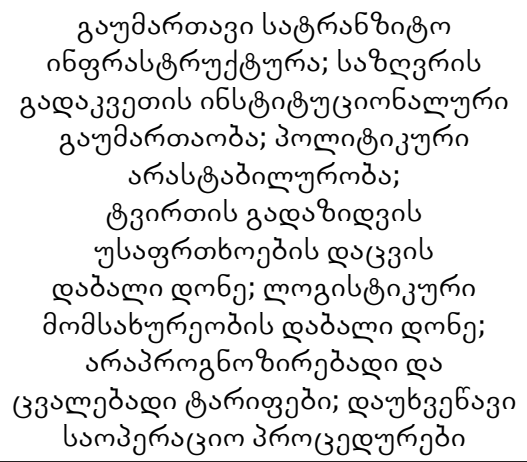 \\
\hline 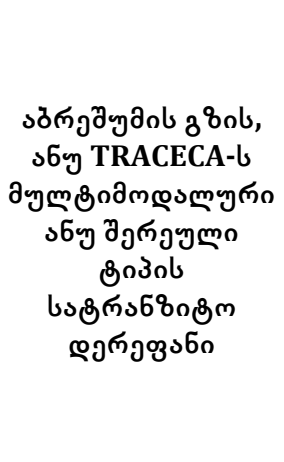 & 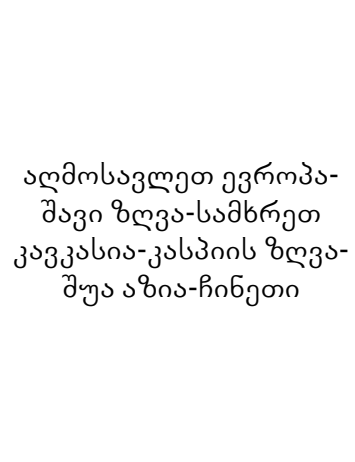 & 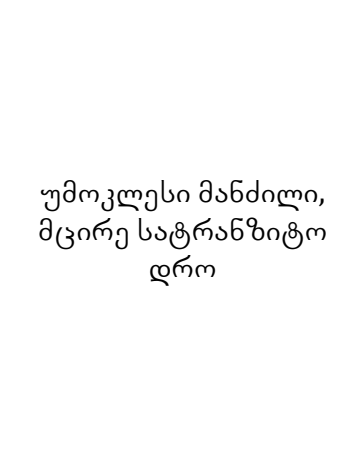 & 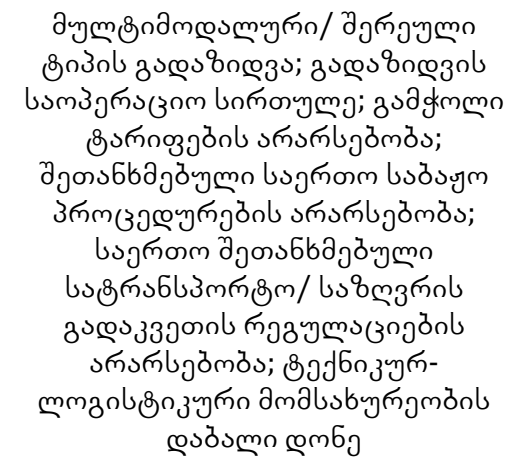 \\
\hline
\end{tabular}




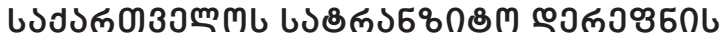

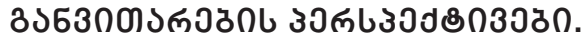

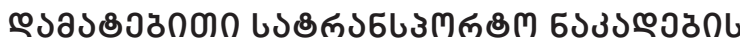

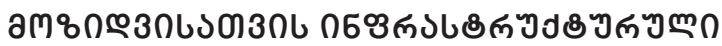

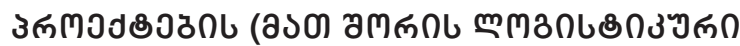

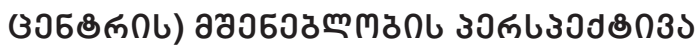

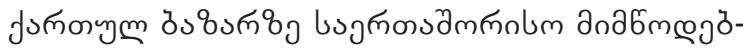

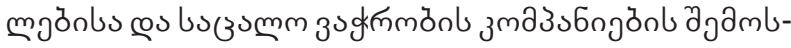

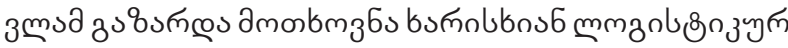

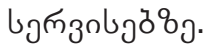

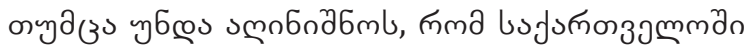

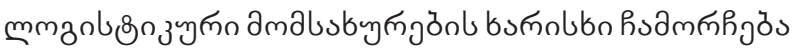

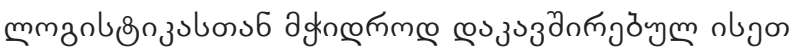

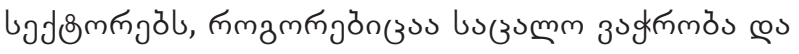

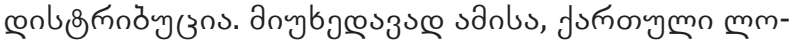

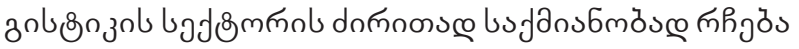

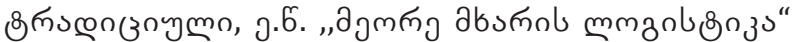

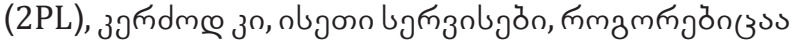

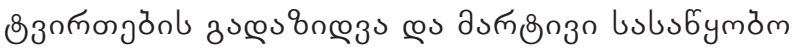

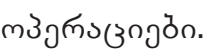

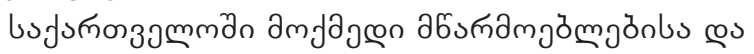

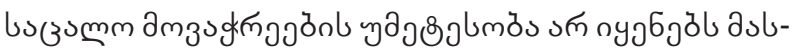

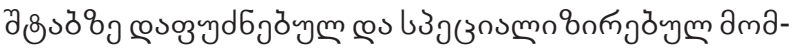

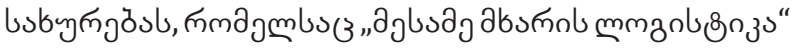

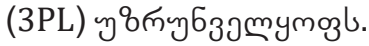

3PLajbsag absmol mmanb \&n zu smol amalusbyng-

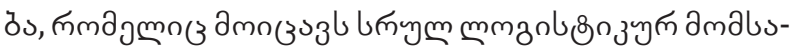

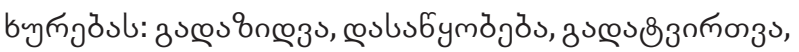

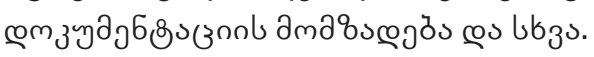

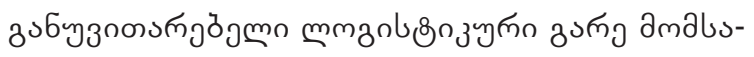

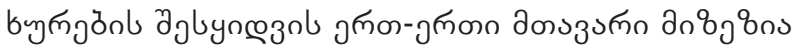
3PL amabsbyn

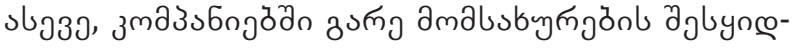

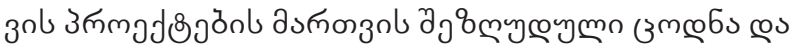

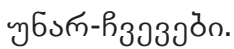

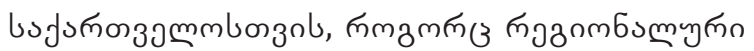

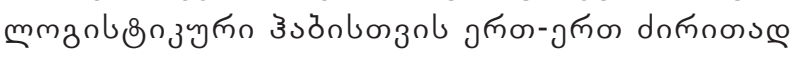

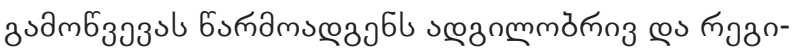

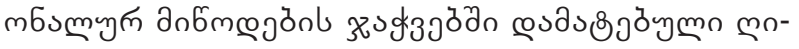

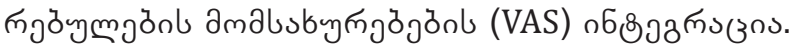

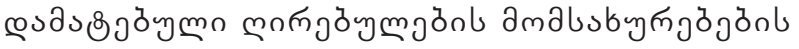
nб

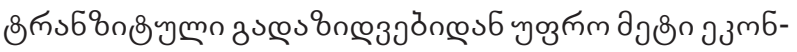

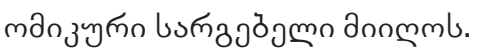

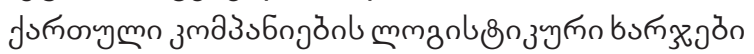

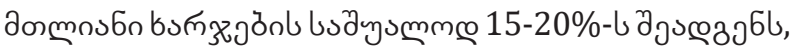
поз

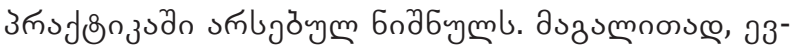

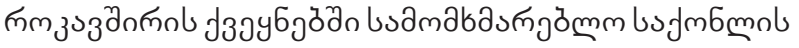

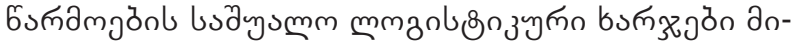

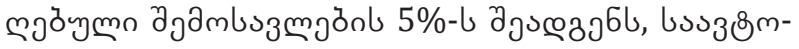

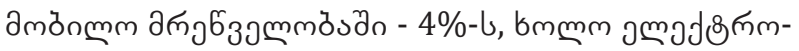

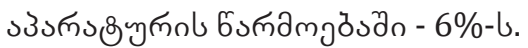

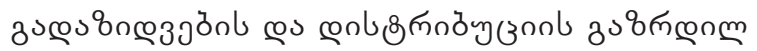

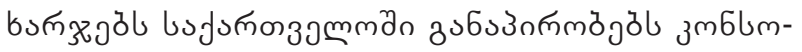

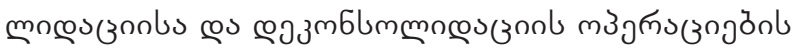

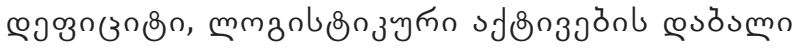

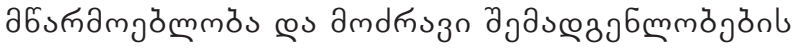

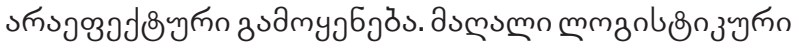

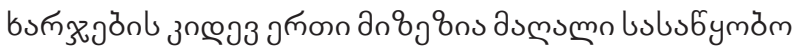

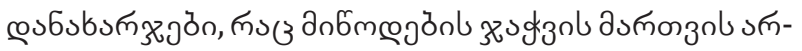

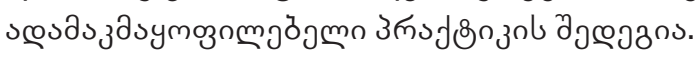

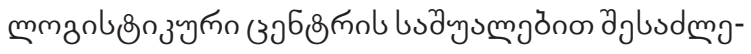

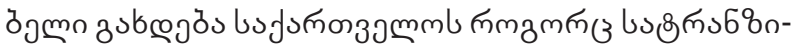

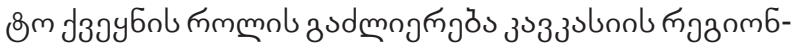

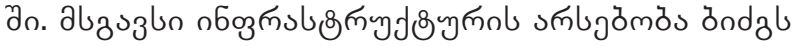

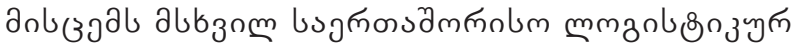

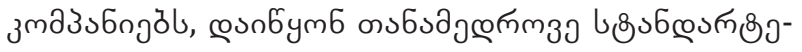

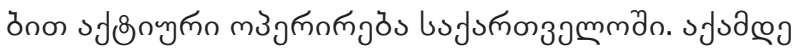

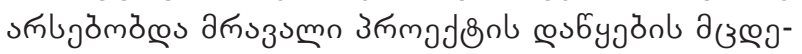

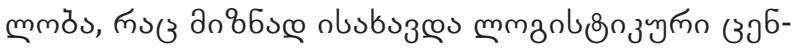

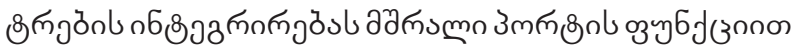

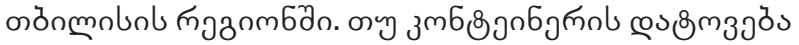

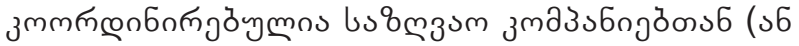

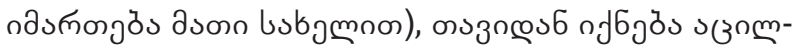

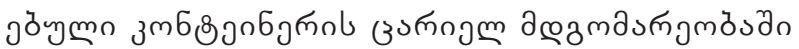

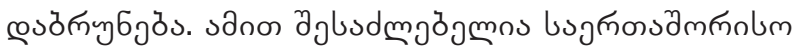

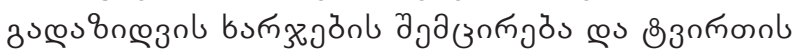

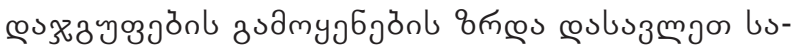

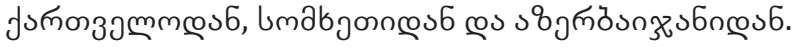

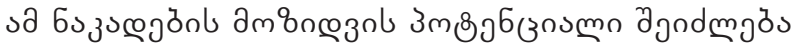

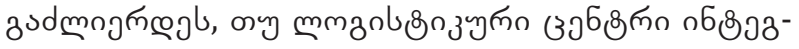

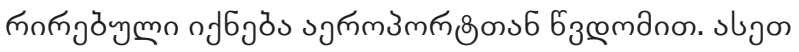

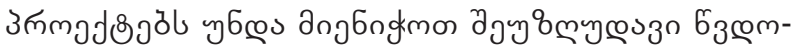

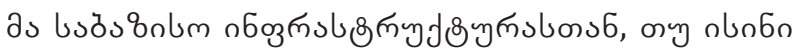
дj

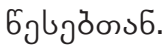

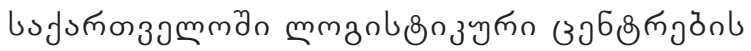

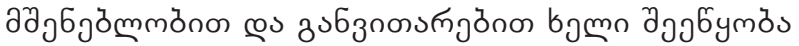

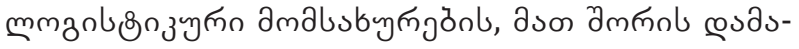

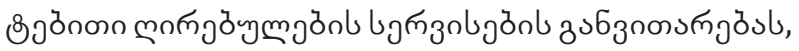

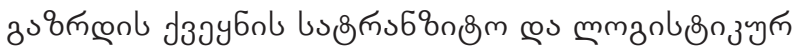

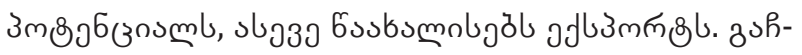

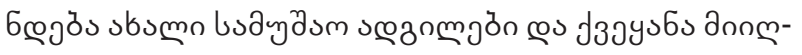

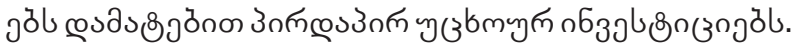

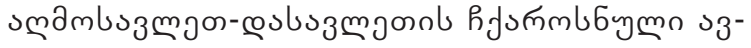

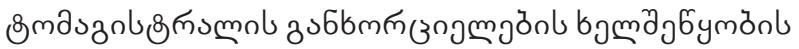
उஙm 


\section{ISSN 2667-9752(Online)}

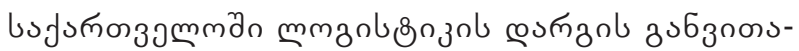

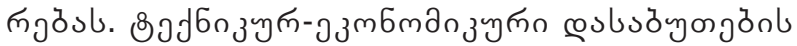
œмзудаб

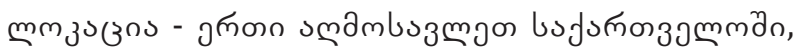

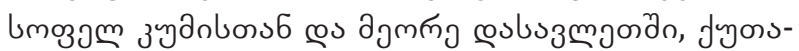

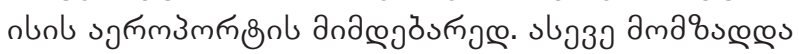

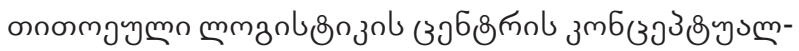

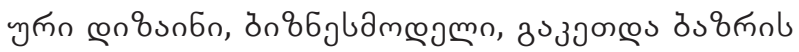
ง6umn

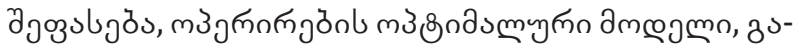

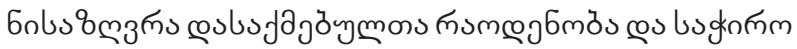
ззомповозовол.

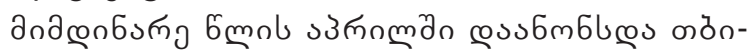

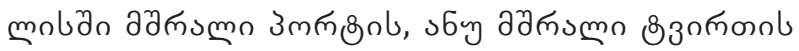

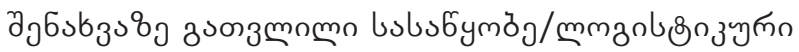

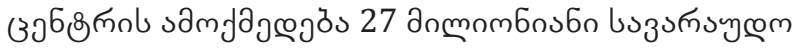

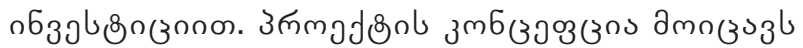

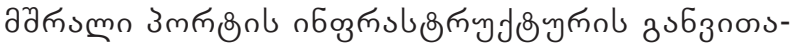

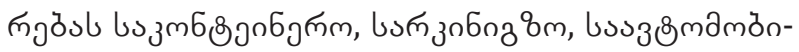

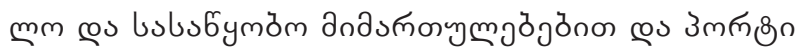
amgalsubyng

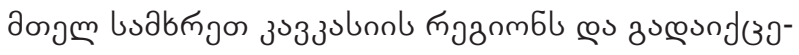

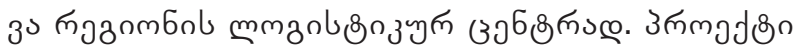

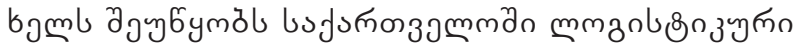

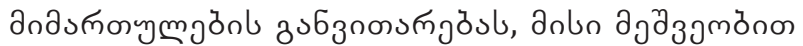

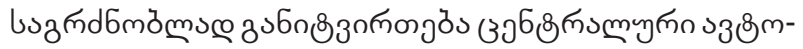

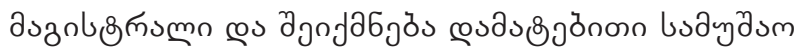

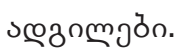

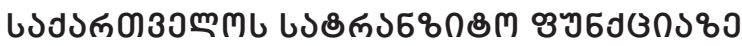

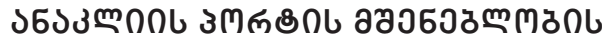

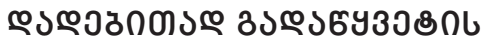

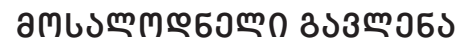

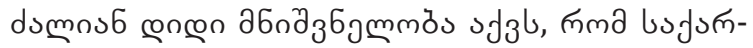

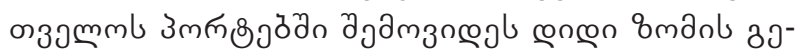
ة

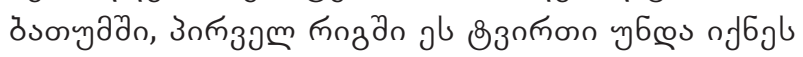

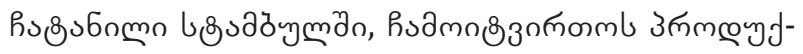

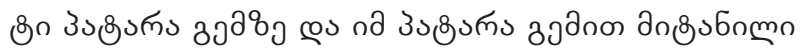

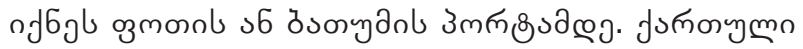

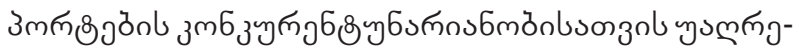

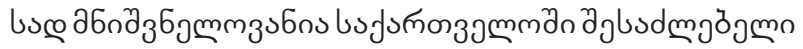

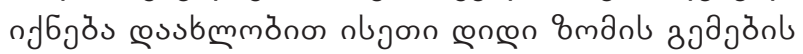

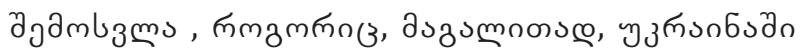

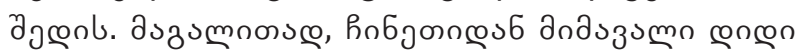

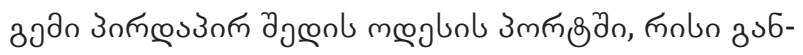

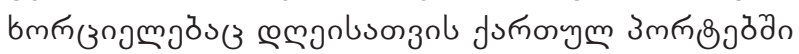

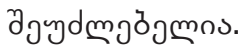

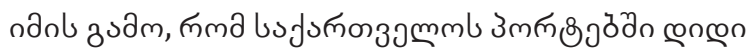

з ว

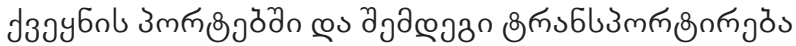

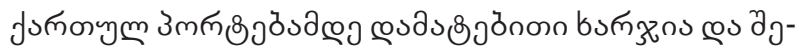

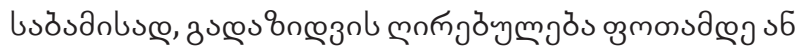

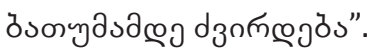

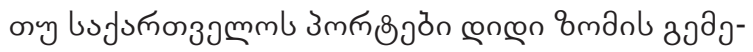

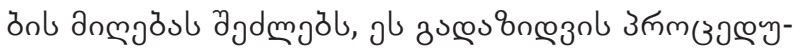

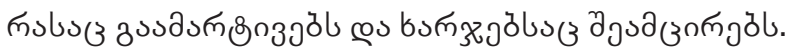

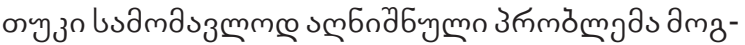

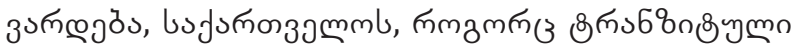

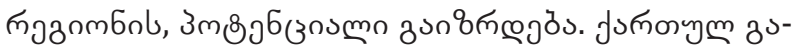

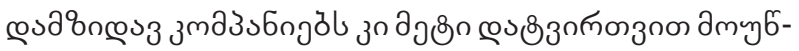

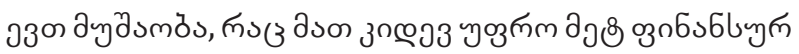
しง пே

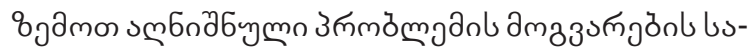

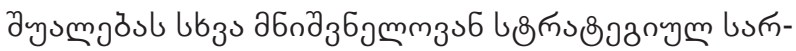

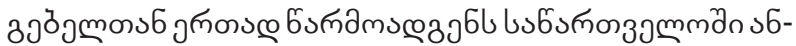

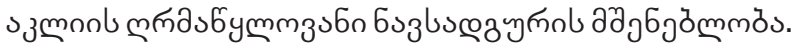

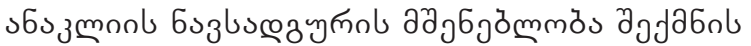

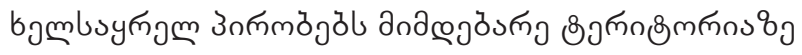

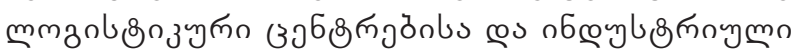

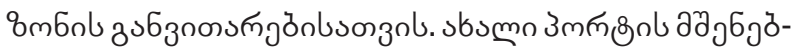

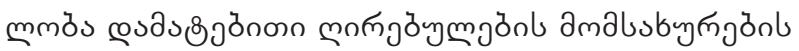

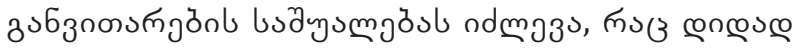

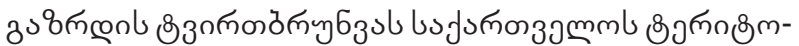

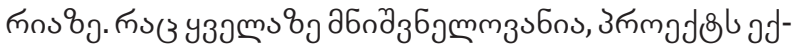

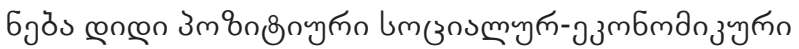

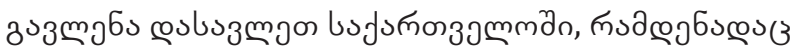

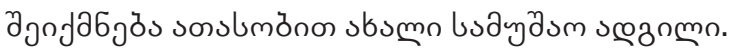

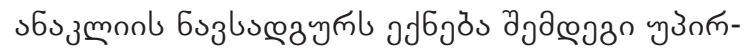

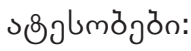

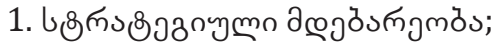

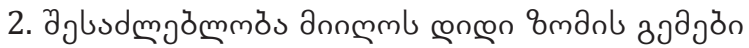

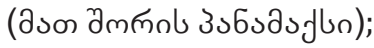

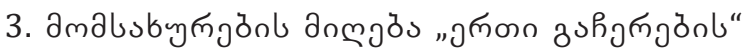
зйб(зозักо;

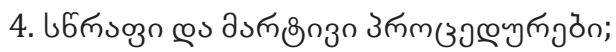

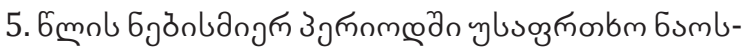

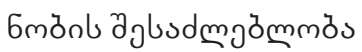

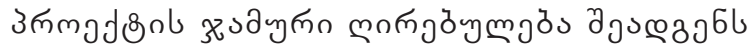

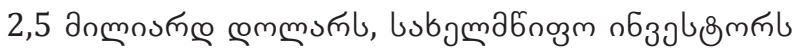

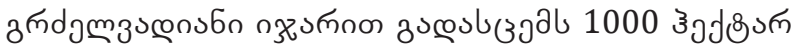

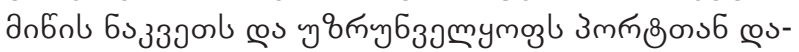

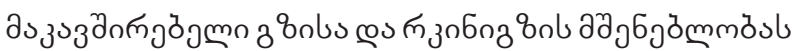

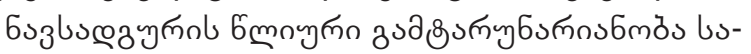

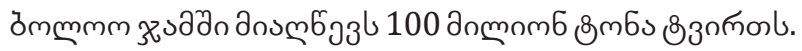

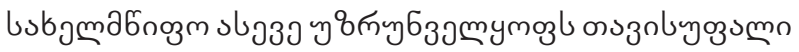

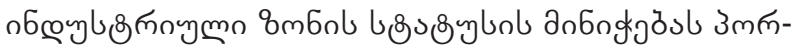

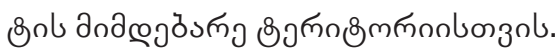




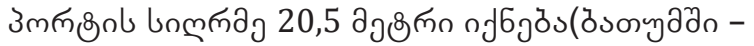

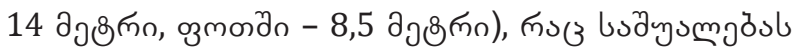
ة

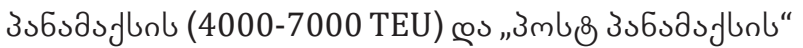

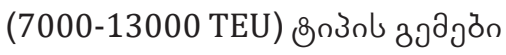

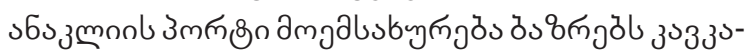

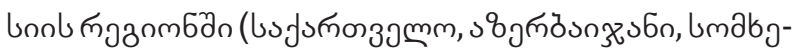

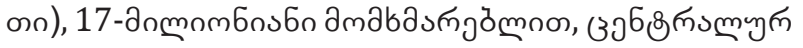

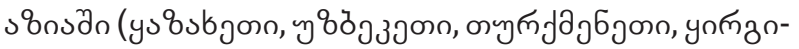

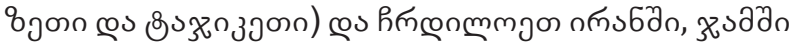

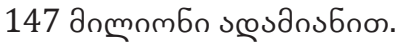

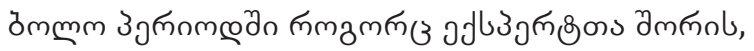
ا

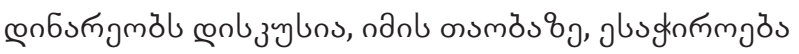

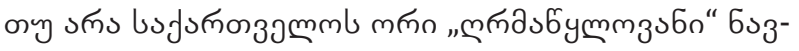

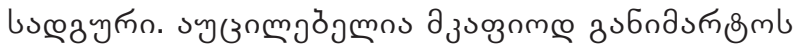

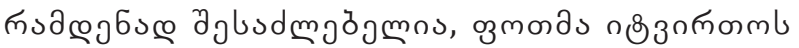

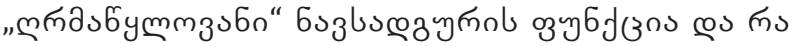

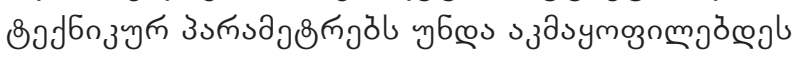

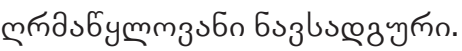

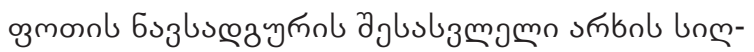

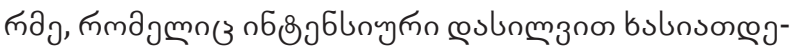

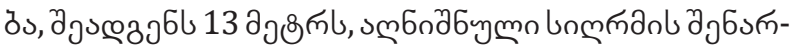

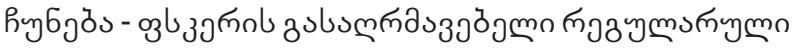

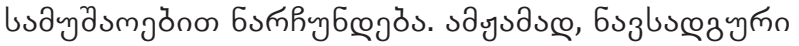

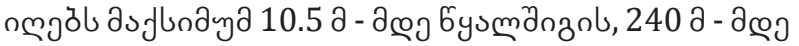

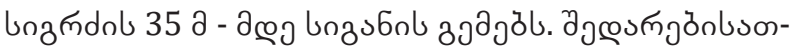

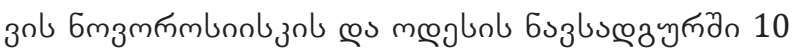

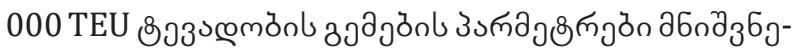

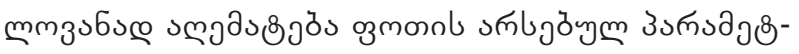
的岂 $12.72-316 \partial-45 \partial$.

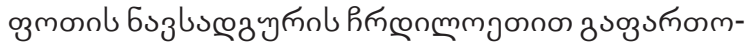

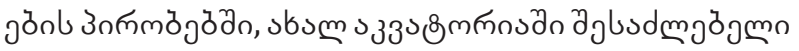
n

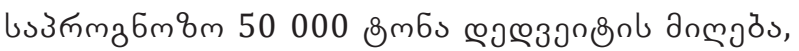

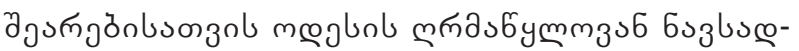

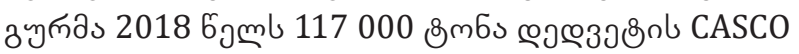
Shiping PANAMA зm68ిวnб

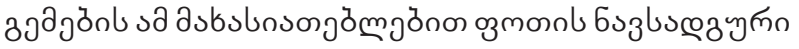

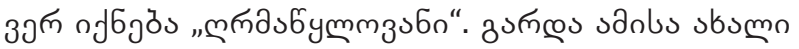

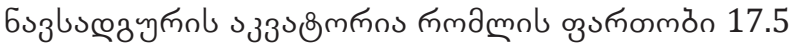

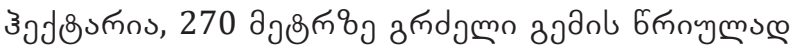

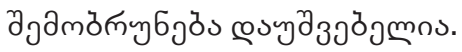

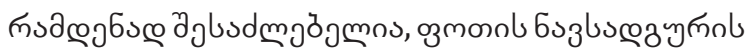

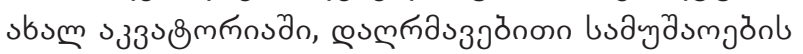

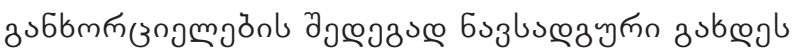

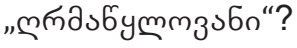

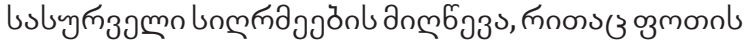

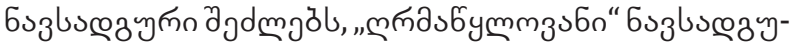

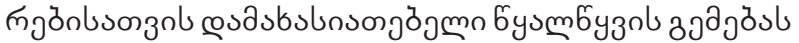

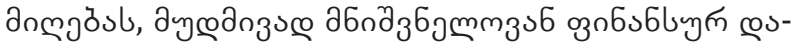

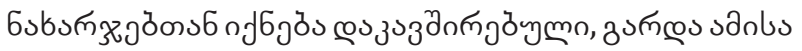

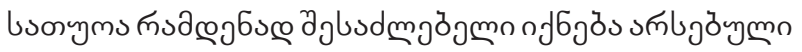

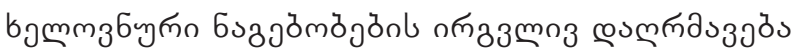

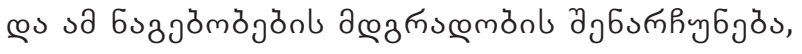

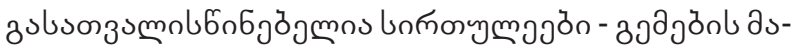
б бз

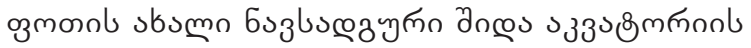

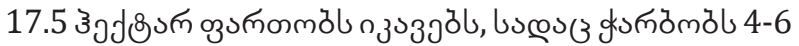
ة

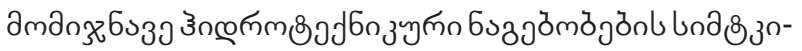

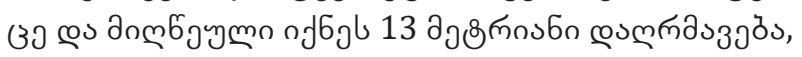

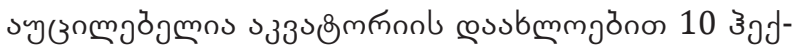

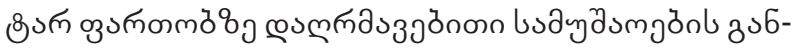

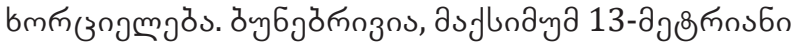
œuмп

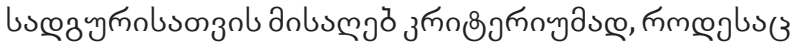

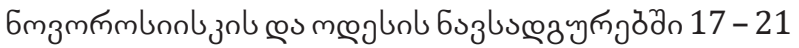

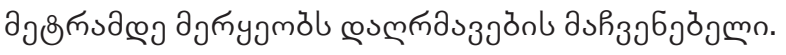

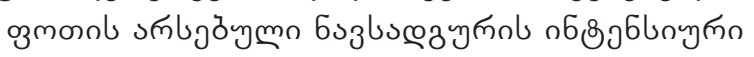

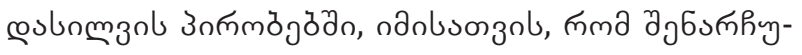

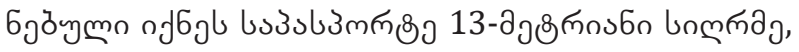

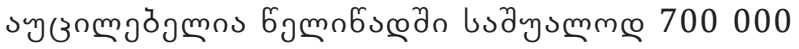

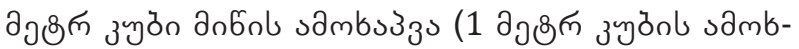

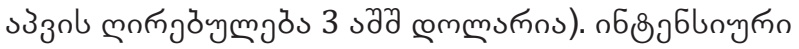

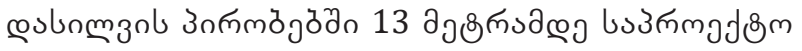

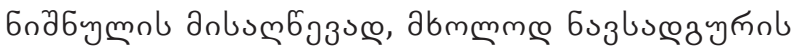

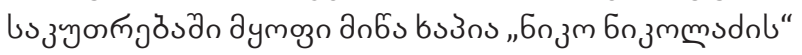

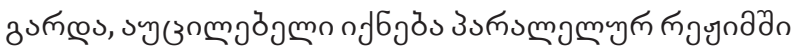

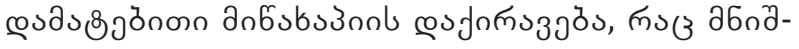

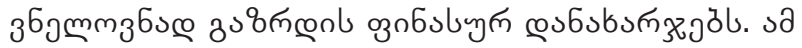

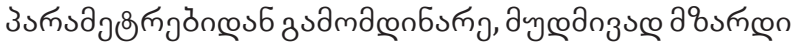

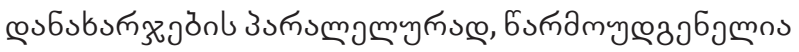

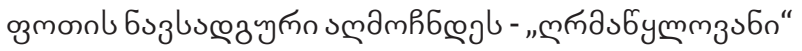

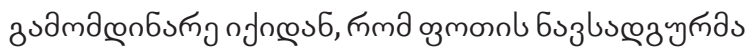

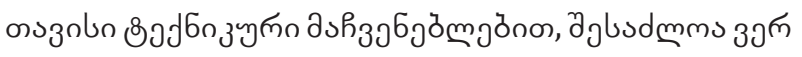

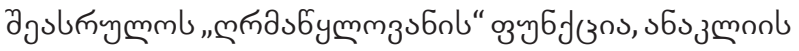

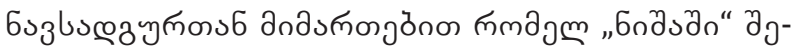

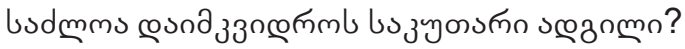

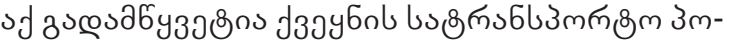

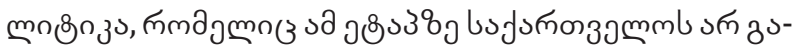

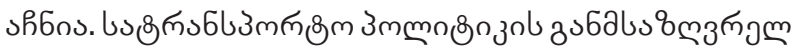

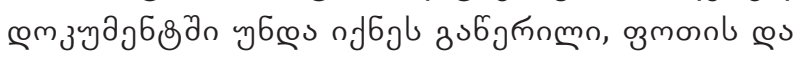

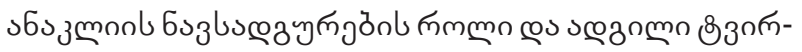

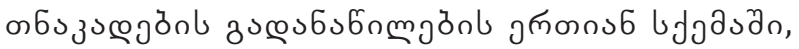

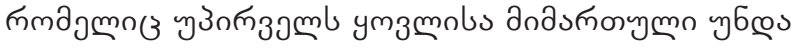

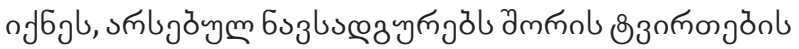

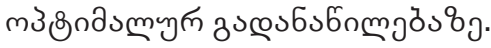




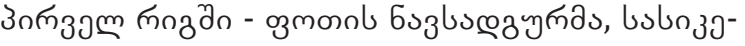

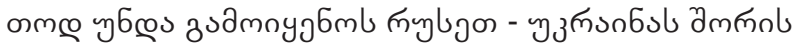

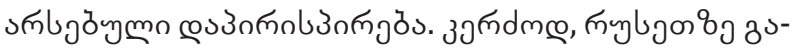

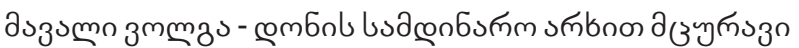

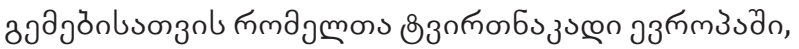

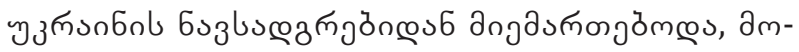

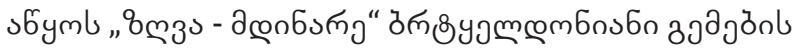

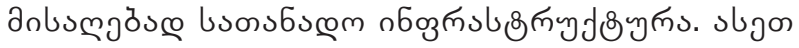

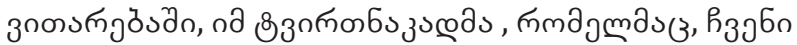

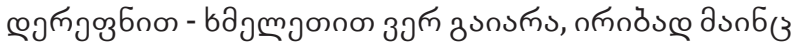

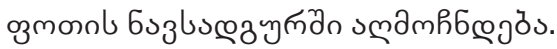

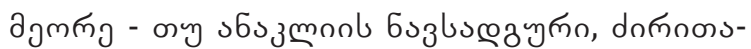

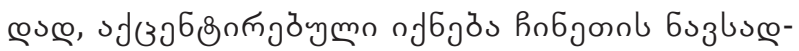

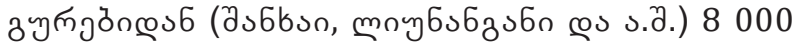

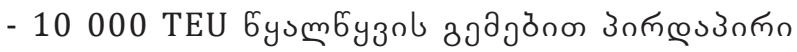

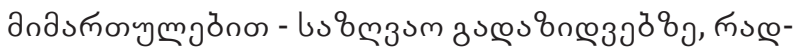

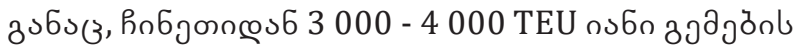

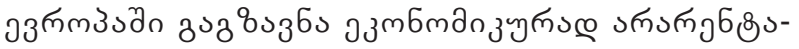

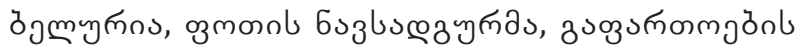

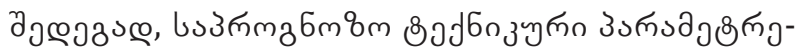

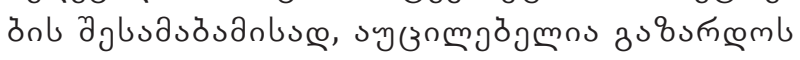

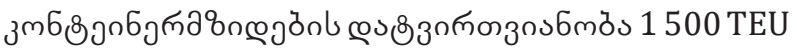

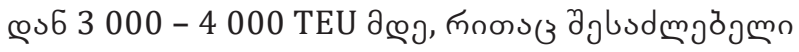

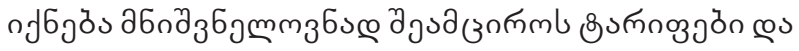

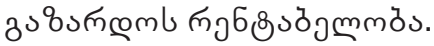

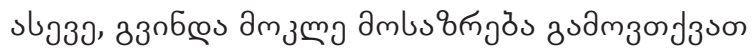

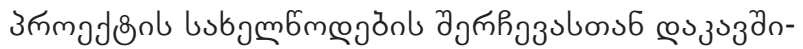

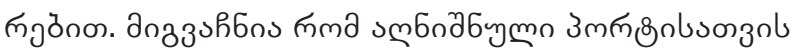

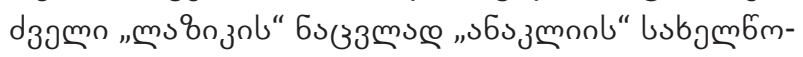

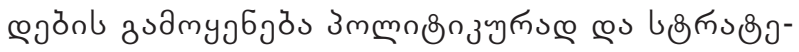

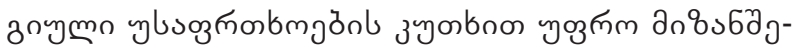

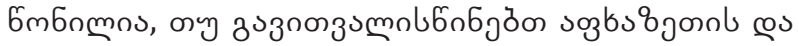

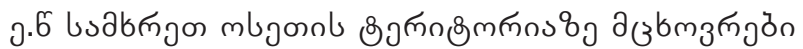

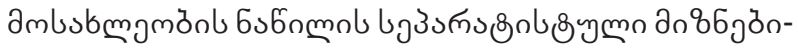

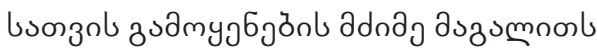

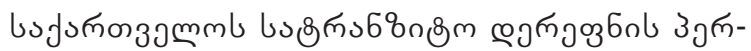

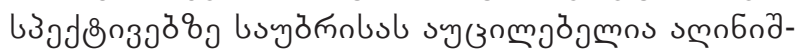

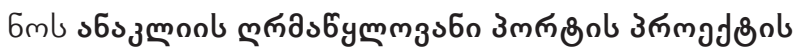

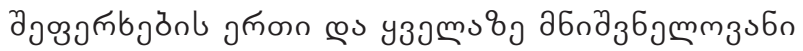

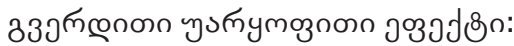

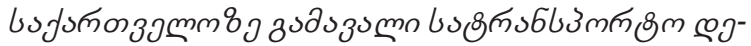

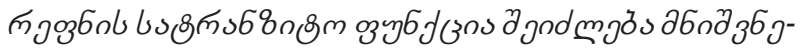

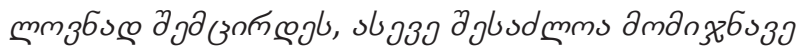

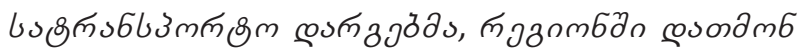

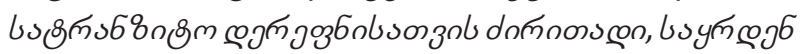

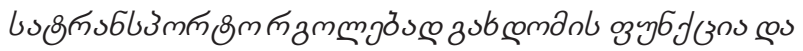

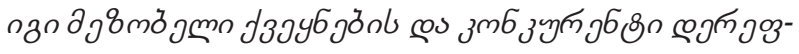

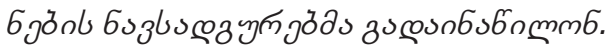

\section{6১}

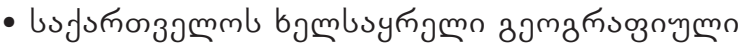

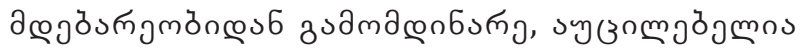

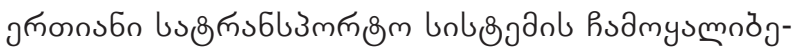

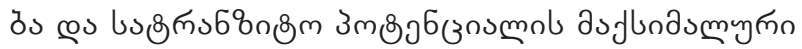
зงวмyวбวอัง.

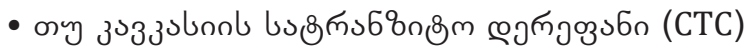

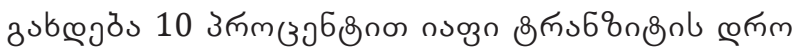

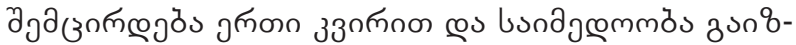

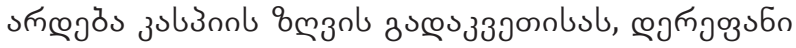

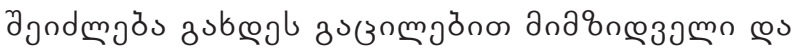

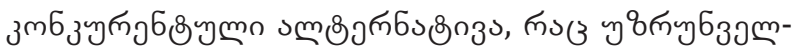

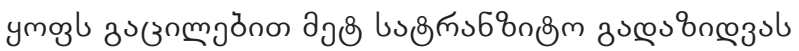

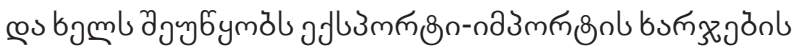
дे

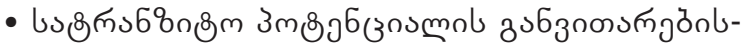

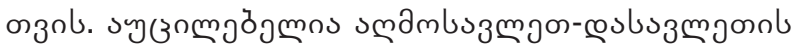

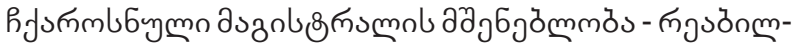

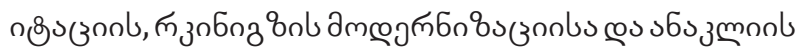

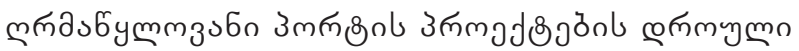

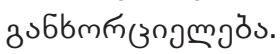

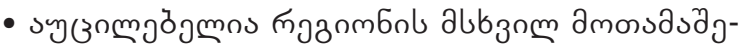

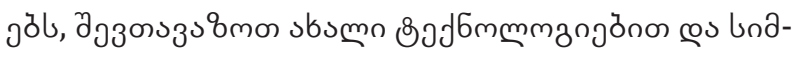

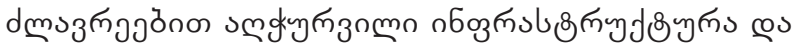
з Зз dmn

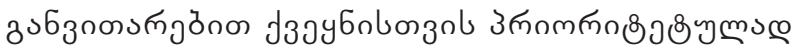

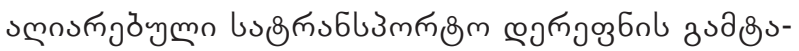

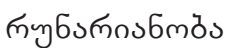

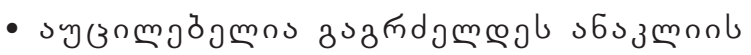

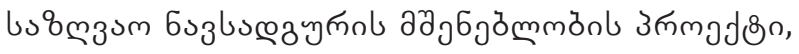

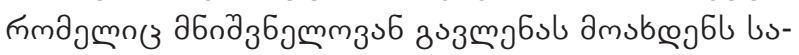

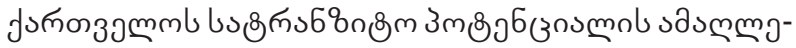
วงใg, з s

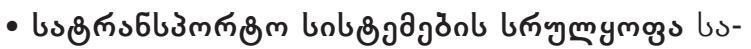

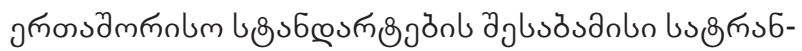

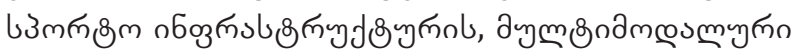
œu nб8ภ

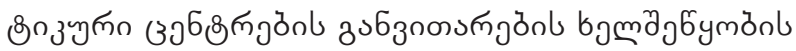
उใกo;

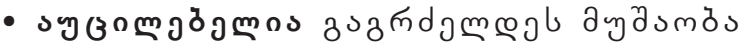

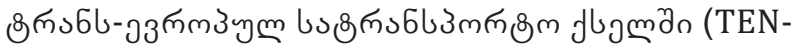

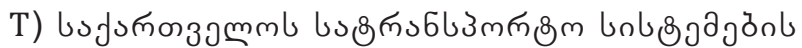

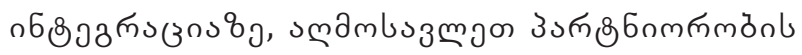

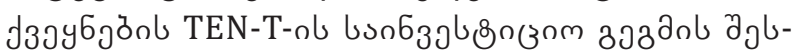

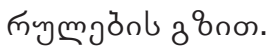

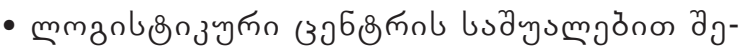

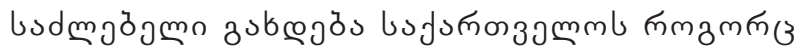

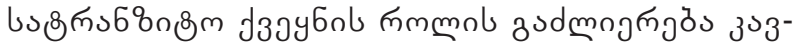




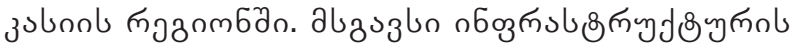
s

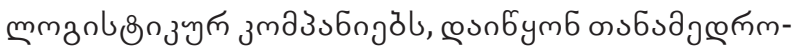

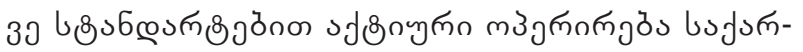
๓зэмmman.

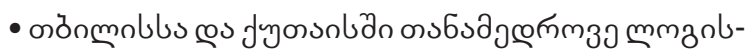

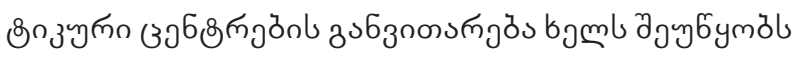

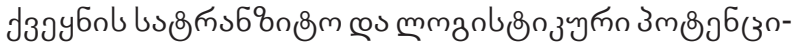
smol zu\&nূal

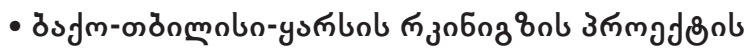

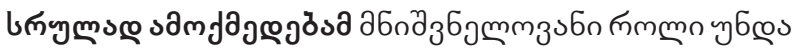

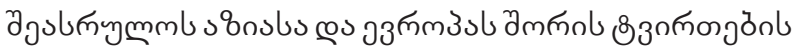

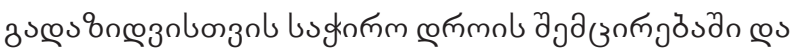

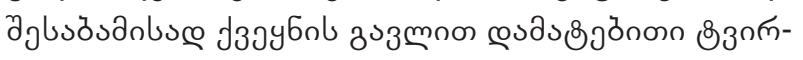

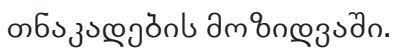

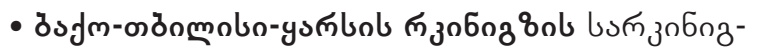

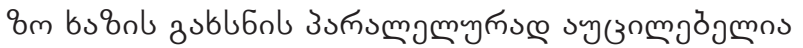

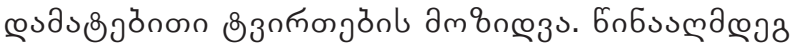

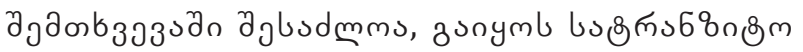

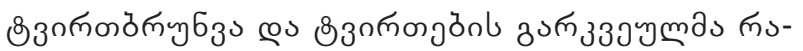

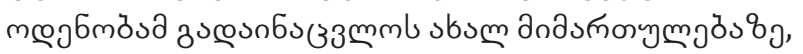

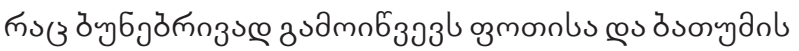

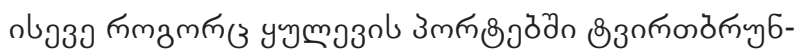

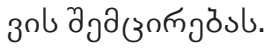

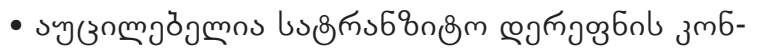

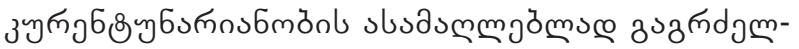

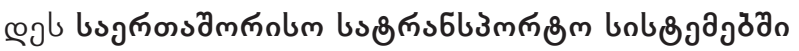
ం6ఠి

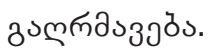

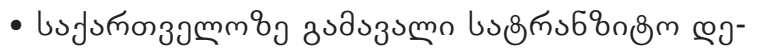

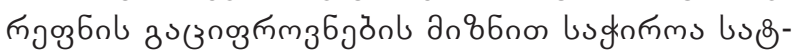

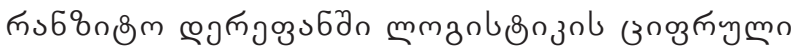

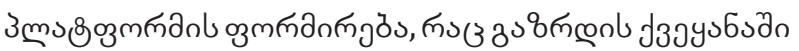

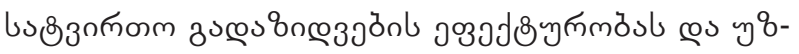

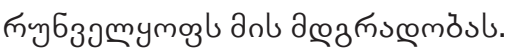

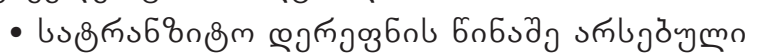

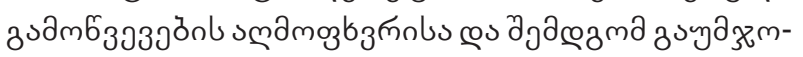

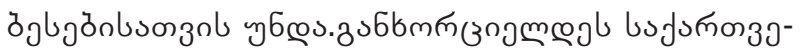

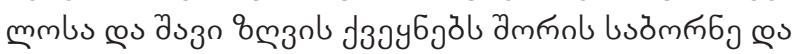

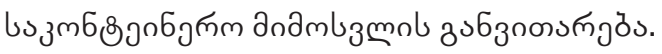

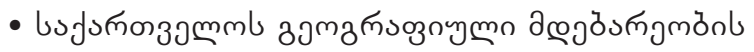

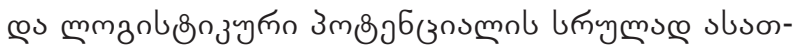

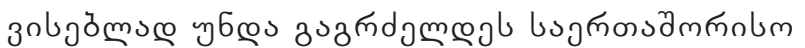

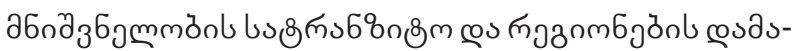

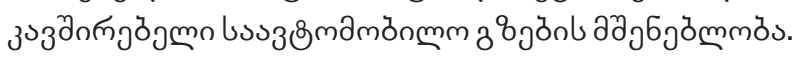

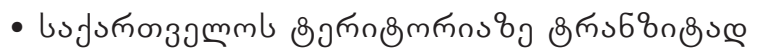

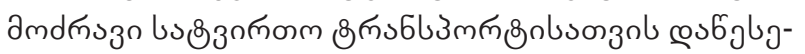

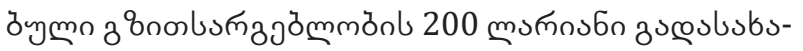

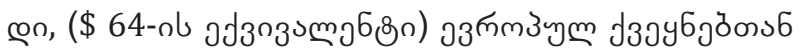

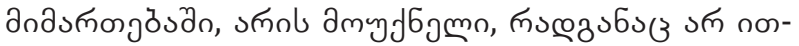

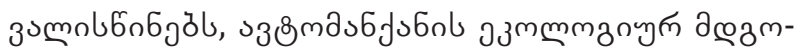

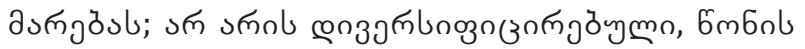

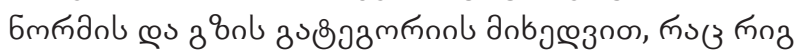

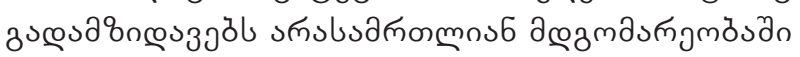

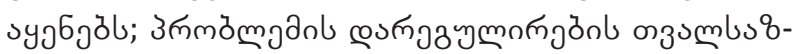

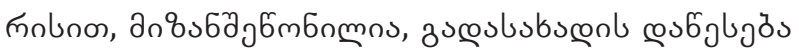

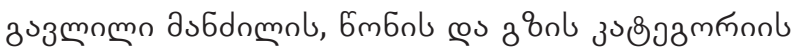

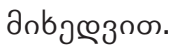

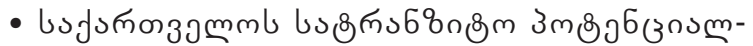
o zuбbuznos

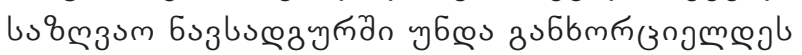

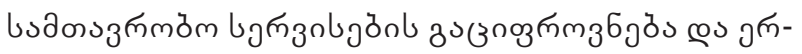

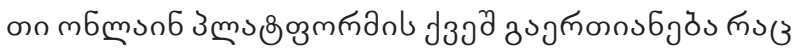

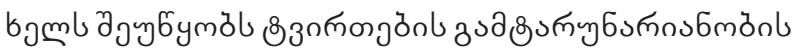

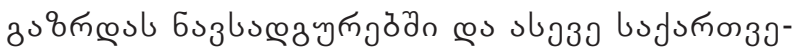

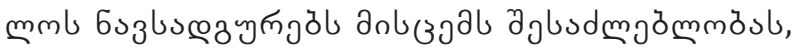

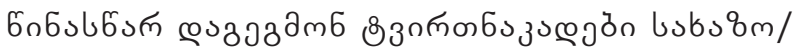

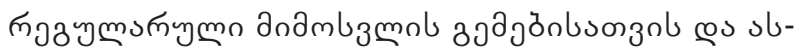

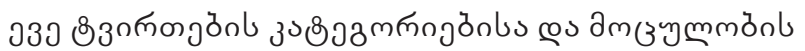

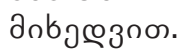

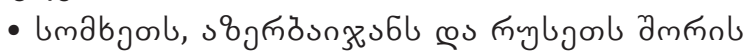

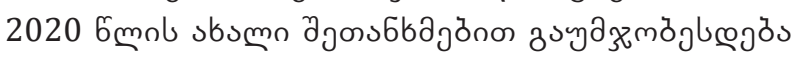

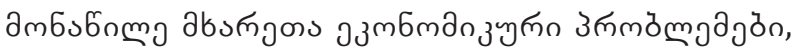

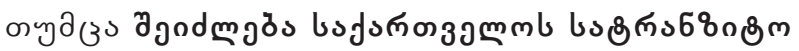

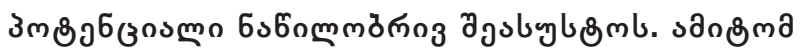

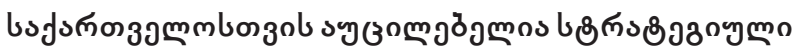

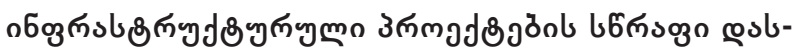

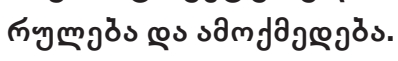

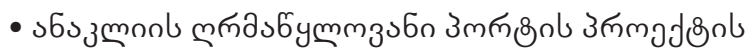

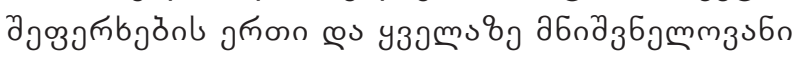

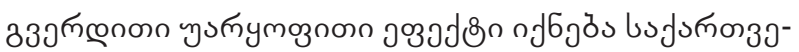

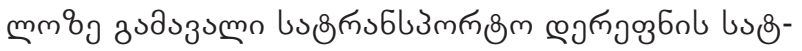

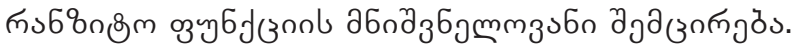

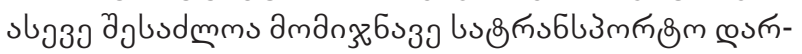

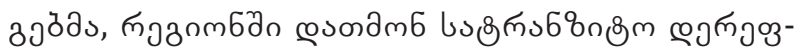

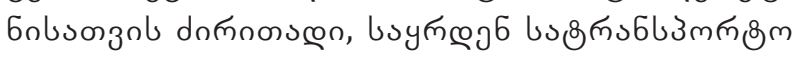
пзмm

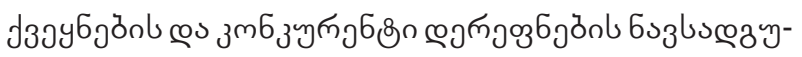

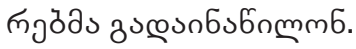

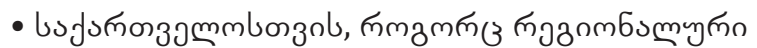

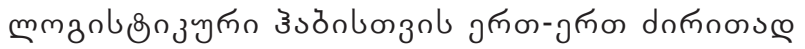

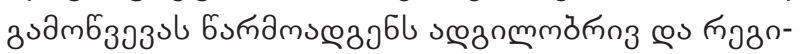

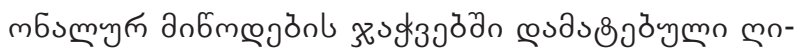

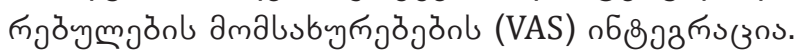

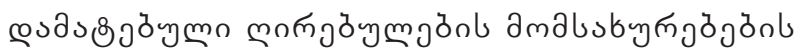

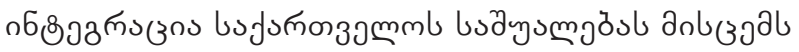

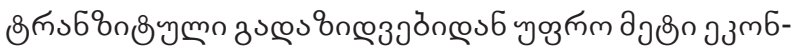

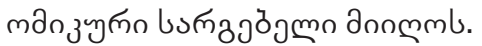




\section{REFERENCES:}

1. Ministry of Economy and Sustainable Development of Georgia, Department of Transport and Logistics Development, http://www.economy.ge;

2. Ministry of Economy and Sustainable Development of Georgia, National Transport and Logistics Strategy of Georgia for 2021-2030, http://www.economy.ge;

3. Ministry of Regional Development and Infrastructure of Georgia, Regional Development - Roads, projects in the regions, https://mrdi.gov.ge/en;

4. Roads Department, 2018-2024 projects, current, completed and future projects, http://www.georoad.ge;

5. USAID, 2017, Strengthening the Public-Private Sector Dialogue Platform in Parliament Research Report: Transportation and Logistics in Georgia: Key Challenges and Development Perspectives USAID Governance for Development, USAID Project Governance for Development. « Deloitte Consulting ", www.usaid.gov;

6. National Statistics Office of Georgia, 2021, Foreign Trade of Georgia 2021. January-April (preliminary), www.geostat.ge;

7. Poti Seaport, 2021, APM TERMINALS, Poti - News - https://www.apmterminals.com/poti;

8. World Bank, 2014, https://www.worldbank.org/en;

9. Georgian Railway., 2021, Georgian Rail Transportation Tariffs, http://www.railway.ge/en;

10. Government of Georgia, 2020, Socio-Economic Development Strategy of Georgia, gov.ge;

11. Georgian Railway, 2019 Infrastructure, Projects - Anaklia, http://www.railway.ge/en;

12. Maritime Transport Agency of Georgia, ports - http://mta.gov.ge/?lng=eng;

13. ADB, 2014-2018, Sector Assessment: Transport, www.adb.or;

14. Georgian Railway., Infrastructure, Statistics, http://www.railway.ge/en.

15. Veshapidze, S., Zubiashvili, T., (2020). About the Origins of Modern Geoeconomic Foundations of Georgia. Ecoforum Journal 9 (2).

16. Zubiashvili, T. and Veshapidze, S., 2019. Labour Emigration and Employment in Georgia. Humanities and Social Sciences Review, 9(01), pp.127-136. 


\title{
EARLY EFFECTS OF THE COVID-19 PANDEMIC ON THE REAL ESTATE MARKET IN GEORGIA
}

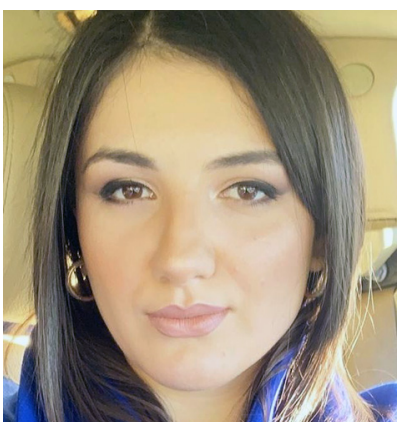

\author{
Natia Terterashvili \\ TSU Doctoral student \\ in the Faculty of Economics and Business \\ E-mail: natia.terterashvili467@eab.tsu.edu.ge
}

DOI suffix: 10.36962/NEC6102202158

\begin{abstract}
The article analyzes the early effects of the COVID_19 pandemic on the Georgian real estate market. There are studies examining the impact of health deterioration and pandemic shocks on housing markets. Based on the analysis of the economic consequences caused by similar events in the past and the tools of state regulation in a crisis situation, parallels are drawn with the modern Covid pandemic. In studying the crisis situation in the real estate market, we also rely on the experience of the global financial crisis of 2007-2008, which is most often associated with the current situation. The research is mainly based on the data of the Georgian Public Registry on the real estate market, which is updated by months. The article also discusses the role and importance of government decision-making in the development business, which has helped the real estate market to some extent.

Crises are particularly damaging to the construction and real estate sectors, but we also expect that the real estate market will recover rapidly with the lifting of restrictions. This is based on the recent experience of Georgia, in particular, our simultaneous analysis of the results of the first wave of the COVID_19 pandemic. The situation was different during the crisis of 2007-2008, which was accompanied by war with Russia. Then the turn over of the construction sector decreased by $12 \%$ per year and it took about three years for the market to fully recover. At the same time, before 2008, the prices in the real estate market were very high, which confirmed the existence of a real estate "bubble". In modern conditions, it is difficult to talk about the existence of a "bubble", because before the pandemic real estate prices were balanced and stable. This fact allows for additional optimism.
\end{abstract}

The paper summarizes the main findings, identifies all the challenges the market are facing and also provides relevant recommendations for market participants.

Keywords: COVID_19 pandemic, Real Estate, Crisis, development business, State regulations.

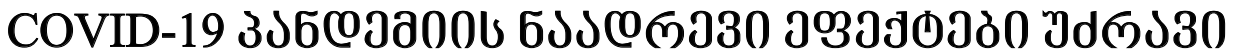

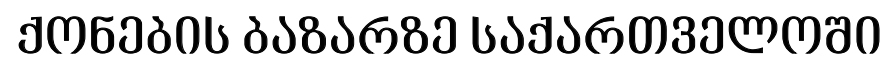

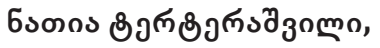
oby jumbmanzols

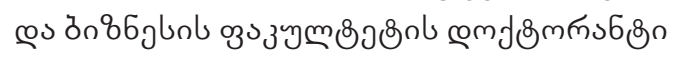
8эm: 551-11-11-32;

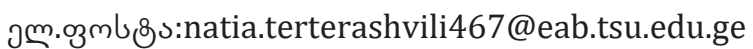

\begin{abstract}
১EMBSBOS

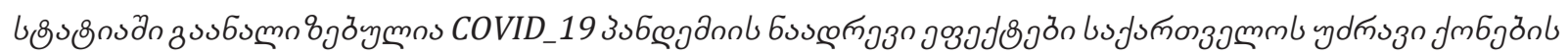
ठง 8ง

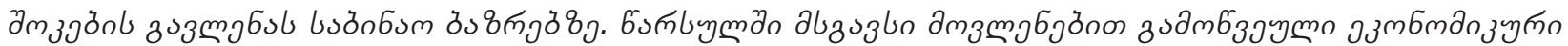

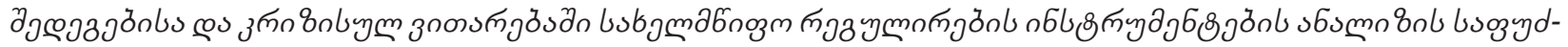

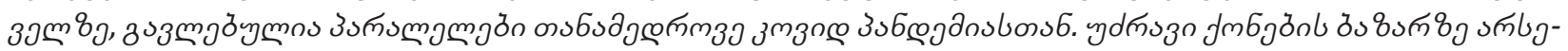




\section{ISSN 2667-9752(Online)}

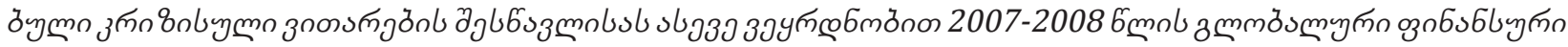

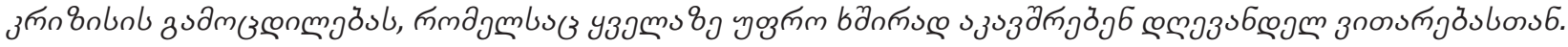

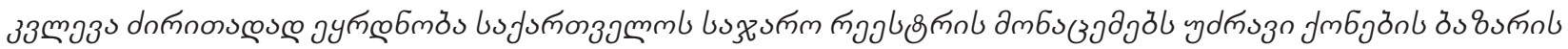

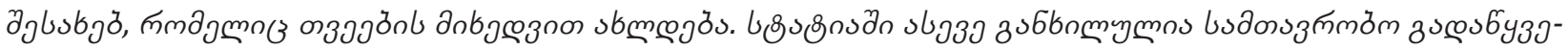

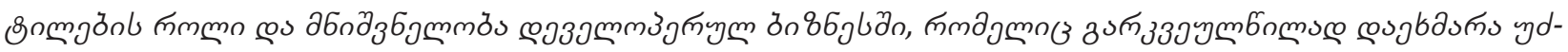

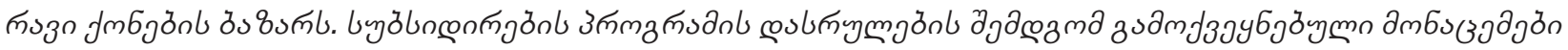

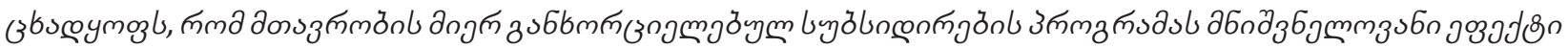

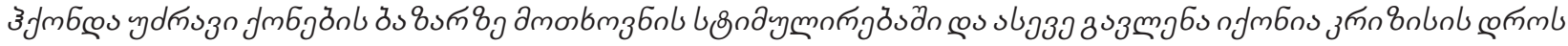

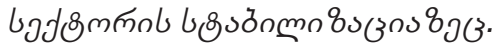

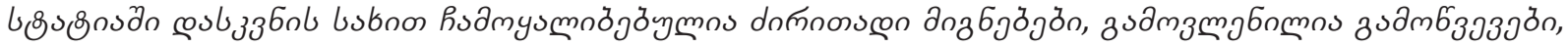

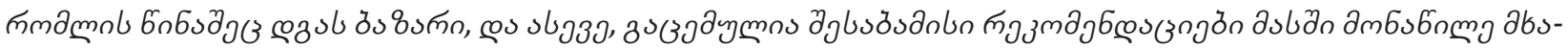
пुjö̌blonzol.

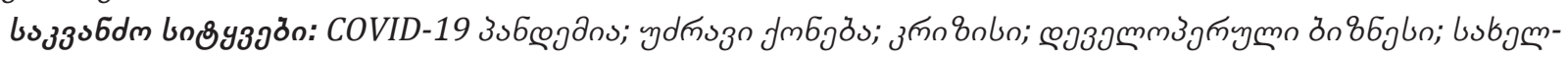

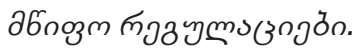

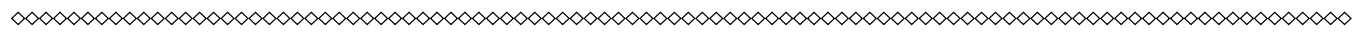

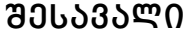

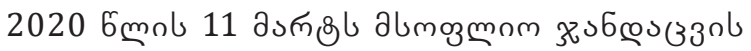

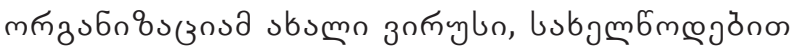

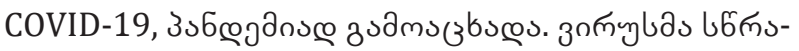

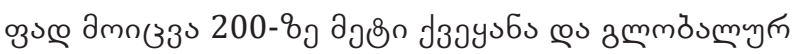

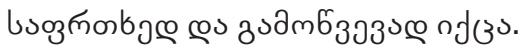

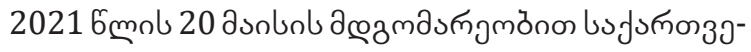

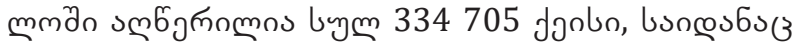

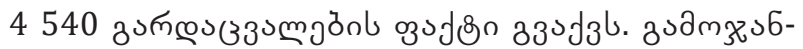
д полм

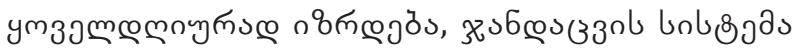

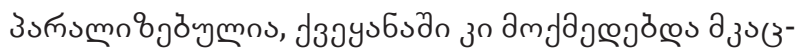

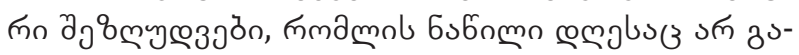

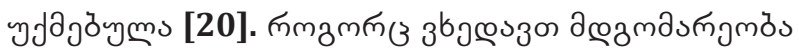

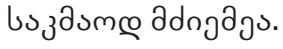

งங்

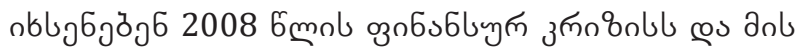

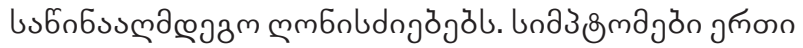

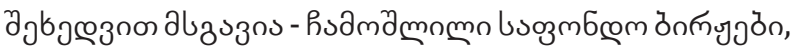

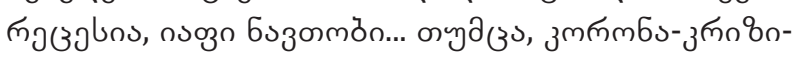

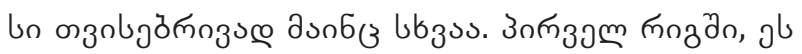

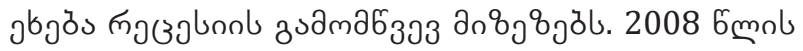
з

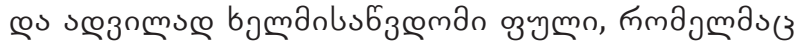

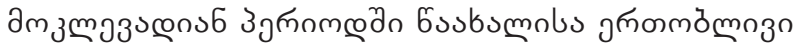

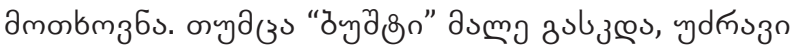

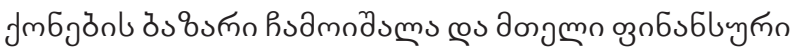

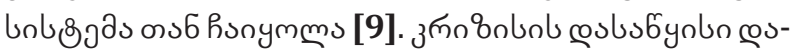
उз3 ๑.6. ᄂyы̆ onb jmo

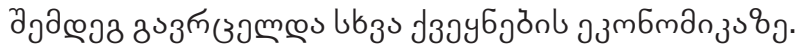

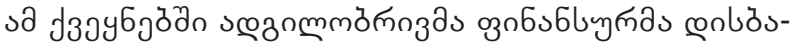

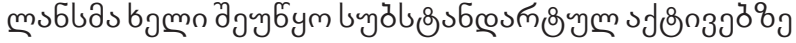

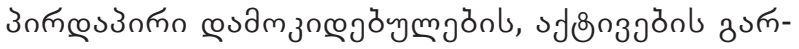

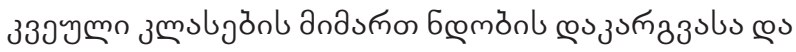

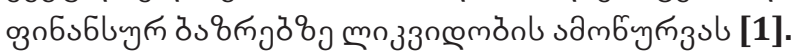

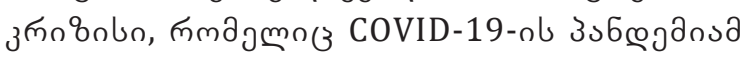

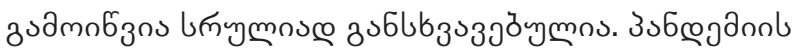

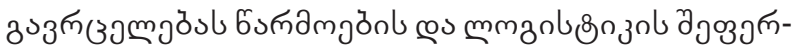

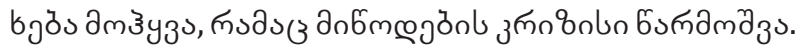
ndymjönonas n

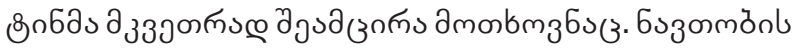

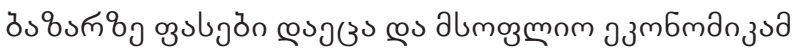

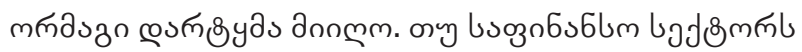

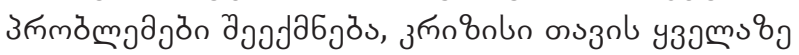

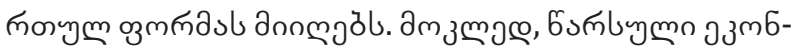

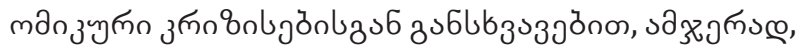

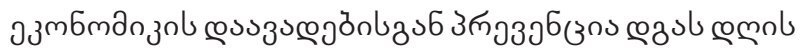
бुओm

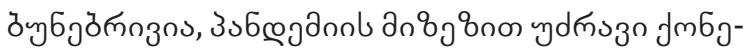

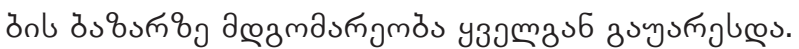

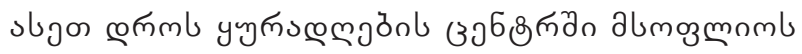

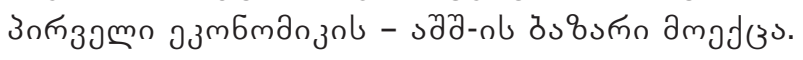

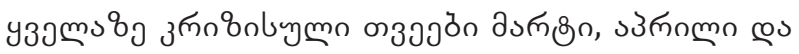

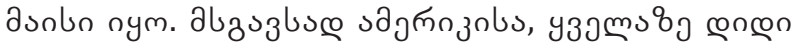

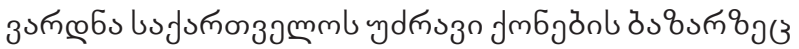

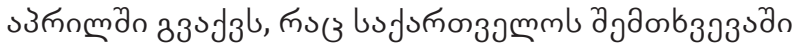

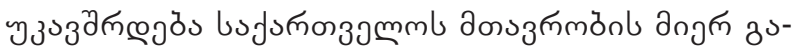

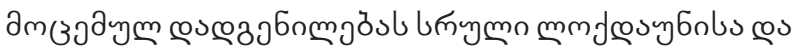

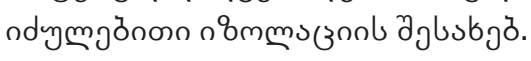

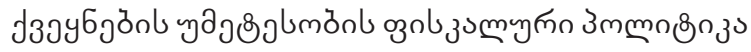

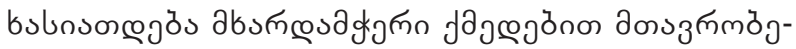

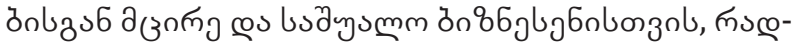

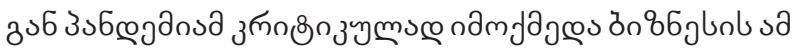

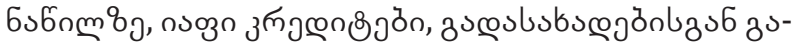




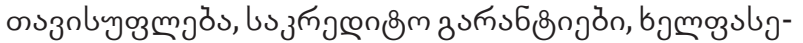

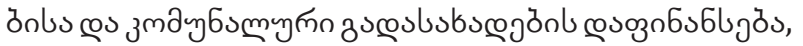

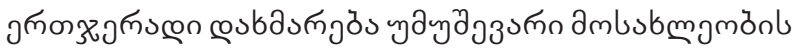

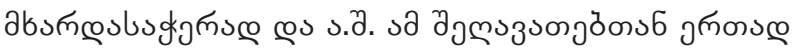

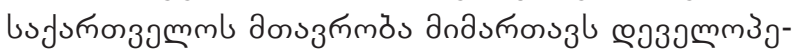

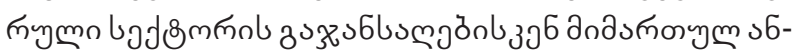

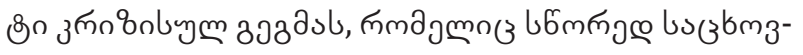

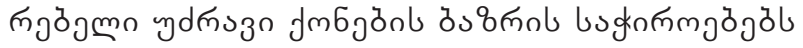

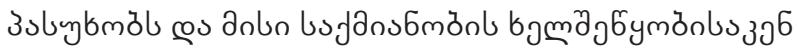

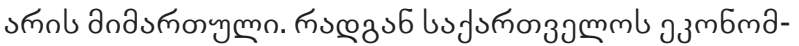

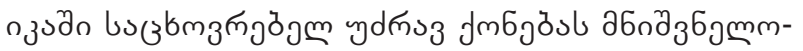

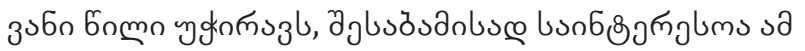

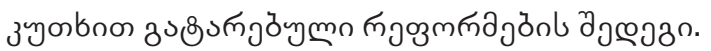

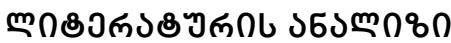

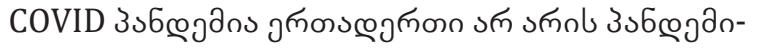

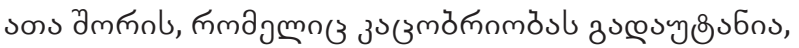

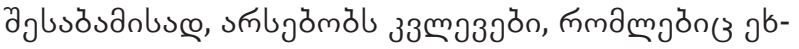

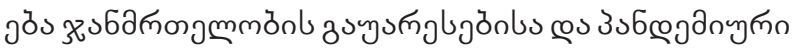

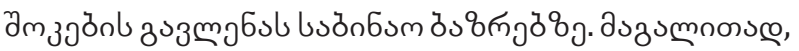

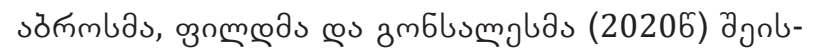

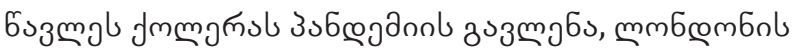

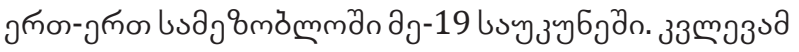

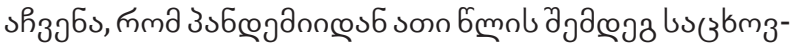

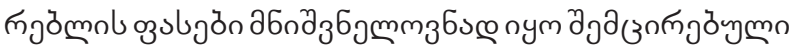

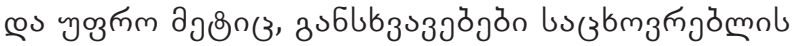

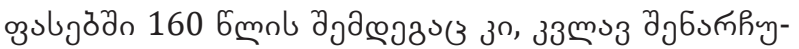
6ృð̌ymos [3].

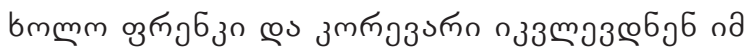

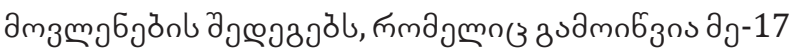

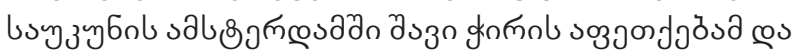

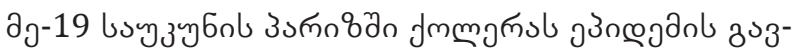

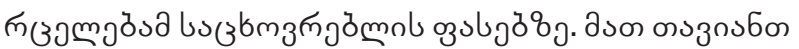

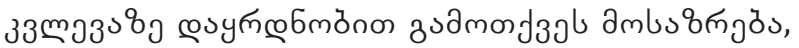

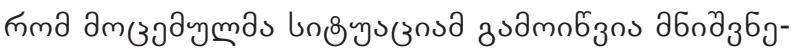

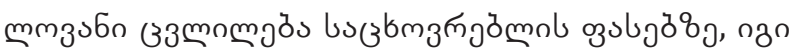

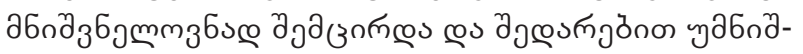

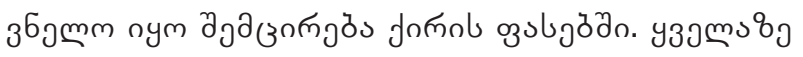

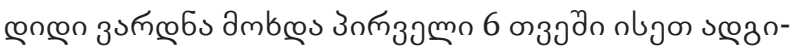

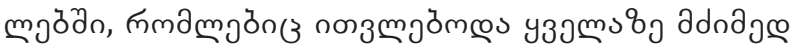

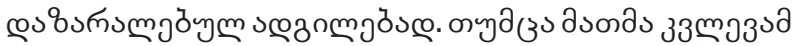

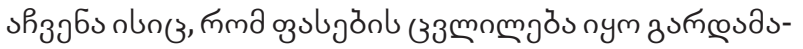

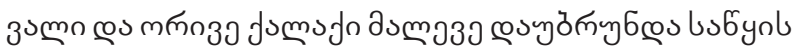

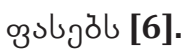

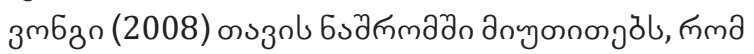

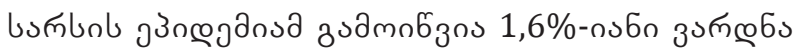

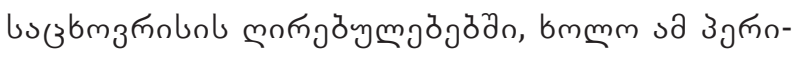
mœðั зง Зెm6-змбаชิว [8].

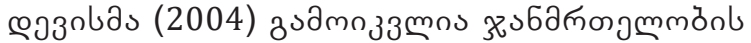

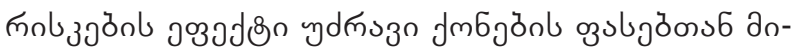

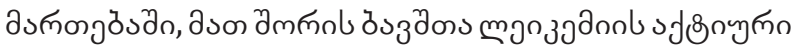

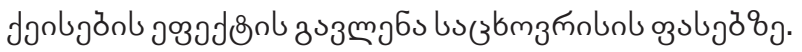

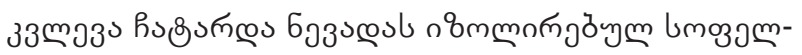

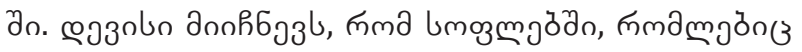

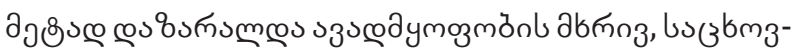

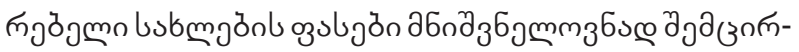

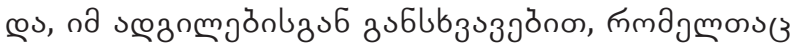

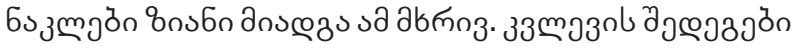

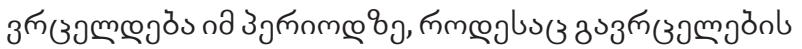

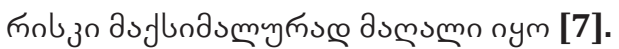

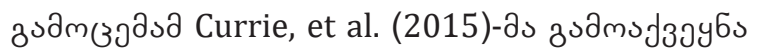

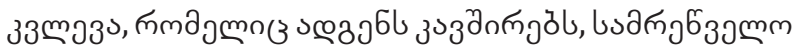

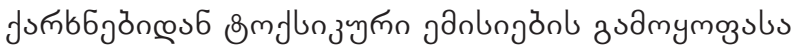

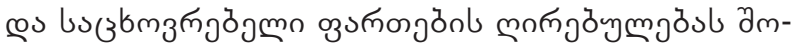

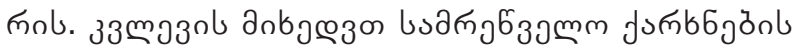

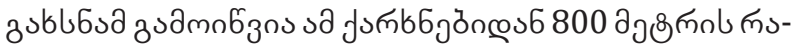

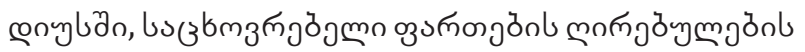

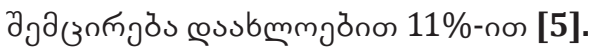

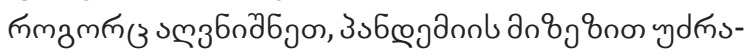

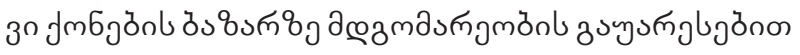

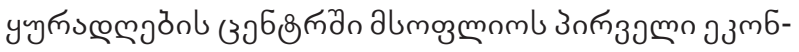

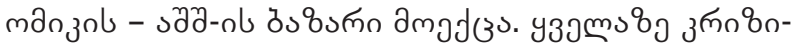

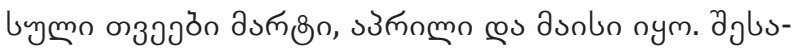

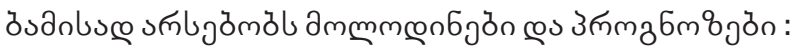

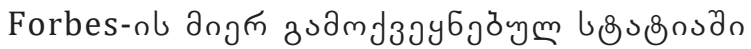

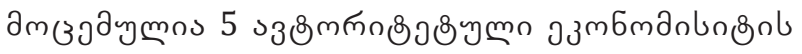

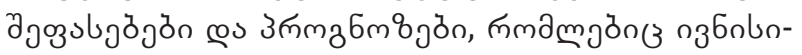

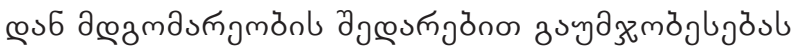

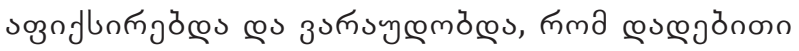
৪

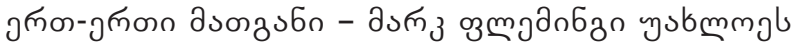

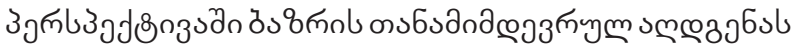

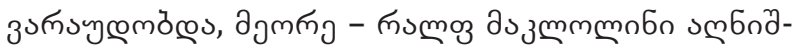

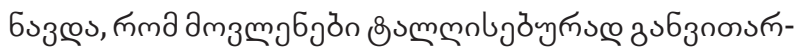

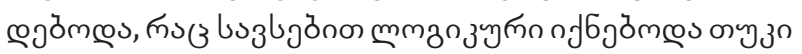

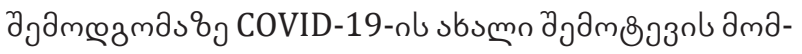

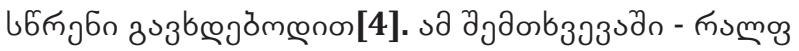

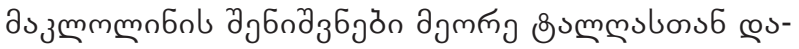

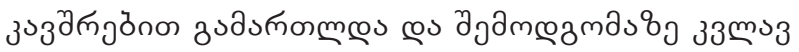

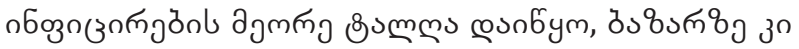

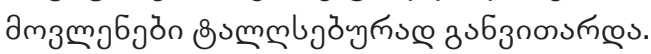

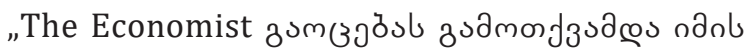

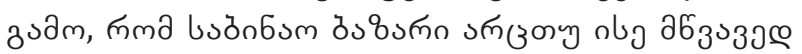

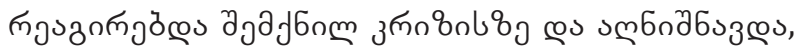

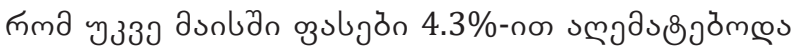

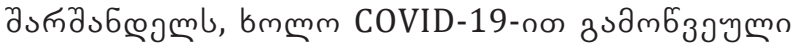

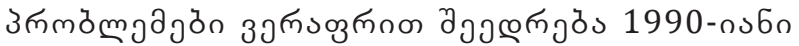




\section{ISSN 2667-9752(Online)}

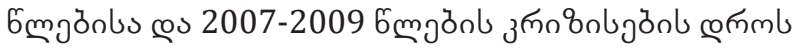

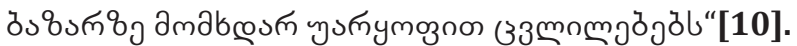

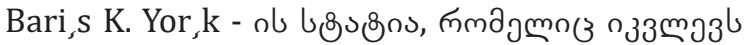

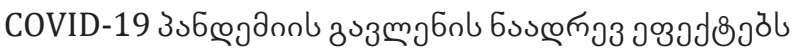

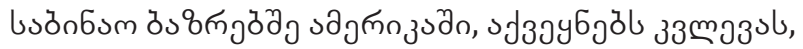
mmajmă

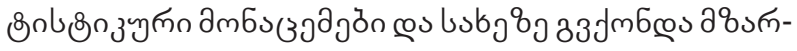

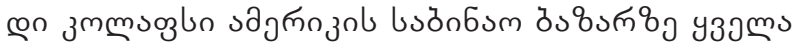

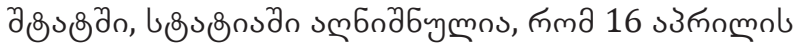

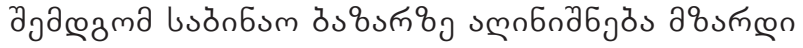

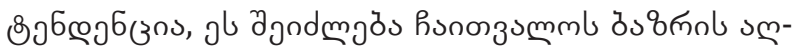

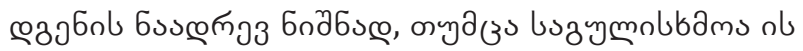

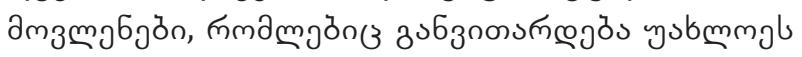

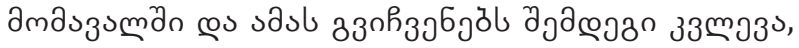

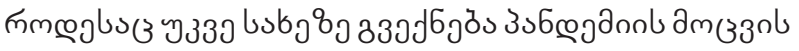
ง6змmo

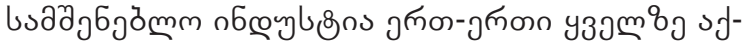

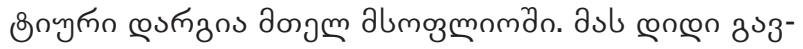

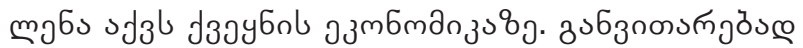

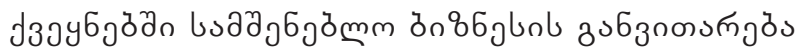

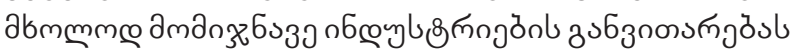

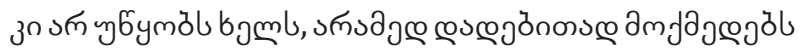

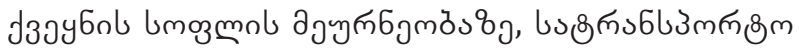

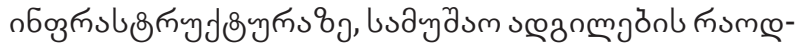

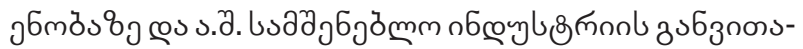

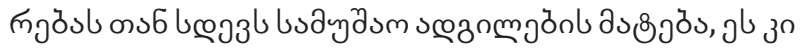

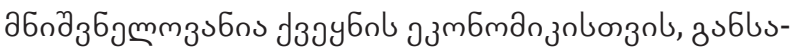

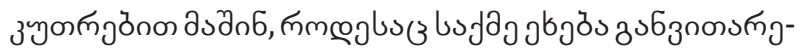

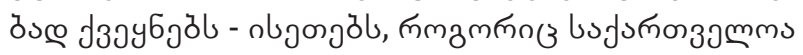

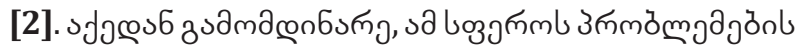
ง6ummo

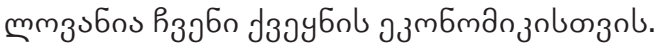

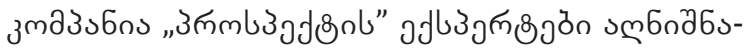

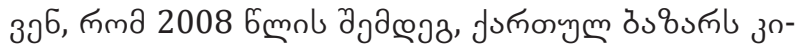

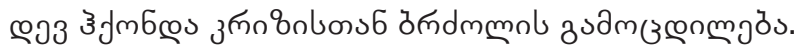

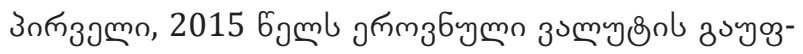

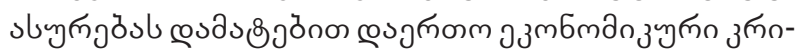

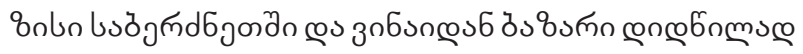

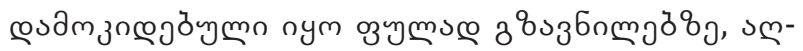

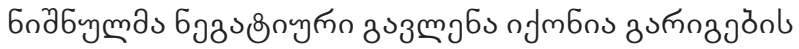

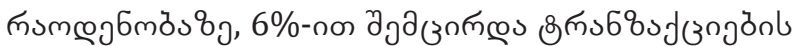

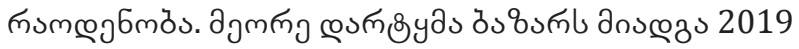

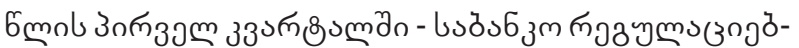

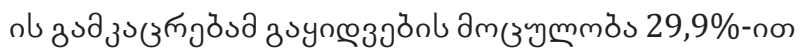
yjua उoms.

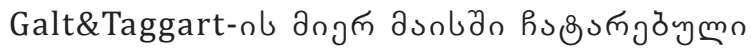

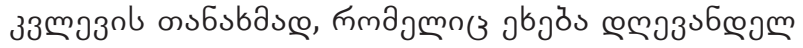

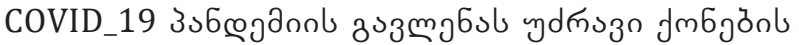
ठ̀

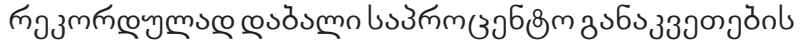

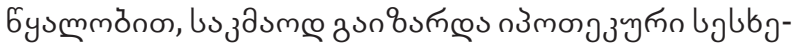

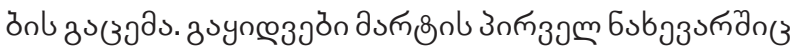

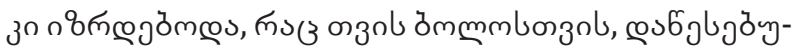

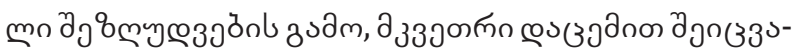

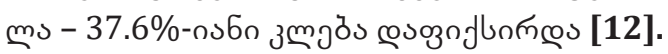

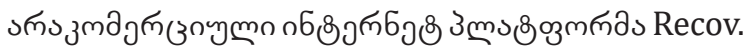

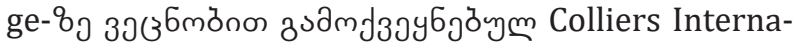

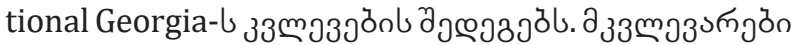

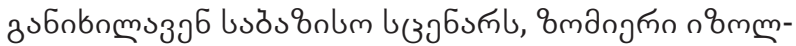

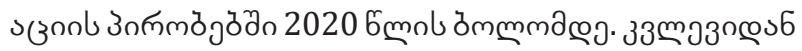

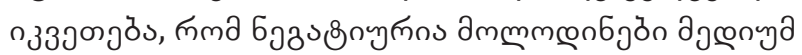

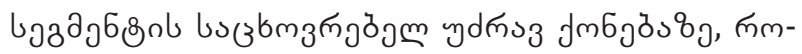

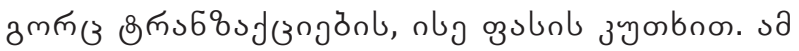

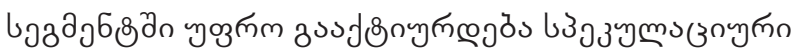

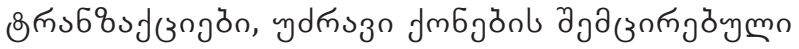

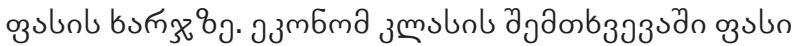
удбпдз

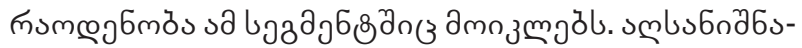

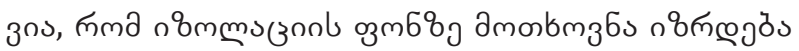

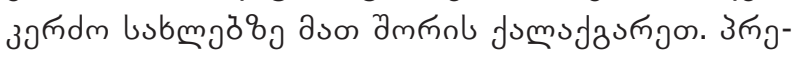

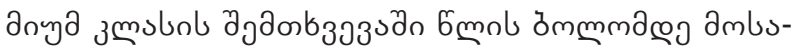

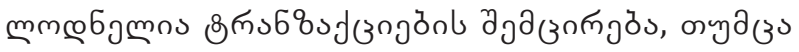

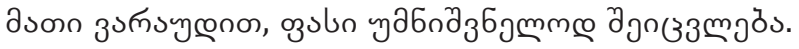

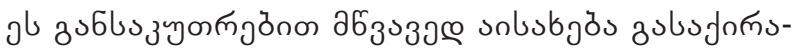

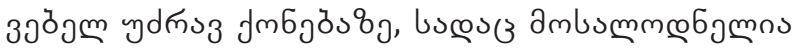
mmammi 8 m

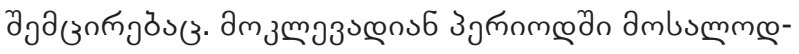

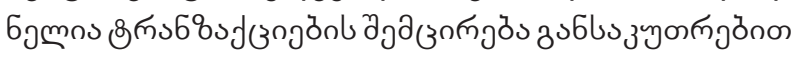

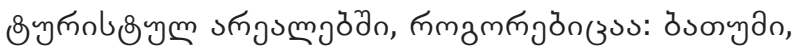

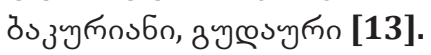

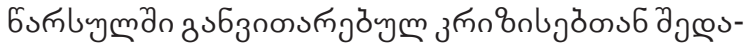

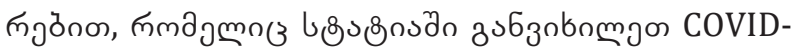

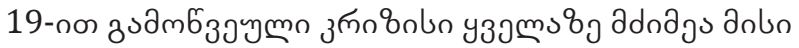

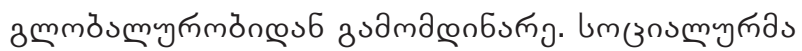

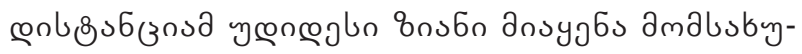

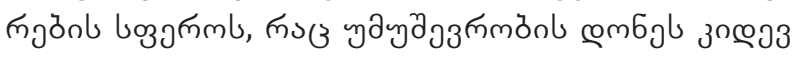

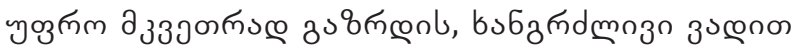

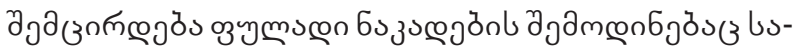

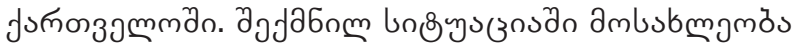
उলnnmmก \&

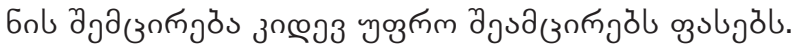

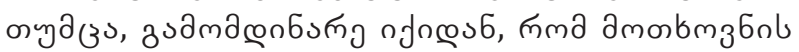

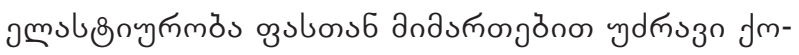

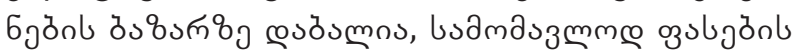

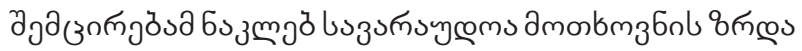
зدаmo6 3 กml [14].

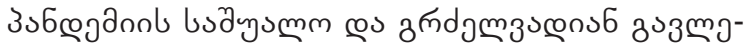

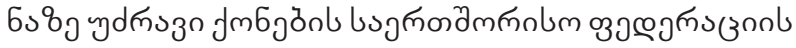


FIABCI-し З๓ృ

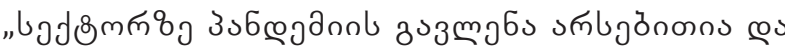

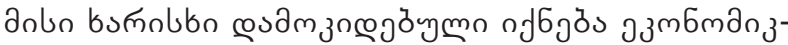

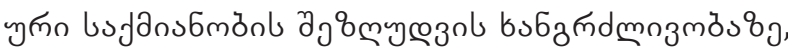

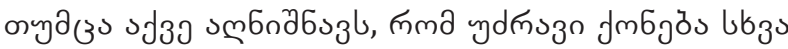

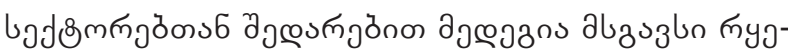

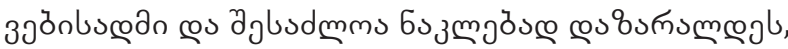

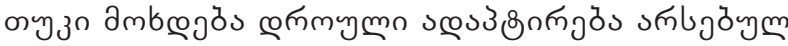
пூummỏsbos6" [15].

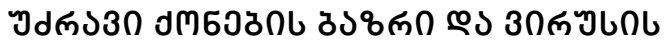

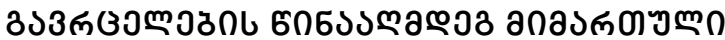

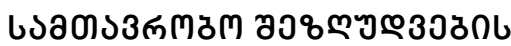

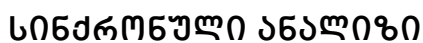

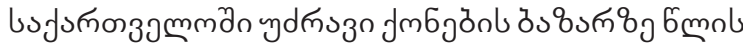

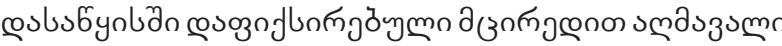

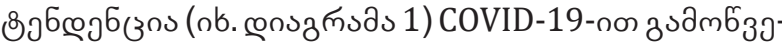

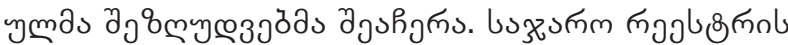

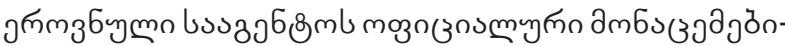

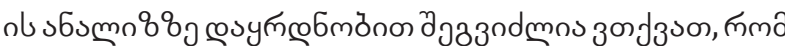

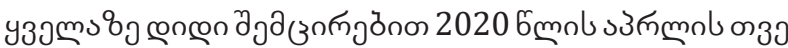

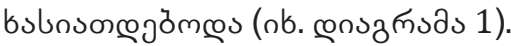

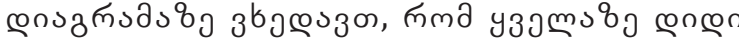

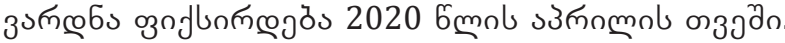

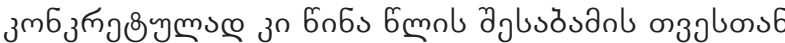

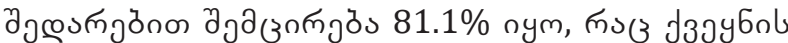

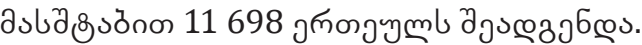

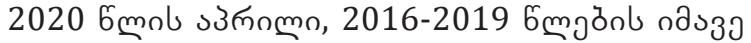

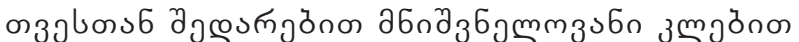

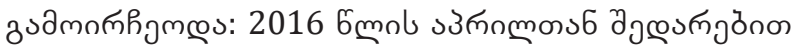

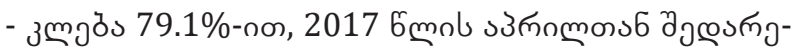

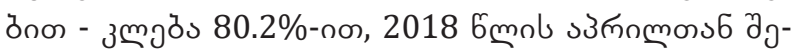

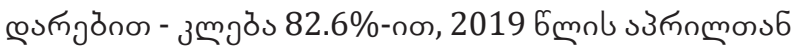

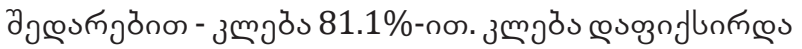

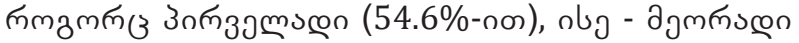

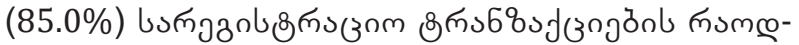
jбmỏsวn.

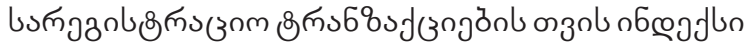

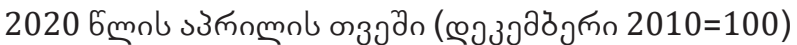

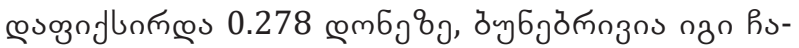

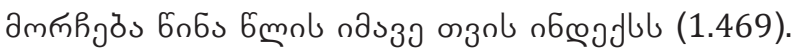

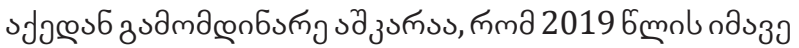

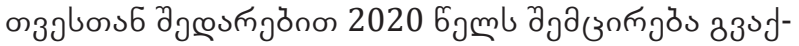

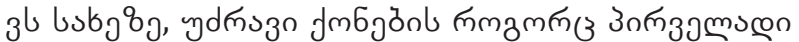

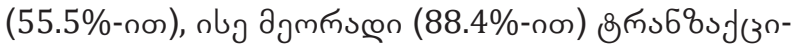

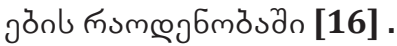

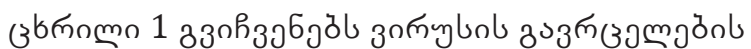

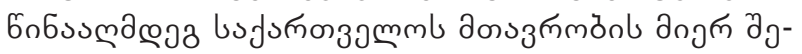

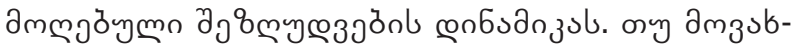

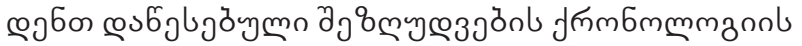

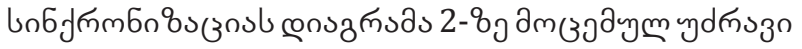

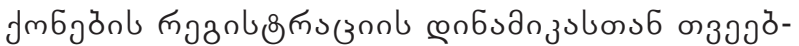

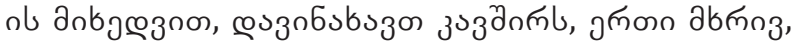

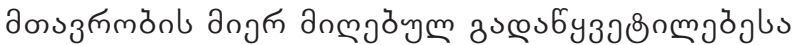

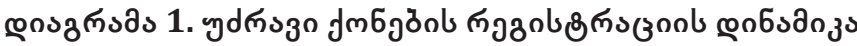

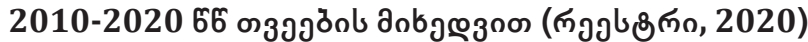

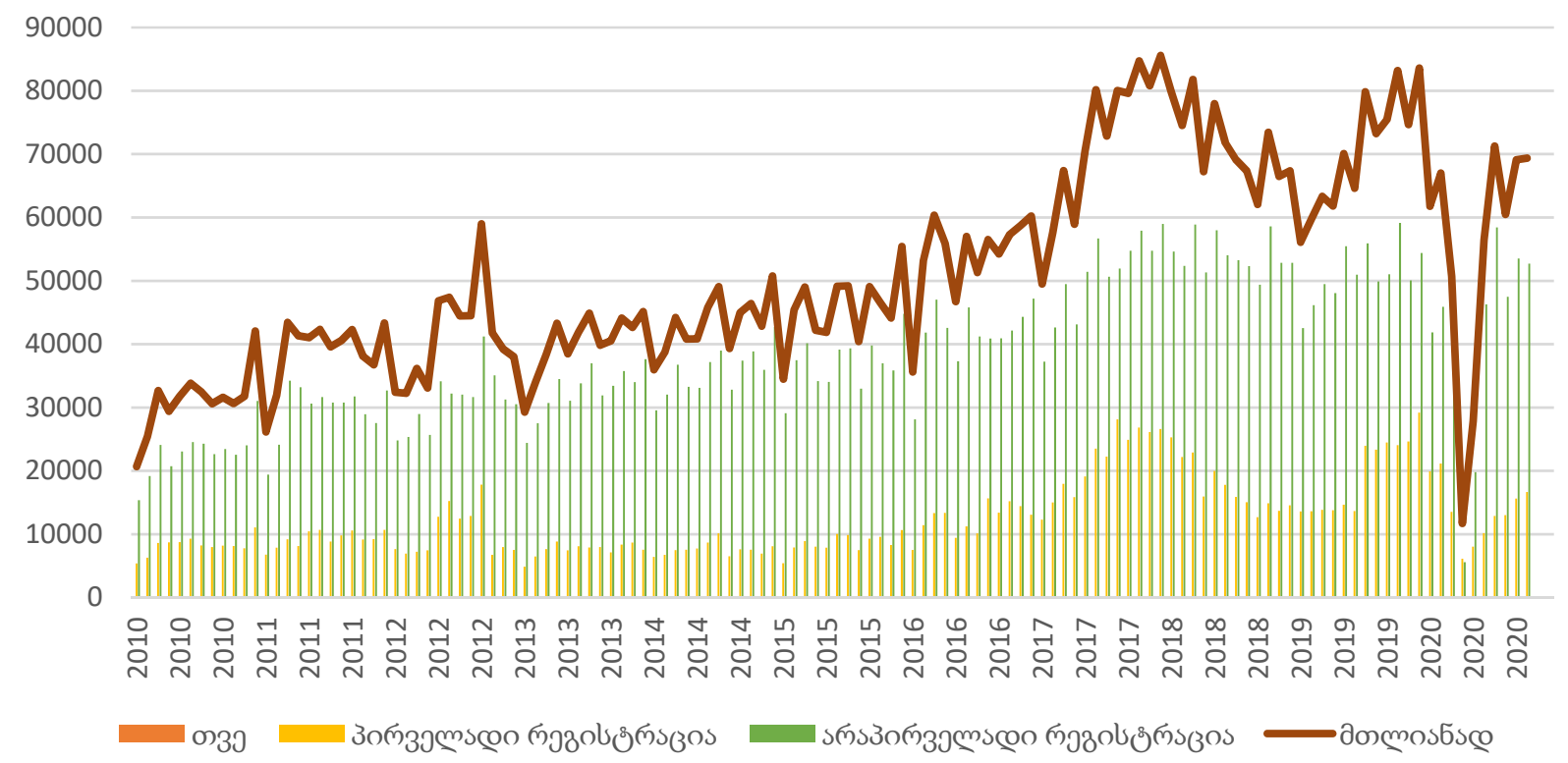

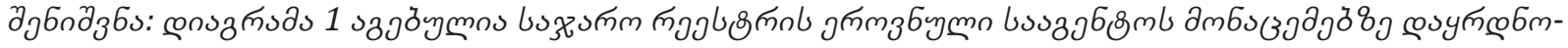
ònon [16]. 


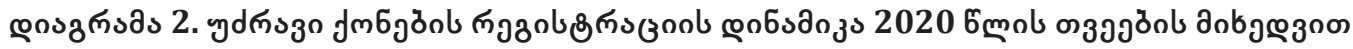

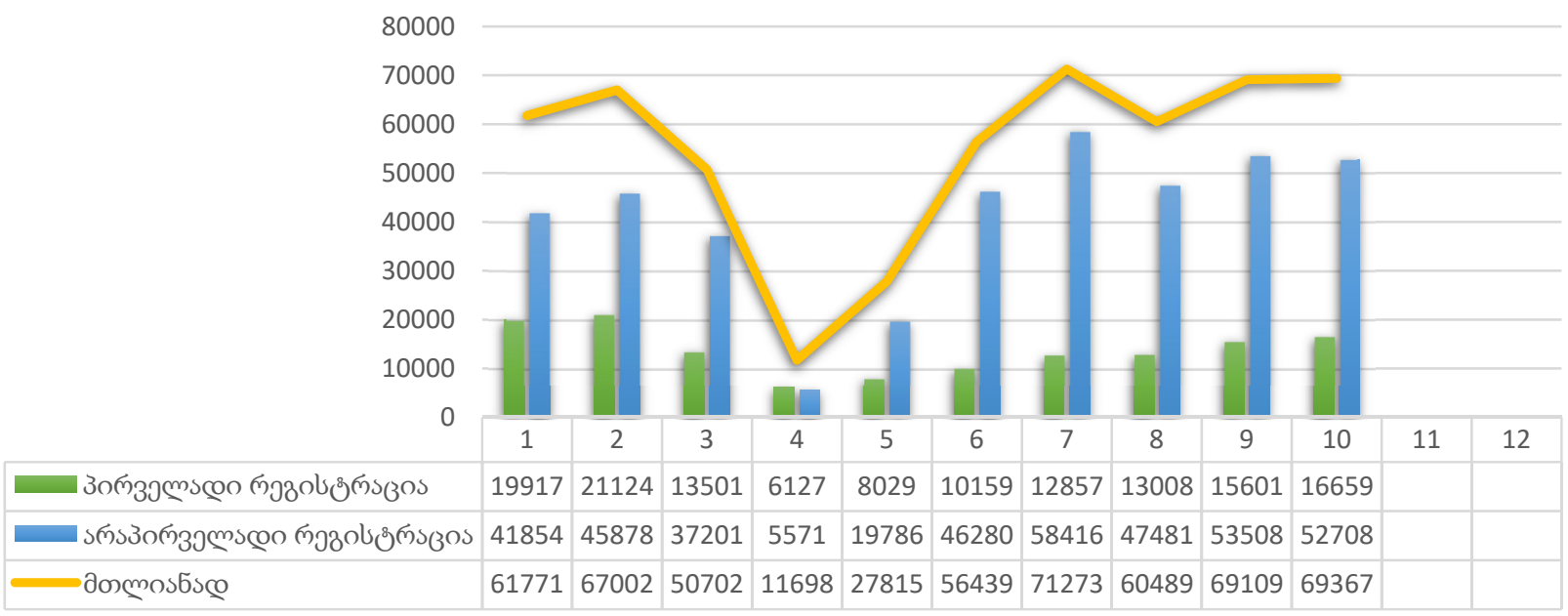

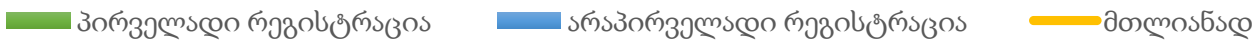

әృбпдз

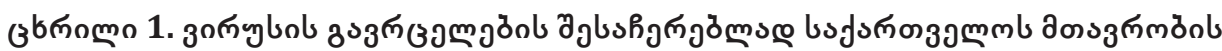

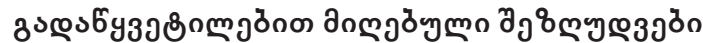

\begin{tabular}{|c|c|}
\hline 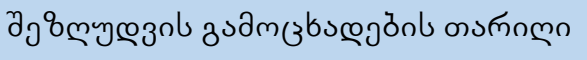 & 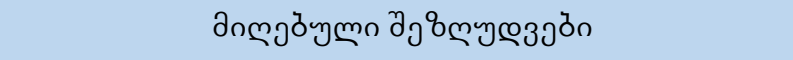 \\
\hline 18 дงল 8 م & 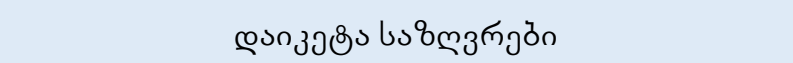 \\
\hline 21 дงm৪ & 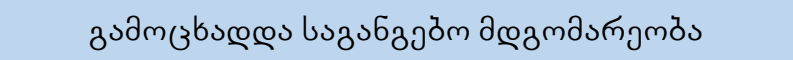 \\
\hline 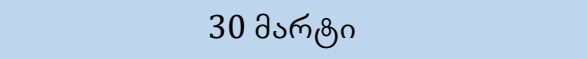 & 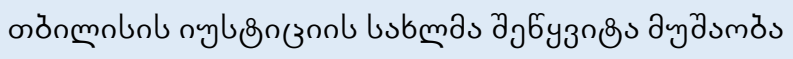 \\
\hline 31 дงm\&n & 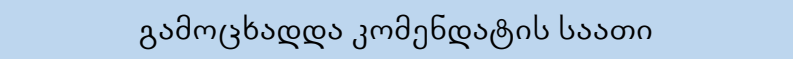 \\
\hline 16 ১Зங்nmn & 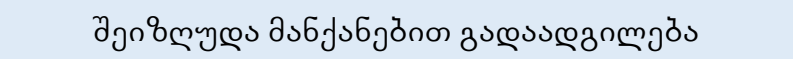 \\
\hline 27 ১Зங்nূm & 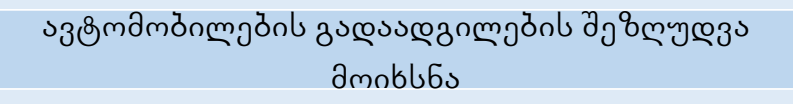 \\
\hline 6 dunbn & 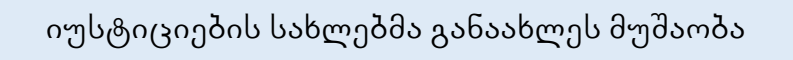 \\
\hline 23 dunbn & 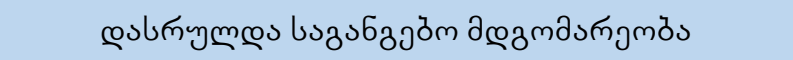 \\
\hline
\end{tabular}

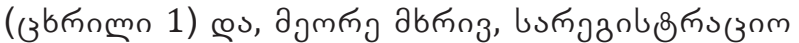

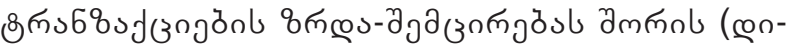

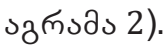

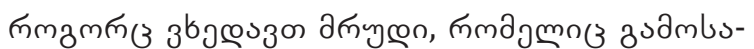

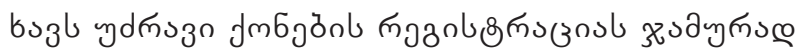

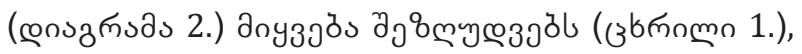

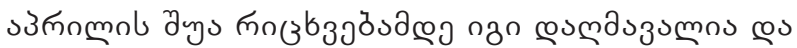

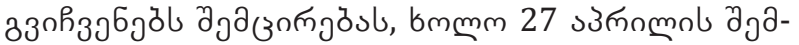

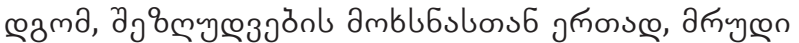
n6yj

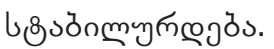

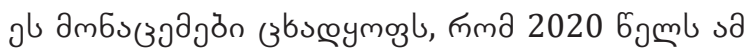

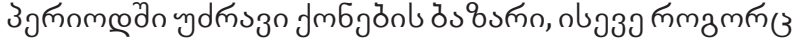

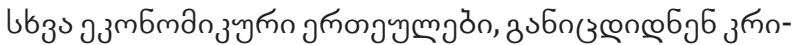

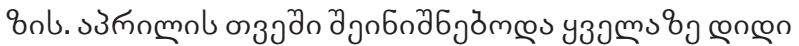

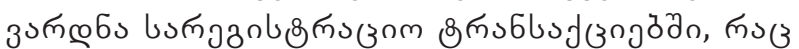

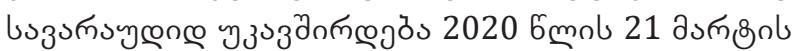

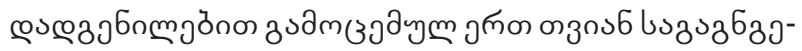

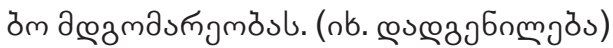

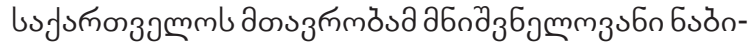

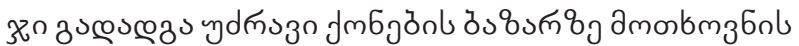

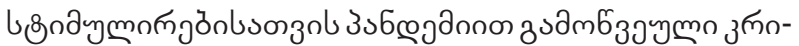

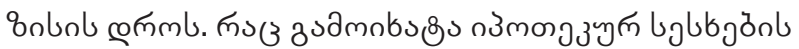

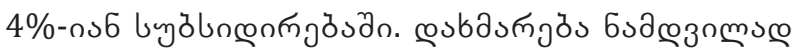




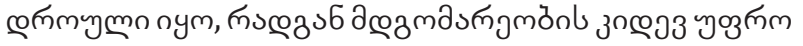

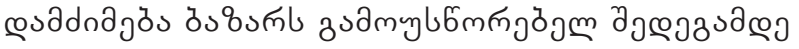
апnуззбюs.

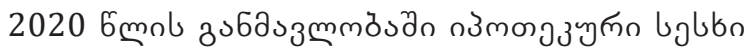

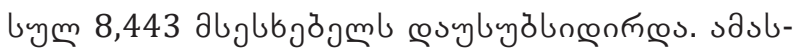

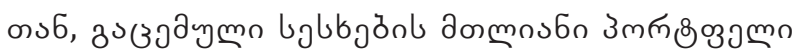

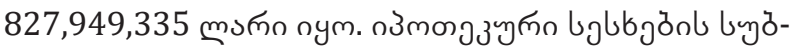
unূn

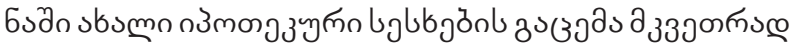

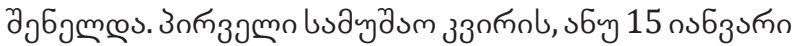

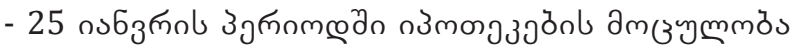

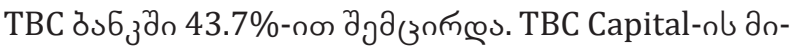

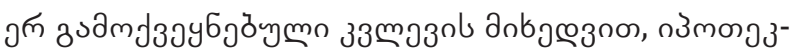
yளn

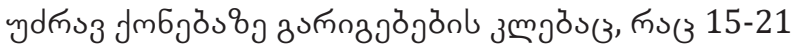

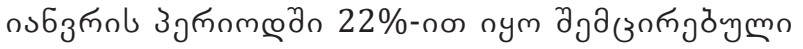

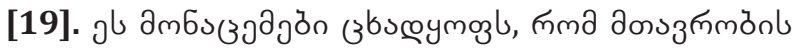

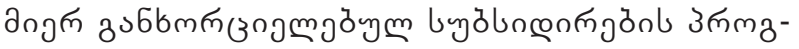

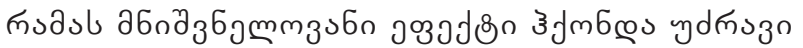

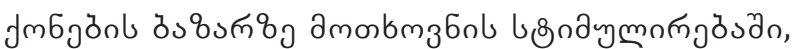

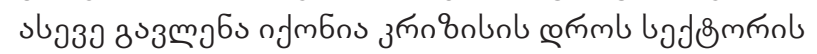

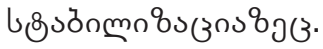

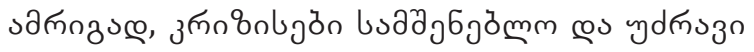

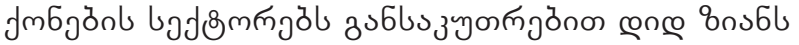

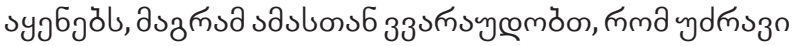

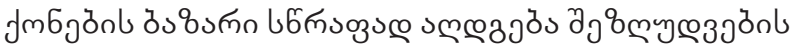

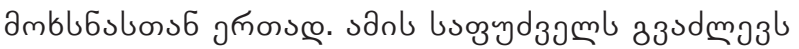

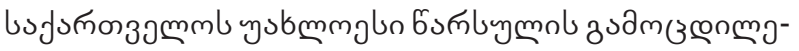

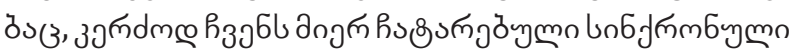
s6ımo

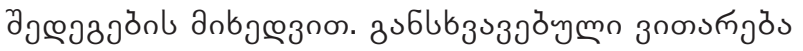

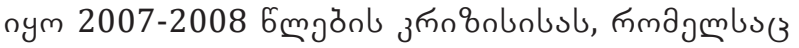

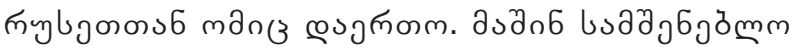

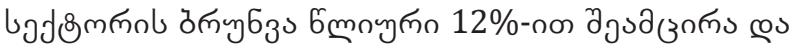

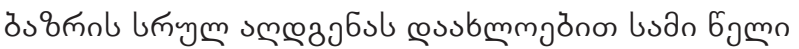

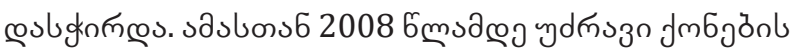

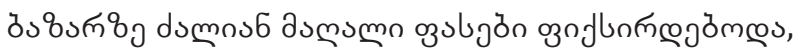

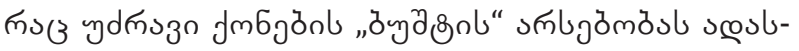

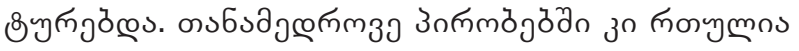

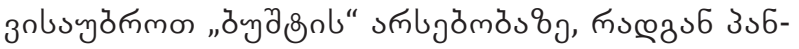

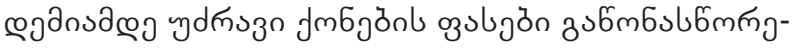

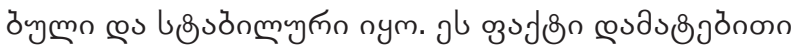

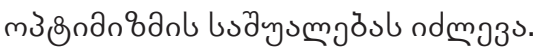

\section{œ১৬336১}

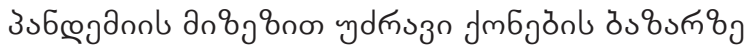

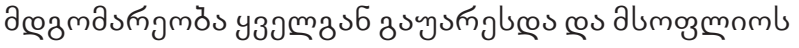

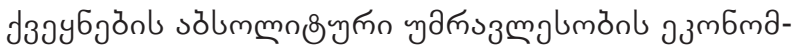

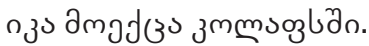

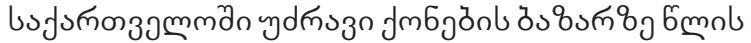

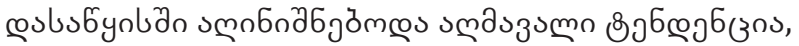

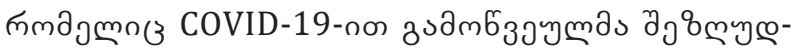

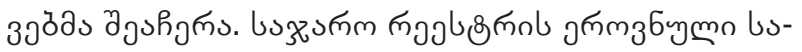

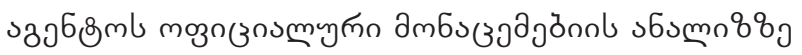

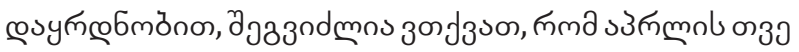
bubnsoœว

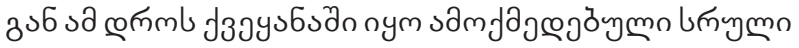

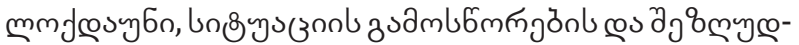

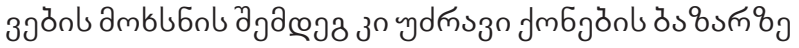

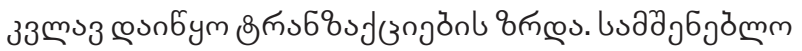

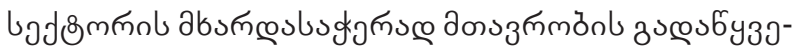

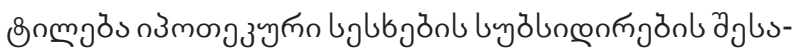

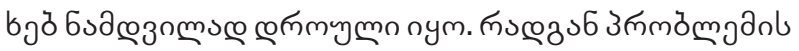

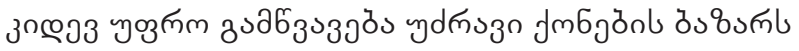

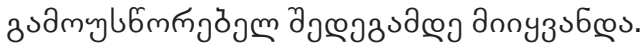

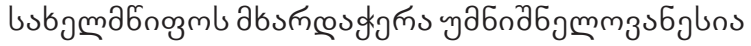

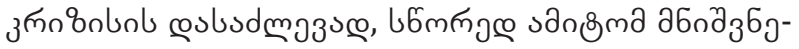

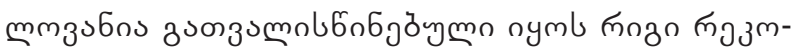

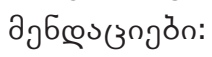

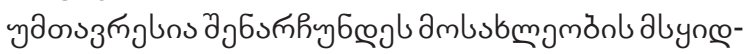

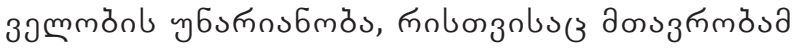

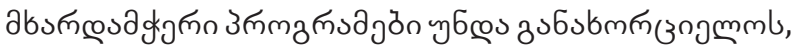

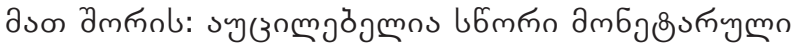

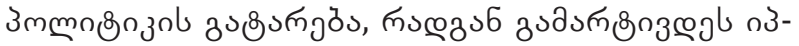

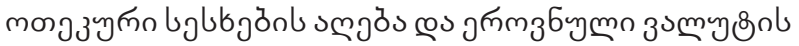

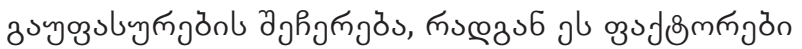

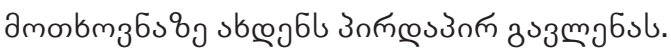

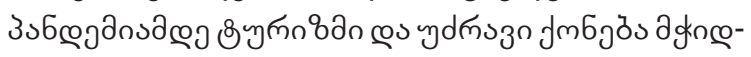

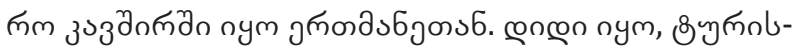

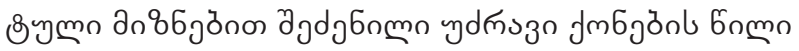

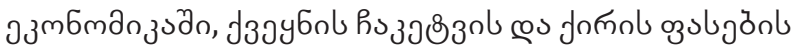

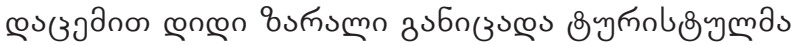

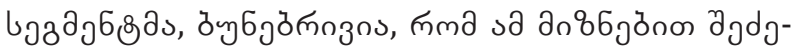

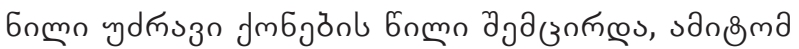

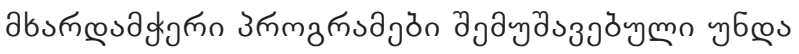

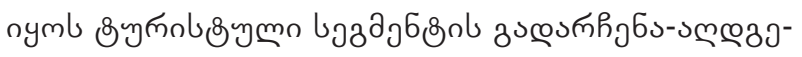
bol zymbnoss.

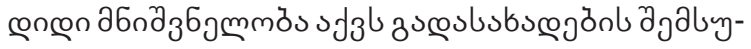

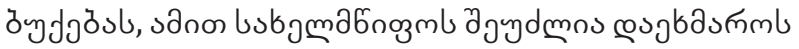

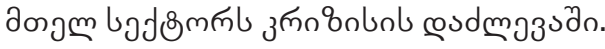

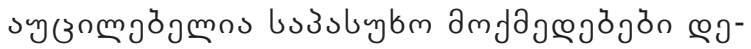

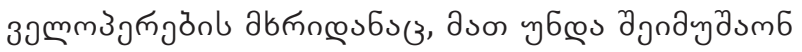

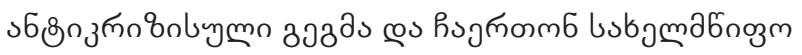

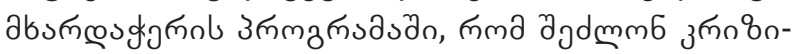

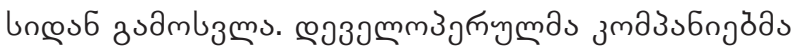

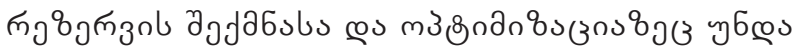

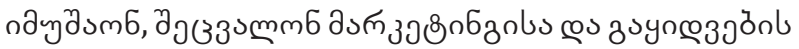

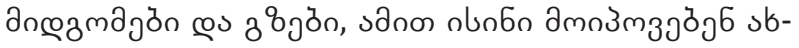




\section{ISSN 2667-9752(Online)}

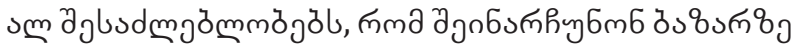

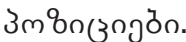

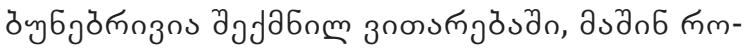

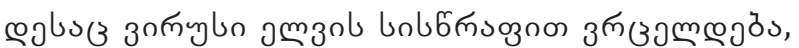

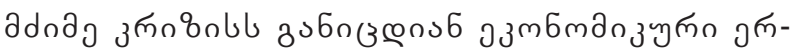

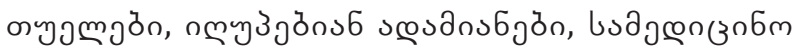

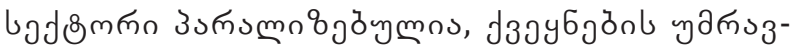

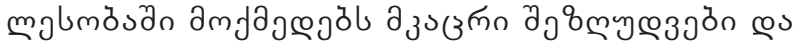

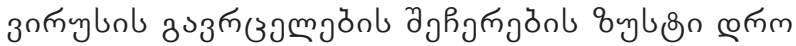

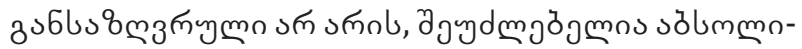

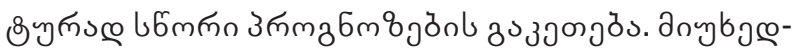

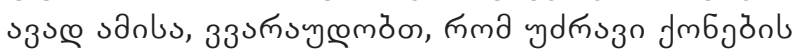

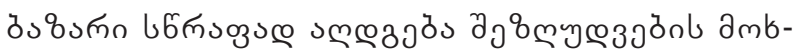

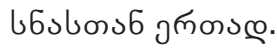

\section{REFERENCES:}

1. Bakradze G. (2014) “The Global Financial Crisis of 2007-2008: Causes and Interpretations”, Economics and Banking, Volume 2, N2;

2. Zurashvili N. (2018) Ways to Improve the Management Efficiency of Development Companies in Georgia, Dissertation, Tbilisi;

3. Ambrus, A., E. Field, R. Gonzalez, 2020, Loss in the time of cholera: Long-run impact of epidemic adisease on the urban landscape, American Economic Review, 110, 475-525.

4. Baris K. Yörük, 2020, Early effects of the COVID-19 pandemic on housing market in the United States, pp 10-11;

5. Currie J, Davi l., Greenstone M., Walker R., 2015, Environmental health risks and housing values: Evidence from 1,600 toxic plant openings and closings, American Economic Review, 105, 678-709.

6. Francke, M. and M. Korevaar, 2020, Housing markets in a pandemic: Evidence from historical outbreaks, Unpublished Manuscript. Available at: http://dx.doi.org/10.2139/ssrn.3566909.

7. Lucas W. Davis, 2004, The effect of health risk on housing values: Evidence from a cancer cluster, American Economic Review, 94, 1693-1704.

8. Wong G, 2008, Has SARS infected the property market? Evidence from Hong Kong, Journal of Urban Economics, 63, 74-95.

9. https://forbes.ge/blog/504/frTxilad-sabazro-ekonomika Last look. 11.02.2021

10. https://businessfeed.ge/udzraoba-udzravi-qonebis-bazarze-da-damaimedebeli-nishnebi/ Last view. 11.02.2021

11. https://www.prospect.ge/post/real-estate-during-covid-19-pandemic Last look. 11.02.2021

12. 11707.pdf (galtandtaggart.com) Last view. 11.02.2021

13. RECOV.GE - by Colliers: Residential (Tbilisi) Last view. 11.02.2021

14. https://www.prospect.ge/post/real-estate-during-covid-19-pandemic Last look. 11.02.2021

15. COVID-19-advice-to-GR_-Final-Kate. April 15 (propertygeorgia.ge) Last seen. 11.02.2021

16. NAPR - Property Registration Statistics Last View. 11.02.2021

17. http://www.parliament.ge/ge/ajax/downloadFile/135879/sruli Last viewed 15.12.2021

18. http://cushwake.ge/storage/files/doc/Snapshots\%20Q3_2020.pdf Last view. 11.02.2021

19. $\bullet$ https://bm.ge/ka/article/subsidirebis-dasrulebis-shemdeg-axali-ipotekebis-raodenoba-43-it-shemcirda--tbc-capital-/74453 Last view. 11.02.2021

20. https://stopcov.ge/ Last viewed 20.05.2021 


\title{
INFLUENCE OF BASIC ACTORS IN GLOBALIZATION IN DEVELOPING AND POST-SOVIET COUNTRIES ON THE EXAMPLE OF GEORGIA
}

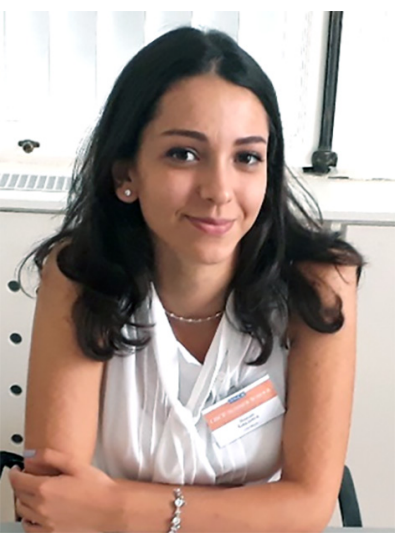

\author{
Mariam Galdava \\ Ph.D. Candidate in Political Science \\ Caucasus International University \\ E-mail: mar.galdava@gmail.com
}

DOI suffix: 10.36962/NEC6102202166

\begin{abstract}
In the 21st century, globalization is becoming more and more extensive as a phenomenon. The article discusses the popularization of globalization and its transformation as a world dilemma caused by new world rules.

The aim of our research is to determine what kind of impact globalization has had, because judging by the example of our country, the country has undergone a transformation as well as political, economic, cultural, and social factors.

One of the main integration processes is the goal of bringing individual groups into a single social and cultural framework of self-identification, which has become irreversible and poses a major problem for the identity of countries, as well as economic, social, political stability and security of the population.

In this article, we use the following research methods: Qualitative research methods, which include a qualitative study of written documents, focus groups, monographic research, etc. A specific case study, on the example of Georgia, the same Case study method that helped us to better study and analyze the facts. The processes of globalization have somewhat reduced the sovereignty of states. Modern states are powerless to control or deal with globalization, to reduce or limit its scope.

However, this did not lead to the destruction of the states. By the way, some states have become more powerful. This is especially noticeable in the example of post-Soviet countries, one of the highlights of which is Georgia. Globalization, like all events, has its pros and cons, but the most worthy positive side of globalization is that it has created and established independent and sovereign states that are becoming stronger day by day in the scientific, social, cultural, political, and economic spheres.
\end{abstract}

Keywords: Independence, Georgia, Globalization, Post-Soviet Country, State.

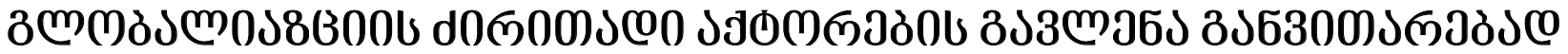

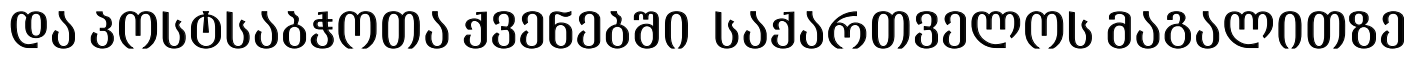

\author{
asmosa zumøuss

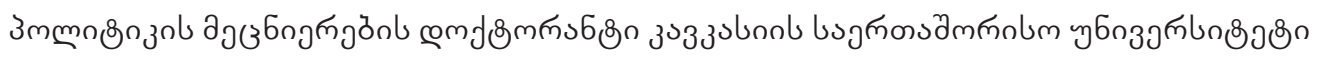 \\ jm. oुmbఠs: mar.galdava@gmail.com \\ ১EMBSßOS
}

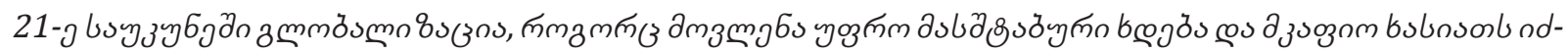

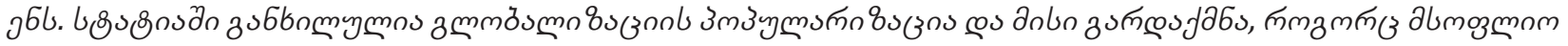
œом

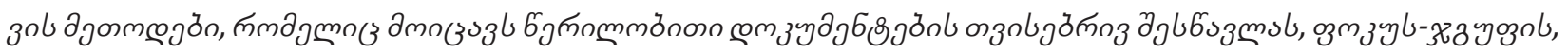

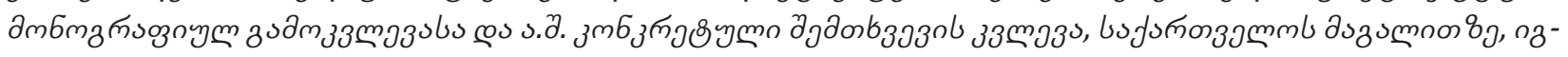




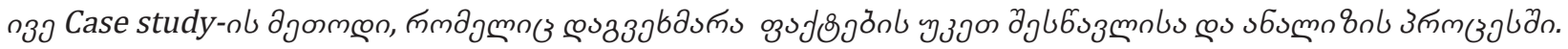

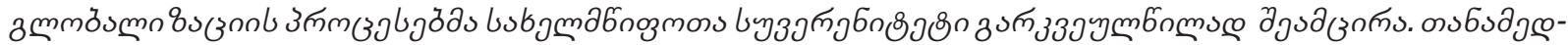

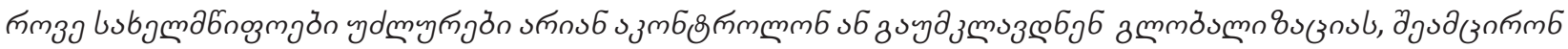

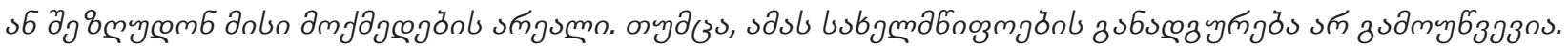

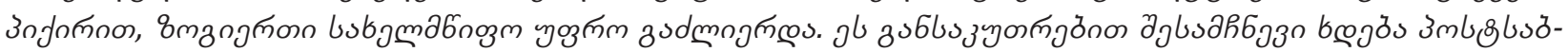

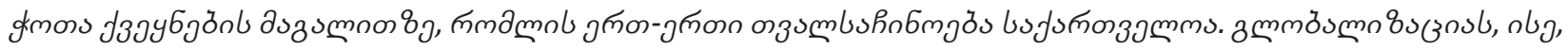

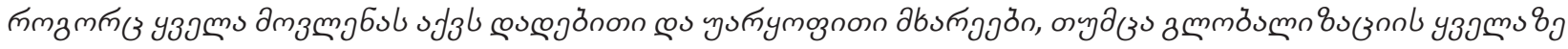

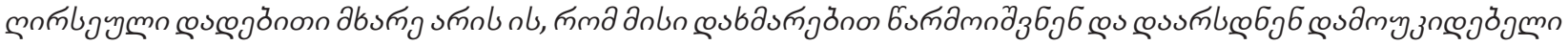

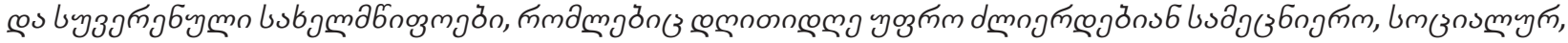

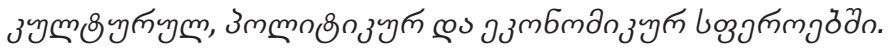

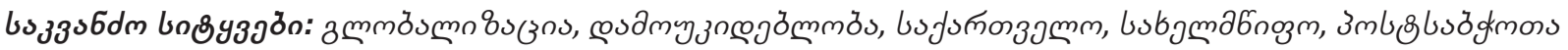
J39yง6s.

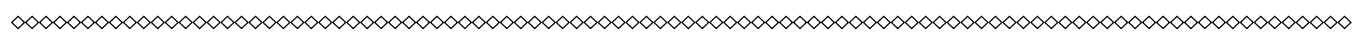

\section{วอเ১З১m0}

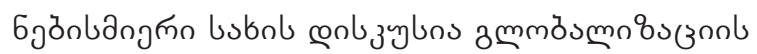

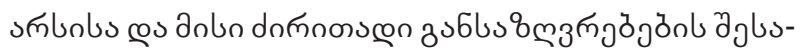

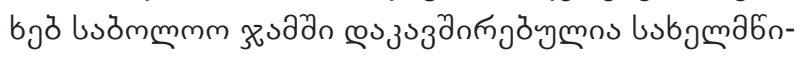

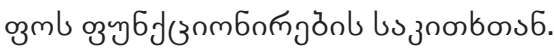

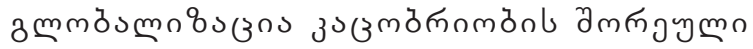

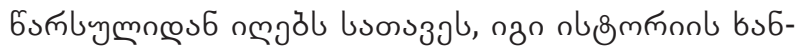

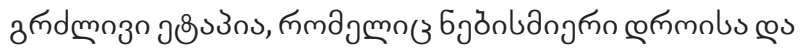

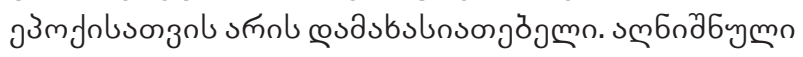

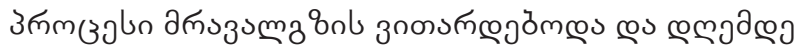

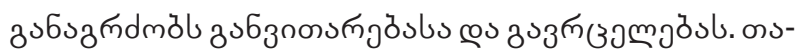

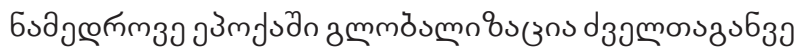

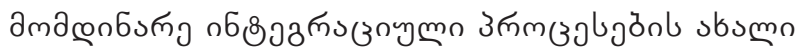

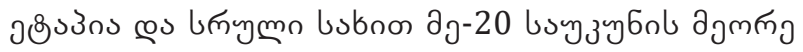
бงbวзง

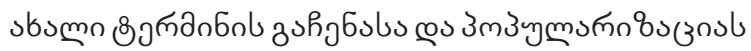

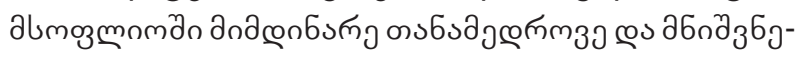

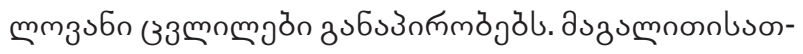

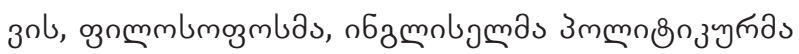

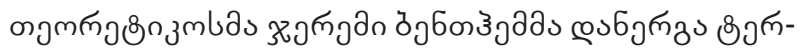

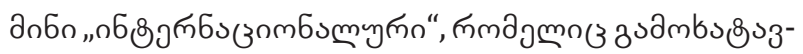

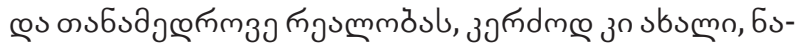

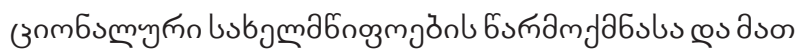

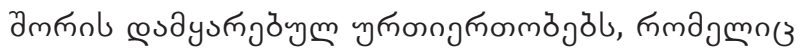

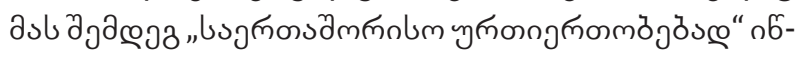

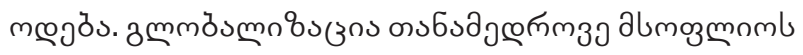

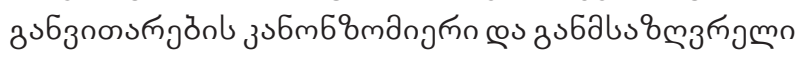

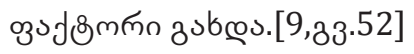

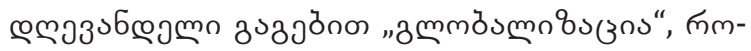
змпु

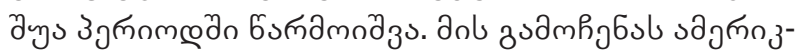

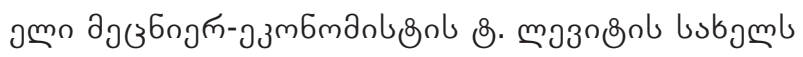

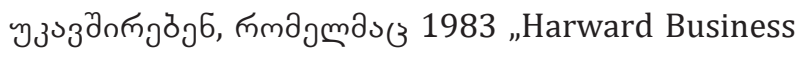

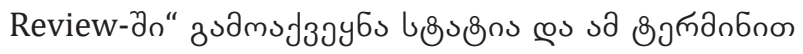

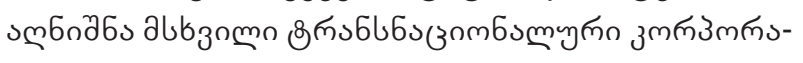

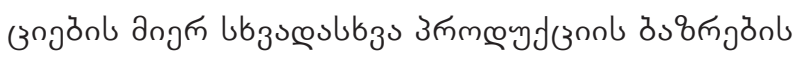

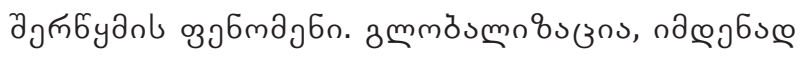

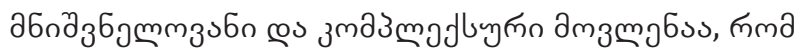

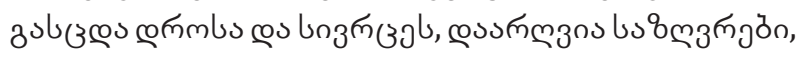

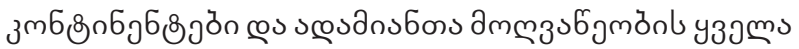

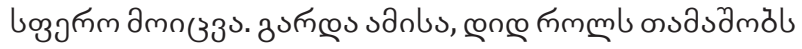

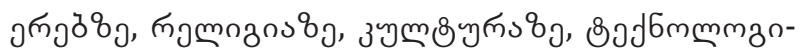

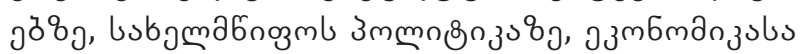

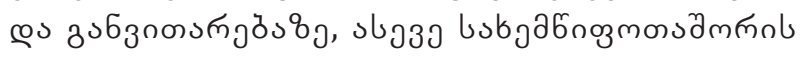

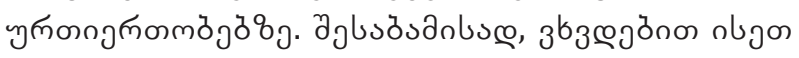

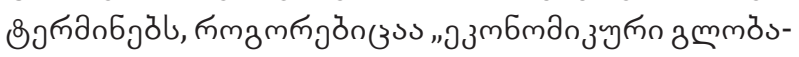

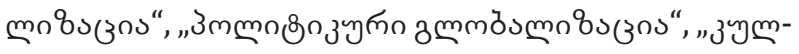

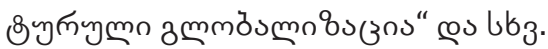

h

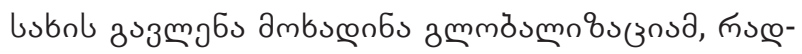

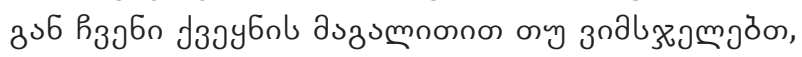

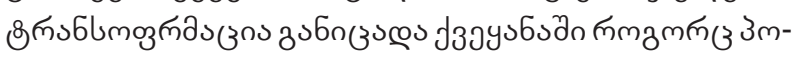

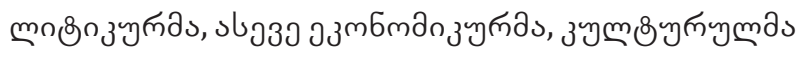

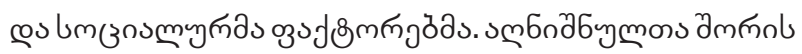

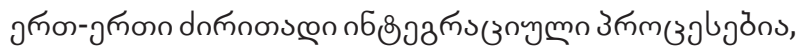
пmдmn

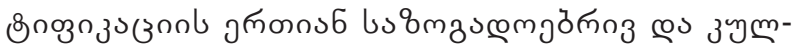

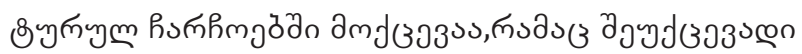

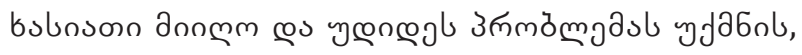

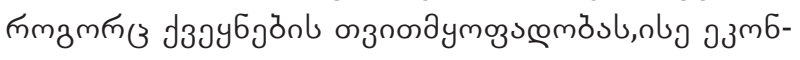

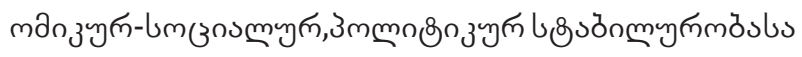

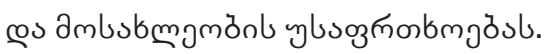

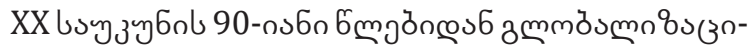

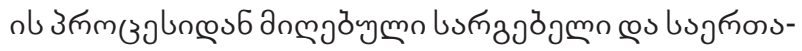

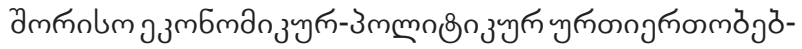

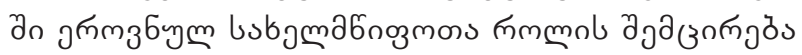

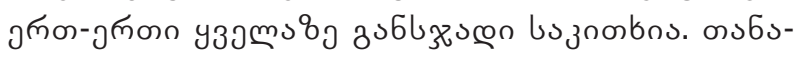

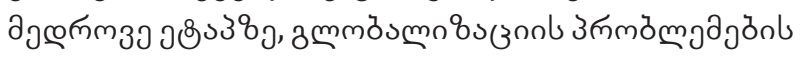

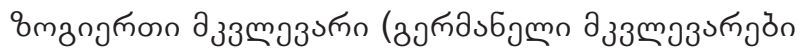

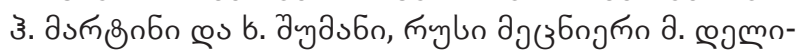




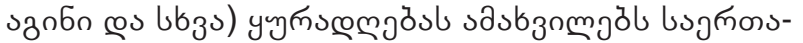

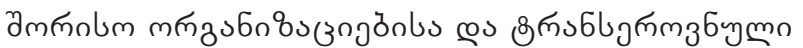

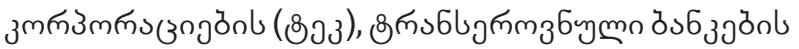

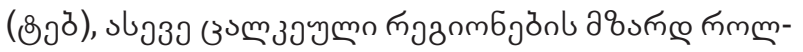

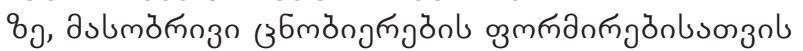

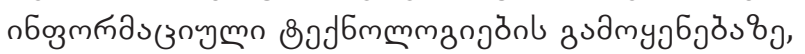

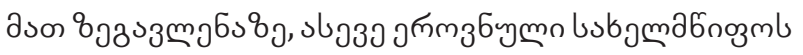

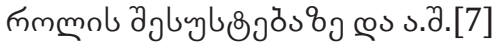

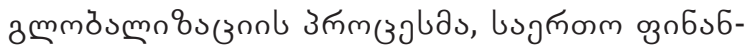

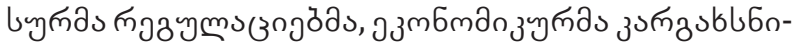

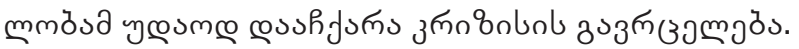
sanb 6soлm

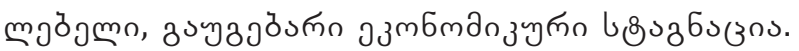
“almozmnm azmbmanzol zmmöumnosuzonl zum-

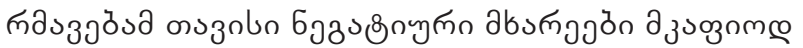

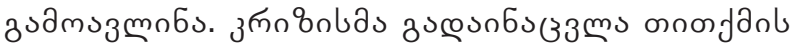

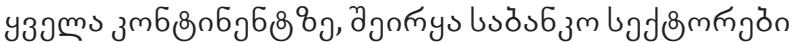

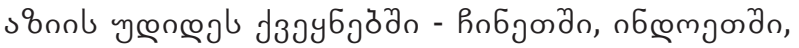

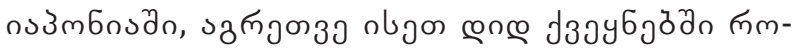

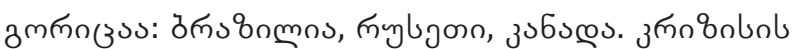

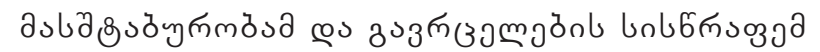

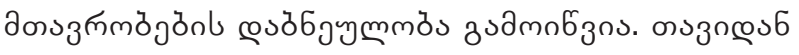

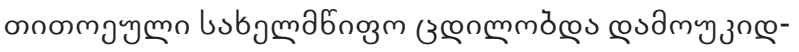

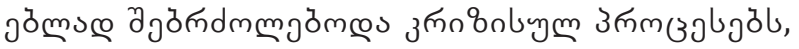

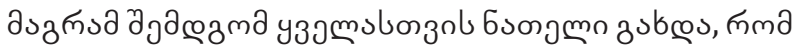

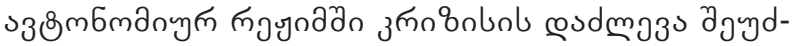

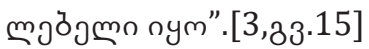

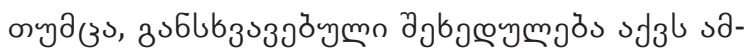

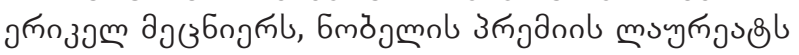

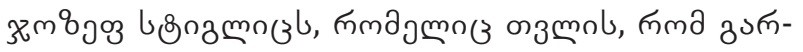

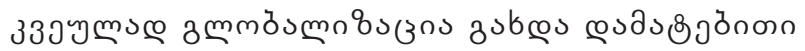

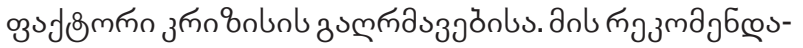

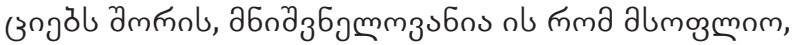

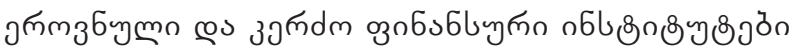

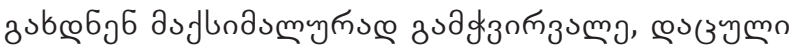

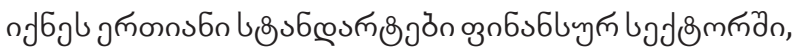

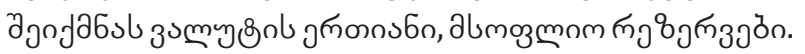

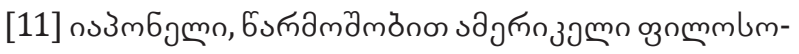

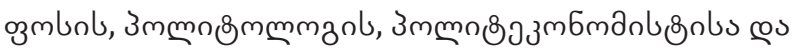

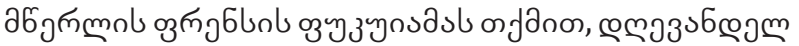

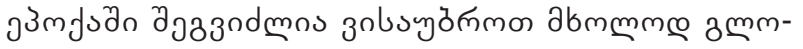

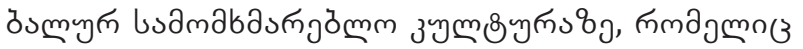

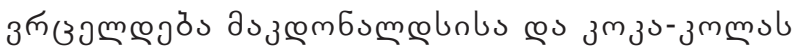

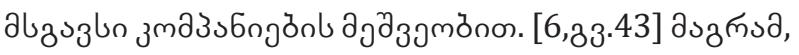

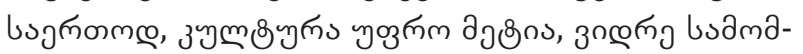

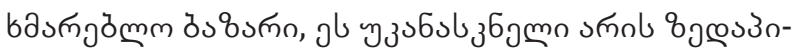

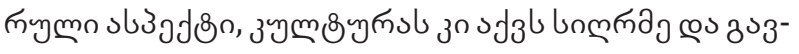

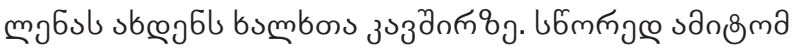

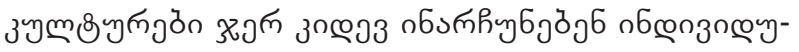

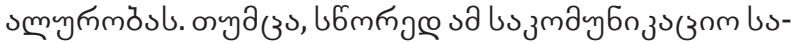

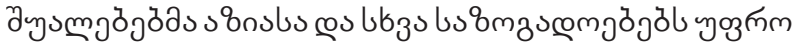

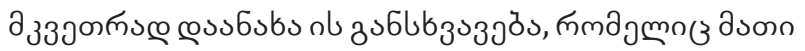

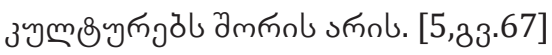

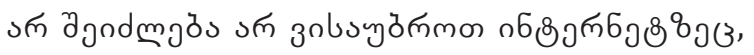

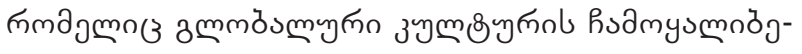

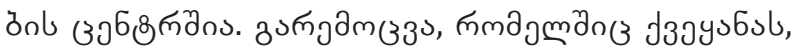

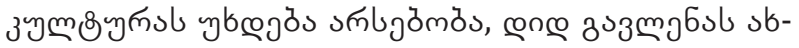

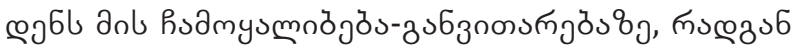

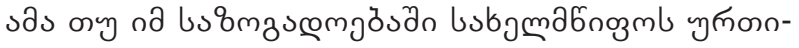

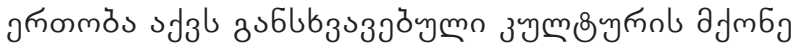

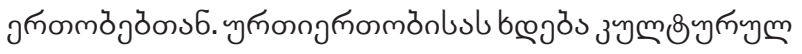

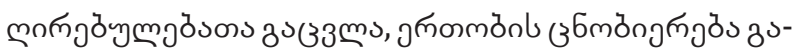

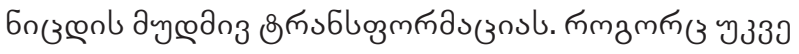

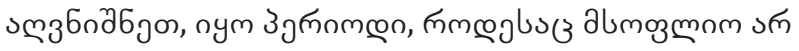

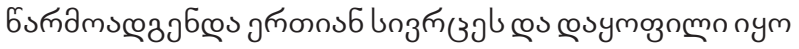

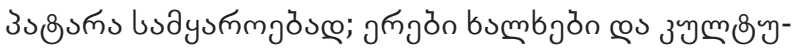

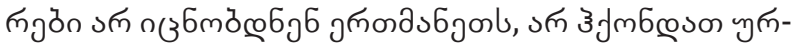

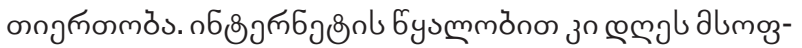

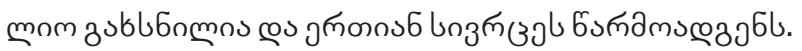

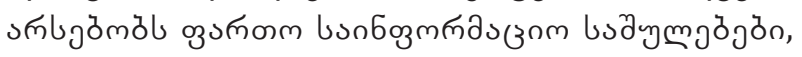

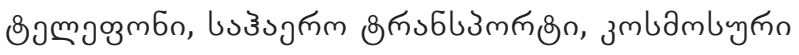

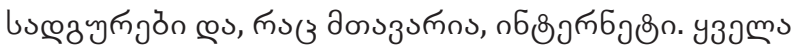

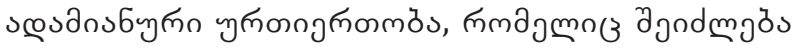

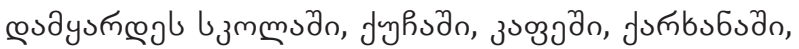

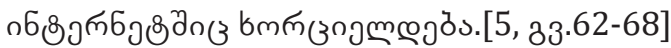

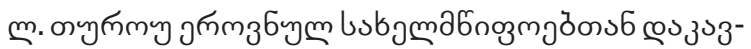

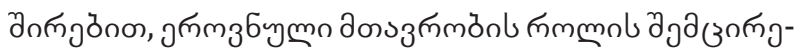

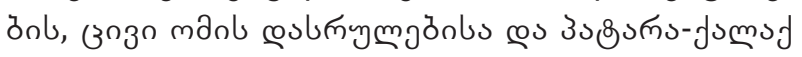

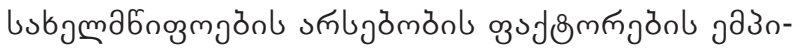

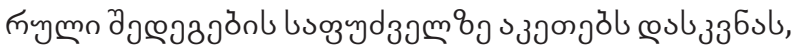

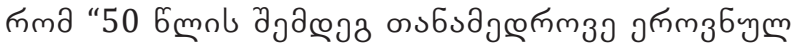

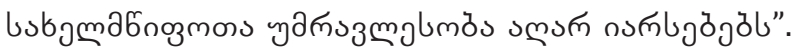

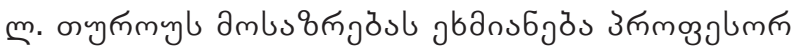

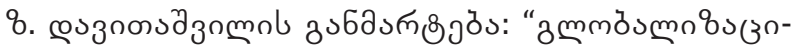

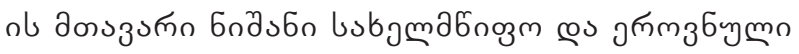
৬s

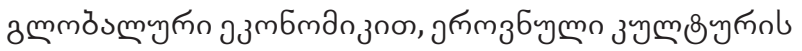

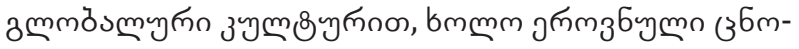

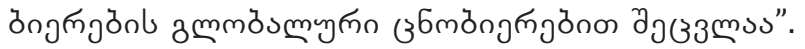

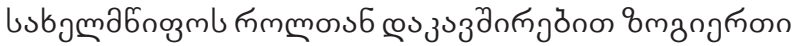

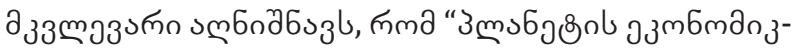

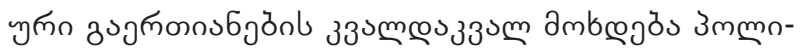

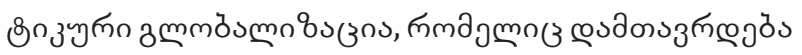
aumozmom doş

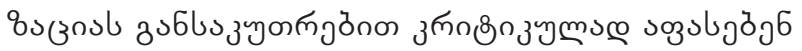

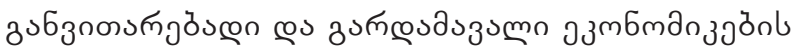

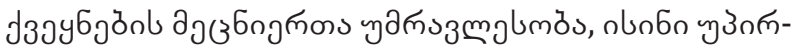

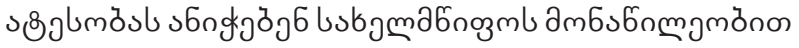




\section{ISSN 2667-9752(Online)}

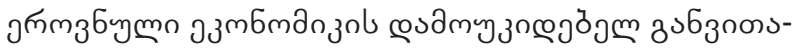

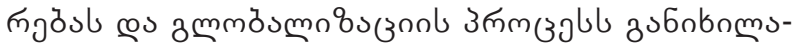

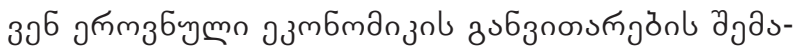

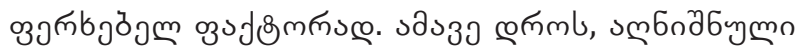
ذзауб

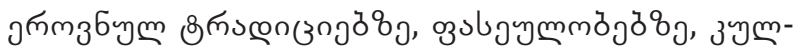

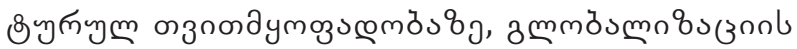

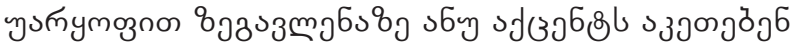

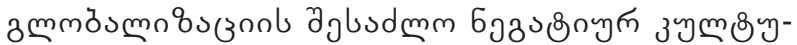

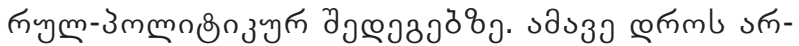

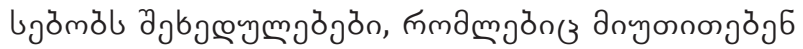

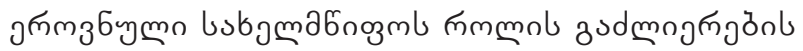

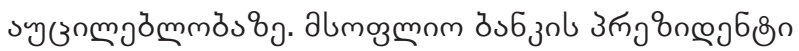

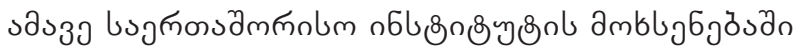

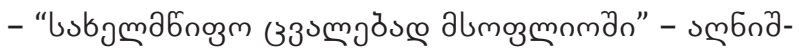

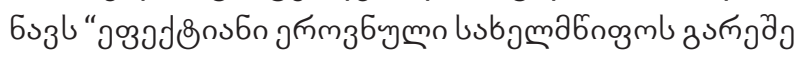

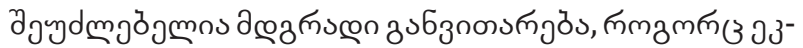

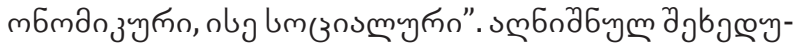

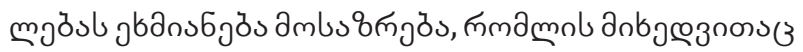

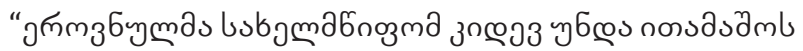

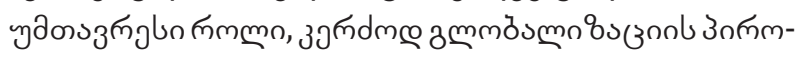

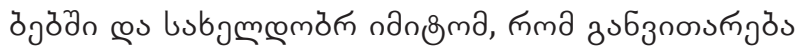

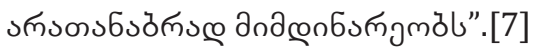

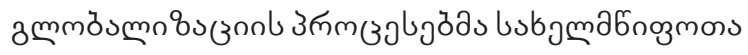

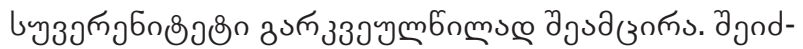

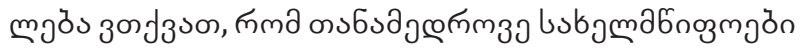

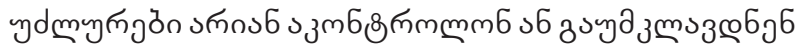

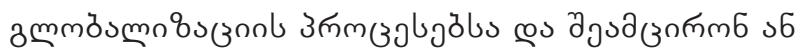

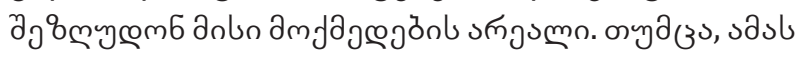

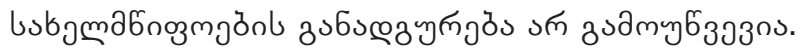

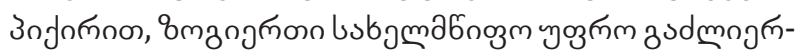

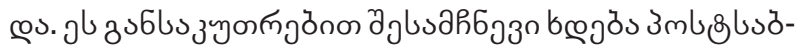

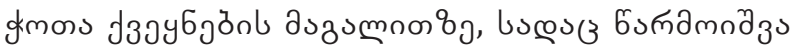

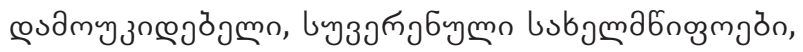

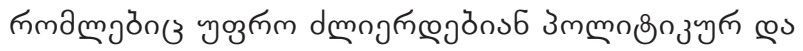

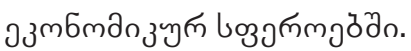

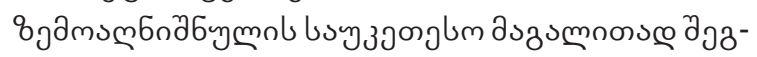

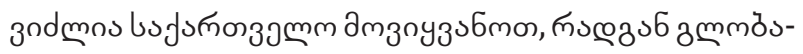

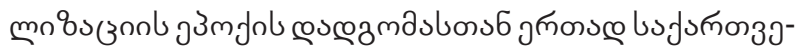

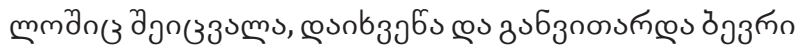
n

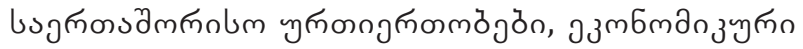

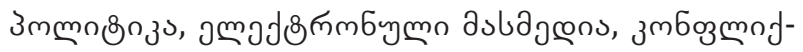

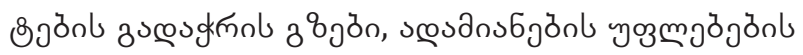

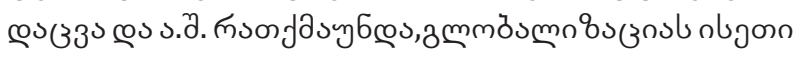
उஙm зз пmaว

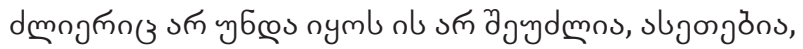

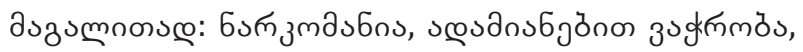

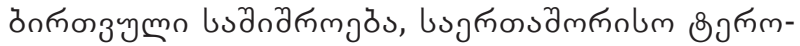

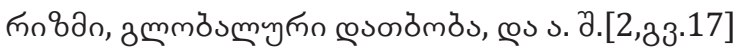

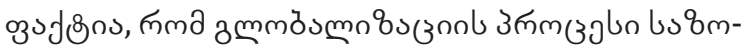

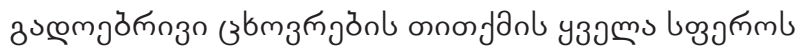

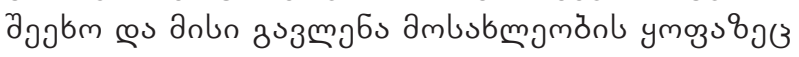

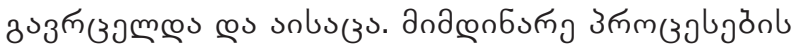

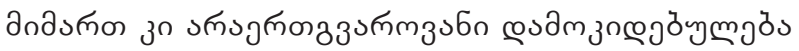

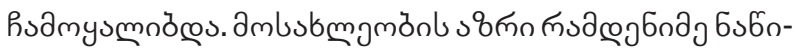

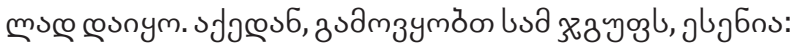

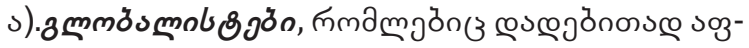

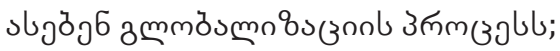

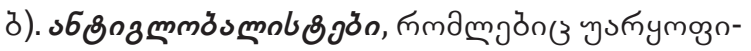

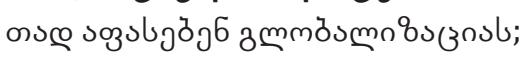

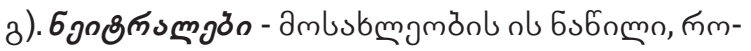

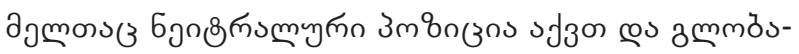

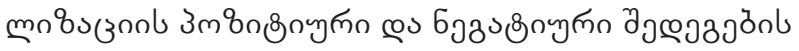

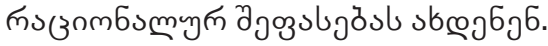

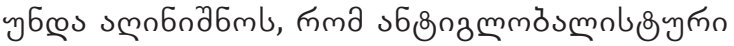

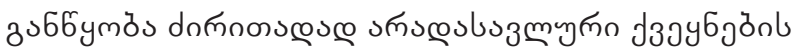

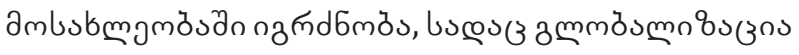

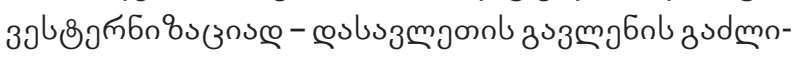

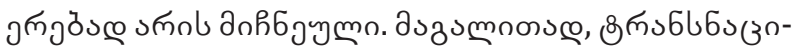

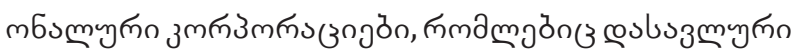

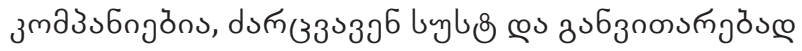

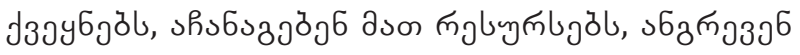

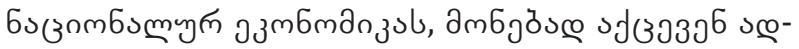

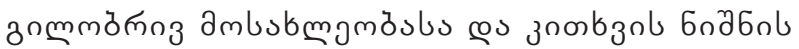

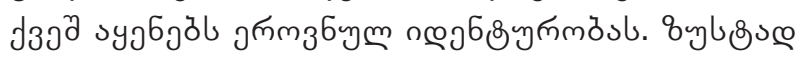

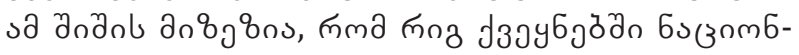

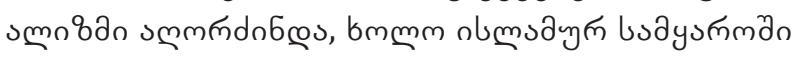

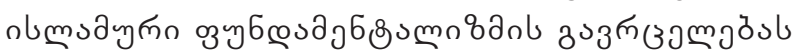

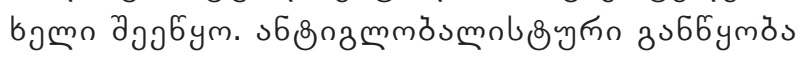

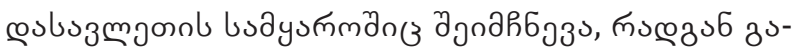

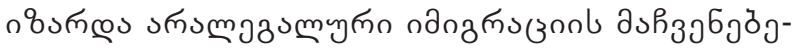

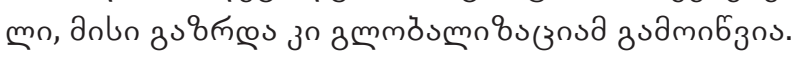

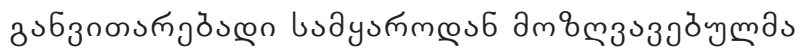

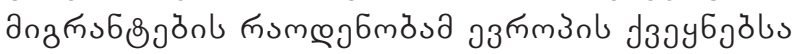

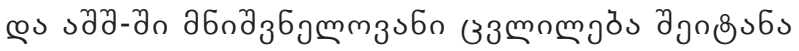

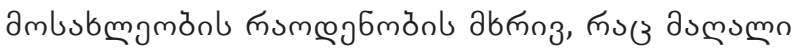

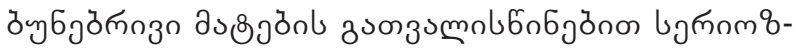

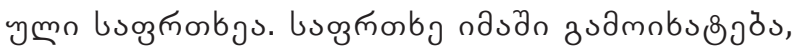

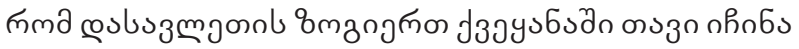

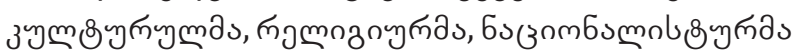

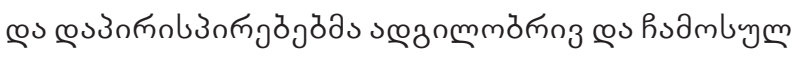

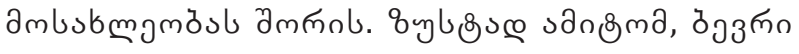

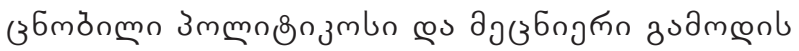

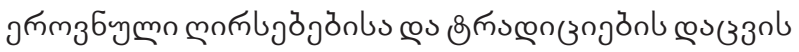
amorkmz 6 non. [1, 33.25]

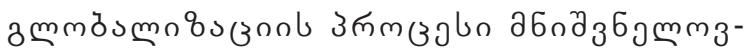




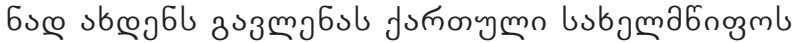

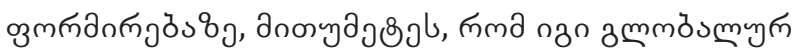

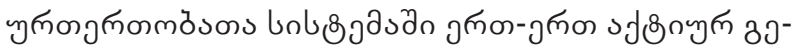

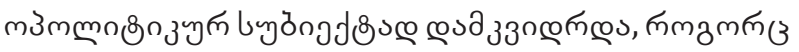

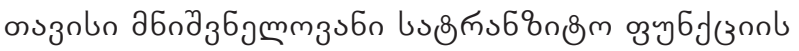

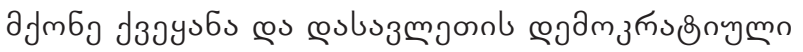

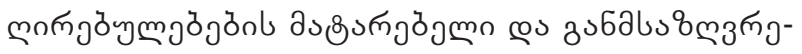

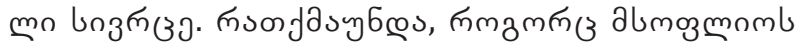

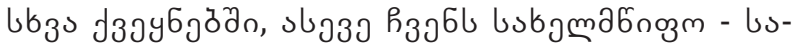

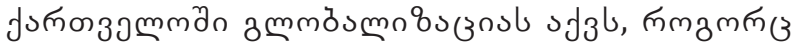

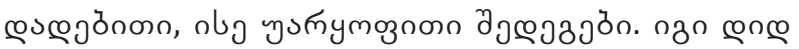

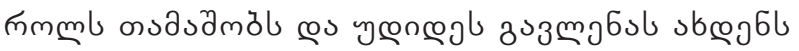

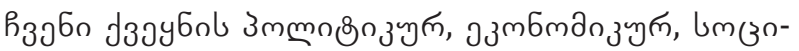

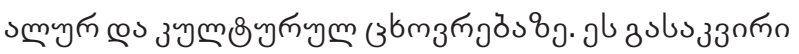

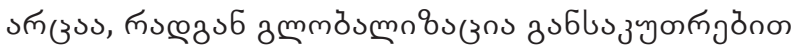

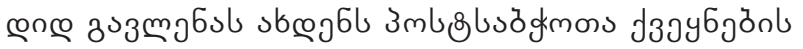

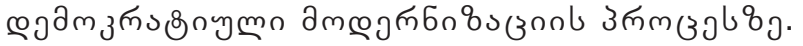

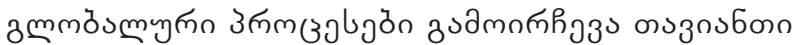

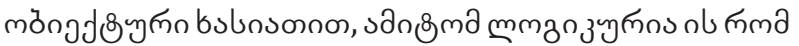

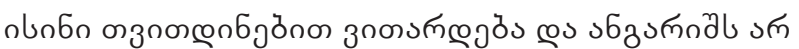

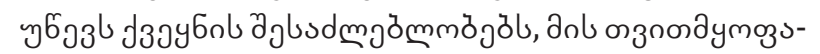

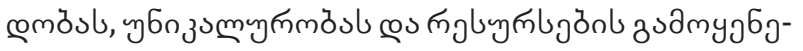

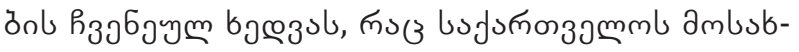

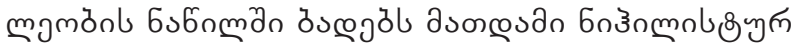

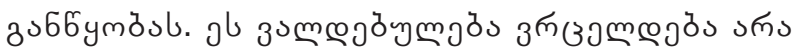

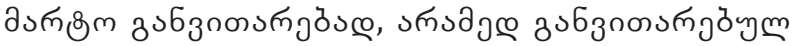

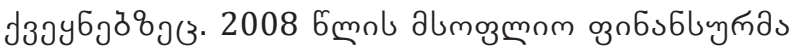

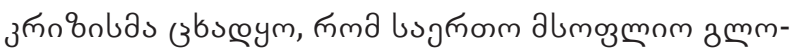

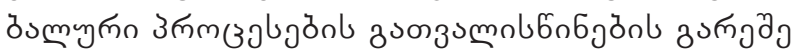

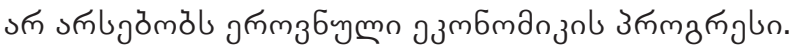

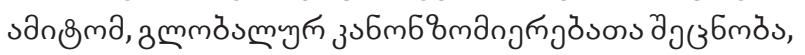

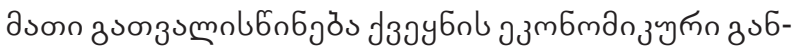

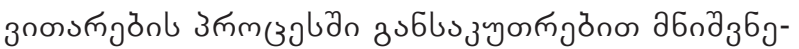

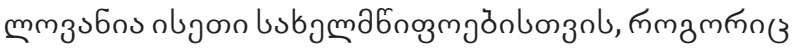

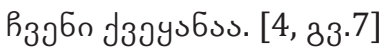

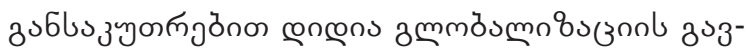

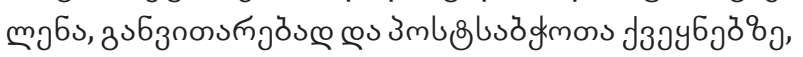

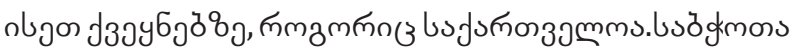

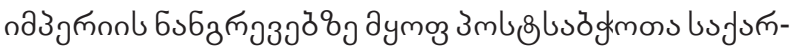

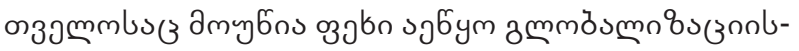

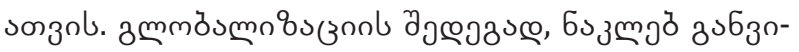

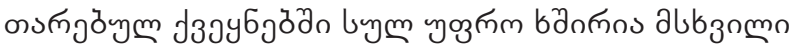

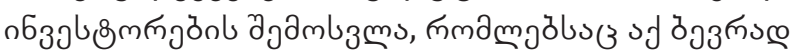

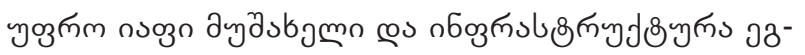

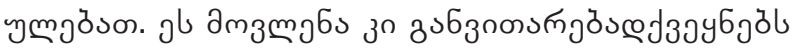

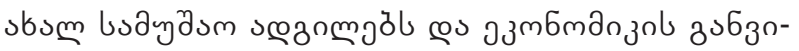

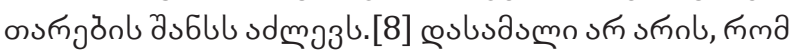

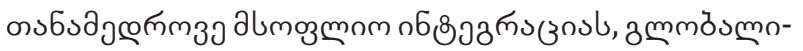

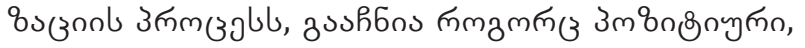

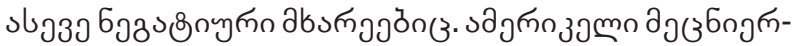

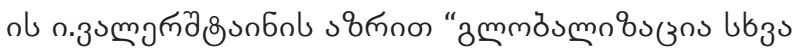

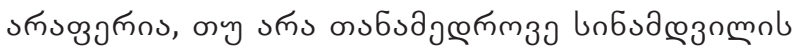

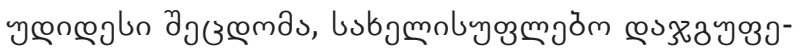

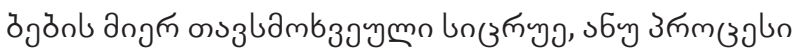

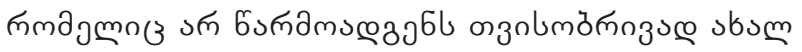

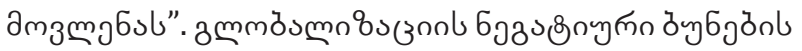

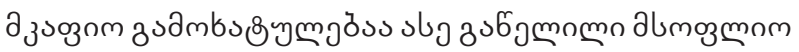

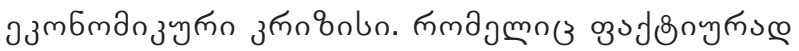

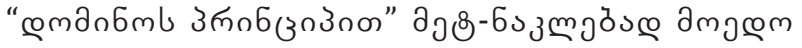

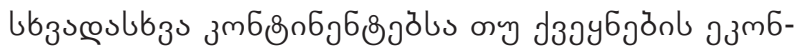

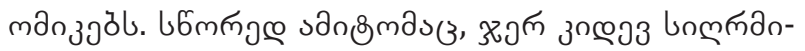

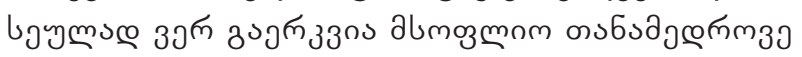

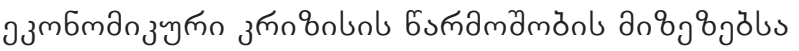

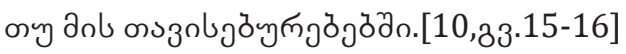

\section{உ১৬336১}

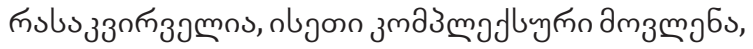

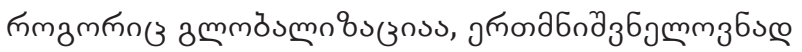

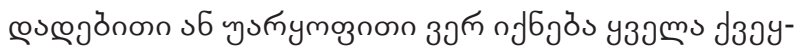
Enbonzol oy s@osans6nborzol.

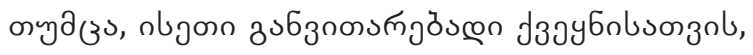

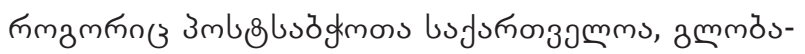

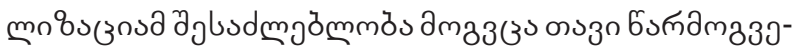

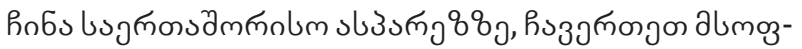

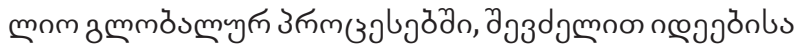

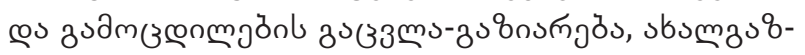

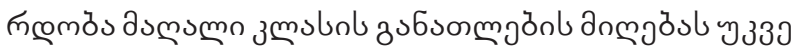

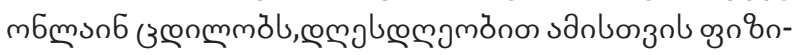

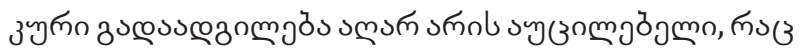

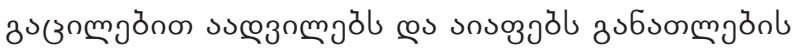
ة

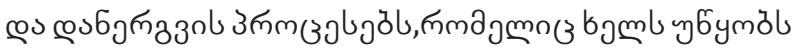

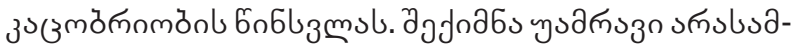

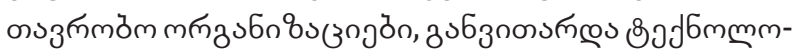

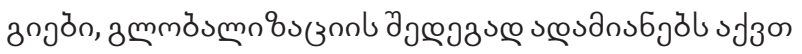

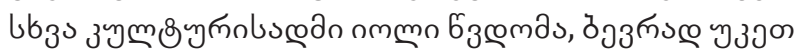

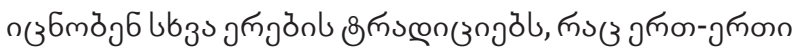

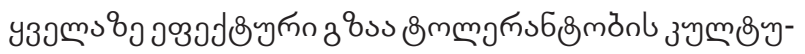
mol zubznonsm jönbonzol.

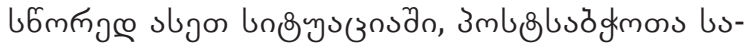

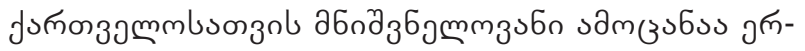

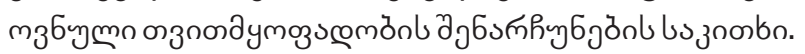

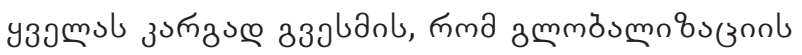

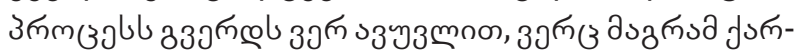

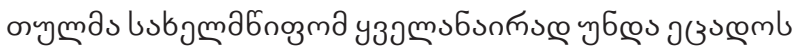

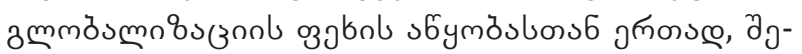

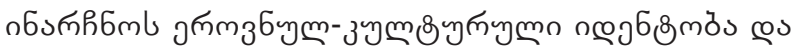

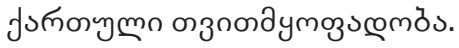




\section{REFERENCES:}

1.Abesadze G.,Abesadze T., “Globalization and Peculiarities of Georgia’s Transit Function”, Tbilisi, 2011 https:// www.tsu.ge/data/file_db/economist_faculty/global.pdf p.682 - last checked on 23.06. 2021

2. Lobzhanidze D., Lobzhanidze G., "World Order and International Legal Systems", "Mandaria Printing House" Kutaisi, 2007 p.336

3. Meskhia I., "Global Crisis and the Georgian Economy”, Journal of Social Economy №2. Tbilisi, 2009

4. Shengelia T., Globalization, Current Problems and Development Trends in International Business, "Universal”, Tbilisi, 2012 - https://www.tsu.ge/data/file_db/economist_faculty/Konfi.pdf, p.558 - last Checked -23.06.2021

5. Tsereteli I., Kakitelashvili St., "Culture and Modernization”, Center for Social Sciences, 2006 p.119

6. "Globalization, modern problems of international business and development trends", "Universal”, Tbilisi, 2012, https://www.tsu.ge/data/file_db/economist_faculty/Konfi.pdf - last checked on 23.06 .2021 p. 558

7. Eteria E., "Globalization and the Nation-State: Theoretical Aspects", https://geoeconomics.ge/?p=3674Last checked -23.06.2021

8. Civic education for all, "Globalization" https://www.civiceducation.ge/ka/lessons/3-30 - last checked 23.06.2021;

9. Lewitt T., Globalization:Knowledge and Society. London. "Sage”, 1990 p.280;

10. 10. Wahlerstein I. Globalization or transition period. Economic strategies 2000, No. 2, M.2000;

11. Stiglitz D., "The Market Failed," https://neftegaz.ru/news/finance/334094-dzhozef-stiglits-rynok-nespravilsya/- Last checked 23.06.2021. 


\title{
THE GEOPOLITICAL DIMENSION OF THE CONFLICTS IN ABKHAZIA AND THE TSKHINVALI REGION
}

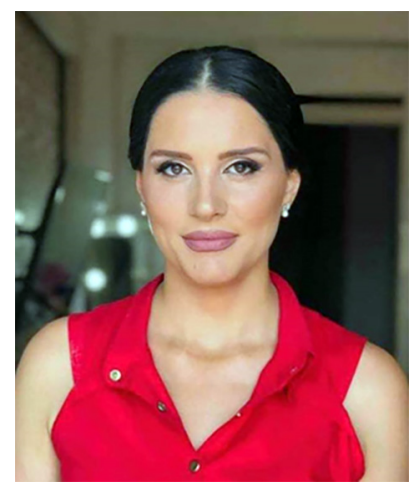

Gvantsa Chkheidze

$\mathrm{PhD}$ student of Doctoral Educational

Programme in Political Science at Caucasus

International University, Coordinator

of the Faculty of Social Sciences

at Caucasus International University

e-mail: gvantsa.chkheidze@ciu.edu.ge

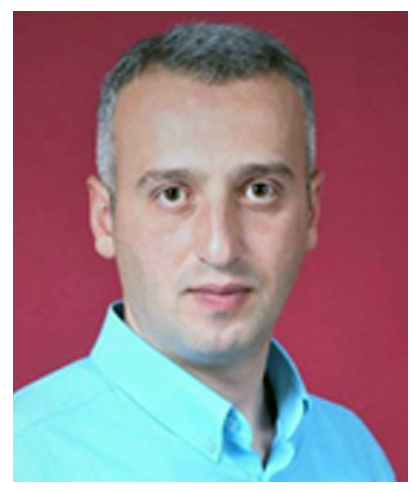

Irakli Mekvevrishvili

PhD student of Doctoral Educational Programme in Political Science at Caucasus

International University,

Director - LEPL Alexander Tsintsadze

Tbilisi Public School

e-mail: irakli.mekvevrishvili@ciu.edu.ge

DOI suffix: 10.36962/NEC6102202172

\begin{abstract}
Conflicts remain an unresolved problem in the modern world, which is particularly difficult for such a small and developing country like Georgia. The collapse of the Union of Soviet Socialist Republics and the emergence of new sovereign states led not only to an adjustment of a world map but also global geostrategic changes. As a consequence, new centers of power appeared, operating through a variety of strategies to obtain a dominant position in the post-bipolar world. The South Caucasus region turned out to be especially "attractive" in this regard. Two of the conflicts in the abovementioned area arose directly in the territory of Georgia, and these conflicts remain unresolved. Research, analysis and search for settlement mechanisms of the conflicts in Abkhazia and Tskhinvali region are the most important challenges for Georgia, therefore, the purpose of our article is to present the impact of Russia's soft power in the existing conflicts and possible mechanisms for resolving the problem.
\end{abstract}

Keywords: Geopolitics, Abkhazia, Tskhinvali, Conflicts. 


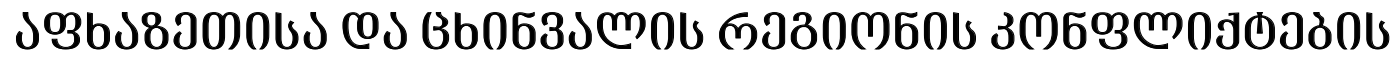

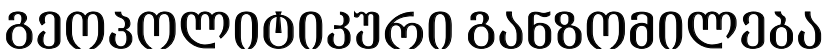

\section{$330 \mathrm{c} 30 \mathrm{hbgodg}$}

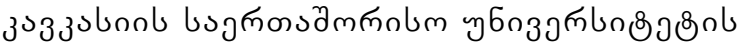
उmmnв̊zol

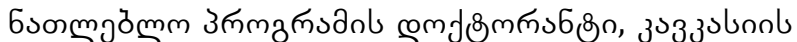

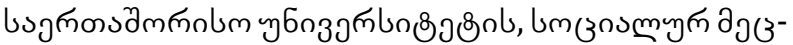

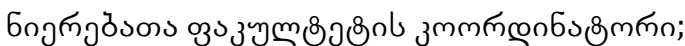

\section{окозмо дәјзәз подзомо}

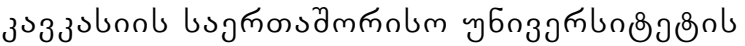

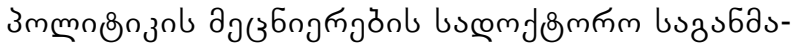

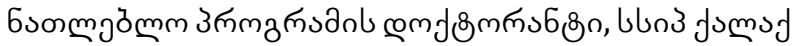

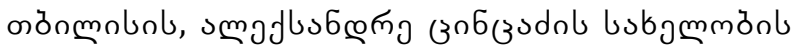

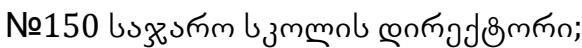

\section{EM\&SBOS}

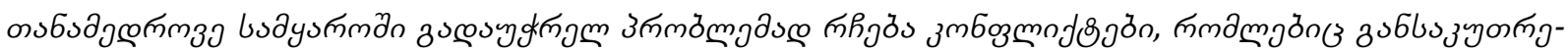

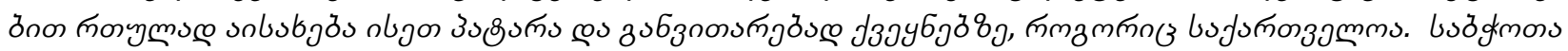

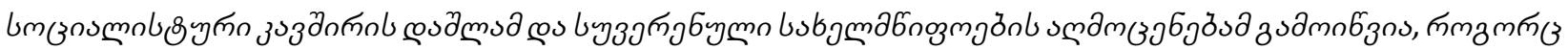

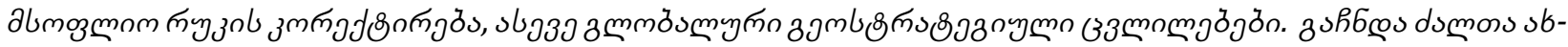

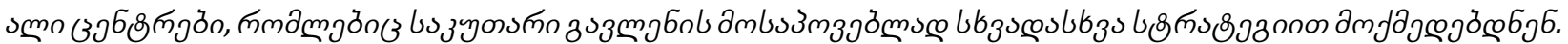

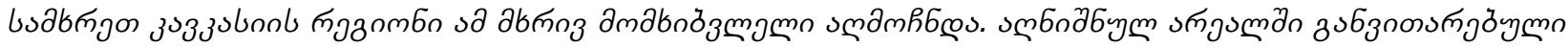

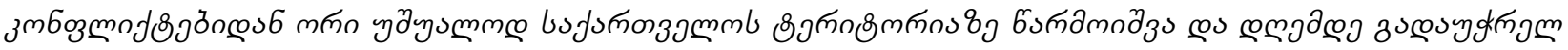

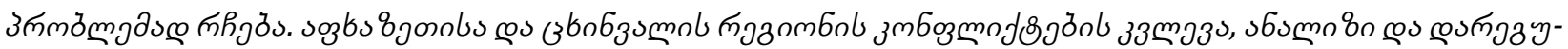

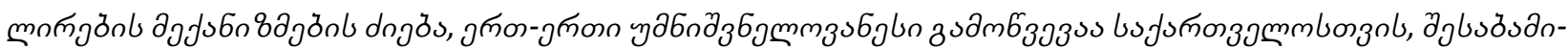

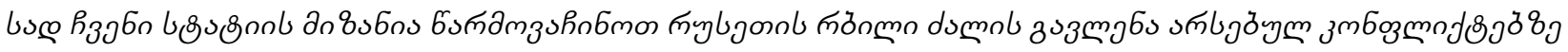

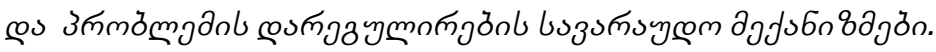

lı ззง6dm lов

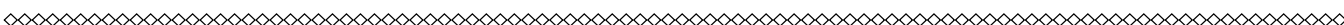

\section{วอ৬১૩১m 0}

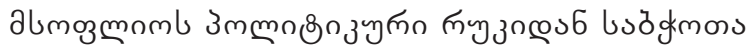

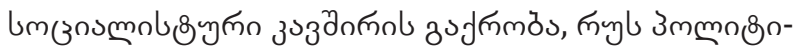

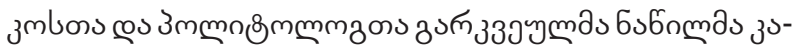

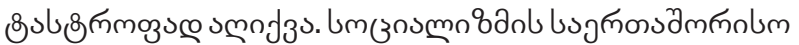

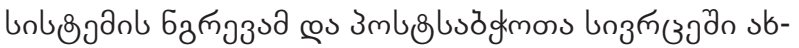

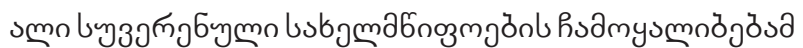

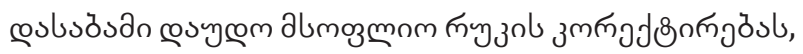

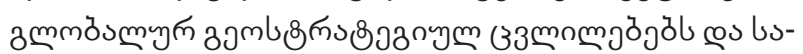

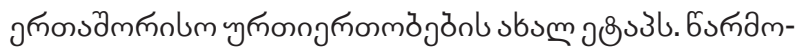

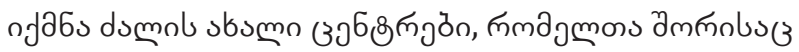

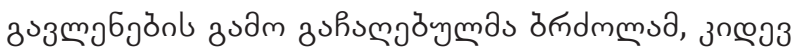

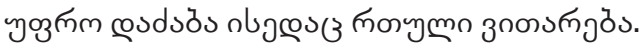

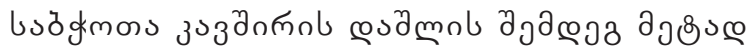

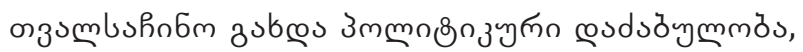

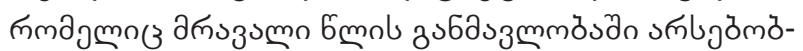

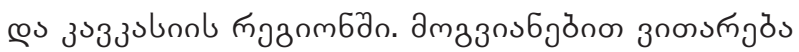

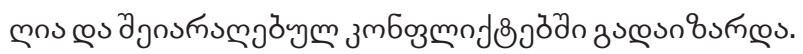

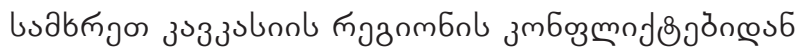

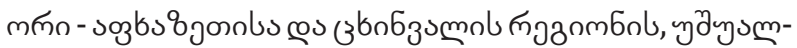

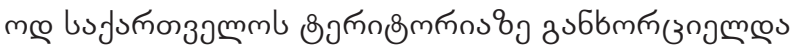

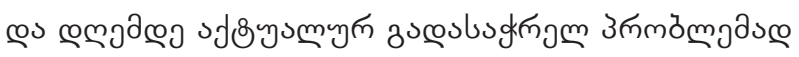

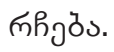

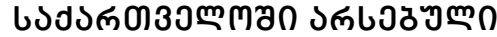

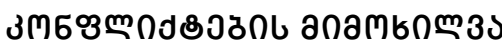

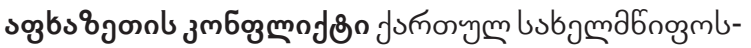

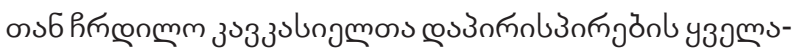

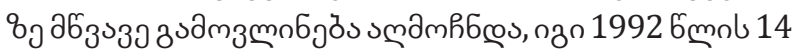
s उ3

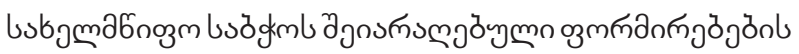

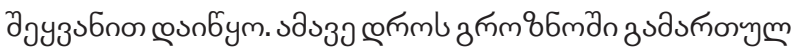

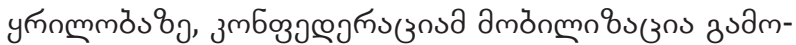

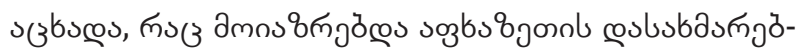

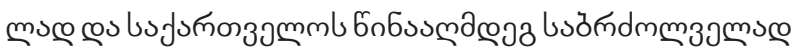

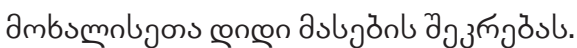

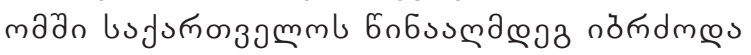

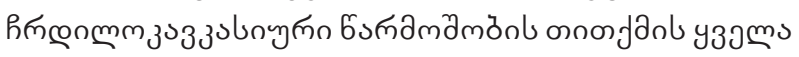

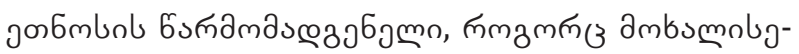
jön ח

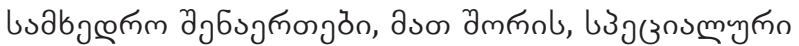

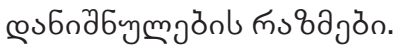




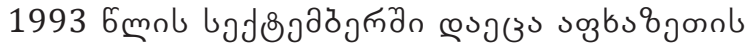

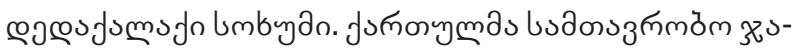

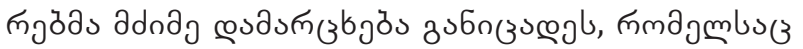

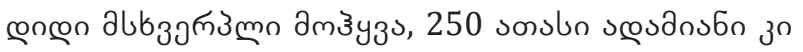

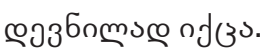

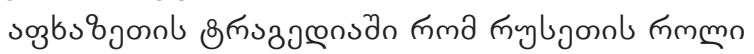

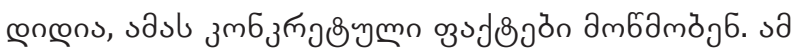

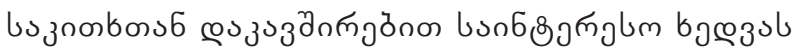

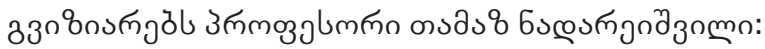

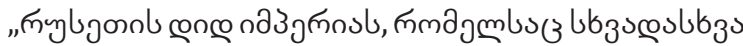

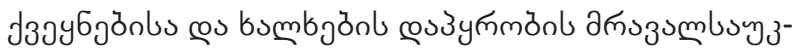
эб

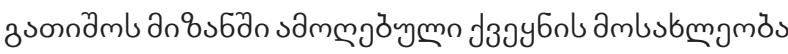

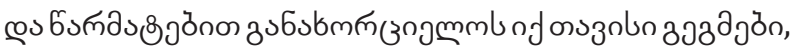
sm 3 h

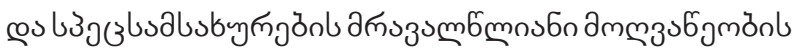

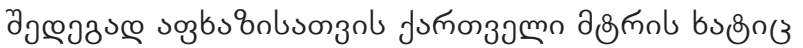

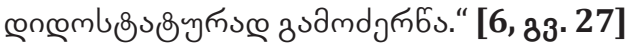

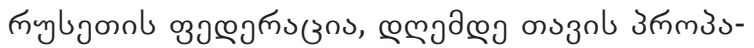

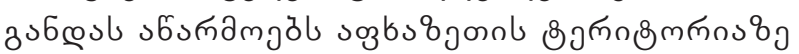
aßkmз

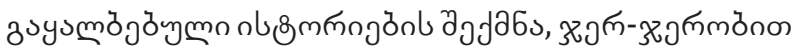

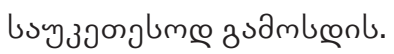

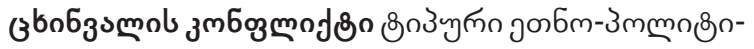

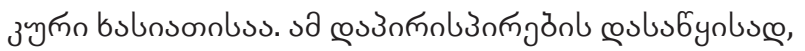

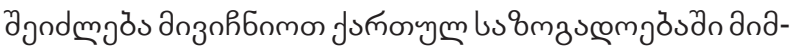

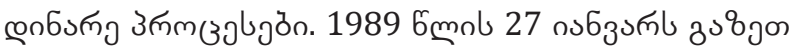

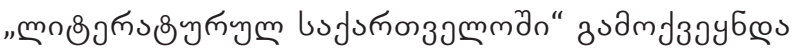

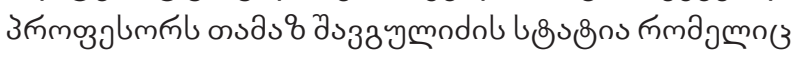

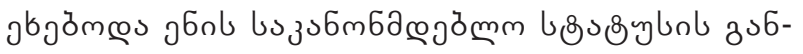
Luqnз mbjonol s s 8 \&mbmany

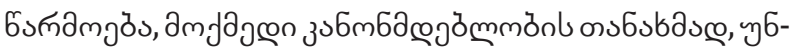

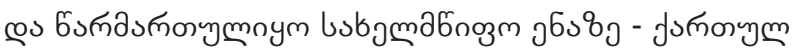

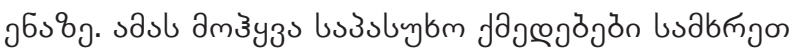

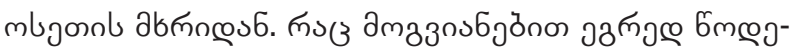

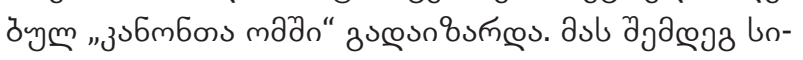

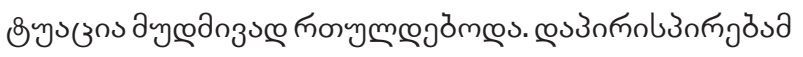

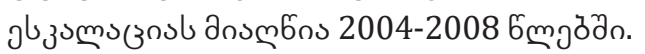

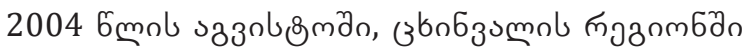

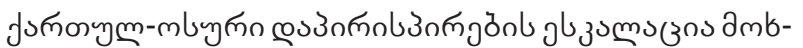

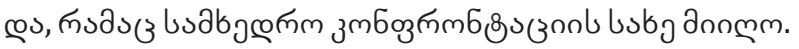

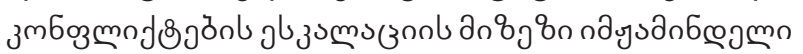
doss

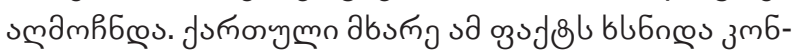

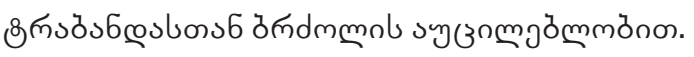

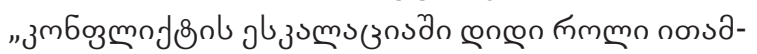

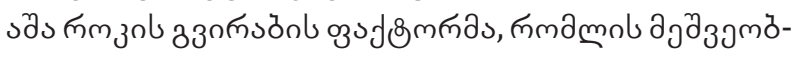

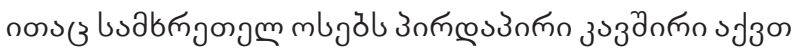

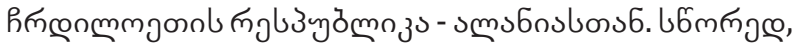

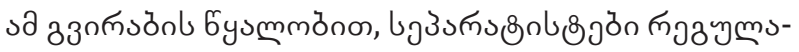

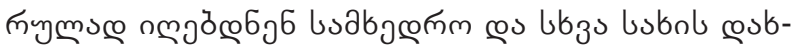

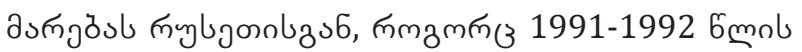

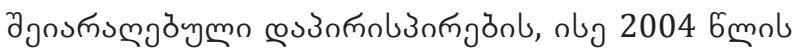

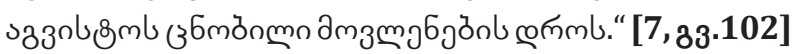

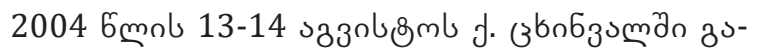

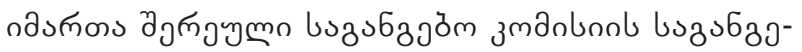

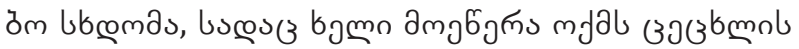

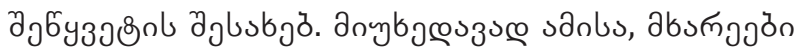

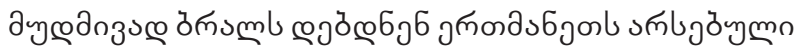

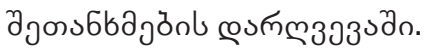

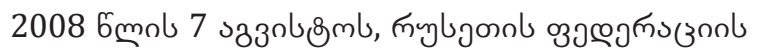

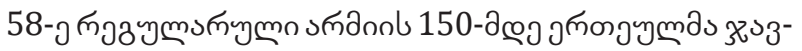

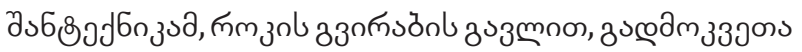

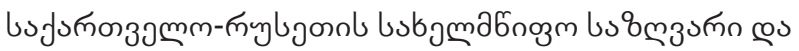

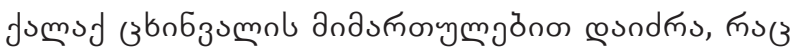

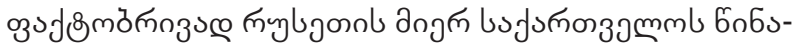

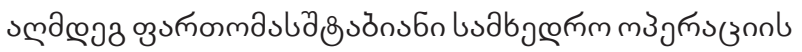

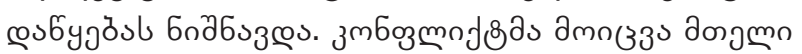

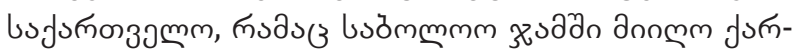
оym-пй

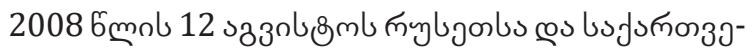

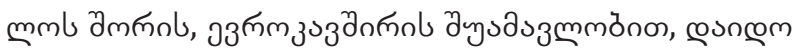

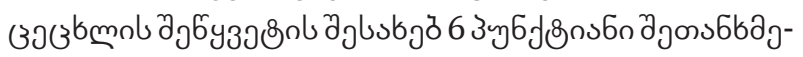

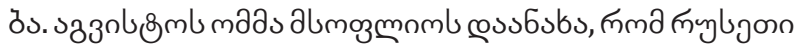

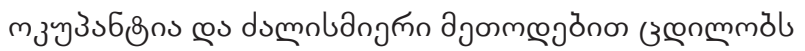

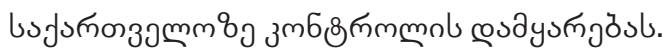

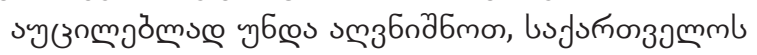

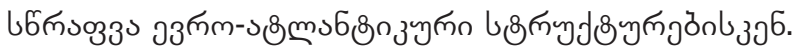

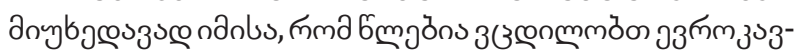

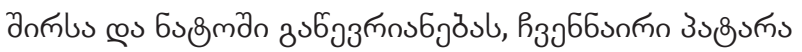

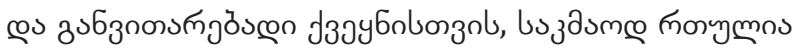

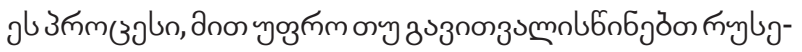

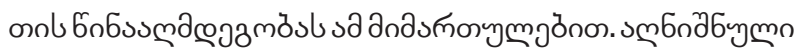

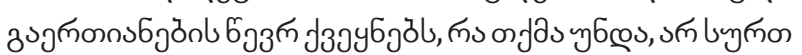

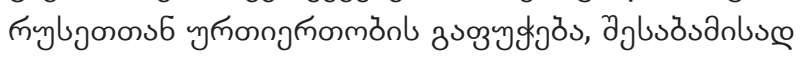

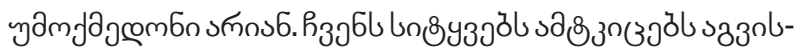

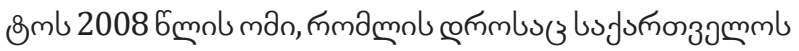

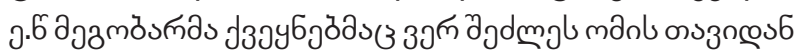

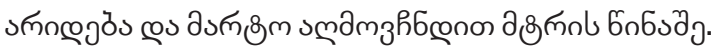

\section{ৎ১৬336১}

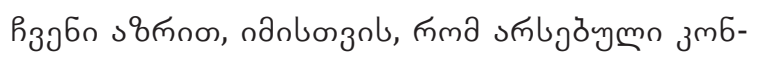

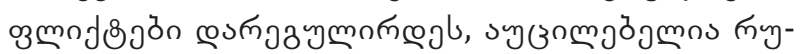

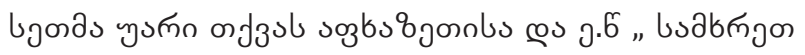

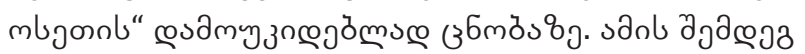

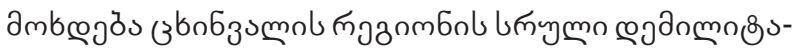

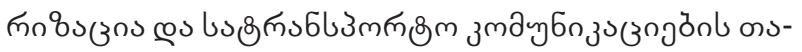




\section{ISSN 2667-9752(Online)}

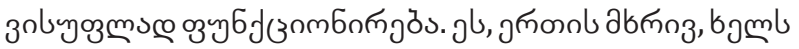

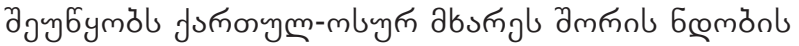
sм⿻а

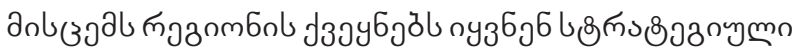

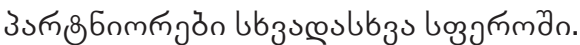

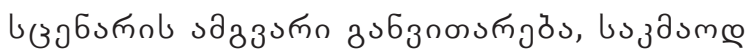

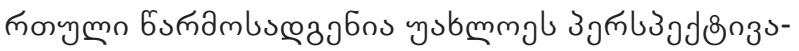

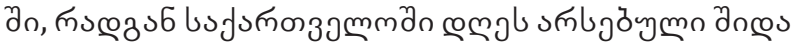

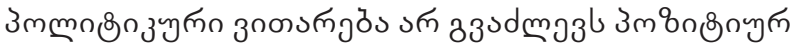

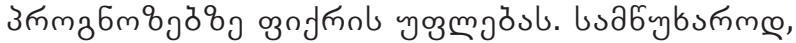

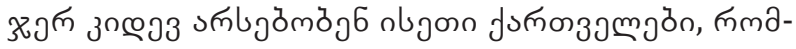

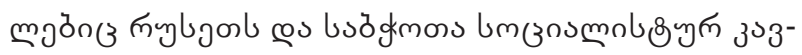

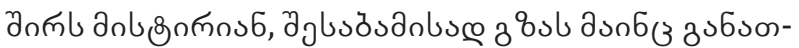

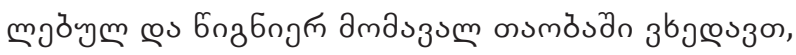
пmам

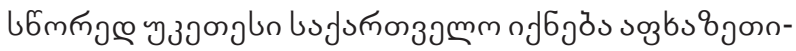

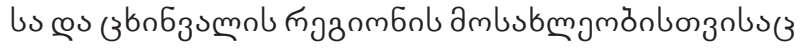
bsbyn̆зэmo.

\section{REFERENCES:}

1. Art R., Jervis R., "International Politics", article "Global Trends 2025", Ilia State University Press, Tbilisi, 2011;

2. Brzezinski Z., "Big Chessboard" Publishing House "Artanuji”, Tbilisi, 2014;

3. Gasviani G., "Abkhazia at the end of the XIX century and XX century", "Chronicle" Publishing House, Tbilisi, 2004;

4. Gachechiladze R., "Georgia in the World Context”, Second Updated Edition, Bakur Sulakauri Publishing House, Tbilisi, 2017;

5. Gegeshidze A., "Global Processes and Georgia", "Guide to Democratization of Georgia”, Club of Independent Experts, Tbilisi, 2012;

6. Nadareishvili T., "Conspiracy against Georgia”, JSC “First Printing House” Tbilisi, 2000;

7. Pipinashvili D., "Conflicts in the South Caucasus and Problems of Regional Stability", Tbilisi, 2009;

8. Samadbegishvili S., GC Analysis "Intermediate End of the Nagorno-Karabakh Conflict as a Landmark Event for the Caucasus Region”, Tbilisi, 2020. 


\title{
POLLING STATIONS - THE MAIN CORNERSTONE OF ELECTIONS
}

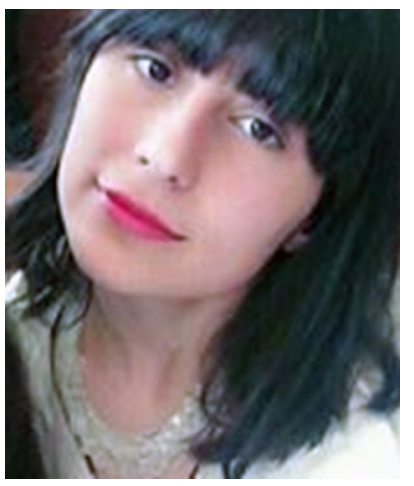

Tsiala Meladze

Georgian Technical University Faculty

of Business Technology PhD student in

Public Management Program

cia.meladze@mail.ru

DOI suffix: 10.36962/NEC6102202176

\begin{abstract}
In this article, we discuss the legislative proposal submitted by us, according to which we request an amendment to Article 61, Part 1, Paragraph 1 of the Election Code of Georgia, according to which a new function was added to the member of the flow regulation commission - if necessary, electronically check In the list. In addition, Article 58 (4) (b) of the law requires a change, according to which special places for election observers and party representatives will be allocated at the polling station on polling day, from where they will be able to freely observe the election process at the polling station.

With these changes, the Election Code of Georgia will be able to better regulate the problems existing at the polling station on the polling day; It serves to create a safe, comfortable electoral environment for both the voter and the other persons involved in the election process in the line of duty.
\end{abstract}

Keywords: Elections, Legislative Proposal, Election Code.

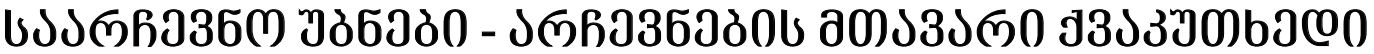

\author{
३๐osmo $\partial_{\text {gmodo }}$

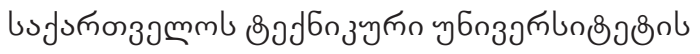

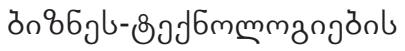

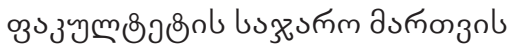

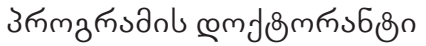

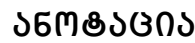

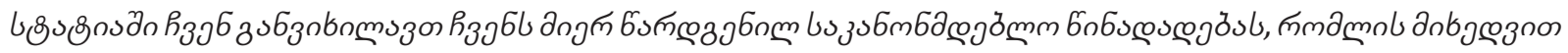

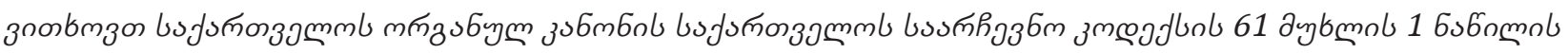

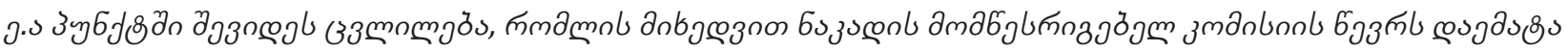

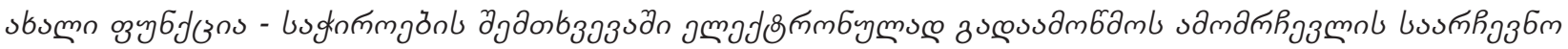

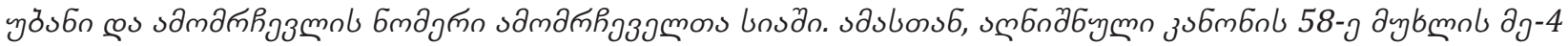

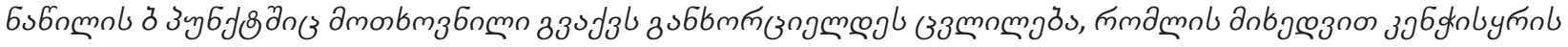

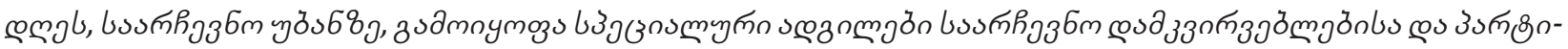

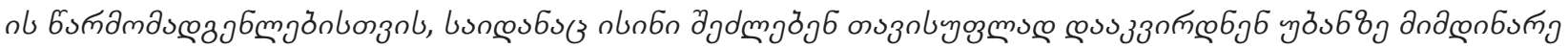

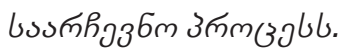

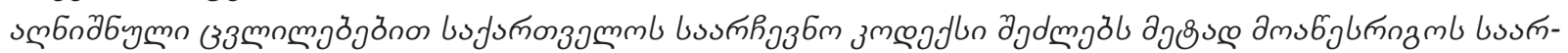

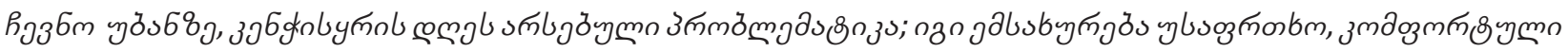

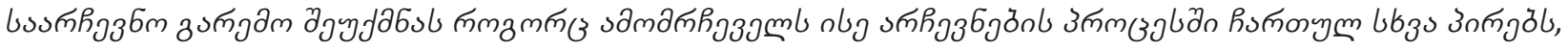

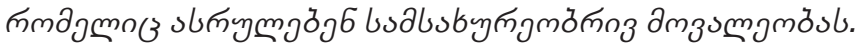

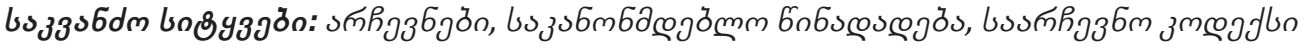




\section{INTRODUCTION}

Elections are one of the most important forms of democracy, and the quality of its development determines the level of development of the country. Consequently, elections have always played an important role in the history of democracies. It is part of a political process for which it is important to create an effective electoral environment. Properly analyzed election laws and procedures are one of the prerequisites for fair, transparent elections. It is necessary to take steps that will guarantee the flow of the electoral process in the right direction in terms of organizational management [1, p. 155].

The election administration plays a key role in determining the degree of electoral democracy. The Election Administration of Georgia is an independent administrative body established in accordance with the Election Code of the Law of Georgia and, importantly, it is independent of other state bodies within its authority.

The Election Administration of Georgia includes:

A) the CEC and its staff;

B) the SEC and its apparatus;

C) district election commissions;

D) Precinct election commissions.

The highest body of the election administration of Georgia is the CEC. It is responsible for the work of election commissions at all levels and determines the uniform application of Georgian election legislation throughout Georgia.

The election administration should be an impartial body; To do this, attention should be paid to the rules of election of this body. The writings of the administration should be selected not on the basis of their political affiliation but on the basis of their professional qualities. What is meant by professional skills? They should be aware of election issues and be able to organize elections effectively. These are the main characteristics that the election administration should rely on. It is important to periodically train them on issues such as: electoral processes, international obligations, human rights standards.

Abraham Lincoln said, "The ballot is stronger than a bullet." And if we want the voter to make his choice in a free environment, the polling stations must be arranged first.

According to the Election Code of Georgia, a polling station is an administrative election unit, which is established in accordance with the rules established by the legislation of Georgia, during elections and referendums for voting and counting of votes [2].

On June 28, 2021, the Parliament adopted amendments to the Code of Georgia, which introduced a
100 -meter radius at polling stations and sanctions for violating the 100-meter radius rules. Accordingly, paragraph 12 of Article 45 of the Code was amended as follows:

"It is inadmissible to place agitation material 25 meters from the entrance of the polling station. This material is subject to removal / dismantling / seizure. It is also inadmissible to physically obstruct the movement of voters in the polling station or within 100 meters of the polling station on the polling day. It is also inadmissible to gather people or register voters within 100 meters of the polling station on polling day. "[3]

On June 14, 2021, we proposed a legislative proposal to the Parliament (N 1-7964 / 21/10), according to which we request an amendment to Article 61, Part 1 , Paragraph 1 of the Organic Law of Georgia, according to which a new function was added to the member of the flow regulation commission. In this case, electronically verify the polling station and the number of voters in the voter list. The flow regulator is a member of the commission that regulates the flow of voters to the polling station. It asks the voter to present a Georgian citizen ID card or a Georgian citizen's passport; It then checks with an ultraviolet lantern whether the voter is marked, and after making sure that the voter is not marked, gives the right to participate in the voting. Also, the flow regulator monitors how many voters stand at the voter registration desk. If more than two voters are standing at the registration table, the next voter enters the polling station temporarily. In addition, Article 58 (4) (b) of the law requires a change, according to which special places for election observers and party representatives will be allocated at the polling station on polling day, from where they will be able to freely observe the election process at the polling station.

As you know, until 2013, voter cards were issued only once, 2-3 days before the elections. And then, for more voter awareness, its distribution was defined in two stages before the election. Voters arriving at the polling station in some cases do not remember which polling station they have the opportunity to vote at, and most often they do not know their number on the voter list. They may not have received this information for some reason. This makes it difficult for all voter registrars, as they spend most of their time searching for voters on voter lists. And finding voters by numbering makes their job much easier. In practice, voter registrars interfere with voter lists when searching by name and surname, and in addition to improper time management, this makes it even more difficult for them. The election process is flawed and a large number of voters are gathered at the polling station. In order for the registrars to simplify the work process 
and save time, it is necessary for the voter to present his / her number to the registrar. Given the pandemic environment, the delay of voters at the polling station, especially inside the polling station, endangers the health of those inside the building.

Observers and party representatives often interfere with the election process and escalate the election. In practice, we encounter cases where observers or party representatives sit close to voter registrars and are not allowed to work in a quiet environment. This inconvenience will be caused not only by the registrars, but also by other members of the commission, who have a significant responsibility on polling day. Accordingly, specially designated polling stations will reduce ongoing controversy at polling stations. Also, a certain distance will be maintained, which, in the context of today's pandemic environment, is completely acceptable and necessary.

The bill may have a positive impact on children's rights, as voters who go to the polls with their children will not have to spend much time in the polling station, and the improved voting environment proposed by the bill will have a positive impact on children. The UK is positive about voters coming to the polls with their children, as it believes that with this move, children are becoming accustomed to respecting the democratic process.

During the election process, attention should be paid to the social distance of voters and the observance of appropriate hygienic norms. The election environment should be regulated so that the voter feels protected; Otherwise it will lead to a decrease in voter turnout.

France held local elections in 2020. Voters were instructed to bring their own pens and keep a one-meter distance in the rows. Despite security measures, turnout fell by about $20 \%$ compared to previous elections.

In the US, voters who wished to run in the election and had symptoms were able to cast their ballots by mail. Consequently, to reduce the risks posed by the development of coronavirus, many states have begun to popularize the mail voting system so that more voters can vote[4].

South Korea has increased its staff in connection with the election. To what extent can Georgia do the same? Given that we are talking about the protection of social distance, and often elections in Georgia are held in a small room, the increase in the number of employees in election commissions can not have a positive impact on the pandemic situation in Georgia. The role of the flow regulator (one of the members of the election commission), which controls the number of voters in the territory of the polling station, will be important in the protection of social distance.

\section{CONCLUSION}

With these changes, the Election Code of Georgia will be able to better regulate the problems existing at the polling station on the polling day; It serves to create a safe, comfortable electoral environment for both the voter and the other persons involved in the election process in the line of duty. Amendments to this law will regulate hitherto incompletely regulated election legal issues. Legislative changes provide:

$\checkmark$ Simplify the work process for voter registrars,

$\checkmark$ Reducing the number of voters at the polling station,

$\checkmark$ Improving time management,

$\checkmark$ To neutralize a politically difficult background,

$\checkmark$ Adherence to safety rules.

\section{REFERENCES}

1. Demetrashvili A., Kvachadze M., Kublashvili K. Etc., "Handbook of Constitutional Law”, Tbilisi, 2005, p. 155 (In Georgian)

2. Paragraph 2 of Article 2 of the Election Code of Georgia, N5636-rs, Legislative Herald of Georgia, 10.01.2012 (last verification 17. 07. 2021) (In Georgian)

3. Paragraph 12 of Article 45 of the Election Code of Georgia, N5636-rs, Legislative Herald of Georgia, 10.01.2012, (last verification 17. 07. 2021) (In Georgian)

4.https://sector3.ge/Libraries/Download/391?fbclid=IwAR3yl6Kjzzb7BkhdvnlIk3BPcM1NCtyueOrBNiTe YT_5Lzrq32mz4ttx0N8, (last verification 22. 07. 2021)

5..https://cesko.ge/geo/list/show/13745-27-seqtembridan-saarchevno-administracia-amomrchevlebistvissainformacio-baratebis-darigebas-iwyebs-16716(last verification 22. 07. 2021)

6.https://www.bbc.com/news/explainers-56849080, (last verification 22. 07. 2021). 


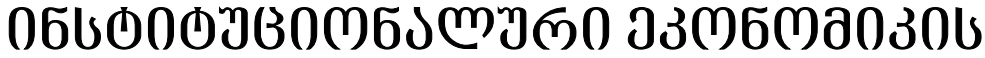

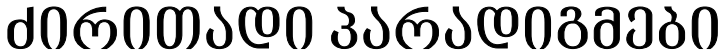

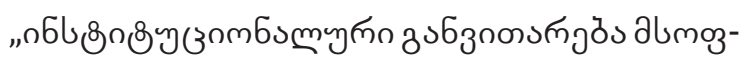

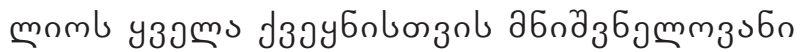

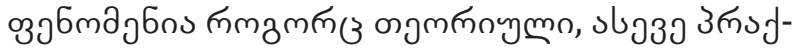

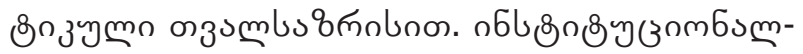

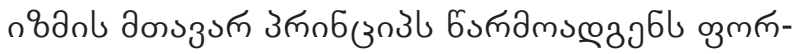

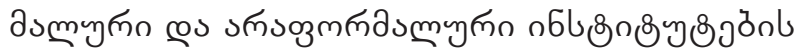

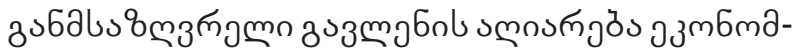

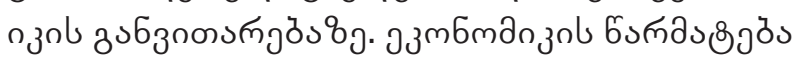

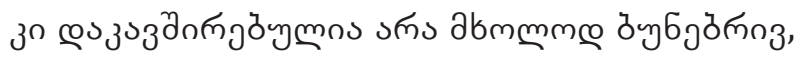

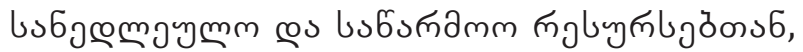

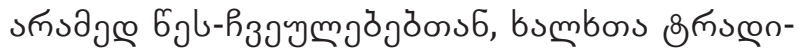

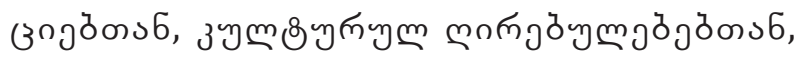

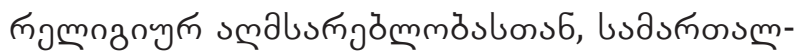

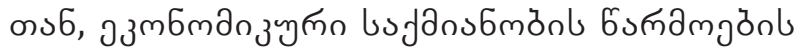

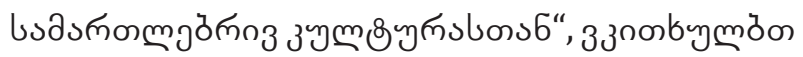

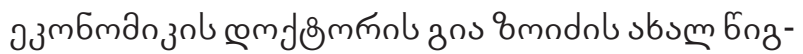

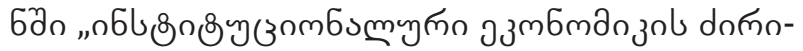

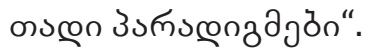

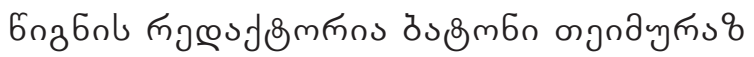

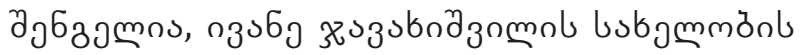

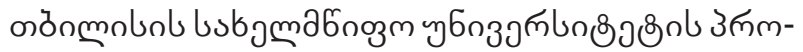

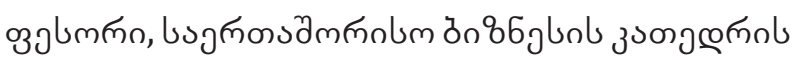

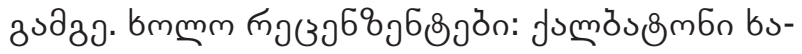

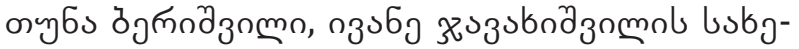

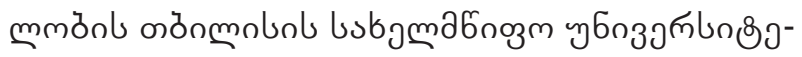

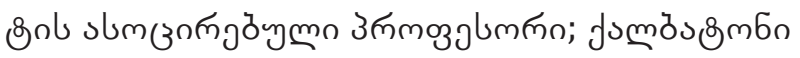
œumo bjb6nsżз

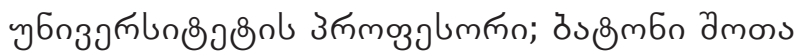

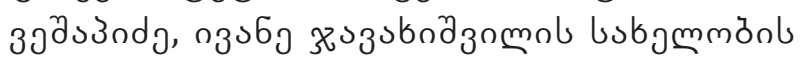

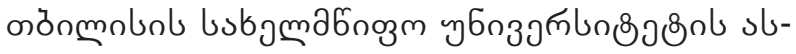

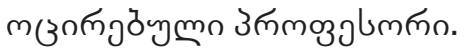

งз৪мm s

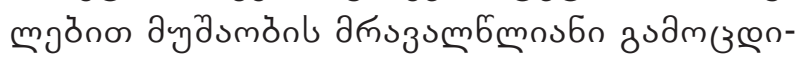

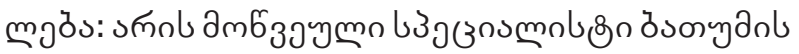

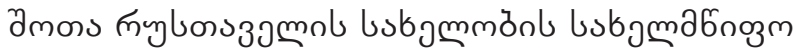

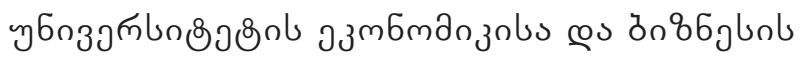

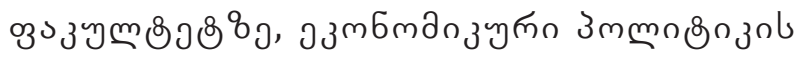

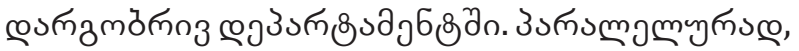
s

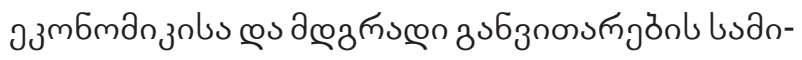

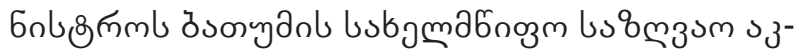

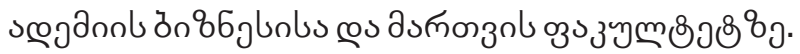

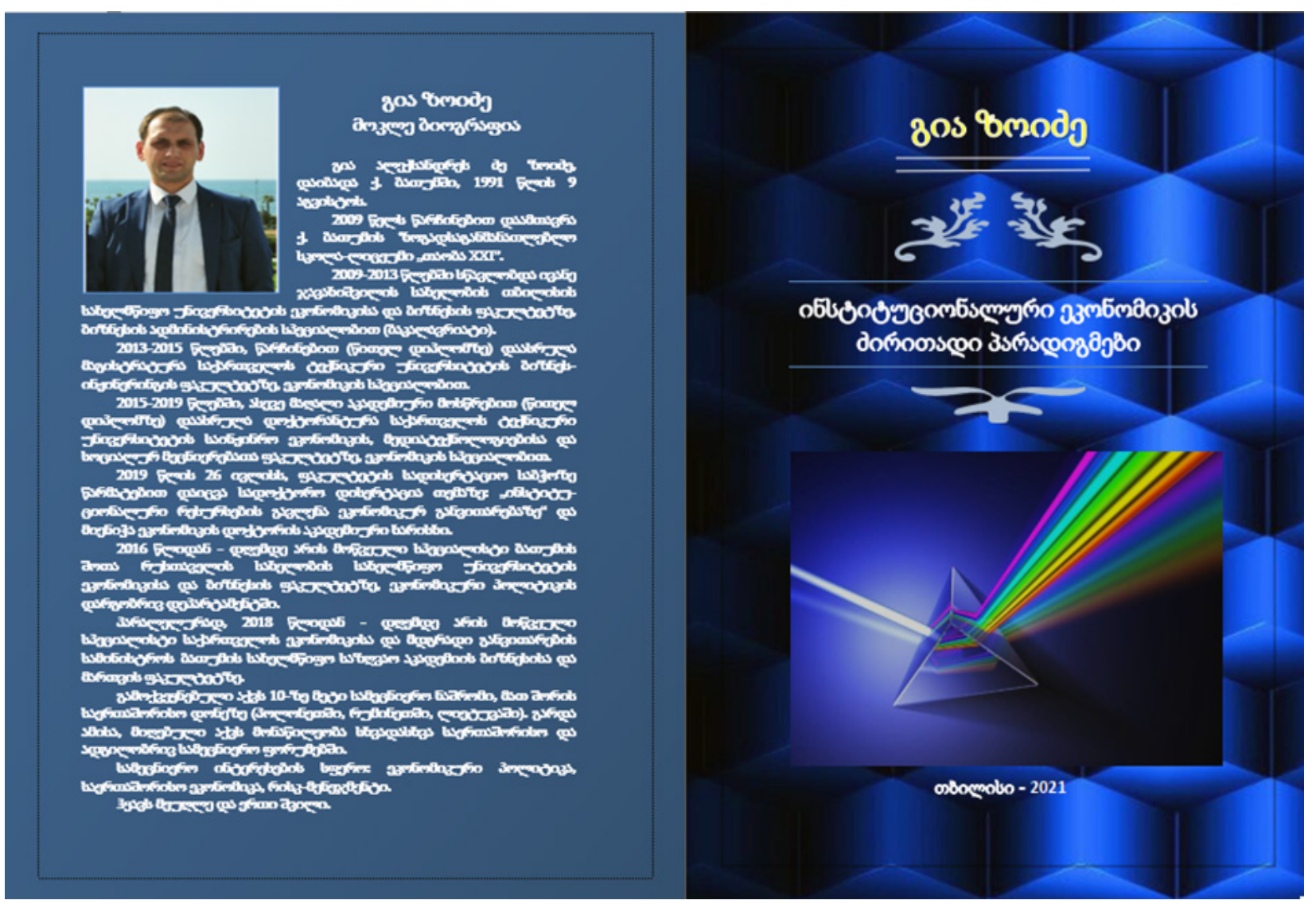




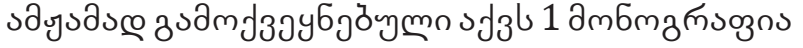

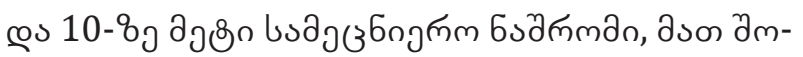

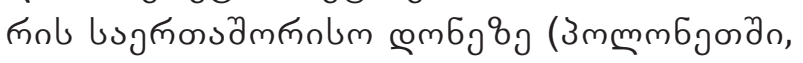

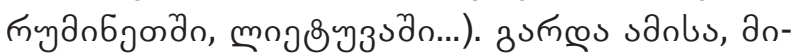

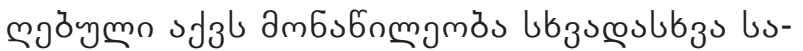

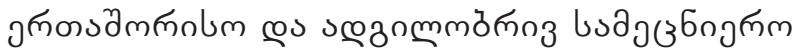

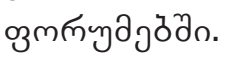

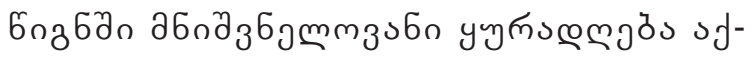

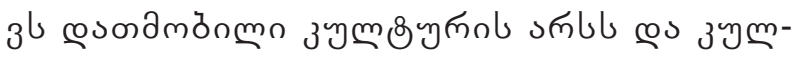

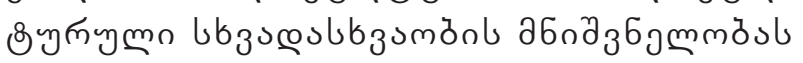

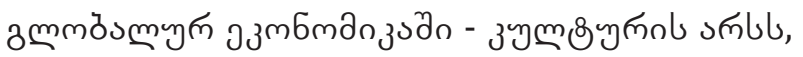

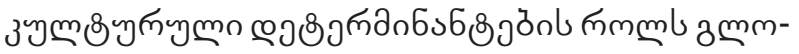

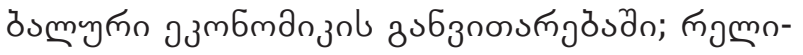

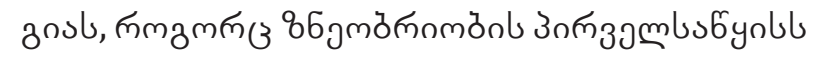

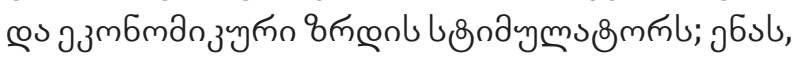

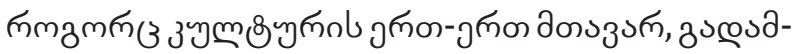

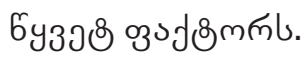

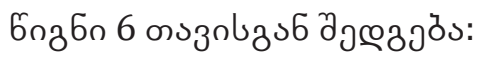

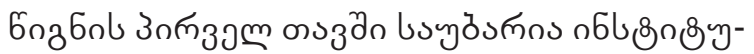

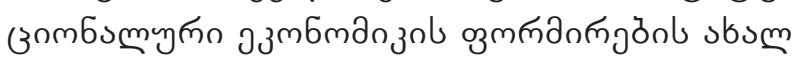

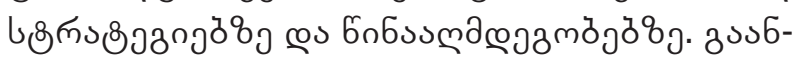

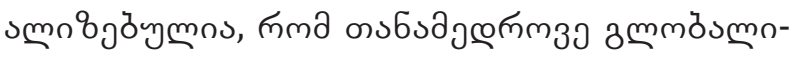

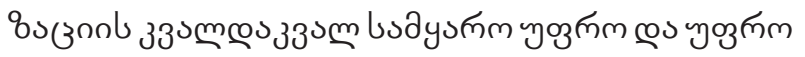

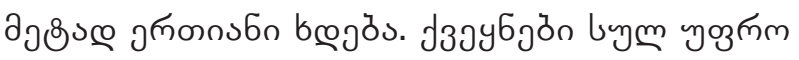

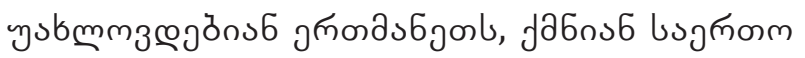

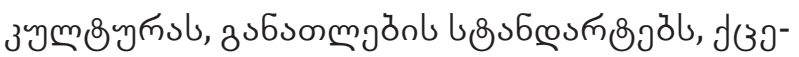

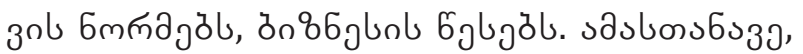

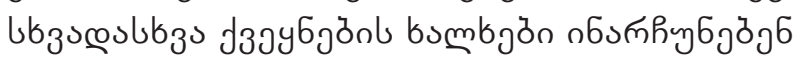

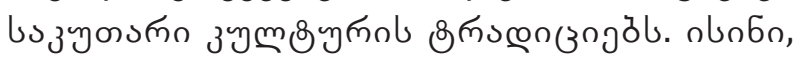

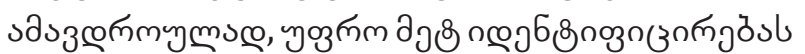

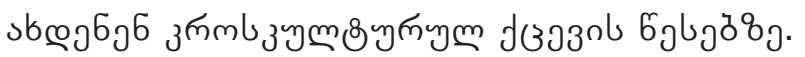

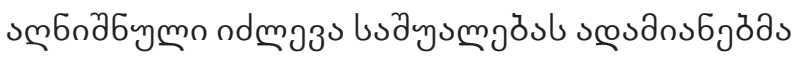

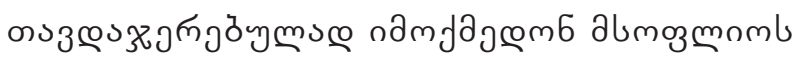

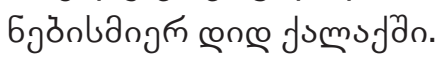

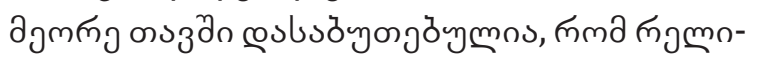

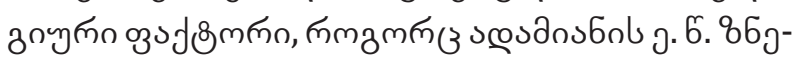

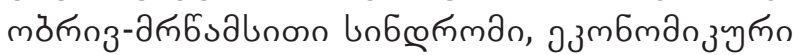

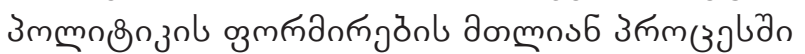

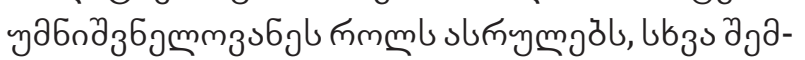

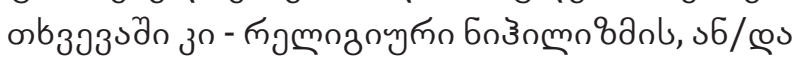

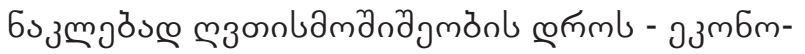

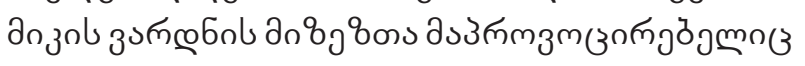

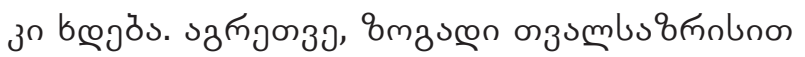

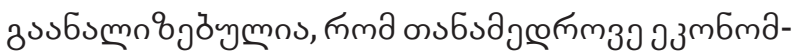

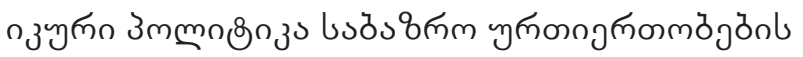

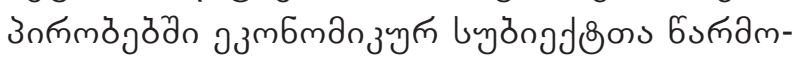

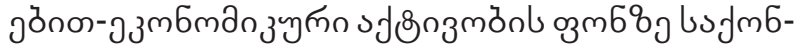

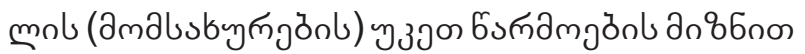

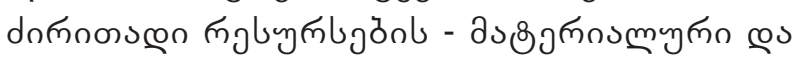

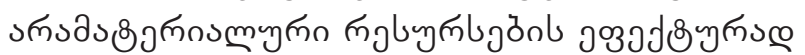
asamyj6jönbs 3 j $^{6}$ s

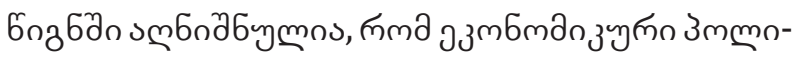

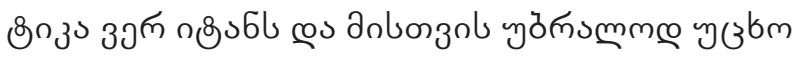

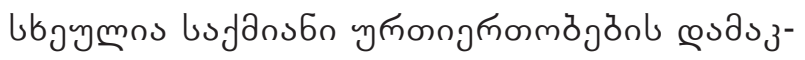

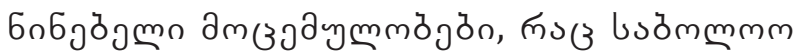

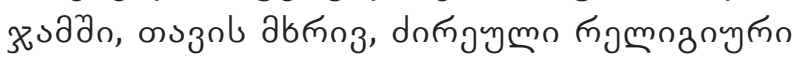

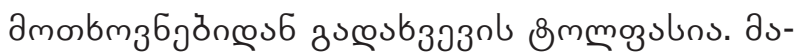

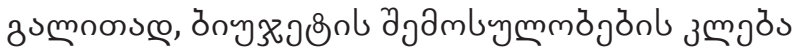

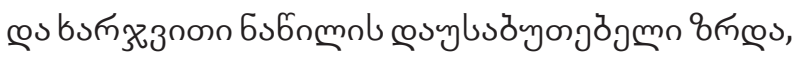

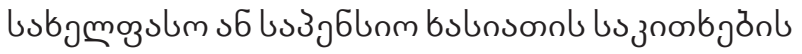

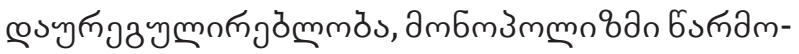

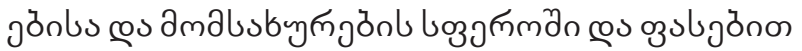

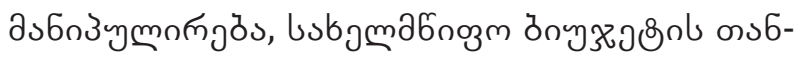

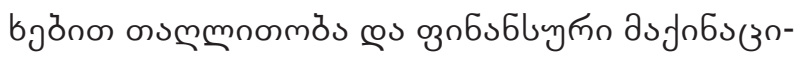

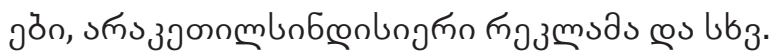

ة

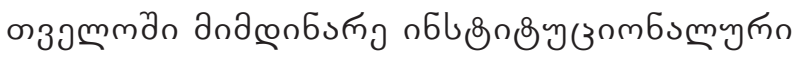

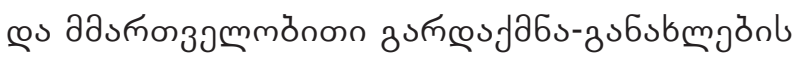

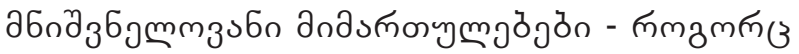
œงœృ

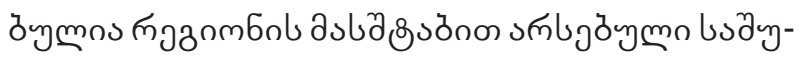

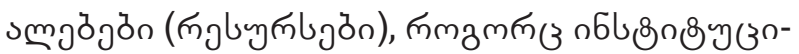

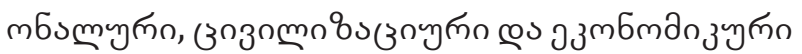

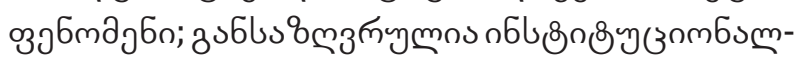

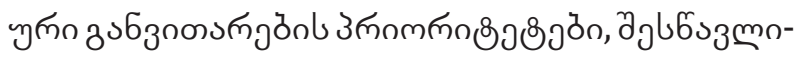

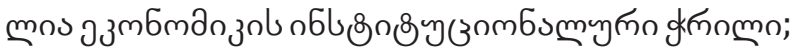

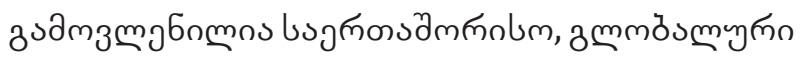

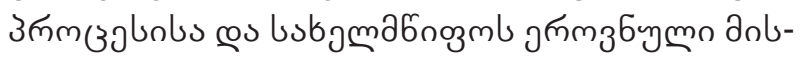

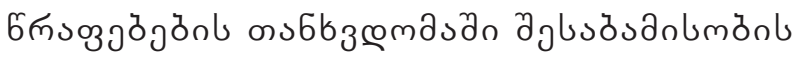

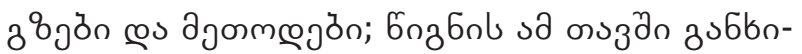

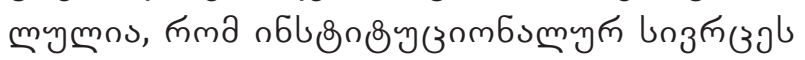

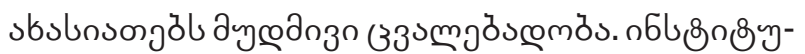

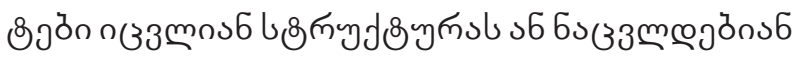

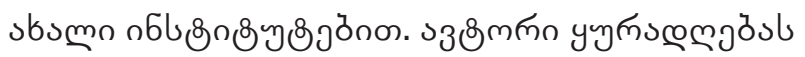

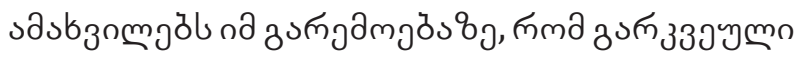

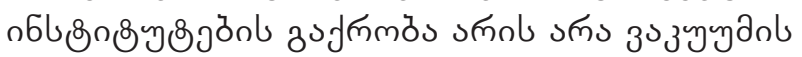

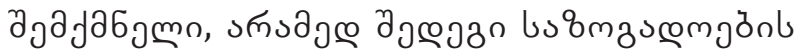

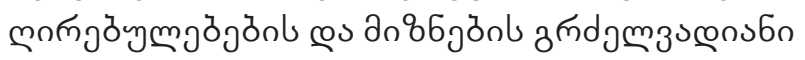

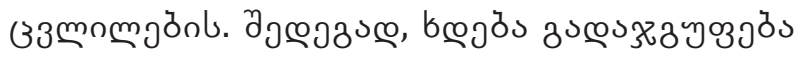

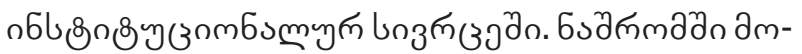

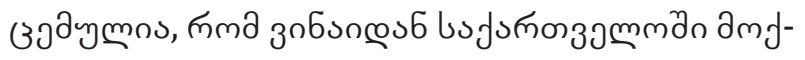

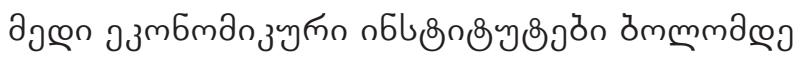




\section{ISSN 2667-9752(Online)}

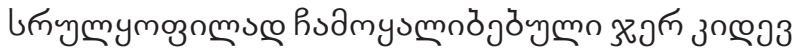

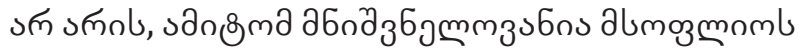

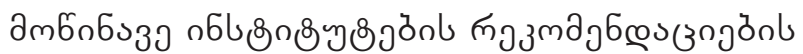

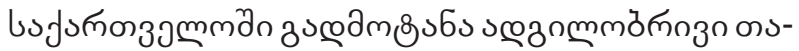

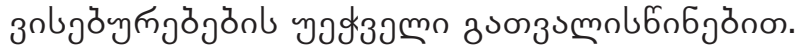

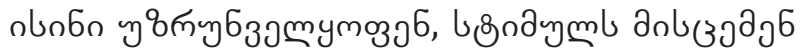

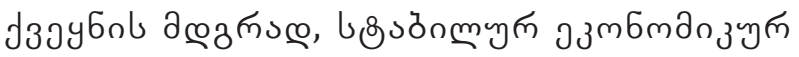

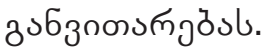

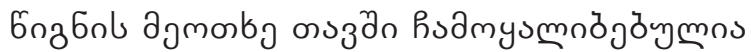

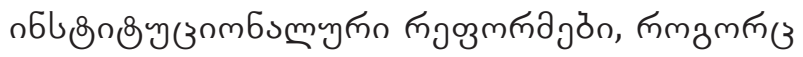

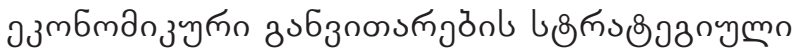

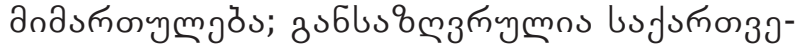

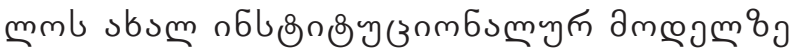

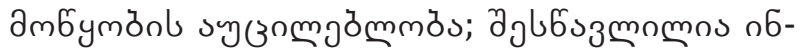

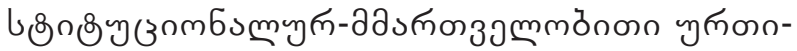

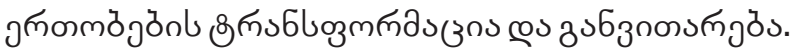

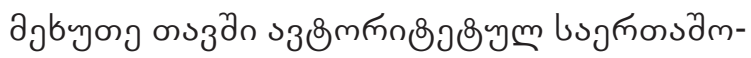

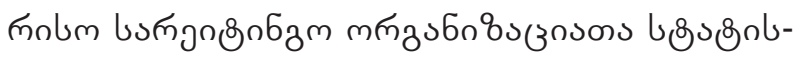

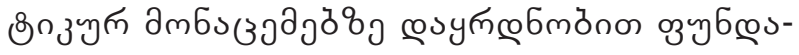

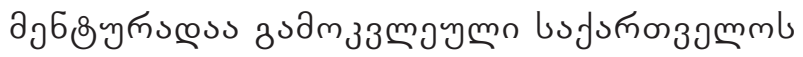

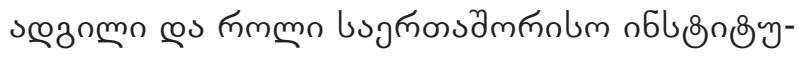

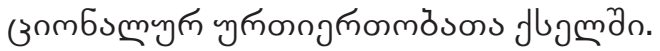

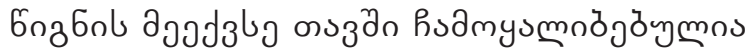

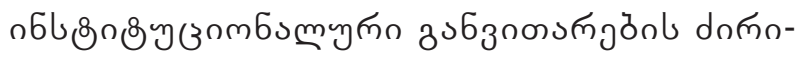

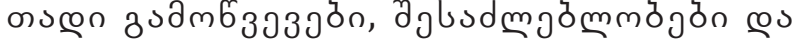

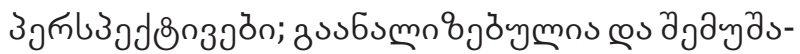

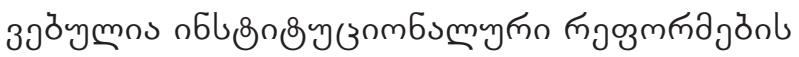

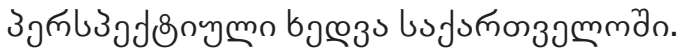

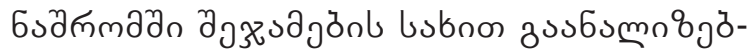

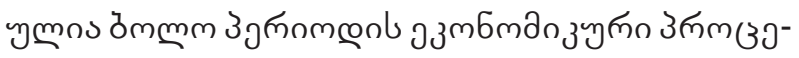

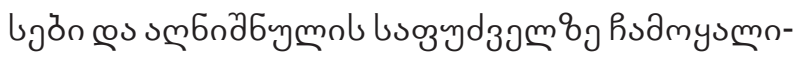

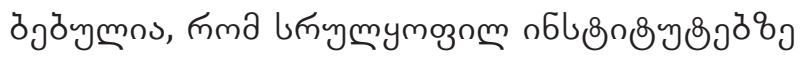

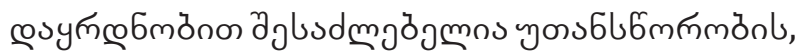

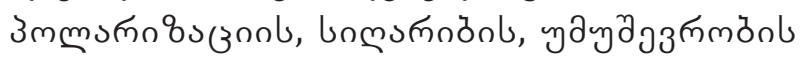

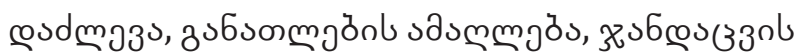

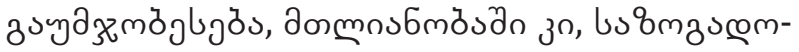

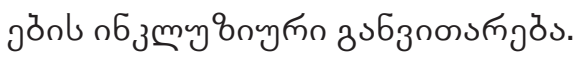

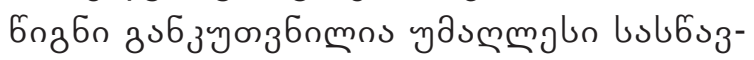

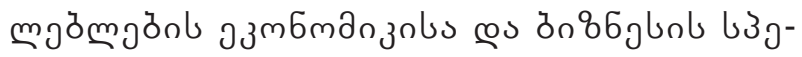

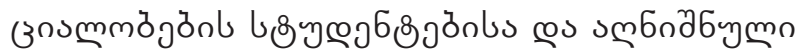

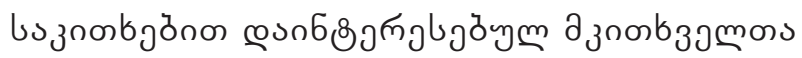
oुstromm 6rnolsonzol.

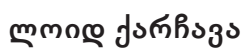

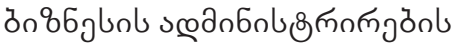

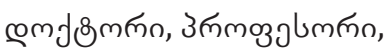
vyøn6sm ,The New Economist"-nl

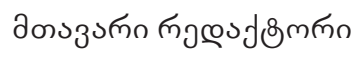




\section{REVIEW OF IRAKLI JANGAVADZE'S TEXTBOOK: "FUNDAMENTALS OF INNOVATIVE ECONOMY"}

World trends in the education system clearly point to the fact that innovative development and a successful state are intellectually synonymous, while innovative activity (entrepreneurship) is a constant search for opportunities beyond the resources currently controlled.

Both theoretically and practically, the research of the innovative sector of the economy is a priority in the modern world. Moreover, the innovative development of countries is recognized as a non-alternative factor of global economic progress.

The world is progressing at a rapid pace, as evidenced by the rapid "moral depreciation" of scientific and technical achievements. Developing countries are making significant strides in shaping and modernizing national innovation systems. They develop state innovation policies, create appropriate legal bases and innovative infrastructure, strengthen the propaganda of small innovative entrepreneurship and science-based production, colossally increase investments in scientific research and development and etc.

In this regard, the textbook of Irakli Jangavadze, a young researcher, Doctor of Economics and lecturer at the Georgian Technical University, entitled "Fundamentals of Innovative Economics" is noteworthy.

It should be noted that the paper fully meets the basic standards of the textbook and is intended for the target audience - predominantly undergraduate students. Namely: the book consists of 16 chapters, at the end of which we find thematic questions. There are used numerous briefcases, tables, diagrams, saturated with modern information and up to 200 sources.

As for the content of the textbook, the main focus is on the stages of development of the society, the theoretical aspects of innovative development, the concept of innovation, the essence and prehistory, also, on the peculiarities of innovations, indicators and opportunities for innovative development of Georgia.

Besides, it analyzes the role and importance of innovation policy in modern conditions, worldproven national innovation systems and strategic models, features of eco-innovation, intellectual capital and innovative infrastructure, etc.

Based on the above, as a reviewer, I strongly support the use of Irakli Jangavadze's intellectual work - "Fundamentals of Innovative Economy" as a guide by higher education universities in the study of economics and business students.

Revaz Shengelia Doctor of Economics, Professor of Georgian Technical University, Editor-in-Chief of the scientific journal "Economics".

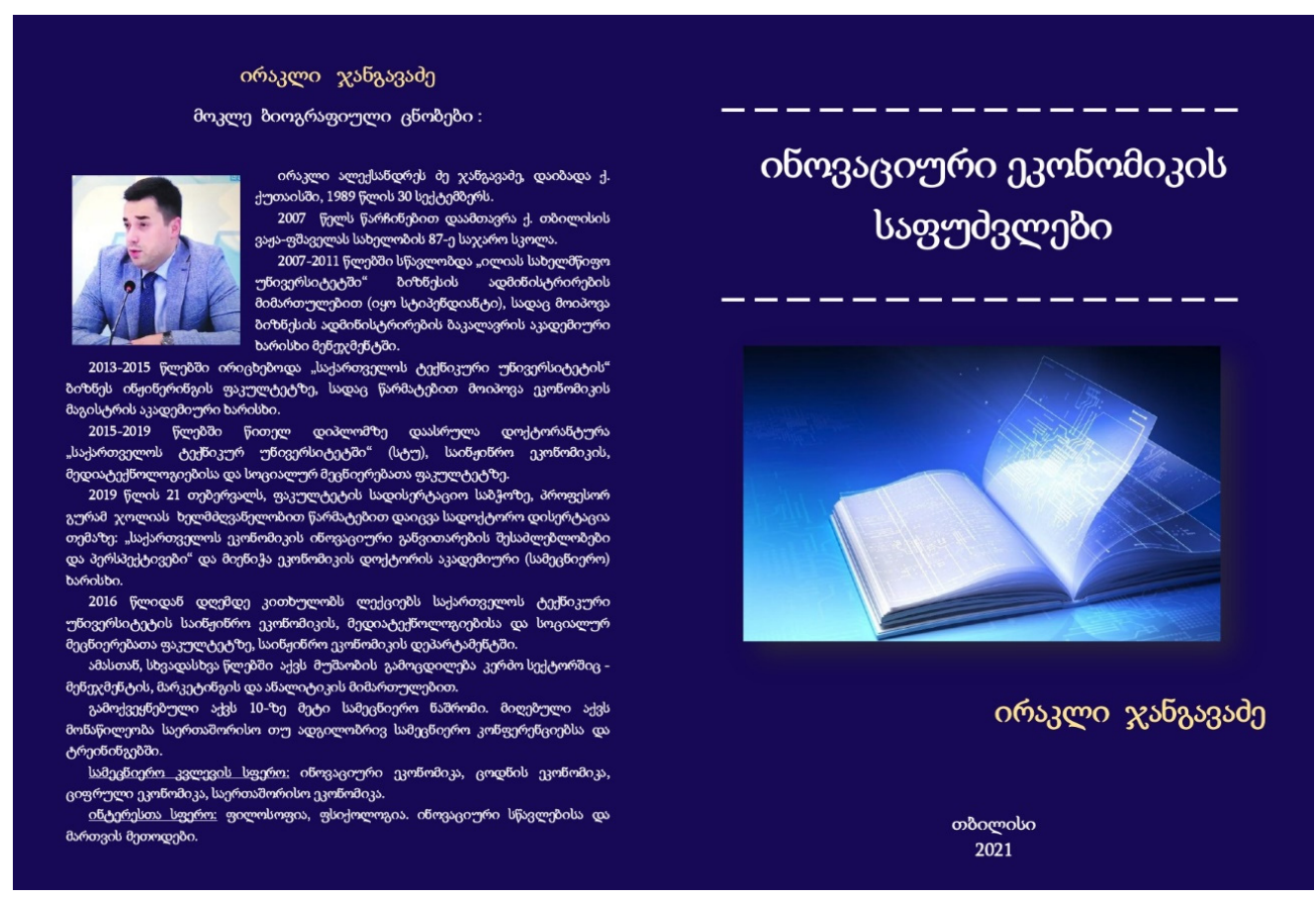




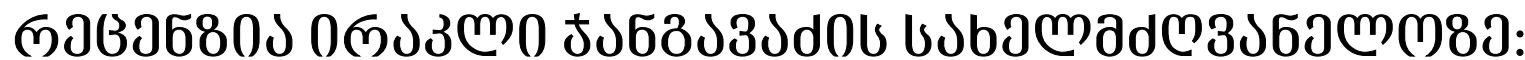

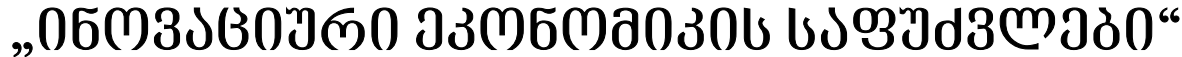

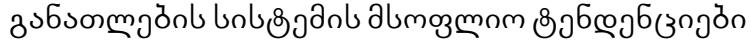

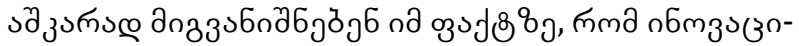

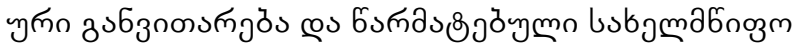

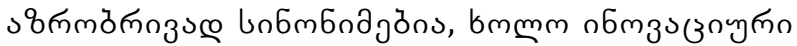

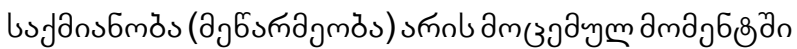

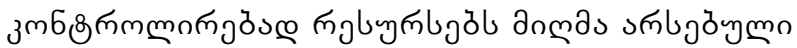

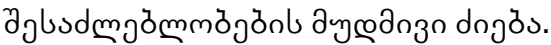

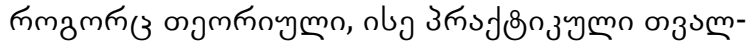

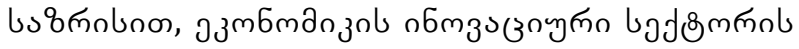

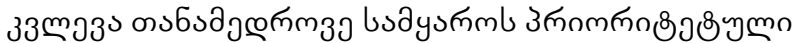

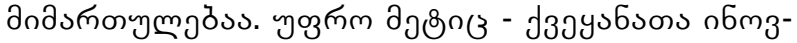

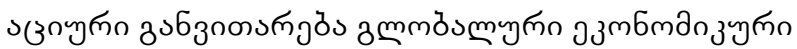

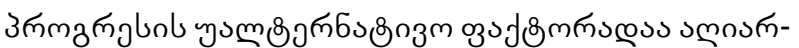
jờmo.

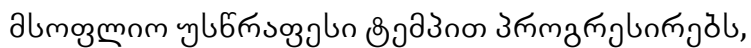

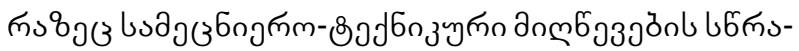

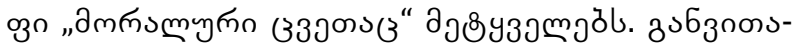

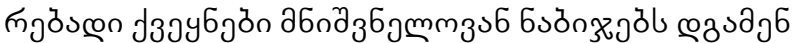

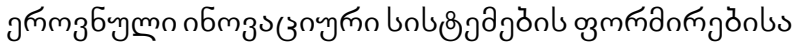

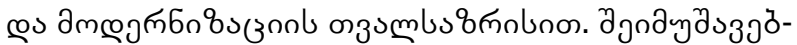

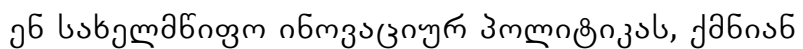

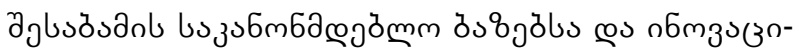

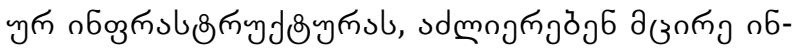

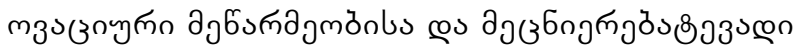

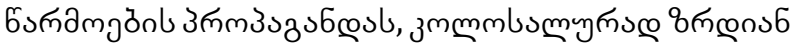

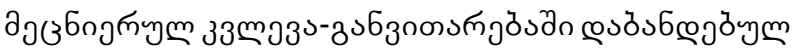

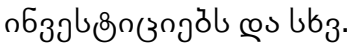

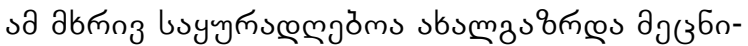

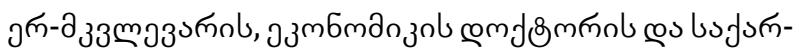

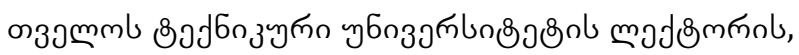

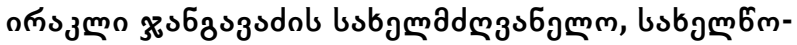

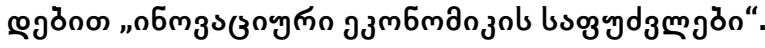

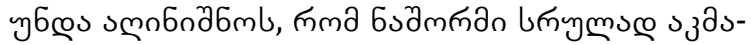

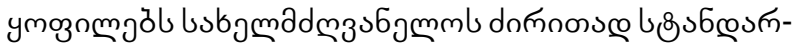

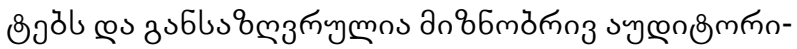

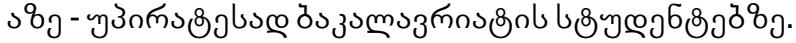

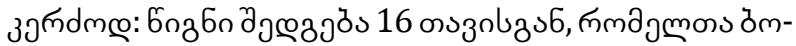

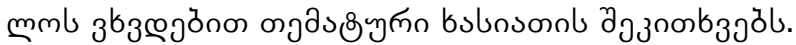

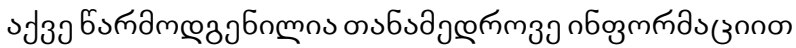

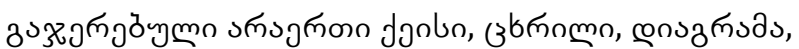

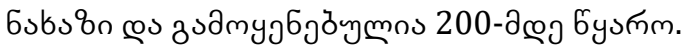

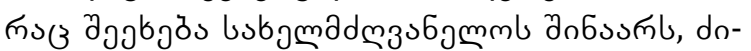

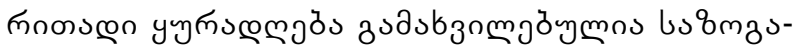

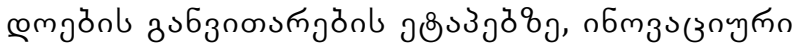

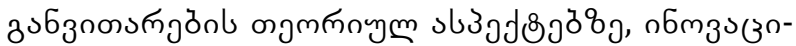

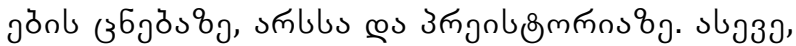

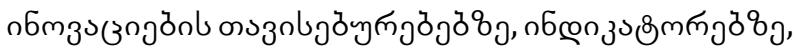

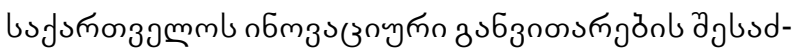

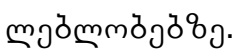

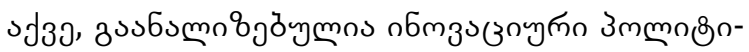

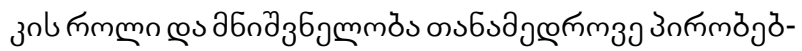

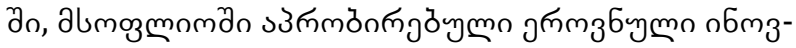

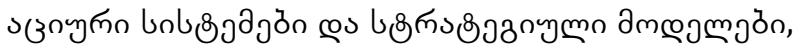

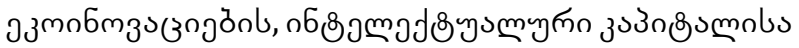

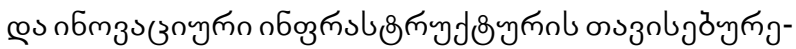

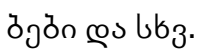

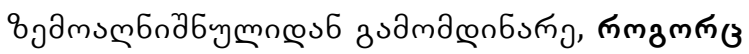

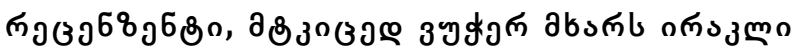

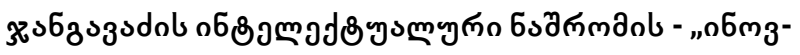

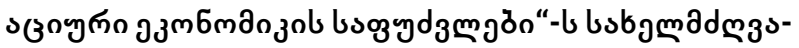

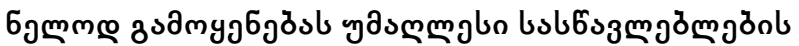

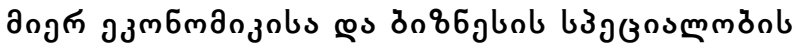

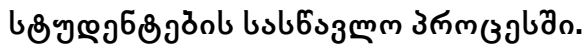

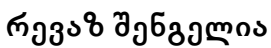
ग3m6manzol œm

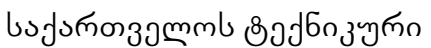

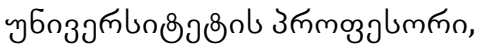

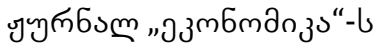
доงวง 


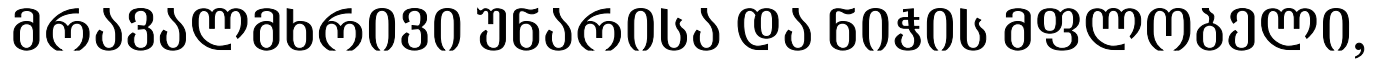

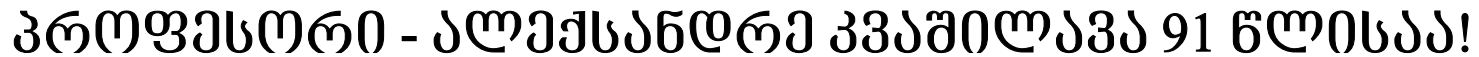

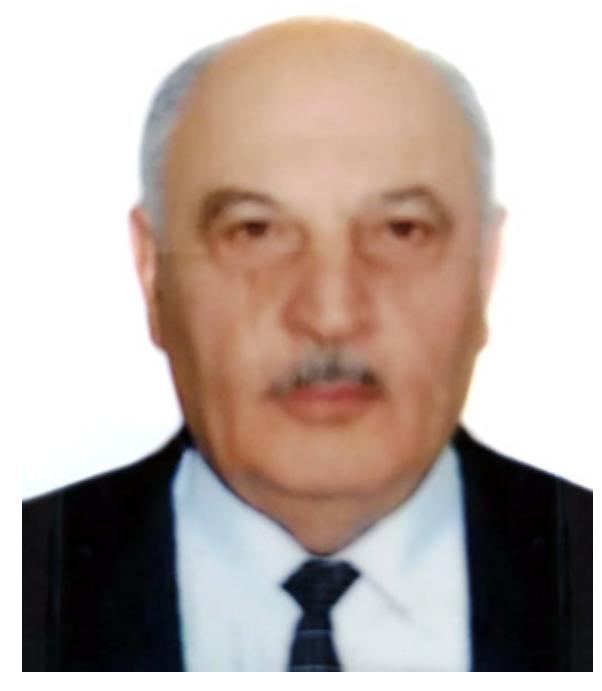

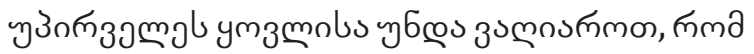

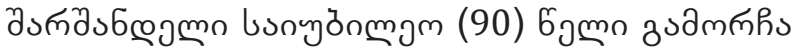

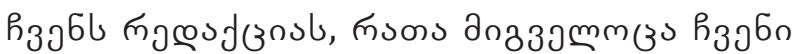

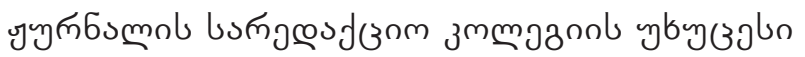

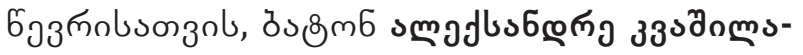

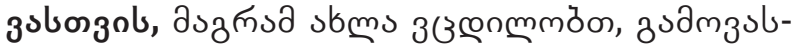
6mmmon.

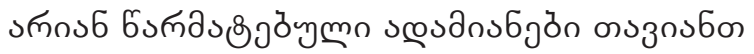

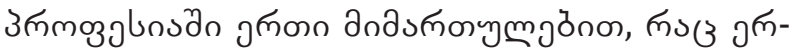

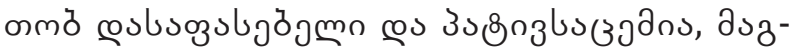

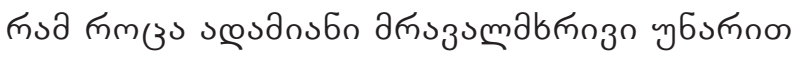

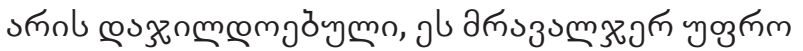

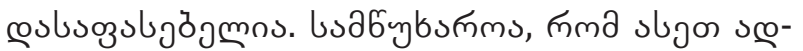

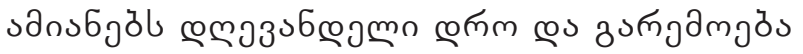

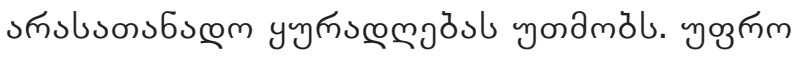

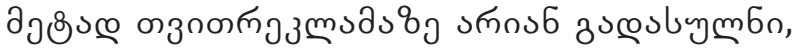

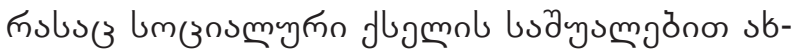

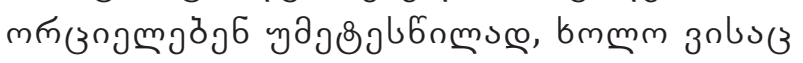

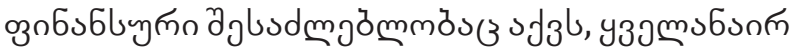

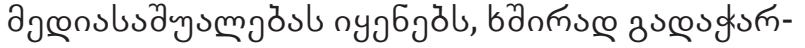

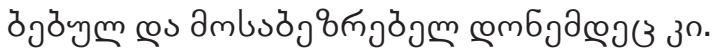

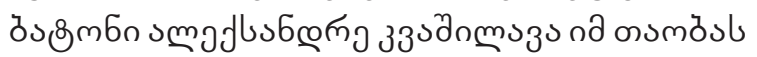

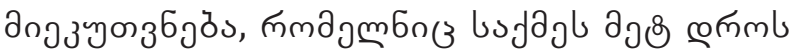

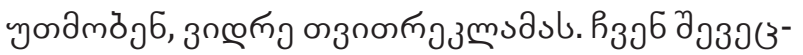

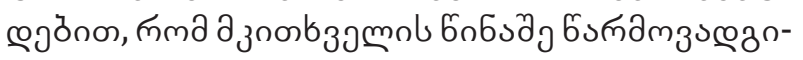

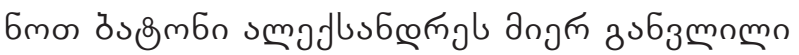
мnґا

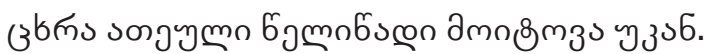

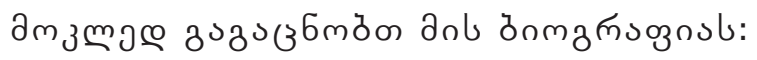

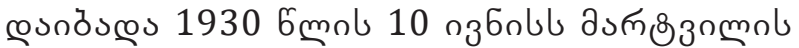

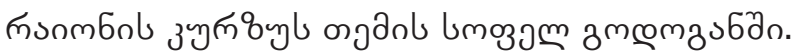

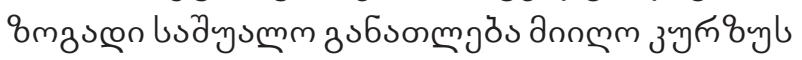

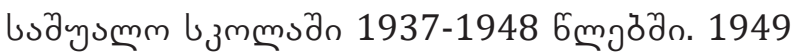

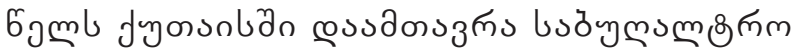

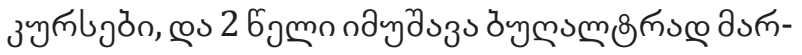

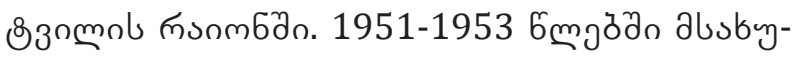

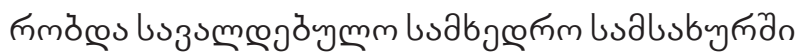

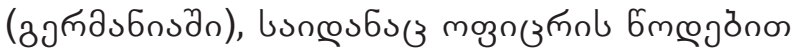

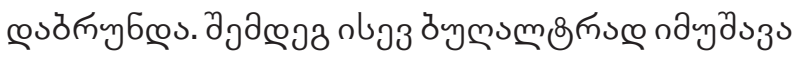

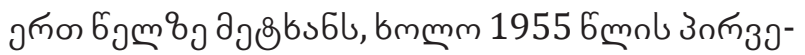

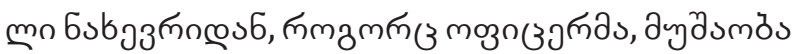

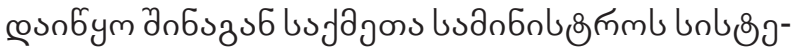

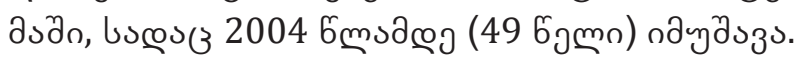

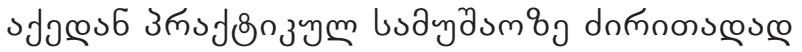

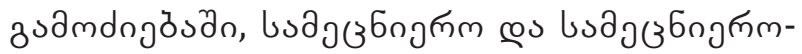

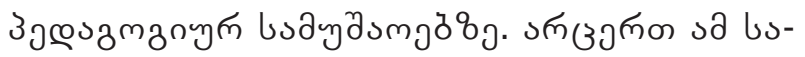

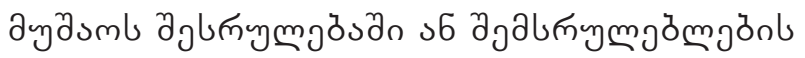

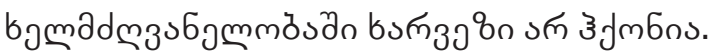

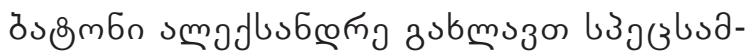

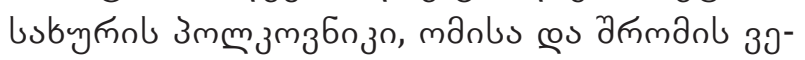

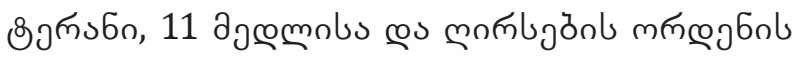

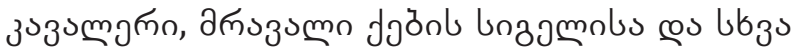

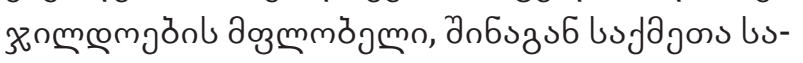

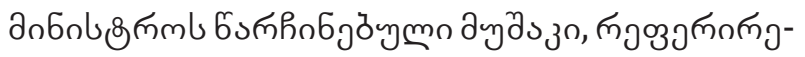

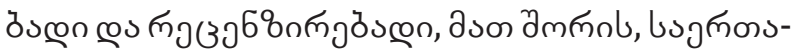

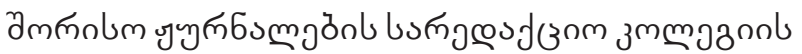

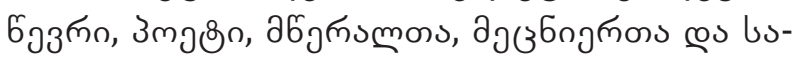

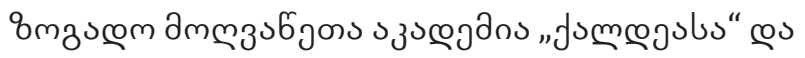

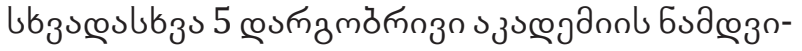

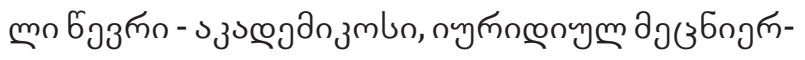

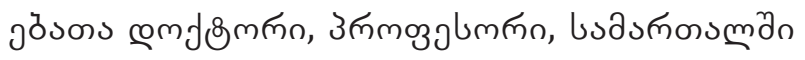

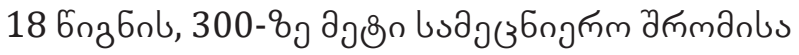
œs 700-\& g a

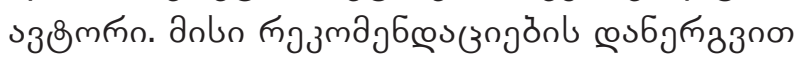
дпщృ

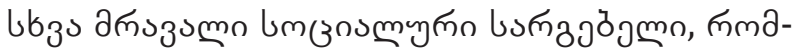

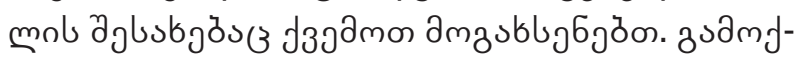

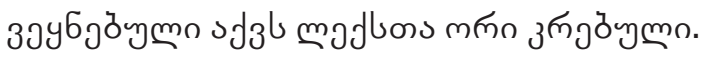

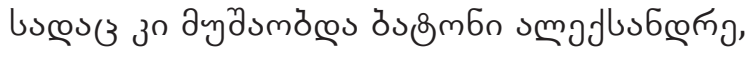
y3эmsз

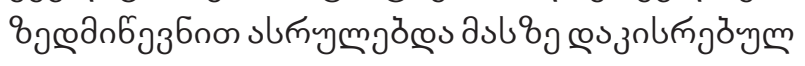




\section{ISSN 2667-9752(Online)}

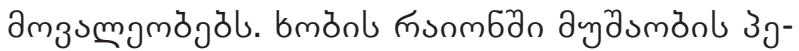

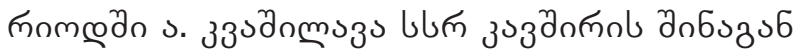

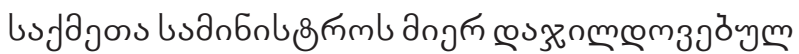

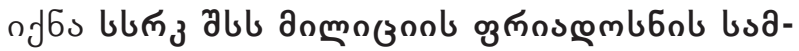

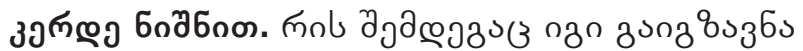

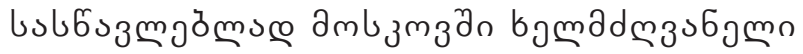

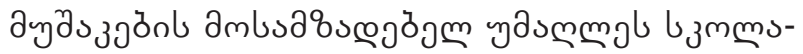

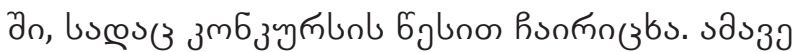
uзmms

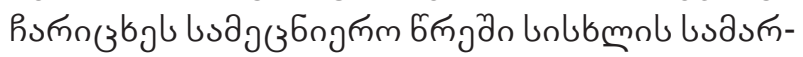

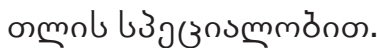

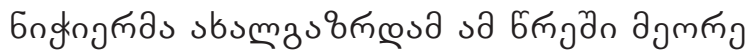

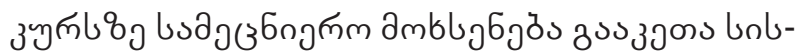

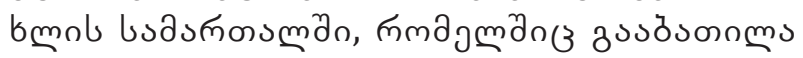

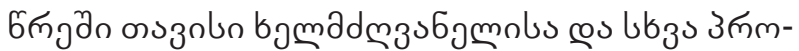

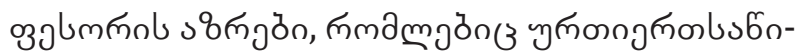

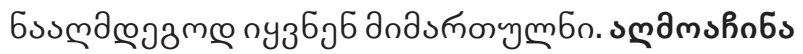

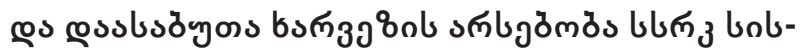

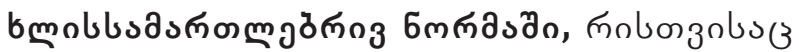

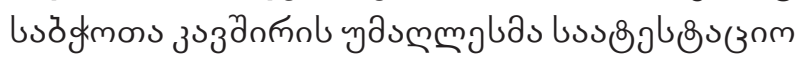
зmanbnsa 6nбsu6s an, agm

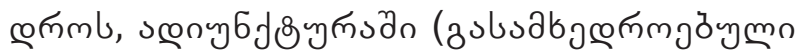

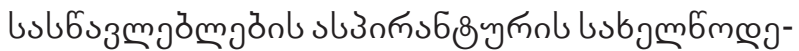

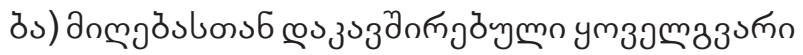

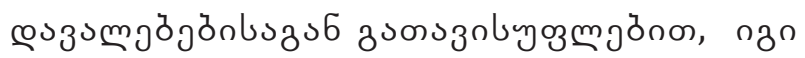

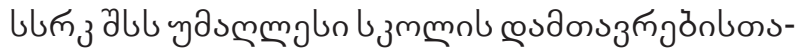

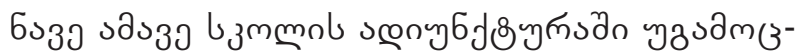

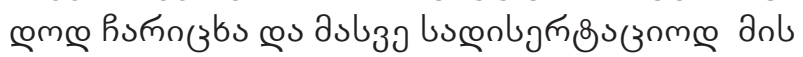

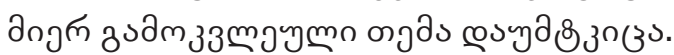

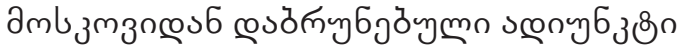

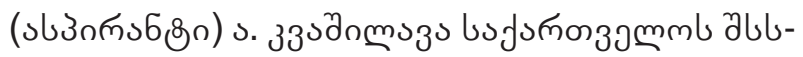

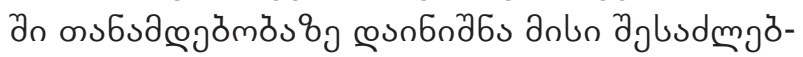

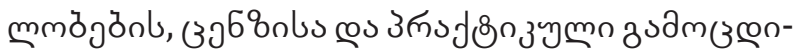

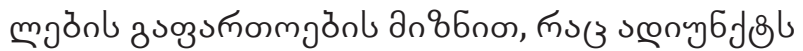

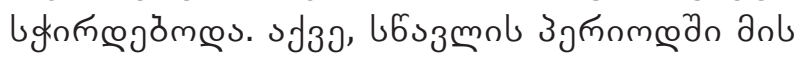
дпэк

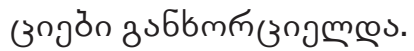

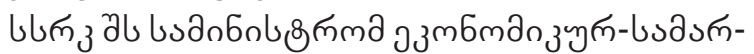

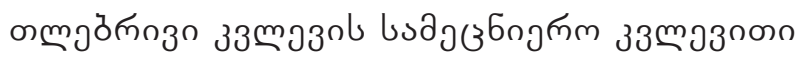

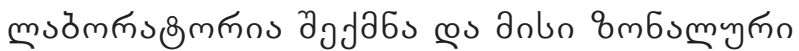

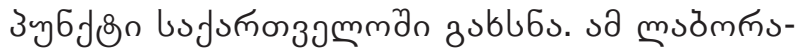

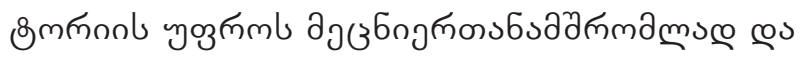

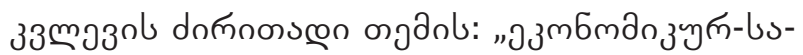

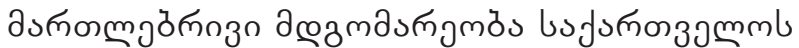

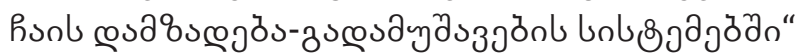

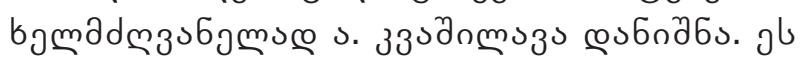

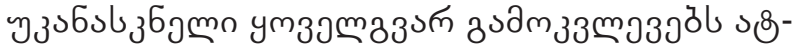

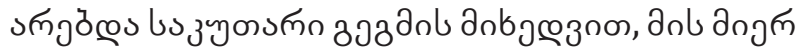

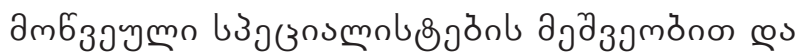

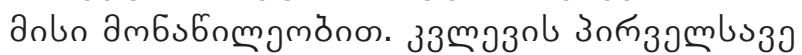

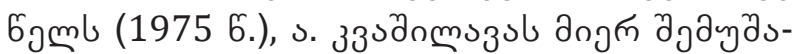

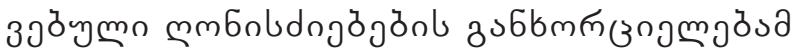

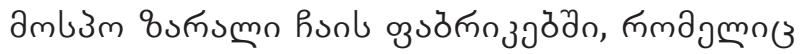

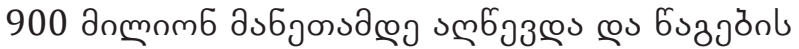

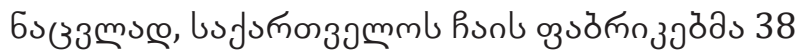
anmombn asбjon amajỏs annmgl.

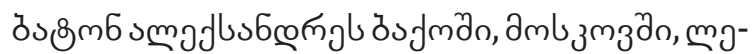

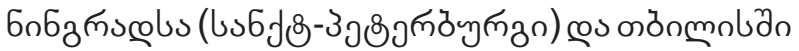

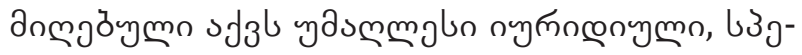

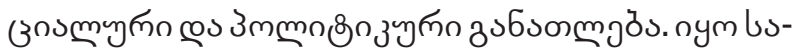

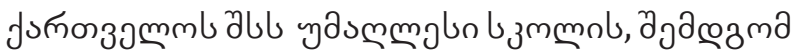

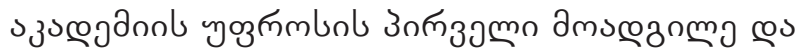

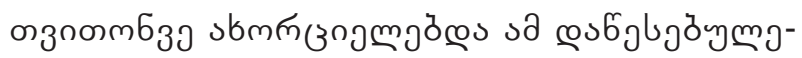

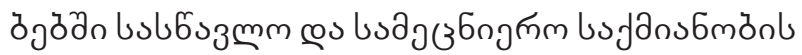

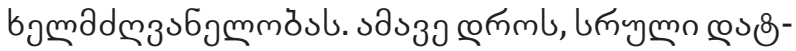

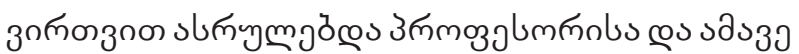

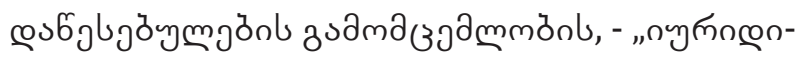

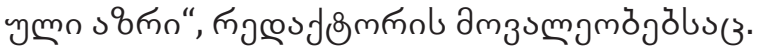

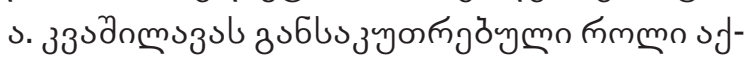

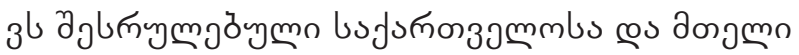

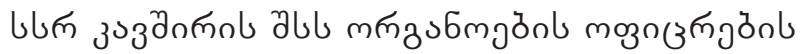

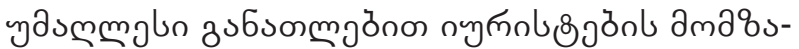

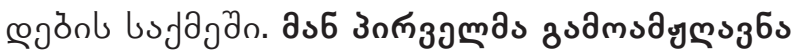

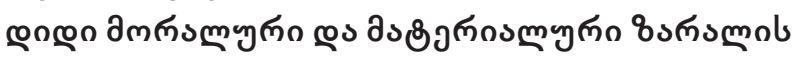
sলn

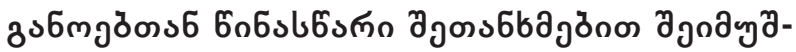

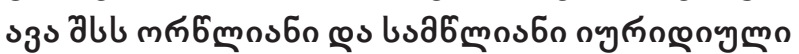

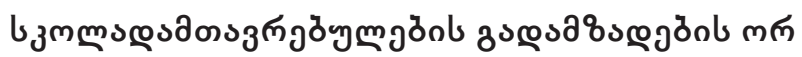

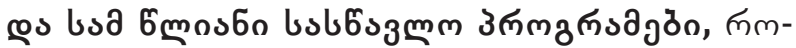

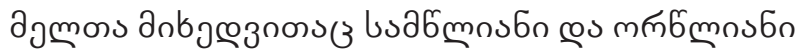

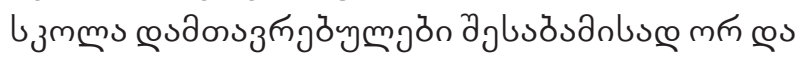

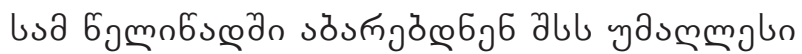

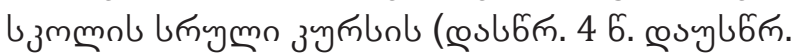

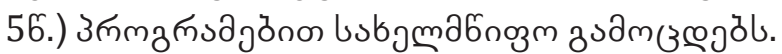

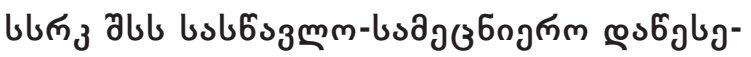

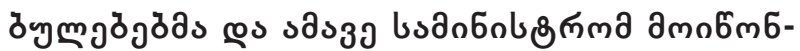

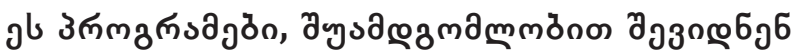

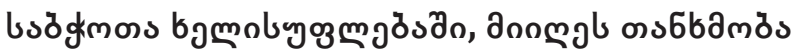

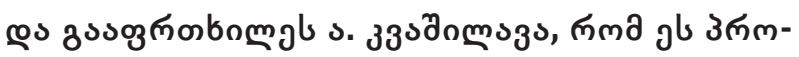

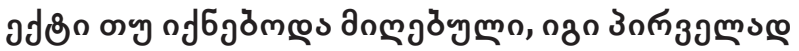

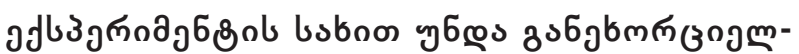

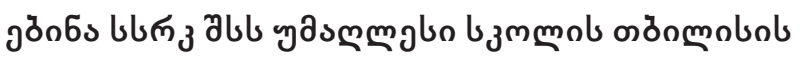




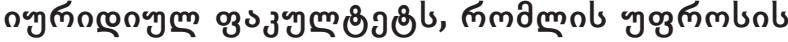

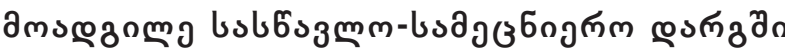

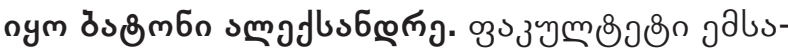

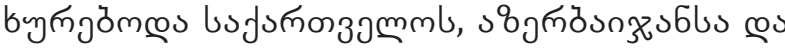
umabjonl.

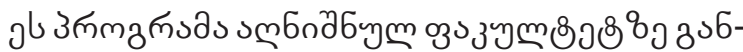

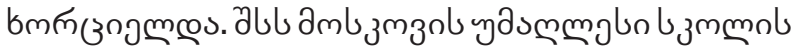

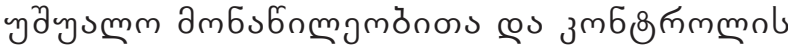

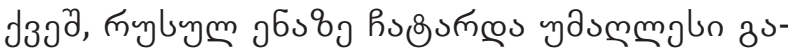

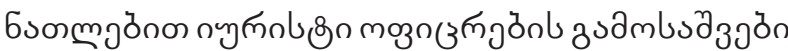

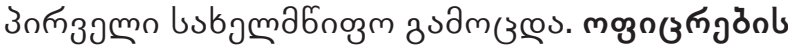

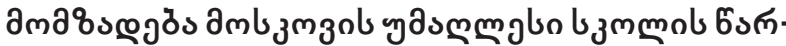

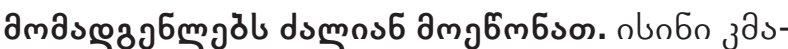
ymoुnmn œuпh उங

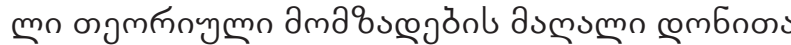

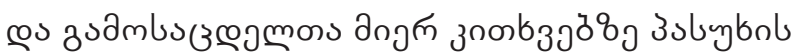

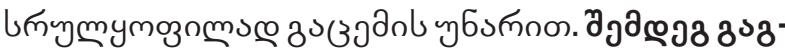
๓nd

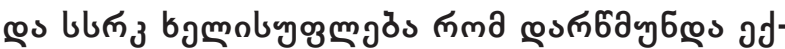

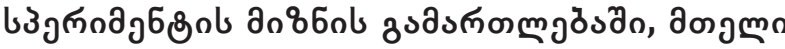

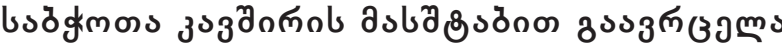

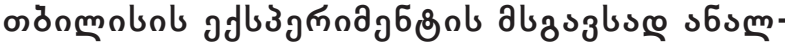

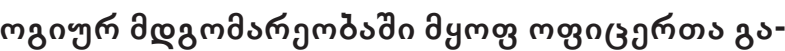

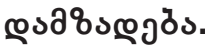

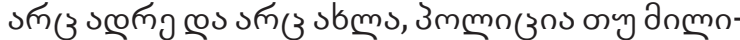

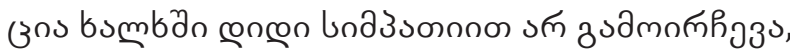

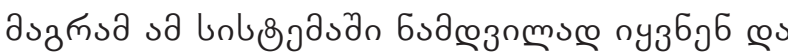

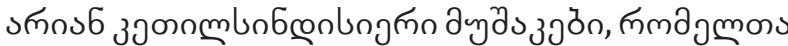

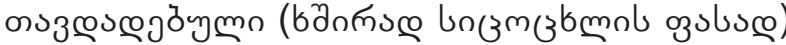

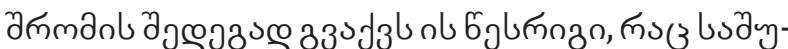

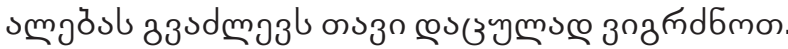

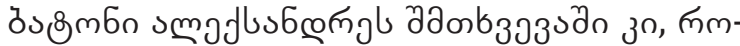

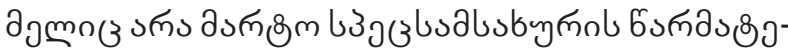

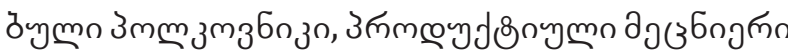

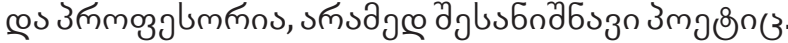

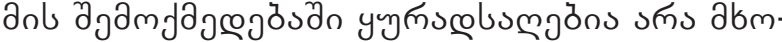

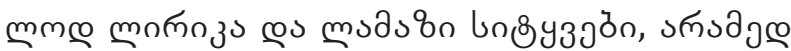

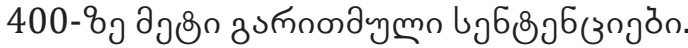

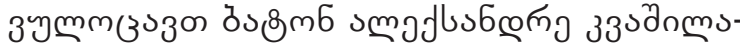

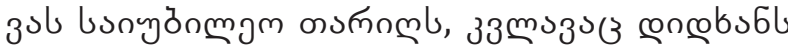

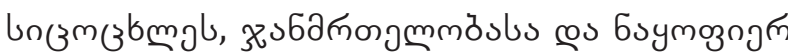

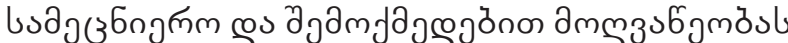

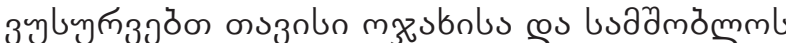

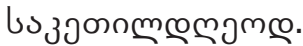

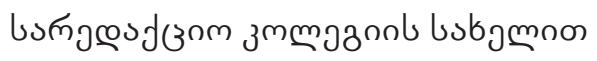

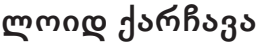
The New Economist-ol

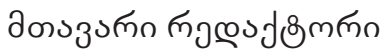




\section{strent งзПб๓วกเชก ECONOMIST

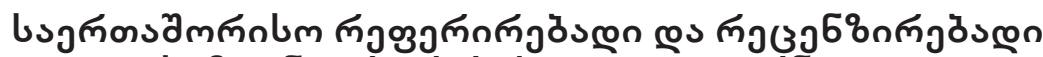

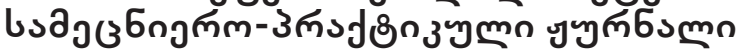

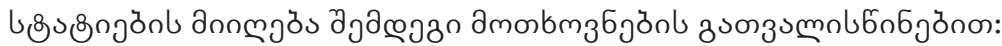

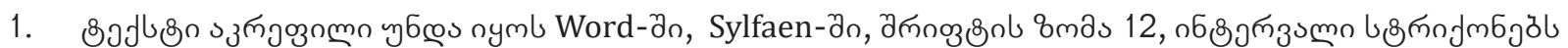

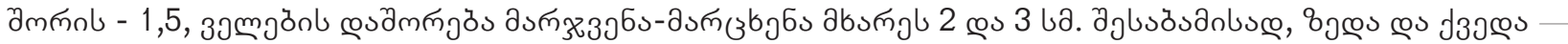

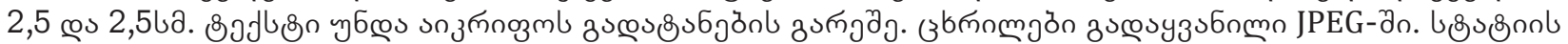

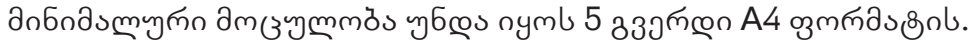

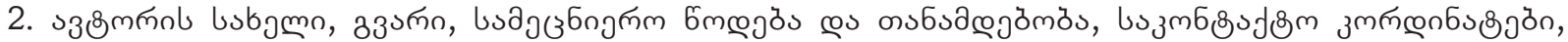

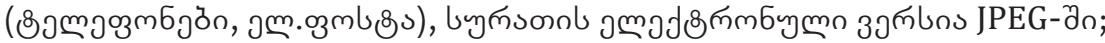

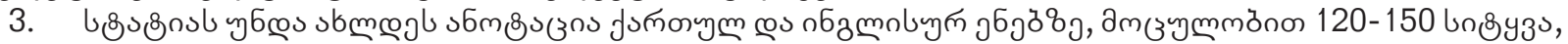

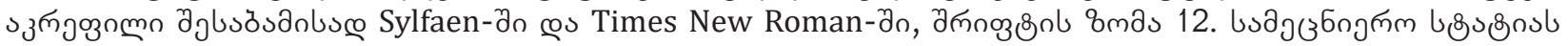

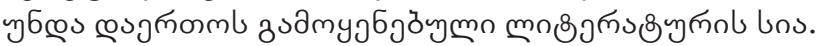

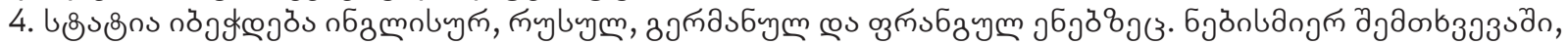

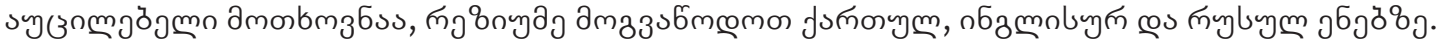

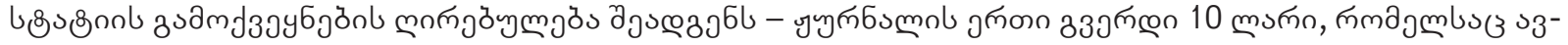

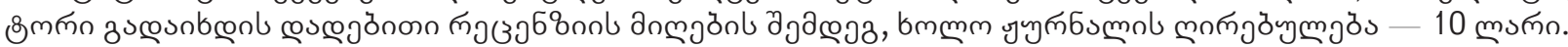

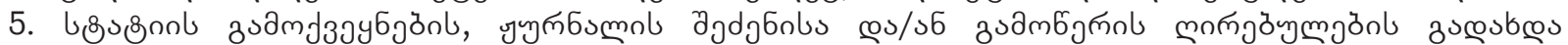

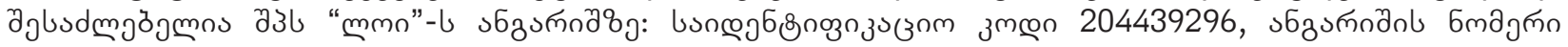

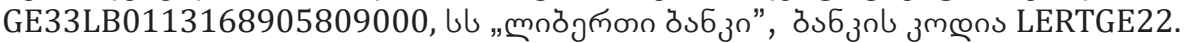

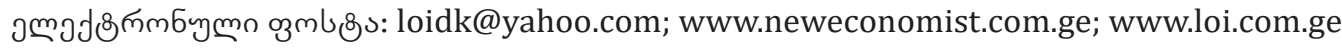 tel: 299-05-76; 555277 554; 558944800.}

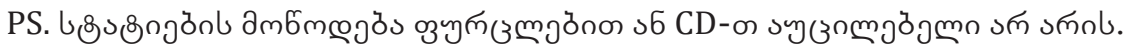

\section{International Precise and Review Scientific-Practical Magazine}

Continues taking articles according to this requests:

1. Text must be gathered in Word, size of Sylfaen font 12 , interval between lines -1.5 , borders in right and left side- 3 and $2 \mathrm{sm}$. Also, up and down borders- 2.5 and $2.5 \mathrm{sm}$. Text must be picked up without transferring. Tables should be in JPEC. The minimal size of article is 5pages of A4 format.

2. The first name of author, last name, scientific rank and post, coordinates (telephone number, e-mail), electronic version of photo in JPEG.

3. The article must have abstract in Georgian and English languages. The size should be 120-150 words, it must be picked up in Sylfaen and in Times New Roman, size of font-12. Scientific article must have list of used literature.

4. The article will published in English, Russian, German and France languages. So, it is also our request to prepare reziume in Georgian, Egnlish and Raussian languages too.

The price of article's publication is- one page of journal 10 GEL (Lari) or 5 USD. And auhor will pay this price after getting positive respond. Also, the price of journal is $10 \mathrm{GEL}$ (Lari) or $5 \mathrm{USD}$.

5. The price of publication article, buying journal and subscription fee could be payed in Ltd. LOI's account: identification code - 204439296, the account number- GE33LB0113168905809000," "liberty bank", central office, bank code LERTGE22.

Our coordinates:

e-mail: loidk@yahoo.com; www.neweconomist.com.ge; www.loi.com.ge Tel: 29905 76; 555277 554; 558944800.

PS. Giving articles with papres or CD-s, is not necessary. 

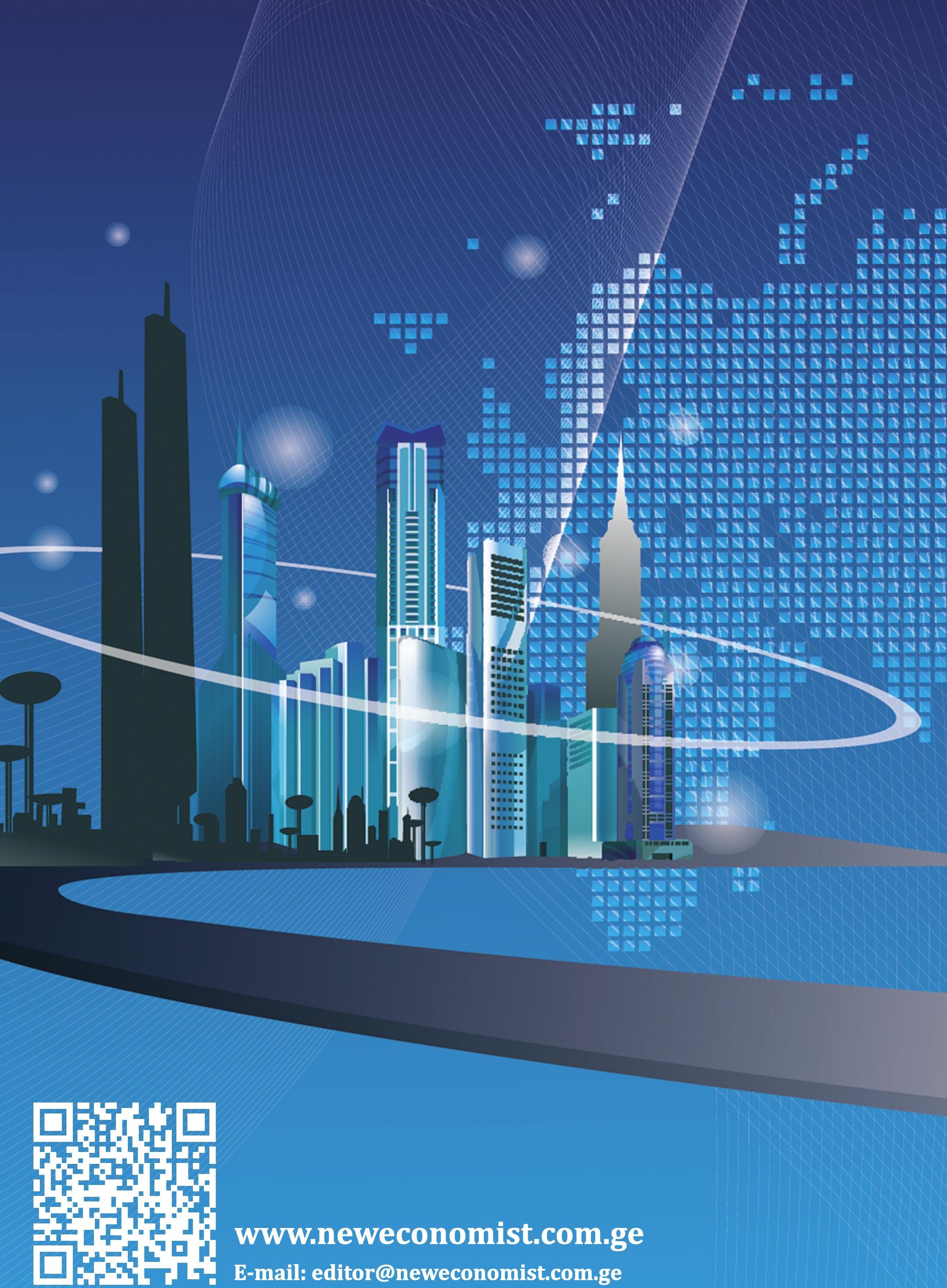

wwwneweconomist.com-ge

E-mail: editor@neweconomist.com.ge 